Wolfgang Melzer

\title{
Gewalt als soziales Problem in Schulen
}

Untersuchungsergebnisse und Präventionsstrategien

Verlag Barbara Budrich

"OPEN ACCESS" 
Wolfgang Melzer/Wilfried Schubarth

Gewalt als soziales Problem an Schulen 
Wolfgang Melzer

Wilfried Schubarth

Gewalt als soziales Problem

an Schulen

Untersuchungsergebnisse

und Präventionsstrategien

Ein eBook im Open Access

Verlag Barbara Budrich, Opladen 2006 


\title{
Dieses Werk ist urheberrechtlich geschützt! \\ Dieses Buch wurde kostenlos heruntergeladen und ist nicht für die kommerzielle Verwertung freigegeben.
}

\author{
Alle Fragen bezüglich der Verwertung des Werkes und aller \\ seiner Teile bitte direkt an den Verlag Barbara Budrich. \\ Stauffenbergstr. 7. D-51379 Leverkusen \\ Tel +49. (0) 2171.344.594 \\ Fax +49 (0) 2171.344.693 \\ info@budrich-verlag.de
}

Gedruckt auf säurefreiem und alterungsbeständigem Papier.

Die Deutsche Bibliothek - CIP-Einheitsaufnahme

Ein Titeldatensatz für die Publikation ist bei Der Deutschen Bibliothek erhältlich.

Alle Rechte vorbehalten.

(C) 2006 Verlag Barbara Budrich, Opladen

www.budrich-verlag.de

ISBN 3-938094-36-2

Das Werk einschließlich aller seiner Teile ist urheberrechtlich geschützt. Jede Verwertung außerhalb der engen Grenzen des Urheberrechtsgesetzes ist ohne Zustimmung des Verlages unzulässig und strafbar. Das gilt insbesondere für Vervielfältigungen, Übersetzungen, Mikroverfilmungen und die Einspeicherung und Verarbeitung in elektronischen Systemen.

Umschlaggestaltung: disegno visuelle kommunikation, Wuppertal - www.disenjo.de Satz: Johanna Boy, Brennberg 


\section{Inhalt}

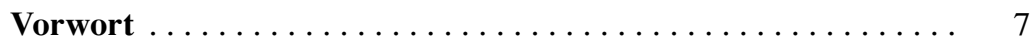

1. Gewalt als gesellschaftliches Phänomen

und soziales Problem in Schulen - Einführung

(Wolfgang Melzer) ....................... 11

1.1 Relevanz des Themas und Stand seiner wissenschaftlichen

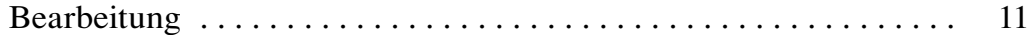

1.2 Definitionen und Konnotationen zum Gewaltbegriff . . . . . . . . . 21

1.3 Eigene Untersuchungen im Überblick . . . . . . . . . . . . . . . . . 27

1.4 Das Konzept einer sozialökologisch orientierten

Gewaltforschung in Theorie und Empirie............ 32

2. Schulische Gewaltformen und Konfliktsituationen im Ost-West-Vergleich

(Wilfried Schubarth, Christoph Ackermann) ............. 46

2.1 Schulische Gewaltformen in der Wahrnehmung von Schülern ... . 49

2.2 Analyse konkreter Gewaltsituationen . . . . . . . . . . . . . . . 59

2.3 Wahrnehmung, Selbstreport und Einstellungen von Schülern

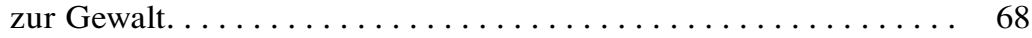

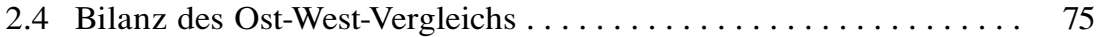

3. Jungengewalt - Mädchengewalt - ein Exkurs

(Dorit Stenke, Sandra Bergelt, Franziska Börner) . . . . . . . . . 78

3.1 Ergebnisse der quantitativen Befragung von Schülerinnen

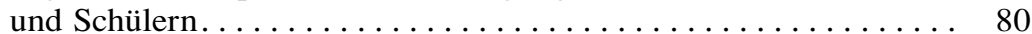

3.2 Ergebnisse der Interview-Studie mit Schülerinnen und Schülern . 96

3.3 Zusammenfassung ....................... 104

4. Rollengefüge von Tätern und Opfern

(Parviz Rostampour) ............................ 106

4.1 Interaktionale Gewaltformen von Schülern untereinander -

Selbstreport von Tätern und Opfern . . . . . . . . . . . . . . . . . . . 109

4.2 Rollenverteilung bei Gewalthandlungen unter Schülern:

Wer ist Täter, wer ist Opfer und wer beides zugleich? . . . . . . . 112

4.3 Täter-Opfer-Typologie:

Charakterisierung der einzelnen Schülergruppen. . . . . . . . . . 120

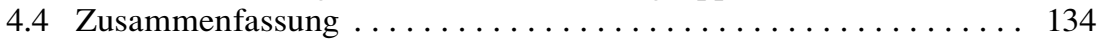


5. Prädiktoren schulischer Gewalt im außerschulischen Bereich (Wolfgang Melzer, Parviz Rostampour).................. 138

5.1 Bedingungen familialer Sozialisation . . . . . . . . . . . . . . . . 139

5.2 Medieneinflüsse . . . . . . . . . . . . . . . . . . . 152

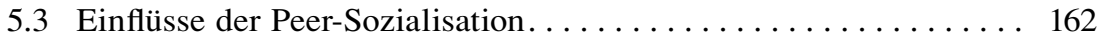

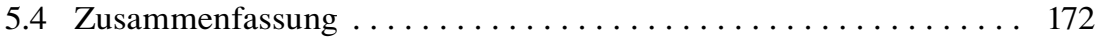

6. Schulkultur und ihre Auswirkung auf Gewalt

(Wolfgang Melzer, Manuela Mühl, Christoph Ackermann) . . . . . . 175

6.1 Schulkultur und Gewalt - ein Modell . . . . . . . . . . . . . . . . 180

6.2 Lehrerprofessionalität, Sozial-räumliche Schulumwelt und Schülerbefindlichkeiten als Aspekte der Schulkultur . . . . . . 183

6.3 Schul- und Erziehungskultur als Prädiktoren für das Gewalthandeln von Schülern. . . . . . . . . . . . . . . . . 195

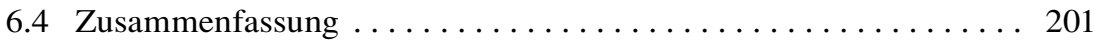

7. Schulische Gewalt im Lebenszusammenhang von Schülern - Gesamtmodell (Mehrebenenanalyse)

(Parviz Rostampour, Wolfgang Melzer, Wilfried Schubarth) . . . . . 205

8. Gewaltprävention im Kontext von

Schulentwicklungsprozessen - eine Pilotstudie

(Kerstin Darge). . . . . . . . . . . . . . . . . . . . . . . . . 221

8.1 Prävention und Intervention - Definitionen und Programme. . . . 223

8.2 Ergebnisse der Pilotstudie an einer Mittelschule . . . . . . . . . . . . 229

8.3 Perspektiven der Gewaltprävention und -intervention sowie der Präventionsforschung . . . . . . . . . . . . . . . . . 247

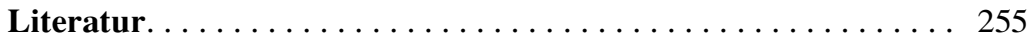

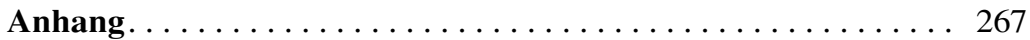




\section{Vorwort}

Bei der als eBook erscheinenden Studie „Gewalt als soziales Problem an Schulen“ handelt es sich um den unter gleichem Titel von der Forschungsgruppe Schulevaluation im Jahre 1998 im Verlag Leske und Budrich publizierten und mittlerweile vergriffenen Band.

Wegen der nach wie vor bestehenden Nachfrage wird die Studie in elektronischer Form einem neuen Leserkreis zugänglich gemacht. Wir haben uns zu diesem Schritt entschlossen, weil

- mit diesem neuen Medium eine noch größere Verbreitung der Untersuchungsergebnisse und unserer Vorschläge für die Gewaltprävention erreicht werden kann,

- die Untersuchung eine der wenigen ist, die die Gewaltthematik im OstWest-Vergleich behandelt,

- die Befunde zu Gewaltformen und -häufigkeiten, zur Täter-Opfer-Typologie und zu den Ursachen von Gewalt bis heute an Aktualität nicht verloren haben,

- der Band sich offenbar auch als Lehrbuch bewährt hat.

Die empirische Untersuchung, die dieser Veröffentlichung zugrunde liegt, ist als Survey angelegt, umfasst zwei repräsentative Schülerbefragungen in der Sekundarstufe I und wurde im Rahmen eines DFG-Projekts in Kooperation einer Bielefelder und einer Dresdner Projektgruppe durchgeführt.

Unser Dank geht an das Bielefelder Team mit Klaus-Jürgen Tillmann, Heinz Günter Holtappels, Ulrike Popp u.a., die uns den Hessischen Datensatz für den Ost-West-Vergleich zur Verfügung gestellt und von denen wir auch bei der Umsetzung des Gesamtkonzepts profitiert haben.

Wir bedanken uns weiterhin bei allen, die an der Dresdner Studie mitgearbeitet haben: Christoph Ackermann, Sandra Bergelt, Kerstin Darge, Franziska Herrmann (geb. Börner), Manuela Mühl, Dorit Stenke und Parviz Rostampour.

Dresden und Potsdam, im Februar 2006 


\section{Vorwort}

Gewalt als eine zielgerichtete physische oder psychische Schädigung anderer Personen oder Sachen bildet gemeinsam mit weiteren Verhaltensweisen, die gesellschaftlich als nicht opportun, sondern als ,abweichend“ gelten bzw. als delinquentes Verhalten unter Strafandrohung stehen, ein Syndrom, das nach Auffassung der öffentlichen Meinung heutzutage ein gravierendes gesellschaftliches Problem darstellt. Die allgemeine Kriminalitätsentwicklung, durch die ordnungspolitische Diskussionen auf den Plan gerufen werden, die Reaktionen der Bevölkerung darauf in Form von Kriminalitätsfurcht sowie Ausgrenzung und Abwertung von Personengruppen, die man für potentielle Täter hält, die Inszenierung von Gewalt in den Medien, durch welche diese Konsequenzen suggeriert werden, die Forderung nach neuer geistig-moralischer Orientierung und Werteerziehung etc. sind Facetten dieser Thematik im öffentlichen Raum.

Ein relevanter Teil dieser oben mit angesprochenen Befürchtungen entspricht leider der Wirklichkeit, auch die pädagogischen Institutionen sind - trotz ihrer höheren Sozialkontrolle - nicht vor gewaltförmigem bzw. gewaltaffinem Verhalten geschützt. Nach unseren Untersuchungen läßt sich der harte Kern einer an sich noch größeren Tätergruppe im schulischen Gewaltkontext der Sekundarstufe $\mathrm{I}$, in der entwicklungsbedingt die Gewaltspitzen liegen, mit etwa $3-4 \%$ und die Gruppe der Opfer mit etwa $7-10 \%$ beziffern. D. h., daß bei insgesamt ca. 5 Millionen Schülern dieser Schulstufe in Deutschland etwa 425.000 Schüler unter den Drangsalierungen anderer Schüler leiden und etwa 175.000 Schüler zum Kreis der notorischen Täter zu rechnen sind. Diese Daten, die in der Studie differenziert interpretiert und in ihren spezifischen Konsequenzen dargestellt werden, sollen hier zunächst den pädagogischen Handlungsbedarf und die Notwendigkeit von Präventions- und Interventionsstrategien begründen helfen.

Um derartige Programme gezielt $-\mathrm{d}$. h. in Übereinstimmung mit den wissenschaftlichen Analysen - entwickeln zu können, ist die schulbezogene Gewaltforschung, die seit Beginn der 90er Jahre erhebliche Fortschritte gemacht hat, aufgefordert, empirische Befunde zum Ausmaß und zur Entwicklung von Gewalt in der Schule, zu den Ursachen aggressiver und gewaltförmiger Verhaltensweisen im personalen, schulischen und außerschulischen Bereich, zur Struktur von Handlungssituationen und zum Verlauf von Gewalthandeln vorzulegen. Es müssen verläßliche Befunde dazu erarbeitet werden, welche Schulformen, Altersjahrgänge und Schülergruppen besonders betroffen sind und welche Verhaltensweisen jeweils die Schwerpunkte bilden, wie stark die Interventionsbereitschaft der Lehrer ist und ebenso die Tolerierung von Gewalt durch die Schüler.

Auf die meisten dieser Fragen werden in dieser Studie Antworten gegeben - auch in bezug auf die Konsequenzen für die pädagogische Prävention und Intervention, die nicht nur konzeptualisiert, sondern auch in einer Pilotstudie erprobt worden ist. 
Die Forschungsgruppe Schulevaluation, bei der die Autorenschaft für den vorliegenden Band liegt, wurde im Jahre 1993 an der TU Dresden mit dem Ziel gegründet, eine Forschungsinfrastruktur im Bereich der Sozialisations- und Bildungsforschung sowie der Schulentwicklung in den ostdeutschen Bundesländern zu schaffen. In größeren und kleineren Einzelprojekten wurden Publikationen, Forschungsberichte und Expertisen zu den Schwerpunkten: (1) Schulorganisation und Schulentwicklung, (2) Soziale Probleme und soziale Verantwortung in Schulen und (3) Interkulturelle Sozialisation vorgelegt. Im Bereich (2), in dem die im folgenden dargestellte Untersuchung angesiedelt ist, wurden in den vergangenen Jahren eine Reihe von quantitativen und qualitativen empirischen Erhebungen durchgeführt, deren Ergebnisse und Erfahrungen mit in die aktuelle Studie - eine Repräsentativbefragung von Schülerinnen und Schülern - einfließen. Diese ist als Ost-West-Vergleich konzipiert, konnte mit Unterstützung der Deutschen Forschungsgemeinschaft im Rahmen des SFB 227 „Prävention und Intervention im Kindes- und Jugendalter" durchgeführt werden und wurde in Kooperation mit einem Bielefelder Kooperationsprojekt unter Leitung von Klaus-Jürgen Tillmann und Heinz Günter Holtappels (jetzt Vechta) realisiert. Unser Dank gilt den beiden Projektleitern und allen Mitgliedern der Projektgruppe (Ulrike Popp, Ulrich Meier und Birgit Holler-Nowitzki), von deren Unterstützung wir vor allem durch erhebliche Vorleistungen bei der Instrumentenentwicklung profitiert haben. Bei der Kooperation kristallisierten sich nach und nach verschiedene Schwerpunktsetzungen heraus; dabei ist uns der Ost-West-Vergleich zugefallen. Bei den Auswertungsstrategien und der Bildung von Faktorvariablen sind die beiden Projekte z.T. unterschiedliche Wege gegangen, so daß sich die publizierten Ergebnisse der einen und der anderen Seite nicht in allen Fällen numerisch vergleichen lassen.

Das Manuskript wurde in Teamarbeit erstellt, gleichwohl zeichnen die genannten Autoren für ihre jeweiligen Kapitel individuell verantwortlich. In unserer internen Diskussion wurde viel Mühe für konstruktive Kritik und Anregungen zu den Texten der anderen aufgebracht - dafür bedanke ich mich bei meinen Mitarbeitern, außerdem für Unterstützung bei der Manuskripterstellung bei Brigitte Scharf sowie bei Christoph Ackermann und Dirk Adomat, die das Typoskript vorbereitet haben.

Zwischenergebnisse unserer Untersuchung sind in Vorlesungen und Seminare eingeflossen und wurden von den Studierenden mit Interesse aufgenommen. Die dabei aufgeworfenen Fragen und Probleme haben die Forschungsgruppe angeregt und uns bei unseren Interpretationen weitergeholfen. In Lehrforschungsprojekten wurden unter Mitwirkung von Studentinnen und Studenten qualitative Untersuchungsteile realisiert, die sich in ein Gesamtbild unserer Untersuchungen zur Gewaltemergenz einfügen.

Schließlich bedanke ich mich bei allen Ungenannten recht herzlich, die direkt oder indirekt zur Fertigstellung und Publikation dieses Bandes beigetragen haben, nicht zuletzt bei den Schulen sowie den Schülerinnen und Schülern, die sich an unseren Befragungen beteiligt, und den Eltern, die dazu ihre Zustimmung gegeben haben. 


\section{Die Autoren:}

Wolfgang Melzer, Professor für Schulpädagogik: Schulforschung und Leiter der Forschungsgruppe Schulevaluation an der Technischen Universität Dresden, Arbeitsschwerpunkte: Schul- und Sozialisationsforschung, Gewalt in der Schule, Schule und Gesundheit

Wilfried Schubarth, Professor für Erziehungs- und Sozialisationstheorie am Institut für Erziehungswissenschaft der Universität Potsdam, Arbeitsschwerpunkte: Jugend- und Schulforschung, Evaluations- und Präventionsforschung

Seit dem Erscheinen des Bandes sind weitere Veröffentlichungen der Autoren zum Themenbereich „Gewalt und Gewaltprävention in der Schule“ erschienen:

- Melzer, W./Schubarth, W./Ehninger, F.: Gewaltprävention und Schulentwicklung. Analysen und Handlungskonzepte. Bad Heilbrunn 2004

- Schubarth, W.: Gewaltprävention in Schule und Jugendhilfe. Theoretische Grundlagen, empirische Ergebnisse, Praxismodelle. Neuwied/Kriftel 2000

- Melzer, W./Schwind, H.-D. (Hrsg.): Gewaltprävention in der Schule. Grundlagen - Praxismodelle - Perspektiven. Baden-Baden 2004

- Simsa, Ch./Schubarth, W. (Hrsg.): Konfliktmanagement an Schulen Möglichkeiten und Grenzen der Schulmediation. Frankfurt a. M.: Deutsches Institut für Internationale Pädagogische Forschung 2001 


\section{Gewalt als gesellschaftliches Phänomen und soziales Problem in Schulen - Einführung (Wolfgang Melzer)}

\subsection{Relevanz des Themas und Stand seiner wissenschaftlichen Bearbeitung}

Staat und Gesellschaft haben als komplexe kulturelle Gebilde in ihrer Geschichte verschiedene Strukturformen durchlaufen, in denen Gewalt in einer je spezifischen Weise ausgetragen wurde bzw. legitimiert war. Es scheint so, daß diese Entwicklung dem Prinzip eines Zivilisationsmodells der inneren Befriedung folgt (vgl. Elias 1976), das in modernen Gesellschaften durch eine Monopolisierung der Gewalt durch den Staat abgesichert wird. Dieses staatliche Gewaltmonopol bedarf in unserer Gesellschaft der demokratischen Legitimation und wird letztlich mit dem Argument der Sicherung des Gemeinwohls und der individuellen Rechte, die der Staat garantiere, begründet. Die Verwirklichung dieses Prinzips obliegt in industriell-bürokratischen Gesellschaften der Administration (vgl. Weber 1924, S. 498ff). Die Bürokratie als Vermittlungsinstanz zwischen politischen Entscheidungsträgern und Bürgern ist für Max Weber aber nicht nur ein legitimes Herrschaftsmittel, durch das die Beziehungen der Menschen untereinander versachlicht und rational gestaltet werden, sondern auch ein Instrument, mit dem der widerständige Wille des einzelnen u.U. gebrochen und eine ,dauerhafte Herrschaft des Apparats über den Menschen“ (Schluchter 1985, S. 9) begründet werden kann. Webers zwiespältige Einschätzung bürokratischer Herrschaft, seine Furcht vor dem Zugriff einer universellen Bürokratie, die er vor mehr als 80 Jahren formulierte, kann angesichts des gegenwärtigen Zustandes staatlicher Administration - ihrer Selbstreferenz und Abgehobenheit - als durchaus berechtigt und vorausschauend gewertet werden. Allerdings haben große Teile der Bevölkerung dies ebenfalls registriert und zeigen entsprechende Einstellungen und Verhaltensweisen, so daß beide Aspekte zwei unterschiedliche Seiten der politischen Kultur in unserem Lande darstellen.

„Politische Kultur“ bezeichnet die Gesamtheit der gesellschaftsbezogenen Einstellungen und Orientierungen sowie die politischen Wertvorstellungen, Glaubenshaltungen und Weltbilder innerhalb eines politischen Systems und in bezug auf dieses. Analysiert man unter diesem Gesichtspunkt die neuere Entwicklung der Bundesrepublik Deutschland, so läßt sich eine „Ambivalenzkultur“ (Baacke 1989) feststellen, die mit dem ehemals existierenden vorindustriellen Modell einer einheitlichen „Volkskultur“, der ein traditionelles „Gemeinschafts"-Muster und auch ein relativ geschlossenes Bild von der 
Obrigkeit zugrunde lag, nichts mehr gemein hat. Unterschiedliche Wertorientierungen und Lebenswelt-Deutungen, sehr verschiedenartige ästhetische und politische Auffassungen konkurrieren gleichberechtigt miteinander, ergänzen sich möglicherweise oder stehen vielleicht sogar im Widerspruch zueinander. Verbindliche gemeinsame Werte sind nur noch ein „Abstraktum“, das zwar verfassungsrechtlichen Bestand, aber eine stark verringerte Relevanz für das Alltagshandeln besitzt und nahezu nach Belieben für den eigenen Bedarf ausgelegt und umdefiniert werden kann. Verhaltensnormen konstituieren sich in „Sub-Kulturen“, aber auch hier stehen den Individuen Optionen frei, sich einer anderen Gesinnungsgruppe anzuschließen oder einen gänzlich individuellen Lebensstil zu praktizieren.

Diese Pluralität der Kulturen und Lebensweisen hat einerseits ein Anwachsen der Toleranz für Unterschiedlichkeit und Vielfalt zur Folge, das mit einer Zunahme an Individualität korrespondiert, andererseits bedeuten eben diese Individualisierungsprozesse immer auch eine „Totalisierung des Ich“ (Rammstedt 1981) und damit eine Entgrenzung von Freiheit sowie eine Zunahme an Konkurrenz und Entfremdung, die auf der Grundlage der Auflösung traditioneller sozialer Milieus gedeihen (Arbeitsgruppe Bielefelder Jugendforschung 1990, Melzer 1987, S. 41ff). In empirischen Studien kommt diese ambivalente Entwicklung ebenso in einer Zunahme an individualisierten und postmaterialistischen Einstellungen (vgl. u.a. Heitmeyer/ Olk 1990, Melzer/ Lukowski/Schmidt 1991, Jugendwerk 1997) wie in einem Anwachsen an Konkurrenzverhalten und Ellenbogenmentalität (vgl. u.a. Richter 1990, Schmidtchen 1997) zum Ausdruck. In seiner politischen Dimension führen diese Veränderungsprozesse zu einer Dualität und Distanz zwischen Individuum und Staat, zu einem skeptischen, mißtrauenden und z.T. verweigernden Einstellungs- und Verhaltenspotential gegenüber der Politik, den staatlichen Institutionen und insbesondere gegenüber den Personen, die diese Bereiche repräsentieren.

Dieses Phänomen einer staatlichen Legitimationskrise (vgl. Habermas 1973) ist sowohl theoretisch als auch empirisch hinreichend belegt und kommt besonders deutlich in den Einstellungen der heranwachsenden Generation zum Ausdruck. In einer neueren Jugendstudie (vgl. Schmidtchen 1997), die als Ost-West-Vergleich angelegt ist, wurde ermittelt, daß sich das Parteieninteresse im Zeitraum von 1980 bis 1994 mehr als halbiert hat, von 32\% auf $14 \%$ zurückgegangen ist und in Ostdeutschland bei nur 10\% liegt (S. 266). Die politischen Einflußmöglichkeiten werden von den Jugendlichen als unzureichend bewertet: mehr als die Hälfte der Befragten äußert dies im Jahre 1994 gegenüber weniger als einem Viertel in den 80er Jahren (S. 265). Vertrauen in die Problemlösungsfähigkeit der Demokratie - angesichts drängender Zukunftsprobleme - hat nur eine Minderheit unter den Jugendlichen; der Prozentsatz derjenigen, die angeben, kein Vertrauen zu besitzen, liegt in Westdeutschland bei $30 \%$ und in Ostdeutschland bei 45\% (S. 267), und nur etwa 4\% (West: $6 \%$, Ost: $2 \%$ ) sind der Auffassung, ,daß die führenden Leute in der Politik, 
in Regierung und Opposition, klare Ziele ansprechen und den Bürgern eine sichere Orientierung geben“" (S. 267).

Insgesamt besteht - so der Tenor dieser Studie - ein Unbehagen über die Entwicklung der politischen Kultur, das sich in einem Absinken des ,gesellschaftspolitischen Wohlbefindens" äußert: Während von 1986 bis 1995 das persönliche Wohlbefinden in Westdeutschland auf einem hohen Niveau nahezu konstant geblieben ist (1986: 74\%; 1995: 73\%), befindet sich das gesellschaftspolitische Wohlbefinden auf einem deutlich niedrigeren Niveau (S. 263) und ist im Vergleichszeitraum sogar noch etwas gesunken (1986: 57\%; 1995: 50\%). In Ostdeutschland ist sowohl das persönliche (1995: 50\%) wie das gesellschaftliche Wohlbefinden (1995: 40\%) der befragten Jugendlichen wesentlich niedriger als in Westdeutschland - allerdings wiederum mit den dargestellten Abstufungen der beiden Indikatoren. Ein Grund für diese Ost-West-Unterschiede ist das im Osten sehr viel höhere Frustrationsniveau mit entsprechenden Erfahrungen in allen Lebensbereichen (S. 268, 89), die sich auch auf den politischen und sozialen Bereich beziehen. ${ }^{1}$

Frustrationen stellen jedoch als psychische Reaktionen, die aus persönlichen Problemlagen resultieren (vgl. Dollard u.a. 1973), und auch, wenn sie auf Erfahrungen gesellschaftlicher Randständigkeit und Desintegration basieren (vgl. Heitmeyer u.a. 1995), nur einen Teilaspekt der Erklärung von Gewalt dar. Denn erstens nehmen entsprechende Erklärungsansätze - so unterschiedlich sie auch sein mögen - nicht auf Gewaltsituationen und deren äußere - gesellschaftliche und institutionelle - Regulative Bezug, und zweitens werden die Möglichkeiten unterschiedlicher Verarbeitungsformen durch das Individuum zu wenig in Betracht gezogen: denn Reaktionen in ähnlichen Situationen können sehr verschiedenartig ausfallen, Aggressionen einer Person können sowohl nach außen als auch gegen sich selbst gerichtet sein (vgl. Mansel/Hurrelmann 1994) oder „sublimiert“ werden.

Nach außen gerichtete Aggressionen kommen eher in solchen makrosozialen Verhältnissen und gesellschaftlichen Bereichen zum Tragen, in denen das Verhalten weniger normiert, gesteuert, kontrolliert und sanktioniert wird. Kommunikatives Handeln läßt sich aber von außen nicht erzwingen. Zwar kann der äußere Schein der Gewaltfreiheit durch Anwendung rigider Kontrollmechanismen erzeugt werden, bei nächster sich bietender Gelegenheit würden die Aggressionen aber ihren Lauf nehmen, wenn das Handlungsrepertoire nicht intrapersonal reguliert und gegengesteuert würde.

Schon Kant hat in seiner Abhandlung „Über Pädagogik“ (1803) darauf hingewiesen, daß die moralische Kultur sich gründen müsse auf Maximen,

1 So wurde in der Untersuchung nicht nur nach Frustrationen im persönlichen und familiären, sondern auch im politischen und sozialen Bereich gefragt, z. B.: andere haben mehr Geld; niemand kümmert sich um junge Leute; schlechte Berufsaussichten; zu viel Egoismus in der Wirtschaft. 
nicht auf Disziplin (Pädagogik und Ethik 1996, S. 36); diese Auffassung wird im Bereich der Psychologie, der Erziehungs- und Sozialwissenschaften durch viele gut begründete Ansätze gestützt, in denen die Notwendigkeit einer sozialisatorischen Herausbildung von „Ich-Identität“ artikuliert wird (vgl. zusammenfassend Döbert/Nunner-Winkler 1975). Die sich ,allmählich anreichernde Ich-Identität (sei) das einzige Bollwerk gegen die Anarchie der Triebe wie gegen die Autokratie des Gewissens“, bemerkt dazu Erik K. Erikson (1973, S. 112).

Dieser Grundgedanke eines Zusammenspiels von Außen- und Innensteuerung wird uns im Rahmen eines sozialisationstheoretischen Ansatzes in der gesamten Studie begleiten. Danach wird Gewalt dann zu einem gravierenden Problem für eine Gesellschaft, wenn die äußeren und inneren Regulationsmechanismen in großem Maßstab versagen. Daß in beiden Bereichen - im makrosozialen wie im personalen - gewaltbegünstigende Faktoren während der letzten Dekade prononcierter hervorgetreten sind, ist eine Gesamteinschätzung unserer Forschungsgruppe, die wir auf der Grundlage unserer verschiedenen Untersuchungen zur Gewaltemergenz vornehmen (vgl. unser Theoriemodell in Kap. 1.4, Abb. 1-3). Ein Indikator dafür sind die deutlichen Steigerungsraten bei der Kriminalitätsentwicklung, besonders bei den Heranwachsenden. So schwierig die Datenlage zur Gewaltdynamik bei Kindern und Jugendlichen auch sein mag, ein gewisser Anstieg des Gewaltvorkommens - auch in der Schule - wird von Seiten der meisten Forscher für wahrscheinlich gehalten, wenngleich er aus methodischen Gründen nicht exakt beziffert werden kann (vgl. u.a. Olweus 1995, Tillmann 1997b, Dann 1997, Mansel/ Hurrelmann 1997).

Auf jeden Fall haben diese Entwicklungen eine breite gesellschaftliche Diskussion entfacht, die die Rolle des Staates als Ordnungsmacht sowie der Familie und Schule als Sozialisationsinstanzen mit einschließt. An diesem Diskurs haben sich seit Beginn der 90er Jahre auch vermehrt Wissenschaftler unterschiedlicher Disziplinen mit kulturkritischen Essays, empirischen Sekundäranalysen, eigenen Erhebungen und mit Ursachenforschung beteiligt (vgl. zusammenfassend Reuband 1996, Holtappels/Heitmeyer/Melzer/ Tillmann 1997). Die wissenschaftliche Auseinandersetzung mit diesen Phänomenen setzte allerdings sehr spät ein und erfolgte erst unter dem Druck der öffentlichen Meinung und der Medien, die - damals wie heute -in Teilen den vollkommen falschen Eindruck erwecken, daß Gewalt den gesamten Alltag, alle möglichen lebensweltlichen und pädagogischen Zusammenhänge und auch die Erziehungsinstitutionen beherrsche. Ob das Aggressionspotential insgesamt gestiegen ist, läßt sich aber von wissenschaftlicher Seite nicht mit Gewißheit sagen, weil keine exakten Zeitreihenanalysen vorliegen und auch, weil sich dieses Konstrukt der empirischen Erfassung schwer erschließt; wohl aber, daß sich die äußeren Regulationsmechanismen (geringere gesellschaftliche Normierung von Handlungsprozessen), die allgemeinen Einstellungen zur Gewalt und der Umgang mit ihr (,Wegschaugesellschaft“) sowie die persönlichkeits- 
internen Steuerungsprozesse verändert haben - in einer Gesellschaft, die sich nicht mehr auf obrigkeitsstaatliche Leitideen festlegen läßt, in der individuelle Selbstverwirklichung und entsprechendes Durchsetzungsvermögen des einzelnen als gültige, ja sogar erstrebenswerte Handlungsmaximen anerkannt und Werte wie Altruismus und Gemeinsinn in Minderheitenpositionen anzutreffen sind.

Die Schule, die als staatlich organisiertes System der Reproduktion den Einflüssen dieses allgemeinen gesellschaftlichen Wandels unterliegt, hat in Westdeutschland Phasen der Restauration (nach dem 2. Weltkrieg) und der Innovation (Bildungsreform der 70er Jahre) durchlaufen. Debatten über schulische Reformen waren von parteipolitischen Konflikten begleitet und haben die Bevölkerung in zwei große Lager gespalten: Verfechter eines vorwiegend auf fachliche Qualifizierung und Auslese gerichteten und in seiner Organisationsstruktur vertikal gegliederten Schulsystems stehen einer anderen Gruppe gegenüber, für die intellektuelle Förderung und Persönlichkeitsentwicklung, Ganzheitlichkeit des Unterrichts und eine Pädagogik der Vielfalt im Rahmen einer einheitlichen Organisationsstruktur die wichtigsten Ziele sind. Diese zweite Position hat in ganz Deutschland durch die „Reformpädagogische Bewegung" der Jahrhundertwende eine gemeinsame Tradition und gelangte in Westdeutschland durch die Bildungsreformphase der 70er Jahre zu einer erneuten Blüte. Als Folge einer umfassenden gesellschaftlichen, ökonomischen, und politischen Krise Ende der 60er Jahre konnten sich zeitweise Veränderungen der politischen Kultur durchsetzen, die z.T. bis heute nachwirken und seinerzeit vor allem in einer Stärkung der Rechte und Partizipationsmöglichkeiten der Bürger zum Ausdruck kamen (vgl. zur Ambivalenz dieser Prozesse Greiffenhagen 1991). In bezug auf Schule hat dies zu einer Neufassung der Schulgesetze, zur Verstärkung von Eltern- und Schülerrechten sowie zu einer Demokratisierung der Schulverfassung insgesamt geführt. Zu diesem Bild gehört auch, daß sich die Erziehungseinstellungen von Lehrern und Eltern liberalisiert haben, der Unterricht schülerzentrierter geworden ist und sich das Schulklima verbessert hat. Dieser strukturelle und interaktionale Wandel von Schule korrespondiert mit jugendspezifischen Entwicklungen, welche die Peers zu einer komplementären Sozialisationsinstanz neben Elternhaus und Schule avancieren läßt. Die neu entstandene ,präfigurative Kraft“ der heranwachsenden Generation (vgl. Mead 1973) hat die Tendenz zur Ausbreitung und wirkt durch eine Orientierung der Schülerrolle an allgemeinen Jugendstandards auch auf die Schule ein, die sich ihrerseits dem veränderten Anspruchsniveau an Lehren, Lernen und Leben in der Schule stellen muß. Die Ambivalenz dieser Prozesse kommt bei der Gewaltproblematik in den Schulen der alten Bundesländer darin zum Ausdruck, daß einerseits die äußeren Regulationsmechanismen zurückgenommen worden sind und damit Handlungsspielräume und höhere Freiheitsgrade für Schüler entstanden sind, die aber auch die Gefahr von Überforderungen und Grenzüberschreitungen beinhalten; auf der anderen Seite sind im Bereich der Schulkultur Potentiale herangewachsen, die geeignet 
zu sein scheinen, die sich gegenwärtig zeigenden sozialen Probleme in der schulischen Lebenswelt zu bewältigen.

Demgegenüber ist die Entwicklung in Ostdeutschland eine andere. In der DDR wurde bis zuletzt am obrigkeitsstaatlichen System der Schule festgehalten. Der Erziehungsstil von Schule und Elternhaus war stärker von Disziplin, Kontrolle, Konformität und Autorität geprägt (vgl. Schubarth/Darge/ Mühl/Ackermann 1997, S. 122f). Die vorherrschende Unterrichtsform war der Frontalunterricht; politische Wertvorstellungen wurden ritualisierend eingeübt. Insgesamt war die Sozialkontrolle stärker und die Möglichkeit der Selbstbestimmung der Schüler geringer. Die Jugendphase in den sozialistischen Ländern kann - nach den Befunden der kulturvergleichenden Jugendforschung - bis in die 80er Jahre hinein als ,restringiertes Moratorium“ charakterisiert werden (vgl. Zinnecker 1991, Zinnecker/Molnar 1988, Melzer/ Lukowski/ Schmidt 1991, Schröder/Melzer/Salustowicz 1995). Die festgestellte Parallelität in den Erscheinungsformen jugendtypischen Verhaltens in dieser Epoche zur westeuropäischen Jugend vor Beginn der 60er Jahre läßt die Annahme einer ,verzögerten Entwicklung“ - kulturspezifische Überformungen in einzelnen Ländern in Rechnung gestellt - berechtigt erscheinen. Primäre Bezugs- und Kontrollinstitutionen waren die Familie, das Schulsystem und die staatlichen Jugendorganisationen. Insgesamt orientierten sich die Jugendlichen stärker an den Maßstäben der Erwachsenengesellschaft, deren Rollen sie auch für sich selbst für erstrebenswert hielten. Der Jugendphase dieser Epoche ist damit eine tendenziell postfigurative, nur zum Teil eine konfigurative und im Gegensatz zur westeuropäischen Jugend kaum eine präfigurative Funktion zuzuschreiben (vgl. Zinnecker 1991, S. 12ff).

Die meisten der seit der Wende in Deutschland durchgeführten Ost-WestVergleichsstudien kommen zu dem grundsätzlichen Ergebnis einer konvergenten Entwicklung in den Einstellungen und Orientierungen der Jugendlichen im Sinne einer „,nachholenden Modernisierung“ (vgl. Kap. 2). Wenn dies zutrifft, wäre die Annahme naheliegend, daß sich auch das Gewaltniveau in den neuen tendenziell an das der alten Bundesländer angleicht und sich somit die Situation in Ost und West nicht mehr wesentlich voneinander unterscheiden dürfte. Aber selbst wenn diese Hypothese bestätigt würde, könnten nicht trotzdem in bezug auf die familiale, schulische oder Peer-Sozialisation unterschiedliche Bedingungen vorliegen, z. B. eine höhere Aggressionsbereitschaft bei ostdeutschen Kindern und Jugendlichen, die durch eine strengere Außenregulation als Residuum der ehemaligen DDR-Schule an diesem Ort nicht zum Ausdruck kommt oder durch eine Verinnerlichung kollektiver Wertorientierungen als Folge dieser Sozialisation ausgeglichen wird, so daß numerische Ost-West-Unterschiede nur aus diesen Gründen nicht in Erscheinung treten?

Dieser Schwerpunkt der Transformation des Bildungssystems in Ostdeutschland und ihrer Auswirkungen auf das Sozialverhalten der Schülerinnen und Schüler wird von unserer Forschungsgruppe seit 1993 in einer Reihe von vergleichenden Studien in Ost- und Westdeutschland bearbeitet (vgl. 1.3). 
Mit diesen Untersuchungen wurde durch die Art ihrer Konzeptualisierung weiterhin die Absicht verfolgt, an die bestehenden Forschungen anzuknüpfen, theoretische und empirische Entwicklungslinien aufzunehmen und sie pointiert fortzusetzen. Denn es mangelte bis einschließlich der 70er Jahre an Forschungen - geschweige denn einer Forschungstradition - in bezug auf die Untersuchung von Gewaltphänomenen in der Schule. Wohl existierten Studien zu anderen sozialen Problemen, die z.T. in die Gewaltproblematik hineinwirken, so z. B. zu Disziplinproblemen und Unterrichtsstörungen, zur Schulangst, zum Schulverdruß und zu allgemeinen Problembelastungen von Schülerinnen und Schülern (vgl. z. B. Züghart 1963, Höhn 1967, Blackham 1971, Melzer 1987, S. 13). Mit Ausnahme vereinzelter Studien zum delinquenten und devianten Verhalten von Kindern und Jugendlichen (z. B. Brusten/Hurrelmann 1973) sowie einer Debatte im Anschluß an Johann Galtungs „Theorie struktureller Gewalt“ (Galtung 1975), in der das Verhältnis von Schulgewalt und Schülergewalt diskutiert wurde (vgl. Grauer/Zinnecker 1978), ist die Gewaltthematik von den schulbezogenen Wissenschaften aber nicht systematisch bearbeitet worden.

Die psychologische Forschung hat sich fortlaufend mit Aggressionsphänomenen - häufig in Experimenten und großen theoretischen Entwürfen - beschäftigt, selten jedoch ihre Ergebnisse auf gesellschaftliche Verhältnisse zurückbezogen und die Bedeutung der Befunde für die Sozialisationsprozesse reflektiert. ${ }^{2}$ Ein explizierter Schulbezug fehlt dieser psychologischen Forschungslinie, die bis heute fortbesteht, völlig. Das gilt mit Ausnahme der Präventions- und Interventionsforschung in dieser Disziplin für alle Facetten psychologischer Aggressionsansätze (z. B. Triebtheorien, Lerntheorien, psychoanalytische Theorien, Frustrationstheorien). Auch im Feld der sozialwissenschaftlich orientierten Theorien ist eine Vielfalt und Bandbreite feststellbar (z.B. Anomietheorie, Etikettierungstheorie, Subkulturtheorie, Individualisierungstheorie), die von ihren „Erfindern“ nicht primär zum Zweck der Erklärung schulischer Gewalt formuliert und ausgearbeitet worden sind. Jedoch haben beide Theoriestränge ihre explizite oder implizite Bedeutung für die Theorie und Empirie, die sich mit der Erfassung schulischer Gewaltphänomene befaßt. Die folgende Synopse gibt dazu einen Überblick, zeigt die klassischen Theoreme im Bereich der Aggressions- und Gewaltforschung mit jeweils kurzen Charakterisierungen ihrer Grundideen und Ziele - ohne Anspruch auf Vollständigkeit. ${ }^{3}$

2 Eine Ausnahme ist die Studie von Dollard u.a. (1994) über „Frustration und Aggression“, in der auch Fragen des Jugendalters, der Kriminalitätsentwicklung und gesellschaftlicher Entwicklung überhaupt („Demokratie, Faschismus, Kommunismus“) im Zusammenhang der eigenen Forschungsbefunde thematisiert sind. Die erste Auflage dieses Buches erschien 1939.

3 Ein systematischer Vergleich einschließlich einer Überprüfung der Leistungsfähigkeit dieser Ansätze würde den Rahmen dieser Arbeit sprengen. Ein Werk mit diesem Anspruch und Zuschnitt wird in Kürze von einem Mitglied der Forschungsgruppe Schulevaluation publiziert. Aus diesem Kontext stammt auch die Synopse (Abb. 1-1) (vgl. Schubarth 1998b). 
Eine nähere Beschäftigung mit Gewalt- und Aggressionstheorien macht deutlich, daß es keine einheitliche Theorie zur Erklärung von aggressiven und gewaltförmigen Verhaltensweisen gibt, keine - wie Luhmann (1984) es formulierte - „Supertheorie in ungewöhnlicher Abstraktionslage“ in Sicht ist. Jede der hier skizzierten Theorien ist ein in sich logisches und widerspruchsfreies Erklärungsmodell mit Stärken und Schwächen, mit einem spezifischen, d.h. immer begrenzten Aussagewert. Wie Schubarth (1998b) aber herausarbeitet, gibt es auch etliche Gemeinsamkeiten: „So spielen z. B. Lernprozesse bei verschiedenen Theorien eine Rolle: nicht nur in den Lerntheorien, sondern z.B. auch bei sozialisations-, individualisierungstheoretischen oder psychoanalytischen Ansätzen. Gleiches gilt für die anomischen Strukturen, die in verschiedenen Konzepten von Bedeutung sind. Die Gemeinsamkeit zwischen den Theorien drückt sich auch darin aus, daß gleiche oder ähnliche Sachverhalte mit unterschiedlichen Begriffen oder in unterschiedlichen Kontexten auftreten. (...) Insgesamt geht die Entwicklung von eher einfachen Ansätzen (z.B. nur ein Ursachenfaktor, wie der Trieb) hin zu immer komplexeren Modellen, in denen verschiedene, auch aus unterschiedlichen Theoriezusammenhängen stammende Elemente integriert werden" (Schubarth 1998b, S. 86).

Was bezüglich der Entwicklung der Theoriebildung in den Sozialwissenschaften im allgemeinen feststellbar ist, trifft auch für die Aggressions- und Gewaltforschung im besonderen zu. Die Phase der Schulenbildung und des theoretischen und methodischen Purismus ist beendet; es herrscht eine Suche nach Mehrebenenkonzepten vor, in die bewährte Theorieelemente „eingebaut“ werden können. Man versucht die Ebene genau zu bemessen, auf der eine Theorie kürzerer oder mittlerer Reichweite greift, bemüht sich, sie in einen Zusammenhang mit Analysen auf anderen Ebenen zu bringen und mit Hilfe von theoretischen Leitlinien zu integrieren (vgl. hierzu und zum folgenden Geulen/Hurrelmann 1980).

Im Feld des aggressiven, auffälligen, devianten, delinquenten Verhaltens von Schülerinnen und Schülern hat sich der Gewaltbegriff als theoretisches Leitkonzept durchgesetzt. Daran hat neben den eingangs skizzierten gesamtgesellschaftlichen Prozessen die Entwicklung der Methodologie mit ihrem Trend zu Mehrebenenanalysen erheblichen Anteil: der Gewaltbegriff kann bei Analysen auf der Mikro-, Meso- und Makroebene gleichermaßen theoretisch und in operationalisierter Form auch empirisch wirksam werden. Dementsprechend wird versucht, gewaltförmige Verhaltensweisen auf individuelle und personale Merkmale und/bzw. auf institutionelle und interaktionale Bedingungen, unter deren Maßgabe die Individuen kommunizieren, und/bzw. auf makrosoziale Einflüsse (z. B. ,strukturelle Gewalt“, allgemeine Kriminalitätsentwicklung, -furcht und -bekämpfung) zurückzuführen und vor diesem breiten Hintergrund zu erklären. Dabei können - je nach Analyseebene und Untersuchungsaspekt - spezifische Theorien mittlerer Reichweite (vgl. Abb. 1-1) als Interpretationsanleitung der erhobenen Schüler-, Lehrer- und Schuldaten zum Tragen kommen. 
Abb. 1-1: Ausgewählte Aggressions- und Gewalttheorien

Theorie

Psychologische Ansätze

Triebtheorien

Frustrationstheorien

Lerntheorien

Psychoanalytische

Theorien

Soziologische Ansätze

Anomietheorie

Subkulturtheorie

Theorien des differentiellen Lernens

Etikettierungstheorien

Individualisierungstheorie
Kurzcharakterisierung

Aggression wird auf spontane Impulse im menschlichen Organismus zurückgeführt.

Aggression entsteht reaktiv durch Frustration.

Aggression beruht auf Lernvorgängen (z. B. Lernen am Modell, Lernen am Erfolg, kognitives Lernen).

Aggression als Ausdruck komplizierter Störungen der gesamten

Persönlichkeit (z. B. Traumatisierungen in der Kindheit).

Abweichendes Verhalten (z.B. Gewalt) entsteht durch "Anpassung" der Gesellschaftsmitglieder an die widersprüchlichen kulturellen und sozialen Verhältnisse der Gesellschaft.

Abweichendes Verhalten wird als "Anpassung" an widersprüchliche Anforderungen seitens der Gesamt- und der Subkultur verstanden (z. B. kann Gewalt innerhalb der Gruppe als konform gelten).

Sowohl abweichendes als auch konformes Verhalten wird erlernt (z. B. durch Interaktion und Kommunikation).

Abweichendes Verhalten entsteht vor allem durch gesellschaftliche Definitions- und Zuschreibungsprozesse.

Gewalt wird als Folge gesellschaftlicher Modernisierungsprozesse (z. B. Auflösung traditioneller Milieus, Wertewandel) und den damit verbundenen Erfahrungen von Desintegration und Verunsicherung aufgefaßt.

Am Idealbild eines mehrebenenanalytisch orientierten Forschungsdesigns werden sich künftige Studien zur Gewalt in der Schule messen lassen müssen. Wenn in der zweiten Hälfte der 90er Jahre Forschungsgruppen bestehen, die mit derart weitgesteckten Ansprüchen antreten (vgl. zum Überblick über derartige Ansätze Holtappels/Heitmeyer/Melzer/Tillmann 1997), ist das ein Indiz dafür, daß seit den spärlichen Anfängen schulbezogener Aggressionsund Gewaltforschung in den 70er Jahren erhebliche Fortschritte erzielt worden sind. Dabei hat sich eine intensive wissenschaftliche Beschäftigung mit der Thematik erst aufgrund eines gravierenden Handlungsbedarfs der Schulpraxis und einer deutlich formulierten öffentlichen Anforderungsstruktur ergeben. Seit Anfang der 90er Jahre bis heute hat kaum ein im weitesten Sinne pädagogisch relevantes Thema die Medien so nachhaltig beschäftigt wie das der Gewalt an Schulen. Kritisch dazu muß angemerkt werden, daß Teile von Presse und Fernsehen mit Übertreibungen und verfälschenden Darstellungen das Bild eines von Brutalität gegen Personen und Sachen bestimmten Schulalltags gezeichnet haben, das mit der Wirklichkeit nicht übereinstimmt (vgl. zur Medienkritik Schubarth 1995). Solchen dramatisierenden Gewaltbeschreibungen stehen bis etwa 1993 so gut wie keine wissenschaftlich fundierten Forschungen 
gegenüber. Das änderte sich jedoch in den Folgejahren aus den oben genannten Gründen sehr rasch: bis Mitte der 90er Jahre werden aus rund einem Dutzend Forschungsprojekten umfassende Untersuchungsbefunde vorgelegt; es bilden sich interdisziplinäre Forschungsgruppen, an denen Kriminologen, Soziologen, Psychologen und Erziehungswissenschaftler beteiligt waren und sind; auch zwischen den Teams entwickelte sich eine intensive Zusammenarbeit, in die auch internationale Erfahrungen einbezogen wurden, ${ }^{4}$ so daß man davon ausgehen kann, daß heute ein sehr breit arbeitendes und alle Felder der Gewaltthematik in Schulen betreffendes Forschungsnetzwerk besteht. ${ }^{5}$

Resümiert man den bisherigen Erkenntnisstand, so besteht weitgehend Konsens hinsichtlich der Formen und Häufigkeiten schulischer Gewalt sowie des Einflusses von Schulform, Alter und Geschlecht (vgl. Tillmann 1997b). Diesbezügliche Befunde sind mehrfach sekundäranalytisch aufgearbeitet und in verschiedenen Übersichtswerken dargestellt worden (vgl. u.a. Schubarth/ Melzer 1993/95, Melzer/Schubarth/Tillmann 1995, Holtappels/ Heitmeyer/ Melzer/Tillmann 1997). Diese Bilanz zeigt ebenfalls, daß schulbezogene Gewaltforschung auch in anderen Bereichen Fortschritte macht: das Rollengefüge von Tätern und Opfern ist mittlerweile sehr differenziert beschrieben (vgl. u.a. Dettenborn/Lautsch 1993, Olweus 1995, 1997, Melzer/Rostampour 1996, Rostampour/Melzer 1997, Lösel/Bliesener/Averbeck 1997), und auch in der Präventions- und Interventionsforschung gibt es positive Entwicklungen (vgl. u.a. Olweus 1995, Hanewinkel/Knaack 1997, Petermann/Jugert/Tänzer/Verbeek 1997, Busch/Todt 1997, Dann 1997).

Dagegen zeigt sich weiterhin Forschungsbedarf in bezug auf ,die innerschulischen und außerschulischen Faktoren, die gewaltauslösend oder -hemmend wirken, (...) den relativen Einfluß familiärer und schulischer Faktoren auf die Ausbildung des Aggressionspotentials, (...) die Bedeutung, die das Schulklima und die Lernkultur einer Schule hier besitzen, (...) den Einfluß, den gesamtgesellschaftliche Prozesse (etwa die zunehmende Jugendarbeitslosigkeit) hier ausüben, und (...) die Wirksamkeit unterschiedlicher Präventions- und Interventionskonzepte“ (Tillmann 1997b, S. 25). Derselbe Autor mahnt - wie wir - eine bessere theoretische Fundierung der Forschung an, die bei einer kritischen Rückfrage nach dem Gewaltbegriff beginnt und auch Modelle zum Verhältnis von Gewalthandeln, Gewaltbereitschaft und situativen Auslösern einschließt. Trotz der festgestellten Fortschritte verbleiben also nach wie vor anspruchsvolle Forschungsdesiderate, zu deren Lösung mit der vorgelegten Studie ein kleiner Beitrag geleistet werden soll.

4 Gemeint sind insbesondere die Forschungen und Aktivitäten des Norwegers Dan Olweus (vgl. u.a. Olweus 1995, 1997).

5 Ein Beispiel für den Netzwerkgedanken war die Fachtagung des SFB 227 „Prävention und Intervention im Kindes- und Jugendalter“ zum Thema Gewalt an Schulen, die im September 1996 in Bielefeld stattfand. 


\subsection{Definitionen und Konnotationen zum Gewaltbegriff}

Eine Klärung des Gewaltbegriffs als Bestandteil der Theoriebildung ist als wissenschaftliche Entwicklungsaufgabe postuliert worden. In seiner methodenkritischen Analyse schulischer Gewaltforschung stellt Krumm (1997) eine „,unbefriedigende Reflexion zentraler Konzepte“ (S. 66) fest und fragt: „Warum fassen die meisten Autoren das, was sie untersuchen wollen, unter 'Gewalt' zusammen? Wenn so Heterogenes wie 'Lächerlichmachen' und 'Schwere Körperverletzung', 'Beschimpfung' und 'Erpressen' unter einem Begriff zusammengefaßt werden soll, ist dann der 'erschreckende' Begriff 'Gewalt' geeigneter als das harmlosere 'abweichende', 'unerwünschte', 'auffällige' Handeln? Ist der Gewaltbegriff gerechtfertigt, wenn man beachtet, daß das, was in der Öffentlichkeit unter Gewalt verstanden wird - nämlich schwere Schädigung durch Normverletzung - glücklicherweise selten vorkommt, 'verbale Gewalt' aber vielfach häufiger?" (S. 67f).

\section{Zur Semantik des Gewaltbegriffs in der Alltagssprache}

In dem o.a. Zitat ist der öffentliche Sprachgebrauch des Begriffes „Gewalt“ thematisiert worden. Gewalt hat in der Alltagssprache heutzutage einen durchgängig negativen Bedeutungsgehalt ${ }^{6}$ und wird als Bezeichnung für soziale Handlungsweisen und Phänomene verwendet, die als gesellschaftlich inopportun gelten. Auf der anderen Seite besitzt Gewalt als Konsumgut und Handlungsform für einen bestimmten Personenkreis, insbesondere unter den Heranwachsenden, eine gewisse Attraktivität und animiert zum Nachahmen (vgl. Kap. 2.3). So gehört in einigen jugendlichen Subkulturen gewaltförmiges Verhalten als Initiationsritus und Medium der Gruppenkohäsion in den Peers zu den akzeptierten und idealisierten Verkehrsformen - dies gilt aber nur für eine kleine Minderheit unter den Jugendlichen. $\mathrm{Da}$ in der Bevölkerung kein homogenes Begriffsverständnis von Gewalt vorliegt, stellt auch die Gewaltkommission der Bundesregierung fest. In ihrem Gutachten bezieht sie sich auf eine international vergleichende Untersuchung, in der zur Begriffsklärung ein „semantisches Differential“ nach Osgood/Suci/Tannenbaum (1957) empirisch eingesetzt wurde: Gewalt sollte als nötig/unnötig, stark/schwach, schlecht/gut, häßlich/schön, aufregend/langweilig, links/ rechts, unwirksam/wirksam und gefährlich/ungefährlich bewertet werden. Ein Hauptergebnis dieser Studie war, daß der ,allgemein-gesellschaftliche Begriff von Gewalt in den untersuchten westlichen Demokratien (...) umfassend negativ etikettiert (ist) (...). Bezüglich

6 Historisch betrachtet hat „Gewalt“ aber durchaus auch positive Bedeutungszuschreibungen (vgl. u.a. Gottschalch 1997, S. 14ff). 
des Paars 'stark - schwach' ergeben sich eindeutige Unterschiede zwischen den romanisch-südeuropäischen Ländern sowie Luxemburg einerseits und den anderen Ländern (einschließlich der Bundesrepublik) andererseits insofern, als in der erstgenannten Ländergruppe Gewalt signifikant mehr als 'stark' erlebt wird. Es fällt schwer, hier nicht an kulturspezifische Besonderheiten (Machismo) zu denken. Bemerkenswert ist das Ergebnis, daß in der Bundesrepublik mehr als in (jedem) anderen EG-Land Gewalt als aufregend gilt; gleiches trifft übrigens auch für die Einschätzung der Wirksamkeit zu. (...) Gewalt entspricht in seiner situativen Einbettung offenbar in weiten Bereichen geltenden Aufmerksamkeitsregeln und Handlungslogiken unserer Gesellschaft; dabei besitzt in der Bundesrepublik offenbar die Vorstellung, Gewalt sei wirksam und man könne mit ihr einiges erreichen, ein überdurchschnittlich großes Gewicht" (Schwind/Baumann u.a. 1990, S. 44).

Dagegen hat „Gewalt“ im Verfassungsrecht und im politischen Raum eine positive Grundbedeutung (,Alle Gewalt geht vom Volke aus.“), der Verfassungsgrundsatz der Gewaltenteilung gilt als Garant der Demokratie. Aber schon beim Terminus „Gewaltverhältnis“, der das Rechtsverhältnis des einzelnen zum Staat bezeichnet, läßt sich ein Bedeutungswandel ablesen: angesichts der Tatsache, daß staatliche Einrichtungen, wie Verwaltung, Schule, Kinder- und Jugendhilfe u.a., heute immer häufiger als Dienstleistung für den Bürger begriffen werden, wirkt dieser Terminus ziemlich anachronistisch. In bezug auf das Rechtsverhältnis von Eltern und Kindern ist eine ähnliche Entwicklung feststellbar: so wurde bereits Ende der 70er Jahre der Begriff der elterlichen Gewalt durch den der elterlichen Sorge ersetzt und bei der Novellierung des Kindschaftsrechtes von 1997 ein Verbot der Mißhandlung von Kindern durch Erziehungsberechtigte explizit in den Gesetzestext aufgenommen. Danach sind entwürdigende Erziehungsmaßnahmen, insbesondere körperliche und seelische Mißhandlungen ausdrücklich für unzulässig erklärt. Weitergehende Forderungen, die in den parlamentarischen Beratungen des Gesetzes erhoben worden sind, kommentiert die zuständige Jugendministerin wie folgt: „Der Begriff der sog. gewaltfreien Erziehung, den die Opposition ins Gesetz hineinschreiben will, können wir vernünftigerweise nicht einführen. Denn nach gängigem Gewaltverständnis ist eine gewaltfreie Erziehung schlichtweg nicht möglich. Nach allgemeinem Rechtsverständnis wende ich bereits Gewalt an, wenn ich z. B. ein Kind durch Festhalten daran hindere, auf die Straße zu laufen, damit es nicht überfahren wird. Eine gewaltfreie Erziehung ist daher eine Illusion und könnte dazu führen, daß Eltern, die nichts anderes tun, als ihrer elterlichen Verantwortung für ihre Kinder nachzukommen, kriminalisiert würden“" (Nolte 1997).

Die Antithese zu dieser machiavellistischen Ausdeutung des Gewaltbegriffs ist die Position ,,struktureller Gewalt“. Diese aus der Friedens- und Konfliktforschung auf pädagogische Verhältnisse übertragene Auffassung beinhaltet eine Kritik daran, daß den Erziehungsverhältnissen und -institutionen aufgrund ihrer hierarchischen Struktur prinzipiell Gewalt innewohnt und 
zwischen Personen in derartigen Rollenhierarchien Herrschaft ausgeübt wird. Parsons kommt zu ganz ähnlichen Auffassungen, wenn er eine Machtachse mit einem entsprechenden Machtgefälle und eine Polarisierung im Verhältnis von Erwachsenen und Heranwachsenden, Lehrern und Schülern (vgl. Parsons 1968, S. 168, Parsons/Bales 1955), die das Gefüge von Familie und Schule strukturell bestimmen, feststellt. Gewalt ist das Mittel zur Aufrechterhaltung dieser Konstellation. In dieser Perspektive hat der Gewaltbegriff neben der analytischen auch eine Kritik-Funktion: die Schule als ,anomische Struktur“ zu begreifen (Böhnisch 1995), bedeutet dann, die strukturelle Gewalthaltigkeit und entsprechende Defizite im sozialen Klima sowie bei der Wahrnehmung von Sozialisations- und Bildungsfunktionen der Schule herauszuarbeiten.

Der Gewaltbegriff und die Gewaltphänomene, auf die er sich bezieht, sind somit sehr facettenreich, und es ist eine große Herausforderung für die schulbezogene Gewaltforschung, dieses Feld in Theorie und Empirie angemessen zu bearbeiten. Ein Problem dabei ist, daß Schülerinnen und Schüler die Beschreibung und Erklärung von Gewaltphänomenen im Kontext ihrer Werturteile und Einstellungen zur Gewalt vornehmen. Eine aus heuristischen Gründen anzustrebende Trennschärfe dieser Dimensionen ist de facto kaum zu erzielen - auch wenn im Instrumentarium unserer Untersuchung zwischen Beobachtung und Selbstreport auf der einen Seite und Bewertung einer Handlungssituation auf der anderen Seite deutlich unterschieden wird. Denn: wer Gewalt häufiger als andere beobachtet, ist möglicherweise auch mehr daran beteiligt und rechtfertigt dies eher als andere (vgl. Kap. 2) oder: es ist nicht ausgeschlossen, daß ein Teil der Schüler, die angeben, als Schlichter aufzutreten, dies aus legitimatorischen Gründen äußern, in Wirklichkeit aber in Gewaltsituationen mit dem Motiv eingreifen, um „mitmischen“ zu können (vgl. Melzer/Rostampour 1996, S. 135ff und Kap. 4). Um derartig komplexe Sachverhalte überhaupt dechiffrieren zu können, ist es notwendig, die Verwendung des Gewaltbegriffs in verschiedenen Schülergruppen auszuloten.

\section{Empirische Inhaltsanalysen zum Gewaltbegriff bei Schülern und Lehrern}

Das Begriffsverständnis von Gewalt bei Schülern und Lehrern wurde im Rahmen unserer Fallanalysen mit Hilfe von Gruppendiskussionen und kleinen quantitativen Erhebungen untersucht. Zur Frage, was sie unter Gewalt verstehen, erfahren wir von Mittelschülern der 9. Klasse:

„Gewalt ist für mich, wenn ich zu jemandem hingehe, dem eine reinziehe, jemandem echt weh tue. Wenn ich jemanden erpresse (...) oder so was. Oder wenn ich jemanden anmache, kleine Kinder anmache. So was, das ist für mich Gewalt.“

„(...) das fängt schon beim Schlagen an, schon wenn du einem eine donnerst oder so, das ist für mich schon Gewalt - bis hin zum Verletzen.“ 
Das hierin zum Ausdruck kommende Verständnis von Gewalt als physische Aggression ist bei den Kindern und Jugendlichen vorherrschend. Von einer Minderheit werden aber auch psychische und subtilere Formen als Gewalt definiert:

„Aber es gibt ja auch noch andere Gewalt, wenn man unter Druck gesetzt wird oder so,
wenn sie dich erpressen, das ist psychische Gewalt oder wie man das nennen mag.“
„Na, zum Beispiel, wenn ein Lehrer einen total unter Druck setzt, das ist - finde ich
- auch Gewalt, oder z.B. in der Toilette einsperren, das kann man sehen wie man will,
wenn's irgendwie Spaß macht, ist o.k. Aber wenn es dann wirklich mit Geldausrauben
ist und so Schutzgeld verlangen, das ist für mich totale Gewalt.“

Um diese Aussagen einzelner Schüler quantitativ abzusichern, haben wir die in Gruppendiskussionen und Interviews mit Schülern und Lehrern ermittelten Gewaltdefinitionen als Items operationalisiert, die Handlungssituationen beschreiben, und den Schülerinnen und Schülern sowie den Lehrerinnen und Lehrern zur Bewertung mit der Frage vorgelegt, ob sie die jeweilige Situation als Gewaltausübung bezeichnen würden. Der Fragebogen umfaßte insgesamt 23 Handlungssituationen, in denen Vandalismus (3 Items), Beschädigungen von Sachen anderer (2), Hänseln (2), sexuelle Belästigung (2), Fremdenfeindlichkeit (1), körperliche Angriffe (2), Erpressung (2), Gruppenprügelei (1), psychische Aggression gegen Lehrer (2), Zerstörung von Lehrereigentum (1), Beleidigung durch Lehrer (2), Lehreraggression (1), Lehrerwillkür (1), handgreifliche Aggression durch Lehrer (1) thematisiert waren (vgl. Anhang). Es zeigen sich folgende Ergebnisse: Kinder und Jugendliche haben - verglichen mit Erwachsenen - ein eher enges Gewaltverständnis, das oft nur Formen physischer Gewalt einschließt. Allerdings wird der Gewaltbegriff von den verschiedenen Subgruppen der Heranwachsenden nicht einheitlich verwendet. Weitestgehende Übereinstimmung besteht bei ihnen zwar darüber, daß es sich bei allen manifesten gewaltförmigen Handlungen, wie körperlichen Angriffen, Erpressungen und Vandalismus, um Gewalt handelt. Sehr unterschiedlich beurteilen sie dagegen Formen psychischer und verbaler Gewalt. Beschimpfungen und Verspotten Gleichaltriger, aber beispielsweise auch psychische Aggressionen von Lehrern gegen Schüler werden von den Heranwachsenden nicht generell als Gewalthandlungen, sondern zum Teil als normale Umgangsformen oder allenfalls als Vorstufe zu einer Gewalthandlung angesehen. Unsere Untersuchungen zeigen weiter, daß Jungen Gewalt - qua definitione - eher tolerieren als Mädchen. Die deutlichsten geschlechtsspezifischen Unterschiede zeigen sich in Bereichen der psychischen und verbalen Gewalt sowie der sexuellen Belästigungen. Hänseleien von Gleichaltrigen, verbale Aggressionen gegen Fremde oder Beleidigungen von Lehrern werden von Jungen eher selten, von Mädchen dagegen recht häufig als Gewalthandlung betrachtet. Ähnliche Tendenzen zeigen sich bei der sexuellen Belästigung, die von Mädchen häufiger als gewaltförmige Handlung verstanden wird. Unterschiedliche Auffassungen über den Gewaltbegriff zeigen auch Schüler verschiedener Schulformen. Der 
Gewaltbegriff von Mittelschülern ist eher eng und tendenziell auf Formen physischer Gewalt beschränkt, der von Gymnasiasten ist weiter gefaßt und schließt auch psychische Aggression ein.

Die Tabelle 1-1 zeigt die beschriebenen Tendenzen am Beispiel von vier ausgewählten Items. Im ersten Item geht es um die Beschädigung von Sachen anderer, im zweiten um Hänseln von Mitschülern, im dritten um grobe Prügelei und im vierten um Erpressung. Die Ergebnisse zeigen, daß bei den weicheren Formen aggressiven Verhaltens (Items 1 und 2) die Unterschiede zwischen den Vergleichs- und Subgruppen sehr hoch sind. Die meisten Lehrer haben ein im Vergleich zu den Schülern weitgefaßtes Gewaltverständnis, das diese beiden Situationen mit einschließt, was nur für eine Minderheit von Schülern zutrifft. Bei der Gruppe der Schüler zeigt sich eine ähnliche Differenzierung zwischen Jungen und Mädchen sowie Mittelschülern und Gymnasiasten, wobei die Ergebnisse für die Mädchen und Gymnasiasten näher bei denen der Erwachsenen liegen. Bei den härteren Gewaltformen wurde eine Nivellierung dieser dargestellten Gruppenunterschiede ermittelt, d.h. hier gibt es eine tendenzielle Übereinstimmung und somit einen weiten Konsens darüber, daß es sich bei aggressiven Handlungen, wie Gruppenprügelei oder Erpressung, um Gewalt handelt.

Tab. 1-1: Gewaltbegriff von Schülern und Lehrern* (Angaben in Prozent)

\begin{tabular}{|c|c|c|c|c|c|c|}
\hline & Schüler & Lehrer & Jungen & Mädchen & $\begin{array}{l}\text { Mittel- } \\
\text { schüler }\end{array}$ & $\begin{array}{l}\text { Gymna- } \\
\text { siasten }\end{array}$ \\
\hline $\begin{array}{l}\text { 1. Wenn Christian nach der Pause } \\
\text { in das Klassenzimmer zurück- } \\
\text { kommt, liegt der Inhalt seiner } \\
\text { Tasche oft verstreut am Boden. }\end{array}$ & 47,1 & 83,3 & 42,7 & 57,6 & 40,2 & 60,7 \\
\hline $\begin{array}{l}\text { 2. Mitschüler der kleinen } \\
\text { Angela, die seit kurzem eine } \\
\text { Brille trägt, sagen nur noch } \\
\text { Brillenschlange zu ihr. }\end{array}$ & 40,9 & 72,9 & 27,6 & 55,6 & 29,5 & 56,1 \\
\hline $\begin{array}{l}\text { 3. Die Mitglieder zweier Cliquen } \\
\text { von Jungen prügeln sich fast } \\
\text { täglich auf dem Schulgelände. }\end{array}$ & 89,9 & 97,9 & 88,2 & 93,6 & 90,8 & 91,2 \\
\hline $\begin{array}{l}\text { 4. Stefan hält dem jüngeren David } \\
\text { die Faust unter die Nase und } \\
\text { sagt: "Morgen will ich Geld } \\
\text { sehen, sonst passiert was!“ }\end{array}$ & 95,3 & 100,0 & 93,8 & 96,9 & 93,7 & 97,4 \\
\hline
\end{tabular}

* Wortlaut der Frage: Handelt es sich bei den folgenden Situationen und Beispielen um Gewalt? (ja/nein) 


\section{Gewalt als theoretischer Leitbegriff}

Ist es angesichts dieser unterschiedlichen Definitionen und Interpretationen des Gewaltbegriffs in der Alltagssprache und der vorgebrachten Einwände von wissenschaftlicher Seite zur Phänomenologie von „Gewalt“ - die Vorkommnisse dazu in der Schule seien sehr facettenreich und es dominierten „weichere“ Formen, die man auch anders bezeichnen könnte - überhaupt noch sinnvoll, am Gewaltbegriff als zentralem analytischen Terminus festzuhalten? Welche Argumente lassen sich demgegenüber für die Verwendung dieses theoretischen Leitkonzeptes anführen?

Um zunächst bereits genannte Argumente aufzugreifen: Der Gewaltbegriff eignet sich deswegen besser als andere Termini, weil er eine Analyse auf der personalen, interaktionalen, institutionellen und makrosozialen Ebene am ehesten gewährleistet und damit für das Programm einer Integration dieser Analyseebenen steht (vgl. 1.1). Zum anderen wird er bevorzugt, weil er die eingangs skizzierten gesellschaftlichen Implikationen der Thematik - „Gewalt entsteht im Zentrum der Gesellschaft“" (vgl. Richter/Sünker 1997) - besser als andere Begriffe ausdrückt. Ein weiteres Argument für dieses Konzept ergibt sich, wenn man den Entstehungszusammenhang von Gewalt berücksichtigt. Kriminelle Karrieren beginnen in der Regel mit Vernachlässigungen und Verwahrlosungen im Elternhaus, sind häufig begleitet von restriktiven Sozialisationsbedingungen und Gewalterfahrungen in Familie, Schule und Gleichaltrigengruppen und zeigen bestimmte Vorformen, bei denen die Grenze zur Delinquenz noch nicht überschritten ist. Diese biographische Entwicklung läßt sich - unter Einbeziehung personaler, struktureller und situativer Bedingungen - im Gesamtmodell als Prozeß der „Gewaltemergenz“ begreifen, an dessen Ende durch Kumulations- und Synergieeffekte zwar eine zielgerichtete schwere Schädigung durch Normverletzung steht, der aber schon bei weniger gravierenden Verhaltensweisen seinen Anfang nimmt. Wendet man ein derartiges Verständnis von Gewalt auf pädagogische Alltagssituationen an, ermöglicht dies auch schon Vorformen manifester Gewalt als solche zu erkennen und sich damit auseinanderzusetzen, die Entstehung einer möglichen „Gewaltspirale“ $\mathrm{zu}$ antizipieren, geeignete pädagogische Maßnahmen dagegen zu ergreifen und früher Grenzen zu ziehen. „Gewaltemergenz“ und „Gewaltprävention“ bedingen sich also gegenseitig und können somit als Leitbegriffe für Analyse und pädagogische Maßnahmen gleichermaßen festgehalten werden. Bevor dieser Ansatz noch etwas elaborierter dargestellt wird (vgl. 1.4), sollen im folgenden Abschnitt die bisherigen Untersuchungen unserer Forschungsgruppe, die zu dieser Konzeptualisierung beigetragen haben, in knapper Form dargestellt werden. 


\subsection{Eigene Untersuchungen im Überblick}

In den ersten beiden Abschnitten sind Ansprüche und Zielsetzungen formuliert, denen wir uns seit 1993 mit multimethodisch und komparatistisch orientierten Verfahrensweisen Schritt für Schritt anzunähern versucht haben. Bislang wurden folgende Studien in Angriff genommen: vergleichende Schulleiterbefragungen in Sachsen, Hessen, Thüringen und Baden-Württemberg, Täter- und Opferanalysen im Anschluß an Repräsentativbefragungen von Schülern, Fallstudien an Einzelschulen zur Transformationsproblematik und zum Umgang mit Gewalt sowie eine Pilotstudie zur Gewaltprävention, die im Zusammenhang mit einer weiteren Untersuchung steht, einer Repräsentativbefragung von Schülern und Lehrern in Ost- und Westdeutschland (vgl. Abb. 1-2). Im folgenden wird in einer kurzen Zusammenfassung über bereits an anderer Stelle publizierte Ergebnisse der Studien 1 - 5, also der vergleichenden Schulleiterbefragungen (1. und 2.), der Täter-Opfer-Analysen (4.) und der Fallstudien (2., 3. und 5.), berichtet (vgl. auch Schubarth 1998b). Die Untersuchungen Nr. 6 und 7 bilden die Grundlage der aktuellen Studie und werden mit ihren Ergebnissen in diesem Band zum ersten Mal im Zusammenhang eines umfassenden Theorie- und Methodenkonzepts interpretiert und dargestellt.

\section{Ergebnisse der vergleichenden Schulleiterbefragungen}

Die vergleichenden Schulleiterbefragungen wurden im Schuljahr 1993/1994 in den Bundesländern Sachsen, Hessen, Thüringen und Baden-Württemberg durchgeführt, wobei alle Schulformen der Sekundarstufe I, also Hauptschulen, Realschulen, Regelschulen, Mittelschulen, Gymnasien, Förderschulen für Lernbehinderte und Erziehungshilfe, und die Berufsschulen einbezogen waren. Ziel war es, sich mit einem relativ geringen Aufwand einen schnellen Überblick über die Problemlage in unterschiedlichen Schulformen und Bundesländern zu verschaffen. Folgende Hauptergebnisse wurden auf diesem Wege ermittelt (vgl. ausführlich Melzer/Schubarth/Tillmann 1995; Schubarth/Kolbe/Willems 1996, Kap. 1; Ackermann 1996):

- Die Gewaltbelastung war - aus der Perspektive der Schulleitungen - an den Schulen in Sachsen und Thüringen niedriger als in Hessen und Baden-Württemberg. Die Unterschiede durchzogen (fast) alle Untersuchungsaspekte; sie betrafen alle einbezogenen Schulformen, wobei die Ost-WestDifferenzen bei den Gymnasien besonders groß waren.

- Zugleich ließen sich Anzeichen von Angleichungstendenzen finden: z. B. lag die wahrgenommene Gewaltzunahme in Sachsen und Thüringen z.T. über der in den beiden alten Bundesländern; ca. ein Drittel der sächsischen und thüringischen Schulleitungen gaben beispielsweise beim Vandalismus 
Abb. 1-2: Eigene Untersuchungen im Überblick

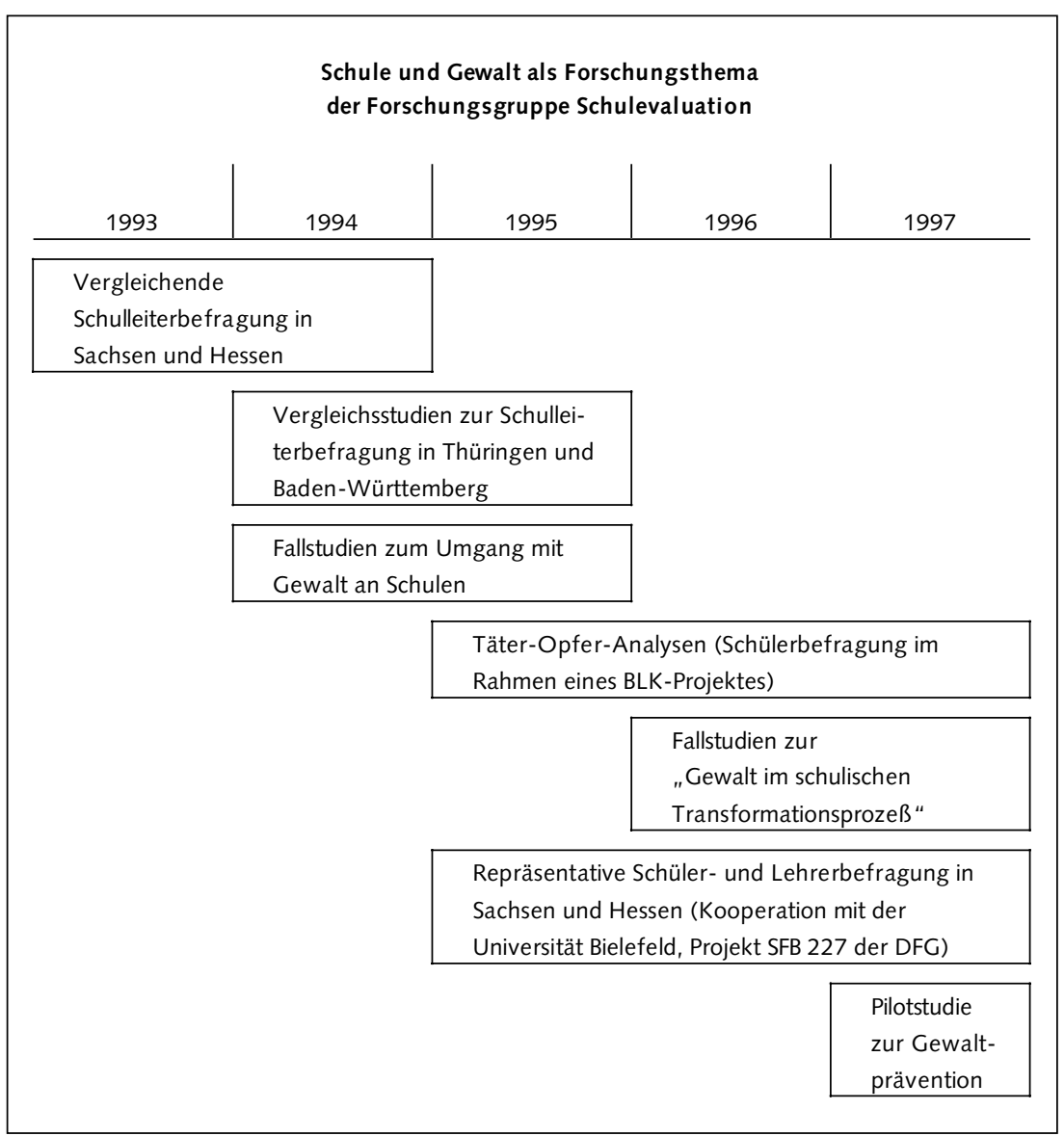

eine steigende Tendenz an, so daß wir damals vermuteten, daß es wahrscheinlich nur eine Frage der Zeit sein würde, bis beim Gewaltausmaß „Westniveau“ erreicht sei.

- Die häufigsten schulischen Gewaltphänomene in allen untersuchten Bundesländern waren: Unterrichtsstörungen, Beschimpfungen, nonverbale Provokationen sowie Vandalismus, während Schutzgelderpressung, Prügeleien zwischen Schülergruppen oder das Tragen von Schußwaffen in Ost wie West eher die Ausnahme waren. Bei fast allen Gewaltformen, mit Ausnahme des Rechtsextremismus, der im Osten stärker verbreitet war, lagen die durchschnittlichen Werte in den alten Bundesländern höher. 
- Ein übereinstimmender Befund ergab sich hinsichtlich der Schulformen: Förderschulen und Mittelschulen bzw. Haupt- und Realschulen waren stärker belastet als Gymnasien. Der sich in der Schulform ausdrückende Status eines Schülers, der aus unterschiedlichen Sozialisationserfahrungen resultiert, erwies sich somit als wichtige Erklärungsvariable für schulisches Gewaltvorkommen.

- Folgt man dem Bild, das die Schulleiter vom „typischen Täter“ zeichneten, so ist dieser vorwiegend männlich, von eher geringerem Leistungsvermögen, durch familiäre und soziale Probleme belastet und in allen Altersgruppen, insbesondere aber in den 7. und 8. Klassen, zu finden.

- Im Umgang mit den „Tätern“ setzen die Schulen vor allem auf pädagogische Gespräche mit den auffälligen Jugendlichen und deren Eltern. Administrative Maßnahmen (z. B. Umsetzung in eine Parallelklasse, Schulverweis) kommen selten vor. Die Jugendhilfe wird ebenfalls nur selten eingeschaltet, noch seltener die Polizei. Die Schulen sind also bestrebt, die auftretenden Konflikte innerhalb der eigenen Institution zu klären.

Die ermittelten deutlichen Ost-West-Unterschiede, die im Durchschnitt höhere Belastungen für die Schulen in Westdeutschland anzeigten, waren nicht unbedingt zu erwarten gewesen. Aufgrund des gesellschaftlichen Umbruchs in Ostdeutschland und der Transformation des dortigen Bildungssystems - mit all ihren Wirren und Unsicherheiten für die an Schule Beteiligten - hätte man auch die entgegengesetzte Konstellation erklären können. Den tatsächlichen Befund interpretierten wir - aus sozialisations- und modernisierungstheoretischer Perspektive - mit dem Beharrungsvermögen von Sozialisationserfahrungen und der Zählebigkeit der früheren Schul- und Erziehungskultur in Ostdeutschland, die den Systemumbruch (noch) überdauert hat. Wir prognostizierten seinerzeit Angleichungsprozesse und unterstrichen die Dringlichkeit und den gewaltpräventiven Charakter innerschulischer Reformprozesse gerade in den neuen Bundesländern.

Insgesamt machte die Schulleiterbefragung deutlich, daß es einerseits für eine Dramatisierung von Gewalt in der Schule und für einen Gewaltanstieg „auf breiter Front“, wie dies von den Medien z.T. suggeriert wird, keine empirischen Belege gibt, andererseits aber auch eine Bagatellisierung unverantwortlich wäre, da ein Teil der Schulen - in der sächsischen Stichprobe 6\% - mit Gewalterscheinungen massiv konfrontiert ist und dort ein dringender Unterstützungs- und Beratungsbedarf besteht.

\section{Befunde aus Täter-Opfer-Analysen}

Mit den Täter-Opfer-Analysen wurde auf der Basis einer BLK-Studie zur Schulqualität in sächsischen Schulen begonnen (vgl. Melzer/Stenke 1996). Die Auswertungen zeigen, daß eine scharfe Trennung zwischen „Tätern“ und 
„Opfern“ nicht immer möglich ist. Vielmehr können Täter- und Opferstatus schnell ineinander übergehen (vgl. Melzer/Rostampour 1996). Es gibt eine Reihe von Prädiktoren, die sowohl für die Opfer- als auch für die Täterrolle konstitutiv sind: Täter und Opfer sind eher Jungen als Mädchen, kommen in den Gymnasien und in bestimmten Bereichen der Berufsschulen signifikant weniger vor und auch an den Schulen, an denen - unabhängig von der Schulform - das Gewaltvorkommen insgesamt niedriger ist. Außerdem zeigen sich familiale Sozialisationseinflüsse: Kinder, die in einem positiven Familien- und Erziehungsklima aufwachsen, sind weniger als Täter und Opfer in Gewalthandlungen involviert (vgl. hierzu und zum folgenden Melzer 1997c).

Die Tätergruppe besteht aus durchschnittlich etwas leistungsschwächeren Schülern, die auch den Sinn des Lernens nicht einsehen und nur wenig Schulfreude empfinden. Sie betrachten das Verhältnis zu ihren Lehrern häufiger als gestört und fühlen sich von ihnen „abgeschrieben“. Die Schule kümmere sich zu wenig um sie, der Unterrichtsstoff werde nicht genügend erklärt und auf Fragen von Schülern würde zu wenig eingegangen, sind häufig geäußerte Auffassungen von Tätern. Überraschenderweise beklagen sie auch, daß die Schule und die Lehrer sie nicht genügend zur Einhaltung von traditionellen Lerntugenden, wie Ordnung, Pünktlichkeit oder Sauberkeit, erziehen würde. Im Unterschied zu den Opfern rechtfertigen die Täter ihr Verhalten als „,notwendig“ und ,natürlich“. Während sie Gruppenmenschen sind - in der Tat mit anderen vereint und durch ihre Ausübung wenigstens von einem Teil der Klasse bewundert, von einem anderen gefürchtet -, ist das typische Opfer isoliert, hat das Gefühl, von anderen nicht akzeptiert zu werden und keine Freunde zu haben. Das gilt auch für die Situation in ihrer Klasse, wo sie sich nicht wohl fühlen, keinen Zusammenhalt feststellen, nur Konkurrenz und Egoismus sehen. Opfer fühlen sich in der Schule überdurchschnittlich unwohl und haben keinen Spaß am Lernen. Obwohl ihre Schulnoten in der Regel besser sind als die der Täter, haben sie ein geringeres Zutrauen in ihre Leistungsfähigkeit; das Opfer ist ein Angsttyp mit geringem Selbstwertgefühl, starker Unzufriedenheit und starker negativer Selbstattribuierung - sowohl im schulischen als auch im außerschulischen Bereich -, so daß von einer biographischen Kontinuität der Opfererfahrung ausgegangen werden kann.

In neueren Untersuchungen wurden diese Ergebnisse bestätigt und weiter ausdifferenziert. Mit Hilfe von Clusteranalysen wurde eine statistisch abgesicherte Typologie entwickelt (vgl. Rostampour/Melzer 1997); danach gehören etwa 3-4\% zum harten Kern der Täter und etwa 7-10\% zur Gruppe der Opfer. Bemerkenswert ist jedoch, daß über die Hälfte der Schülerschaft zur Gruppe der „Unbeteiligten“ zu rechnen ist, die also in der Regel weder als Opfer noch als Täter auftritt. In der Tatsache, daß die Mehrheit der Schülerschaft nicht an Gewalthandlungen beteiligt ist, liegt ein starkes, aktivierbares Potential für Präventions- und Interventionsansätze.

Die Untersuchung des Täter-Opfer-Status im schulischen Gewaltkontext und einer auf die jeweilige Rolle bzw. die spezifischen Statusgruppen abgestimm- 
ten Präventions- und Interventionsstrategie wird uns auch in dieser Studie beschäftigen (vgl. Kap. 4 und 8).

\section{Die Möglichkeiten der Einzelschule: Ergebnisse von Fallstudien}

Parallel zu den quantitativen Studien wurden zwei vergleichende Fallstudien durchgeführt (vgl. Schubarth/Stenke 1996, Schubarth/Darge/Mühl/Bergelt/ Börner/Hannich 1997). Mit Hilfe eines multimethodischen Ansatzes (neben der quantitativen Erhebung auch Gruppen- und Einzelinterviews, Beobachtungen sowie soziometrische Tests) wurden gewaltfördernde und -hemmende schulische Bedingungen transparent gemacht und entsprechende Präventionsund Interventionsmöglichkeiten entwickelt. Ein besonderer Akzent lag dabei zum einen auf den „Transformationsanalysen“, d.h. auf Untersuchungsfragen zum Einfluß des gesellschaftlichen Umbruchs auf Aggression und Gewalt und zum anderen auf der Erarbeitung von Präventions- und Interventionskonzepten als immanenter Bestandteil von Schulentwicklung.

Anhand der Fallstudien wurden - neben dem Gewaltverständnis und den Gewaltformen - vor allem folgende Bedingungsfelder für Gewalt näher beleuchtet: Schulleben und Schulerleben, Lehrer-Schüler-Verhältnis, SchülerSchüler-Verhältnis, Verhalten in gewalthaltigen Konfliktsituationen und außerschulische Aspekte. Die Untersuchungen bezogen sich sowohl auf die Ebene der Einzelschule, als auch auf die Klassen- sowie die Individualebene. Die ermittelten Ergebnisse wurden den Schulen zurückgespiegelt, so daß Schulentwicklungsprozesse initiiert bzw. befördert wurden.

Die Fallstudien verdeutlichen, wie unterschiedlich Schulen mit Problemen von Aggression und Gewalt umgehen. Schulen, die sich mit der Problematik offensiv auseinandersetzen und aktive Schulentwicklung betreiben, stehen Schulen gegenüber, die anscheinend resignieren bzw. hilflos wirken und Hilfe „von außen“ benötigen. Als wesentliche Einflußfaktoren für eine erfolgreiche gewaltpräventive Arbeit kristallisierten sich heraus:

- das Engagement, die Überzeugungskraft und die Durchsetzungsfähigkeit der Schulleitung,

- das pädagogische Konzept bzw. die „Philosophie“ der Schule, einschließlich bestimmter Präventions- und Interventionsstrategien,

- das gemeinsame, geschlossene Handeln des Lehrerkollegiums, insbesondere bei Konflikt- bzw. Gewaltsituationen,

- bei Gewalt nicht wegschauen, sondern eingreifen und pädagogisch angemessen reagieren,

- ein positives Lehrer-Schüler-Verhältnis, ein partnerschaftlicher und vertrauensvoller Umgang miteinander,

- das regelmäßige pädagogische Gespräch mit Schülern, das Vermeiden von Überreaktionen, Stigmatisierungen und Etikettierungen (keine verletzende Bemerkungen seitens der Lehrer gegenüber den Schülern), 
- die aktive Einbeziehung der Schüler in die Gestaltung des Unterrichts sowie des Schullebens insgesamt,

- ein interessanter, nicht monotoner, sondern didaktisch-methodisch und inhaltlich vielfältiger Unterricht,

- ein abwechslungsreiches Schulleben mit vielfältigen Kontakten nach „draußen“" (Öffnung von Schule) und

- eine aktive Zusammenarbeit mit den Eltern.

Insgesamt wird in den Fallstudien eine gewisse Hilflosigkeit der Lehrerschaft beim Umgang mit Aggression und Gewalt deutlich. Um dieses Problem zu lösen, ist die Entwicklung und Bereitstellung pädagogischer Konzepte, die Durchführung von schulinternen Fortbildungsmaßnahmen, von Trainingskursen und/oder pädagogischen Tagen ein dringendes Erfordernis. Der Erwerb pädagogischer, insbesondere sozialer Kompetenzen, muß sich dabei auf den Bereich der Interaktion und Kommunikation insgesamt erstrecken. Die Befunde verweisen allerdings auch darauf, daß die Gewalt an Schulen ebenso von außerschulischen Bedingungen im Bereich der Familie, des Freizeitverhaltens und der Jugendkultur beeinflußt wird. Gleichwohl sollten die bestehenden schulischen Möglichkeiten der Prävention und Intervention in bezug auf Gewalt keinesfalls unterschätzt werden. Diese - das zeigen unsere Analysen - sind durchaus vorhanden, werden aber nach wie vor viel zu wenig genutzt. Schulische Gewaltprävention kann allerdings nur dann ihre Wirkungen voll entfalten, wenn sie auf der Ebene der Einzelschule angesiedelt ist, die spezifischen Bedingungen, die dort vorherrschen, berücksichtigt und in allgemeine Strategien der Schulentwicklung eingebunden ist. Maßnahmen der Organisationsentwicklung und Qualitätssicherung schulischen Lehrens und Lernens zeigen somit - wenigstens bei einem Teil der Problemlagen - Effekte in bezug auf die Eindämmung schulischer Gewalt - dies ist das wichtigste Ergebnis der Fallstudien, das in den übrigen Untersuchungen unserer Forschungsgruppe ebenfalls bestätigt worden ist.

\subsection{Das Konzept einer sozialökologisch orientierten Gewaltforschung in Theorie und Empirie}

In diesem letzten Teil der Einführung sollen zunächst die an verschiedenen Stellen bereits direkt oder implizit angesprochenen theoretischen Aspekte und Bezüge der Gewaltthematik zusammengefaßt und zu einem Modell der Gewalt in der Schule verdichtet werden. Bei der Heranführung an dieses Gesamtmodell wird gleichzeitig beschrieben, in welcher Weise die Operationalisierung erfolgt, durch die eine empirische Überprüfung der Hypothesen gewährleistet ist. Der zweite Punkt dieses Abschnitts enthält Hinweise zur Durchführung 
der empirischen Untersuchung, zum Untersuchungsdesign und zur Qualität der Stichprobe. Zum Abschluß wird ein Überblick über die Gliederung des Bandes, den Gegenstand der einzelnen Kapitel und den Gesamtablauf der Argumentation gegeben.

\section{Theoretische Aspekte und ihre Operationalisierung}

Aus den bisherigen Ausführungen und den referierten Voruntersuchungen ergibt sich, daß der vorliegenden Studie ein sozialökologischer Untersuchungsansatz (im Sinne eines Mehrebenenmodells) zugrunde liegt, für den der Gewaltbegriff leitend ist. Gewalt wird als zielgerichtete direkte Schädigung begriffen, die unter körperlichem Einsatz und/oder mit psychischen und verbalen Mitteln erfolgt und sich gegen Personen und Sachen richten kann. Gewalt in der Schule wird in konkreten Alltagssituationen in der Klasse, in der Schule, auf dem Schulweg etc. ausgeübt, meistens unter direkter oder indirekter Mitwirkung von Mitschülern und ist - mit Ausnahme von Vandalismus - in der Regel auf andere Personen bzw. deren Sachen/Eigentum gerichtet. Auch sind unter diesem interaktionalen Gesichtspunkt Lehrer als Adressaten, Verursacher, Verhinderer oder Akteure mit in diese Prozesse involviert und somit ebenfalls ein wichtiger Aspekt der Analyse.

Die empirische Erfassung dieser Gewaltformen und -interaktionen erfolgt mit Hilfe eines Schülerfragebogens, der für die älteren Schüler aus insgesamt 240 Fragen besteht und die Perspektiven der Gewaltbeobachtung (Wie häufig hast Du z. B. eine ernsthafte Prügelei zwischen zwei Jungen in den letzten 12 Monaten beobachtet?), des Täter-Selbstreports (Wie oft hast Du das selbst gemacht?), des Opfer-Selbstreports (Wie oft bist Du in diesem Zeitraum von anderen geschlagen worden?), der Einstellungen zur Gewalt (z. B. Gewalt ist etwas völlig Normales) umfaßt und des weiteren eine Stellungnahme zu konkreten Handlungssituationen beinhaltet. Dazu wurden vier Gewaltsituationen skizziert und in einem Item beschrieben - z. B.: Ein Junge/ein Mädchen tritt jemanden in böser Absicht - und den Probanden mit der folgenden Aufforderung zur Stellungnahme vorgelegt: Hast Du das innerhalb oder außerhalb der Schule beobachtet oder selbst getan? Wie verhalten sich die Lehrer in dieser Situation? Wie reagierst Du selbst darauf (ist normal, habe Angst, ärgere mich, versuche das Verhalten zu beenden).

Diese verschiedenen empirischen Zugänge zu dem komplexen Phänomen der Gewalt beziehen sich auf ein Spektrum ,weicherer" und „härterer" Gewaltformen, die in der Schule ausagiert werden. Nach unserer Auffassung könnte sich über den „Einstieg“ in weniger gravierende Formen u.U. ein schwerwiegenderes Fehlverhalten entwickeln. Dazu gibt es gesicherte Erkenntnisse, die von Schmidtchen wie folgt zusammengefaßt werden: „Angriffe auf das Selbstwertgefühl, das Selbstkonzept einer Person führen in aller Regel zu aggressiven Reaktionen, im Grenzfall auch zu Gewalt. Beleidigungen seien 
die wirksamste Art, aggressive Reaktionen hervorzurufen, das ist die Meinung der amerikanischen Forscher. Angriffe auf das Selbstkonzept, Beleidigungen, Herabsetzungen gehören zu den geläufigsten gesellschaftlichen Aggressionsfiguren. (...) Am Ende der Beleidigung steht häufig die Gewalt (Schmidtchen 1997, S. 275f).

Um die gesamte Bandbreite aggressiven und abweichenden Verhaltens in ihren Wechselbeziehungen analysieren zu können, wurde neben den oben bereits genannten Verhaltensformen nach weiteren Aspekten gefragt: nach Vorformen der Gewalt im Bereich der Schuldevianz (z. B. Unterrichtsstörungen, Mogeln, Schuleschwänzen), nach möglichen komplementären Formen wie Alkohol-, Zigaretten- und Drogenkonsum und schließlich nach Tatbeständen außerschulischer Delinquenz (z. B. Diebstahl, Schlägereien, Einbruch, Zerstörung fremden Eigentums). Die allgemeine Hypothese hierzu lautet, daß es sich bei diesen Dimensionen eines Gewalthandelns im weitesten Sinne um ein Geflecht nicht opportunen Verhaltens mit systemischen Wechselbezügen handelt. Gewaltförmige und gewaltaffine Verhaltensweisen werden von der Gesellschaft und auch von der Schule - sofern sie entdeckt werden - in entsprechender Weise negativ sanktioniert. Die mit diesen Sanktionen verbundenen Etikettierungsprozesse verstärken die aus anderen Gründen ohnehin bestehende Tendenz dazu, daß Personen, die sich einmal in einem Netz ,abweichenden Verhaltens" verstrickt haben, es schwerer haben, sich daraus zu befreien, als darin gefangen zu bleiben. Olweus sieht diese Annahme in seinen und anderen internationalen Studien bestätigt und stellt zusammenfassend fest: „Gewalttätigkeit kann (...) als eine Komponente eines allgemeineren sozialfeindlichen und regelverletzenden (,verhaltensgestörten“) Verhaltensmusters angesehen werden. Aus dieser Sicht kann man leicht vorhersagen, daß Jugendliche, die aggressiv sind und anderen gegenüber Gewalt anwenden, ein deutlich höheres Risiko laufen, später in weitere Problembereiche wie Kriminalität und Alkoholmißbrauch zu geraten“ (Olweus 1997, S. 289).

Wenn sich so Unterschiedliches wie Täter- und Opfersein im schulischen Gewaltkontext gegenseitig bedingt (vgl. 1.3), liegt das neben den erwähnten systemischen Wechselbezügen auch daran, daß für beides zu einem erheblichen Teil dieselben Prädiktoren zutreffen (vgl. Melzer/Rostampour 1996, S. 143ff). Bei der Ermittlung dieser Ursachen von Gewalt folgen wir einschlägigen Sozialisations- und Entwicklungsmodellen, nach denen neben den bio-physischen und personalen Voraussetzungen vor allem die primäre Sozialisation innerhalb des emotionalen Zentrums der Familie, die sekundäre Sozialisation mit dem Einfluß schulischer Prägungen, Erfahrungen und Auseinandersetzungen sowie die insbesondere in der Jugendphase relevante Peer-Sozialisation mit ihren vergleichsweise höheren Freiheitsgraden von grundlegender Bedeutung für die „Ökologie menschlicher Entwicklung“ (Bronfenbrenner 1989) sind. Dementsprechend werden im Hinblick auf die Erklärung des Gewaltverhaltens mögliche Ursachen im Bereich des familialen Erziehungsstils und des Familienklimas, der Lern-, Erziehungs- und Kommunikationskultur in den Schulen 
sowie der Verkehrsformen der Peers einschließlich des Medien- und Freizeitverhaltens der Jugendlichen erwartet. Dem Postulat von Heitmeyer/UlbrichHerrmann (1997) an die Adresse der schulbezogenen Gewaltforschung, die Gesamtheit der Lebens- und Erfahrungswelt als möglichen Erklärungsfundus schulischer Gewalt mit heranzuziehen, ist zuzustimmen.

Aus den bisherigen Ausführungen ergeben sich die Konturen eines Theoriemodells, in das neben den dargestellten noch weitere Einfluß-, Steuerungsund Vermittlungselemente einfließen (vgl. Abb. 1-3). Das schematisch dargestellte Modell ist zugeschnitten auf die Erklärung von „Gewaltemergenz“. Dieser Terminus aus der Systemtheorie und der Makrosoziologie bezeichnet die Komplexität von Gewalt mit ihren verschiedenen Gewaltformen, den Wechselbeziehungen zwischen ihnen, den Situationen, in denen sie in Erscheinung tritt, den personalen, sozialisatorischen und sozialökologischen Bedingungsfaktoren sowie den äußeren Steuerungs- und (intrapersonalen) Selbstregulationsmechanismen. „Emergenz“ bedeutet „das Hervortreten neuer Eigenschaften (emergent properties) beim Übergang von einer niederen zu einer höheren Ebene eines Systems, die nicht auf Eigenschaften der Elemente auf der niedrigeren Ebene rückführbar sind. So sollen bestimmte Eigenschaften von Gruppen oder Organisationen (z. B. Kohäsion oder Effektivität) nicht durch die Eigenschaften der beteiligten Mitglieder, sondern nur durch andere Gruppen- oder Organisationseigenschaften oder Strukturen erklärt werden können“" (Schütze 1978, S. 185). Daher ist es im Hinblick auf die Analyse von Gewalt bedeutsam, auf welcher Ebene sie sich artikuliert und welche neue Qualität sich z.B. bei Gewaltsituationen im Klassenverband, auf Schulebene oder in verschiedenen Schulformen zeigt. Empirisch einlösbar scheint uns der mit dem Emergenzbegriff verbundene hohe methodische Anspruch nur durch eine sog. Mehrebenenanalyse (vgl. Kap. 7). Einen Schritt in diese Richtung unternehmen wir in Kapitel 6, in dem die Ursachen für Gewalt auf der Schulund Klassenebene untersucht werden, wenn in die Analyse nicht mehr - wie zuvor - Individualdaten, sondern auf Klassenebene aggregierte Daten einbezogen werden.

Gewaltemergenz fungiert in unserem Modell als abhängige, zu erklärende Variable. Unabhängige Variablen sind der außerschulische Sozialisationskontext mit den bereits mehrfach angesprochenen Prädiktoren (Familien-, Peers-, Medien- und Freizeiteinflüsse), die „Schulkultur“, für die sich in vorangegangenen Untersuchungen zur Schulqualität vor allem die Lehrerprofessionalität und die sozial-räumliche Schulumwelt als evidente Indikatoren erwiesen haben (vgl. Melzer/Stenke 1996), und schließlich bestimmte Persönlichkeitsmerkmale, die schon in vielen Untersuchungen bestätigt worden sind (vgl. Abb. 1-3). Zwischen den abhängigen und den unabhängigen Variablen werden zwei vermittelnde Ebenen angenommen, in denen sich Interaktions- und Steuerungsprozesse vollziehen und die als „Prädiktoren und äußere Regulationsmechanismen“ bzw. als „Prädiktoren und Selbstregulationsmechanismen“ bezeichnet werden. All diese Prozesse vollziehen sich im Kontext makrosozialer 
Bedingungen, für die in Ostdeutschland die Transformation des Gesellschaftsund Schulsystems geradezu paradigmatisch ist.

\section{Abb. 1-3: Sozialökologie schulischer Gewalt (Gesamtmodell)}

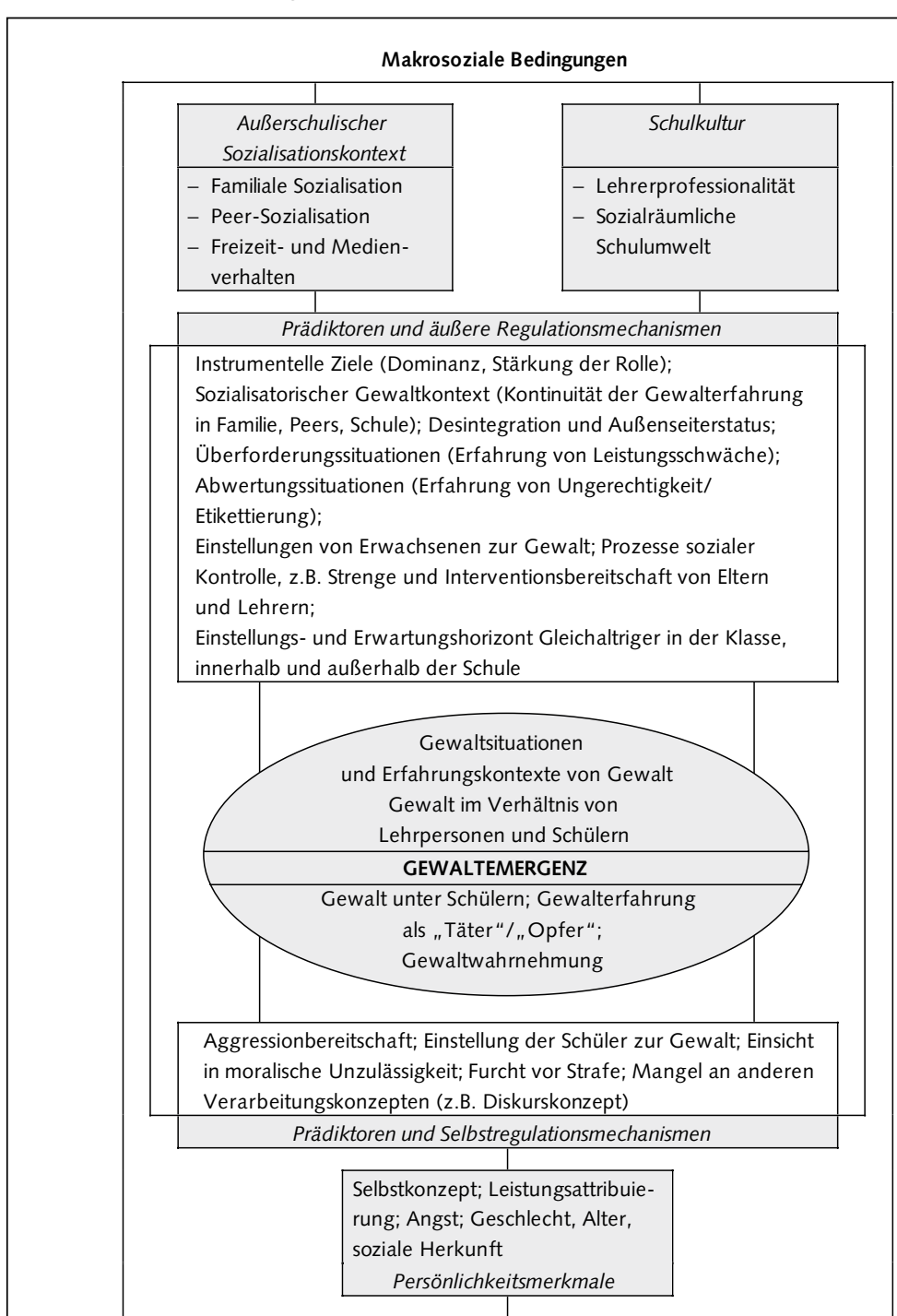

Makrosoziale Bedingungen 
Auf die makrosozialen Bedingungen von Gewalt ist bereits eingegangen worden. Eine detaillierte Analyse dieser allgemeinsten Einflußebene in bezug auf Gewalt in der Schule kann - so bedeutsam sie im Prinzip sein mag - nicht Gegenstand einer empirischen Spezialstudie zur Schüler- und Schulgewalt sein. Aus den bisherigen Ausführungen dürfte aber schon deutlich geworden sein, daß Individualisierungs-, Modernisierungs- und Kommunitarismustheorien (vgl. Habermas 1985a, Beck 1986, Bell 1990, Vobruba 1991, Etzioni 1997) makroanalytische Bezugspunkte unseres Gesamtforschungsansatzes darstellen und als Hintergrund bei Interpretationen von Daten, bei der Entwicklung von Perspektiven oder bei der „Deduktion“ von Konsequenzen implizit oder explizit mit herangezogen werden. Außerdem sind wir im Hinblick auf die methodische Problematik des Ost-West-Vergleichs auf Theorien zur gesellschaftlichen Transformation angewiesen (vgl. Kap. 2). Der Focus der Untersuchung liegt - wie in der Abb. 1-3 dargestellt - im Bereich von Theorien mittlerer und kürzerer Reichweite. Dabei werden zwei Akzente gesetzt: der eine im Bereich der Komparatistik, also der Frage nach möglichen Unterschieden im Ost-West-Vergleich; der andere auf der (vergleichenden) Analyse der Bedingungen für Gewalt in der Institution Schule selbst, weil diesbezüglich eine Forschungslücke besteht und Schule eher dazu neigt, Ursachen von Schülerproblemen den familialen und außerschulischen Sozialisationsbedingungen zuzuschreiben, als eine mögliche eigene Beteiligung zu reflektieren. Im Anschluß an die neuere Debatte zur Schulentwicklung und Schulautonomie (vgl. u.a. Rolff 1996, Paschen 1996, Melzer 1997a) stellt sich die Frage nach dem Zusammenhang von Schulqualität und sozialen Problemen in der Schule: führt die Verbesserung der Schulqualität in zentralen Dimensionen, wie Lehrerprofessionalität, Schulklima, Schulökologie und Schülerpartizipation (vgl. Melzer/Stenke 1996), zu einer Gewaltminderung, so daß Strategien der Schulentwicklung auch in der Gewaltprävention wirksam werden? Die Ergebnisse unserer bisherigen Untersuchungen sprechen dafür; falls sich dieser Zusammenhang hier wiederum bestätigt, würde sich der Bogen von der Analyse zur Präventions- bzw. Interventionsforschung schließen.

\section{Untersuchungen und Stichprobenqualität}

Die empirischen Untersuchungen, die der vorliegenden Studie zugrunde liegen, wurden im Rahmen eines DFG-Projekts (SFB 227 „Prävention und Intervention im Kindes- und Jugendalter") von zwei Teilprojekten durchgeführt. Die Untersuchungen unserer Dresdner Forschungsgruppe bezogen sich auf den Freistaat Sachsen, die Vergleichsuntersuchungen, die mit demselben Instrumentarium von unserem Bielefelder Partnerprojekt ${ }^{7}$ realisiert wurden, auf

7 Das Bielefelder Teilprojekt wird gemeinsam von Klaus-Jürgen Tillmann und Heinz Günter Holtappels geleitet. Mitarbeiter/innen in dem Projekt sind: Ulrich Meier, Birgit Holler- 
das Bundesland Hessen. Diese beiden Bundesländer wurden deswegen für den Vergleich ausgewählt, weil in ihnen nach den Ergebnissen der vergleichenden Schulleiterbefragung die größten Unterschiede bestanden. Da sich die Gewaltsituation an Schulen in Thüringen und Sachsen auf der einen und in Baden-Württemberg und Hessen auf der anderen Seite jeweils sehr ähnlich darstellt, kann der jetzt in der repräsentativen Schülerbefragung vorgenommene Vergleich der Extremgruppen als Kontrolle möglicher Ost-West-Unterschiede überhaupt gelten.

Die im folgenden dargelegten Informationen zum Untersuchungsdesign, zur Durchführung und zu den Methoden der Untersuchung beziehen sich vorwiegend auf den sächsischen Untersuchungsteil und gelten analog für die hessische Befragung, über deren Ergebnisse eine gesonderte Publikation (vgl. Tillmann u.a. 1998) erfolgt, der auch Angaben zur Methodik und Stichprobenqualität der hessischen Untersuchung zu entnehmen sind. Insgesamt wurden Ende 1995/Anfang 1996 3.147 Schüler und 311 Lehrer in Sachsen sowie 3540 Schüler und 448 Lehrer in Hessen befragt. Eine vergleichende Stichprobe wurde durch ein mehrstufiges Auswahlverfahren in beiden Bundesländern (Auswahl typischer Regionen, Zufallsauswahl der Schulen und der Klassen, Totalerhebung der Schüler dieser Klassen, Totalerhebung aller Lehrer der ausgewählten Schulen) gewonnen. Befragt wurden die Jahrgangsstufen 6,8 und die Abschlußklassen der Sekundarstufe I (9. bzw. 10. Jahrgang) in allen Schulformen des allgemeinbildenden Schulsystems. Dieses ist im Freistaat Sachsen relativ übersichtlich und kann am besten mit dem Begriff ,Zwei-Säulen-Modell“ allgemeiner Bildung charakterisiert werden (vgl. Melzer 1997b). Neben Förderschulen bestehen im Bereich der Sekundarstufe I lediglich Gymnasien und Mittelschulen. Letztere sind teilintegrierte Systeme, die den Hauptschul- und Realschulbildungsgang umfassen, entsprechende Abschlüsse ermöglichen und die Schülerinnen und Schüler mit unterschiedlichen Differenzierungskonzepten (homogene, heterogene Lerngruppen) zu diesem Ziel führen. Die Differenzierung der Bildungsgänge setzt mit dem Jahrgang 7 ein. Die Schulen unterscheiden sich nicht nur untereinander in der Wahl des Differenzierungsmodells; auch zwischen den Jahrgängen innerhalb der Schulen bestehen in dieser Hinsicht unterschiedliche Regelungen. Die Repräsentanz dieser Differenzierungskonzepte in der Stichprobe wird durch Zufallsauswahl der Klassen bzw. Lerngruppen in den Mittelschulen hergestellt. Außer Förder-, Mittelschulen und Gymnasien bestehen keine weiteren Schulformen, also insbesondere keine kooperativen oder integrativen Gesamtschulen, wie sie in Hessen zu finden sind. Für die hessische Bildungslandschaft ist dagegen eine plurale Bildungsstruktur typisch, deren Komplexität durch Zusammenfassung

Nowitzki und Ulrike Popp. Das quantitative Instrumentarium wurde in Vorleistung dieser Projektgruppe entwickelt. Wir bedanken uns dafür ebenso wie für die Überlassung des hessischen Datensatzes für den Ost-West-Vergleich. 
einiger Formen (Hauptschulen/Realschulen) im Hinblick auf den Ost-WestVergleich reduziert worden ist. Aus dieser unterschiedlichen Struktur ergeben sich methodische Probleme und Einschränkungen beim Schulformvergleich (vgl. Kap. 2).

Eine weitgehende Verallgemeinerbarkeit der Untersuchungsergebnisse wird durch das bereits erwähnte Verfahren der Stichprobenkonstruktion gewährleistet. Dieses sieht im ersten Schritt eine gezielte Auswahl von Regionen und Städten vor, die für das jeweilige Bundesland charakteristisch sind und neben einer Metropole sowohl städtische als auch ländliche Strukturen berücksichtigt. Eine Repräsentativität für Sachsen wurde auf dieser Ebene durch eine - diesen regionalen Strukturen entsprechende - Auswahl der Schulstandorte zu erreichen versucht. Der zweite Schritt beruht auf einer Zufallsauswahl der Schulen in den Regionen, wobei für den Vergleich der Schulformen an den zufällig ausgewählten Standorten möglichst nahe beieinander liegende Mittelschulen, Gymnasien und Förderschulen in die Untersuchung einbezogen wurden. Die Lehrerbefragung wurde als Totalerhebung an den so ausgewählten Schulen durchgeführt. Für die Schülerbefragung folgte eine weitere Stufe der Zufallsauswahl der Klassen in den zur Befragung anstehenden Jahrgängen. In Sachsen gab es im Schuljahr vor Durchführung der Befragung (1994) insgesamt 395.070 Schüler/innen, die 965 staatliche Schulen in den genannten Schulformen besuchten. Insgesamt ergibt sich folgende Verteilung der Grundgesamtheit: 663 Mittelschulen mit 217.299 Schüler/innen, 191 Gymnasien mit 158.945 Schüler/innen, 94 Förderschulen für Lernbehinderte mit 17.490 Schüler/innen und 17 Förderschulen für Erziehungshilfe mit 1.336 Schüler/innen.

Um aus dieser Grundgesamtheit eine repräsentative Stichprobe zu erhalten, wurde versucht, in den ausgewählten Regionen eine, den tatsächlichen Verteilungen der Schülerschaft (z. B. bezüglich der Proportionen zwischen den Schulformen und der Geschlechteranteile in den jeweiligen Schulformen) entsprechende Auswahl von Schulen und Klassen zu erreichen. In mehrzügigen Schulen wurden die Klassen, in denen die Befragungen stattfanden, innerhalb der Jahrgänge nach dem Zufallsprinzip ausgewählt. In den (kleineren) Mittelschulen wurde eine Befragung von in der Regel zwei und den (größeren) Gymnasien in der Regel drei Schulklassen pro Jahrgang durchgeführt, um auch klassenspezifische Unterschiede und Besonderheiten im Rahmen der Einzelschulen, der Schulformen und des gesamten Schulsystems differenziert herausarbeiten zu können.

Die Qualität der Stichprobe sollte - wie bereits angesprochen - u.a. durch eine Auswahl von Regionen und Städten gewährleistet werden, die für den Freistaat Sachsen typisch sind. Zu dieser Typik zählen: die Gliederung in drei Regierungsbezirke mit entsprechenden Schulaufsichtsstrukturen (Oberschulämter), die sich regional an den drei Großstädten Dresden, Leipzig und Chemnitz ausrichten; das Fehlen weiterer Großstädte unterhalb dieser Größenordnung; eine relativ geringe Bevölkerungsdichte im ländlichen Raum und das Vorhandensein strukturschwacher Gebiete im Grenzbereich zu Polen 
und Tschechien. Etwa $30 \%$ der Bevölkerung lebt in den Großstädten, die als politisches und kulturelles Zentrum (Dresden) und als Wirtschafts- und Handelszentrum (Leipzig) Metropolencharakter besitzen sowie einen wichtigen Industriestandort darstellen (Chemnitz). Aus der Ortsklassengröße 1 (Großstadt mit Metropolencharakter) wurde die Stadt Dresden als Untersuchungsstandort (entspricht einem Oberschulamtsbereich) ausgewählt. Etwa $20 \%$ der Bevölkerung lebt in Städten mit Einwohnerzahlen zwischen 100.000 und 20.000. Typisch für Sachsen ist die Vielzahl von kleineren Städten am unteren Rand dieser Marge. Als Untersuchungsstandorte wurden drei kleinere Städte mit Einwohnerzahlen bis 26.000 (Ortsklassengröße 2) aus einem zweiten Oberschulamtsbereich (Leipzig) ausgewählt: Borna, Delitzsch, Eilenburg. Wesentlich größere Städte gibt es im Regierungsbezirk Leipzig nicht, so daß diese Auswahl eine Repräsentanz für diese Region darstellt. Etwa $50 \%$ der sächsischen Bevölkerung lebt in Gemeinden unter 20.000 Einwohner, davon ca. 12 $\%$ in Dörfern unter 2.000 Einwohnern. Da letztere jedoch kaum über eigene Schulen im Sekundarbereich verfügen, haben wir uns für eine breit gefächerte Erhebung in einem überwiegend ländlichen Kreis des Regierungsbezirkes Chemnitz (dritter Oberschulamtsbereich) entschieden. Ausgewählt wurde der Aue-Schwarzenberg-Kreis im Erzgebirge mit zwei größeren und sieben kleineren Gemeinden: Schwarzenberg (19.013 Einwohner), Schneeberg (18.210 Einwohner), Johanngeorgenstadt (8.253 Einwohner), Bockau (unter 5.000 Einwohner), Bernsbach (unter 5.000 Einwohner), Eibenstock (6.419 Einwohner), Schlema (6.340 Einwohner), Lauter (5.499 Einwohner), Zschorlau (unter 5.000 Einwohner). Diese Gemeinden werden der Ortsklassengröße 3 zugerechnet; bei den Regionalanalysen werden aber die beiden größeren Gemeinden dieser Gruppe von Schulstandorten mit denen der Ortsklasse 2 abgeglichen. Tabelle 1-2 gibt eine Übersicht über diese Stichprobenkonstruktion.

Nach der Genehmigung der Untersuchung durch das Sächsische Staatsministerium für Kultus wurden die ausgewählten Schulen angeschrieben, über unser Vorhaben informiert und um Mitarbeit gebeten. Nach zwei Wochen nahmen wir telefonischen Kontakt mit den Schulleitungen auf, um uns zu vergewissern, ob diese bereit waren, an der Untersuchung mitzuwirken. Bei positiver Resonanz schickten wir den entsprechenden Schulen Briefe mit ausführlichen Informationen über die Ziele und den Ablauf der Befragung. Diese Informationen richteten sich in getrennten Schreiben an Lehrer, Schüler und Eltern. Bestandteil des Elternbriefes war eine Einverständniserklärung, mit der von den Erziehungsberechtigten eine Teilnahme ihrer Kinder an der Untersuchung ermöglicht, aber auch verweigert werden konnte. Einige wenige Schulen sahen sich nicht in der Lage, an der Untersuchung teilzunehmen. Die Nichtbeteiligung wurde mit Zeitproblemen und Belastungen durch andere Befragungen oder Modellversuche begründet. Als Ersatz wurden Schulen derselben Schulform, Region und Ortsklassengröße ausgewählt.

Die Hauptuntersuchung fand vom 26. Oktober 1995 bis 16. Januar 1996 statt. Die Schülerbefragung wurde von jeweils einem Projektmitarbeiter an 
Tab. 1-2: Verteilung der Schulen, Klassen und Schüler nach Region und Schulform

\begin{tabular}{|c|c|c|c|c|}
\hline Region & $\begin{array}{l}\text { Bereich } \\
\text { Oberschulamt }\end{array}$ & $\begin{array}{l}\text { Bereich } \\
\text { Oberschulamt }\end{array}$ & $\begin{array}{l}\text { Bereich } \\
\text { Oberschulamt }\end{array}$ & Insgesamt \\
\hline Schulform & Dresden* & Leipzig** & Chemnitz ${ }^{* * *}$ & \\
\hline \multirow{3}{*}{ Mittelschulen } & Schulen: 3 & Schulen: 3 & Schulen: 10 & Schulen: 16 \\
\hline & Klassen: 19 & Klassen: 18 & Klassen: 56 & Klassen: 93 \\
\hline & Schülerzahl: 354 & Schülerzahl: 324 & Schülerzahl: 1019 & Schülerzahl: 1697 \\
\hline \multirow[t]{3}{*}{ Gymnasien } & Schulen: 2 & Schulen: 2 & Schulen: 3 & Schulen: 7 \\
\hline & Klassen: 18 & Klassen: 18 & Klassen: 24 & Klassen: 60 \\
\hline & Schülerzahl: 383 & Schülerzahl: 367 & Schülerzahl: 515 & Schülerzahl: 1265 \\
\hline \multirow[t]{3}{*}{ Förderschulen } & Schulen: 2 & Schulen: 2 & Schulen: 3 & Schulen: 7 \\
\hline & Klassen: 6 & Klassen: 7 & Klassen: 13 & Klassen: 26 \\
\hline & Schülerzahl: 48 & Schülerzahl: 46 & Schülerzahl: 91 & Schülerzahl: 185 \\
\hline \multirow[t]{3}{*}{ Insgesamt } & Schulen: 7 & Schulen: 7 & Schulen: 16 & Schulen: 30 \\
\hline & Klassen: 42 & Klassen: 42 & Klassen: 91 & Klassen: 179 \\
\hline & Schülerzahl: 785 & Schülerzahl: 737 & Schülerzahl: 1625 & Schülerzahl: 3147 \\
\hline
\end{tabular}

* Schulen in der Stadt Dresden

** Schulen in mittelgroßen Städten nahe dem Ballungsgebiet Leipzig (Borna, Delitzsch, Eilenburg)

*** Schulen in Kleinstädten und ländlichen Gemeinden des Oberschulamtsbereiches Chemnitz (Schneeberg, Schwarzenberg, Johanngeorgenstadt, Eibenstock, Schlema, Raschau, Zschorlau, Lauter, Bernsbach, Bockau)

den jeweiligen Schulen im Klassenverband durchgeführt, wobei für die Beantwortung der ausführlichen Version des Fragebogens - Schüler der 6. Jahrgänge und Förderschüler erhielten eine Kurzfassung - zwei Schulstunden benötigt wurden. Im Anschluß an die Klassenbefragungen führten wir ein Schulleiterinterview und eine Schulbegehung durch, bei der Eindrücke über das Schulgebäude, Schulgelände und Pausensituationen in einem Inspektionsbogen festgehalten wurden. Im Anschluß an den jeweiligen Schulbesuch wurde von dem Erhebungsteam ein kurzes Schulportrait angefertigt.

Nach erfolgter Datenübertragung und Prüfung der Stichprobenqualität wurden einige Nacherhebungen durchgeführt, um die Repräsentanz der Schulformen, der ländlichen Schulen und die Geschlechterverteilungen - der Grundgesamtheit entsprechend - in der Stichprobe zu verbessern. Die folgenden Tabellen geben Auskunft über die Stichprobenkonstruktion und die Qualität der endgültigen Stichprobe. Eine Überprüfung der Stichprobenqualität erfolgte nach den Kriterien Region, Ortsklassengröße, Schulform, Klassenstufe und Geschlecht. Bei allen Parametern bestanden keine oder nur geringfügige Differenzen zwischen Grundgesamtheit und Stichprobe. Bei den Schulformen sind die Mittelschulen leicht überproportioniert und in dieser Schulform der Mädchenanteil ebenfalls. Für die Interpretation der Daten zur Gewaltbeobachtung und zum Täter-Selbstreport bedeutet das, daß unsere Aussagen zur Be- 
lastung des sächsischen Schulsystems und insbesondere zur Mittelschule sehr realistisch sind und keinesfalls eine Übertreibung darstellen. In der hessischen Stichprobe finden sich entsprechende Verschiebungen (Anteil der Haupt- und Realschulen ist etwas zu klein), so daß für den Ost-West-Vergleich in dieser Hinsicht keinerlei methodische Probleme bestehen. Bei bi- und multivariaten Verfahren fallen derartige Verschiebungen in der Stichprobe (Abweichungen von 3 bis 5 Prozentpunkten bei den Qualitätskriterien) kaum noch ins Gewicht und können bei der Interpretation vernachlässigt werden. Der Rücklauf der Schüler- bzw. Lehrerbefragungen wird in den Tabellen 1-3 und 1-4 dargestellt.

Tab. 1-3: Rücklauf der Schülerbefragung nach Schulform und Klassenstufe

\begin{tabular}{lccccccc}
\hline & \multicolumn{3}{c}{ Schulform } & \multicolumn{3}{c}{ Klassenstufe } & Gesamt \\
& $\begin{array}{c}\text { Gymna- } \\
\text { sium }\end{array}$ & $\begin{array}{c}\text { Mittel- } \\
\text { schule }\end{array}$ & $\begin{array}{c}\text { Förder- } \\
\text { schule }\end{array}$ & Kl. 6 & Kl. 8 & Kl. 9/10 & \\
Soll & 1441 & 2194 & 325 & 1329 & 1342 & 1289 & 3960 \\
Ist & 1265 & 1697 & 185 & 1028 & 1104 & 1015 & 3147 \\
Prozent & 88 & 77 & 57 & 77 & 82 & 79 & 79 \\
\hline
\end{tabular}

Tab. 1-4: Rücklauf der Lehrerbefragung (in Prozent)

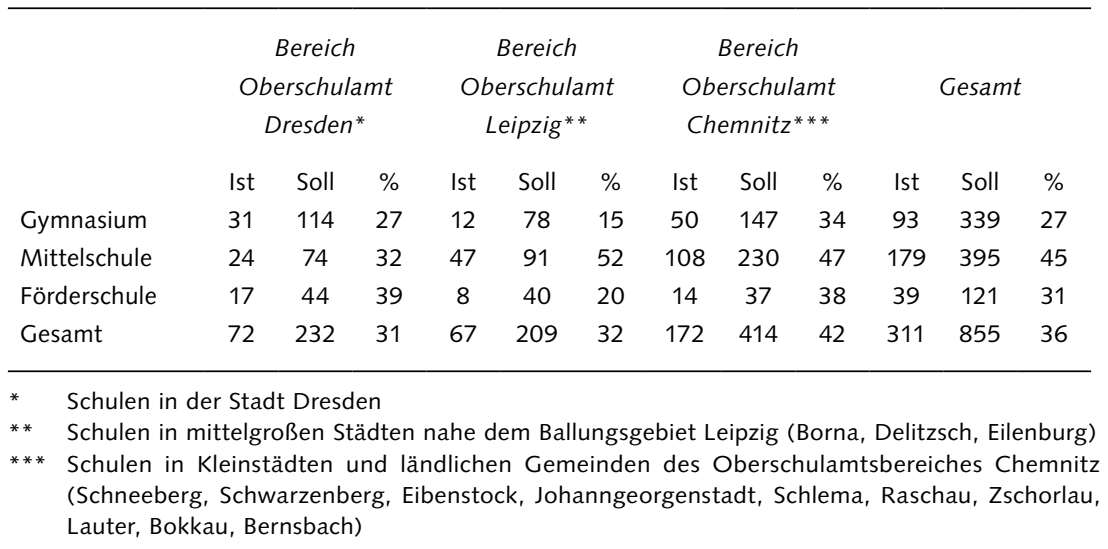


Zur Befragung der Lehrerschaft der jeweiligen Schule übergaben wir der Schulleitung die erforderliche Anzahl von Fragebögen. Die ausgefüllten Exemplare wurden von der Schulleitung gesammelt und an uns zurückgesandt. Die Anonymität blieb dadurch gewahrt, daß jeder Lehrerfragebogen in einem verschlossenen Briefumschlag abgegeben wurde. Die Lehrer hatten ebenso die Möglichkeit, den Fragebogen direkt an die Forschungsgruppe zurückzuschicken. Wie die Tabelle 1-4 zeigt, ist der Rücklauf der Lehrerbefragung trotz des betriebenen Aufwandes sehr niedrig. Insgesamt konnten bei der Überprüfung der Stichprobenqualität für die Schülerbefragung sehr gute, für die Lehrerbefragung weniger gute Werte ermittelt werden. Daraus wurde die Konsequenz gezogen, die Lehrerdaten nur flankierend und nicht durchgängig systematisch-vergleichend bei der Analyse mit heranzuziehen.

Die bislang dargestellten Verfahren und Methoden der Erhebung beziehen sich auf die quantitativen Schüler- und Lehrerbefragungen, die im Rahmen einer simultanen Komparation in Sachsen und Hessen umgesetzt wurden. Über die Ergebnisse dieser quantitativen Untersuchungen wird in den folgenden Kapiteln berichtet (Kap. 1 bis 7).

Die in Kapitel 8 dargestellten Ergebnisse einer Pilotstudie zur Gewaltprävention basieren zum einen ebenfalls auf den quantitativen Erhebungen (vgl. Darstellung der Qualitäts- und Gewaltprofile), zum anderen auf einer Reihe von qualitativen Untersuchungsverfahren und Instrumenten (z. B. Gruppendiskussionen, soziometrische Tests, Beurteilungen der Klassensituation und Gruppenstruktur durch Lehrer), die in dem entsprechenden Kapitel dargestellt bzw. im Anhang dokumentiert sind.

\section{Inhalt und Gliederung des vorliegenden Bandes}

Der vorliegende Band ist wie folgt aufgebaut. In Kapitel 2 werden die ermittelten Gewaltformen im Ost-West-Vergleich dargestellt und im Hinblick auf weitere Differenzierungen nach Schulform, Jahrgang und Geschlecht interpretiert. Die empirische Grundlage für dieses Kapitel sind zunächst die Beobachtungen von Schülern. Danach geht es um unterschiedliche Aspekte der Gewaltphänomenologie, festgemacht an konkreten Handlungssituationen: Gewalt von Schülern innerhalb und außerhalb der Schule, Selbstreport als Täter, Beurteilung der Interventionsbereitschaft der Lehrer durch die Schüler, eigene Einstellungen und Verhalten der Schüler. Unterschiede der beiden untersuchten Bundesländer, der Schulform, des Jahrgangs und des Geschlechts sollen hier ebenso herausgearbeitet werden, wie beim anschließenden Punkt, in dem die verschiedenen Perspektiven von Gewalt - die der Wahrnehmung, des Selbstreports und der situationsunabhängigen Einstellungen zur Gewalt - miteinander verglichen werden. Welche Bedeutung dem Ost-West-Vergleich bei der schulischen Gewaltthematik insgesamt zukommt, soll als Resümee des Kapitels 2 festgehalten werden. 
Bereits bei ersten Interpretationen zu den Gewaltformen und Gewaltsituationen werden geschlechtsspezifische Unterschiede zwischen Jungen und Mädchen deutlich, die im Kapitel 3 unter dem Titel ,Jungengewalt - Mädchengewalt“ systematisiert werden. Dargestellt werden - über die vertiefende Interpretation bereits vorgestellter Skalen hinaus - Ergebnisse zu spezifischen Fragen, die von Jungen und Mädchen im quantitativen Fragebogen getrennt zu beantworten waren (z.B nur für Jungen: „Ich schlage mich besonders gern, wenn Mädchen dabei zuschauen.“; nur für Mädchen: „Wenn sich Jungen prügeln, schaue ich gerne zu."). Hinzu kommt die Auswertung qualitativen Materials aus Gruppendiskussionen mit Mädchen und Jungen. Das Kapitel basiert ausschließlich auf sächsischem Datenmaterial.

Ein Schwerpunkt unserer Forschungsgruppe sind Täter-Opfer-Analysen. In Kapitel 4 wird eine Täter-Opfer-Typologie vorgestellt, die mit Hilfe von Clusteranalysen erstellt wurde. Weiterhin werden mit Hilfe von Mittelwertvergleichen die Prädiktoren für den Täter- bzw. Opfer-Status auf individueller Ebene herausgearbeitet. In Ergänzung zu bereits vorgelegten Publikationen sollen hier die unterschiedlichen Gruppen (Täter, Opfer, Episoden-Täter, TäterOpfer, Unbeteiligte) genau beschrieben und mit Hilfe individueller Merkmale charakterisiert werden.

Die herausgefundenen Prädiktoren des Täter-Opfer-Status werden als Systematik für die folgenden beiden Kapitel 5 und 6 genutzt. In den Abschnitten über das außerschulische Bedingungsgefüge wird in Kapitel 5 nach den Einflüssen der familialen Sozialisation, der Gleichaltrigengruppen und des Freundeskreises sowie des Medienkonsums in der Freizeit gefragt. Bei der Analyse der schulischen Lernbedingungen in Kapitel 6 geht es um Lern-, Erziehungsund Kommunikationskultur als hypothetische Prädiktoren schulischer Gewalt, die - falls sich die Annahmen bestätigen - gleichzeitig Ansatzpunkte schulischer Präventions- und Interventionsmaßnahmen darstellen. Um in bezug auf den Einfluß schulischer Lernbedingungen auf die Gewalt zu „objektiveren“ Ergebnissen zu gelangen, werden die Daten in diesem Kapitel auf Klassenebene aggregiert, d.h. Grundlage der Interpretation sind hier intersubjektive Befunde bzw. Parameter der Klassensituation und nicht mehr bloß individuelle Schülerwahrnehmungen.

In Kapitel 7 erfolgt die Prüfung eines Gesamtmodells durch eine Mehrebenenanalyse, in der die Gewaltemergenz im Kontext des Lebenszusammenhangs von Schülerinnen und Schülern, der institutionellen Bedingungen sowie der äußeren bzw. inneren Regulationsmechanismen erklärt werden soll. Mit diesem Kapitel wird die Systematik der empirischen Analyse (Hypothesen in Kapitel 1, Modellprüfung in den Kapiteln 2, 4, 5 und 6) abgeschlossen.

Thema des Kapitel 8 sind die Möglichkeiten und Erfahrungen mit Gewaltprävention und -intervention. Hierzu stellen wir ein von uns entwickeltes Modell vor, das in einer Pilotstudie eingesetzt und erprobt wurde. Es schließt an die Ergebnisse der quantitativen Untersuchung an, spiegelt diese den Lehrern und der Schulleitung einer Einzelschule in Form eines „Gewalt- und Schulqua- 
litätsindexes" zurück und führt im Diskurs von Wissenschaft und Praxis bzw. von wissenschaftlicher Analyse und den Alltagstheorien der Pädagogen und anderer Gruppen, die an Schule beteiligt sind, zu möglichen gemeinsamen Schlußfolgerungen und Maßnahmen im Hinblick auf die Verbesserung der Schulkultur. Dieser schulumfassende Ansatz wird ergänzt durch klassenspezifische Analysen und Interventionen, die - bei entsprechender Notwendigkeit - auch zu einer individuellen Betreuung von Schülerinnen und Schülern führen. Um ein zentrales Ergebnis vorwegzunehmen: Verbesserungen der Schulkultur sind notwendig, reichen nach unseren Befunden jedoch nicht aus, da sich durch eine Entwicklung der Schulqualität und Schulkultur zwar eine Reduzierung des Gewaltvorkommens an Schulen erreichen läßt, Konzepte, wie das der „Lebenswelt Schule“, haben aber einen nur geringen Einfluß auf den Opfer-Status und die Anzahl der Opfer - und dies sind doch die eigentlichen Leidtragenden von Gewalt. 


\section{Schulische Gewaltformen und Konfliktsituationen im Ost-West-Vergleich \\ (Wilfried Schubarth, Christoph Ackermann)}

Im Rahmen der Studien zur Gewalt an Schulen, die von der Forschungsgruppe Schulevaluation in den letzten Jahren durchgeführt wurden, stellt der OstWest-Vergleich einen zentralen Forschungsschwerpunkt dar (vgl. Kap. 1). Gestützt auf transformationstheoretische Überlegungen ließen wir uns bei den komparativen Studien von der Annahme leiten, daß der radikale Umbruch der Lebensverhältnisse im Osten Deutschlands, darin eingeschlossen die Umstrukturierung des Schulsystems, nicht ohne Folgen für die Betroffenen, d.h. für Schüler und Lehrer, geblieben sein dürfte. Insbesondere gingen wir davon aus, daß es infolge von Modernisierungs- bzw. Individualisierungsprozessen auch im Bereich der Schule nicht nur zu einem Zugewinn an Freiheitsgraden (z.B. mehr Wahlmöglichkeiten, erhöhte Eigenverantwortung), sondern auch zu neuartigen Risiken und Belastungen (z. B. infolge verstärkter Selektion und erhöhten Erwartungsdrucks) kommen würde (vgl. z. B. Melzer/Hurrelmann 1990, Flösser/Otto/Tillmann 1996, Schubarth/ Stenke/Melzer 1996).

Unabhängig davon, ob die seit dem Untergang der DDR vollzogene Systemtransformation als ,nachholende Revolution“ (Habermas 1990), als „Kolonialisation“ (Dümcke/Vilmar 1995) oder - wie von den meisten Sozialwissenschaftlern - als ,nachholende“ bzw. „weitergehende Modernisierung“ (Zapf 1991) gedeutet wird, herrscht Konsens darüber, daß die tiefgreifenden Veränderungen in nahezu allen Lebensbereichen von den Menschen in den neuen Bundesländern enorme Anpassungsleistungen erforderten bzw. noch erfordern. Wenn in einer solchen anomischen Situation die neuen Anforderungen die vorhandenen sozialen Bewältigungskompetenzen und Ressourcen des Einzelnen übersteigen, können Aggression und Gewalt - zumal im Kindes- und Jugendalter - wahrscheinlich werden. Aggressionen können dabei sowohl nach außen als auch nach innen gerichtet sein (vgl. Mansel/ Hurrelmann 1994). In diesem Sinne sehen wir im Auftreten von Aggression und Gewalt eine mögliche Bewältigungsform des Umbruchs, wobei der Umbruch selbst - unter modernisierungstheoretischer Perspektive betrachtet - als ein besonderer Individualisierungsschub mit überaus ambivalenten Wirkungen für die Betroffenen interpretiert werden kann.

Sozialwissenschaftler in Ost und West haben seit dem Zusammenbruch der DDR den Prozeß der deutschen Vereinigung als ,,sozialen Großversuch“ (Gießen/Leggewie 1991) wissenschaftlich begleitet und in Form von empirischen Studien vielfach zu beschreiben versucht. ${ }^{1}$ Nicht nur in der Jugendfor-

1 Allein im Rahmen der Kommission für die Erforschung des sozialen und politischen Wan- 
schung war dabei die Debatte um die Integration der ostdeutschen Jugend in das vereinte Deutschland von zentraler Bedeutung. Damit war auch die Frage bzw. die Sorge verbunden, ob aus den bestehenden Integrationsproblemen eine neue neonationale, gewaltbereite Jugendprotestbewegung erwachsen könnte (vgl. Zinnecker 1995). Auch wenn solche Ängste - nach dem Abklingen der Welle spektakulärer, exzessiver fremdenfeindlicher Gewalt in der ersten Hälfte der neunziger Jahre - zwischenzeitlich weitgehend unberechtigt scheinen, und sich die befürchtete Jugendprotestbewegung nicht abzeichnet, so lassen doch einige Befunde und Entwicklungstendenzen auf ein nicht unerhebliches latentes Problem- bzw. Protestpotential unter ostdeutschen Jugendlichen schließen. Als solche Indizien können z. B. gelten:

- die seit dem Umbruch angestiegene Kriminalitätsrate bei ostdeutschen Jugendlichen, die mittlerweile z.T. über der des Westens liegt (vgl. z. B. Kury/Obergfell-Fuchs 1995, Frehsee 1995),

- der starke Anstieg von Rechtsextremismus und fremdenfeindlicher Gewalt in den neuen Ländern zu Beginn der neunziger Jahre (vgl. z. B. Otto/Merten 1993, Willems 1993),

- die in verschiedenen Studien registrierte höhere Gewaltakzeptanz und Gewaltbereitschaft bei ostdeutschen Jugendlichen (Bundesministerium für Frauen, Familie, Jugend und Senioren 1994, Schmidtchen 1997),

- das geringe Vertrauen der Jugendlichen in den neuen Bundesländern in die Politik und gegenüber den etablierten Parteien, was als „Politik- bzw. Politikerverdrossenheit" thematisiert wird (vgl. z. B. Melzer 1992, Hoffmann-Lange 1995, Jugendwerk 1997).

Um Mißverständnissen vorzubeugen: Gewalt ist weder ein typisch ostdeutsches Phänomen, noch ein typisches Phänomen, das ausschließlich Jugendliche betrifft. Gewalt ist vielmehr ein gesamtgesellschaftlich zu verantwortendes Problem. So sind unter den Jugendlichen in den alten Bundesländern recht ähnliche Tendenzen festzustellen. Hinsichtlich der angeführten Befunde gibt es keine prinzipiellen, sondern höchstens graduelle Differenzen. Dennoch ist das Problempotenial bzw. der Problemdruck in den neuen Bundesländern offensichtlich größer. Unser zugrunde gelegter Modernisierungs- und Transformationsansatz, ließ uns (gestützt auf die oben genannten Überlegungen und vorhandene empirische Befunde) deshalb zu der Hypothese gelangen, da - bezogen auf unsere Schulstudien zu Gewalt - auch die Gewaltbelastung an Schulen in den neuen Bundesländern im Vergleich zu der in den alten Bundesländern größer sein müßte.

dels in den neuen Bundesländern (KSPW) entstanden von 1992 bis 1996 ca. 150 Kurzstudien, 60 größere Projekte und 120 Expertisen (vgl. Buhlmann 1997). Fragen von Aggression und Gewalt spielen dabei allerdings eine eher untergeordnete Rolle. Zum Transformationsprozeß im Schulbereich vgl. neben dem Sammelband von Flösser/Otto/Tillmann (1996) z. B. auch Dudek/Tenorth 1993, Neunter Jugendbericht 1994, Weishaupt/Zedler 1994, Döbert 1995a, Hoyer 1996 und Stenke/Melzer 1996. 
Unsere bisherigen Schulstudien zum Ost-West-Vergleich bestätigten diese Annahme jedoch nicht. Sowohl die vergleichende Schulleiterbefragung unter Einbeziehung der Bundesländer Sachsen, Thüringen, Hessen und Baden-Württemberg (vgl. Melzer/Schubarth/Tillmann 1995, Schubarth 1996, Kolbe 1996, Weishaupt 1996) als auch erste Auswertungen der in diesem Band ausführlich vorgestellten repräsentativen Schülerbefragung in Sachsen, die mit einer entsprechenden Befragung in Hessen verglichen werden kann, ergaben vielmehr, daß das Gewaltniveau in Ostdeutschland unter dem in Westdeutschland liegt (vgl. Schubarth 1995, Schubarth/Darge/Mühl/Ackermann 1997). Auch einige andere vergleichende Untersuchungen bestätigten ein geringeres Gewaltniveau an ostdeutschen Schulen (vgl. z. B. Dettenborn/ Lautsch 1993, Hurrelmann/Pollmer 1994). Bei der Interpretation dieser Befunde verwiesen wir auf zwei Erklärungsmuster: zum einen auf die Zählebigkeit und das Beharrungsvermögen der früheren Schul- und Erziehungskultur Ostdeutschlands (z. B. relativ hoher Stellenwert von Disziplin, Kontrolle, Konformität, Autorität) und zum anderen auf das Vorhandensein bestimmter sozialer Elemente aus der DDR-Schule (z. B. Arbeitsgemeinschaften, Förderangebote, Lernpatenschaften; zu bewahrenswerten Elementen der DDRSchule vgl. auch Prüß/Bettmer 1996, S. 241ff.). Allerdings wurden in unseren Vergleichsuntersuchungen bereits auch gewisse Angleichungstendenzen sichtbar. So waren nur bei den extremen, ,härteren“ Gewaltformen die Ost-West-Differenzen noch deutlich, während diese bei den eher alltäglichen Formen wie Beschimpfungen und Unterrichtsstörungen nahezu nivelliert waren.

Im vorliegenden Beitrag soll - aufbauend auf den bisherigen Ergebnissen und Erkenntnissen - der Ost-West-Vergleich vor allem in zweifacher Hinsicht weitergeführt und vervollständigt werden, indem:

1. das Ausmaß der verschiedenen schulischen Gewaltformen in der Wahrnehmung von Schülern in Sachsen und Hessen verglichen und dabei der Einfluß der Merkmale Geschlecht, Jahrgangsstufe und Schulform geprüft werden soll (erster Teil),

2. der Ost-West-Vergleich auch auf die Analyse konkreter Gewaltsituationen und des Interventionsverhaltens von Schülern und Lehrern in diesen Situationen ausgedehnt wird (zweiter Teil) und

3. Reaktionsweisen auf Gewalthandeln (z. B. Nachahmung oder Angst) einbezogen werden (dritter Teil).

Mit diesen weitergehenden Ost-West-Analysen soll vor allem geklärt werden, ob der bisher diagnostizierte Befund von der geringeren Gewaltbelastung im Osten erhärtet wird oder ob er relativiert bzw. modifiziert werden muß. Da wir entsprechend unseres modernisierungs- und transformationstheoretischen Ansatzes perspektivisch betrachtet, von einer Angleichung des Gewaltniveaus der ostdeutschen Schulen an das westdeutscher Schulen ausgehen, war daneben von besonderem Interesse, ob sich Befunde ermitteln lassen, die auf eine solche Angleichung hindeuten könnten. Damit sollten ggf. auch Prognosen über künftige Gewaltentwicklungen ermöglicht werden. 
Die empirische Basis bilden die repräsentativen Schülerbefragungen in Sachsen und Hessen, wobei wir uns im ersten Teil auf die Wahrnehmungen der verschiedenen schulischen Gewaltformen aus Sicht der Schüler konzentrieren und im zweiten Teil auch Ergebnisse aus dem Schüler-Selbstreport und Befunde aus der Lehrerbefragung mit einbeziehen. ${ }^{2}$

\subsection{Schulische Gewaltformen in der Wahrnehmung von Schülern}

Die zahlreichen Schulstudien zu Aggression und Gewalt, die seit Anfang der neunziger Jahre durchgeführt wurden, haben dazu beigetragen, daß mittlerweile ein empirisch recht fundiertes Bild von der Situation an Schulen vorliegt. Dies gilt insbesondere für die Formen und die Häufigkeiten von Gewalterscheinungen sowie für die Schulform-, Alters- und Geschlechtsunterschiede (vgl. z. B. Funk 1994, Schwind u.a. 1995, Fuchs/Lamnek/Lüdtke 1996, Tillmann 1997a, Schubarth 1998). So ist durch die bisherigen Schuluntersuchungen gut belegt, daß unter den schulischen Gewaltphänomenen die verbalen Aggressionen dominieren, während extreme Formen (z. B. Erpressung oder Waffeneinsatz) relativ selten sind. Dabei gilt folgende Regel: Je härter die Gewaltformen sind, desto seltener treten sie auf und umgekehrt. Das zeigt sich auch bei den Gewaltformen, die wir in unsere Analysen zum Ost-West-Vergleich einbezogen haben (vgl. Abb. 2-1).

Die einzelnen Gewaltphänomene werden unter einem Oberbegriff zusammengefaßt, der einen Faktor im statistischen Sinne darstellt, d.h. eine von anderen Phänomenen unterscheidbare Situation. Im folgenden wollen wir das Ausmaß der in die Befragung einbezogenen Gewaltformen anhand von Stellvertreter-Items veranschaulichen. Dazu wurden diejenigen Items ausgewählt, die den entsprechenden Faktor besonders gut repräsentieren.

Abbildung 2-1 macht deutlich, daß die Rangreihung der wahrgenommenen Gewaltformen aus der Schülerperspektive in Sachsen und Hessen weitgehend übereinstimmt: An der Spitze stehen in Sachsen verbale Aggressionen, es folgen leichte körperliche Auseinandersetzungen (,Spaßkampf“), verbale Aggressionen gegenüber Lehrern, dann ernsthafte Prügeleien unter Schülern, Vandalismus, sexuelle Belästigungen, Erpressungen und schließlich Angriffe mit einer Waffe. Die Rangreihung der einzelnen Gewaltformen unterscheidet sich zwischen Sachsen und Hessen lediglich in einer Position: In Sachsen treten verbale Aggressionen gegenüber Lehrern häufiger auf als Prügeleien

2 Zu Ergebnissen des Vergleichs von Schüler- und Lehrerperspektive hinsichtlich schulischer Gewaltphänomene vgl. Schubarth 1997. 


\section{Abb. 2-1: Wahrgenommene schulische Gewaltformen in Sachsen und Hessen (Prozentanteil der Schüler, der mindestens mehrmals im Monat die angeführte Gewaltform beobachtet hat) ${ }^{*}$}

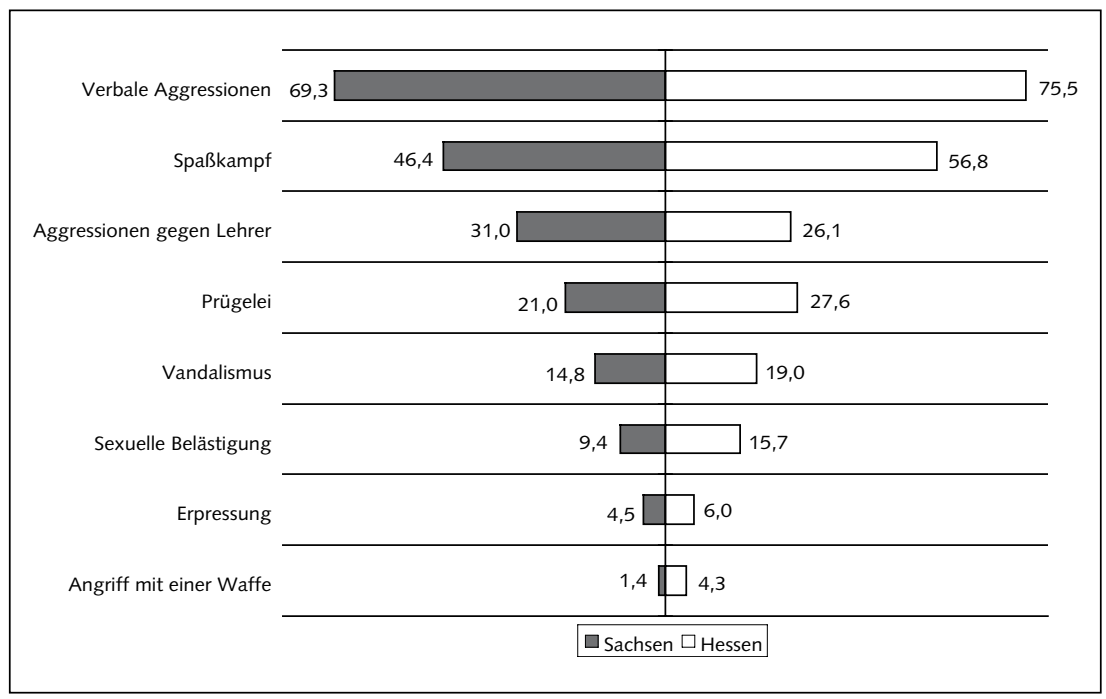

Legende:

Verbale Aggressionen:

Spaßkampf:

Aggressionen gegen Lehrer:

Prügelei:

Vandalismus:

Sexuelle Belästigung:

Erpressung:

Angriff mit einer Waffe:
Beschimpfen/Gemeine Ausdrücke. (V157)

Spaßkampf zwischen zwei Jungen. (V136)

Schüler/innen beschimpfen oder beleidigen Lehrpersonen in deren Gegenwart. (V146)

Ernsthafte Prügelei zwischen zwei Jungen. (V137)

Unterrichtsmaterial wurde absichtlich beschädigt. (V133)

Ein oder mehrere Jungen bedrängen ein Mädchen $u$. fassen es gegen ihren Willen an (z. B. an den Busen). (V144)

Jemand fordert Geld und droht mit Strafe (z.B. Prügel), wenn nicht gezahlt wird. (V162)

Jemand wird von einem (einer) anderen mit einer Waffe (Schlagring, Messer usw.) angegriffen. (V143)

* Bei den Balken handelt es sich um die zusammengefaßten Werte „fast täglich“, ,,mehrmals wöchentlich“ und „mehrmals im Monat“ einer 6- bzw. 5-stufigen Skala. In Sachsen und Hessen wurden nicht völlig identische Skalen verwendet. Während in Sachsen eine sechsstufige Skala eingesetzt wurde (,fast täglich“, „mehrmals wöchentlich“, „,mehrmals im Monat“, ,,alle paar Monate“, ,,seltener“, „,nie“), wurde in Hessen eine fünfstufige Skala ohne die Kategorie „seltener“ verwendet. 
- in Hessen ist es umgekehrt. Auffällig ist in beiden Bundesländern der relativ hohe Stellenwert (verbaler) Aggressionen gegenüber Lehrern. Auch die Differenzierung bei körperlichen Auseinandersetzungen zwischen Spaßkampf und Prügelei ist bemerkenswert. Während Spaßkampf eher ein alltägliches, latent gewalthaltiges Phänomen ist, kommen ernsthafte Prügeleien weitaus weniger vor, wobei die Trennschärfe zwischen beiden Phänomenen nicht immer eindeutig gegeben ist. Körperliche Attacken (in leichterer oder eher schwerer Form) werden in Sachsen wie Hessen häufiger wahrgenommen als Gewalt gegen Sachen. ${ }^{3}$ Im Gegensatz zu manchen Mediendarstellungen kommen Erpressung und Waffeneinsatz in beiden Bundesländern selten vor. Nur eine kleine Minderheit hat dies öfter beobachtet.

Hinsichtlich des Ost-West-Vergleichs ist des weiteren festzustellen, daß - von einer Ausnahme abgesehen (Aggressionen gegen Lehrer) - alle angeführten Gewaltformen in Sachsen weniger beobachtet werden als in Hessen. Die Differenzen liegen mit Ausnahme des Spaßkampfes zwischen 1,5\% und $6,6 \%$, sind also (obwohl statistisch signifikant) nicht allzu groß. Am größten sind die Unterschiede noch bei den „härteren“ Formen wie Angriff mit einer Waffe und bei den sexuellen Belästigungen. Ob die Ost-West-Differenzen bezogen auf die sexuellen Belästigungen möglicherweise auf die größere Sensibilisierung der hessischen Jugendlichen bei diesem Thema oder auf das eher ,problemlos-natürliche“ Verhältnis zur Sexualität bei den sächsischen Jugendlichen zurückzuführen ist, muß offen bleiben. Beide Annahmen schließen sich zudem nicht aus.

Was die Aggressionen gegenüber den Lehrern betrifft, so ist hier eine weitere Differenzierung erforderlich: Während die gegen Lehrer gerichteten verbalen Aggressionen in Sachsen etwas stärker verbreitet sind, ist es bei den „härteren“ Gewaltformen wie Beschädigung von Lehrereigentum oder Telefonterror wieder umgekehrt. Somit kann insgesamt festgehalten werden, daß das Gewaltniveau an sächsischen Schulen - in der Wahrnehmung der Schüler - geringer ist, womit unsere bisherigen Ergebnisse zum Ost-West-Vergleich, sowohl aus der Schulleiterbefragung als auch aus der Schülerbefragung, bestätigt werden. ${ }^{4}$

3 Im Unterschied zu Schülern und auch Lehrern nehmen Schulleiter mehr Vandalismus wahr als körperliche Gewalt. Die Nähe zum Tatgeschehen beeinflußt offensichtlich die Gewaltwahrnehmung. So ist anzunehmen, daß Schüler und Lehrer näher am körperlichen Gewaltgeschehen sind als Schulleiter. Umgekehrt haben Schulleiter schon aufgrund ihrer Dienststellung eine sensiblere Wahrnehmung gegenüber Erscheinungen von Vandalismus entwickelt. Ähnliches gilt für sexuelle Belästigungen (vgl. Schubarth 1997).

4 Dies gilt auch für die Ergebnisse aus dem Schüler-Selbstreport der „Täter“ bzw. „Opfer“ (vgl. Schubarth/Darge/Mühl/Ackermann 1997). 
Bei der Frage nach dem Einfluß des Geschlechts auf die Wahrnehmung schulischer Gewalt gingen wir - gestützt auf bisherige Ergebnisse - davon aus, daß Jungen aufgrund ihrer stärkeren Involvierung in gewalthaltige Auseinandersetzungen auch mehr Gewalt beobachten. Andererseits ist zu berücksichtigen, daß Mädchen sensibler gegenüber Gewalt sind (zum Gewaltbegriff von Mädchen vgl. Kap. 1) und geschlechtsspezifische Differenzen bei der Wahrnehmung von Gewalt geringer ausfallen als bei der selbstberichteten Gewalt (vgl. Kap. 3). Bezüglich des Ost-West-Vergleichs gingen wir aufgrund bisher vorliegender Untersuchungsergebnisse (vgl. Hoffmann-Lange 1995, Shell-Jugendstudie 1992) davon aus, daß geschlechtsspezifische Differenzen im Osten stärker ausgeprägt sein würden als im Westen.

Abb. 2-2: Wahrgenommene Gewaltformen in Sachsen und Hessen, differenziert nach Geschlecht (Prozentanteil der Schüler, der mindestens mehrmals im Monat die angeführte Gewaltform beobachtet hat)*

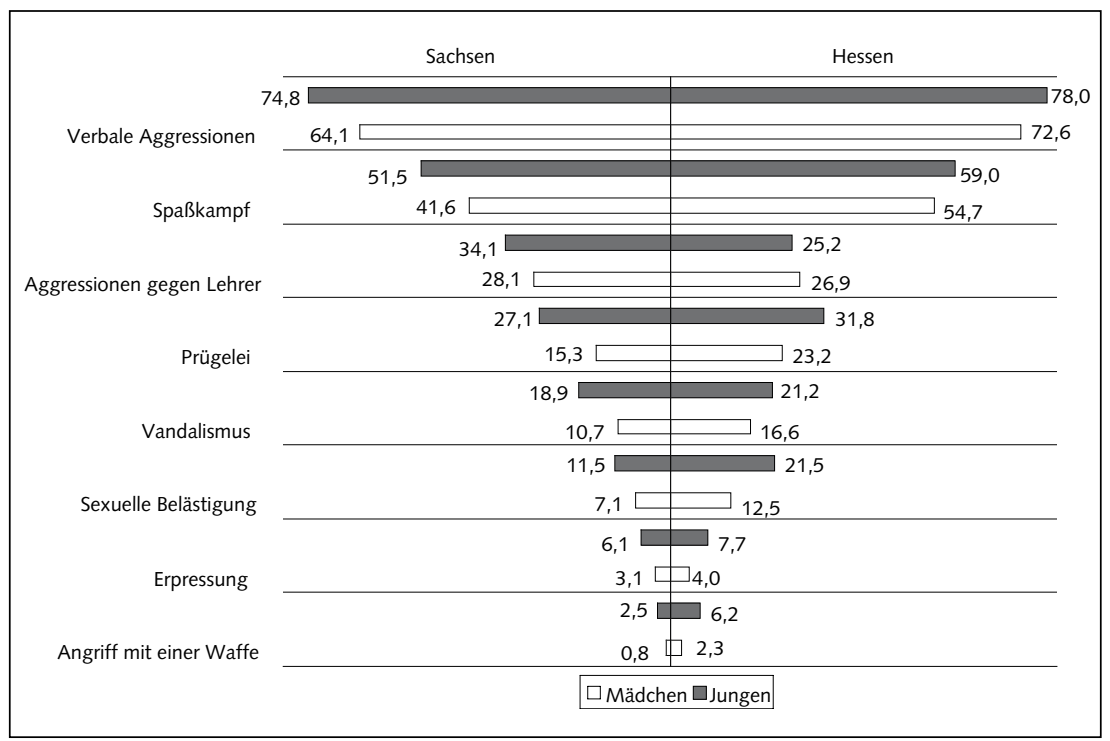

* Legende vgl. Abb. 2-1

Wie die Abbildung 2-2 zeigt, werden (mit einer Ausnahme) in Ost wie West die angeführten Gewaltformen häufiger von Jungen als von Mädchen wahrgenommen. Dieser Befund entspricht unseren Erwartungen. Auch der zweite Befund, daß - abgesehen von den verbalen Aggressionen gegenüber Lehrern - sowohl von den hessischen Jungen im Vergleich mit den sächsischen Jungen als auch von den hessischen Mädchen im Vergleich mit den sächsischen Mädchen 
mehr Gewalt beobachtet wird, liegt völlig im Rahmen unserer Erwartungen und bestätigt bisherige Forschungsergebnisse. Die verbalen Schüleraggressionen gegenüber Lehrern sind ein Sonderfall und zwar in doppelter Hinsicht: zum einen beobachten die hessischen Mädchen diese Gewaltform öfter als die hessischen Jungen, zum anderen wird dieses Gewaltphänomen als einzige Gewaltform in Sachsen sowohl von Jungen als auch von Mädchen stärker wahrgenommen als in Hessen.

Auch unsere andere Annahme, daß die Geschlechterdifferenzen im Osten größer ausfallen würden als im Westen, wird bestätigt. Die Differenzen in der Wahrnehmung zwischen Jungen und Mädchen sind in Sachsen in der Regel etwas größer als in Hessen (Ausnahmen sind Aggressionen gegen Lehrer und sexuelle Belästigungen). Daß die modernisierungsbedingten Angleichungsprozesse zwischen Jungen und Mädchen in bezug auf die Gewalt in Sachsen weniger vorangeschritten sind, könnte mit dem noch vorhandenen „Individualisierungsrückstand" bzw. mit dem Nachwirken eines konventionelleren Erziehungsklimas zu tun haben, wodurch Geschlechterstereotype verstärkt wurden bzw. werden. Darauf deutet auch der Befund hin, daß die Ost-West-Differenzen bei den Jungen tendenziell geringer sind als bei den Mädchen, so daß geschlußfolgert werden kann, daß bei den sächsischen Jungen die Angleichung bereits weiter fortgeschritten ist als bei den Mädchen. Auch hierfür könnte als Erklärung dienen, daß Frauen und Männer in der DDR - aufgrund des fehlenden öffentlichen Diskurses über geschlechtsspezifisches Rollenverhalten - auf der individuellen Ebene (im Gegensatz zur staatlich ,,verordneten“ Gleichberechtigung) eher traditionellen Geschlechterstereotypen verhaftet blieben (vgl. neben dem nachfolgenden Beitrag z. B. auch Hellwig/Nickel 1993, Gille 1995). Dies könnte auch heute noch Konsequenzen bezüglich der Affinität zur Gewalt haben, die traditionell eine Männerdomäne ist.

Bisherige Untersuchungen mit Blick auf die Rolle der Jahrgangsstufe ergaben, daß die Gewalt an Schulen in Ost wie West bis zur 8. bzw. 9. Klasse ansteigt und danach wieder abfällt. Dies ist zugleich ein wichtiger Hinweis darauf, daß Gewalthandeln auch Ausdruck eines systemübergreifenden, entwicklungspsychologisch bedingten Phänomens in der Phase der Pubertät und der jugendlichen Identitätsfindung ist. Unsere jahrgangsspezifischen Analysen der einzelnen Gewaltformen sollten die These von der „Gewaltspitze“ in der 8./9. Klasse (Tillmann 1997a, S. 41) überprüfen. Gleichzeitig gingen wir mit Blick auf den Ost-West-Vergleich davon aus, daß - aufgrund der vergleichbaren entwicklungspsychologischen Bedingungen in Ost und West - keine prinzipiellen Ost-West-Differenzen zu erwarten seien. 
Abb. 2-3: Wahrgenommene Gewaltformen in Sachsen und Hessen, nach Jahrgangsstufe differenziert (Prozentanteil der Schüler, der mindestens mehrmals im Monat die angeführte Gewaltform beobachtet hat)*

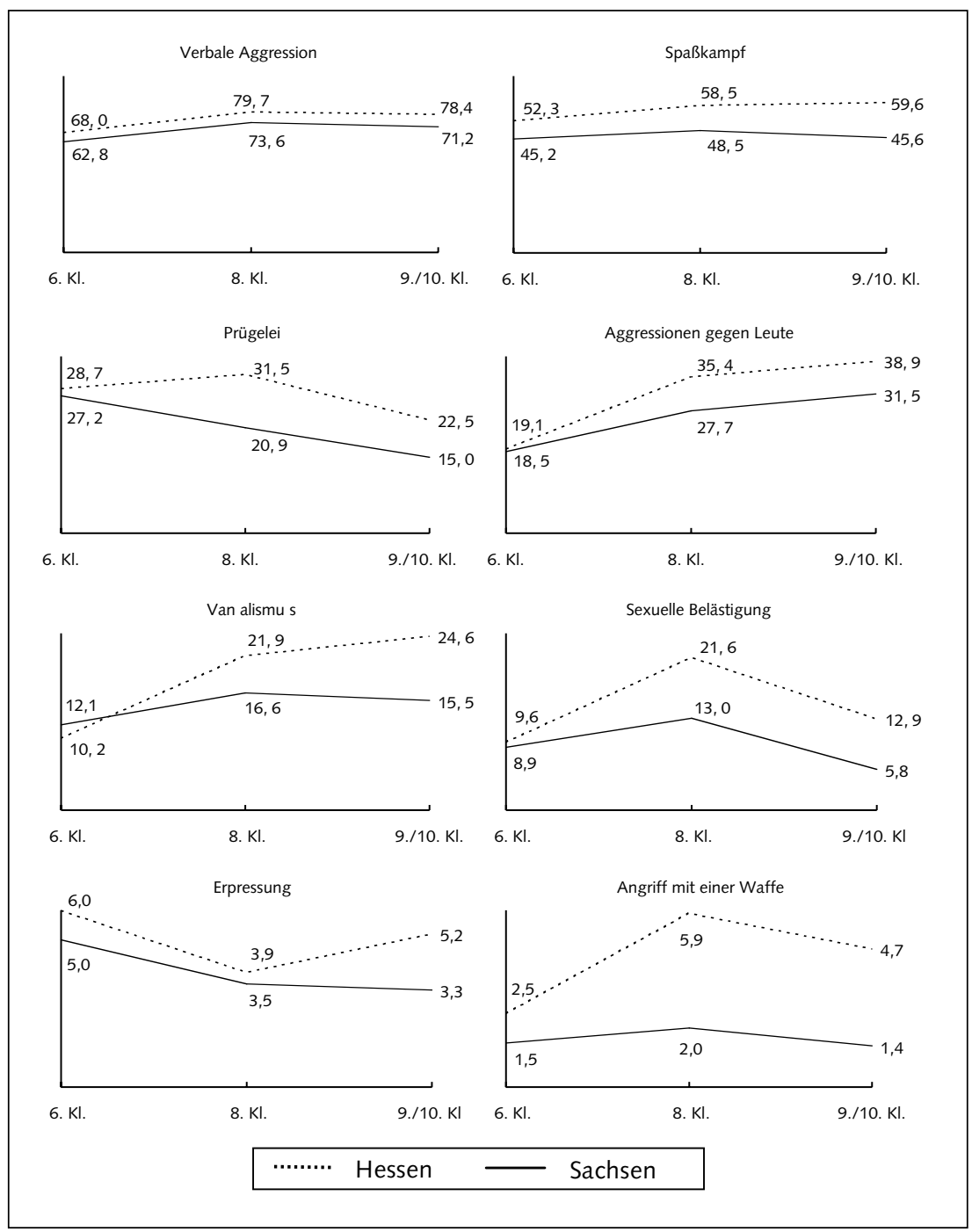

* Skala: 1 = nie $\ldots 6$ = fast täglich; Legende vgl. Abb. 2-1 
Abb. 2-3 zeigt für jede der einbezogenen schulischen Gewaltformen die entsprechende Verlaufskurve von der 6. über die 8. bis zur 9./10. Klasse. ${ }^{5}$ Dabei fällt auf, daß sich kein einheitliches Bild, kein typischer Verlauf für alle Gewaltformen ergibt. Vielmehr hängt die Verlaufskurve von der jeweiligen Gewaltform ab. Nur bei einigen Gewaltformen (verbale Aggression, sexuelle Belästigung, Angriff mit einer Waffe) zeigt sich die erwartete Verlaufskurve mit der „Gewaltspitze“ in den 8. Klassen. Daneben gibt es bei Aggressionen gegenüber Lehrern eine ansteigende Tendenz und bei anderen Gewaltformen, z.B. Prügeleien, umgekehrt eine eher fallende Tendenz. Sehr uneinheitlich ist - wiederum entgegen unserer Annahme - auch das Bild hinsichtlich des Ost-West-Vergleichs. Nur bei wenigen Gewaltformen ist die Verlaufskurve in Sachsen und Hessen weitgehend identisch (z.B. bei verbaler Aggression und bei Aggression gegen Lehrer). Bei verbaler Aggression ist zudem, ähnlich wie beim „Spaßkampf“, ein relativ hohes Niveau über alle Jahrgangsstufen hinweg zu erkennen. Beschimpfungen sowie leichte körperliche Auseinandersetzungen sind somit - zumindest in der Sekundarstufe I - unabhängig vom Alter und gehören aufgrund ihrer beträchtlichen Ausprägung quasi zum Schulalltag. Bei den übrigen Erscheinungsformen schulischer Gewalt variiert dagegen der Kurvenverlauf in Sachsen und Hessen. In Sachsen fällt z. B. bei ernsthaften Prügeleien der Anteil derer, die dies beobachten, von der 6. bis zur 9./10. Klasse deutlich ab, in Hessen hingegen nimmt er bis zur 8. Klasse zu und fällt erst danach ab. Bei Vandalismus hingegen steigt in Sachsen die Wahrnehmung von der 6. zur 8. Klasse an und fällt anschließend leicht ab. In Hessen jedoch steigt er von der 6. bis zur 9./ 10. Klasse deutlich an. Ähnliches gilt für „Spaßkampf“. Holtappels und Meier begründen den Anstieg bei der Beobachtung von Gewaltformen mit zunehmendem Schüleralter vor allem mit einer entwicklungsbedingt wachsenden Sensibilität für Gewalt (vgl. Holtappels/Meier 1997, S. 51). Allerdings muß die Aussage dahingehend differenziert werden, daß es nicht nur Gewaltformen gibt, die eine Zunahme zu verzeichnen haben (z.B. Aggressionen gegenüber Lehrern), sondern auch solche, die in etwa konstant bleiben (z. B. verbale Aggressionen) und solche, deren Ausmaß mit steigender Jahrgangsstufe abnimmt (z. B. ernsthafte Prügeleien). Insbesondere die deutliche Zunahme der wahrgenommenen Aggressionen gegen Lehrer und das tendenzielle Anwachsen des Vandalismus mit steigendem Jahrgang weisen auf schulinterne Problemlagen und ihre Verschärfung im Rahmen der Schullaufbahn hin. Der wachsende Stellenwert von Schüleraggressionen ge-

5 Hierbei ist allerdings zu berücksichtigen, daß es sich nicht um einen Längsschnitt einer identischen Population handelt, sondern um einen Querschnittvergleich (Quasi-Panel). Ein echter Längsschnitt ist von unserer Forschungsgruppe in nächster Zeit geplant. Als älteste Jahrgangsstufe wurden die Abschlußklassen der einzelnen Schulformen bzw. Bildungsgänge (9. Klasse Hauptschulbildungsgang der Mittelschule bzw. Förderschule und 10. Klasse Realschulbildungsgang der Mittelschule) untersucht. Zu Vergleichszwecken wurde auch im Gymnasium die 10. Klasse befragt. 
gen Lehrer sowie gegen das Schuleigentum (Vandalismus) kann auch als zunehmender Protest gegen die zugemutete ,institutionelle“ bzw. ,strukturelle“ Gewalt der Schule gedeutet werden. ${ }^{6}$ Zugleich ist darin auch ein Protest gegen nichterfüllte Partizipationserwartungen zu sehen, die mit zunehmendem Alter der Schüler wachsen (vgl. Melzer 1998).

Neben dem Geschlecht und der Jahrgangsstufe spielt bei den Schulstudien zur Gewalt auch die Frage nach dem Einflu $\beta$ der Schulform eine große Rolle. Hierzu liegen mittlerweile zahlreiche empirische Befunde vor (vgl. z. B. Fuchs/Lamnek/Luedtke 1996, Knopf 1996, Kolbe 1996). Diese bestätigen die allgemeine Erwartungshaltung, daß das Gewaltniveau an Förderschulen bzw. Sonderschulen am höchsten, an Gymnasien hingegen am niedrigsten ist, während sich die übrigen Schulformen zwischen diesen beiden Polen bewegen. Ein analoges Bild erwarteten wir auch von unserer Analyse der wahrgenommenen schulischen Gewaltformen und zwar sowohl für Sachsen als auch für Hessen. Dabei muß jedoch berücksichtigt werden, daß der Sachsen-Hessen-Vergleich in bezug auf die Schulformen aufgrund der ganz unterschiedlichen Schulsysteme nur mit Einschränkungen möglich ist. ${ }^{7}$ Deshalb können auch nur einige grundlegende Tendenzen beschrieben werden.

Entsprechende Schulformanalysen zeigen auch für Sachsen und Hessen die bekannten Unterschiede, wonach - insgesamt betrachtet - an Förderschulen bzw. Sonderschulen am meisten und an Gymnasien am wenigsten Gewalt wahrgenommen wird (vgl. auch Holtappels/Meier 1997, S. 51, Schubarth 1997, S. 68). Das gilt allerdings nicht für alle Gewaltformen: So sind in Sachsen wie in Hessen die Schulformunterschiede bei den verbalen Aggressionen, beim Spaßkampf und auch beim Vandalismus erstaunlich gering. Vulgäre Beschimpfungen, leichte körperliche Auseinandersetzungen sowie Vandalismus werden an allen Schulformen in etwa gleichem Maße wahrgenommen. Anders ist dies jedoch bei den eher ,härteren“ Gewaltformen (z. B. bei ernsthaften Prügeleien und bei Erpressung), bei denen sich das erwartete Bild zeigt, d.h. die Förderschulen bzw. Sonderschulen am meisten und die Gymnasien am wenigsten belastet sind.

Hinsichtlich des Ost-West-Vergleichs kann festgestellt werden, daß - wiederum mit Ausnahme der verbalen Aggressionen gegen Lehrer - die hessischen Schulformen im Vergleich mit den entsprechenden sächsischen in der Tendenz die etwas höheren Werte aufweisen. Am größten sind die Ost-

6 Darauf verweisen vor allem unsere qualitativen Untersuchungen und Fallstudien an Schulen (vgl. Schubarth/Stenke 1996, Schubarth/Darge/Mühl/Bergelt/Börner/Hannich u.a. 1997).

7 Die Schwierigkeiten eines Schulformvergleichs zeigen sich vor allem darin, daß in den beiden Vergleichspopulationen die vergleichbaren Schulformen unterschiedlich stark vertreten sind: So stellt z. B. das sächsische Gymnasium einen Anteil von 40\%, das hessische jedoch nur von $25 \%$ der Schüler. Die sächsische Mittelschule ist mit $54 \%$ an der sächsischen Gesamtpopulation vertreten, die vergleichbaren hessischen Haupt- und Realschulen dagegen nur mit 20\%, während die hessischen Gesamtschulen - eine in Sachsen nicht existente Schulform - über die Hälfte der Population ausmachen (vgl. Tillmann u.a. 1998). 
West-Differenzen noch bei den Förderschulen bzw. Sonderschulen, bei den Gymnasien sind die Unterschiede ebenfalls meist noch spürbar, während bei den sächsischen Mittelschulen im Vergleich zu den hessischen Haupt- und Realschulen die Differenzen kaum noch wahrnehmbar sind. Unterstellt man im Rahmen des schulischen Transformationsprozesses eine Angleichung des Ostens an den Westen, so scheint die Ost-West-Angleichung hinsichtlich der Gewaltbelastung in den einzelnen Schulformen unterschiedlich zu verlaufen; am schnellsten offenbar bei den Mittelschulen. Insgesamt drückt jedoch die unterschiedliche Gewaltbelastung der Schulformen in Ost wie in West auch das unterschiedliche gesellschaftliche Prestige der jeweiligen Schulform, deren unterschiedlichen individuellen Karrieremöglichkeiten für ihre Schüler und die sich dahinter verbergende soziale Selektion der Schülerschaft aus (vgl. z. B. Kolbe 1996, S. 62ff., Tillmann 1997a, S. 41).

Eine differenzierte Analyse innerhalb der sächsischen Mittelschule nach den Bildungsgängen (Realschulbildungsgang und Hauptschulbildungsgang ab Klasse 7) zeigt folgendes: Von den Hauptschülern werden die meisten hier untersuchten Gewaltformen häufiger wahrgenommen als von den Schülern im Realschulbildungsgang; einige Gewaltformen werden von den Hauptschülern in der Extremposition (,fast täglich“) sogar doppelt so häufig beobachtet (Aggressionen gegen Lehrer, Prügelei, Vandalismus, sexuelle Belästigung $)^{8}$. Eine ähnliche Tendenz zeigt sich innerhalb der Förderschulen, bei denen in Sachsen (entsprechend der spezifischen Problemlagen der Schüler) Förderschulen für Lernbehinderte und solche für Erziehungshilfe unterschieden werden. Hier sind es die Förderschulen für Erziehungshilfe, an denen alle Gewaltformen (mit Ausnahme des Angriffs mit einer Waffe) von den Schülern deutlich häufiger beobachtet werden.

An Schulen gibt es bekanntlich nicht nur Aggressionen und Gewalt, die von den Schülern ausgehen, sondern auch aggressives und gewaltförmiges Handeln von Lehrern (vgl. Schwind u.a. 1995, Knopf 1996, Scherer 1996, Krumm 1997). Neben den Schüleraggressionen wurden deshalb auch verschiedene Formen der Lehreraggressionen in unsere Analysen einbezogen. Dabei unterscheiden wir zwischen subtil-aggressivem bzw. pejorativem Lehrerhandeln und manifesten Lehreraggressionen (vgl. Tab. 2-1). ${ }^{9}$

8 Zu Fragen der Differenzierung zwischen Schülern im Hauptschul- und im Realschulbildungsgang hinsichtlich ihres Sozialverhaltens und ihrer Einstellung zur Schule vgl. Adomat 1997, Melzer/Adomat 1998.

9 Die subtilen/pejorativen Lehreraggressionen werden hier durch die Stellvertreter-Items „Wenn bei uns jemand etwas falsch macht, dann stellen ihn die Lehrer als schlecht hin“ und „Es gibt Lehrer/innen die einen vor der ganzen Klasse blamieren“ repräsentiert, die manifesten Aggressionen durch die Items „Bei uns gibt es Lehrer, die gegen Schüler schon mal handgreiflich werden“ sowie „Bei uns kommt es nur selten vor, daß uns Lehrer/innen anschreien.“. Da beim letztgenannten Item im Unterschied zu den anderen die Handlung verneint wird, wurde die Skala zu Vergleichszwecken gedreht. 
Wie Tabelle 2-1 zeigt, ist keine klare Aussage hinsichtlich der Lehreraggressionen im Ost-West-Vergleich möglich. Einige Formen von Lehreraggression werden in Sachsen stärker wahrgenommen (Schüler/innen schlechtmachen, anschreien), andere in Hessen (Schüler/innen blamieren, gegen sie handgreiflich werden). Dieses wenig eindeutige Ergebnis findet sich auch beim Vergleich von Jungen und Mädchen entlang der Ost-West-Dimension wieder. Insbesondere bei den subtil-aggressiven bzw. pejorativen Formen des Lehrerhandelns sind allerdings die Differenzen sowohl in der Gesamtstichprobe als auch bei den Jungen gering, während sich sächsische und hessische Mädchen stärker unterscheiden. Einen Sonderfall stellt die physische Lehreraggression (handgreiflich werden) dar, die von Jungen in Ost wie West deutlich mehr als von Mädchen wahrgenommen wird. Dies läßt sich jedoch zum einen dadurch erklären, daß Jungen vermutlich stärker von solchen Lehreraggressionen betroffen sind; zum anderen kann ihre stärkere Gewaltbeobachtung in bezug auf das Lehrerhandeln auch als Legitimation für eigenes Gewalthandeln gegenüber Lehrern interpretiert werden.

Tab. 2-1: Wahrgenommene Lehreraggressionen im Ost-West-Vergleich (Mittelwertvergleich gesamt und nach Geschlecht differenziert)*

\begin{tabular}{|c|c|c|c|c|c|c|}
\hline \multirow[b]{2}{*}{$\begin{array}{l}\text { subtil-aggr./pejoratives } \\
\text { Lehrerhandeln }\end{array}$} & \multicolumn{2}{|c|}{ gesamt } & \multicolumn{2}{|c|}{ Mädchen } & \multicolumn{2}{|c|}{ Jungen } \\
\hline & Sa. & $\mathrm{He}$. & Sa. & $\mathrm{He}$. & Sa. & $\mathrm{He}$. \\
\hline $\begin{array}{l}\text { Schüler/innen vor der } \\
\text { Klasse schlechtmachen } \\
\text { (V180) }\end{array}$ & 2,62 & 2,56 & 2,57 & 2,44 & 2,68 & 2,66 \\
\hline $\begin{array}{l}\text { Schüler/innen vor der } \\
\text { Klasse blamieren } \\
\text { (V185) }\end{array}$ & 3,01 & 3,11 & 3,00 & 3,16 & 3,01 & 3,06 \\
\hline \multicolumn{7}{|l|}{$\begin{array}{l}\text { Manifeste } \\
\text { Lehreraggression }\end{array}$} \\
\hline $\begin{array}{l}\text { Schüler/innen } \\
\text { anschreien } \\
\text { (V181) }\end{array}$ & 3,12 & 2,97 & 3,10 & 3,01 & 3,14 & 2,93 \\
\hline $\begin{array}{l}\text { gegen Schüler/innen } \\
\text { handgreiflich werden } \\
\text { (V182) }\end{array}$ & 1,84 & 2,00 & 1,65 & 1,87 & 2,05 & 2,12 \\
\hline
\end{tabular}

* Skala: 1 = stimmt gar nicht ... 5 = stimmt ganz genau (in beiden Stichproben)

Bemerkenswerter als die Differenzen zwischen Jungen und Mädchen ist aber das Ausmaß, in dem solche Lehreraggressionen sowohl in Ost wie West wahrgenommen werden: Etwa jeder zweite sächsische wie hessische Schüler stimmt der Aussage, daß Schüler bei einem Fehler als schlecht hingestellt werden, zumindest teilweise, d.h. ganz, überwiegend oder teils/teils, zu (vgl. 
ausführlicher Schubarth/Darge/Mühl/Ackermann 1997). Dies läßt auf Defizite im professionellen Lehrerhandeln, vor allem hinsichtlich der Gestaltung pädagogisch angemessener Lehrer-Schüler-Interaktionen, schließen (vgl. Kap. 6). Auf ein beachtliches Niveau von Lehreraggressionen wird auch in anderen Untersuchungen hingewiesen (vgl. Krumm 1997).

Um als Zwischenbilanz den Einfluß der drei Faktoren Geschlecht, Jahrgangsstufe und Schulform auf die Gewaltwahrnehmung zu bestimmen, wurde eine erste multiple Regressionsanalyse, getrennt nach sächsischer und hessischer Stichprobe, durchgeführt. Dabei war von vornherein klar, daß die drei einbezogenen Faktoren nur einen kleinen Teil der möglichen Einflußfaktoren für Gewalt darstellen und die entsprechende Varianzaufklärung nicht allzu hoch liegen würde. Mit einer Varianzaufklärung von 5-10\% wurde dies auch bestätigt. Die Regressionsanalyse ergab sowohl für Sachsen als auch für Hessen, daß von den drei Faktoren - über alle schulische Gewaltformen hinweg gesehen - der Schulform das größte Gewicht zukommt, gefolgt von dem Geschlecht und der Jahrgangsstufe. In den weiteren Analysen werden wir der Bedeutung dieser Faktoren im Gesamtensemble der Einflußfaktoren ausführlicher nachgehen (vgl. dazu Kap. 4 und 6).

\subsection{Analyse konkreter Gewaltsituationen}

Nachdem im ersten Teil unserer Ost-West-Analysen der Schwerpunkt auf der Wahrnehmung schulischer Gewaltformen aus der Schülerperspektive lag, wird im folgenden anhand verschiedener fiktiver gewalthaltiger Konfliktsituationen neben der Beobachtung von Gewaltkonflikten zusätzlich das Reagieren in solchen Situationen, vor allem das Interventionsverhalten, untersucht. Dabei wird nicht nur die Perspektive der Schüler, sondern auch die der Lehrer einbezogen, so daß eine doppelte Vergleichsperspektive möglich ist: Schüler - Lehrer sowie Ost - West. Im einzelnen sollen folgende Dimensionen im Ost-West-Vergleich analysiert werden:

- Wahrnehmung konkreter Gewaltsituationen durch Schüler und Lehrer

- Gewaltverhalten von Schülern in konkreten Situationen (Selbstreport)

- Schülerintervention aus Sicht von Schülern und Lehrern

- Lehrerintervention aus Sicht von Schülern und Lehrern

Forschungsleitend ist wiederum die Frage, ob die bisher festgestellte etwas geringere Gewaltbelastung an ostdeutschen Schulen bestätigt wird oder ob diesbezüglich Differenzierungen bzw. Modifizierungen vorgenommen werden müssen. Von besonderem Interesse ist auch, ob Schüler bzw. Lehrer in Sachsen und Hessen in gewalthaltigen Situationen ähnlich reagieren oder ob Unterschiede auftreten. Zugleich soll weiterhin die Frage verfolgt werden, ob sich Anzeichen von Angleichungstendenzen finden lassen. 
Aufgrund der bisherigen Befunde erwarten wir für Sachsen auch bei der Wahrnehmung konkreter schulischer Gewaltsituationen und dem realen Gewaltverhalten sowohl aus Schüler- wie auch aus Lehrersicht ein im Vergleich zu Hessen etwas niedrigeres Gewaltniveau. Hinsichtlich des Interventionsverhaltens von Schülern und Lehrern gehen wir aufgrund der unterschiedlichen Entwicklungen in den BRD- und DDR-Schulen (vgl. Kap. 1.1) von einem höheren Grad der Sozialkontrolle an ostdeutschen Schulen aus, zu der Lehrer wie Schüler mit einer stärkeren Interventionsbereitschaft in Konfliktsituationen beitragen. Allerdings ist auch der Umgang mit dem Anstieg gewaltförmigen Verhaltens nach der Wende ein besonderes Problem, auf das viele Lehrer noch nicht vorbereitet sind und das zu Verunsicherungen führen kann.

\section{Wahrnehmung konkreter Gewaltsituationen durch Schüler und Lehrer}

Ähnlich wie bei den im ersten Teil dargestellten schulischen Gewaltformen soll zunächst am Beispiel von vier konkreten gewalthaltigen Konfliktsituationen die Häufigkeit der Wahrnehmung solcher Konflikte aus Schüler- wie Lehrersicht ${ }^{10}$ ermittelt und dargestellt werden. Dabei handelt es sich um folgende vier Konfliktsituationen:

- Treten:

Ein Junge/ein Mädchen tritt jemanden in böser Absicht.

- Erpressung:

Ein Junge/ein Mädchen fordert Geld von jemandem und droht ihm/ihr Strafe an, wenn er/sie nicht bezahlt.

- Prügelei:

Zwei prügeln sich so stark, daß es Verletzungen (z. B. blutige Nasen, blaue Augen gibt).

- Sexuelle Übergriffe:

Ein Junge bedrängt ein Mädchen und faßt es gegen ihren Willen an den Busen oder zwischen die Beine.

$\mathrm{Zu}$ jeder der vier Situationen wurden verschiedene Reaktionen und Einstellungen erfragt. Dabei wird zwischen der Wahrnehmung der jeweiligen Gewalthandlung in der Klasse und in der gesamten Schule (außerhalb der Klasse vor und nach dem Unterricht, in der Pause usw.) unterschieden. Der Ost-WestVergleich, auf den wir uns im folgenden konzentrieren, wird anhand eines Mittelwertvergleichs vorgenommen (vgl. Tab. 2-2).

10 Bei der Lehrerbefragung wurde auf die Konfliktsituation „Erpressung“ verzichtet, da angenommen wurde, daß diese in der Regel nicht vor den Augen der Lehrer vollzogen wird. 
Tab. 2-2: Wahrnehmung konkreter Gewaltsituationen durch Schüler und Lehrer im Ost-West-Vergleich (Mittelwertvergleich)*

\begin{tabular}{lcccccc}
\hline So etwas habe ich beobachtet & \multicolumn{2}{c}{ in der Klasse } & \multicolumn{4}{c}{ in der Schule } \\
& \multicolumn{2}{c}{ Schüler } & \multicolumn{2}{c}{ Schüler } & \multicolumn{2}{c}{ Lehrer } \\
& Sa. & He. & Sa. & He. & Sa. & He. \\
Treten & 2,43 & 2,48 & 2,62 & 2,71 & 2,71 & 2,70 \\
Erpressung & 1,21 & 1,21 & 1,35 & 1,40 & - & - \\
Prügelei & 1,56 & 1,55 & 1,78 & 1,89 & 1,78 & 1,78 \\
Sexuelle Übergriffe & 1,49 & 1,61 & 1,62 & 1,74 & 1,44 & 1,46 \\
\hline
\end{tabular}

* Skala: $1=$ nie $\ldots .5$ = sehr oft (in beiden Stichproben)

Die Ost-West-Analysen bei der Schülerschaft ergeben folgendes Bild: Sächsische und hessische Schüler nehmen die vier angeführten Gewaltsituationen im gleichen bzw. ganz ähnlichen Maße wahr. Hinsichtlich der einzelnen Konfliktsituationen ergeben sich allerdings gewisse Differenzierungen. So sind bei den Situationen „Treten“, „Erpressen“ und „Prügelei“ kaum Ost-West-Differenzen auszumachen, während sich bei den sexuellen Übergriffen ein leichtes Übergewicht in Hessen ergibt. Als ein weiteres Ergebnis ist festzuhalten, daß sich in Ost wie West die Wahrnehmung schulischer Gewaltsituationen innerhalb und außerhalb der Klasse unterscheidet. Außerhalb der Klasse, d.h. vor allem vor und nach dem Unterricht sowie in der Pause, wird mehr Gewalt beobachtet. Das kann dadurch erklärt werden, daß außerhalb des Unterrichts die Kontrolle durch Lehrer weitaus geringer ist, da die Schule eine größere soziale und räumliche Einheit darstellt.

Die Differenzierung zwischen unterrichtlicher und außerunterrichtlicher Gewalt hat auch Auswirkungen für den Ost-West-Vergleich: Während bei der Gewaltwahrnehmung innerhalb der Klasse (mit Ausnahme der sexuellen Übergriffe) keine signifikanten Ost-West-Unterschiede auftreten, sind diese Unterschiede bei den wahrgenommenen Gewaltsituationen außerhalb der Klasse durchgängig signifikant. Das heißt, daß im Osten besonders außerhalb des Unterrichts, z.B. auf dem Schulhof, vor Schulbeginn und nach Schulschluß, weniger Gewalt wahrgenommen wird. Eine Erklärung dafür könnte sein, daß die außerunterrichtliche Kontrolle und Disziplinierung der Schüler seitens der Lehrer im Osten infolge des konventionelleren Erziehungsstils im Vergleich mit Hessen (noch) stärker ist. Schulische Erziehung war in der DDR nicht nur auf den Unterricht beschränkt, sondern sollte möglichst viele Bereiche einbeziehen. Möglicherweise wirkt dies in Form stärkerer Erziehungsbemühungen und Kontrolle auch außerhalb des Unterrichts noch nach. ${ }^{11}$

11 Eine weitere, eher methodenkritische Erklärung liegt darin, daß die Anzahl der Schüler an hessischen Schulen im Durchschnitt etwas größer ist als an sächsischen Schulen und aus diesem Grund auch mehr Gewaltvorfälle registriert werden. 
Bei den Jungen sind die Ost-West-Differenzen im Unterschied zu den Mädchen nicht signifikant, was wiederum für eine größere Ähnlichkeit im Verhalten der sächsischen und hessischen Jungen im Unterschied zu den Mädchen spricht. Sächsische Gymnasien und Förderschulen weisen im Vergleich zu den entsprechenden hessischen Schulformen die geringeren Beobachtungsquoten auf. Eine eher umgekehrte Tendenz zeigt sich beim Vergleich der sächsischen Mittelschule mit den hessischen Real- bzw. Hauptschulen: Hier sind sogar die sächsischen Mittelschulen etwas mehr belastet, womit die Tendenz bestätigt wird, daß die sächsische Mittelschule und die hessische Realschule hinsichtlich der Gewaltbelastung ähnlicher sind als die anderen verglichenen Schulformen.

Für die Jahrgangsstufen ergibt sich ein sehr differenziertes Bild: In Klasse 6 sind die Ost-West-Unterschiede bei der Gewaltwahrnehmung nicht signifikant. In dieser Klassenstufe wird entgegen dem Gesamttrend in Sachsen sogar mehr Gewalt innerhalb der Klasse beobachtet. In Klasse 9 bzw. 10 werden in Sachsen weniger Gewalthandlungen wahrgenommen, während in Klasse 8 keine eindeutige Aussage möglich ist (vgl. Tab. 2-3).

Etwas anders verhält es sich bei der beobachteten Gewalt in der gesamten Schule (außerhalb der Klasse): Hier läßt sich der Gesamttrend einer etwas höheren Gewaltwahrnehmung in Hessen bestätigen. Insgesamt gilt aber: Je höher die Klassenstufe, umso deutlicher die Ost-West-Unterschiede. Umgekehrt könnte dies als ein Zeichen der Angleichung in den unteren Klassenstufen gedeutet werden, wodurch unsere Angleichungs-Hypothese eine erneute Bestätigung erhalten würde. Dies führt zu der folgenreichen Annahme, daß sich ältere Schüler, die die DDR-Schule wenigstens teilweise noch durchlaufen haben, in ihrem sozialen Verhalten (z. B. in bezug auf Aggression und Gewalt) anscheinend von den nachwachsenden Jahrgängen unterscheiden. ${ }^{12}$ Die Unterschiede, die zwischen Sachsen und Hessen in den höheren Jahrgangsstufen noch nachweisbar sind, würden dann bei den nachwachsenden Jahrgängen verschwinden.

Die ermittelten Befunde haben auch nachhaltige Konsequenzen für die Bewertung des Ost-West-Vergleichs, denn die bisher festgestellte etwas niedrigere Gewaltbelastung im Osten ist dann in erster Linie auf die geringere Belastung in den höheren Klassenstufen, vor allem den 9. bzw. 10. Klassen, zurückzuführen. Somit ergibt sich an dieser Stelle ein weiteres Indiz dafür, daß eine baldige Angleichung des Gewaltniveaus im Osten an das des Westens $\mathrm{zu}$ erwarten ist.

12 Auf das unterschiedliche soziale Verhalten von Schülern in Abhängigkeit davon, ob oder wie lange die DDR-Schule durchlaufen wurde, wird auch von Schülern in Gruppendiskussionen immer wieder hingewiesen (vgl. z. B. Schubarth/Stenke 1996). 
Tab. 2-3: Wahrnehmung konkreter Gewaltsituationen durch Schüler im Ost-West-Vergleich (Mittelwertvergleich differenziert nach Jahrgangsstufen)*

So etwas habe ich in meiner Klasse in den letzten 12 Monaten beobachtet.

\begin{tabular}{lcccccc} 
& \multicolumn{2}{c}{6. Klasse } & \multicolumn{2}{c}{ 8. Klasse } & \multicolumn{2}{c}{$9 . / 10$. Klasse } \\
& Sa. & He. & Sa. & He. & Sa. & He. \\
Treten & 2,57 & 2,52 & 2,56 & 2,66 & 2,14 & 2,24 \\
Erpressung & 1,24 & 1,21 & 1,26 & 1,24 & 1,12 & 1,19 \\
Prügelei & 1,64 & 1,55 & 1,61 & 1,61 & 1,42 & 1,49 \\
Sexuelle Übergriffe & 1,49 & 1,43 & 1,79 & 1,94 & 1,48 & 1,53 \\
\hline
\end{tabular}

* $\quad$ Skala: $1=$ nie $\ldots .5$ = sehr oft (in beiden Stichproben)

Im Gegensatz zu den Befunden aus Schülersicht, die ingesamt den Gesamttrend einer etwas geringeren Gewaltbelastung im Osten bestätigen, sind die Ergebnisse aus Sicht der Lehrer einigermaßen überraschend: Sächsische und hessische Lehrer beobachten - bezogen auf die angegebenen Gewaltsituationen - Aggression und Gewalt in etwa gleichem Ausmaß (vgl. Tab. 2-2). Dies wirft die Frage auf, wie die Differenzen zwischen der Schüler- und der Lehrersicht im Ost-West-Vergleich zu interpretieren sind. Folgende Erklärung bietet sich an: Ostdeutsche Lehrer könnten durch den von ihnen seit der Wende wahrgenommenen Gewaltanstieg (vgl. Schubarth 1997, S. 69f) stärker verunsichert sein als ihre Schüler, so daß sie Gewalt selektiv und dadurch häufiger wahrnehmen als diese. Dafür spricht vor allem der höhere Wert der sächsischen Lehrer bei der Beobachtung „böswilligen Tretens“. Für sächsische Lehrer scheint ein derartig ausgetragener Konflikt im Vergleich zu ihren Schülern noch weniger „,normalisiert“ zu sein. Hessische Lehrer könnten demgegenüber stärker an Gewaltphänomene „gewöhnt“ sein, was sich auf ihre Wahrnehmungsperspektive dergestalt auswirkt, daß sie im Endeffekt in der Einschätzung der Gewaltsituation an der Schule mit ihren Schülern übereinstimmen. Die in beiden Ländern mit steigendem Gewaltniveau größer werdende Differenz zwischen Schüler- und Lehrersicht (in Hessen freilich deutlicher als in Sachsen) verweist jedoch auch darauf, daß sich stärkere Gewaltformen eher dem Blick der Lehrer entziehen.

Als Konsequenz läßt sich festhalten, daß durch die Einbeziehung der Lehrerperspektive die bisherigen Aussagen zum Ost-West-Vergleich relativiert werden müssen. Die aus Schülerperspektive etwas geringere Gewaltbelastung an sächsischen Schulen läßt sich durch die Lehrersicht nicht bestätigen. 


\section{Gewaltverhalten von Schülern in konkreten Situationen}

Die Befunde aus dem Schüler-Selbstreport bestätigen den Ergebnistrend der bisherigen Analysen. Auch bei der Frage nach der Häufigkeit der selbst ausgeführten Gewalthandlung (Treten, Erpressen, Prügelei, sexuelle Übergriffe ${ }^{13}$ ) sind die Unterschiede zwischen den sächsischen und hessischen Schülern nur gering. Insgesamt ergibt sich wiederum für Hessen eine leicht höhere Belastung, wobei für die Situationen „Erpressung“ und „Prügelei“ die Unterschiede signifikant sind. Bei der Situation des „Tretens“ weist Sachsen sogar die etwas höhere Gewaltbelastung auf (vgl. Tab. 2-4).

Die Differenzierung nach Geschlecht zeigt, daß sächsische Jungen signifikant häufiger ,treten“ als die hessischen. Umgekehrt ist das Bild bei den Mädchen: Solche Gewalthandlungen, wie ernste Prügeleien und Erpressen, werden von den hessischen Mädchen signifikant häufiger ausgeführt. Für den OstWest-Vergleich bedeutet dies, daß das geringere Gewaltniveau im Osten vor allem auf dem „friedfertigeren“ Verhalten der Mädchen basiert. Als Erklärung kann wiederum das stärkere (Nach-)Wirken entsprechender Geschlechterstereotype angeführt werden, die Mädchen z. B. untersagen, körperliche Gewalt anzuwenden (vgl. Kap. 3). Bei den Analysen zu den Jahrgangsstufen ergaben sich lediglich für die 9. bzw. 10. Klassen einige signifikante Unterschiede (in Hessen mehr Gewaltwahrnehmung als in Sachsen), womit der Befund der Angleichung in den unteren Jahrgangsstufen bestätigt wird. Auch die Schulformanalysen bestätigten die bei der Gewaltwahrnehmung angeführten Tendenzen, bei allerdings insgesamt nur geringen Differenzen (vgl. 2.1).

Tab. 2-4: Gewaltverhalten von Schülern in konkreten Situationen im Ost-West-Vergleich (Mittelwertvergleich des Selbstreports)*

So etwas habe ich in diesem Jahr selbst getan.

\begin{tabular}{lcc} 
& Sa. & He. \\
Treten & 1,65 & 1,63 \\
Erpressung & 1,13 & 1,16 \\
Prügelei & 1,26 & 1,31 \\
Sexuelle Übergriffe & 1,37 & 1,42 \\
\hline
\end{tabular}

* Skala: $1=$ nie ... 5 = sehr oft (in beiden Stichproben)

Zum Ausmaß des selbstberichteten Gewaltverhaltens ist generell festzuhalten, daß es nur eine kleine Minderheit von Schülern betrifft, die die vorgegebenen

13 Zum Täterverhalten bei der Konfliktsituation „,sexuelle Übergriffe“ wurden nur Jungen befragt. 
Gewalthandlungen häufiger ausführt. So beträgt der Anteil derer, die die o.g. Handlungen ,oft" bzw. ,sehr oft" ausführen, in Sachsen wie Hessen weniger als $7 \%$, bei Erpressung weniger als $2 \%$. Nimmt man jedoch auch diejenigen hinzu, die dies nur manchmal tun, steigt der Anteil bei „,sexuellen Übergriffen“ auf $10-12 \%$ und bei „Treten“ auf $15-20 \%$ an.

\section{Schülerintervention aus Sicht von Schülern im Ost-West-Vergleich}

Wie reagieren nun Schüler und Lehrer in konkreten gewaltförmigen Konfliktsituationen? Versuchen sie zu intervenieren oder schauen sie lieber weg? Und die uns in diesem Zusammenhang besonders interessierende Frage: Gibt es beim Interventionsverhalten Ost-West-Unterschiede? Betrachten wir zunächst das Interventionsverhalten der Schüler.

Aus der Sicht der Schüler greifen die sächsischen Schüler bei Gewalt mehr ein als die hessischen und versuchen, den Konflikt zu beenden (vgl. Tab. 2-5). Dieses Ergebnis entspricht unserer Annahme, vermuteten wir doch an ostdeutschen Schulen einen höheren Grad von Sozialkontrolle, was auch ein stärkeres Interventionsverhalten der Schüler zur Folge haben könnte. Vergleicht man jedoch den absoluten Anteil derer, die bei Gewalt schlichtend eingreifen, so sind diese in Ost wie West noch in der Minderheit: 15-20\% geben an, öfter einzugreifen, weitere $20-30 \%$ tun dies gelegentlich, während die Mehrheit selten oder gar nicht interveniert. Bei dem Faktor, der das intervenierende Handeln der Schüler mißt (V203, V213, V223, V233), überprüften wir auch, ob es sich beim „Eingreifen“ nicht etwa eher um eine aktive Beteiligung an gewaltförmigen Auseinandersetzungen im Sinne eines „Mitmischens“ handelt. ${ }^{14}$ Ein geringfügiger, positiver Zusammenhang von $\mathrm{r}=.08$ zwischen dem „Interventions-Faktor“ und einem Faktor, der Items zur stimulierenden Wirkung von Gewalt (,Wenn ich konkrete Gewaltsituationen beobachte, reizt es mich; ich würde am liebsten das Gleiche machen."; V201, V211, V221, V231) beinhaltet, läßt diese Annahme nicht unberechtigt erscheinen. Da jedoch die Zusammenhangsmaße zwischen dem „Interventions-Faktor“ und den Faktoren „Ärger über Gewalt“ (V200, V210, V220, V230) bzw. „Ablehnung gewaltförmigen Schülerverhaltens" (V202, V212, V222, V232) mit r=.27** bzw. $r=.22 * *$ deutlich höher liegen, gehen wir davon aus, daß mit unserem „Interventions-Faktor“ überwiegend ein Verhalten beschrieben wird, das der Beendigung von gewaltförmigen Auseinandersetzungen dient. ${ }^{15}$

14 Bei einer früheren, vergleichbaren Untersuchung (vgl. Melzer/Rostampour 1996) wurde die Intervention mittels dreier Items differenzierter erfaßt. Als Ergebnis zeigte sich, daß Interventionshandeln verschiedenen Intentionen unterliegt. Deshalb lag eine Überprüfung der Intentionen nahe, die hinter dem hier verwendeten „Interventions-Faktor“" stehen.

15 Die für die sächsische Stichprobe separat ermittelten Zusammenhänge gelten im wesentlich auch für die hessische Stichprobe. 
Die mangelnde Interventionsbereitschaft unter der Schülerschaft ist somit ein ost-west-übergreifendes Problem. Auch nach Geschlecht, Jahrgangsstufe und Schulform treten nur geringe Unterschiede auf. Mädchen intervenieren bei Gewalt etwas weniger als Jungen. Dafür lassen sich mindestens zwei Gründe anführen: Zum einen sind sie weniger in Gewaltsituationen verwickelt und nehmen demzufolge auch weniger gewaltförmige Auseinandersetzungen wahr; zum anderen trauen sich Mädchen auch weniger einzugreifen, da sie entsprechend sozialisiert sind und sie dabei aufgrund ihrer körperlichen Voraussetzungen eher „,den Kürzeren ziehen“ würden. Der Befund, daß Schüler der 6. Klassen mehr eingreifen als die höherer Jahrgänge, stellt allerdings dem sozialen Lernprozeß in der Schule kein gutes Zeugnis aus. Dies ist ein deutlicher Hinweis darauf, daß dem sozialen Lernen in der Schule (z. B. dem sozialverantwortlichen Verhalten in Gewaltsituationen) mehr Aufmerksamkeit geschenkt werden muß (vgl. Schubarth 1998a).

Tab. 2-5: Schülerintervention aus Sicht von Schülern* im Ost-WestVergleich (Mittelwertvergleich)

\begin{tabular}{lcc}
\hline \multicolumn{2}{c}{ Wenn ich so etwas beobachte, dann greife ich ein und versuche, das Verhalten zu beenden. } \\
& Sa. & He. \\
Treten & 2,41 & 2,29 \\
Erpressung & 2,41 & 2,19 \\
Prügelei & 2,36 & 2,17 \\
Sexuelle Übergriffe & 2,59 & 2,27 \\
\hline
\end{tabular}

* Für Sachsen: Skala: 1 = stimmt gar nicht ... 5 = stimmt ganz genau,

für Hessen: Skala: $1=$ nie $\ldots 5=$ sehr oft; Item-Formulierungen vgl. Tab. 2-2

\section{Lehrerintervention aus Sicht von Schülern und Lehrern im Ost-West-Vergleich}

Neben der Schülerintervention bei konkreten Gewaltsituationen haben wir auch das entsprechende Lehrerverhalten im Ost-West-Vergleich erfragt. Bei unseren Hypothesen waren wir dabei aufgrund des angenommenen höheren Grades der Sozialkontrolle von einem stärkeren Interventionsverhalten bei ostdeutschen Lehrern ausgegangen. Wie die Tabelle 2-6 zeigt, muß diese generelle Annahme jedoch modifiziert werden (vgl. Tab. 2-6): Nimmt man die selbstberichteten Lehrerinterventionen, treffen unsere Vorannahmen noch eher zu. Bei der Situation des ,Tretens in böser Absicht“" ist die Interventionsbereitschaft der Lehrer nach eigenen Angaben in Sachsen höher als in Hessen; in bezug auf 
Prügelei und sexuelle Übergriffe besteht eine relativ hohe Interventionsbereitschaft bei allen Lehrern. Vielleicht liegt es an der Besonderheit dieser beiden Formen (z. B. extreme Aggressionen, die zudem im Osten weniger vorkommen und für die Lehrer noch weniger sensibilisiert bzw. vorbereitet sind), daß diesbezüglich die Interventionsbereitschaft sächsischer Lehrer nicht stärker ist. Bezieht man die Schülermeinungen zu Lehrerintervention in den Vergleich mit ein, wird die Interpretation noch komplizierter, und die generelle Hypothese läßt sich nicht mehr halten. Es zeigen sich aber auf den zweiten Blick ähnliche Strukturen. Bei den extremen Formen (Erpressung, Prügelei), die in Hessen als vergleichsweise gravierende Probleme in Erscheinung treten, greifen - nach Meinung der Schüler - die hessischen Lehrer stärker ein als die sächsischen. Dies gilt aus den genannten Gründen auch für sexuelle Übergriffe, während in bezug auf die Alltagssituation „Treten“ keine signifikanten Unterschiede bestehen.

\section{Tab. 2-6: Lehrerintervention aus Sicht von Schülern und Lehrern im Ost-West-Vergleich (Mittelwertvergleich)*}

\begin{tabular}{lcccc}
\hline \multicolumn{4}{c}{ Wenn unsere Lehrer solches Verhalten beobachten, } \\
dann greifen sie ein und versuchen, dieses Verhalten zu beenden. \\
Wenn meine Kollegen solches Verhalten beobachten, \\
dann greifen sie ein und versuchen, dieses Verhalten zu beenden. \\
\multicolumn{4}{c}{ Schüler } & \multicolumn{3}{c}{ Lehrer } \\
\multicolumn{1}{c}{ Sa. } & He. & Sa. & He. \\
Treten & 3,70 & 3,71 & 4,35 & 4,19 \\
Erpressung & 3,24 & 3,49 & - & - \\
Prügelei & 3,70 & 3,92 & 4,50 & 4,50 \\
Sexuelle Übergriffe & 3,33 & 3,55 & 4,31 & 4,33
\end{tabular}

* Skala: 1 = nie ... 5 = sehr oft (in beiden Bundesländern)

Die Frage ist jedoch noch offen, warum sächsische Lehrer, die z.T. noch durch eine eher lehrerzentrierte und autoritäre Schule geprägt sind, nicht noch stärker intervenieren. Vielleicht, weil das System als Ganzes noch immer funktioniert? Dafür würde auch die (noch) geringere Gewaltbelastung und die höhere Interventionsbereitschaft der Schüler in sächsischen Schulen sprechen. Ein weiterer Grund könnte die gewisse Unsicherheit beim Umgang mit Gewaltproblemen sein - schließlich ist das für sie eine relativ neue Situation in der Nachwendezeit, die den Lehrern viel abverlangt. ${ }^{16}$ Auf Grund ihrer Ausbildung und

16 Etwa zwei Drittel der von uns befragten Lehrer sind der Auffassung, daß die Gewaltentwicklung seit der Wende leicht $(41,4 \%)$ bzw. stark (24,9\%) zugenommen hat; für die Mittelschulen wird diese negative Entwicklung von über drei Viertel der Lehrer bestätigt. 
Fortbildung sind sie nur in Ausnahmefällen mit Strategien der Konfliktlösung vertraut. Hier scheint unter der ostdeutschen Lehrerschaft ein entsprechender Nachholebedarf zu bestehen; darauf verweist auch die im Vergleich zu Hessen negativere Bewertung verschiedener Aspekte des professionellen Lehrerhandelns durch die Schüler (vgl. Kap. 6). ${ }^{17}$

Nach Auskunft der Schüler wird in den unteren Klassen mehr interveniert als in den oberen Klassen, in sächsischen Gymnasien mehr als in der Mittelschule und Förderschule. Doch auch in höheren Klassen und gerade an den stärker belasteten Schulformen wie Mittelschule oder Förderschule bedarf es des konsequenten Interventionshandelns, der bewußten Grenzziehung bei Gewalt und der Förderung gewaltfreier Konfliktlösungskompetenzen. Für Sachsen kristallisieren sich somit vor allem die Mittelschule und die Förderschule als Schwerpunkte der Gewaltprävention und -intervention heraus - mit allerdings großen Unterschieden zwischen den Einzelschulen.

Darüber hinaus bleibt - länderübergreifend - festzustellen, daß Schüler und Lehreraussagen zum Interventionsverhalten von Lehrern nicht übereinstimmen: Lehrer in Ost wie West glauben öfter zu intervenieren als das nach Ansicht der Schüler der Fall ist. Das Selbstbild der Lehrerschaft ist in dieser Beziehung recht trügerisch, mehr Zivilcourage und entsprechende Kompetenzen sind deshalb gefragt (vgl. Holtappels/Meier/Tillmann 1996).

\subsection{Wahrnehmung, Selbstreport und Einstellungen von Schülern zur Gewalt}

In diesem abschließenden dritten Teil unserer Ost-West-Analysen sollen Einstellungen und Reaktionsweisen von Schülern gegenüber Gewalt untersucht werden, die sich jeweils auf die bereits dargestellten vier konkreten gewaltförmigen Konfliktsituationen beziehen, und zu den bisherigen Perspektiven der Gewaltwahrnehmung und des Selbstreports in Beziehung gesetzt werden. Dabei handelt es sich um folgende Einstellungen bzw. Reaktionsweisen:

Wenn ich so etwas (Treten, Erpressen, Prügelei, sexuelle Übergriffe) beobachte, dann ...

- sehe ich das als ganz normal an; ich denke mir nichts dabei.

Gewalt normal (V198, V208, V218, V228)

17 Ein weiterer Grund könnte in der stärkeren Feminisierung des Lehrerberufs in Ostdeutschland liegen. Die damit verbundene These, daß Lehrerinnen seltener intervenierend eingreifen als Lehrer, wird allerdings durch unsere Analysen nicht gestützt: Der Mittelwertvergleich des selbstberichteten Interventionshandelns, differenziert nach Geschlecht, erbringt in Ost und West keine signifikanten Unterschiede. 
- empfinde ich Angst.

Angst vor Gewalt (V199, V209, V219, V229)

- ärgere ich mich.

Ärger über Gewalt (V200, V210, V220, V230)

- reizt es mich (ich würde am liebsten das Gleiche machen).

Animation durch Gewalt (V201, V211, V221, V231)

- wünsche ich mir, daß sich Schüler nicht so verhalten.

Unerwünschtheit von Gewalt (V202, V212, V222, V232)

Diese fünf Reaktionsformen lassen sich inhaltlich komplementär zueinander strukturieren. Den zwei eher Gewalt befürwortenden Reaktionen „Gewalt normal“ und „Animation durch Gewalt" sind drei eher ablehnende Reaktionsformen (Ärger, Unerwünschtheit, Angst) entgegengestellt. „Angst“ verweist allerdings nicht eindeutig, sondern eher indirekt bzw. diffus auf eine gewaltablehnende Haltung, da diese Reaktionsweise aus einer entsprechenden allgemeinen psychischen Disposition resultiert. Darüber hinaus unterscheiden sich die Reaktionsformen in zwei stark emotional gefärbte (Angst, Ärger) und zwei weniger emotional gefärbte Reaktionen (Gewalt normal, Unerwünschtheit), während die „Animation durch Gewalt“ das Phänomen ,sozialer Ansteckung" (Olweus) beinhaltet.

Bevor wir jedoch zur vergleichenden Betrachtung der einzelnen Reaktionsweisen in den konkreten Gewaltsituationen kommen, soll zunächst die Frage geklärt werden, wie stark diese Reaktionen mit den in den vorigen Abschnitten dargestellten Wahrnehmungen von Gewalt sowie den selbstberichteten Gewalthandlungen im Zusammenhang stehen. Dazu wurden acht verschiedene Faktoren (im statistischen Sinne) gebildet und eine Korrelationsmatrix erstellt, die über die Stärke des Zusammenhangsmaßes zwischen den einzelnen Faktoren Auskunft gibt (vgl. Tab. 2-7). ${ }^{18}$

Tabelle 2-7 zeigt sowohl die Zusammenhänge zwischen der Gewaltwahrnehmung, dem Gewalthandeln und den Reaktionen auf Gewalt als auch innerhalb der einzelnen Dimensionen. Neben dem starken Zusammenhang zwischen den selbstberichteten „harten“ und selbstberichteten „weichen“ Ge-

18 Der Faktor „Wahrnehmung“ (Faktor 2. Ordnung) setzt sich aus den Dimensionen (Faktoren) Vandalismus (V130-V134), Prügelei (V137, V139-V141), Prügelei in konkreten Situationen (V194, V195, V214, V215), Sexuelle Belästigung (V144, V145, V224, V225), Aggressionen gegen Lehrpersonen (V147, V149, V151), Psychische Aggressionen gegen Mitschüler (V156-V158a) und Erpressung gegen Mitschüler (V162-V164, V204, V205) zusammen. Beim Selbstreport konnten zwei Dimensionen unterschieden werden: Der Faktor selbstberichtete „Harte Aggressionen“ beinhaltet die Items V263-266, V268, V272, V275 und V279. In den Faktor selbstberichtete „Weiche Aggressionen“ sind die Items V260-V262, V267, V273, V274 und V278 eingeflossen. Mit der Einbeziehung dieser Faktoren wollten wir über die konkreten Gewaltsituationen hinausweisend eine objektivere Aussage erzielen. Die Faktoren, die im folgenden Einstellungen und Reaktionen in Gewaltsituationen wiedergeben, wurden im obenstehenden Kasten bereits eingeführt. 
walthandlungen sowie zwischen selbstausgeführten Gewalthandlungen und der Gewaltwahrnehmung lassen sich hinsichtlich der Gewalteinstellungen vor allem folgende Zusammenhänge herausstellen:

- Je mehr Gewalt als „,normal“ angesehen wird, desto mehr Gewalthandlungen hat man selbst ausgeführt und desto mehr hat man auch Gewalt beobachtet. Die Korrelationskoeffizienten ( $\mathrm{r}=.50 / \mathrm{r}=.49$ bzw. $\mathrm{r}=.40)$ weisen dabei auf einen relativ starken Zusammenhang hin.

- Je mehr Angst man vor Gewalt hat, desto weniger ist man selbst „Täter“ und desto weniger Gewalt nimmt man auch wahr, wobei diese Zusammenhänge geringer ausfallen.

- Je mehr man sich über Gewalt ärgert, desto weniger ist man „Täter“ und desto weniger beobachtet man Gewalt.

- Je mehr Gewalt (zum Nachahmen) reizt, desto mehr begeht man auch Gewalthandlungen und desto mehr nimmt man Gewalt wahr.

- Je mehr Gewalt als unerwünscht angesehen wird, desto weniger Gewalt wird angewendet und desto weniger Gewalt wird auch beobachtet.

Tab. 2-7: Zusammenhang zwischen Faktoren der Einstellungen/Reaktion auf Gewalt, Gewaltwahrnehmung und selbstberichteten Gewalthandlungen (Korrelationsmatrix für die sächsische Stichprobe ${ }^{19}$ )

\begin{tabular}{|c|c|c|c|c|c|c|c|c|c|}
\hline & & \multirow[b]{2}{*}{$\begin{array}{c}\text { Wahr- } \\
\text { neh- } \\
\text { mung }\end{array}$} & \multicolumn{2}{|c|}{ Selbstreport } & \multicolumn{5}{|c|}{$\begin{array}{l}\text { Einstellungen/Reaktionen } \\
\text { in Gewaltsituationen }\end{array}$} \\
\hline & & & $\begin{array}{l}\text { Harte } \\
\text { Aggr. }\end{array}$ & $\begin{array}{l}\text { Weiche } \\
\text { Aggr. }\end{array}$ & $\begin{array}{l}\text { Gewalt } \\
\text { normal }\end{array}$ & Angst & Ärger & $\begin{array}{l}\text { Anima- } \\
\text { tion }\end{array}$ & $\begin{array}{l}\text { Gew. } \\
\text { unerw. }\end{array}$ \\
\hline \multirow{3}{*}{ 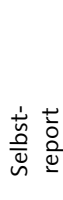 } & $\begin{array}{l}\text { Wahrneh- } \\
\text { mung }\end{array}$ & & $.62^{* *}$ & $.56^{* *}$ & $.40 * *$ & $-.10^{* *}$ & $-.14^{* *}$ & $.44 * *$ & $-.31 * *$ \\
\hline & Harte Aggr. & $.62^{* *}$ & & $.77 * *$ & $.50 * *$ & $-.16^{* *}$ & $-.19 * *$ & $.51 * *$ & $-.40^{* *}$ \\
\hline & $\begin{array}{l}\text { Weiche } \\
\text { Aggr. }\end{array}$ & $.56^{* *}$ & $.77^{* *}$ & & $.49 * *$ & $-.21^{* *}$ & $-.19 * *$ & $.46^{* *}$ & $-.39 * *$ \\
\hline \multirow{5}{*}{ 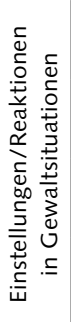 } & $\begin{array}{l}\text { Gewalt } \\
\text { normal }\end{array}$ & $.40^{* *}$ & $.50^{* *}$ & $.49 * *$ & & $-.22^{* *}$ & $-.34 * *$ & $.57 * *$ & $-.54^{* *}$ \\
\hline & Angst & $-.10^{* *}$ & $-.16^{* *}$ & $-.21^{* *}$ & $-.22^{* *}$ & & $.54 * *$ & $-.09 * *$ & $.39 * *$ \\
\hline & Ärger & $-.14 * *$ & $-.19 * *$ & $-.19 * *$ & $-.34 * *$ & $.54^{* *}$ & & $-.14^{* *}$ & $.50 * *$ \\
\hline & Animation & $.44 * *$ & $.51 * *$ & $.46^{* *}$ & $.57 * *$ & $-.09 * *$ & $-.14^{* *}$ & & $-.42 * *$ \\
\hline & unerwünscht & $-.31 * *$ & $-.40^{* *}$ & $-.39 * *$ & $-.54 * *$ & $.39 * *$ & $.50 * *$ & $-.42 * *$ & \\
\hline
\end{tabular}

19 Die Werte für die hessische Stichprobe sind ähnlich. 
Darüber hinaus zeigen sich - wie erwartet - positive Zusammenhänge zwischen den Reaktionen „Gewalt normal“ und „Animation durch Gewalt" sowie zwischen „Angst vor Gewalt“, „Ärger über Gewalt“ und „Gewalt unerwünscht": Wer also Gewalt für normal ansieht, den reizt es auch mehr, bei Gewalt „mitzumischen“, und wer Angst vor Gewalt hat, der ärgert sich auch eher über Gewalt und lehnt Gewalt eher ab. Insgesamt läßt sich somit ein „gewaltaffines Syndrom" erkennen, zu dem sowohl selbstbegangene Gewalthandlungen und eine verstärkte Gewaltwahrnehmung als auch bestimmte Einstellungen gegenüber Gewalt gehören. Dies stimmt auch mit Befunden aus der Aggressionsforschung überein, nach denen aggressive Kinder dazu neigen, ihre Aufmerksamkeit selektiv auf bedrohlich eingestufte Handlungen zu richten, anderen eine feindliche Absicht zu unterstellen und aggressives Verhalten eher als positiv zu bewerten (vgl. Petermann/Jugert/Verbeek/ Tänzer u.a. 1997, S. 325). Aus weiteren Datenanalysen wissen wir, daß in dieses Geflecht gewaltförmiger und gewaltaffiner Verhaltensweisen und Einstellungen auch Aspekte der Schuldevianz, der Delinquenz im außerschulischen Raum und z.T. des Alkohol-, Nikotin- und Drogenkonsums mit hineinspielen, so daß eine forschungsleitende Annahme unsererseits als bestätigt gelten kann (vgl. auch Kap. 6).

Im folgenden wenden wir uns den differenzierten Schülerreaktionen im Kontext der bereits bekannten Konfliktsituationen zu (vgl. Abschnitt 2.2). In Tabelle 2-8 sind die fünf erfragten Reaktionsformen, bezogen auf die unterschiedlichen gewaltförmigen Konfliktsituationen, im Ost-West-Vergleich und nach Geschlecht differenziert dargestellt (vgl. Tab. 2-8). Unabhängig vom OstWest-Vergleich zeigt sich zunächst, daß die Reaktionen in Gewaltsituationen von der jeweiligen Gewaltform abhängig sind: Sie steigen mit der Härte der Gewaltform in Richtung einer zunehmenden Ablehnung. In der Gesamttendenz besteht gleichwohl ein eher gewaltablehnendes Klima: Die Mittelwerte für Unerwünschtheit, Ärger und Angst liegen im Vergleich zu Animation und Normalität höher. Für ein eher gewaltablehnendes Klima spricht auch, daß über alle Situationen hinweg die Mehrheit der Schüler in Sachsen wie Hessen möchte, daß sich Schüler nicht so verhalten, wie es in den Gewaltsituationen beschrieben wird (volle bzw. überwiegende Zustimmung). Diese Ergebnisse relativieren die These von Heitmeyer u.a., wonach Gewalt unter Jugendlichen als normal gilt und im menschlichen Zusammenleben nahezu selbstverständlich angewandt wird (vgl. Heitmeyer u.a. 1995, S. 133).

Die stärkste Ablehnung erhalten sexuelle Übergriffe und Erpressungen - allerdings reizen sexuelle Belästigungen relativ stark zum Nachmachen, obwohl sie am wenigsten als normal angesehen werden und am unerwünschtesten sind. Eher gemäßigt sind dagegen die Reaktionen auf das Treten, womit diese Gewaltform schon eher als Alltagserscheinung gelten kann. 
Tab. 2-8: Schülerreaktionen in gewaltförmigen Konfliktsituationen im Ost-West-Vergleich, differenziert nach Geschlecht (Mittelwertvergleich)

Situation

\begin{tabular}{lcc} 
& \multicolumn{2}{c}{ Gesamt } \\
& Sa. & He. \\
Treten & 2,55 & 2,13 \\
Erpressung & 1,88 & 1,55 \\
Prügelei & 1,99 & 1,65 \\
Sex. Übergriffe & 1,86 & 1,59
\end{tabular}

Gesamt $\mathrm{Sa}$.

Treten

Erpressung

Prügelei

Sex. Übergriffe

Treten

Erpressung

Prügelei

Sex. Übergriffe

\begin{tabular}{lcc} 
& \multicolumn{2}{c}{ Gesamt } \\
& Sa. & He. \\
Treten & 1,84 & 1,58 \\
Erpressung & 1,48 & 1,28 \\
Prügelei & 1,59 & 1,36 \\
Sex. Übergriffe & 1,59 & 1,40
\end{tabular}

Gesamt Sa.

Treten

Erpressung

Prügelei

3,61

3,89

3,80

3,88

1,94

Gesamt

Sa. He.

$2,62 \quad 2,40$

2,66

2,51

2,70

Sex. Übergriffe
Einstellungen/Reaktionen

Gewalt normal

$$
\text { Mädchen }
$$

Sa. He.

$2,31 \quad 2,03$

$1,69 \quad 1,45$

1,72

1,53

1,44

1,57

Angst vor Gewalt

Mädchen

$\mathrm{Sa}$.

$\mathrm{He}$.

2,59

2,04

2,28

2,26

2,83

2,44

2,97

Ärger über Gewalt

Mädchen

Sa.

$\mathrm{He}$.

2,80

2,54

3,05

2,81

3,04

2,67

3,14

3,40

Animation durch Gewalt

\section{Mädchen}

Gewalt unerwünscht

3,56

3,53
Sa.

1,54

1,33

1,34

$-$

\section{Mädchen}

\begin{abstract}
Sa.
\end{abstract}
3,87

4,13

4,10

$\mathrm{He}$.

1,41

1,18

1,23

$-$

4,26
$\mathrm{He}$.

3,62

3,85

3,79

3,88

Jungen

Sa. He.

$2,04 \quad 1,68$

$2,16 \quad 1,85$

$2,14 \quad 1,79$

$1,79 \quad 1,47$

1,64

1,78

1,73

Jungen

Sa. He.

$2,43 \quad 2,27$

$2,65 \quad 2,51$

$2,54 \quad 2,35$

$2,56 \quad 2,28$

* Skala: 1 = stimmt gar nicht ... 5 = stimmt ganz genau (in beiden Stichproben) 
In diesem Zusammenhang stellt sich die Frage, inwiefern sich die Reaktionen nach bestimmten Kriterien (Geschlecht, Jahrgang) unterscheiden. Zu erwarten wäre z. B., daß sich nach diesen Kriterien jeweils eher gewaltablehnende und eher gewaltbefürwortende Gruppen herauskristallisieren. Diese Überlegung trifft beispielsweise auf die Differenzierung nach dem Geschlecht zu. Mädchen tendieren eher zu gewaltablehnenden Reaktionsformen, Jungen zu befürwortenden. Hinsichtlich der Jahrgänge ist zwar das gewaltbefürwortende Klima der 8. Klassen markant, jedoch lassen sich bei der Betrachtung der 6. und 10. Klassen keine eindeutigen Strukturen ausmachen: Zwar beurteilen die Schüler der 6. Klassen die Gewaltsituationen im Vergleich zu den älteren seltener als normal und haben tendenziell mehr Angst vor Gewalt; gleichzeitig fühlen sie sich durch Gewalt aber auch stärker animiert.

Bei der Unterscheidung nach den Bundesländern ergibt sich das zunächst verblüffende Ergebnis einer sowohl stärkeren Gewaltbefürwortung als auch stärkeren Gewaltablehnung durch die sächsischen Schüler. Die Schüler in Sachsen reagieren also auf Gewalt emotional viel extremer und widersprüchlicher. Die im Vergleich zu Hessen stärkere Befürwortung von Gewalt auf der Einstellungsebene steht dabei im Kontrast zu den in Sachsen vorliegenden etwas niedrigeren Werten auf der Handlungsebene, also den tatsächlich ausgeübten Gewalttaten. Als Erklärung für diesen paradoxen Befund kann die gesellschaftliche Umbruchsituation im Osten angeführt werden. So hat der Umbruch durch den Wegfall bisheriger Kontrollinstanzen dazu geführt, daß bisherige Normen und Werte erodierten, was einerseits als „Freisetzung“ und „Normalisierung" von Gewalt wahrgenommen wird. Andererseits scheinen aber auch noch bestimmte Normvorstellungen weiter zu existieren, so daß Gewalthandlungen eigentlich eher abgelehnt werden. Dies könnte die für ostdeutsche im Vergleich zu westdeutschen Jugendlichen feststellbare größere Differenz von Gewalteinstellungen und Gewalthandlungen, über die auch in anderen Studien berichtet wird, erklären (vgl. z. B. Friedrich/Förster 1996, Schmidtchen 1997): Obwohl ostdeutsche Jugendliche Gewalt als etwas „Normales“ ansehen und Gewalt eher billigen, vollziehen sie vergleichsweise (noch?) weniger Gewalthandlungen. Anscheinend verfügen sie noch über höhere Hemmschwellen bzw. über stärkere Kontroll- und Steuerungsdispositionen, die sie am Vollzug entsprechender Gewalthandlungen stärker hindern. Ob es auch hier künftig zu einer größeren Annäherung zwischen Einstellungs- und Handlungsebene entsprechend den westdeutschen Verhältnissen kommt, ist vorerst zwar noch Spekulation, u.E. aber doch wahrscheinlich.

Darüber hinaus fällt auf, daß die Ost-West-Unterschiede bei der Gewaltbefürwortung (Gewalt normal, Animation durch Gewalt) bei den Jungen größer sind als bei den Mädchen. Umkehrt sind die Differenzen bezüglich der Gewaltablehnung (Angst, Ärger, Ünerwünschtheit) bei den Mädchen größer als bei den Jungen (vgl. Tab. 2-8). Die gewaltbefürwortende Haltung wird also von den sächsischen Jungen stärker als von den hessischen demonstriert, während die sächsischen Mädchen stärker ihre gewaltablehnende Einstellung 
artikulieren. Dies verweist ein weiteres Mal auf eine stärkere Verbreitung von geschlechtsstereotypen Vorstellungen bei sächsischen Schülern. Auch hier gehen wir davon aus, daß die Umbruchsituation, in der verstärkt auf traditionelle Weiblichkeits- und Männlichkeitsbilder zurückgegriffen wurde, mit in Rechnung gestellt werden muß (vgl. auch Kap. 3).

Tab. 2-9: Teilmodell zur Erklärung der Gewaltemergenz am Beispiel selbstberichteter Gewalthandlungen (Mutiple Reggressionsanalyse)

So etwas habe ich in den letzten 12 Monaten selbst getan.

Ein Junge/ein Mädchen tritt jemanden in böser Absicht. ${ }^{+}$
Zwei prügeln sich so stark, daß es Verletzungen (z. B. blutige Nasen, blaue Augen) gibt. ${ }^{+}$

\begin{tabular}{lcc} 
& $\beta^{++}$ & $\beta^{++}$ \\
Klasse 6 & $-.05^{* *}$ & $-.05^{* *}$ \\
Klasse $10^{++}$ & $-.10^{* *}$ & $-.05^{* *}$ \\
Geschlechtt++ & $.26^{* *}$ & $.23^{* *}$ \\
Ost-West & -.02 & .02 \\
\hline
\end{tabular}

$+\quad$ Es handelt sich um zwei getrennt durchgeführte Regressionsanalysen.

++ standardisierter Regressionskoeffizient

+++ Bei den Jahrgangsstufen wurden Dummy-Variablen gebildet. Die Klasse 8 stellt in beiden Analysen die Referenzkategorie dar. Die der Dimension "Geschlecht" zugrundeliegende DummyVariable beinhaltet die Werte $0=$ Mädchen und $1=$ Jungen. Für die Ost-West-Dimension gelten die Werte $0=$ Sachsen und $1=$ Hessen.

** $p<.01$

Zum Abschluß der Ost-West-Analysen wurde mittels des Verfahrens der multiplen Regression innerhalb einer Gesamtstichprobe der Schüler, d.h. Sachsen und Hessen in einer gemeinsamen Stichprobe, geprüft, welches der bisher untersuchten Merkmale (,Geschlecht“, ,Jahrgangsstufe“ oder „Bundesland“) die größten bzw. geringsten Effekte auf Gewaltemergenz aufweist. Anliegen dieser Analyse war es nicht, ein umfassendes multivariates Modell zur Aufklärung der Gewaltemergenz zu überprüfen (vgl. dazu Kapitel 7), sondern den relativen Einfluß der genannten Faktoren auf einer multivariaten Ebene im Ost-West-Vergleich zu bestimmen ${ }^{20}$. Die statistischen Berechnungen ergaben, daß dem Merkmal „Bundesland“ (Sachsen - Hessen) von den in die Regressionsanalysen einbezogenen Variablen das geringste Gewicht zukommt (vgl. Tab. 2-9). Dies läßt darauf schließen, daß bei der Erklärung für Gewalt an

20 Der Anteil der aufgeklärten Varianz liegt dementsprechend bei beiden Regressionen in einem niedrigen Bereich ( $8 \%$ bzw. 6\%). 
Schulen solche Faktoren, wie „Geschlecht“, vermutlich aber auch Faktoren der familialen und/oder schulischen Sozialisation sowie die "Schulform“21 relevanter sind als der Faktor „Bundesland“. Deshalb werden bei den weiteren Analysen diese Faktoren auch im Mittelpunkt der Betrachtungen stehen, während eine Untersuchung der Ost-West-Unterschiede nicht systematisch weiterbetrieben wird.

\subsection{Bilanz des Ost-West-Vergleichs}

Was hat nun der Ost-West-Vergleich an substantiellen Ergebnissen gebracht? Welche Folgerungen ergeben sich daraus für die weitere Forschung sowie für die Schulpraxis und die Schulpolitik?

Im folgenden sollen zusammenfassend vor allem fünf Hauptergebnisse hervorgehoben werden:

1. Als Hauptergebnis bleibt festzuhalten, daß die Gewaltbelastung an sächsischen Schulen im Vergleich zu der an hessischen Schulen etwas geringer ist. Damit wurden die bisherigen Untersuchungsergebnisse zum OstWest-Vergleich (sowohl aus der vergleichenden Schulleiterbefragung als auch aus bisherigen Analysen der repräsentativen Schülerbefragung) bestätigt. Allerdings sind die Differenzen meist sehr gering. Die geringen Differenzen (ermittelt durch die Wahrnehmung des Gewaltausmaßes als auch durch den Selbstreport) betreffen nahezu alle untersuchten Dimensionen gewaltförmigen Verhaltens und darüber hinaus auch das Interventionsverhalten in Gewaltsituationen. Die weitgehenden Ähnlichkeiten zwischen den Schülerpopulationen in bezug auf gewaltförmiges und abweichendes Verhalten werden auch durch andere Befunde der Studie, z. B. zur Schuldevianz und zur Delinquenz, bestätigt. ${ }^{22}$

2. Ein weiteres Ergebnis der Ost-West-Analysen ist, daß die bisherige Annahme von der geringeren Gewaltbelastung in Sachsen im Vergleich zu

21 Eine Regressionsanalyse in der gemeinsamen Stichprobe für Sachsen und Hessen unter Einbeziehung der Schulform war aufgrund der in diesen beiden Bundesländern jeweils völlig anders strukturierten Sekundarstufe I nicht möglich. Wie jedoch bereits unter Abschnitt 2.1 gezeigt, hat sich bei einer Regressionsanalyse nur für Sachsen die Bedeutsamkeit der Schulform für die Erklärung des selbstberichteten Gewaltverhaltens in den konkreten Gewaltsituationen herausgestellt.

22 So gibt es in der Regel nur geringe Differenzen bei der Schuldevianz (allerdings schwänzen deutlich mehr hessische Schüler die Schule als sächsische) und bei Delinquenz (z. B. Einbruch). Signifikante Unterschiede treten dagegen bei autoritären und nationalistischen Orientierungen auf: Sächsische Schüler sind im Vergleich zu hessischen Schülern stärker autoritär und nationalistisch eingestellt (vgl. auch Kap. 4). 
Hessen relativiert und modifiziert werden muß. Eine Modifizierung und Differenzierung macht sich erforderlich, denn die Unterschiede im Gewaltniveau betreffen

- eher die extremen als die alltäglichen Gewaltformen,

- eher die Gewalthandlungen außerhalb als innerhalb der Klasse,

- eher die Mädchen als die Jungen,

- eher die oberen als die unteren Jahrgangsstufen der Sekundarstufe I,

- eher die wahrgenommenen als die selbstberichteten Gewalthandlungen und

- eher die Befunde aus Schülersicht als die aus Lehrersicht.

Daraus kann u.a. geschlußfolgert werden, daß die geringere Gewaltbelastung in Sachsen in erster Linie auf das geringere Gewaltniveau bei den Mädchen und den höheren Jahrgangsstufen zurückzuführen ist, was für den von uns unterstellten Angleichungsprozeß weitreichende Konsequenzen hat.

3. Für unsere Angleichungs-Hypothese, d.h. für die Angleichung des Gewaltniveaus des Ostens an das des Westens, lassen sich in unseren Analysen einige Anhaltspunkte finden. Da die Unterschiede in den 6. Klassen nahezu verschwunden sind, kann eine künftig weiter voranschreitende Angleichung prognostiziert werden. Die Angleichung vollzieht sich dabei von den eher alltäglichen zu den extremeren Gewaltformen. Auf eine mögliche Angleichung deuten auch die Befunde zu den Reaktionen in Gewaltsituationen hin.

Die in unserer Studie festgestellte geringe Relevanz der Ost-West-Dimension für die Gewaltentwicklung sollte allerdings nicht darüber hinwegtäuschen, daß Einflußfaktoren, die im Rahmen einer Schulstudie nicht zu erfassen waren - wie beispielsweise die wirtschaftliche und soziale Infrastruktur - als moderierende Variablen die Ost-West-Gegensätze auch im Bereich der Gewalt womöglich dahingehend verschärfen könnten, daß der hier festgestellten Angleichungstendenz eine „Überflügelung“ folgt. Gleichzeitig gilt es zu bedenken, daß die zunehmende Verschärfung der wirtschaftlichen und sozialen Probleme ein ost-west-übergreifendes Phänomen ist - allerdings eines mit einem besonderen Problemdruck im Osten, der auf absehbare Zeit kaum zu beheben sein wird.

4. Unsere Befunde machen außerdem deutlich, daß angesichts von Aggression und Gewalt ein professionelles pädagogisches Handeln in zunehmendem Maße gefragt ist. Die Analysen belegen, daß in Ost wie West - bei einem noch größeren Nachholebedarf im Osten - die sozialen Kompetenzen und die notwendige Zivilcourage mitunter nicht ausreichen, pädagogisch angemessen in Gewaltsituationen zu reagieren. Damit ist ein wichtiges Feld für die Aus- und Fortbildung benannt. Darüber hinaus ist dem prosozialen Lernen in der Schule insgesamt größere Bedeutung zuzumessen.

5. Schließlich zeigen erste Regressionsanalysen zum Erklärungswert der einbezogenen Dimensionen im Hinblick auf die Gewaltemergenz, daß in un- 
serer Untersuchung dem Merkmal „Bundesland“ eine eher untergeordnete Bedeutung zukommt. Bei den weiteren Analysen zur Aufhellung von Ursachen und Bedingungsfaktoren für Gewalt wird der Ost-West-Vergleich daher nicht im Mittelpunkt stehen. Vielmehr wird im Rahmen unseres Modells (vgl. Kap. 1) die Analyse von Aspekten der familialen Sozialisation, der außerschulischen Einflüsse (vgl. Kap. 5) und der Schulkultur (vgl. Kap. 6 ) den zentralen Forschungsschwerpunkt bilden.

Auch wenn die Ost-West-Unterschiede im Gewaltausmaß gering sind und ebenso die Erklärungskraft der Variable „Land“ relativ bescheiden ausfällt, heißt das nicht, daß Probleme der Gewaltentwicklung in den neuen Bundesländern künftig keiner besonderen politischen, pädagogischen und wissenschaftlichen Begleitung bedürfen (vgl. dazu insbesondere das 3. Hauptergebnis). Vielmehr bleibt als Konsequenz festzuhalten, daß die spezifischen Problemkonstellationen an Schulen in den neuen Bundesländern (vgl. auch Kap. 6) für Wissenschaft, Politik, vor allem aber für Lehrer und Erzieher auch weiterhin eine große Herausforderung darstellen. Für die schulbezogene Gewaltforschung heißt das auch, neben der Analyse der Gewalt in der Schule die gesamte Gewaltentwicklung bei Kindern und Jugendlichen im Kontext gesellschaftlicher, soziokultureller Wandlungsprozesse stärker in den Blick zu nehmen (vgl. auch Heitmeyer/Ulbrich-Herrmann 1997). 


\section{Jungengewalt - Mädchengewalt - ein Exkurs (Dorit Stenke, Sandra Bergelt, Franziska Börner)}

Es wird mittlerweile allgemein davon ausgegangen und auch in den vorliegenden Untersuchungsergebnissen zur Gewalt an Schulen bestätigt, daß Jungen als Täter in Gewalthandlungen wesentlich stärker involviert sind und auch als Opfer wesentlich häufiger von Gewalt betroffen sind als Mädchen. „Gewalt“ - so stellt auch Böhnisch 1994 fest - „hat ein männliches Gesicht.“ Zweierlei ist an diesem Befund allerdings auffällig:

Zum einen wird der - wenn auch geringe - Anteil der Mädchen übersehen, die inzwischen - und wie festgestellt wird, zunehmend (vgl. Mansel 1995, S. 110) - zu physischen Gewalthandlungen greifen und ein deutlich aggressives Verhalten zeigen. Der im vorangegangenen Kapitel bereits vorgestellte Befund, daß die Differenz im Gewalthandeln bei hessischen Jungen und Mädchen geringer ist als bei den sächsischen Jungen und Mädchen, läßt sich ebenfalls in diese Richtung interpretieren, wenn man der These zustimmt, daß sich die Jugendlichen in den neuen Bundesländern noch in der Phase der nachholenden Modernisierung befinden. Es ist dann für die Zukunft zu erwarten, daß auch hier der Abstand geringer werden wird.

Zum anderen wird dem Umstand, daß es sich um ein männliches Phänomen handelt, gerade in der pädagogischen Diskussion zu wenig Bedeutung beigemessen. Nur selten werden geschlechtsspezifische Erklärungsmuster herangezogen und noch weniger wird eine geschlechterbezogene Pädagogik eingefordert, die sich mit den Ursachen von Gewalt gerade im männlichen Lebenszusammenhang beschäftigt. So wird von der Frauenforschung noch immer zu Recht kritisiert, daß die bisherige Gewaltforschung eher geschlechtsunspezifisch erfolgt und die soziale Kategorie Geschlecht vernachlässigt wird (vgl. zur Kritik z.B. Meyer 1993, zuletzt Popp 1997). Gewalt wird zwar vorwiegend als männliches bzw. männerspezifisches Problem definiert, eine Auseinandersetzung über männerspezifische Zugangsweisen zur Gewalt und die Bedeutung von Gewalthandlungen für die männliche Sozialisation beginnt jedoch erst zögerlich. Wenn es aber tatsächlich geschlechtsspezifische oder geschlechtsbezogene unterschiedliche Zugänge zum Gewalthandeln gibt, dann gilt es auch stärker als bisher nach der Beteiligung von Mädchen und ihrem spezifischen Umgang mit Gewalt und der Bedeutung von Gewalthandeln für die Sozialisation von Mädchen zu fragen.

Wie im folgenden zu zeigen sein wird, kann diesen zuletzt formulierten Fragen anhand der vorliegenden standardisierten Befragung von sächsischen Schülerinnen und Schülern nur eingeschränkt nachgegangen werden, weil zahlreiche angesprochene Aspekte für die Anlage der Untersuchung keine Rolle gespielt haben und sich demzufolge auch keine Ergebnisse formulieren lassen. So wurde nicht nach unmittelbar geschlechtsbezogenem Gewalthandeln 
gefragt, weder nach verbalen Aggressionen von Jungen und Mädchen, die das jeweils andere Geschlecht diskreditieren, noch nach aggressivem Gewalthandeln gegenüber Jungen und Mädchen, die sich geschlechtsuntypisch (z.B. als „Schlampe“, „Streber“, „Feigling“) verhalten. Eine Ausnahme in diesem Zusammenhang stellen zwei Items dar, die unter dem Begriff „Sexuelle Belästigung" zusammengefaßt werden. In beiden Items stecken allerdings wiederum einseitige geschlechtertypische Zuschreibungen (Jungen fassen Mädchen an den Busen, Mädchen fassen Jungen zwischen die Beine). Der Fall, daß auch Aggressionen von Jungen gegenüber anderen Jungen in der beschriebenen Art vorkommen, ist nicht vorgesehen. Ebensowenig körperliche, auch sexualisierte Übergriffe von Mädchen gegenüber anderen Mädchen. Schließlich läßt sich auch noch der Fall denken, daß ein Mädchen von anderen gezwungen wird, einen Jungen sexuell zu belästigen oder ein Junge von anderen aufgefordert wird, sich in der beschriebenen Weise einem Mädchen - oder einem Jungen - zu nähern. Gegen diese Aufzählung läßt sich mit Recht einwenden, daß immer noch weitere zahlreiche Situationen denkbar sind, die im Rahmen eines notwendigerweise im Umfang beschränkten Instrumentes nicht alle erfaßt werden können. Es sollte allerdings darauf hingewiesen werden, daß die Konstruktion von Untersuchungsinstrumenten schon von bestimmten geschlechtertypischen Vorstellungen ausgeht und eine Vielzahl von geschlechterbezogenen Erkenntnissen ausschließt. Nun war dies auch nicht das zentrale Anliegen der vorliegenden Befragung.

Gleichwohl soll im folgenden ersten Abschnitt nach den Unterschieden von Mädchen und Jungen im Hinblick auf Ausmaß und Involviertheit in Gewalthandeln gefragt und die Einstellungen der Schülerinnen und Schülern zu Gewalt insgesamt dargestellt werden. In einem zweiten Abschnitt wird anhand von Interviews in Jungen- und Mädchengruppen zum Thema Schule und Gewalt ein anderes methodisches Verfahren vorgestellt und der Zugang der Jungen und Mädchen zu Gewalt thematisiert. Welche Bedeutung hat Gewalthandeln für die männliche und weibliche Sozialisation? Außerdem wird untersucht, wie sich die Jugendlichen ihren eigenen Umgang mit Gewalt und den des jeweils anderen Geschlechts erklären. Abschließend sollen Forschungsdesiderate aufgezeigt und die Konsequenzen für eine pädagogische Gewaltprävention und -intervention erörtert werden. 


\subsection{Ergebnisse der quantitativen Befragung von Schülerinnen und Schülern}

\section{Beobachtungen von Gewalthandlungen bei Jungen und Mädchen}

Nachdem im Kapitel 2 geschlechtsspezifische Analysen zur Wahrnehmung von Gewalt vor allem im Rahmen des Ost-West-Vergleichs vorgenommen wurden, soll an dieser Stelle ein Überblick über die geschlechtsspezifischen Unterschiede an sächsischen Schulen gegeben werden.

Mit den Ergebnissen dieser Analyse schließen wir an die Aussagen aus Kapitel 2 an: Jungen nehmen gewalthaltige Auseinandersetzungen stärker wahr als Mädchen. Den Jungen fallen ebenso wie den Mädchen verbale Aggressionen am ehesten auf, gefolgt von Spaßkampf (zwischen zwei Jungen oder zwei Mädchen), Aggressionen gegen Lehrer und Prügeleien. Dagegen werden Angriffe mit einer Waffe, Erpressung, sexuelle Belästigung und Vandalismus weniger häufig beobachtet und von ihnen berichtet.

Betrachtet man nun die Häufigkeit der Wahrnehmung von Gewalt genauer, so fällt ein signifikanter geschlechtsspezifischer Unterschied auf. Unabhängig von den Gewaltformen, nehmen Schülerinnen weniger Gewalt an Schulen wahr als Schüler. Das hängt zum einen damit zusammen, daß Jungen insgesamt mehr in Gewalthandlungen involviert sind und zum anderen sich Gewalthandlungen oft in Jungengruppen abspielen, in denen Mädchen sie gar nicht wahrnehmen können. Es ist aber auch davon auszugehen, daß Jungen oftmals zu Übertreibungen („Angebereien“) neigen, um in ihrem Ansehen als „,echte“ Jungen/Männer zu steigen bzw. als jemand wahrgenommen zu werden, den man ernst nehmen muß (vgl. Kap 4). Daraus folgernd können wir vermuten, daß sich die Schüler entsprechend den Erwartungen der Lehrerinnen und Lehrer, der Mitschülerinnen und Mitschüler, der Eltern usw. an die Jungenrolle verhalten. Wie sich noch zeigen wird, ist Gewalt für Jungen eher ein normales Phänomen alltäglichen Handelns als für Mädchen. Außerdem könnten Jungen, gerade wenn sie selbst gewalttätig sind, ein Interesse daran haben, darauf hinzuweisen, daß die anderen ebenfalls zu Gewalthandlungen neigen. Damit wird die unterstellte Normalität des Gewalthandelns wiederum bestätigt.

Auffällig ist allerdings die geschlechtsspezifisch unterschiedliche Wahrnehmung von Gewalt je nach Deliktart. Während sich bei der Wahrnehmung von Aggressionen gegen Lehrer nur eine Differenz von 6 Prozentpunkten ergibt (28,1\% der Mädchen und 34,1\% der Jungen), zeigen sich bei der Wahrnehmung von verbalen Aggressionen und Spaßkampf jeweils 10 Prozentpunkte Differenz zwischen den Geschlechtern, bei den Deliktarten Erpressung und Angriffen mit einer Waffe sind es jeweils doppelt so viele Jungen wie Mädchen, die dieses wahrnehmen. Die geringste Differenz ergibt sich bei der Wahrnehmung von Aggressionen gegen Lehrer, vermutlich weil bei dieser Deliktform Jungen wie Mädchen gleichermaßen anwesend sind. 
Abb. 3-1: Beobachtung schulischer Gewaltformen durch Mädchen und Jungen (Prozentanteil der Schülerinnen und Schüler, die die angeführten Gewaltformen mindestens mehrmals im Monat beobachtet haben)*

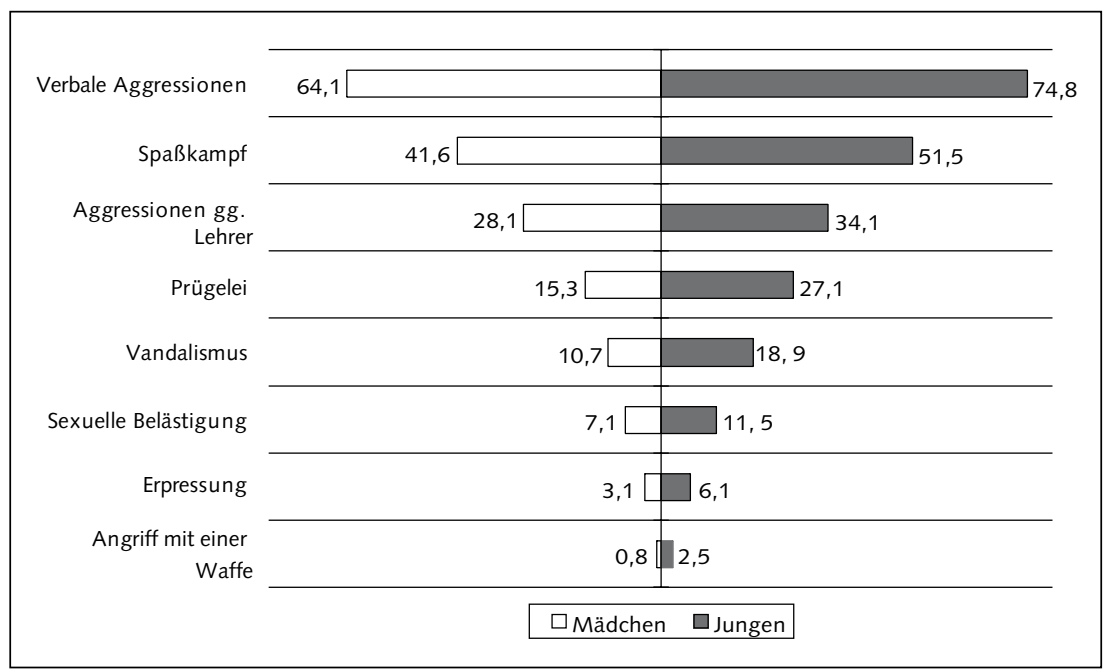

* Die Rangreihe wurde nach den Mittelwerten der Gesamtstichprobe erstellt.

Legende:

Verbale Aggressionen:

Spaßkampf:

Aggressionen gegen Lehrer:

Prügelei:

Vandalismus:

Sexuelle Belästigung:

Erpressung:

Angriff mit einer Waffe:
Beschimpfen/Gemeine Ausdrücke. (V157)

Spaßkampf zwischen zwei Jungen. (V136)

Schüler/innen beschimpfen oder beleidigen Lehrpersonen in deren Gegenwart. (V146)

Ernsthafte Prügelei zwischen zwei Jungen. (V137)

Unterrichtsmaterial wurde absichtlich beschädigt. (V133)

Ein oder mehrere Jungen bedrängen ein Mädchen und fassen es gegen ihren Willen an (z.B. an den Busen). (V144)

Jemand fordert Geld und droht mit Strafe (z.B. Prügel), wenn nicht gezahlt wird. (V162)

Jemand wird von einem (einer) anderen mit einer Waffe (Schlagring, Messer usw.) angegriffen. (V143)

Wie sieht die geschlechtsspezifische Beteiligung an Gewalthandlungen aus der Perspektive von Jungen und Mädchen aus? Die folgende Tabelle 3-1 zeigt den Anteil der Jungen und Mädchen, die die angegebenen Gewalthandlungen im Verlauf des letzten Schuljahres an der jeweiligen Schule bei Jungen und/ oder Mädchen beobachtet haben. Die Zahl der Schülerinnen und Schüler, die auf die Frage antworteten, variiert - entsprechend der Häufigkeit der Beobachtung - sehr stark zwischen den verschiedenen Items (Vandalismus wurde 
Tab. 3-1: Beobachtung von Gewalthandlungen nach Jungen und Mädchen (Angaben in Prozent)

\begin{tabular}{|c|c|c|c|c|c|c|}
\hline \multirow[b]{2}{*}{$\begin{array}{c}\text { So etwas habe ich } \\
\text { beobachtet ... }\end{array}$} & \multicolumn{3}{|c|}{ Mädchen } & \multicolumn{3}{|c|}{ Jungen } \\
\hline & $\begin{array}{l}\text { fast nur bei } \\
\text { Jungen }\end{array}$ & $\begin{array}{l}\text { bei Jungen } \\
\text { / Mädchen }\end{array}$ & $\begin{array}{l}\text { fast nur bei } \\
\text { Mädchen }\end{array}$ & $\begin{array}{l}\text { fast nur bei } \\
\text { Jungen }\end{array}$ & $\begin{array}{l}\text { bei Jungen } \\
\text { / Mädchen }\end{array}$ & $\begin{array}{l}\text { fast nur bei } \\
\text { Mädchen }\end{array}$ \\
\hline $\begin{array}{l}\text { (non)verbale Attacken } \\
\text { zw. Schüler/innen }{ }^{1} \\
(n=2871)\end{array}$ & 13 & 82 & 5 & 19 & 78 & 3 \\
\hline $\begin{array}{l}\text { ausländerfeindliche } \\
\text { Sprüche }{ }^{2} \\
(n=2328)\end{array}$ & 36 & 63 & 1 & 40 & 59 & 2 \\
\hline $\begin{array}{l}\text { subtile Aggression } \\
\text { gegen Lehrer } \\
(n=2466)\end{array}$ & 25 & 72 & 3 & 31 & 66 & 4 \\
\hline $\begin{array}{l}\text { manifeste Aggressionen } \\
\text { gegen Lehrer }{ }^{4} \\
(n=1318)\end{array}$ & 37 & 62 & 1 & 36 & 61 & 3 \\
\hline $\begin{array}{l}\text { Vandalismus }{ }^{5} \\
(\mathrm{n}=2837)\end{array}$ & 37 & 62 & 1 & 53 & 45 & 2 \\
\hline $\begin{array}{l}\text { Erpressung von } \\
\text { Mitschüler/ innen } \\
(\mathrm{n}=1635)\end{array}$ & 54 & 45 & 1 & 58 & 41 & 2 \\
\hline
\end{tabular}

1 Die Beobachtungen beziehen sich auf die Items „Auslachen/Spotten“ (V156), „Beschimpfen/ Gemeine Ausdrücke“ (V157), „Gemeine Gesten/Zeichen“(V158), „Daß jemanden von anderen ausgeschlossen (geschnitten) wird.“ (V158a).

2 Die Beobachtungen beziehen sich auf das Einzelitem „Ausländerfeindliche Sprüche“ (V169).

3 Die Beobachtungen beziehen sich auf die Items ,Schüler/innen beschimpfen oder beleidigen Lehrpersonen in deren Gegenwart.“ (V146) und „Telefonterror gegen Lehrpersonen.“ (V147).

4 Die Beobachtungen beziehen sich auf die Items ,Schüler/innen bedrohen Lehrer/innen (z.B. mit Schlägen).“ (V149), „Schüler/innen schlagen absichtlich eine Lehrperson.“ (V150) u. „Schüler/ innen entwenden oder beschädigen absichtlich Sachen, die einer Lehrperson gehören.“(V151).

5 Die Beobachtungen beziehen sich auf die Items ,Einrichtungsgegenstände wurden absichtlich beschädigt.“ (V130), „Sachen, die anderen Schülern gehören, wurden absichtlich beschädigt.“ (V131), „Wände wurden absichtlich beschmiert.“ (V132), „Unterrichtsmaterial wurde absichtlich beschädigt.“ (V133), „Toiletteneinrichtungen wurden absichtlich beschädigt.“(V134).

6 Die Beobachtungen beziehen sich auf die Items, ,Jemand fordert Geld und droht mit Strafe (z.B. Prügel), wenn nicht gezahlt wird.“ (V162), ,Jemand fordert, Sachen abzugeben (z.B. eine schöne Jacke) und droht mit Strafe (z.B. Prügel), wenn nicht abgegeben wird.“ (V163), „Jemand droht mit Strafe (z.B. Prügel), falls dem Lehrer etwas mitgeteilt wird.“(V164). 
häufiger beobachtet als Lehrerschikane, vgl. Abb. 3-1). Aus diesem Grund ist die Summe (n) der Schülerinnen und Schüler, die jeweils antworteten, in jeder Zeile aufgeführt. Die Einschätzungen der Beteiligung der Geschlechter beinhaltet demzufolge keine Angabe über die Vorkommenshäufigkeit an den Gewalthandlungen.

Insgesamt sprechen die Aussagen für die schon oben erwähnten Ergebnisse der vorangegangenen Analysen, daß Jungen Gewalthandlungen tendenziell öfter wahrnehmen und stärker in Gewalthandlungen involviert sind als Mädchen. Das zeigt der große Anteil der Schülerinnen und Schüler, die abweichendes Verhalten „fast nur bei Jungen“ oder „bei Jungen und Mädchen“ beobachteten. Die meisten der Mädchen und Jungen sind sich darin einig, daß mit Ausnahmen von Vandalismus und Erpressung von Mitschülerinnen und Mitschülern beide Geschlechter an Gewalthandlungen beteiligt sind und aggressive Handlungen sehr selten ,fast nur bei Mädchen“ zu beobachten waren. Über die Hälfte der Schüler (53\%) gibt an, Vandalismus ,fast nur bei Jungen“ beobachtet zu haben. Die meisten Schülerinnen (62\%) dagegen haben diese Art des gewalttätigen Verhaltens ,bei Jungen und Mädchen“ beobachtet.

Darüber hinaus gibt es wenige Unterschiede zwischen den einzelnen Gewalthandlungen hinsichtlich der wahrgenommenen Beteiligung der Geschlechter. Erwähnenswert ist die Form der (non)verbalen Attacken zwischen Schülerinnen und Schülern. Da sind es prozentual mehr Mädchen und auch Jungen, die diese Handlung eher beiden Geschlechtern zuordnen als ,nur den Jungen“ oder „,nur den Mädchen“. Diese Form der Gewalt wurde von den Mädchen im Vergleich zu allen anderen als diejenige eingeschätzt, die am ehesten „,fast nur von Mädchen“ ausgeführt wurde. Zwar ist das Auftreten mit $5 \%$ im Vergleich zu den anderen beiden Kategorien (,fast nur bei Jungen“, „,bei Jungen und Mädchen“) sehr gering, bezogen auf die anderen Gewalthandlungen ist diese Beobachtung jedoch auffallend. Vielleicht ist das auch als ein Hinweis darauf zu interpretieren, daß verbale Attacken Gewaltformen sind, die unter den Mädchen auch dann vorkommen, wenn keine Jungen zugegen sind - also eine Form von Gewalt, die von Mädchen - eher als anderes Gewalthandeln - auch untereinander verübt wird.

\section{Täterselbstreport}

Während auf der Seite der Beobachtung der Unterschied zwischen Jungen und Mädchen hinsichtlich der Häufigkeit bereits stark ausgeprägt ist, weisen die Täterselbstreporte noch deutlichere geschlechtsspezifische Differenzen auf. In Kapitel 2 basieren die Analysen zum Gewalttäterverhalten auf der Auseinandersetzung mit vorgegebenen, konkreten Situationen. Bei der folgenden Untersuchung, bei der verschiedene Einzelitems betrachtet werden, ist der situationsunabhängige Täter-Selbstreport Gegenstand der Analyse. In der Abbildung 3-2 sind alle gewalttätigen Handlungen aufgeführt, nach deren 
Beteiligung in der Untersuchung gefragt wurde. Die Schülerinnen und Schüler sollten anhand einer sechsteiligen Skala (von $1=$ nie, $6=$ fast täglich) angeben, wie oft sie die angeführten aggressiven Handlungen in den letzten 12 Monaten ausgeführt haben. In der Abbildung sind die Prozentanteile der Schülerinnen und Schüler angegeben, die die Handlungen mindestens mehrmals im Monat durchgeführt haben.

Es fällt auf, daß Mädchen von einem deutlich geringeren Anteil an den in Schulen durchgeführten Gewalthandlungen berichten als Jungen. Ihre Beteiligung ist allerdings an jeder angeführten Handlung gegeben, Mädchen sind also nicht ausschließlich unbeteiligt. Auffällig ist jedoch die geschlechtsunabhängige Differenz zwischen den einzelnen Arten von Aggressionen. Mädchen wie Jungen führen die ,härteren“ Aggressionen, wie beispielsweise Vandalismus und Diebstahl, seltener aus als aggressive Handlungen wie Schlagen, Hänseln, Ärgern. Erstaunlich ist die Ausnahme ,des Beschimpfens anderer mit gemeinen Ausdrücken“: Diese Deliktform wird von Jungen (26,2\%) wesentlich häufiger ausgeführt als von Mädchen (9,4\%), obwohl sie sicherlich auch zu den eher ,weicheren“ Aggressionen zu zählen ist, aufgrund ihres offensiven Charakters aber vermutlich nicht zu den bevorzugten Verhaltensformen von Mädchen zählt. Für Jungen wie auch für Mädchen gilt, daß aggressives Verhalten häufiger durch sogenannte weichere Aggressionsformen zum Ausdruck kommt.

Bei Betrachtung der Mädchen und ihrer Beteiligung an aggressiven Handlungen ist festzustellen, daß die häufigste ausgeführte Gewalttätigkeit mit $21 \%$ das Ärgern im Unterricht ist. Zum gleichen Resultat (über ein Drittel der Jungen) führt auch die Analyse der männlichen gewalttätigen Handlungen. Mit Blick auf das Geschlecht ist auch die Position des Hänselns in der Rangreihung der Gewalthandlungen gleich. Während dies die Mädchen mit 15\% mindestens mehrmals monatlich tun, sind es bei den Jungen 28\%. An dritter Stelle steht bei den Mädchen das Ärgern von Lehrern und bei den Jungen das Beschimpfen anderer und das Ärgern von Lehrern.

Abbildung 3-2 verdeutlicht, wie unterschiedlich die Ausprägungen von Gewalt bezüglich der Geschlechterbeteiligung ist - so sind z.B. die Jungen an allen von uns als ,härtere“ Aggressionsformen bezeichneten Handlungen dreibis viermal so häufig beteiligt wie die Mädchen. Es gibt demgegenüber keine Deliktform, die von Mädchen häufiger als von Jungen angewendet wird. Demzufolge kann nach den hier vorliegenden Ergebnissen auch nicht behauptet werden, daß Mädchen spezifisch andere Gewaltformen bevorzugen als Jungen. Dies war allerdings im Rahmen einer geschlossenen Befragung und bei den vorhandenen Items auch nicht zu erwarten: Weder werden spezifische Aggressionsformen aufgeführt, die eher bei Mädchen vermutet werden (z.B. auto-aggressive Handlungen), noch konnten die befragten Schülerinnen und Schüler eventuell vorhandene, weitere Gewalthandlungen zusätzlich anführen. 


\section{Abb. 3-2: Täterselbstreport (Prozentanteil der Schülerinnen und Schüler, die die angeführten Gewalthandlungen mindestens mehrmals im Monat durchgeführt haben)*}

\begin{tabular}{|c|c|c|}
\hline andere im Unterricht ärgern & $21,0 \square$ & 35,9 \\
\hline andere hänseln & $15,3 \square$ & 27,8 \\
\hline andere beschimpfen & $9,4 \square$ & 26,2 \\
\hline Lehrpersonen ärgern & $13,1 \square$ & 26,1 \\
\hline andere bewerfen & $6,3 \square$ & 16,2 \\
\hline Streit anfangen & $4,0 \square$ & 10,5 \\
\hline anderen Sachen verstecken & $3,8 \square$ & 11,3 \\
\hline Prügelei & $3,0 \square$ & 12,1 \\
\hline anderen Sachen wegnehmen & $3,1 \square$ & $\square 10,6$ \\
\hline andere unter Druck setzen & $1,9 \square$ & 6,8 \\
\hline Beschädigung im Schulgebäude & $2,9 \square$ & 9,2 \\
\hline zusammen jemanden verprügeln & $2,3 \square$ & 7,4 \\
\hline Schulinventar zerstören & $2,1 \square$ & 7,0 \\
\hline anderen Sachen zerstören & $1,6 \square$ & 5,2 \\
\hline Belästigung, Bedrohung & $1,3 \square$ & 5,5 \\
\hline \multirow[t]{2}{*}{ Waffen mitführen } & $1,3 \square$ & 5,3 \\
\hline & $\square$ Mädchen & Jungen \\
\hline
\end{tabular}

* Die Rangreihe wurde nach den Mittelwerten der Gesamtstichprobe erstellt.

Legende:

andere im Unterricht ärgern:

andere hänseln:

andere beschimpfen:

Lehrpersonen ärgern:

andere bewerfen:

Streit anfangen:

anderen Sachen verstecken:

Prügelei:

anderen Sachen wegnehmen: andere unter Druck setzen: Beschädigung im Schulgebäude: zusammen jemanden verprügeln: Schulinventar zerstören:

anderen Sachen zerstören:

Belästigung, Bedrohung:

Waffen mitführen:
Andere im Unterricht geärgert, beworfen oder beschossen. (V260)

Andere gehänselt oder mich über sie lustige gemacht. (V262)

Andere mit gemeinen Ausdrücken beschimpft. (V278)

Einen Lehrer oder eine Lehrerin geärgert oder provoziert. (V261)

Andere mit Sachen (z.B. Lineal, Mäppchen) beworfen. (V274)

Mit anderen bewußt Streit angefangen, sie angeschrien, beschimpft. (V273)

Anderen Sachen versteckt, so daß sie sie nicht wiederfinden konnten. (V276)

Mich mit einem (einer) anderen geprügelt. (V264)

Anderen etwas gewaltsam weggenommen. (V279)

Andere unter Druck gesetzt. (V267)

Im Schulgebäude etwas absichtlich beschädigt. (V263)

Mit anderen einen Jungen/ein Mädchen verprügelt. (V265)

Sachen absichtlich zerstört, die der Schule gehören (z.B. Stühle, Bücher). (V275)

Sachen von anderen absichtlich kaputtgemacht. (V266)

Anderen auf dem Schulweg aufgelauert, sie belästigt, bedroht oder verprügelt. (V268)

Waffen (Schreckschußpistole, Reizgas) mit in die Schule gebracht. (V272) 


\section{Opferselbstreport}

Nachdem gezeigt wurde, inwieweit Mädchen und Jungen an Gewalthandlungen aktiv beteiligt sind bzw. wie oft sie solche Handlungen beobachtet haben, soll nun danach gefragt werden, mit welcher Häufigkeit Schülerinnen und Schüler Opfer von Aggressionen werden. Dies ist unter geschlechtsspezifischen Gesichtspunkten eine besonders interessante Frage, wird doch in der Frauenforschung häufig davon ausgegangen, daß Mädchen und Frauen in ganz besonderer Weise Opfer von (männlichen) Aggressionen und Gewalthandlungen sind (vgl. z.B. Kavemann 1992).

In der Abbildung 3-3 werden die Ergebnisse des Opferselbstreports in der Reihenfolge ihrer Häufigkeit dargestellt; dabei werden zum Täterselbstreport vergleichbare Handlungsformen aus der Opferperspektive betrachtet. Die Schülerinnen und Schüler wurden aufgefordert ihre Antworten auf die Frage, ob sie selbst an der Schule oder auf dem Schulweg in den letzten 12 Monaten Opfer von personen- oder sachbezogenen Angriffen geworden sind, auf einer 6-stufigen Skala zwischen den Ausprägungen „fast täglich“ und „,nie“ einzustufen. Für die folgende Darstellung wurden die Kategorien „fast täglich“, „mehrmals wöchentlich“ und „mehrmals im Monat“ zusammengefaßt. Dabei zeigt sich, daß Jungen wie Mädchen häufiger Opfer von personenbezogenen als von sachbezogenen Angriffen werden.

Auffällig ist wiederum - ähnlich dem Täterselbstreport - der Unterschied in der Häufigkeit zwischen den Geschlechtern, mit der sie angeben, Opfer zu sein. Die Jungen sind öfter als die Mädchen Ausübende und Leidtragende von Gewalthandlungen anderer (vgl. auch Melzer/Rostampour 1996, S. 140; Popp 1997, S. 77ff). Allerdings ist die Rangreihung der Häufigkeit bei Jungen und Mädchen etwas unterschiedlich. Die häufigste Form der Gewalt, bei der Mädchen Opfer sind, ist mit 6,3\% das Hänseln/Ärgern, dem folgt mit 5,6\% das Beschimpfen/Beleidigen und mit 3,1\% das Wegnehmen von Sachen anderer. Unerwartet ist das Ergebnis, daß 10\% der Jungen angeben, gehänselt bzw. geärgert zu werden. Seltener werden Jungen Opfer von Beschimpfungen bzw. Beleidigungen (9,1\%), 6\% der Jungen werden Sachen versteckt und 5\% werden geschlagen. Gehänselt zu werden ist somit nicht ausschließlich ein Problem der Mädchen, sondern betrifft auch die Jungen in einem hohen Ausmaß. Und wie aus dem Täterselbstreport zu erkennen war, sind die Ausführenden dieser Gewaltform ebenfalls Jungen wie Mädchen.

Jungen sind allerdings doppelt so häufig Opfer von Belästigungen, Bedrohungen, von sachbezogenen Beschädigungen sowie von Erpressung. Dreimal so häufig wie Mädchen müssen sie sich sogar einer Schlägerei stellen. Immerhin 5\% aller Jungen gaben an, mehrmals im Monat und öfter Opfer einer Schlägerei zu sein.

Inwieweit es sich hierbei jeweils um geschlechtsbezogene Aktivitäten handelt (jemand wird geärgert, weil sie ein Mädchen ist oder als Junge oder 
Mädchen ein nicht rollenkonformes oder zu rollenkonformes Verhalten zeigt), läßt sich mit dem vorliegenden Instrument nicht beantworten. Inwieweit Schule also einen Spiegel der gesellschaftlichen Machtverhältnisse und einen Ort der Gewalt gegen Mädchen darstellt, Mädchen also männlichen Dominanzstrukturen ausgesetzt sind, wie dies befürchtet wird (vgl. z.B. Kavemann 1992), läßt sich ebenfalls mit den vorliegenden Daten nicht beantworten.

Abb. 3-3: Opferselbstreport (Prozentanteil der Schülerinnen und Schüler, die mindestens mehrmals im Monat Opfer von Gewalthandlungen geworden sind)*

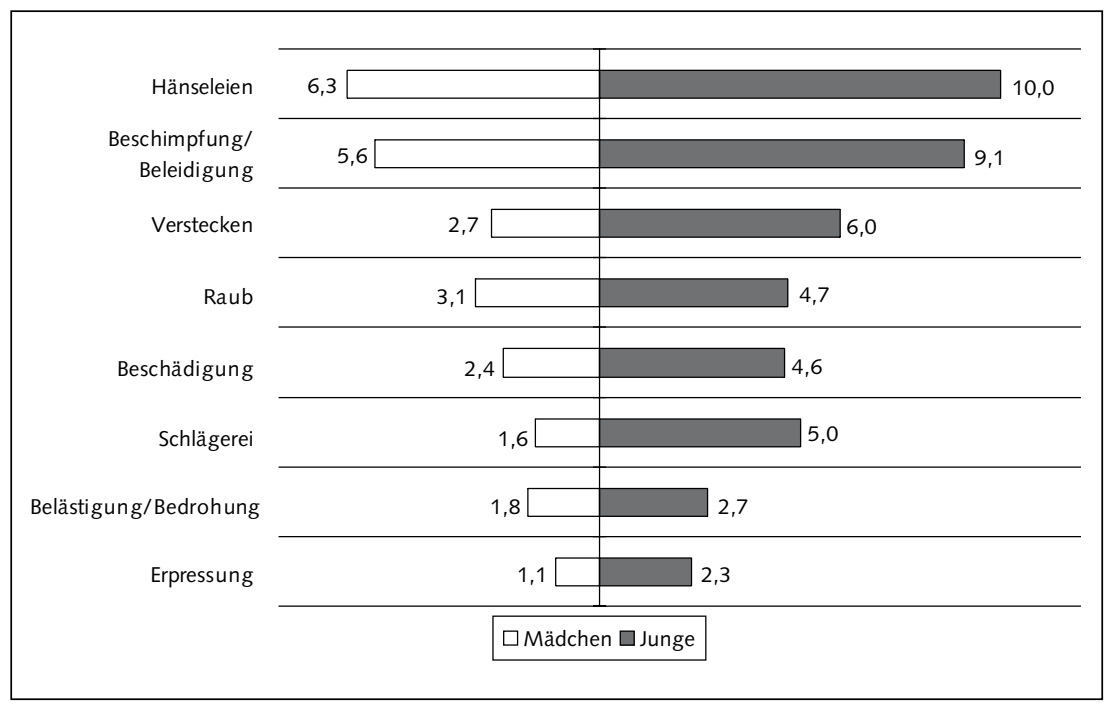

* Die Rangreihe wurde nach den Mittelwerten der Gesamtstichprobe erstellt.

Legende:

Hänseleien:

Beschimpfung/Beleidigung:

Verstecken:

Raub:

Beschädigung:

Schlägerei:

Belästigung, Bedrohung:

Erpressung:
Wie oft bist $\mathrm{Du}$ in den letzten 12 Monaten von anderen ... gehänselt oder geärgert worden? (V281) angeschrien, beschimpft, beleidigt worden? (V283) Sachen versteckt, so daß Du sie nicht wiederfinden konntest? (V287) gewaltsam etwas weggenommen? (V286) Sachen absichtlich kaputtgemacht? (V285) geschlagen worden? (V280)

Auf dem Schulweg belästigt, bedroht worden? (V282) unter Druck gesetzt, erpreßt worden? (V284) 


\section{Einstellungen zur Gewalt}

An die Untersuchung zur Beobachtung und zum Verhalten der Täter bzw. Opfer anschließend sollen in diesem Abschnitt die Einstellungen der Jungen und Mädchen zur Gewalt näher betrachtet werden. Dies geschieht durch drei unterschiedliche Herangehensweisen.

Zum einen werden Einstellungen untersucht, die von den Schülerinnen und Schülern getrennt auf einer 5-stufigen Zustimmungsskala ${ }^{1}$ angegeben wurden. Es handelt sich hierbei um Aussagen, die an traditionelle Geschlechtsrollenstereotype anknüpfen. In der Tabelle 3-2 sind die prozentualen Verteilungen der Zustimmung von „teils/teils“ bis "stimmt ganz genau“ aufgeführt. Dabei ist zu beachten, daß die Aussagen ausschließlich Gewalthandlungen, die von Jungen ausgehen, thematisieren. Es lassen sich also wiederum keine Erkenntnisse darüber gewinnen, welche Einstellungen Mädchen bzw. Jungen zu Gewalthandlungen von Mädchen und Frauen haben.

\section{Tab. 3-2: Geschlechtsspezifische Einstellungen zur Gewalt (Angaben in Prozent)}

\begin{tabular}{|c|c|c|c|}
\hline & teils/teils & $\begin{array}{l}\text { stimmt } \\
\text { überwiegend }\end{array}$ & $\begin{array}{l}\text { stimmt ganz } \\
\text { genau }\end{array}$ \\
\hline \multicolumn{4}{|c|}{ Mädchen } \\
\hline $\begin{array}{l}\text { Wenn sich Jungen prügeln, schaue ich gerne zu. } \\
\text { (V191) }\end{array}$ & 23,8 & 6,3 & 7,7 \\
\hline $\begin{array}{l}\text { Wenn sich zwei Jungen meinetwegen schlagen, } \\
\text { fühle ich mich geschmeichelt. (V192) }\end{array}$ & 21,4 & 10,1 & 10,5 \\
\hline $\begin{array}{l}\text { Ich habe oft Mitleid mit Jungen, die sich nicht so } \\
\text { richtig wehren können. (V193) }\end{array}$ & 33,5 & 29,1 & 16,6 \\
\hline \multicolumn{4}{|c|}{ Jungen } \\
\hline $\begin{array}{l}\text { Ich schlage mich besonders gerne, wenn Mädchen } \\
\text { dabei zuschauen. (V153) }\end{array}$ & 14,3 & 4,5 & 3,9 \\
\hline $\begin{array}{l}\text { Meistens gerate ich mit einem Jungen wegen } \\
\text { Mädchen in Streit. (V154) }\end{array}$ & 13,5 & 4,7 & 3,9 \\
\hline $\begin{array}{l}\text { Die Mädchen nehmen die hilflosen und schwachen } \\
\text { Jungen gerne in Schutz. (V155) }\end{array}$ & 30,3 & 10,8 & 9,5 \\
\hline
\end{tabular}

1 Skala von $1=$,stimmt gar nicht“ bis $5=$,stimmt ganz genau“; $3=$,teils/teils“. 
Bei den in der Tabelle dargestellten Items wurden die ersten drei Aussagen ausschließlich den Mädchen vorgestellt und die letzten drei Aussagen ausschließlich den Jungen, so daß sich die Ergebnisse nicht unmittelbar aufeinander beziehen lassen und auch die jeweilige Entsprechung fehlt. So können z.B. keine Aussagen darüber gemacht werden, ob Jungen auch gern zuschauen, wenn sich andere Jungen prügeln, oder ob Jungen Mitleid mit anderen Jungen haben, die sich nicht wehren können. Betrachtet man das Zustimmungsverhalten der Mädchen, so fällt ein Unterschied zwischen der ersten und zweiten im Vergleich zur dritten Aussage auf. 14\% bzw. 20,6\% der Mädchen geben hier zu, daß sie gern zuschauen, wenn sich Jungen prügeln bzw. daß sie sich geschmeichelt fühlen, wenn sich zwei Jungen ihretwegen schlagen. Demgegenüber stimmen allerdings 45\% der Mädchen der Aussage zu, daß sie Mitleid mit Jungen haben, die sich nicht wehren können. Auf den ersten Blick scheint es sich hierbei um einen Ausdruck der Gewaltakzeptanz von Mädchen zu handeln, die aufgrund eines traditionellen Geschlechtsrollenstereotypes zu erklären ist.

Eine nähere analytische Betrachtung zeigt allerdings, daß es sich bei diesen Ergebnissen weniger um geschlechtsspezifische Zugangsweisen zur Gewalt oder um geschlechtsspezifisch unterschiedliche Akzeptanz von Jungen- und Mädchenrollen handelt (Mädchen sollen zugucken, Jungen sollen prügeln), sondern daß sich hinter diesen geradezu plakativen Geschlechtsstereotypen ganz andere Aspekte verbergen. Für die beiden ersten Items zeigt eine Korrelationsanalyse - sowohl bei den Mädchen als auch bei den Jungen -, daß es sich weniger um die Zustimmung zu geschlechterbezogenen Verhaltensweisen handelt, sondern um eine Zustimmung zum Gewalthandeln allgemein. So stimmen allen vier Aussagen (V191, V192, V153, V154) - im Gegensatz zur jeweils dritten Aussage (V193, V155) - vor allem diejenigen Mädchen und Jungen eher zu, die selbst eher Täter und seltener Opfer sind, die häufig Gewalt beobachten und generell Gewalt befürworten. Mittels Faktorenanalyse läßt sich zeigen, daß die Items für Gewaltakzeptanz insgesamt stehen und daß mit dem dritten Item bei Jungen und Mädchen ein eigener Faktor ,Mitleid“ repräsentiert wird: Es geht also weniger um den Aspekt geschlechtsrollenstereotypischen Verhaltens in bezug auf Gewalt, sondern um die von Jungen wie Mädchen zum Ausdruck gebrachten Einstellungen: Mädchen haben mit Gewaltopfern, die sich nicht wehren können, Mitleid bzw. Jungen nehmen an, daß Mädchen mit den Gewaltopfern Mitleid haben. Damit läßt sich die Einstellung für die Mädchen „Ich habe oft Mitleid mit den Jungen, die sich nicht richtig wehren können." nicht ausschließlich als Zustimmung zu einer bestimmten männlichen Rollenerwartung interpretieren (z.B. „Ein richtiger Junge weiß, sich zu wehren“).

Gerade dieses Beispiel zeigt, daß selbst dann, wenn das Geschlecht der Akteure ausdrücklich thematisiert wird, und sogar geschlechtsbezogene Antwortvorgaben gemacht werden, noch nicht notwendigerweise etwas über die geschlechtsspezifischen Einstellungen der Befragten zum Ausdruck 
kommt. Es ist auch fraglich, ob mit den angesprochenen Items wirklich „geschlechtsbezogene Interaktionen“ untersucht werden (vgl. Popp 1997, S. 217). Möglicherweise überlagert das Thema „Gewalt und aggressives Verhalten“ andere mögliche Erklärungsdimensionen. Für eine Aufklärung der Frage, wie Mädchen zum Gewalthandeln von Jungen stehen und ob sie selber nicht auch eine „klammheimliche Freude“ beim Zuschauen empfinden, lassen sich aus diesem Untersuchungsdesign keine Aussagen treffen.

Abb. 3-4: Einstellungen zur Gewalt (Prozentanteil der Schülerinnen und Schüler, die den Aussagen teilweise oder ganz zustimmen)*

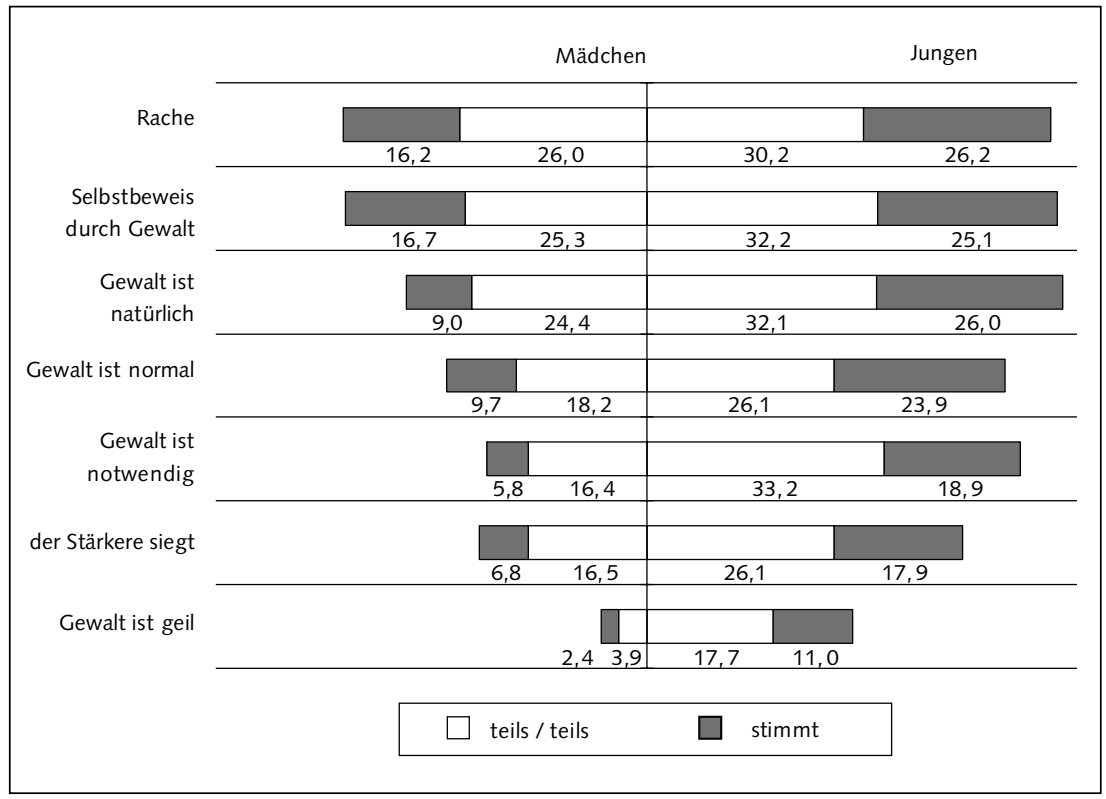

* Die Rangreihe wurde nach den Mittelwerten der Gesamtstichprobe erstellt.

Legende:

Rache:

Selbstbeweis durch Gewalt:

Gewalt ist natürlich:

Gewalt ist normal:

Gewalt ist notwendig:

der Stärkere siegt:

Gewalt ist geil:
Auge um Auge, Zahn um Zahn, so ist nun einmal das Leben. (V190) Es ist völlig normal, wenn Männer sich im körperlichen Kampf mit anderen selbst beweisen wollen. (V189)

Gewalt gehört selbstverständlich zur menschlichen Natur. (V186)

Gewalt ist etwas völlig normales, weil sie überall vorkommt. (V188) Um seine Interessen durchzusetzen, muß man manchmal Gewalt anwenden. (V186b)

Der Stärkere muß sich durchsetzen, sonst gibt es keinen Fortschritt. (V187)

Gewalt ist geil. (V186a) 
Die zweite Verfahrensweise zur Erfassung der Gewalteinstellung von Jungen und Mädchen basiert auf der Untersuchung verschiedener Items zur Akzeptanz von und zur Einstellung zur Gewalt (Abb. 3-4). Die Schülerinnen und Schüler konnten auf einer 5-stufigen Skala ihre Zustimmung zu den verschiedenen vorgegebenen Aussagen über Gewalt zwischen „,stimmt ganz genau“ und „stimmt gar nicht“ abstufen. Für die Darstellung sind die Kategorien „stimmt ganz genau“ und „stimmt überwiegend“" unter „stimmt“ zusammengefaßt worden.

Die Unterschiede zwischen den Mädchen und den Jungen bezüglich der Einstellung zu Gewalt sind deutlich zu erkennen. Über alle Items hinweg stimmen die Mädchen den vorgegebenen Aussagen weniger zu als die Jungen. Die Untersuchung nach geschlechtsspezifischen Differenzen ergab bei allen Items einen signifikanten Unterschied.

Mädchen sehen demzufolge weniger als die Jungen Gewalt als normal, gut und notwendig in unserer Gesellschaft an. Am größten sind die Zustimmungen von Jungen und Mädchen bei der Aussage, daß es normal sei, wenn Männer sich im körperlichen Kampf selbst beweisen wollen. Über die Hälfte der Schüler und immerhin 42\% der Mädchen können dieser Aussage teilweise und ganz zustimmen. Das ist ein deutlicher Hinweis darauf, daß Schülerinnen genauso wie Schüler Gewalt für die Entwicklung der Jungen als angemessen ansehen. Ob und was Mädchen und Jungen über die Notwendigkeit gewaltförmigen Verhaltens von Mädchen innerhalb ihrer Sozialisation denken, bleibt unerwähnt, da im Fragebogen die Items ausschließlich auf männliche Lebensentwürfe bezogen sind. Es können also keine Angaben darüber gemacht werden, ob Mädchen den traditionellen Geschlechterrollen folgen oder nicht.

Jungen wie Mädchen stellen fest, daß Gewalthandeln auch eine interaktionelle Dimension im Hinblick auf den Umgang mit dem jeweils anderen Geschlecht hat und daß Stereotypen über das richtige männliche Verhalten existieren, die Gewaltakzeptanz und Gewalthandeln befördern. Es wurde allerdings in dieser Untersuchung nicht danach gefragt, wie sich soziale Kontrolle der Mädchen untereinander auswirkt und ob diese dazu führt, offenes Gewalthandeln zu diskreditieren. Ebenso wenig wird für die Seite der männlichen Schüler untersucht, ob sie nicht unterstützend beim Ausagieren von Gewalt tätig sind, z.B. durch Zugucken oder Anfeuern - also bei Handlungsformen, die eher den Mädchen zugeschrieben wurden (vgl. z.B. Holzkamp/ Rommelspacher 1991, Stenke 1996).

In einem weiteren Schritt werden im folgenden die Aspekte Autoritarismus, Nationalismus und Gewalt als Konfliktlösung im Rahmen des ,gewaltaffinen Syndroms" (vgl. Kap. 1) untersucht. Die Abbildung 3-5 beinhaltet die Zustimmungen der Schülerinnen und Schüler ab Klasse 8 zu autoritären und nationalistischen Aussagen. In der Darstellung sind die Kategorien „stimmt ganz genau“ und „stimmt überwiegend“ abermals in „stimmt“ zusammengefaßt. Wie erwartet unterscheiden sich Jungen und Mädchen in der Zustimmung zu den nationalistischen und autoritären Einstellungen dahingehend, daß die Mädchen 


\section{Abb.3-5: Einstellungen zu Autoritarismus, Nationalismus und Gewalt (Prozentanteil der Schülerinnen und Schüler, die den Aussagen teilweise oder ganz zustimmen - gestapelt)*}

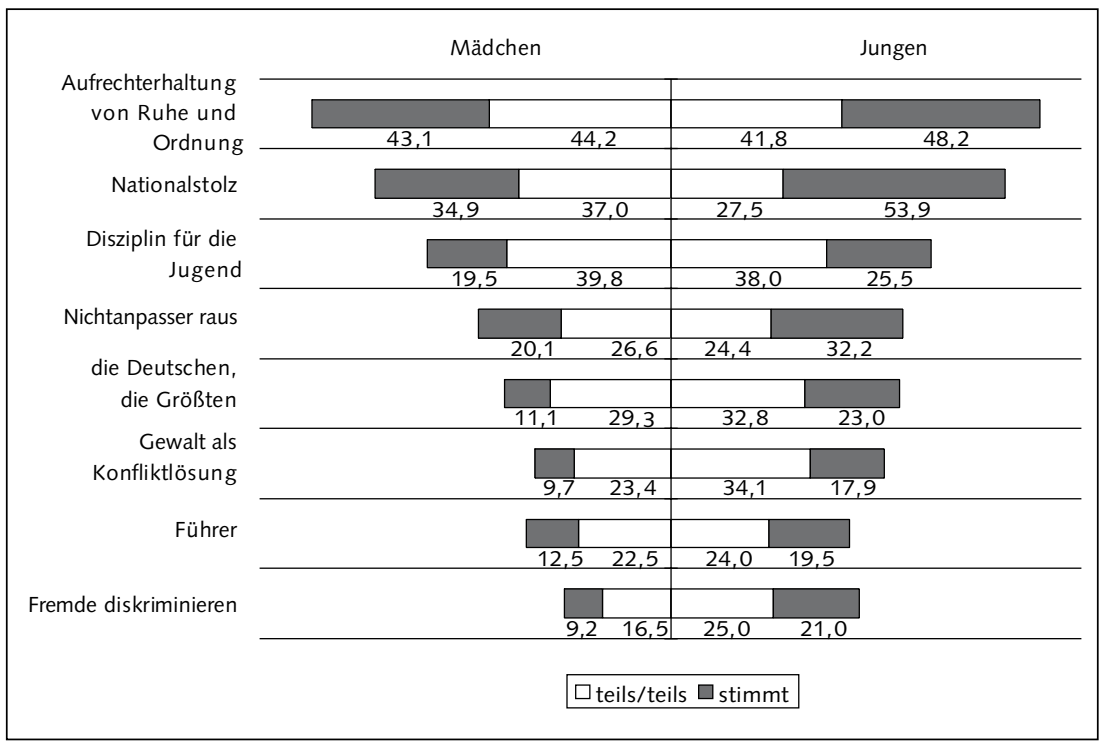

* Die Rangreihe wurde nach den Mittelwerten der Gesamtstichprobe erstellt.

Legende:

Aufrechterhaltung von Ruhe und Ordnung:

Das wichtigste in der heutigen Zeit ist die Aufrechterhaltung von Ruhe und Ordnung. (V301)

Nationalstolz:

Nur für die Deutschen: Ich bin stolz, ein/e Deutsche/r zu sein. (V307a)

Disziplin für die Jugend:

Es ist höchste Zeit, daß die Jugend wieder Disziplin lernt. (V304)

Nichtanpasser raus:

Wer sich in Deutschland nicht anpassen kann, soll das Land verlassen. (V306)

die Deutschen, die Größten:

Die Deutschen waren schon immer die Größten in der Geschichte. (V303)

Gewalt als Konfliktlösung:

In jeder Gesellschaft gibt es Konflikte, die nur mit Gewalt gelöst werden können. (V305)

Führer:

Unser Volk sollte wieder einen Führer haben, der mit starker Hand regiert. (V302)

Fremde diskriminieren:

Ich bin durchaus bereit, Fremde schlechter zu behandeln, damit sie wissen, wo hier ihre Grenzen sind. (V307)

insgesamt etwas geringere Zustimmungsquoten aufweisen, die Rangreihung allerdings identisch ist. Die größten Differenzen zeigen sich bei dem Item ,Ich bin durchaus bereit, Fremde schlechter zu behandeln, damit sie wissen, wo ihre Grenzen sind." - einer Aussage, die eine Bereitschaft zum aktiven Eingreifen 
beinhaltet. Deutlich geringer ist die Zustimmung der Mädchen ebenfalls bei der Aussage, daß Gewalt ein Mittel der Konfliktlösung darstelle. Das bestätigt die Annahmen über die interaktionelle Dimension des Gewalthandelns, wie sie bereits beim Täterselbstreport zum Ausdruck kommen.

Am wenigsten unterscheiden sich Jungen und Mädchen im Hinblick auf die autoritäre Forderung nach Aufrechterhaltung von Ruhe und Ordnung (43,1\% der Mädchen und 48,2\% der Jungen) sowie bei der Aussage, es sei höchste Zeit, daß die Jugend wieder Disziplin lernt. Im Vergleich zu den Einstellungen zur Gewalt und zur Gewaltbilligung fällt die hohe Zustimmung zu den autoritären Aussagen auf. Im Hinblick auf die Einstellungen der Mädchen deuten sich ähnlich Befunde an, wie sie Utzmann-Krombholz (1994) in ihrer Studie zu „Mädchen und Rechtsextremismus“ gefunden hat. Sie stellt fest, daß Mädchen und junge Frauen, auch wenn sie von männlicher Macht und Stärke fasziniert sind, oft gleichzeitig Angst vor Gewalt haben und offenbar wenig psychische Ressourcen besitzen, mit Gewalt um zugehen, die sich direkt gegen sie richtet oder sich in ihrer unmittelbaren Umgebung ereignet. Sie vermutet deshalb, daß das Orientierungsmuster „Autoritarismus“ die Grundlage einer weiblichen Variante des Rechtsextremismus darstellt, wobei die entscheidende Barriere zum rechtsextremen Einstellungsmuster im gewalttätigen Ausländerhaß zu sehen ist (vgl. Utzmann-Krombholz 1994, Stenke 1996).

\section{Zusammenfassung des quantitativen Teils}

Die folgende Abbildung 3-6 gibt einen Überblick über die bisher vorgestellten Perspektiven geschlechtsbezogener Analysen. Dazu wird die Differenz zwischen den Mittelwerten von Jungen und Mädchen bei den einzelnen Gewaltund Einstellungsfaktoren dargestellt.

In die Abbildung sind verschiedene Oberfaktoren bzw. Faktoren aufgenommen, die der Forschungsgruppe zur quantitativen Datenanalyse dienten. Die Mittelwerte lassen einen deutlich größeren Zustimmungsanteil der Jungen erkennen. Die Differenzen zwischen Jungen und Mädchen sind über alle Faktoren hinweg hochsignifikant. Am stärksten unterscheiden sich die männlichen von den weiblichen Jugendlichen hinsichtlich der Billigung von Gewalt (vgl. Legende Abb. 3-4). Hier stimmen die Mädchen - im Vergleich mit den übrigen Faktoren - den Aussagen zu Gewalt weitaus weniger zu als die Jungen. Dem Faktor Gewaltbilligung folgen die Täterfaktoren in der Rangreihung der Mittelwertdifferenzen, wobei der Unterschied zwischen Jungen und Mädchen um so größer wird, je härter die Aggressionen sind. An vierter Stelle steht der Einstellungsfaktor zum Nationalismus (vgl. Legende Abb. 3-5). Die Mittelwertunterschiede bei dem Faktor „Beobachtung““2 bringen zum Ausdruck,

2 Der Oberfaktor „Beobachtung“ setzt sich zusammen aus sieben verschiedenen Faktoren: Vandalismus, Prügelei, Prügelei in konkreten Situationen, sexuelle Belästigung, Aggressio- 
daß Mädchen weniger Gewalt wahrnehmen. Auch bei der Analyse der Unterschiede zwischen Jungen und Mädchen beim Opfer-Status ${ }^{3}$ von personen- bzw. sachbezogenen Angriffen zeigt sich, daß Mädchen auch seltener Opfer von gewalttätigen Angriffen sind, als sie Gewalt wahrnehmen. Sie werden nach eigenen Angaben nicht so oft Opfer von Angriffen wie Jungen. Am kleinsten allerdings sind die Unterschiede zwischen Jungen und Mädchen beim Faktor „Einstellungen zu Autoritarismus“.

Abb. 3-6: Mittelwertvergleich zwischen Jungen und Mädchen bezüglich verschiedener Gewaltfaktoren

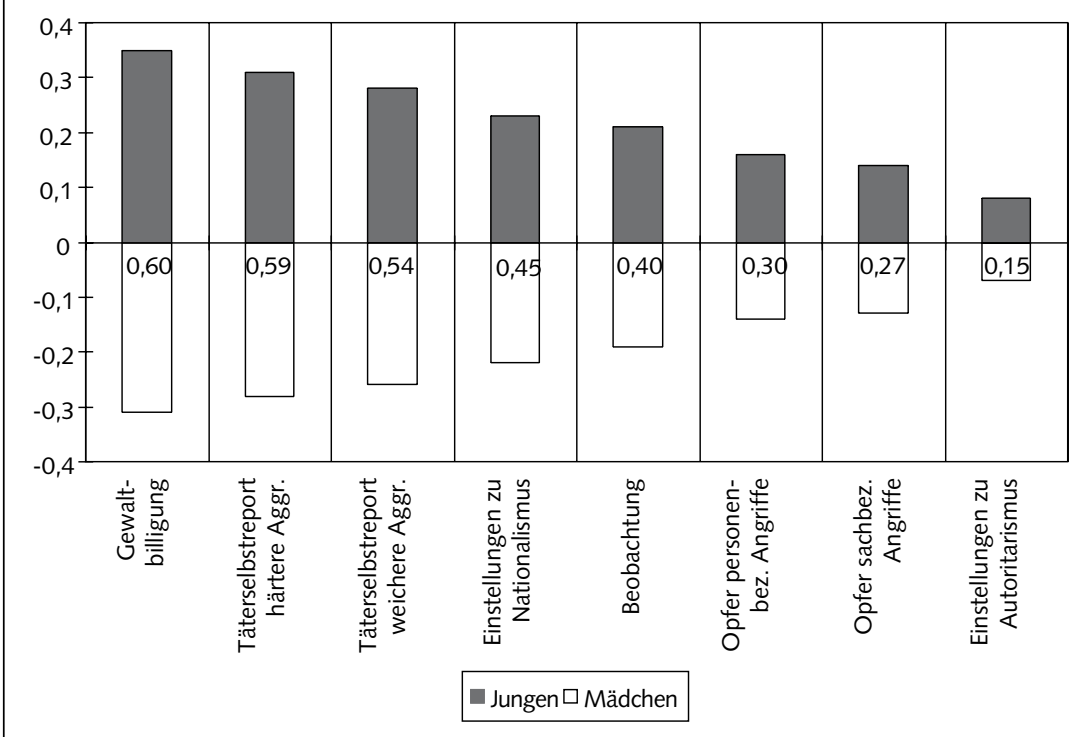

nen gegen Lehrer, Aggressionen gegen Mitschüler und Erpressung gegen Mitschüler. Die verschiedenen Items dieser Faktoren sind nicht identisch mit den Items, die zur Analyse der Wahrnehmung von Gewalt im Kapitel 2 und 3 verwendet wurden.

3 Der Oberfaktor „Opfer“ setzt sich zusammen aus den personen- und sachbezogenen Angriffen. $\mathrm{Zu}$ den personenbezogenen Angriffen zählen die Items ,von anderen geschlagen worden“ (V280), ,von anderen gehänselt oder geärgert worden“(V281), ,auf dem Schulweg belästigt, geschlagen worden“ (V282), ,von anderen angeschrien, beschimpft, beleidigt worden“ (V283), ,von anderen unter Druck gesetzt worden“ (V284). Zu den sachbezogenen Angriffen zählen die Items: ,,von anderen Sachen absichtlich kaputt gemacht“ (V285), ,von anderen gewaltsam etwas weggenommen“ (V286), ,von anderen Sachen versteckt, so daß Du sie nicht wiederfinden konntest“" (V287). 
Die Auswertung der Schülerinnen- und Schülerbefragung bringt als Ergebnis, daß sich Jungen und Mädchen in der Wahrnehmung von Gewalt, im Ausüben von Gewalt und im Opfer-Status bezüglich gewalttätiger und aggressiver Handlungen stark unterscheiden. Auf die naheliegende Frage, wie sich diese Unterschiede erklären lassen, ohne auf tautologische oder geschlechtsrollenstereotype Erklärungen zurückzugreifen, ermöglicht das vorliegende Instrument keine hinreichend differenzierten Antworten. Selbst die wenigen Items, in denen das Geschlecht der Personen ausdrücklich thematisiert wird, eignen sich nur eingeschränkt, um wirklich zu geschlechterbezogenen Aussagen zu gelangen. So findet sich in der dafür eingeführten Fragenbatterie, wie anhand der Faktorenanalyse gezeigt wurde - wider Erwarten und gegen die Absicht der Untersuchung - die Dimension Mitleid anstatt eines geschlechtsspezifischen Faktors. Auch läßt sich im Rahmen der quantitativen Untersuchung nicht feststellen, ob die Differenzen zwischen Jungen und Mädchen auf unterschiedliche Gewaltbegriffe der beiden Geschlechter zurückzuführen sind. Aus anderen Untersuchungen ist bekannt, daß Mädchen (wie auch Erwachsene) ein anderes Gewaltverständnis als Jungen haben (vgl. Kap. 1.2). Sie sehen bereits Handlungen als Gewalt an, die von den Jungen verharmlosend als solche nicht wahrgenommen werden. Bei den Jungen scheint eher die Meinung vorzuherrschen, daß alle Handlungen, die nicht blutig ausgehen, eher nicht zur Gewalt zählen (vgl. dazu z.B. Schubarth/Ackermann 1997). Auch diesem Erklärungsansatz läßt sich hier nicht weiter nachgehen, da die Inhaltsanalyse zum Gewaltbegriff nicht Bestandteil der Repräsentativbefragung war.

Gleichwohl ist festzustellen, daß Mädchen an Gewalt nicht völlig unbeteiligt sind; sie sind weder ausschließlich noch überwiegend Opfer von Gewalt. Der Frage, inwieweit und in welchem Ausmaß und vor allem mit welchen Motiven Mädchen in schulische Gewalthandlungen verstrickt sind - wie sie z.B. von Popp 1997 formuliert wird -, läßt sich allerdings mit den Daten der vorliegenden Untersuchung nicht überprüfen. Wenn es spezifisch weibliche Ausdrucksformen von Gewalt gibt, wie z.B. von Olweus ${ }^{4}$ vermutet wird, dann lassen sich diese im allgemeinen nur feststellen, wenn auch explizit nach solchen den Mädchen unterstellten Verhaltensweisen gefragt wird und diese dann von beiden befragten Gruppen berichtet oder zurückgewiesen werden können.

Neben der Frage nach „typisch weiblichen“ Gewaltformen bleibt allerdings auch bei Jungen der Bezug zur geschlechtsspezifischen Sozialisation offen. Welche Bedeutung Gewalt und Aggression bei der Entwicklung der männlichen Geschlechtsrolle haben und inwiefern die männliche Geschlechtsrollensozialisation zur Akzeptanz von Gewalt und zur Förderung aggressiven

4 Olweus nennt als für die Mädchen typische Verhaltensweisen z.B. hinterlistige Schikanen wie üble Nachrede, Verbreitung von Gerüchten, Manipulation von Freundschaftsbeziehungen (vgl. Olweus 1995, S. 65). 
Verhaltens von Jungen beiträgt, läßt sich ebenfalls nicht beantworten. Was Tillmann (1997) in Bezug auf die Methodologie schulbezogener Gewaltforschung postuliert, gilt auch und erst recht für die geschlechtsbezogene Forschung: Zwar wisse die Gewaltforschung mittlerweile recht viel darüber, welche Waffen Jungen mit in die Schule brächten und wie häufig dies von den Lehrkräften bemerkt würde. Es sei aber wenig darüber bekannt und auch noch wenig danach gefragt worden, welche symbolische Bedeutung es innerhalb der Schülerkultur habe, Waffen unterschiedlichster Art mitzuführen. Dies erfordere, so Tillmanns Schlußfolgerung, eine Hinwendung zu anderen Forschungsmethoden, nämlich z.B. dazu, die Anregungen der ethnomethodologischen (Jugend-)Forschung aufzugreifen und den vielen schriftlichen Befragungen methodisch vielfältige Fallstudien folgen zu lassen (vgl. Tillmann 1997, S. 25). Unter geschlechterbezogener Perspektive ist nicht nur danach zu fragen, welche Jungen welche Waffen aus welchen jugendkulturellen Gründen mit sich tragen, sondern auch welche Bedeutung das Tragen und/oder Zeigen von Waffen für die männliche Sozialisation hat bzw. ihm bei der $(\mathrm{Re})$ Konstruktion von Männlichkeit zukommt. Welche Rolle spielen Waffen oder Waffenbesitz in der Gestaltung der Interaktionen mit dem eigenen und dem ,anderen“ Geschlecht, wie bewußt oder unbewußt gehen diese Prozesse vonstatten, ließe sich der Fragenkatalog geschlechterbewußt fortsetzen. Auch eignen sich dabei u.E. offene Methoden, die den Jugendlichen Raum lassen, ihre Positionen aus ihrer alltäglichen Kommunikation heraus zu entwickeln weitaus besser als geschlossene Instrumente, wenn sehr differenzierte Sachverhalte ausgelotet werden sollen. Dies muß allerdings auch entsprechende Konsequenzen für die Auswertungsverfahren haben. Ein erster Ansatz, der in diese Richtung zielt, soll mit der folgenden Untersuchung vorgestellt werden.

\subsection{Ergebnisse der Interview-Studie mit Schülerinnen und Schülern}

Innerhalb eines Lehrforschungsprojektes wurden - gemeinsam mit Studierenden - die Forschungen zu den schulischen und außerschulischen Entstehungsbedingungen von Gewalt vertieft (vgl. Schubarth/Darge/Mühl/Bergelt/ Börner/Hannich 1997). Mit Hilfe von Einzel- und Gruppeninterviews sollte ein Perspektivwechsel vorgenommen werden und stärker nach dem individuellen Umgang mit Gewalterfahrungen gefragt werden; der Interaktionsdynamik des schulischen Gewalthandelns sollte mit der Durchführung von Gruppendiskussionen Rechnung getragen werden. Auf diese Weise sollte das Bild aus der standardisierten Befragung in einzelnen Details vertieft werden.

Die Schülerinnen und Schüler für die Gruppendiskussionen und Einzelinterviews wurden an den zwei beteiligten Schulen anhand von soziometrischen 
Tests ausgewählt ${ }^{5}$. Den beiden Interviewsituationen lag ein Leitfaden zugrunde, der die folgenden Themenkomplexe beinhaltete: Schulfreude/ Schulfrust, Lehrer-Schüler-Verhältnis, Schüler-Schüler-Verhältnis, Gewalt und abweichendes Verhalten, biographische und außerschulische Einflüsse. Die Gruppendiskussionen und Einzelinterviews wurden themenbezogen in einer Querschnittsanalyse ausgewertet. Für den folgenden Abschnitt werden vor allem die Passagen ausgewertet, die Zusammenhänge zwischen den aggressiven Aspekten schulischen Handelns und Interaktionen mit dem anderen Geschlecht im Hinblick auf Aggression und Gewalt zum Ausdruck bringen. Dabei sollen auch die gegenseitigen geschlechterbezogenen Wahrnehmungen thematisiert werden. Um eine gewisse Vergleichbarkeit zu den Analysen des quantitativen Teils zu gewährleisten, erfolgt die Darstellung auch jeweils unter den Gesichtspunkten „Beobachtung bzw. Wahrnehmung von Gewalt“, „Täterselbstreport“ und „Opferselbstreport“. Im Mittelpunkt steht allerdings jeweils die Frage, inwieweit im Sprechen über Gewalthandeln jeweils geschlechterbezogene Aspekte eine Rolle spielen. Diese hier vorgestellten Analysen der Gruppendiskussionen bzw. Einzelinterviews zeigen allerdings auch, daß gerade in den Situationen, in denen es um das Beobachten von Gewalt geht, oftmals die Rolle der Erzählenden und der Grad ihres Involviertseins nicht deutlich wird. Eine Beteiligung an gewalttätigen Aktionen wurde offenbar nicht immer zugegeben und von den Interviewern auch nicht immer ausdrücklich nachgefragt. Stärker rekonstruktive und tiefenhermeneutische Auswertungsverfahren könnten möglicherweise noch bessere Ergebnisse erbringen, dies war allerdings im Rahmen des hier dargestellten Forschungszusammenhanges nicht möglich.

\section{Jungen und ihr Umgang mit Aggression und Gewalt}

In einem Interview betont ein männlicher Jugendlicher (Klassenstufe 9) vor allem die „Rangeleien“ untereinander, die oftmals ,wegen Kleinigkeiten, wegen Sachgegenständen" stattfinden. Diejenigen, gegen die sich die Gewalt richtet, sind oft ,,die Schwächsten und das sind mehr die Jungen.“ Von den Mädchen wird berichtet: „Die Mädchen, die hängen sich dann einfach immer bei de-

5 Bei den Schulen handelt es sich um zwei überdurchschnittlich belastete Mittelschulen, eine davon ist in Kapitel 8 porträtiert. An beiden Schulen wurden insgesamt 13 Einzelinterviews (darunter eines mit einem Mädchen) und sechs Gruppeninterviews in der 9. Klassenstufe durchgeführt. Pro Schule wurden jeweils mit einer geschlechtsheterogenen und zwei geschlechtshomogenen - je einer Mädchengruppe und einer Jungengruppe - Gruppendiskussionen durchgeführt. Die Gespräche dauerten bis zu 90 Minuten und wurden auf Tonband aufgezeichnet und anschließend von den Interviewern transkribiert.

6 Bei allen kursiv gesetzten Passagen handelt es sich um Zitate aus den Interviews bzw. Gruppendiskussionen. Aus Gründen der besseren Lesbarkeit wird auf einen direkten Verweis auf die Fundstellen verzichtet. Ein genauer Nachweis und Abdruck zusammenhängender Interviewpassagen findet sich bei Bergelt (1997). 
nen (den kämpfenden Gruppen, d. A.) dazwischen. Das eine Mädchen sagt: 'ich will zu der Gruppe, weil die mir besser gefällt und die andere will zu der (anderen Gruppe, d. A.).“ Mädchen werden als eher indirekt an gewalttätigen Aktionen Beteiligte wahrgenommen. Ein Mädchen, das öfter durch aggressives Verhalten auffällt, hat es schwer in der Klasse ernstgenommen zu werden. Man nimmt sich zwar vor ihr in acht, vor allen Dingen, wenn man das Gefühl hat, sie macht nun keinen Spaß mehr, sondern ist bereit zuzuschlagen, gleichzeitig wird eher abfällig über sie gesprochen: „Sie hat einen komischen Charakter“. Eine Reaktion, die ein aggressiver Junge nur in den seltensten Fällen erfährt - ihm wird selbst von seinen tatsächlichen oder potentiellen Opfern noch mit Respekt oder gar mit Unterwerfung begegnet: „(...) das ist unser kleiner Franz, der wird immer von dem einen verprügelt, so in der Art (...) und halt immer so geärgert. Der denkt, daß das seine Freunde sind und läuft einfach immer mit denen, obwohl der immer eine drauf bekommt." Die Demonstration von Macht erfolgt dabei nicht unbedingt mit ,roher Gewalt“, vielmehr wird die gesamte Palette vom Hänseln, bis Sachen wegnehmen, Anrempeln usw. vorgeführt: „Da haben sich drei Typen zusammengeschlossen und die können einen irgendwie nicht leiden, aber einfach nur so, weil sie mal wieder was raus lassen wollen. Da wollen sie mal wieder zeigen, sie sind die Könige der Klasse, was sie aber im Grunde nicht sind und da suchen sie sich mal einen aus und da fällt ein Füller, eine Schiefermappe und so. Und manchmal verschwindet ein Ranzen, aber selten, der ist bald wieder da." Obwohl an der Passage die Machtdemonstration deutlich spürbar ist, wird sie im gesamten Interviewkontext eher heruntergespielt. Zwar ärgert sich der berichtende Jugendliche über das Verhalten der „drei Typen“, aber so richtig eingestehen will er das nicht. Auch wird nicht deutlich, ob der berichtende Junge zu den Opfern der ,drei Typen“ gehört oder nicht. Die Täter haben in aller Regel mit ihrer Machtdemonstration Erfolg, sie werden weder zur Rechenschaft gezogen noch wird ihnen richtige Gegenwehr entgegengebracht. Dies deckt sich auch mit den Täter-Opfer-Analysen wie sie im folgenden Kapitel vorgestellt werden (vgl. auch Rostampour/Melzer 1997), wonach die Täter nicht unbedingt zu den Außenseitern der Klasse oder der Schule gehören und im Gegensatz zu manchen Opfern gut in das Sozialgefüge der Klasse integriert sind. Selbst wenn die Macht demonstrierenden Jungen nicht immer von allen geliebt werden, so ist doch eindeutig klar, daß sie die „,richtigen Männer“ sind und sich durchzusetzen wissen. Zeigen Mädchen ein ähnliches Verhalten, werden sie abgewertet, marginalisiert und weder gefürchtet noch anerkannt. Der Anspruch auf aggressives Verhalten liegt eindeutig bei den männlichen Jugendlichen.

Im allgemeinen wehren sich auch die männlichen Opfer von Gewalt nicht, da sie sonst befürchten müssen, daß sie erneut der Gewalt ausgesetzt sind und bei einer Auseinandersetzung mit den Stärkeren eine schlechte Position haben: „Na erstens Mal, die sind etwas größer wie ich und eben älter. Und allgemein nimmt man sich ja vor denen allgemein in acht, die irgendwie bissel Macht auf die anderen ausüben." 
Auf der anderen Seite wissen auch die Täter, daß es ihnen kaum Anerkennung bringt, Macht und körperliche Gewalt gegenüber jüngeren Schülern oder Mädchen zu demonstrieren. Wichtig ist für die Beteiligten, daß der Gegner sich wehrt, nur dann ist er ein lohnender Gegner - und es bringt Anerkennung, ihn besiegt zu haben. Im Gegenteil, die Auseinandersetzung mit dem falschen Gegner führt eher zu Spott und Verachtung. Hier gilt es ganz offenbar, den richtigen männlichen Kodex im Umgang mit Gewalt zu kennen, umgekehrt stellt das Gewalthandeln selbst eine „extreme Form von Bewältigungshandeln“ (Böhnisch 1994, S. 111) dar. Dabei hängt es von dem sozialen Umfeld ab, ob diese Formen zur Verbesserung des eigenen Selbstwertgefühls zur Anwendung kommen oder nicht. „Gewalt wird dann zum Bewältigungsmuster, wenn andere soziokulturelle Ressourcen - also z.B. die Kompetenz der Kommunikation und Fähigkeit der Empathie - nicht verfügbar sind. Aber auch dort, wo die soziale Kontrolle und Akzeptanz von gegenseitiger Integrität - sei sie über sozialen Diskurs oder kulturelle Bedingungen entstanden - abhanden gekommen ist, kann der soziale Raum für Gewalt durchlässiger werden“ (Böhnisch 1994, S. 111).

Gewalt dient aber auch der Herstellung einer „hegemonialen Männlichkeit“ (Kersten o.J.): danach resultieren Strukturen von Herrschaftsanwendung und Herrschaftsunterworfenheit aus dem Geschlechterverhältnis, dem Verhältnis der kulturell dominanten Mehrheit gegenüber den Minderheiten in einer gegebenen Gesellschaft und dem Verhältnis zwischen sozialen Klassen: „Hegemonie - d.h. die Aufrechterhaltung einer Vorrangstellung erfolgt nicht durch alltägliche brutale Gewaltanwendung, sondern durch abgesicherte und sich selbst reproduzierende Strukturen der Dominanz - entsteht im Zusammenwirken von Geschlechter-, Rassen- und Klassenverhältnissen. Hegemoniale Männlichkeit beruht nicht ausschließlich auf der Unterordnung von Frauen, sondern besteht zentral in der Unterordnung von ökonomisch und sozial abhängigen Männlichkeiten" faßt Kersten (ebd., S. 26) dieses Phänomen zusammen, welches sich bereits in den wenigen hier zitierten Interviewpassagen andeutet. Schule gelingt es bislang offenbar nur wenig, dieser hegemonialen Männlichkeit etwas entgegenzusetzen. Sie wird noch nicht einmal thematisiert, dringt nicht in das Bewußtsein der in Schule handelnden Lehrerinnen und Lehrer - diese sind vielmehr Teil dieses Prozesses und Lehrerinnen bieten den männlichen Jugendlichen noch eine Folie, auf der sie ihr männliches Imponiergehabe austragen können, indem diesen Jugendlichen nichts entgegengesetzt wird. Aber auch Lehrer haben ein virulentes Interesse, diesen in den genannten Hierarchien ablaufenden „Auseinandersetzungen um Rechtmäßigkeit und Kontrolle“ (ebd., S. 26) zustimmend - wenn auch manchmal nur heimlich - zur Seite zu stehen, da dies auch ihren Status in einer so oft als feminisiert beschriebenen Institution festigt. Dies ist zugleich eine Erklärung für die so oft abwesenden Lehrer, die wegschauen, anstatt einzugreifen, marginalisieren, anstatt den Ernst der Lage zu erkennen. Die mehr oder weniger stillschweigende Toleranz gegenüber den gewalttätigen Jungen, bleibt nicht ohne sozialisatorischen Effekt 
auf die anderen männlichen Jugendlichen und vor allem zeitigt es Folgen bei den Mädchen, denen der heimliche Lehrplan der Koedukation so deutlich vor Augen geführt wird - sie lernen neben allem anderen auch, was es heißt, in dieser Gesellschaft eine Frau zu sein.

\section{Mädchen und ihr Umgang mit Aggression und Gewalt}

Einige Mädchen (der Klassenstufe 9) bringen in den Interviews eine ambivalente Einstellung zum Ausdruck: einerseits beschweren sie sich über die Jungen in ihrer Klassen, daß sie so kindisch seien, ,die sind halt ein bissel anders, spielen noch Fußball und dann haschen die sich (...) wie Kinder, so meine ich das jetzt". Andererseits sind die Mädchen traurig darüber, daß die Jungen so sind und stellen fest: „Guckt euch mal die Kerle an, mir gefällt kein Kerl mehr an der Schule, kein einziger." Früher, gemeint ist die Zeit vor ein, zwei Jahren, als die Schule aufgrund ihres hohen Gewaltvorkommens auffällig war, waren noch „,echte Kerle“ an der Schule, ,die waren viel älter und viel erwachsener" - und viel gewalttätiger. Denn obwohl immer wieder betont wird, daß Gewalt ,eklig“ sei, geht von den erwachsenen älteren Jungen eine gewisse Faszination aus. Auf der einen Seite werden Jungen abgelehnt, die offene, direkte Gewalt ausüben (vor allem wenn sich diese gegen Kleinere richtet, weil dies nicht dem Verhalten eines „richtigen“ Mannes entspricht), gleichzeitig imponieren und gefallen einigen Mädchen die Jungen, die sich durchsetzen können, die sich nicht reinreden lassen und die etwas zu sagen haben (z.B. in der Auseinandersetzung mit Lehrern). Diese Wahrnehmung stellt das direkte weibliche Pendant zur Sicherung der hegemonialen Männlichkeit dar und bestätigt Jungen wie Mädchen wechselseitig, daß es lohnend ist, sich aggressiv und männlich dominant in der schulischen Welt zu bewegen und nicht nur ein "bissel kindisch Haschen zu spielen."

Die aktive Beteiligung am Ärgern und Herabsetzen von sogenannten AuBenseitern wird dann auch quasi anthropologisch erklärt: „es gibt halt immer Außenseiter, die runtergemacht werden, eigentlich von allen. Wenn die halt nicht so toll aussehen, z.B. so ein Kleiner, der hat abstehende Ohren und so, den machen sie immer ziemlich runter, auch so, die sehen auch immer so ziemlich plebsig aus." Die Interviewpartnerinnen räumen zwar ein, daß sie früher auch „Außenseiter geärgert haben“, aber in letzter Zeit würden sie das nicht mehr tun. Die Aggressivität weicht plötzlich einer Hilflosigkeit: „Klar ist das irgendwo Scheiße, wenn du dir das anguckst, das tut einem auch leid und so, blo $\beta$ ich muß auch sagen, man kann eigentlich gar nichts machen (...). Also ich saß schon in einer Bahn, da wurde halt ein Kumpel von mir verprügelt. So, ich saß daneben und konnte im Endeffekt gar nichts machen. Das ist eigentlich so ein Gefühl von Hilflosigkeit irgendwo, aber was will man machen? Was denn? Kannste nichts machen. (...) Und so ist das mit den Außenseitern auch, was willste großartig machen. Da biste dann selber noch 
irgendwie (...).“ Ihre Freundin bestätigt sie: „Das wäre auch schön blöde.“ Auf die Nachfrage, ob die beiden schon einschlägige Erfahrungen gemacht hätten, antworten sie: „Ich hab die Erfahrung schon gemacht. Also gerade zur Gewalt, daß du im Endeffekt selber eine dran kriegst." Ihre Handlungsspielräume werden von beiden Mädchen als beschränkt und einer männlichen Dominanz unterworfen eingeschätzt. Obwohl sie das Verhalten der Täter als unrechtmäBig und gemein ansehen, wehren sie sich nicht und setzen sich auch nicht für die Opfer ein. Dabei spielt die Angst davor, selber Opfer eines körperlichen Angriffes zu werden, eine eben so große Rolle wie die Angst, ausgegrenzt und auf der Seite der Opfer, der Außenseiter verortet zu werden, also ihr Ansehen bei den Tätern zu verlieren. Dieses Verhalten wiederum bestätigt die männliche Dominanz, der eben nichts entgegengesetzt wird.

Sprechen Mädchen über Gewalt, dann wird die gesamte Ambivalenz deutlich. Zur Veranschaulichung soll hier ein längerer Gesprächsausschnitt wiedergegeben werden:

Frage: Habt Ihr auch mal das Bedürfnis, draufzuhauen, irgend jemanden?

Rica: Nee, das würde ich auch nie machen.

Bettina: Also ich kenne viele Leute, die welche mal eben verkloppen und so.

Rica: Echt eklig.

Bettina: Aber wenn ich (...) mich mal so viehisch ärgere, sage ich auch manchmal, ich haue dir eine aufs Maul oder so. Aber das kann ich dann doch nicht, ich stehe dann im Endeffekt vor der, und ich kann das einfach nicht, weil ...

Rica: Nee, man könnte ja jemandem weh tun.

Bettina: Nee, daß geht da überhaupt nicht drum, sagen wir mal, ich hab den Mut nicht, der eine zu klatschen oder so, daß geht wirklich nicht. (...) Da muß ich richtig ausrasten.

Nicht ausschließlich die moralische oder intellektuelle Ablehnung von Gewalt wird in dieser Passage angesprochen, von Seiten Bettinas geht es auch weniger darum, das potentielle Opfer zu schonen, sondern um die Unfähigkeit, um den fehlenden Mut, sich zu wehren, sich jemandem mit Gewalt zu nähern - Mädchen schlagen sich nicht, selbst wenn sie mal die große Wut haben sollten, und selbst wenn die Gegnerin zu besiegen wäre. Auch Gewaltopfer können nicht immer nur mit Mitleid rechnen. Zwar wird einem männlichen Opfer von Hänseleien zugute gehalten, ,,daß er nicht gleich heult“, mehr Mitleid wird allerdings nicht aufgebracht, denn er ist ,einfach doof", was letztlich heißen soll, es geschieht ihm schon recht. Viele Passagen der durchgeführten Interviews deuten darauf hin, daß Mädchen bei gewalttätigen Auseinandersetzungen unterschiedlicher Art häufig zugegen sind und sich mit unterschiedlichem Engagement auch einmischen, meistens verbal und meistens hinterher. Nicht in allen Fällen wird dabei die Anwendung von Gewalt verurteilt, oft wird dem Opfer eine Teilschuld gegeben und nicht immer wird die eigene Gewaltabstinenz mit dem Motiv der Gewaltächtung erklärt oder mit der Wunsch, einen oder eine andere nicht zu verletzten, sondern mit dem fehlenden Mut und den fehlenden persönlichen Voraussetzungen, wirklich zuzuschlagen, mal so 
richtig aus sich herauszugehen. Gewaltanwendung ist nur im Affekt legitim - „da muß ich wirklich ausrasten“ - wenn alle anderen gesellschaftlichen, geschlechterbezogenen und moralischen Filter aussetzen. Für ein ähnliches affektives Verhalten bei Jungen zeigen die Mädchen in den Gesprächen auch Verständnis: „,muß seine Wut abreagieren an irgendeinem anderen;“ ein derartiges Motiv für Gewalt, das sich vor allem gegen Schwächere richtet, wird von den befragten Mädchen häufig berichtet.

Mädchen erwarten dabei allerdings auch, wenn sie Gewalt als ungerecht erleben, daß dem betreffenden Gewalttäter von einem Jungen mal richtig die Meinung gesagt wird - ,wenn ich ein Junge wär, ich hätte dem lange schon die Meinung gesagt, nur als Mädchen getraut man sich das nicht so, aber als Junge, da hätte ich die dem echt schon voll ins Gesicht gesagt". Von den Mädchen wird erwartet, daß sie über eine enge Beziehung zu demjenigen auch einen gewissen positiven Einfluß ausüben: ,schon durch die Freundin jetzt, ist er wesentlich ruhiger geworden, die hat vielleicht doch einen gewissen Einfluß auf ihn und hat diesen auf ihn schon ausgeübt". Als Mädchen hat man andere Einflußmöglichkeiten als die Jungen und kann diese dann auch entsprechend nutzen. Gerade in dieser Passage werden die unterschiedlichen Rollenerwartungen an Jungen und Mädchen mehr als deutlich. Diese Rollenerwartungen führen nicht immer dazu, daß Mädchen sanft und lieb sind und sich positiv gegen Gewalt wenden, indem sie den Gewalttäter ,zähmen“, wie in dem genannten Beispiel. Beachtet werden muß gerade dabei, daß der Junge für das ihn zähmende Mädchen trotz - oder gerade wegen - seines ,Machogehabes" erst mal interessant sein muß, damit überhaupt eine Freundschaftsbeziehung entsteht. Dies wird von den befragten Mädchen nicht hinterfragt. Es erscheint vielmehr selbstverständlich und nicht weiter bedenkenswert, daß der eben so geschilderte, immer wieder unangenehm auffallende Junge eine Freundin hat. Seine Attraktivität für Mädchen wird dadurch offenbar nicht grundsätzlich beeinträchtigt. Dabei ist es wohl zum einen so, daß die Beziehungen mit diesen Jungen für Mädchen attraktiv sind, weil sie an der Macht teilhaben können, zum anderen scheint es aber auch so, als durchschauten die Mädchen die „Gewaltshows“, die die Jungen abziehen und nehmen diese einfach nicht ernst.

Geschlechtsspezifische Interaktionsmuster zwischen den Mädchen sind oft auch aggressiv und verletzend, in der Regel allerdings in psychischer Hinsicht. Dabei spielt attraktives Aussehen, das Tragen der richtigen Kleidung und Marken, sowie die angenommene und faktische Konkurrenz um Beziehungen zu Jungen eine wichtige Rolle. Auffällig ist selbst in den Interviewsituationen, in denen erwachsene Interviewer/innen anwesend waren, daß einzelne Mädchen ignoriert werden, wenn sie etwas sagen oder auf ihre Äußerungen nicht eingegangen wird bzw. die berichteten Beleidigungen direkt in der Interviewsituation erfolgen. Immer wieder wird auch berichtet, wie leicht der Verbund einzelner Mädchengruppen auseinanderbrechen kann, sich neue Konstellationen ergeben und wie schnell ein Opfer zur Täterin werden kann: „Letztes Jahr 
war ich dran, und dieses Jahr ist es umgekehrt, da haben wir dann die Beate so fertig gemacht, und das tat uns wirklich gut, das mußte auch mal sein."

Gerade das Rivalitätsverhalten der Mädchen untereinander wird als völlig normal geschildert und in keinem Gespräch wirklich bedauert oder kritisiert. Alle betroffenen Mädchen trösten sich offenbar damit, daß sie jederzeit auch in die überlegenere Situation kommen können, oder aber eine Rivalin einen Fehler macht und selbst Neid, Mißgunst und Abwertungen erfährt. Mädchen beschreiben sich dann auch selbst als Täterinnen im Entwerten, Herabsetzen, Ausgrenzen und Ignorieren - vor allem von anderen Mädchen. Die Jungen können zwar berichten, daß da etwas unter den Mädchen läuft, aber sie wissen in den wenigsten Fällen, was das genau ist, ,,denn die Mädchen machen ihr Ding für sich“. Das bedeutet einerseits, daß sie es nicht in Gegenwart der Jungen tun, aber andererseits auch, daß Jungen sich nicht für diese Mädchenauseinandersetzungen interessieren und diese nicht besonders wahrnehmen; verbale Sticheleien sind auch nicht besonders auffällig, vor allem nicht wenn sie Dauerthema sind. Zwar sind auch die Jungen häufig der Meinung, daß die Opfer ihrer Aggressionen selbst schuld sind, aber bei den Mädchen wird das zur Entschuldigung für jegliche Abwertung anderer Mädchen. Wann immer von Auseinandersetzungen berichtet wird, fällt schnell der Satz, ,sie ist aber auch selbst schuld." Entweder ist sie zu mädchenhaft oder zu jungenhaft, entweder sieht sie zu gut aus oder nicht gut genug, entweder paßt sie sich zu wenig an oder sie schmeißt sich an jeden und jede ran, entweder hat sie keinen Freund oder zu viele Freunde (gehabt). Die Beliebtheit bei den eigenen Geschlechtsgenossinnen kommt für manche Mädchen einem Lotteriespiel gleich. Wie sich das auf die Entwicklung einer positiven Geschlechtsidentität auswirkt, konnte im Rahmen der Gruppendiskussionen leider nicht untersucht werden - neben dem bereits untersuchten Thema Mädchenfreundschaften scheint es allerdings auch lohnenswert, dem Thema Mädchenfeindschaften und -rivalitäten nachzugehen.

Wird den Mädchen schon von den Jungen vor Augen geführt, was es heißt in dieser Gesellschaft eine Frau zu sein und daß sie damit rechnen müssen, aggressiven männlichen Angriffen und Abwertungen ausgesetzt zu sein, so wird anhand der Mädchen-Mädchen-Interaktionen deutlich, daß von seiten der weiblichen Mitstreiterinnen in einer Klasse nicht immer mit Solidarität und Unterstützung zu rechnen ist. Im Gegenteil, daß was bei den Jungen gut ankommt, führt zur Abwertung bei den Mädchen und umgekehrt. Manche Jugendliche haben es schwer, sich überhaupt in das Klassen- und Gruppengefüge einzubringen. Dann ist nicht notwendig damit zu rechnen, daß die Mädchen dabei ein unterstützende Rolle einnehmen. Zumindest im Hinblick auf Mobbing bestätigen die Ergebnisse dieser Grupppendiskussionen die Aussagen von Olweus (1995). Offen bleibt hier allerdings, ob die Auswirkungen für die betroffenen Mädchen bereits adäquat erfaßt worden sind. Einzelinterviews, die es ermöglichen auch Betroffenheit in einem relativ geschützten Raum zu artikulieren, würden hierbei wahrscheinlich weiter führen. Es ist ebenfalls 
nicht gelungen, Interaktionen zwischen Jungen und Mädchen so zu rekonstruieren, daß die Bedeutung des gewalttätigen Handels für die Entwicklung von Geschlechtsrollen deutlich gemacht werden konnte ${ }^{7}$.

Allerdings zeigt bereits diese kurze Auswertung einzelner Interviewausschnitte, daß sich für die Gewaltprävention die Aufgabe ergibt, sich mit vorherrschenden Geschlechterrollen und Leitbildern kritisch auseinanderzusetzen, die Interaktionen zwischen Jungen und Mädchen stets zu reflektieren, dabei geschlechtsspezifische Ausdrucksformen von Aggressionen und Gewalt zu berücksichtigen und ggf. in gezielter Jungen- bzw. Mädchenarbeit zu thematisieren. Daneben ist es gerade für die in den sozialen und erzieherischen Berufen Tätigen, - sei es als Lehrer/Lehrerin, Sozialpädagoge/Sozialpädagogin, Vater oder Mutter - wichtig, im Umgang mit dem anderen Geschlecht Vorbild zu sein und gleichberechtigte Beziehungen nicht nur zu postulieren, sondern diese auch selbst zu leben (vgl. auch Schubarth/Ackermann 1997, S. 18). Angesichts sich verändernder und nicht mehr eindeutiger Männer- und Frauenrollen bestünde eine Möglichkeit der neuen Gestaltung des Geschlechterverhältnisses in der gleichberechtigten Verständigung über und letztlich dem „Aushandeln“ von Handlungsspielräumen für Jungen und Mädchen, Männer und Frauen, jenseits männlicher Hegemonie und weiblicher Unterwerfung. Den Beitrag, den die Schule dazu leisten kann, gilt es noch zu bestimmen.

\subsection{Zusammenfassung}

Die Auswertung der Schülerinnen- und Schülerbefragung zeigt wie erwartet, daß sich Jungen und Mädchen in der Wahrnehmung von Gewalt, im Ausüben von Gewalt und im Opferwerden von gewalttätigen und aggressiven Handlungen stark unterscheiden. Dabei sind die Unterschiede zwischen Jungen und Mädchen um so deutlicher, je aggressiver die ausgeführten Gewalthandlungen sind und je eher körperliche Gewalt und Waffeneinsatz vorhanden ist. Mädchen sind nicht völlig unbeteiligt an Gewalthandlungen, allerdings sind sie nicht überwiegend die Opfer schulischen Gewalthandelns, wie dies häufig vermutet wird. Hinweise auf spezifisch weibliche Ausdrucksformen von Gewalt lassen sich anhand der Daten der vorliegenden Untersuchung nicht finden, in keiner der angeführten Gewaltformen stellen die Mädchen die Mehrzahl der Ausübenden. Auch bei den Jungen bleibt der Zusammenhang der ausge-

7 Diese Mängel hängen mit der Anlage der Gruppendiskussionen zusammen, die in erster Linie dazu dienten, die schulische Situation insgesamt zum Thema zu machen. Der Rolle der Geschlechter wurde nicht in allen Interviews gleichermaßen thematisiert, z.T. bot sich das nicht an, z.T. wurde es auch von einzelnen Interviewern als nicht so wichtig erachtet, so daß mit dieser Sekundäranalyse nur ein kleines Schlaglicht geworfen werden kann. 
übten Gewalt zu männlichen Sozialisationsmustern offen. Eine konsequent geschlechterbezogene Auswertung läßt sich nicht vornehmen. In einem zweiten Untersuchungsschritt wurden darum Schülerinnen und Schüler an zwei gewaltbelasteten Schulen in Gruppendiskussionen und Einzelinterviews nach ihren Gewalterfahrungen im schulischen Kontext befragt. Dabei konnten wir zeigen, wie gewaltbezogene Interaktionen den schulischen Alltag von Jungen und Mädchen bestimmen und daß dies durchaus auch im Zusammenhang mit geschlechterbezogener Sozialisation steht. Es wird deutlich, welche Anforderungen an die Mädchen im Umgang mit männlicher Gewalt gestellt werden, welche Möglichkeiten von Seiten der Mädchen bestehen, gewalttätige Angriffe von Jungen zurückzuweisen, wie Gewalt als Bestandteil in den (schulischen) Alltag integriert wird sowie welchen geschlechterbezogenen Schranken sich Mädchen im Umgang mit Gewalt ausgesetzt sehen. Noch stärker als bei den befragten Mädchen wird anhand der Aussagen der Jungen sichtbar, daß Gewalt ein wichtiges Mittel zur Statusabsicherung innerhalb der Klasse bzw. innerhalb der Schule ist und daß diesen Statusauseinandersetzungen von Seiten der Mädchen keine Grenzen gesetzt werden, im Gegenteil: diese sind eingebunden in den Prozeß des Ringens um Macht und Anerkennung. Mädchen und Jungen lernen gerade in gewaltbezogenen Interaktionen, was es heißt, in dieser Gesellschaft eine Frau bzw. ein Mann zu sein; das was sie lernen, ist von traditionellen Geschlechtsrollenstereotypen nicht weit entfernt, wenngleich mittlerweile wesentlich ausdifferenzierter.

Zwei Konsequenzen sollen benannt werden: zum einen müssen Analysen zur Gewalt im schulischen Alltag, wenn sie den geschlechtsspezifischen $\mathrm{Zu}-$ sammenhang untersuchen wollen, methodisch variantenreicher angelegt werden; vor allem müssen vorhandene geschlechterbezogene Überlegungen in der Anlage des Designs, der Hypothesenbildung und der Konstruktion der Instrumente berücksichtigt werden. Zum anderen ergibt sich für die (schulische) Gewaltprävention die Notwendigkeit, sich mit herrschenden Geschlechterrollen und Leitbildern kritisch auseinanderzusetzen und Räume bzw. Situationen zu schaffen, in denen gleichberechtigte Beziehungsmuster eingeübt werden können. Dies gilt für die Arbeit mit Mädchen und Jungen gleichermaßen und setzt voraus, daß stärker als bisher geschlechterbewußte Ansätze Eingang in die Ausbildung bzw. in die Fort- und Weiterbildung von Lehrerinnen und Lehrer sowie von Sozialpädagoginnen und Sozialpädagogen finden. 


\section{Rollengefüge von Tätern und Opfern (Parviz Rostampour)}

Im Kapitel 2 wurde die Häufigkeit verschiedener Gewaltformen in der Wahrnehmung von Schülern sowie als Täter-Selbstreport (in vier konkreten Handlungssituationen) dargestellt. Außerdem wurden Einstellungen, Reaktionen und Interventionsbereitschaft in bezug auf diese Situationen (ebenfalls im Kapitel 2) und die allgemeine Einstellungsstruktur in Zusammenhang mit den geschlechtsspezifischen Analysen thematisiert (vgl. Kap. 3). Zusätzlich zu diesen Perspektiven wurden in einer weiteren Fragedimension die eigenen $\mathrm{Ge}$ walthandlungen der Schüler als Täter (unabhängig von den genannten Situationen) und ihre eigenen Erlebnisse als Opfer erfragt. In diesem Kapitel wollen wir uns auf diesen Aspekt und vor allem auf die Rollenverteilung im Kontext der schulischen Gewalt und die Charakteristika der an der Gewalt beteiligten Schülergruppen konzentrieren. Dabei werden wir auf unsere bisherigen Veröffentlichungen zurückgreifen und diese weiter präzisieren und ergänzen.

Bereits in Melzer/Rostampour (1996) hatten wir anhand der Analyse einer anderen repräsentativen sächsischen Stichprobe festgestellt, daß es bei den Tätern und Opfern sowohl gemeinsame Merkmale bzw. Einflußfaktoren gibt, die ihre Beteiligung an Gewalt erklären, als auch unterscheidende Merkmale und Einflußfaktoren, die für die jeweilige Rolle spezifisch sind. Zu den gemeinsamen Merkmalen und Einflußfaktoren gehören beispielsweise: Geschlecht, Schulform, Gewaltbelastung der Klasse bzw. Schule, aggressives Lehrerverhalten, Leistungskonflikte mit den Eltern und ein schlechtes Familienklima. Demnach sind die Jungen mehr als die Mädchen an der Gewalt beteiligt und zwar sowohl als Täter als auch als Opfer. Täter und Opfer gibt es in den Mittelschulen mehr als in den Gymnasien und insbesondere in den belasteten Schulen und Klassen. Aggressives Verhalten der Lehrer und schlechtes Familienklima (darunter auch Leistungskonflikte mit den Eltern) gehen auch mit der Beteiligung der Schüler an Gewalt einher, und zwar sowohl als Täter als auch als Opfer.

Als typische unterscheidende Merkmale der Täter haben sich folgende Aspekte herausgestellt: geringere Schulfreude, geringeres Vertrauen zu den Lehrern, niedrigerer Leistungsstatus, schlechtere Einschätzung der Fach- und Integrationskompetenz der Lehrer, schlechtere schulökologische Bedingungen und Defizite der Schule bei der Vermittlung von Sozialkompetenz und von traditionellen Lerntugenden (z.B. Ordnung). Als typische unterscheidende Merkmale der Opfer sind folgende festgestellt worden: Außenseiterstatus, Leistungsangst, niedrigeres Alter, negatives Selbstwertgefühl und schlechtere Einschätzung des Klassen- bzw. Schulklimas.

Diese Ergebnisse stehen im Einklang mit Befunden anderer einschlägiger Studien. So sind typische Gewalttäter nach Olweus $(1995,1997)$ nicht nur 
gegenüber Gleichaltrigen, sondern auch oft gegenüber Erwachsenen aggressiv (Konflikte mit den Eltern und Lehrern), haben eine positive Einstellung zur Gewalt und zur Anwendung von gewaltsamen Mitteln. Unter den Jungen sind die Täter meistens körperlich stärker als die anderen, insbesondere im Vergleich zu den Opfern. Täter sind in der Regel impulsiv und weniger ängstlich, zeigen mehr Selbstwertgefühl als die anderen und sind oft von einer kleinen Gruppe von Gleichaltrigen umgeben. Typische Opfer sind dagegen ängstlicher und unsicherer als die anderen Schüler, sind oft vorsichtig, empfindsam, still und leiden oft unter mangelndem Selbstwertgefühl. Sie fühlen sich als Versager, dumm, wenig attraktiv. Sie sind in der Regel einsam und verlassen, sind auch körperlich schwächer (bei den Jungen) und haben häufig eine negative Einstellung zur Gewalt. ${ }^{1}$

Krumm (1993) zitiert einige Befunde aus Studien in angelsächsischen Ländern, die ebenfalls mit den oben genannten Ergebnissen übereinstimmen und sie ergänzen. Danach sind Täter aggressiv gegen Eltern, Lehrer, Geschwister und Peers. Sie sind nicht ängstlich, glauben an ihre Stärke, sind unabhängig, witzig, nie um Ausreden verlegen und gelten als „Machos“. Sie sind populärer als die Opfer und haben wenig Empathie für diese. Körperlich sind sie stark, robust, energisch und sportlich. Schuldgefühle oder Scham sind bei ihnen wenig zu spüren. Sie kommen meistens aus Familien mit chaotischen Verhältnissen oder mit wenig herzlich-empathischen Beziehungen zwischen Eltern und Kindern. Die Kinder werden in diesen Familien entweder vernachlässigt oder mit harten Strafen „diszipliniert“. Opfer dagegen sind ängstlich, ungeschickt, physisch schwach, scheu, ungesellig und nicht kommunikativ. Sie sind kleiner als die Täter und wenig attraktiv. Zu den weiteren Opfermerkmalen gehören Minderwertigkeitsgefühle und Abhängigkeit von der Familie; ohnehin haben sie mehr Familienprobleme als die unauffälligen Schüler. Zur Schule sind Täter eher negativ und Opfer eher positiv eingestellt. Bei den Tätern sind schlechtere Schulleistungen festzustellen, bei den Opfern durchschnittliche Leistungen.

Lösel/Bliesener/Averbeck (1997) haben festgestellt, daß die an der Gewalt Beteiligten - vor allem die Täter - schlechtere Noten in den Kernfächern (Deutsch, Englisch und Mathematik) aufweisen als die anderen. Bei den Sportnoten weisen die Opfer schlechtere Noten auf als die Täter und die anderen. Das deutet nach Ansicht der Autoren darauf hin, daß die Opfer körperlich weniger fit sind. Weiterhin wurde ein deutlicher Zusammenhang zwischen der Beteiligung an Gewalt (in welcher Rolle auch immer) und der „biographischen Belastung“2 festgestellt. Sowohl Täter als auch Opfer kommen in den meisten

1 Das letztere wird in unseren Ergebnissen nicht bestätigt (s. u.).

2 Das umfaßt vor allem familiale Probleme ,wie z.B. Scheidung der Eltern, andere Verluste, Armut, Arbeitslosigkeit, enge Wohnverhältnisse, Alkohol- und Medikamentenmißbrauch der Eltern“ (Lösel/Bliesener/Averbeck 1997, S. 141). 
Fällen aus belasteten Familien. Auch in dieser Studie wurde festgestellt, daß die Täter überdurchschnittlich in Peer-Gruppen integriert sind, während die Opfer eher isoliert und auch sehr unbeliebt sind. Die Täter sind nach Ansicht der Lehrer impulsiver als die Opfer und am meisten dominant; die Opfer am wenigsten. Die Autoren stellen weiterhin fest, daß die Opfer ,,vor allem in den Skalen des internalisierenden Syndroms deutlich erhöhte Werte (haben). Sie berichten über mehr sozialen Rückzug, körperliche Beschwerden, Angst und Depression, soziale Schwierigkeiten, Aufmerksamkeits- und Identitätsprobleme" (ebd., S. 148). Auch die Täter berichten über mehr körperliche Beschwerden (z.B. Kopfweh, Erbrechen), Aufmerksamkeits- und Impulsivitätsprobleme. Im Vergleich zu den Opfern weisen sie aber bei sozialem Rückzug, Angst und Depression, sozialen Problemen und Selbstwert- sowie Identitätsproblemen niedrigere Werte und bei Aggressivität und „,zwanghaft/schizoiden“ Problemen (z.B. Dinge horten, seltsame Gedanken und Ideen haben) höhere Werte auf.

Die genannten Merkmale bzw. Einflußfaktoren gelten für die jeweiligen Typen nur insgesamt und dürfen nicht darüber hinweg täuschen, daß es sowohl bei den Tätern als auch bei den Opfern sehr unterschiedliche Individuen gibt. Busch und Todt (1997) unterteilen die Täter anhand einer hierarchischen Clusteranalyse in vier Täter-Typen, die sich nach zwei Hauptdimensionen „Reaktiv/Proaktiv“ und „niedrige/hohe Problembelastung“ voneinander unterscheiden. Wir wollen uns aber in diesem Beitrag nicht mit den Unterschieden innerhalb der jeweiligen beteiligten Gruppe beschäftigen, sondern mit den Unterschieden und Gemeinsamkeiten im Vergleich der Gruppen und Handlungstypen.

In Rostampour/Schubarth (1997) und Rostampour/Melzer (1997) hatten wir dargestellt, daß sich die Täter- und Opferrollen nicht ausschließen und nicht in jedem Fall eindeutig voneinander zu trennen sind. Es wurde gezeigt, daß die Täter bisweilen zum Opfer werden und daß auch die Opfer hin und wieder zurückschlagen. Darüber hinaus wurden eine Reihe weiterer Determinanten der Rollenverteilung bei schulischen Gewalthandlungen herausgefunden. Wir werden im folgenden diesen Ansatz noch einmal aufgreifen und vertiefen. Im ersten Teil (4.1) vergleichen wir die Häufigkeit verschiedener Gewaltformen in der Schule anhand des Selbstreports der Schüler. Dabei werden wir auch auf eine Asymmetrie zwischen den Täter- und Opferangaben hinsichtlich der Häufigkeit der Gewalthandlungen eingehen. In einem zweiten Teil (4.2) werden wir anhand des Selbstreports der Schüler feststellen, welche Befragten an der Gewalt in der Schule beteiligt sind und welche Rollen sie dabei überwiegend übernehmen. Eine Clusteranalyse wird die Stichprobe in die entsprechenden Untergruppen aufteilen. Im dritten Teil (4.3) werden wir diese Untergruppen unter die Lupe nehmen, um die spezifischen Merkmale der an Gewalt beteiligen Schüler besser erkennen zu können. Im letzten Teil (4.4) fassen wir die Ergebnisse zusammen. 


\subsection{Interaktionale Gewaltformen von Schülern untereinander - Selbstreport von Tätern und Opfern}

Um die Stärke und Art der Verwicklung der Befragten an das schulische Gewaltgeschehen festzustellen, wurden die Schüler nach der Häufigkeit ihrer Beteiligung an unterschiedlichen Gewaltformen in der Schule oder auf dem Schulweg während der letzten 12 Monate gefragt. Die Items umfassen psychisch-verbale Angriffe gegen die Mitschüler (wie Ärgern, Hänseln und Beschimpfen), sachbezogene Angriffe gegen andere Schüler (wie Verstecken, Zerstören und gewaltsame Wegnahme Sachen anderer), körperliche Angriffe (wie Prügelei), Vandalismus (gegen das Schulgebäude und Schuleigentum) und Aggression gegen die Lehrer. ${ }^{3}$ Tabelle 4-1 zeigt die 16 Täter-Items des Fragebogens und zwar sortiert nach ihren Mittelwerten in der sächsischen Stichprobe. In Tabelle 4-2 sind in entsprechender Weise die acht Opfer-Items dargestellt. Die Präsentation der Daten erfolgt, sofern keine bedeutsamen OstWest-Unterschiede bestehen, anhand der sächsischen Befragungsergebnisse. In der Regel sind diese auch in den Tabellen und Abbildungen dokumentiert. Die hessischen Daten werden z.T. mit zur Interpretation herangezogen und sind bei der Darstellung deutlich gekennzeichnet.

Wie die Tabellen 4-1 und 4-2 zeigen, treten die „,harmloseren“ Gewaltformen wie Ärgern (V260), Hänseln (V262, V281) und Beschimpfen (V278 und V283) am häufigsten und die härteren Formen wie Waffenbesitz (V272), gezielte Aggressionen auf dem Schulweg (V268, V282), absichtliches Zerstören (V266, V275, V263 und V285), Verprügeln (V265, V280) und unter Druck setzen (V267 und V284) am seltensten auf. Damit können die Ergebnisse des Selbstreports die der Wahrnehmung weitgehend bestätigen (vgl. Kap. 2). In der hessischen Stichprobe weisen die Opfer-Items dieselbe Reihenfolge der Gewaltformen wie in Sachsen auf. Auch bei den Täter-Items ist die Reihenfolge im Prinzip dieselbe, nur mit einigen kleinen Unterschieden: beispielsweise findet in Sachsen mehr Ärgern (V260) statt als Hänseln (V262); in Hessen ist es umgekehrt.

3 Die Antwortkategorien auf einer 6stufigen Skala lauten: „fast täglich“, ,,mehrmals wöchentlich“, ,mehrmals im Monat“, ,,alle paar Monate“, ,,seltener“ und „nie“. Wir behandeln diese Skala als Intervallskala und nehmen dadurch möglicherweise entstehende Meßungenauigkeiten in Kauf. Allerdings hat ein Versuch zur „Intervallisierung“ dieser Skala zu - im Prinzip - identischen Ergebnissen geführt. 
Tab. 4-1: Täter-Items sortiert nach ihren Mittelwerten*

Wie oft hast Du selbst an Deiner Schule oder auf dem Schulweg

Mittelin den letzten 12 Monaten folgendes gemacht?

wert

V260: Andere im Unterricht geärgert, beworfen oder beschossen.

V262: Andere gehänselt oder mich über sie lustig gemacht.

V278: Andere mit gemeinen Ausdrücken beschimpft.

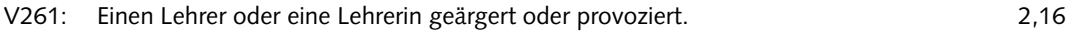

V274: Andere mit Sachen (zum Beispiel Lineal, Mäppchen) beworfen. 1,86

$\begin{array}{ll}\text { V273: } & \text { Mit anderen bewußt Streit angefangen, sie angeschrien, beschimpft. } \\ 1,69\end{array}$

V276: Anderen Sachen versteckt, so daß sie sie nicht wiederfinden konnten. 1,69

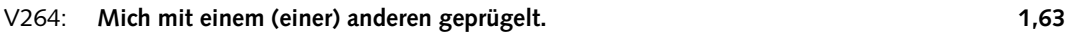

$\begin{array}{lr}\text { V279: Anderen etwas gewaltsam weggenommen. } & 1,54\end{array}$

\begin{tabular}{lr} 
V267: & Andere unter Druck gesetzt. \\
\hline
\end{tabular}

V263: Im Schulgebäude etwas absichtlich beschädigt. $\quad 1,46$

$\begin{array}{lr}\text { V265: Mit anderen einen Jungen/ein Mädchen verprügelt. } & 1,38\end{array}$

V275: Sachen absichtlich zerstört, die der Schule gehören (z.B. Stühle, Bücher). 1,36

V266: Sachen von anderen absichtlich kaputtgemacht. $\quad 1,35$

V268: Anderen auf dem Schulweg aufgelauert, sie belästigt, bedroht, verprügelt. $\quad 1,30$

V272: Waffen (Schreckschußpistole, Reizgas) mit in die Schule gebracht. $\quad 1,22$

* Die interaktionellen Gewaltformen unter den Schülern sind fettgedruckt.

Tab. 4-2: Opfer-Items sortiert nach ihren Mittelwerten*

Wie oft bist Du selbst an Deiner Schule oder auf dem Schulweg

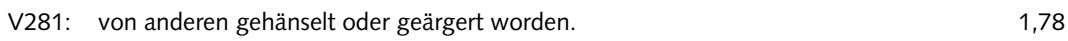

V283: von anderen angeschrien, beschimpft, beleidigt worden. $\quad 1,71$

Wie oft wurde(n) Dir an Deiner Schule oder auf dem Schulweg in den letzten 12 Monaten ...

V287: von anderen Sachen versteckt, so daß Du sie nicht wiederfinden konntest. $\quad 1,42$

$\begin{array}{ll}\text { V286: von anderen gewaltsam etwas weggenommen. } & 1,38\end{array}$

V285: von anderen Sachen absichtlich kaputtgemacht. $\quad 1,36$

Wie oft bist Du selbst an Deiner Schule oder auf dem Schulweg in den letzten 12 Monaten ...

$\begin{array}{ll}\text { V280: von anderen geschlagen worden. } & 1,33\end{array}$

$\begin{array}{lr}\text { V282: } & \text { auf dem Schulweg belästigt, bedroht worden. } \\ 1,23\end{array}$

V284: von anderen unter Druck gesetzt, erpreßt worden. $\quad 1,16$

* Alle Items betreffen interaktionelle Gewaltformen. 
Bei einem Vergleich zwischen den Tabellen 4-1 und 4-2 ist festzustellen, daß die Mittelwerte der Täter-Items insgesamt höher liegen als die der Opfer-Items. Das ist möglicherweise ein Hinweis auf eine Übertreibung bei den Täter-Items und/oder eine Untertreibung bei den Opfer-Items. Um dieses Phänomen genauer darzustellen, vergleichen wir den Mittelwert jedes Opfer-Items mit dem Mittelwert des entsprechenden Täter-Items (bzw. in drei Fällen mit den Mittelwerten von zwei Täter-Items, die in Frage kommen). Abbildung 4-1 zeigt die Ergebnisse dieses Vergleichs in Sachsen. Jedes der acht Kästchen beinhaltet die Mittelwerte einer bestimmten Gewaltform für jeweils ein Opfer-Item (die jeweils linke Säule) und im Vergleich mit einem bzw. zwei Täter-Items. Der Mittelwert des Opfer-Items V281 (gehänselt oder geärgert worden) ist 1,8; die entsprechenden Täter-Items V260 (andere im Unterricht ärgern, bewerfen oder beschießen) und V262 (andere Hänseln oder sich über sie lustig machen) weisen aber höhere Mittelwerte $(2,6$ bzw. 2,4) auf. Der Vergleich mit V260 soll unter dem Vorbehalt bleiben, daß dieses Item auf der einen Seite über das Ärgern hinaus auch physische Attacken (Bewerfen und Beschießen) mit einschließt und möglicherweise deshalb einen höheren Mittelwert aufweist. Auf der anderen Seite führt die Begrenzung auf die Unterrichtssituation möglicherweise zu einem niedrigeren Mittelwert. Der Vergleich mit V262 zeigt aber eindeutig, daß die Täterseite über mehr Fälle berichtet als die Opferseite.

Das zweite Kästchen in der Abbildung 4-1 beinhaltet das Item V283 (von anderen angeschrien, beschimpft, beleidigt worden) als Opfer-Item mit einem Mittelwert von 1,7. Das entsprechende Täter-Item V273 (mit anderen bewußt Streit angefangen, sie angeschrien, beschimpft) hat einen ebenso hohen (oder sogar kleineren) Mittelwert. Hier hat vermutlich die Einschränkung auf das bewußte Handeln den Mittelwert beim Täter-Item nach unten getrieben. Das Täter-Item V278 (andere mit gemeinen Ausdrücken beschimpft) weist aber einen Mittelwert von 2.2 auf, obwohl dieses Item nur auf gemeine Beschimpfung eingeschränkt ist und nicht wie das Opfer-Item Anschreien und andere Formen der Beleidigung mit einschließt. Diese und weitere Vergleiche bei den anderen sechs Kästchen der Abbildung 4-1 zeigen, daß man im allgemeinen niedrigere Werte feststellt, wenn man anstelle von Täter-Items die Opfer-Items als einen Indikator für das Niveau der Gewaltbelastung der Schulen berücksichtigt. Ähnliche Ergebnisse wurden auch in der hessischen Stichprobe festgestellt. Diese Asymmetrie ist in der Regel auch in jeder Einzelschule zu beobachten (mit Ausnahme der Förderschulen, bei denen diesbezüglich keine Systematik zu erkennen ist).

Es stellt sich die Frage nach den Motiven für diese Über- und/oder Untertreibung. Eine Übertreibung bei den Tätern könnte als eine Demonstration der Stärke und Überlegenheit aufgefaßt werden; eine Untertreibung bei den Opfern hingegen könnte man als einen Versuch für die Verdeckung eigener Schwäche und Unterlegenheit sowie als bewußte und unbewußte Verdrängung der unangenehmen Erlebnisse interpretieren. 
Abb. 4-1: Mittelwerte der Opfer-Items im Vergleich mit den entsprechenden Täter-Items*

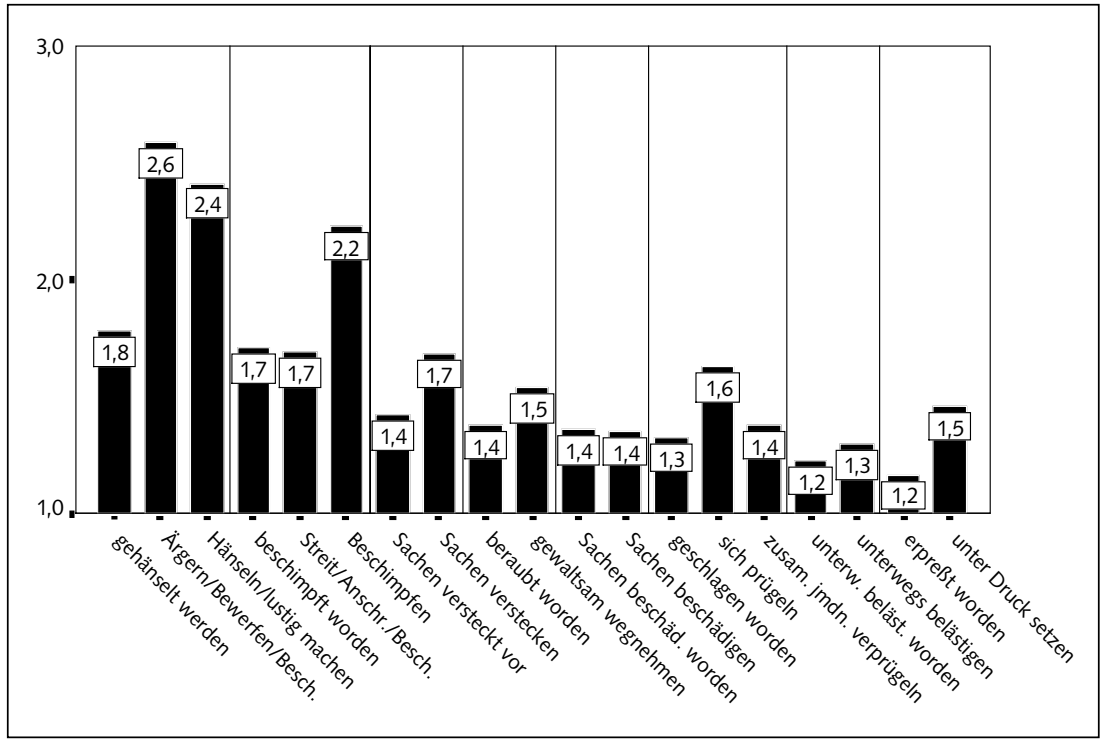

* In jedem Kästchen steht ein Opfer-Item am linken Rand und ein oder zwei entsprechende TäterItems rechts daneben.

\subsection{Rollenverteilung bei Gewalthandlungen unter Schülern: Wer ist Täter, wer ist Opfer und wer beides zugleich?}

In diesem Kapitel wollen wir eine Typologie des Gewalthandelns herausarbeiten sowie die Charakteristika der an der schulischen Gewalt beteiligten Schülergruppen untersuchen und erklären: Wodurch unterscheiden sich die Täter und Opfer voneinander und von den Unbeteiligten? Um derartige Gruppen in unserer Stichprobe zu identifizieren, wurden die Angaben der Befragten über ihre eigene Beteiligung an Gewalt (die Täter- und Opfer-Items) ausgewertet. Allerdings sollten nur diejenigen Gewaltformen in der Analyse berücksichtigt werden, die von einem oder mehreren Schülern ausgehen und sich gegen einen oder mehrere Schüler richten. Denn nur bei diesen interaktionellen (interpersonellen) Gewaltformen kommen die Schüler sowohl als Täter als auch als Opfer in Frage. Damit bleiben die Aggressionen gegen die Lehrer und der Vandalismus gegen das Schuleigentum für die Bestimmung der Rollenverteilung erst einmal unberücksichtigt. Die drei unberücksichtigten 
Täter-Items sind in der Tabelle 4-1 in kursiv gedruckt. Die anderen 13 TäterItems (im Fettdruck) und alle acht Opfer-Items (Tab. 4-2) werden dagegen für die Bestimmung der Rollenverteilung berücksichtigt.

Die Bestimmung der Rollen ist keine einfache Aufgabe, weil die Täterund Opferrollen nicht eindeutig voneinander zu trennen sind. Der Täter von heute kann morgen zum Opfer werden und umgekehrt. Das zeigen vor allem die positiven Korrelationen zwischen den 13 Täter-Items einerseits und den acht Opfer-Items andererseits: alle möglichen $(13 \times 8=104)$ Korrelationskoeffizienten sind positiv und liegen zwischen $\mathrm{r}=.07$ und $\mathrm{r}=.37$ (mit einem Durchschnitt von $\mathrm{r}=.21$ ). Unter diesem Umstand ist die Erkennung der Rollen am besten durch eine gleichzeitige Berücksichtigung sowohl der Täter- als auch Opfer-Items möglich. Dazu kann man sich die Clusteranalyse (k-Means-Verfahren) zunutze machen. Die Befragten werden bei diesem Verfahren anhand ihrer Werte für die 21 klassifizierenden Items ${ }^{4}$ in $\mathrm{k}$ Gruppen (Cluster) aufgeteilt, und zwar so, daß jede Gruppe in sich möglichst homogen ist und die Gruppen untereinander möglichst heterogen sind. Die Anzahl der Cluster (k) kann anhand von statistischen und/oder inhaltlichen Kriterien festgelegt werden. Wir haben uns aus theoretischen und empirischen Gründen, die hier nicht im einzelnen dargelegt werden können, für eine 5-Cluster-Lösung (sowohl in der sächsischen als auch in der hessischen Stichprobe) entschieden.

Abbildung 4-2 zeigt die Mittelwerte der 21 Items der interaktionellen Gewaltformen sowie die der 3 übrigen Täter-Items (Vandalismus und Aggression gegen die Lehrer) in den 5 Clustern der sächsischen Stichprobe. Die meisten Befragten $(55,9 \%)$ fallen in eine Gruppe, die bei allen Items Mittelwerte aufweist, die sich nur geringfügig von 1 (d.h. „nie“) unterscheiden (dickere volle Linie in der Abbildung). Diese Befragten werden im folgenden als Unbeteiligte bezeichnet, weil sie - abgesehen von Ausnahmefällen - weder als Täter noch als Opfer auftreten.

Die zweitgrößte Gruppe (25,8\%) weist niedrige Mittelwerte bei den Opfer-Items und etwas höhere Mittelwerte bei den Täter-Items und insbesondere bei den ,weicheren“ Gewaltformen (Strich-Punkt-Linie) auf. Wir bezeichnen diese Gruppe als Episoden-Täter, weil sie mehr als die Unbeteiligten aber weitaus weniger als die Täter-Gruppe an den Gewalthandlungen beteiligt ist. Die eigentlichen Täter bilden eine relativ kleine Gruppe (8,0\%), die hohe Mittelwerte bei den Täter-Items und niedrige Mittelwerte bei den Opfer-Items hat (Punktlinie). Eine andere kleine Gruppe (7,0\%) zeichnet sich durch niedrige Mittelwerte bei den Täter-Items und hohe Mittelwerte bei den Opfer-Items aus (dünnere volle Linie). Somit werden die Befragten dieser Gruppe im folgenden als Opfer bezeichnet.

4 Die Items wurden vor der Analyse so gewichtet, daß ein Gleichgewicht erstens zwischen den Täter- und den Opfer-Items und zweitens zwischen den personenbezogenen und sachbezogenen Items hergestellt wurde. 
Abb. 4-2: Mittelwerte der Täter- und Opfer-Items in den Clustern bei einer 5-Cluster-Lösung (Sachsen)

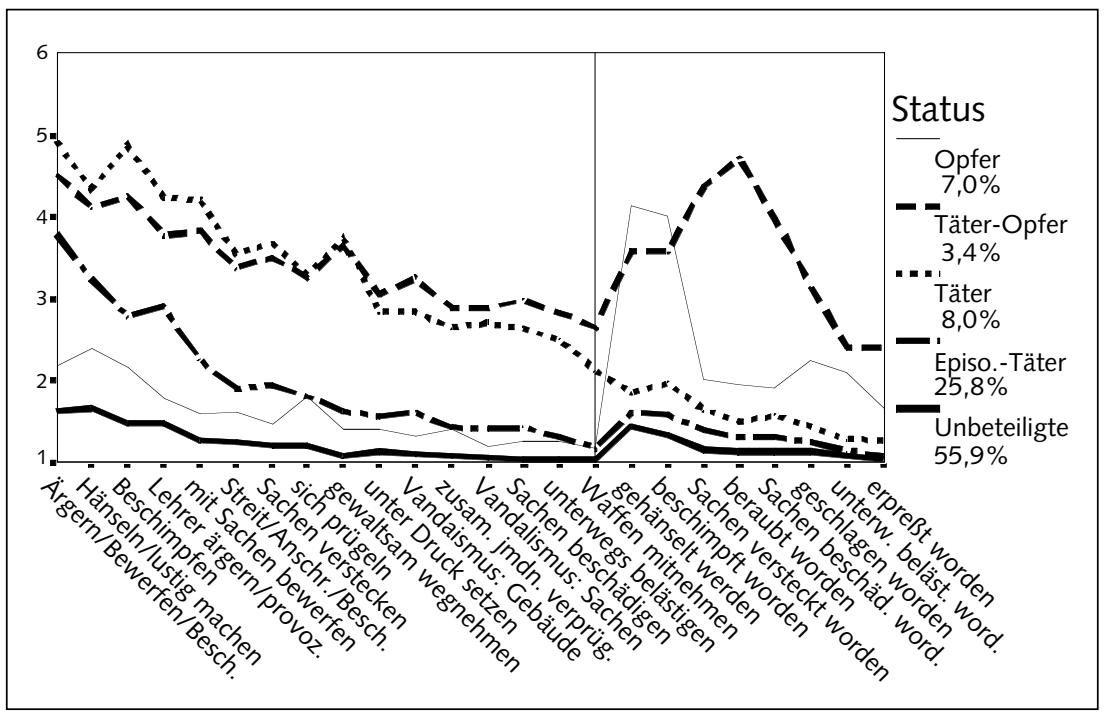

Die kleinste Gruppe $(3,4 \%)$ zeigt hohe Mittelwerte sowohl bei den Täter- als auch bei den Opfer-Items (gestrichelte Linie). Die Befragten dieser Gruppe werden folgerichtig als Täter-Opfer bezeichnet. Bei einer näheren Betrachtung der Abbildung 4-2 kann man feststellen, daß die harmloseren Gewaltformen von den Täter-Opfern etwas weniger als von den Tätern ausgeübt werden und die härteren und selteneren Formen etwas mehr. Parallel dazu kann man feststellen, daß die Täter-Opfer etwas weniger als die Opfer Zielscheibe der harmloseren Angriffe sind und dafür häufiger als diese bei den härteren und selteneren Formen der Gewalt in die Opferrolle geraten. Somit kann diese kleine Gruppe als der harte Kern der schulischen Gewalt eingestuft werden. Gewisse Ähnlichkeiten bestehen zu der von Olweus so bezeichneten Gruppe der ,provokativen Opfer"; allerdings wird durch diese Benennung die Opferrolle stärker betont als die Täterrolle. Die Abb. 4-2 zeigt aber, daß die Täter-Opfer diese beiden Rollen im gleichen Maße ausüben. Außerdem enthält die Bezeichnung „provokative Opfer" implizit eine Kausalrichtung bzw. eine zeitliche Abfolge (Provokation führt zum Opferstatus), die mit den Daten nicht zu überprüfen ist. Man könnte genauso gut von „zurückschlagenden Opfern“ oder „,provozierten Tätern“ sprechen. Wir bleiben deshalb bei der neutralen Bezeichnung „Täter-Opfer“.

Abbildung 4-3 zeigt (analog zu Abbildung 4-2) die Mittelwerte der Täterund Opfer-Items in den Clustern einer 5-Cluster-Lösung mit denselben Klas- 
sifikationsvariablen in der hessischen Stichprobe. Hier ist die gleiche Struktur zu beobachten wie in Sachsen. Das zeigt, daß die gefundene Struktur nicht nur für Sachsen gilt. ${ }^{5}$

Abb. 4-3: Mittelwerte der Täter- und Opfer-Items in den Clustern bei einer 5-Cluster-Lösung (Hessen)

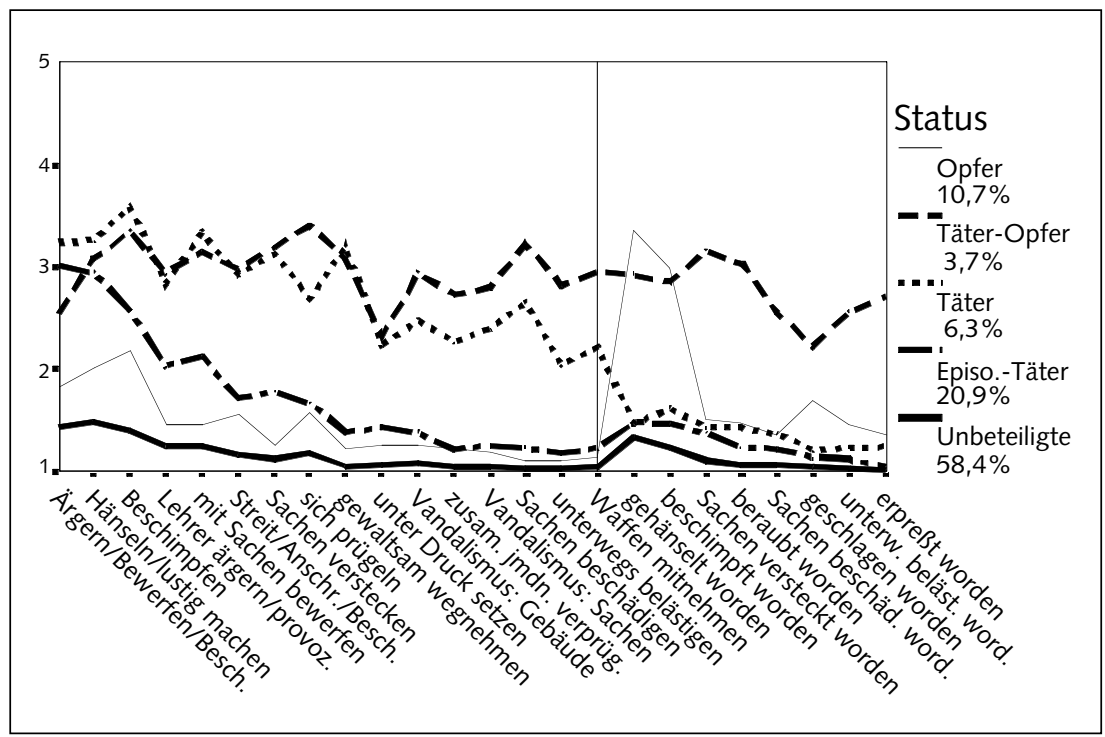

$\mathrm{Da}$ die Täter- und Opferrollen oft ineinander gehen, wird nicht nur mit der Existenz der Gruppe Täter-Opfer begründet. Wie die Abbildungen 4-2 und 4-3 zeigen, gibt es keine „reine“ Täter- oder Opfergruppe. Die Täter werden auch manchmal zum Opfer (auf jeden Fall häufiger als die Episoden-Täter und diese wiederum häufiger als die Unbeteiligten). Auch die Opfer spielen manchmal die Täterrolle (häufiger als die Unbeteiligten). Dieser Wechsel der Rollen zeigt noch einmal, warum für die Erkennung der verschiedenen Typen die Täter- und Opfer-Items nicht getrennt voneinander, sondern in Verbindung miteinander (in einer Clusteranalyse) berücksichtigt werden müssen.

5 Die prozentuale Besetzung der Cluster sowie ihre Mittelwerte in den beiden Stichproben sind nicht ohne weiteres miteinander vergleichbar, weil die Items in Hessen eine 5stufige Skala (,nie“, ,,alle paar Monate“, „,mehrmals im Monat“, „,mehrmals wöchentlich“ und „,fast täglich“) und in Sachsen eine 6stufige (,fast täglich“, „,mehrmals wöchentlich“, „,mehrmals im Monat“, ,,alle paar Monate“, „seltener“ und „,nie“) aufweisen. 
Die Existenz der Episoden-Täter wirft die Frage auf, warum sich auf der Opferseite keine Gruppe der „Episoden-Opfer“ herausgebildet hat. Es ist vorstellbar, daß es auch Schüler gibt, die ab und zu angegriffen werden, wie es ja Schüler gibt, die ab und zu angreifen. Daß sich eine solche Gruppe nicht zu erkennen gegeben hat, ist möglicherweise mit der schon erklärten Vermutung verbunden, da $\beta$ auf der Opferseite vieles verschwiegen und verdrängt wird. Wenn dem so ist, dann kann man sich vorstellen, daß diejenigen, die nicht so oft angegriffen werden, eher in der Lage sind, ihre Erlebnisse zu verschweigen und verdrängen. Dagegen können diejenigen, die häufig und wiederholt angegriffen werden, nicht alles verschweigen und verdrängen.

Die prozentuale Besetzung der Gruppen steht mehr oder weniger im Einklang mit den Ergebnissen weiterer Studien. Olweus (1995) stellt fest, daß 7\% der norwegischen Schülerschaft der Grund- und weiterführenden Schulen als Täter „hin und wieder“ oder „öfter“ an Gewalt beteiligt sind. Weitere 9\% werden als Opfer klassifiziert (1,6\% Täter-Opfer und 7,4\% nur Opfer). Lösel, Bliesener und Averbeck (1997) klassifizieren die Befragten (Klassen 7 und 8) in fünf Gruppen: Täter (5,2\%), Opfer $(5,2 \%)$, Täter-Opfer $(2,1 \%)$, Normalgruppe ( $82 \%$ ) und Kompetente (5,5\%). Die Unterschiede zwischen den einzelnen Studien sind zu einem erheblichen Teil auf unterschiedliche Klassifikationskriterien und unterschiedliche Grundgesamtheiten, insbesondere hinsichtlich der Jahrgangsstufe, zurückzuführen.

Wir haben für die Clusteranalyse - wie schon erwähnt - ausschließlich die Gewaltformen berücksichtigt, die sich direkt gegen die einzelnen Schüler richten. Wir sehen aber in den Abbildungen 4-2 und 4-3, daß auch die Täter der anderen Gewaltformen (Vandalismus und Aggression gegen die Lehrer) in denselben Gruppen zu suchen sind wie bei den interaktionellen Gewaltformen (nämlich in den Clustern der Täter und Täter-Opfer).

Eine andere Frage ist die nach dem Zusammenhang zwischen Gewalt in der Schule und Jugendkriminalität oder Kriminalität im allgemeinen. Olweus (1995) stellt fest, daß etwa 60\% der Jungen, die in den Klassen 6 bis 9 als Täter charakterisiert wurden, mindestens einmal bis zum Alter von 24 Jahren als Straftäter verurteilt worden waren, während dies nur für $10 \%$ der unbeteiligten Jungen gilt. 35\% bis $40 \%$ der Täter (Jungen wie Mädchen) waren sogar mindestens dreimal verurteilt worden. Olweus kommt hier zu dem Ergebnis, daß „Gewalttätigkeit (...) auch als eine Komponente eines allgemeineren sozialfeindlichen und gesetzesbrechenden ('verhaltensgestörten') Verhaltensmusters angesehen werden (kann). Aus dieser Sicht kann man leicht vorhersagen, daß Jugendliche, die aggressiv sind und anderen gegenüber Gewalt anwenden, ein deutlich erhöhtes Risiko laufen, später in weitere Problembereiche wie Kriminalität und Alkoholmißbrauch zu geraten“ (ebd., S. 45). Das, was Olweus als allgemein sozialfeindliches Verhaltensmuster bezeichnet, ist identisch mit dem, was wir in diesem Buch als gewaltaffines Syndrom bezeichnen. Wir können zwar aufgrund der Konzeption unseres Projekts nicht die Entwicklung der einzelnen Schüler bis zum Erwachsenenalter verfolgen, wir können aber 
überprüfen, ob schulische Gewalttäter bereits im Jugendalter mehr als andere Schüler in delinquente Fälle verwickelt und für Alkohol, Zigaretten und Drogen anfällig sind.

Die Schüler wurden gefragt, ob und wie oft sie einige bestimmte Delikte in den letzten 12 Monaten begangen haben und zwar ohne Einschränkung auf die Schule als Tatort. Tabelle 4-3 zeigt die entsprechenden Items und ihre prozentualen Verteilungen in Sachsen. Die Zahlen sind insgesamt alarmierend hoch. 32,8\% der Befragten geben zu, mindestens einmal im Jahr in Schlägereien verwickelt gewesen zu sein; 13,3\% sogar in härteren Schlägereien mit schlimmen Folgen. 18,4\% der Befragten haben innerhalb eines Jahres mindestens einmal absichtlich Sachbeschädigung begangen. 16,1\% geben auch zu, andere mindestens einmal im Jahr um mehr als $10 \mathrm{DM}$ bestohlen zu haben. 7,4\% sind mindestens einmal in einen Einbruch verwickelt gewesen; 7,8\% in Aufbrechen von Automaten. ${ }^{6}$ Dieselben Fragen wurden auch in Hessen gestellt. Die Unterschiede zwischen den beiden Bundesländern sind insgesamt minimal und nur in drei von sieben Fällen statistisch signifikant (Chi²-Test): in Hessen gibt es etwas mehr Rauferei/Schlägerei (V294) und Aufbrechen der Automaten (V299); in Sachsen gibt es dagegen etwas mehr Diebstahl (V297).

Um zu überprüfen, inwieweit die Gewalt in der Schule und die Jugendkriminalität von denselben Tätern begangen werden, können wir die Mittelwerte der eben dargestellten Delinquenz-Items in den fünf Clustern unserer Clusteranalyse miteinander vergleichen. Abbildung 4-4 zeigt diese Mittelwerte in der sächsischen Stichprobe. Auf einer Skala von 1 ('keinmal') bis 4 ('über 4 mal') weisen die Unbeteiligten Mittelwerte auf, die sich kaum von 1 unterscheiden. Das zeigt, daß die Schüler, die nicht in schulische Gewalt verwickelt sind, in der Tat keine Rolle in der Jugendkriminalität spielen. Bei den Opfern und den Episoden-Tätern sind zwar größere Mittelwerte als bei den Unbeteiligten zu verzeichnen. Die Werte liegen aber im Schnitt deutlich unterhalb von 2 ('einmal im Jahr'). Ganz anders ist es aber bei den Tätern und Täter-Opfern, die relativ hohe Mittelwerte bei allen erhobenen Deliktarten aufweisen. Auch in der hessischen Stichprobe wurde dasselbe Muster beobachtet, ${ }^{7}$ so daß wir sagen können, daß die Gewalt in der Schule und die Jugendkriminalität in engem Zusammenhang miteinander stehen.

6 Diese Zahlen müssen unter Vorbehalt interpretiert werden: bei solchen kritischen Fragen kann man nicht davon ausgehen, daß die Befragten alle ihre Straftaten zugeben.

7 Nur durch die höheren Mittelwerte der Delinquenz-Items in dem Cluster „Täter-Opfer“ von Hessen unterscheiden sich die Bundesländer. In Sachsen sind die Täter und Täter-Opfer gleichermaßen in Jugendkriminalität verwickelt. In Hessen weisen die Täter fast dieselben Mittelwerte auf wie in Sachsen, die Täter-Opfer aber höhere Werte. Dieser harte Kern der schulischen Gewalt bildet somit auch (zumindest in Hessen) den harten Kern der Jugendkriminalität. 
Tab. 4-3: Prozentuale Verteilung der Delinquenz-Items

\begin{tabular}{|c|c|c|c|c|}
\hline Hast Du selbst in den letzten 12 Monaten folgendes getan? & keinmal & einmal & 2-4 mal & $\begin{array}{c}\text { über } 4 \\
\text { mal }\end{array}$ \\
\hline V294: Dich mit anderen gerauft, geschlagen. & 67,2 & 17,4 & 9,3 & 6,1 \\
\hline $\begin{array}{l}\text { V295: Eine Schlägerei mitgemacht und dabei jemanden } \\
\text { zusammengeschlagen oder arg zugerichtet. }\end{array}$ & 86,7 & 8,4 & 3,2 & 1,7 \\
\hline $\begin{array}{l}\text { V296: Einer Bande (Gruppe, Club, Clique) angehört, in der } \\
\text { manche unerlaubten "Dingen gedreht" werden. }\end{array}$ & 78,2 & 11,3 & 6,1 & 4,4 \\
\hline $\begin{array}{l}\text { V297: Anderen Gegenstände oder Geld im Wert von mehr } \\
\text { als } 10 \text { DM entwendet. }\end{array}$ & 83,9 & 7,6 & 3,9 & 4,6 \\
\hline $\begin{array}{l}\text { V298: Irgendwo eingebrochen (z.B. in ein Gebäude, ein Auto } \\
\text { usw.), um etwas zu stehlen. }\end{array}$ & 92,6 & 4,1 & 2,1 & 1,3 \\
\hline V299: Einen Automaten aufgebrochen oder "gefilzt". & 92,2 & 5,8 & 1,4 & ,7 \\
\hline $\begin{array}{l}\text { V300: Fremdes (auch öffentliches) Eigentum mit Absicht } \\
\text { zerstört oder erheblich beschädigt. }\end{array}$ & 81,6 & 11,8 & 3,9 & 2,8 \\
\hline
\end{tabular}

Abb. 4-4: Mittelwerte der Delinquenz-Items in den fünf Clustern zum Gewaltstatus

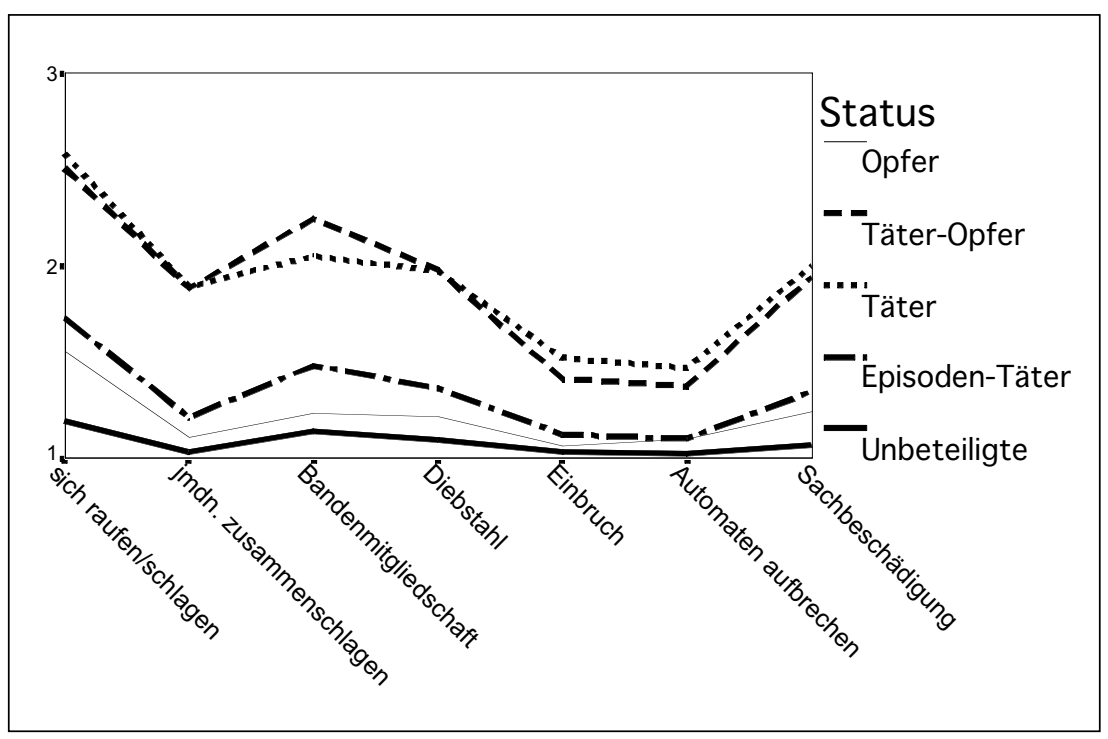


In Sachsen wurden die Schüler auch nach dem Konsum von Alkohol, Zigaretten und Drogen in der Schule und auf dem Schulweg während der letzten 12 Monate gefragt. Mehrmals im Monat oder öfter konsumieren 11,2\% der Befragten Alkohol, 22,5\% Zigaretten und 1,6\% Drogen. Fast täglich ist dies bei 2,7\%, 16,2\% bzw. 0,8\% der Befragten der Fall. Es gibt positive Korrelationen zwischen den drei Items $\left(r_{1-2}=.51 ; r_{1-3}=.33\right.$ und $\left.r_{2-3}=.27\right)$. Tabelle 4-4 zeigt die Mittelwerte dieser drei Items in den fünf Clustern des Gewaltstatus.

Tab. 4-4: Mittelwerte von Alkohol-, Zigaretten- und Drogenkonsum in den fünf Clustern des Gewaltstatus

\begin{tabular}{|c|c|c|c|c|c|c|}
\hline Item-Nr.:* & $\begin{array}{l}\text { a. }{ }^{* *} \\
\text { Opfer }\end{array}$ & $\begin{array}{c}b . \\
\text { Täter-Opfer }\end{array}$ & $\begin{array}{c}\text { C. } \\
\text { Täter }\end{array}$ & $\begin{array}{c}d . \\
\text { Episoden- } \\
\text { Täter }\end{array}$ & $\begin{array}{c}\text { e. } \\
\text { Unbeteiligte }\end{array}$ & $\begin{array}{c}* * * \\
\eta^{2}\end{array}$ \\
\hline $279 a$ & $1,54 \mathrm{bcd}$ & 2,89ade & 3,25ade & 2,06abce & $1,34 \mathrm{bcd}$ & .22 \\
\hline $279 b$ & $1,89 \mathrm{bcd}$ & 3,62ade & 3,90ade & 2,78abce & $1,67 \mathrm{bcd}$ & .15 \\
\hline $279 c$ & $1,10 b c$ & 1,79acde & 1,47abde & $1,12 \mathrm{bce}$ & $1,03 \mathrm{bcd}$ & .08 \\
\hline
\end{tabular}

* Wie oft hast Du selbst an Deiner Schule oder auf dem Schulweg in den letzten 12 Monaten folgendes gemacht? (Skala: $1=$ nie ... 6 = fast täglich)

V279a. Alkohol getrunken.

V279b. Zigaretten geraucht.

V279c. Drogen genommen (z.B. Haschisch).

* Anhand der Buchstaben $a, b, c, d$ und e ist bei jedem Mittelwert zu erkennen, von welchen anderen vier Mittelwerten er sich signifikant unterscheidet $(p<.05)$.

*** Anteil der erklärten Varianz

Die niedrigsten Werte in allen drei Fällen sind bei den Unbeteiligten zu beobachten, gefolgt von den Opfern, wobei in keinem Fall der Unterschied zwischen den Unbeteiligten und den Opfern statistisch signifikant ist. Die höchsten Werte weisen die Täter und Täter-Opfer auf. Sie unterscheiden sich in allen drei Fällen signifikant von den anderen drei Gruppen. Der Unterschied zwischen Tätern und Täter-Opfern ist nur in einem Fall signifikant: die TäterOpfer nehmen mehr Drogen als die Täter. Die relativ hohen $\eta^{2}$-Werte zeigen, daß es zwischen dem Gewaltstatus und dem Konsum von Alkohol, Zigaretten und Drogen einen starken Zusammenhang gibt. Somit kann man sagen, daß bei den Gewalttätern eine höhere Wahrscheinlichkeit besteht, in der einen oder anderen Form abhängig zu werden. 


\subsection{Täter-Opfer-Typologie: Charakterisierung der einzelnen Schülergruppen}

Nachdem die fünf typischen Schülergruppen hinsichtlich der Beteiligung an Gewalt identifiziert worden sind, soll nun der Frage nachgegangen werden, welche Spezifika jede Gruppe aufweist und wodurch sich diese Gruppen voneinander unterscheiden. Dabei konzentrieren wir uns in diesem Kapitel auf die individuellen Merkmale, die schulbezogenen Einstellungen und Leistungsmerkmale. Einflußfaktoren aus den Bereichen Familie, Medien und Peer-Gruppe werden in Kapitel 5 behandelt.

\section{Individuelle Merkmale}

\section{Geschlecht, Jahrgang und Schulform:}

Wir hatten bereits festgestellt, daß sich die Jungen erheblich mehr als die Mädchen an der Gewalt beteiligen (vgl. Kap. 2 und 3). Tabelle 4-5 zeigt diesen Unterschied noch einmal. Während 69,4\% aller Mädchen zur Gruppe der Unbeteiligten gehören, befinden sich nur $41,4 \%$ der Jungen in dieser Gruppe. In allen beteiligten Gruppen sind die Jungen stärker als die Mädchen vertreten, insbesondere bei den Tätern (13,7 vs. 2,6\%) und Episoden-Tätern (31,9 vs. 20,2\%). Bei den Opfern und Täter-Opfern sind die Geschlechterunterschiede aber etwas kleiner (8,3 vs. $5,7 \%$ bzw. 4,6 vs. $2,2 \%) .{ }^{8}$ Eine mögliche Erklärung für diese Disproportionalität ist die, daß ein Teil der Aggressionen zwischen den Geschlechtern stattfindet und dabei in den meisten Fällen die Jungen als Täter und die Mädchen als Opfer auftreten. Eine weitere mögliche Erklärung ist, daß die schon erwähnte Übertreibung bei den Täterangaben und/oder Untertreibung bei den Opferangaben eher für die Jungen gilt als für die Mädchen. Möglicherweise wegen ihrer im Durchschnitt stärkeren Sensibilität vergessen oder verdrängen die Mädchen ihre unangenehmen Opfererfahrungen nicht so schnell wie die Jungen. Diese möglichen Erklärungen schließen sich gegenseitig nicht aus.

Tabelle 4-5 zeigt auch, daß Täter am meisten im 8. Jahrgang vertreten sind $(10,8 \%)$ und am wenigsten im 6. Jahrgang (4,1\%). Ihr Anteil im 9. bzw. 10. Jahrgang unterscheidet sich mit 7,9\% kaum vom Gesamtdurchschnitt. In den höheren Jahrgängen kommen die Episoden-Täter am relativ stärksten vor $(31,0 \%)$, gefolgt von den Schülern des 8 . Jahrgangs $(28,7 \%)$; Sechstkläßler sind (mit 17,3\%) auch hier unterrepräsentiert. Der Anteil der Opfer nimmt mit zunehmender Klassenstufe fast linear ab. Im Jahrgang 6 befinden sich mehr als doppelt so viel Opfer wie in den Jahrgängen 9 und 10 (9,9\% vs. 4,4\%).

8 Ähnliche Ergebnisse sind auch in der hessischen Stichprobe festgestellt worden. 
Tab. 4-5: Verteilung von Geschlecht, Jahrgang und Schulform in den fünf Clustern des Gewaltstatus*

\begin{tabular}{|c|c|c|c|c|c|c|c|c|}
\hline \multirow{2}{*}{ Status } & \multicolumn{2}{|c|}{ Geschlecht } & \multicolumn{3}{|c|}{ Jahrgang } & \multicolumn{3}{|c|}{ Schulform } \\
\hline & Junge & $\begin{array}{l}\text { Mäd- } \\
\text { chen }\end{array}$ & KI. 6 & KI. 8 & KI. 9/10 & $\begin{array}{l}\text { Gymna- } \\
\text { sium }\end{array}$ & $\begin{array}{l}\text { Mittel- } \\
\text { schule }\end{array}$ & $\begin{array}{l}\text { Förder- } \\
\text { schule }\end{array}$ \\
\hline \multirow[t]{2}{*}{ Opfer } & 124 & 92 & 98 & 75 & 44 & 83 & 113 & 21 \\
\hline & $8,3 \%$ & $5,7 \%$ & $9,9 \%$ & $6,8 \%$ & $4,4 \%$ & $6,6 \%$ & $6,8 \%$ & $11,5 \%$ \\
\hline Täter- & 69 & 35 & 41 & 49 & 14 & 22 & 61 & 21 \\
\hline Opfer & $4.6 \%$ & $2,2 \%$ & $4,1 \%$ & $4,5 \%$ & $1,4 \%$ & $1,7 \%$ & $3,7 \%$ & $11,5 \%$ \\
\hline \multirow[t]{2}{*}{ Täter } & 204 & 41 & 47 & 119 & 80 & 65 & 167 & 14 \\
\hline & $13,7 \%$ & $2.6 \%$ & $4,8 \%$ & $10,8 \%$ & $7,9 \%$ & $5,2 \%$ & $10,1 \%$ & $7,7 \%$ \\
\hline Episo.- & 475 & 323 & 171 & 315 & 313 & 308 & 450 & 41 \\
\hline Täter & $31,9 \%$ & $20,2 \%$ & $17,3 \%$ & $28,7 \%$ & $31,0 \%$ & $24,4 \%$ & $27,2 \%$ & $22,5 \%$ \\
\hline Unbe- & 616 & 1111 & 631 & 539 & 558 & 782 & 861 & 85 \\
\hline \multirow[t]{2}{*}{ teiligte } & $41,4 \%$ & $69,4 \%$ & $63,9 \%$ & $49,1 \%$ & $55,3 \%$ & $62,1 \%$ & $52,1 \%$ & $46,7 \%$ \\
\hline & 1488 & 1602 & 988 & 1097 & 1009 & 1260 & 1652 & 182 \\
\hline Gesamt & $48,2 \%$ & $51,8 \%$ & $31,9 \%$ & $49,1 \%$ & $32,6 \%$ & $40,7 \%$ & $53,4 \%$ & $5,9 \%$ \\
\hline
\end{tabular}

* Prozentangaben sind Spaltenprozente, mit Ausnahme der letzten Zeile. in allen Fällen: $p<.00000$

Der Status als Täter-Opfer ist fast ausschließlich in den Jahrgängen 6 und $8 \mathrm{zu}$ beobachten. In den Klassenstufen 9 und 10 ist der Anteil dieser Gruppe (mit $1,4 \%)$ sehr gering. Das bedeutet, daß sich mit zunehmendem Alter die Rollen eindeutiger herausbilden. Tabelle 4-5 zeigt, daß der Anteil der Unbeteiligten in der Klassenstufe 6 am größten $(63,9 \%)$ und in der Klassenstufe 8 am kleinsten $(49,1 \%)$ ist. Das zeigt, daß sich innerhalb der untersuchten Altersspanne der Übergang von Klasse 6 zu Klasse 8 für viele Schüler hinsichtlich der Verwicklung in schulische Gewalt kritisch gestaltet. In Kapitel 2 wurde jedoch darauf verwiesen, daß sich für die einzelnen Gewaltformen ein differenzierteres Bild ergibt, das von diesem generellen Verlaufsmodell abweicht.

Wie Tabelle 4-5 zeigt, sind die Gymnasien mit 62,1\% Unbeteiligten am wenigsten belastet und die Förderschulen mit nur 46,7\% am meisten. Der relativ hohe Anteil der Opfer und Täter-Opfer in den Förderschulen im Vergleich zu den anderen Schulformen ist bemerkenswert, obwohl der Anteil der Täter und Episoden-Täter in dieser Schulform insgesamt $(7,7+22,5 \%)$ fast genauso groß ist wie in den Gymnasien $(5,2+24,4 \%)$ und kleiner als in den Mittelschulen $(10,1+27,2 \%)$. Es ist unwahrscheinlich, daß die Täter in den Förderschulen besonders aggressiv sind und ihnen deshalb mehr Schüler zum Opfer 
fallen. Vielmehr ist anzunehmen, daß die Übertreibung bei den Täter- und/ oder Untertreibung bei den Opferangaben mehr in den beiden anderen Schulformen (vor allem in Mittelschulen) und weniger in Förderschulen stattfindet (vermutlich verbunden mit stärkerer Empfindlichkeit und geringerem Stolz).

\section{Körpergröße:}

Der Zusammenhang zwischen der körperlichen Stärke und Gewalttätigkeit wird bereits von Olweus (1995) betont. Er schreibt: „Insgesamt kann der typische Gewalttäter als einer oder eine beschrieben werden, der oder die ein aggressives Reaktionsmuster (bei Jungen) mit körperlicher Stärke verbindet.“ Ein passives Opfer (d.h. ein Opfer, das sich unsicher und wertlos fühlt und nicht zurückschlägt) wird von ihm charakterisiert ,durch ein ängstliches und zurückgezogenes Reaktionsmuster in Verbindung (im Falle von Jungen) mit körperlicher Schwäche." Wir verfügen zwar über keine direkten Informationen bezüglich der Körperstärke der Befragten. Wir haben aber in Sachsen in den meisten Schulen die befragten Schüler aufgefordert, ihre Körpergröße anzugeben. Wir gehen hier davon aus, daß die Körpergröße stark mit der Körperstärke korreliert und als ein indirekter Indikator für diese benutzt werden kann. Die konkrete Frage, die hier beantwortet werden soll, lautet: Gibt es einen Zusammenhang zwischen der Körpergröße der Schüler und ihrem Gewaltstatus? Wir vermuten, daß die Täter überdurchschnittlich groß und die Opfer überdurchschnittlich klein sind. Wie Tabelle 4-6 zeigt, bestätigen die Daten diese Vermutung. Die Täter sind insgesamt mit einem Durchschnitt von $169,3 \mathrm{~cm}$ am größten, gefolgt von den Episoden-Tätern mit 167,9cm. Die TäterOpfer nehmen mit 167,9cm eine Mittelposition ein, gefolgt von den Unbeteiligten mit 163,6cm. Am kleinsten sind erwartungsgemäß die Opfer mit 162,2cm. Die Differenzen zwischen Tätern und Episoden-Tätern einerseits und Opfern und Unbeteiligten andererseits sind statistisch signifikant (Scheffé-Test). Diese Zahlen müssen aber etwas relativiert werden, weil die Körpergröße der Schüler mit ihrem Alter (bzw. Klassenstufe) und ihrem Geschlecht zusammenhängt. ${ }^{9}$ Es ist deshalb möglich, daß der beobachtete Zusammenhang zwischen der Körpergröße und dem Status nur den Zusammenhang zwischen Alter und Geschlecht einerseits und dem Status andererseits widerspiegelt. Deshalb haben wir diesen Mittelwertvergleich für die Mädchen und Jungen getrennt durchgeführt. Bei den Jungen ist die geschilderte Struktur noch deutlicher zu beobachten. Bei den Mädchen ist dies nicht der Fall. Die Opfer sind zwar auch hier am kleinsten. Sie unterscheiden sich aber nur von den Episoden-Täterinnen signifikant, weil die Unterschiede unter den Mädchen insgesamt kleiner sind als bei den Jungen (vgl. Tab. 4-6). Die Unterschiede zwischen den anderen drei beteiligten Gruppen sind minimal und nicht signifikant.

9 In der Stichprobe sind die Jungen im Schnitt 4,5cm größer als die Mädchen; die Korrelation zwischen Alter und Größe beträgt $\mathrm{r}=.64$. 
Tab. 4-6: Mittelwert der Körpergröße (in $\mathrm{cm}$ ) in den fünf Clustern des Gewaltstatus

\begin{tabular}{lcccccc}
\hline & $\begin{array}{c}a .{ }^{*} \\
\text { Opfer }\end{array}$ & $\begin{array}{c}b . \\
\text { Täter-Opfer }\end{array}$ & $\begin{array}{c}c . \\
\text { Täter }\end{array}$ & $\begin{array}{c}d . \\
\text { Episoden- } \\
\text { Täter }\end{array}$ & $\begin{array}{c}\text { U. } \\
\text { Unbeteiligte }\end{array}$ & ${ }^{* *}$ \\
Alle & $162,2 \mathrm{~cd}$ & 165,6 & $169,3 \mathrm{ae}$ & $167,9 \mathrm{ae}$ & $163,6 \mathrm{~cd}$ & .05 \\
Mädchen & $161,7 \mathrm{~d}$ & 164,1 & 164,5 & $164,9 \mathrm{ae}$ & $162,4 \mathrm{~d}$ & .02 \\
Junge & $162,6 \mathrm{~cd}$ & 166,3 & $170,5 \mathrm{ae}$ & $170,1 \mathrm{ae}$ & $165,6 \mathrm{~cd}$ & .05 \\
\hline
\end{tabular}

* Anhand der Buchstaben $a, b, c, d$ und e ist bei jedem Mittelwert zu erkennen, von welchen anderen vier Mittelwerten er sich signifikant unterscheidet $(p<.05)$.

* Anteil der erklärten Varianz

Um zu prüfen, ob es unabhängig vom Alter einen Zusammenhang zwischen Körpergröße und Gewaltstatus gibt, wurden für Jungen und Mädchen zwei getrennte multiple Regressionsanalysen durchgeführt. ${ }^{10}$ Für Jungen haben wir festgestellt, daß - altersunabhängig und im Vergleich zu den Unbeteiligten) - die Täter im Schnitt 2,8cm, die Episoden-Täter $1,7 \mathrm{~cm}$ und die Täter-Opfer $0,8 \mathrm{~cm}$ größer und die Opfer 0,6cm kleiner sind. Bei den Mädchen sind die Täter-Opfer im Schnitt 2,6cm, die Episoden-Täterinnen 1,4cm und die Täterinnen $1,1 \mathrm{~cm}$ größer als die Unbeteiligten; zwischen Unbeteiligten und Opfern gibt es keinen Größenunterschied. Natürlich können Körpergröße oder Körperstärke nicht als Ursache für Gewalttätigkeit betrachtet werden. Vielmehr gehen wir davon aus, daß das durch andere Faktoren erzeugte Aggressionspotential eher bei den stärkeren bzw. größeren Schülern in konkrete Gewaltaktionen einmündet als bei schwächeren bzw. kleineren.

\section{Gewaltbilligung:}

Die Beteiligung an Gewalt und die Rollenverteilung sind nicht unabhängig von der Einstellung zur Gewalt. Zum einen werden die Hemmschwellen bezüglich der Gewaltanwendung bei einer positiven Einstellung zur Gewalt schneller überwunden und zum anderen dient eine solche Einstellung als eine Rechtfertigung für die schon ausgeübte Gewalt. Um den Zusammenhang zwischen Einstellung und Verhalten bezüglich der Gewalt näher überprüfen zu können,

10 Die abhängige Variable in diesen Regressionen ist die Körpergröße, die durch Alter und Status als erklärende Variable vorhergesagt wird. Da der Status eine kategoriale Variable mit fünf Ausprägungen ist, wird er durch vier Dummy-Variablen repräsentiert, die jeweils einer der beteiligten Gruppen zuzuordnen sind. Jede Dummy-Variable hat den Wert 1, falls der Befragte zur entsprechenden beteiligten Gruppe gehört, sonst den Wert 0. Die Gruppe der Unbeteiligten stellt somit die Referenzkategorie dar. 
haben wir auch die allgemeine Einstellung der Befragten über die Gewalt erhoben. Die Befragten haben anhand von sieben Items angegeben, wie sie dazu stehen (vgl. Legende zu Tab. 4-7). Man könnte erwarten, daß die Täter die Gewalt am ehesten als normal, natürlich, notwendig und gerechtfertigt einschätzen (weil sie für ihre Taten diese Rechtfertigung brauchen) und die Opfer am wenigsten (weil sie am stärksten darunter leiden). Um dies zu überprüfen, haben wir einen Mittelwertvergleich durchgeführt: Tabelle 4-7 zeigt die Mittelwerte der Items für Gewaltbilligung in den fünf Clustern des Gewaltstatus. Erwartungsgemäß sind die Mittelwerte aller sieben Items bei den Tätern am höchsten, gefolgt von den Täter-Opfern, wobei der Unterschied zwischen diesen beiden Gruppen nur in einem Fall signifikant ist.

Tab. 4-7: Mittelwerte der Items der Gewaltbilligung in den fünf Clustern des Gewaltstatus

\begin{tabular}{|c|c|c|c|c|c|c|}
\hline Item-Nr. & $\begin{array}{l}\text { a. }{ }^{*} \\
\text { Opfer }\end{array}$ & $\begin{array}{c}b . \\
\text { Täter-Opfer }\end{array}$ & $\begin{array}{c}c . \\
\text { Täter }\end{array}$ & $\begin{array}{c}d . \\
\text { Episoden-Täter }\end{array}$ & $\begin{array}{c}e . \\
\text { Unbeteiligte }\end{array}$ & $\eta^{*}$ \\
\hline V186 & $2,49 \mathrm{bcde}$ & $3,24 \mathrm{ad}$ & 3,65ade & 2,79abce & $2,04 a b c d$ & .18 \\
\hline $186 a$ & $1,54 \mathrm{bcde}$ & 2,67 acde & 3,02 abde & 1,84abce & $1,25 \mathrm{abcd}$ & .27 \\
\hline $186 \mathrm{~b}$ & $2,30 \mathrm{bce}$ & 3,16ade & 3,48ade & $2,52 \mathrm{bce}$ & $1,78 a b c d$ & .23 \\
\hline V187 & $2,10 \mathrm{bce}$ & 3,04 ade & 3,34ade & $2,35 \mathrm{bce}$ & $1,73 a b c d$ & .18 \\
\hline V188 & $2,35 \mathrm{bce}$ & 3,16 ade & 3,51ade & $2,56 \mathrm{bce}$ & $1,89 a b c d$ & .17 \\
\hline V189 & $2,47 \mathrm{bcde}$ & 3,34ade & 3,61ade & 2,78abce & $2,17 \mathrm{abcd}$ & .13 \\
\hline V190 & $2,48 \mathrm{bce}$ & 3,35ade & 3,67ade & $2,76 \mathrm{bce}$ & $2,20 \mathrm{abcd}$ & .12 \\
\hline
\end{tabular}

* Anhand der Buchstaben a, b, c, d und e ist bei jedem Mittelwert zu erkennen, von welchen anderen vier Mittelwerten er sich signifikant unterscheidet $(p<.05)$.

** Anteil der erklärten Varianz

V186: Gewalt gehört selbstverständlich zur menschlichen Natur.

V186a: Gewalt ist geil.

V186b: Um seine Interessen durchzusetzen, muß man manchmal Gewalt anwenden.

V187: Der Stärkere muß sich durchsetzen, sonst gibt es keinen Fortschritt.

V188: Gewalt ist etwas völlig normales, weil sie überall vorkommt.

V189: Es ist völlig normal, wenn Männer sich im körperlichen Kampf mit anderen selbst beweisen wollen.

V190: Auge um Auge, Zahn um Zahn, so ist nun einmal das Leben.

Skala: 1 = stimmt gar nicht $\ldots 5$ = stimmt ganz genau 
Interessant ist weiterhin, daß - entgegen unserer Vermutung - nicht bei den Opfern die höchste Ablehnung von Gewalt zu verzeichnen ist, sondern bei der Gruppe der Unbeteiligten. Bei allen sieben Items haben die Opfer signifikant höhere Werte als die Unbeteiligten. ${ }^{11}$ Das zeigt, daß die Einstellung der Opfer zur Gewalt nicht unbedingt das Ergebnis ihrer negativen Erlebnisse ist (sonst hätten wir hier bei ihnen eine stärkere Ablehnung der Gewalt feststellen können). Außerdem ist die partielle Diffusion von Täter- und Opferstatus einschließlich eines möglichen Rollentausches ein Grund dafür, daß Täter und Opfer als in Gewalt Involvierte diese Handlungen eher legitimieren als die Unbeteiligten.

\section{Aggressionsbereitschaft:}

Wir wollen nun überprüfen, inwieweit die von den Befragten selbst eingeschätzte Aggressionsbereitschaft mit ihrem Gewaltstatus in Zusammenhang steht. Es wird erwartet, daß die Aggressionsbereitschaft unter den Unbeteiligten am geringsten und unter den Tätern und Täter-Opfern am stärksten ist. Fünf Items (die auch zusammen einen Faktor bilden) dienen hier als Indikatoren der Aggressionsbereitschaft (vgl. Legende zu Abb. 4-5). Wie Abbildung 4-5 zeigt, weisen die Unbeteiligten erwartungsgemäß bei allen fünf Indikatoren die niedrigsten Werte auf. Bei den ersten drei Indikatoren stehen die Täter und Täter-Opfer an der Spitze der Aggressionsbereitschaft, während die Opfer und Episoden-Täter gemeinsam eine Mittelposition einnehmen. Bei den letzten zwei Indikatoren übertreffen die Opfer sogar die Täter und TäterOpfer. Das ist dadurch zu erklären, daß die letzten zwei Items nicht nur die Aggressionsbereitschaft, sondern auch die subjektive Betroffenheit beinhalten, die offensichtlich bei den Opfern ausgeprägter ist.

Eine ähnliche Struktur wurde auch in der hessischen Stichprobe beobachtet. Insgesamt ist jedoch in Sachsen eine größere Aggressionsbereitschaft festzustellen. Ihre fünf Indikatoren weisen in der sächsischen Stichprobe im Gesamtdurchschnitt deutlich höhere Werte auf als in der hessischen. Die Mittelwertdifferenzen sind in vier von fünf Fällen statistisch signifikant (Ausnahme: V128). Der Zusammenhang zwischen der Aggressionsbereitschaft und dem Status ist aber insgesamt in Hessen stärker als in Sachsen (Ausnahme: V105).

11 Ähnliche Ergebnisse wurden auch in der hessischen Stichprobe beobachtet. 
Abb. 4-5: Mittelwerte der Items der Aggressionsbereitschaft in den fünf Clustern des Gewaltstatus

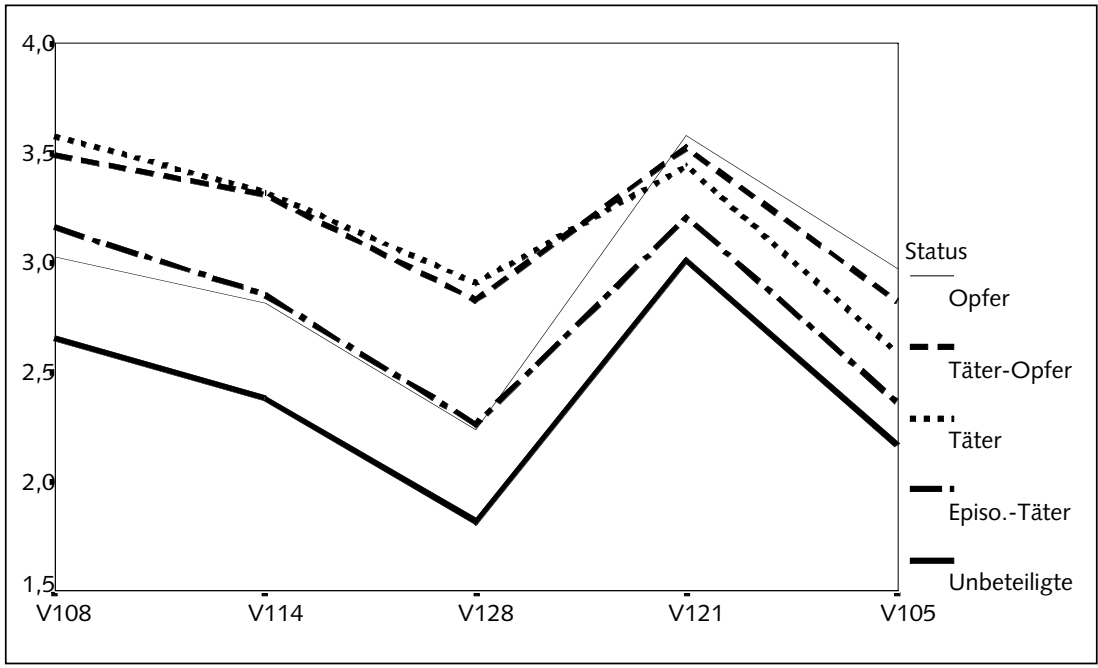

V108: Ich steigere mich leicht in einen Streit hinein.

V114: Ich gerate schnell in Wut.

V128: Bei Streit mit anderen raste ich richtig aus und habe mich nicht mehr unter Kontrolle.

V121: Es bringt mich zum Kochen, wenn andere sich über mich lustig machen.

V105: Ich fühle mich leicht angegriffen.

Skala: 1 = stimmt gar nicht $\ldots .5=$ stimmt ganz genau

\section{Nationalismus/Fremdenfeindlichkeit:}

Die Aggression gegen die Mitschüler steht auch im Zusammenhang mit der Aggression gegen Fremde bzw. mit Nationalismus. Anhand von fünf Items, die als Indikatoren für Nationalismus/Fremdenfeindlichkeit betrachtet werden (vgl. Legende zu Tab. 4-8), wird nun dieser Zusammenhang überprüft. Dafür vergleichen wir wieder die Mittelwerte dieser fünf Items in den fünf Clustern des Status. Die Ergebnisse in Tabelle 4-8 zeigen, daß bei allen fünf Items die höchsten Werte bei Tätern, gefolgt von Täter-Opfern, und die niedrigsten Werte bei Unbeteiligten, gefolgt von Opfern, zu beobachten sind.

Auch für Hessen wurde der Zusammenhang zwischen Nationalismus/ Fremdenfeindlichkeit und Gewalttätigkeit überprüft. Der Zusammenhang ist dort etwas schwächer als in Sachsen. Die Täter-Opfer weisen in Hessen einen signifikant stärkeren Nationalismus als die Täter auf, während es in Sachsen eher umgekehrt ist (nicht signifikant). In Sachsen weisen alle Items für Nationalismus und Fremdenfeindlichkeit hochsignifikant höhere Mittelwerte auf als in Hessen (das letzte Item wurde in Hessen nicht erfragt). 


\section{Autoritarismus:}

Tabelle 4-8 zeigt, daß bei Autoritarismus eine andere Struktur als bei Nationalismus zu beobachten ist. Hier weisen die Opfer, gefolgt von den Unbeteiligten, die höchsten Mittelwerte auf. Vermutlich erhoffen sich die Opfer von Ruhe, Ordnung und Disziplin mehr soziale Kontrolle über die Gewalttäter.

Tab. 4-8: Mittelwerte der Items für Nationalismus/Fremdenfeindlichkeit und Autoritarismus in den fünf Clustern des Gewaltstatus

\begin{tabular}{|c|c|c|c|c|c|c|}
\hline Item-Nr. & $\begin{array}{l}\text { a. }{ }^{*} \\
\text { Opfer }\end{array}$ & $\begin{array}{c}b . \\
\text { Täter-Opfer }\end{array}$ & $\begin{array}{c}c . \\
\text { Täter }\end{array}$ & $\begin{array}{c}d . \\
\text { Episoden-Täter }\end{array}$ & $\begin{array}{c}\text { e. } \\
\text { Unbeteiligte }\end{array}$ & $\begin{array}{l}* * \\
\eta^{2}\end{array}$ \\
\hline \multicolumn{7}{|c|}{ I. Nationalismus/Fremdenfeindlichkeit } \\
\hline V302 & $2,18 b c$ & 2,85ade & 2,97ade & $2,27 \mathrm{bce}$ & $1,99 \mathrm{bcd}$ & .06 \\
\hline V303 & $2,31 b c$ & $3,02 \mathrm{ae}$ & 3,21 ade & $2,58 \mathrm{bce}$ & $2,19 \mathrm{bcd}$ & .07 \\
\hline V306 & $2,71 \mathrm{c}$ & $3,31 \mathrm{e}$ & 3,48 ade & $2,79 \mathrm{ce}$ & $2,36 \mathrm{bcd}$ & .07 \\
\hline V307 & $1,97 \mathrm{bcd}$ & 2,98ade & 3,21 ade & 2,41abce & $1,81 \mathrm{bcd}$ & .14 \\
\hline $307 a$ & $3,14 \mathrm{c}$ & 3,60 & 3,94ade & $3,56 \mathrm{ce}$ & $3,15 c d$ & .04 \\
\hline \multicolumn{7}{|c|}{ II. Autoritarismus } \\
\hline V301 & $3,62 c$ & 3,34 & 3,12 ade & $3,38 c e$ & $3,57 \mathrm{~cd}$ & .02 \\
\hline V302 & $3,08 \mathrm{~cd}$ & 2,82 & $2,62 a$ & $2,59 \mathrm{ae}$ & $2,83 d$ & .01 \\
\hline
\end{tabular}

* Anhand der Buchstaben a, b, c, d und e ist bei jedem Mittelwert zu erkennen, von welchen anderen vier Mittelwerten er sich signifikant unterscheidet $(p<.05)$.

** Anteil der erklärten Varianz

V302: Unser Volk sollte wieder einen Führer haben, der mit starker Hand regiert.

V303: Die Deutschen waren schon immer die Größten in der Geschichte.

V306: Wer sich in Deutschland nicht anpassen kann, soll das Land am besten verlassen.

V307: Ich bin durchaus bereit, Fremde schlechter zu behandeln, damit sie wissen, wo hier ihre Grenzen sind.

V307a: Nur für die Deutschen: Ich bin stolz, ein/e Deutsche/r zu sein.

V301: Das wichtigste in der heutigen Zeit ist die Aufrechterhaltung von Ruhe und Ordnung.

V304: Es ist höchste Zeit, daß die Jugend wieder Disziplin lernt.

Skala: 1 = stimmt gar nicht $\ldots 5$ = stimmt ganz genau

\section{Außenseiterstatus:}

In Melzer/Rostampour (1996) hatten wir anhand einer anderen sächsischen Stichprobe festgestellt, daß Außenseiter- und Opferstatus eng miteinander verbunden sind. Unter allen überprüften erklärenden Variablen wies der Außenseiterstatus den stärksten Zusammenhang mit dem Opferstatus auf. Ähnliche Ergebnisse wurden auch in den anderen Studien berichtet. Die Ergebnisse der 
vorliegenden Stichprobe bestätigen noch einmal diesen engen Zusammenhang. Als Indikatoren für den Außenseiterstatus existieren drei Items (vgl. Legende zu Tab. 4-9), die auch gemeinsam einen Faktor bilden. Die Mittelwerte dieser drei Indikatoren bei den Opfern sind größer als in allen anderen Gruppen, gefolgt von den Täter-Opfern (vgl. Tab. 4-9). Der Unterschied zwischen den Opfern einerseits und den Tätern, Episoden-Tätern und Unbeteiligten andererseits ist in allen Fällen statistisch signifikant. In der hessischen Stichprobe weisen die Opfer und Täter-Opfer gemeinsam die Spitzenwerte bezüglich des Außenseiterstatus auf. Die Unbeteiligten sind in beiden Stichproben am wenigsten isoliert, gefolgt von den Episoden-Tätern und Tätern, wobei der Unterschied zwischen den Unbeteiligten und den Episoden-Tätern in beiden Stichproben und der Unterschied zwischen den Unbeteiligten und den Tätern in Sachsen nicht signifikant sind. Der Zusammenhang zwischen dem Außenseiterstatus und dem Status bezüglich der schulischen Gewalt ist in Hessen etwas stärker als in Sachsen.

Tab. 4-9: Mittelwerte der Items für die Außenseiterrolle in den fünf Clustern des Gewaltstatus

\begin{tabular}{lcccccc}
\hline Item-Nr. & $\begin{array}{c}a{ }^{*} \\
\text { Opfer }\end{array}$ & $\begin{array}{c}\text { Täter-Opfer } \\
\text { V106 }\end{array}$ & $\begin{array}{c}c . \\
\text { Täter }\end{array}$ & $\begin{array}{c}d . \\
\text { Episoden-Täter }\end{array}$ & $\begin{array}{c}\text { U. } \\
\text { Unbeteiligte }\end{array}$ & $\eta^{*}$ \\
V119 & $2,53 \mathrm{cde}$ & $2,20 \mathrm{cde}$ & $1,72 \mathrm{ab}$ & $1,66 \mathrm{ab}$ & $1,59 \mathrm{ab}$ & .07 \\
V126 & $3,02 \mathrm{cde}$ & $2,98 \mathrm{cde}$ & $2,40 \mathrm{ab}$ & $2,33 \mathrm{ab}$ & $2,20 \mathrm{ab}$ & .04 \\
\hline
\end{tabular}

* Anhand der Buchstaben a, b, c, d und e ist bei jedem Mittelwert zu erkennen, von welchen anderen vier Mittelwerten er sich signifikant unterscheidet $(p<.05)$.

* Anteil der erklärten Varianz

V106: Ich habe das Gefühl, Lehrer/innen und andere Schüler behandeln mich wie einen Außenseiter.

V119: Ich glaube, niemand versteht mich so recht.

V126: Ich habe das Gefühl, viele in der Schule meiden den Kontakt mit mir.

Skala: 1 = stimmt gar nicht $\ldots .5$ = stimmt ganz genau

\section{Selbstwertgefühl:}

Wie der Außenseiterstatus ist ein negativeres Selbstwertgefühl für die Opfer bezeichnend (vgl. Melzer/Rostampour 1996). Für das Selbstwertgefühl verwenden wir neun Indikatoren (vgl. Legende zu Abb. 4-6). Die ersten fünf Items beinhalten ein positives Selbstwertgefühl, die letzten vier ein negatives. Abbildung 4-6 zeigt die Mittelwerte dieser Indikatoren in der sächsischen Stichprobe. Bei den Items mit positiven Inhalten weisen die Opfer die niedrigsten und die Täter die höchsten Mittelwerte auf. Die Unterschiede zwischen 
den Gruppen sind aber nur in einigen Fällen signifikant: die Opfer sind mit ihrem Aussehen weniger zufrieden als die Täter und Unbeteiligten (V92a), die Täter sind mehr als die Opfer, Episoden-Täter und Unbeteiligten stolz auf sich (V103) und die Opfer halten sich weniger für erfolgreich als die Täter (V124). Die Unterschiede zwischen den Gruppen kommen aber mit den letzten vier (negativen) Items besser zum Vorschein. Die Opfer und Täter-Opfer weisen hier deutlich höhere Mittelwerte (also mehr negatives Selbstwertgefühl) auf als die anderen. Negatives Selbstwertgefühl ist am wenigsten bei den Unbeteiligten und Episoden-Täter festzustellen.

Abb. 4-6: Mittelwerte der Items für das Selbstwertgefühl in den fünf Clustern des Gewaltstatus

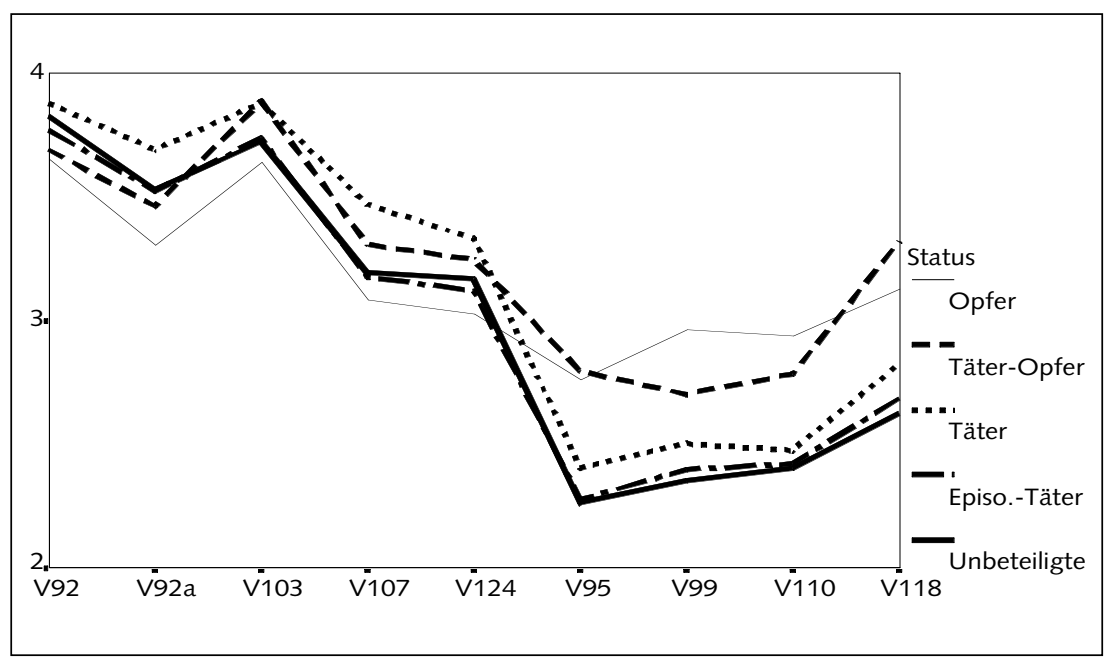

V92: Im großen und ganzen bin ich mit mir zufrieden.

V92a: Ich bin mit meinem Aussehen zufrieden. (Nur in Sachsen erfragt)

V103: Ich glaube, ich habe eine Reihe guter Eigenschaften.

V107: Ich habe Grund, auf mich stolz zu sein.

V124: Im großen und ganzen halte ich mich für erfolgreich.

V95: Ich wünsche mir oft, eine andere/ein anderer zu sein.

V99: Ich fühle mich oft unglücklich.

V110: Manchmal komme ich mir wirklich nutzlos vor.

V118: Ich wollte, ich könnte von mir eine bessere Meinung haben.

Skala: 1 = stimmt gar nicht $\ldots 5=$ stimmt ganz genau

Eine ähnliche Struktur ist auch in der hessischen Stichprobe zu beobachten. Die Werte der hessischen Stichprobe liegen bei den positiven Items im Gesamtdurchschnitt höher und bei den negativen Items niedriger als die Werte 
in der sächsischen Stichprobe. In sieben von acht Fällen sind die Unterschiede statistisch signifikant. Somit können wir schlußfolgern, daß die sächsischen Schüler ein niedrigeres Selbstwertgefühl haben als die hessischen. Wenn wir nur die negativen Items berücksichtigen, können wir feststellen, daß der $\mathrm{Zu}-$ sammenhang zwischen dem Selbstwertgefühl und dem Gewaltstatus in der hessischen Stichprobe stärker ist als in der sächsischen. Bei den positiven Items ist dies nicht eindeutig. Diese Differenzierung überrascht uns deswegen nicht, weil sich die ersten fünf und die letzten vier Items zwei getrennten Faktoren zuordnen lassen. Den ersten bezeichnen wir als positives Selbstwertgefühl. Dieser ist sowohl für den Ost-West-Vergleich als auch für die Erklärung von Gewalt weniger bedeutsam. Den zweiten könnte man als ,defätistisches Selbstkonzept" bezeichnen. Mutlosigkeit und Resignation gehen also mit dem Gewaltstatus einher und sind in Sachsen deutlich stärker ausgeprägt, insbesondere bei Täter-Opfern, Opfern und Tätern.

\section{Schulbezogene Einstellungen und Leistungsmerkmale}

\section{Schulfreude:}

Wir wollen nun untersuchen, ob sich unsere fünf Gruppen hinsichtlich der Schulfreude voneinander unterscheiden. Die Dimension wurde anhand von sechs Items erfragt (vgl. Legende zu Tab. 4-10). Die ersten drei Items beinhalten eine positive und die letzten drei Items eine negative Einstellung zur Schule. Bei der eher allgemeinen Frage, ob man gerne zur Schule geht (V98), weisen die Unbeteiligten den höchsten Mittelwert (vgl. Tab. 4-10) auf, gefolgt von den Opfern (mit einer nicht signifikanten Differenz). Bei den anderen drei Gruppen ist eine signifikant geringere Schulfreude zu beobachten als bei den Unbeteiligten, am geringsten bei den Tätern und Täter-Opfern. Die Unterschiede zwischen den Gruppen sind bei der Frage nach der „guten Laune“ in der Schule (V109) nicht so groß. Bemerkenswert ist hier, daß die Opfer in der Schule am schlechtesten und die Täter-Opfer am besten gelaunt sind, obwohl nach Item V98 die Opfer eine größere Schulfreude haben als die TäterOpfer und Täter. Gehen wir davon aus, daß die schlechtere Laune der Opfer durch die Attacken gegen sie zustande kommt, dann läßt sich dieser Befund so interpretieren, daß die Möglichkeit, sich durchsetzen oder verteidigen zu können, die Täter-Opfer in bessere Laune versetzt. Bei der Frage nach Spaß in der Schule (V120) ist der höchste Mittelwert für die Gruppe der Unbeteiligten festzustellen, gefolgt von den Täter-Opfern. Am wenigsten finden die Täter Dinge in der Schule, die ihnen Spaß machen. Bei den negativen Aussagen über die Schule (V94, V125 und V127) haben die Unbeteiligten in allen Fällen die kleinsten Mittelwerte und die Täter und Täter-Opfer die größten.

In Hessen ist die Schulfreude größer als in Sachsen (bei allen sechs Items hochsignifikant). Auch bei allen fünf Gruppen der hessischen Stichprobe ist insgesamt größere Schulfreude zu beobachten als in den vergleichbaren Grup- 
pen in Sachsen. Der Zusammenhang zwischen der Schulfreude und der Zugehörigkeit zu den Gewalt-Clustern ist aber in Hessen schwächer.

Tab. 4-10: Zusammenhang zwischen der Schulfreude und dem Gewaltstatus (Mittelwertvergleich)

\begin{tabular}{lcccccc}
\hline Item-Nr. & $\begin{array}{c}\text { a. } \\
\text { Opfer }\end{array}$ & $\begin{array}{c}\text { b. } \\
\text { Täter-Opfer }\end{array}$ & $\begin{array}{c}c . \\
\text { Täter }\end{array}$ & $\begin{array}{c}d . \\
\text { Episoden-Täter }\end{array}$ & $\begin{array}{c}\text { e. } \\
\text { Unbeteiligte }\end{array}$ & ${ }^{* *}$ \\
$\eta^{2}$ \\
V98 & $2,44 \mathrm{bc}$ & $1,96 \mathrm{ae}$ & $1,88 \mathrm{ade}$ & $2,24 \mathrm{ce}$ & $2,64 \mathrm{bcd}$ & .05 \\
V109 & $3,14 \mathrm{be}$ & $3,52 \mathrm{a}$ & 3,32 & 3,35 & $3,43 \mathrm{a}$ & .01 \\
V120 & 3,09 & 3,12 & $2,86 \mathrm{e}$ & $3,09 \mathrm{e}$ & $3,33 \mathrm{~cd}$ & .02 \\
V94 & $3,05 \mathrm{e}$ & $3,29 \mathrm{e}$ & $3,34 \mathrm{de}$ & $3,00 \mathrm{ce}$ & $2,69 \mathrm{abcd}$ & .04 \\
V125 & $3,38 \mathrm{c}$ & $3,79 \mathrm{e}$ & $3,80 \mathrm{ade}$ & $3,49 \mathrm{ce}$ & $3,22 \mathrm{bcd}$ & .03 \\
V127 & $3,47 \mathrm{be}$ & $3,98 \mathrm{ade}$ & $3,71 \mathrm{de}$ & $3,34 \mathrm{bce}$ & $3,08 \mathrm{abcd}$ & .04 \\
\hline
\end{tabular}

* Anhand der Buchstaben a, b, c, d und e ist bei jedem Mittelwert zu erkennen, von welchen anderen vier Mittelwerten er sich signifikant unterscheidet $(p<.05)$.

* Anteil der erklärten Varianz

V98: Ich gehe sehr gern zur Schule.

V109: In der Schule bin ich meist gut gelaunt.

V120: Es gibt in der Schule eigentlich viele Dinge, die mir Spaß machen.

V94: Schon der Gedanke an die Schule macht mich oft mißmutig.

V125: Ich bin froh, wenn ich nicht mehr zur Schule gehen muß.

V127: Ich könnte meine Zeit besser außerhalb der Schule nutzen.

Skala: 1 = stimmt gar nicht $\ldots 5$ = stimmt ganz genau

\section{Schulangst:}

Sind die Opfer über die Gewaltsituationen hinaus im allgemeinen ängstliche Typen? Es ist anzunehmen, daß ein Opferstatus dazu führt, daß man sich auch in anderen Situationen ängstlich und unsicher verhält. Es kann auch angenommen werden, daß die ohnehin ängstlichen Typen vorrangig zur Zielscheibe der Gewaltattacken werden. ${ }^{12}$ Wir wollen im folgenden überprüfen, ob sich unsere fünf Gruppen auch hinsichtlich der Schulangst unterscheiden. Die Schulangst wird hier anhand von fünf Items repräsentiert (vgl. Legende zu Tab. 4-11). Die Mittelwerte dieser Items in den fünf Clustern unterscheiden sich nicht so stark voneinander (vgl. Tab. 4-11). Trotzdem kann man feststellen, daß in allen Fällen die Opfer die höchsten Mittelwerte aufweisen. Somit gehen wir

12 Die Korrelation zwischen den beiden Faktoren „Schulangst“ und „Angst in konkreten Gewaltsituationen beträgt $r=.28$. 
davon aus, daß sich die Ängstlichkeit der Opfer nicht auf die Gewaltsituationen beschränkt. Ähnliche Ergebnisse wurden für die hessische Stichprobe ermittelt.

Tab. 4-11: Zusammenhang zwischen der Schulangst und dem Status in der schulischen Gewalt (Mittelwertvergleich)

\begin{tabular}{lcccccc}
\hline Item-Nr. & $\begin{array}{c}* * \\
\text { Opfer }\end{array}$ & $\begin{array}{c}b . \\
\text { Täter-Opfer }\end{array}$ & $\begin{array}{c}c . \\
\text { Täter }\end{array}$ & $\begin{array}{c}d . \\
\text { Episoden-Täter }\end{array}$ & $\begin{array}{c}e . \\
\text { Unbeteiligte }\end{array}$ & $\begin{array}{c}* * \\
\eta^{2}\end{array}$ \\
V316 & $3,63 \mathrm{c}$ & 3,40 & $3,01 \mathrm{ae}$ & 3,29 & $3,37 \mathrm{c}$ & .01 \\
V317 & $3,70 \mathrm{cde}$ & $3,38 \mathrm{c}$ & $2,79 \mathrm{abde}$ & $3,11 \mathrm{ac}$ & $3,25 \mathrm{ac}$ & .02 \\
V318 & 2,72 & 2,64 & 2,49 & 2,41 & 2,47 & .00 \\
V319 & 3,46 & 3,21 & 3,11 & 3,13 & 3,13 & .00 \\
V320 & $3,07 \mathrm{c}$ & $3,06 \mathrm{c}$ & $2,49 \mathrm{abe}$ & 2,75 & $2,86 \mathrm{c}$ & .01 \\
\hline
\end{tabular}

* Anhand der Buchstaben $a, b, c$, d und e ist bei jedem Mittelwert zu erkennen, von welchen anderen vier Mittelwerten er sich signifikant unterscheidet $(p<.05)$.

** Anteil der erklärten Varianz

V316: Ich werde nervös, wenn ich an die Tafel gerufen werde, unabhängig davon, ob ich vorbereitet bin oder nicht.

V317: Ich bekomme Herzklopfen, wenn ein Lehrer/eine Lehrerin mich abfragen will.

V318: Aus Angst, etwas Falsches zu sagen, beteilige ich mich selten am Unterricht.

V319: Es kommt häufig vor, daß ich am Anfang einer Prüfung keinen klaren Gedanken fassen kann.

V320: Ich habe Angst vor Klassenarbeiten.

Skala: 1 = stimmt gar nicht $\ldots 5$ = stimmt ganz genau

\section{Leistungsstatus in der Schule:}

Die fünf Schülergruppen unterscheiden sich auch hinsichtlich der Schulleistung voneinander. In Melzer/Rostampour (1996) hatten wir festgestellt, daß die Täter u.a. durch eine schlechtere Schulleistung zu kennzeichnen sind. Lösel, Bliesener und Averbeck (1997) kommen ebenfalls zu diesem Ergebnis. Hier operationalisieren wir den Leistungsstatus durch die Noten in den Fächern Deutsch, Mathematik und Englisch im letzten Zeugnis. Tabelle 4-12 zeigt die Mittelwerte dieser Noten in jeder Gruppe. Die besten Noten werden von den Unbeteiligten erzielt, gefolgt von den Opfern, und die schlechtesten Noten von den Täter-Opfern, gefolgt von den Tätern.

Ähnliche Ergebnisse wurden auch für die hessischen Stichprobe festgestellt. Die sächsischen Schüler haben im Gesamtdurchschnitt in allen drei Fächern bessere Noten erzielt als die hessischen (hochsignifikant). Auch alle Schülergruppen der sächsischen Stichprobe weisen bessere Noten als ihre hessischen Pendants auf. 
Die vorliegenden Ergebnisse bestätigen die Befunde anderer Untersuchungen insoweit, als die Täter überdurchschnittlich schlechte Noten haben. Wir können dies für das Cluster der Täter und das der Täter-Opfer bestätigen, wobei die letztgenannte Gruppe als harter Kern der Täter sogar noch etwas schlechtere Noten hat. ${ }^{13}$

Tab. 4-12: Zusammenhang zwischen der Schulleistung (Noten) und dem Gewaltstatus (Mittelwertvergleich)

\begin{tabular}{lcccccc}
\hline Item-Nr. & $\begin{array}{c}a{ }^{*} \\
\text { Opfer }\end{array}$ & $\begin{array}{c}\text { b. } \\
\text { Täter-Opfer }\end{array}$ & $\begin{array}{c}c . \\
\text { Täter }\end{array}$ & $\begin{array}{c}d . \\
\text { Episoden-Täter }\end{array}$ & $\begin{array}{c}\text { e. } \\
\text { Unbeteiligte }\end{array}$ & ${ }^{* *}$ \\
$\eta^{2}$ \\
V21 & $2,60 \mathrm{c}$ & $2,92 \mathrm{e}$ & $2,91 \mathrm{ae}$ & $2,74 \mathrm{e}$ & $2,47 \mathrm{bcd}$ & .04 \\
V22 & 2,87 & $3,10 \mathrm{e}$ & $2,94 \mathrm{e}$ & $2,90 \mathrm{e}$ & $2,71 \mathrm{bcd}$ & .01 \\
V23 & $2,76 \mathrm{~b}$ & $3,14 \mathrm{ae}$ & $2,94 \mathrm{e}$ & $2,87 \mathrm{e}$ & $2,60 \mathrm{bcd}$ & .03 \\
\hline
\end{tabular}

* Anhand der Buchstaben a, b, c, d und e ist bei jedem Mittelwert zu erkennen, von welchen anderen vier Mittelwerten er sich signifikant unterscheidet $(p<.05)$.

** Anteil der erklärten Varianz

V21: Note Deutsch im letzten Zeugnis. (Skalen von 1 bis 6)

V22: Note Mathematik im letzten Zeugnis.

V23: Note Englisch im letzten Zeugnis.

\section{Leistungsattribuierung:}

Die fünf Gruppen unterscheiden sich auch hinsichtlich der Einschätzung eigener Leistungsfähigkeit in der Schule. Anhand von drei Items V102, V104 und V173 (s. Tabelle 4-13) ist dies überprüfbar. Ihre Mittelwerte sind bei den Beteiligten am niedrigsten. D.h., alle an Gewalt beteiligten Gruppen zeigen bei den Schulanforderungen negativere Selbstattribuierungen als die Unbeteiligten. Dies ist am meisten bei den Täter-Opfern der Fall und am wenigsten bei den Episoden-Tätern. Nach Olweus $(1995 ; 1997)$ fühlen sich die typischen Opfer als Versager und dumm. Wenn wir davon ausgehen, daß unsere drei Items als Indikatoren für ein solches Gefühl (Versagen bzw. Dummheit) gelten, dann können wir feststellen, daß es diesbezüglich keinen signifikanten Unterschied zwischen Opfern und Tätern gibt. Das bedeutet, daß Täter wie Opfer eine sehr ähnliche negative Leistungsattribuierung zeigen, die bei der Gruppe der Täter aufgrund der schlechteren Noten auch berechtigt ist, bei den Opfern aber dieser Grundlage entbehrt und durch ihr negatives Selbstbild zustande kommt.

13 Die Täter-Opfer weisen zwar schlechtere Noten auf als die Täter. Die Differenz zwischen diesen Gruppen ist aber statistisch nicht signifikant. 
In Sachsen wird die eigene Leistungsfähigkeit insgesamt schlechter eingeschätzt als in Hessen. Der Zusammenhang zwischen der Leistungsattribuierung und dem Status in der schulischen Gewalt ist in Hessen etwas stärker; die Unterschiede zwischen den Gruppen haben aber dieselbe Struktur.

Tab. 4-13: Zusammenhang zwischen der Leistungsattribuierung und dem Gewaltstatus (Mittelwertvergleich)

\begin{tabular}{lcccccc}
\hline Item-Nr. & $\begin{array}{c}a .^{*} \\
\text { Opfer }\end{array}$ & $\begin{array}{c}b . \\
\text { Täter-Opfer }\end{array}$ & $\begin{array}{c}c . \\
\text { Täter }\end{array}$ & $\begin{array}{c}d . \\
\text { Episoden-Täter }\end{array}$ & $\begin{array}{c}\text { e. } \\
\text { Unbeteiligte }\end{array}$ & ${ }^{* *}$ \\
$\eta^{2}$ \\
V102 & $2,59 \mathrm{e}$ & $2,95 \mathrm{cde}$ & $2,53 \mathrm{be}$ & $2,46 \mathrm{be}$ & $2,21 \mathrm{abcd}$ & .03 \\
V104 & $2,46 \mathrm{e}$ & $2,77 \mathrm{de}$ & $2,61 \mathrm{de}$ & $2,30 \mathrm{bc}$ & $2,22 \mathrm{abc}$ & .02 \\
V173 & $2,46 \mathrm{e}$ & $2,63 \mathrm{de}$ & $2,59 \mathrm{de}$ & $2,25 \mathrm{bce}$ & $2,08 \mathrm{abcd}$ & .03 \\
\hline
\end{tabular}

* Anhand der Buchstaben a, b, c, d und e ist bei jedem Mittelwert zu erkennen, von welchen anderen vier Mittelwerten er sich signifikant unterscheidet $(p<.05)$.

* Anteil der erklärten Varianz

V102: In der Schule habe ich oft das Gefühl, daß ich weniger zustande bringe als andere. V104: Schon bevor ich mit einer Aufgabe anfange, weiß ich, daß ich sie nicht gut mache. V173: Ich kann lernen, soviel ich will: Das, was die Schule verlangt, kann ich gar nicht schaffen.

Skala: 1 = stimmt gar nicht $\ldots 5$ = stimmt ganz genau

\subsection{Zusammenfassung}

Die Analyse der Täter- und Opfer-Items hat folgendes verdeutlicht:

1. Täter- und Opferrollen in der schulischen Gewalt sind nicht gänzlich voneinander zu trennen und können vielfach schnell ineinander übergehen. Trotzdem sind bestimmte Typen erkennbar, die überwiegend die eine oder die andere Rolle einnehmen und immer mehr mit diesen Rollen identifiziert werden.

2. Es gibt einen engen Zusammenhang zwischen der Gewalttätigkeit in der Schule und der (außerschulischen) Jugendkriminalität. Die schulischen Gewalttäter (einschließlich Täter-Opfer) begehen erheblich mehr Delikte als die anderen Schüler. Auch Alkohol, Zigaretten und Drogen werden vor allem von Tätern und Täter-Opfern konsumiert.

3. In unseren Analysen haben sich fünf Schülergruppen hinsichtlich der Beteiligung an schulischer Gewalt herauskristallisiert. Es wurde gezeigt, daß die überwiegende Mehrheit der Schüler (etwa 56\%) in der Regel weder als Täter noch als Opfer an Gewalt beteiligt sind (Unbeteiligte). Bei den 
Verhaltensweisen der Beteiligten handelt es sich in den meisten Fällen (knapp 26\%) um eine gelegentliche Verwicklung in Gewalthandlungen und insbesondere in ,weichere“ Gewaltformen (Episoden-Täter). Häufige Beteiligung an Gewalt kommt bei einer Minderheit der Schüler vor (gut 18\%). Ein kleiner Teil davon (gut 3\%) tritt gleichermaßen als Täter und Opfer in Aktion, insbesondere bei härteren Gewaltformen, und kann als harter Kern des Gewalthandelns in der Schule bezeichnet werden. Die restlichen $15 \%$ können in zwei etwa gleich große Gruppen von Tätern (8\%) und Opfern (7\%) eingeteilt werden. Diese in der sächsischen Stichprobe festgestellten Gruppen und ihre Anteile werden durch die Ergebnisse der hessischen Stichprobe und weiterer Studien im großen und ganzen bestätigt.

4. Die Typologie der an Gewalt beteiligten Schüler hat gezeigt, daß fünf Sechstel der Täter männlich sind. Der Anteil der Täter nimmt bis zur 8. Klasse zu und danach etwas ab. In Mittelschulen gibt es fast doppelt so viele Täter wie in den Gymnasien. Die Täter (und insbesondere männliche Täter) sind überdurchschnittlich groß. Sie haben eine positive Einstellung gegenüber Gewalt, weisen eine hohe Aggressionsbereitschaft auf und betrachten Gewalt als normal. Nationalismus und Fremdenfeindlichkeit sind am stärksten unter den Tätern verbreitet. Disziplin und Aufrechterhaltung von Ruhe und Ordnung sind bei ihnen aber nicht besonderes beliebt. Sie fühlen sich - anders als die Opfer - auf keinen Fall als Außenseiter oder minderwertig. Gemeinsam mit den Täter-Opfern haben sie die schlechtesten Noten in den Kernfächern Deutsch, Mathematik und Englisch und die niedrigste Schulfreude. Sie zeichnen sich auch durch relativ niedrige Schulangst aus, schätzen ihre Leistungsfähigkeit aber etwas schlechter ein.

5. Opfer sind im allgemeinen etwas jünger und kleiner und sind an Förderschulen am stärksten vertreten. Etwa $43 \%$ der identifizierten Opfer sind weiblich. Das ist - gemessen an ihrer relativ geringen Beteiligung an der Gewalt - insgesamt sehr hoch. Die Gewaltbilligung ist bei den Opfern signifikant schwächer als bei den Tätern, aber signifikant stärker als bei den Unbeteiligten. Der letztere Befund deutet darauf hin, daß die Opfer mindestens zum Teil an eine Berechtigung für Gewaltanwendung glauben. Die Aggressionsbereitschaft ist bei den Opfern zwar niedriger als bei Tätern, aber stärker als bei den Unbeteiligten. Bei den Opfern ist weiterhin eine hohe Zustimmung zu traditionellen Lerntugenden, wie Disziplin, Ruhe und Ordnung, zu verzeichnen. Prägende Merkmale der Opfer sind ihr Außenseiterstatus und ihr geringes Selbstwertgefühl. Ihre Schulleistungen liegen zwischen denen der Unbeteiligten, die den höchsten Leistungsstatus haben, und denen der Täter und Täter-Opfer, die tendenziell schlechtere Schüler sind. Dasselbe gilt auch für die Schulfreude der Opfer. Bei der Schulangst haben sie aber die höchsten Werte. Auch schätzen sie ihre Leistungsfähigkeit schlechter ein als andere Schülergruppen. 
6. Bei der Gruppe der Episoden-Täter beträgt der Anteil der Mädchen 40\%. Dieser Typus kommt in höheren Klassenstufen häufiger vor. In einer abgeschwächten Form weisen Mitglieder dieser Gruppe im allgemeinen dieselben Merkmale wie Täter auf. Ihre Werte liegen bei fast allen untersuchten Merkmalen zwischen denen der Tätern und denen der Unbeteiligten.

7. Die kleine Gruppe der Täter-Opfer ist die problematischste von allen. Sie teilen fast alle Schwächen der Täter und Opfer zugleich. Sie sind genauso häufig in kriminelle Delikte verwickelt wie die Täter und haben hohe Werte beim Konsum von Alkohol, Zigaretten und besonders Drogen (letzteres sogar signifikant mehr als die Täter). Ihr Anteil nimmt in den höheren Klassenstufen ab. Gut zwei Drittel von ihnen sind männlich. Sie sind selten in den Gymnasien und besonders häufig in den Förderschulen zu treffen. Sie billigen die Gewalt signifikant mehr als Opfer, EpisodenTäter und Unbeteiligte und weisen eine genauso starke Aggressionsbereitschaft wie Täter auf. Für sie ist die Gewalt genauso normal wie für die Täter. In bezug auf den Nationalismus weisen sie höhere Werte als Opfer auf und unterscheiden sich kaum von Tätern. Sie fühlen sich auch überdurchschnittlich als Außenseiter, wenn auch nicht so stark wie die Opfer. Dasselbe gilt für das Selbstwertgefühl. Die schlechtesten Noten werden von den Täter-Opfern erzielt. Sie gehen wie die Täter ungern zur Schule, obwohl sie in der Schule nach ihren Angaben gut gelaunt sind. Sie haben auch die negativsten Urteile über die eigene Leistungsfähigkeit. Bei dieser Gruppe zeigt sich besonders deutlich, daß Täter- und Opferstatus sich nicht ausschließen. Da die Täter-Opfer überdurchschnittlich harte Gewaltformen bevorzugen und gleichzeitig auch Leidtragende extremer Drangsalierungen sind, sehen wir in dieser Gruppe den harten Kern schulischen Gewalthandelns repräsentiert.

8. Die Typologie basiert auf einer Analyse sämtlicher interaktioneller Gewalthandlungen im schulischen Kontext und schließt - im Sinne unserer Definition von Gewaltförmigkeit und Gewaltaffinität - auch Aspekte der Schuldevianz ein, also weniger gravierende Verhaltensweisen, die gegen Mitschüler oder Lehrer gerichtet sind. Wenn bei einem so weit gefaßten Gewaltverständnis etwa 56\% der Schülerinnen und Schüler nicht in abweichende und aggressive Verhaltensweisen verwickelt sind, lassen sich aus diesen Befunden - unseren bisherigen Ergebnissen aus Kapitel 2 entsprechend - zwei Schlußfolgerungen ziehen. Erstens besteht zwar Anlaß, über Gewalthandlungen von Kindern in einer nicht zu vernachlässigenden Größenordnung (15\% Täter und Opfer, ca. 3\% Täter-Opfer) nachzudenken, die Ursachen zu erforschen und geeignete pädagogische Maßnahmen zu ergreifen. Dennoch sind zweitens Dramatisierungen zurückzuweisen. Dies muß um so deutlicher gesagt und mit Daten belegt werden, als von seiten der Medien den Wissenschaftlern teilweise der Vorwurf der Bagatellisierung entgegengebracht wird. Die Tatsache, daß eine deutliche Mehrheit der Schülerschaft nicht in Gewalthandlungen involviert ist, sie ablehnt 
und sich auch nicht von ihnen animieren läßt, stellt in Verbindung mit einer Kompetenz und Bereitschaft zur Schlichtung eine gute Basis für das Gelingen pädagogischer Präventions- und Interventionsmaßnahmen dar.

Wir haben in diesem Kapitel die Analyse auf die Ebene der individuellen und personellen Merkmale (Mikroebene) beschränkt. Es gibt aber eine Reihe Prädiktoren und Risikofaktoren in den Bereichen Familie, Medien, Jugendkultur und Schule. Diese sozialisatorischen und institutionellen Einflüsse werden in den nächsten Kapiteln behandelt. Die in diesem Kapitel dargestellten fünf Typen der Gewaltbeteiligung werden nicht mehr im Mittelpunkt der Analyse stehen; wir werden nur gelegentlich auf sie zurückgreifen. Zielpunkt der weiteren Analysen sind dort also nicht mehr primär die Personengruppen, sondern eher die Strukturen und Deutungsmuster von Gewalt als Syndrom gewaltförmiger und gewaltaffiner Handlungsformen und Einstellungen. 


\section{Prädiktoren schulischer Gewalt im außerschulischen Bereich (Wolfgang Melzer, Parviz Rostampour)}

Nachdem in den vorangegangenen Kapiteln deutlich geworden ist, daß in Ost- und Westdeutschland eine ähnliche Problemlage hinsichtlich der Gewalthäufigkeit, der Verteilung der einzelnen Gewaltformen und in bezug auf das Rollengefüge von Tätern und Opfern besteht und auch bei den individuellen Prädiktoren weitgehende Übereinstimmungen auftreten, geht es bei der weiteren Ursachensuche um mögliche Einflüsse im außerschulischen Bereich. Bei der Analyse dieser Sozialisationsbedingungen, die sich möglicherweise auf das Gewalthandeln auswirken, stellt sich ebenfalls die Frage, ob hier OstWest-Unterschiede zu verzeichnen sind oder nicht.

Das synergistische Zusammenwirken sozialisatorischer, interaktionaler und situativer Einflüsse in der Biographie der Heranwachsenden ist - wie oben bereits an verschiedenen Stellen dargelegt - eine zentrale Hypothese zur Erklärung von gewaltförmigen und gewaltaffinen Verhaltensweisen. In diesem Kapitel geht es um die Einflüsse primärer Sozialisation im sozialökologischen Zentrum der Familie, des Freizeit- und Medienverhaltens und der Peer-Sozialisation - also von möglichen Prädiktoren im nichtschulischen Bereich, die aber gleichwohl - so unsere Hypothese - für das Gewalthandeln innerhalb der Schule bedeutsam sein können. Sozialisation wird dabei nicht nur als Prägung des Individuums durch seine Umwelt, sondern als aktive Auseinandersetzung mit dieser verstanden; dazu gehört auch die Interaktion mit anderen, z. B. den Eltern, Mitschülern und Freunden.

Das Kapitel gliedert sich in drei Teile. Zunächst werden die Familienkontexte analysiert (5.1): die Familienstrukturen (z.B. Vollständigkeit der Familie, Geschwisterkonstellation), das Familienklima (z.B. sich wohlfühlen zu Hause, Unterstützung durch die Eltern), der Erwartungshorizont der Eltern in Verbindung mit ihrem Erziehungsstil (z.B. Erwartungen, Freiheitsgrade, Strenge) und die Erfahrung von Aggressivität im Erziehungshandeln der Eltern (schimpfen, schreien, prügeln). Außerdem ist im Zusammenhang mit dem Ursprungsmilieu der Kinder zu untersuchen, was „Schichtmodelle“, in denen sich die gesellschaftliche Stratifikation niederschlägt, im Hinblick auf die Erklärung von Gewalt leisten.

Im zweiten Teil (5.2) beschäftigen wir uns mit den Medieneinflüssen, wobei der Medienkonsum teilweise in den Familienbereich mit hineinfällt, teilweise im außerschulischen Bereich anzusiedeln ist. Lassen sich, wie allenthalben behauptet, Auswirkungen des Medienverhaltens auf Gewalthandeln feststellen? Was wird an Fernsehsendungen gesehen, was verbieten Eltern und wie wird mit diesen Regelungen umgegangen? Wieviel Zeit wird werktags und 
am Wochenende mit Fernsehen und Video verbracht? Können die Heranwachsenden mit ihrer Freizeit überhaupt etwas anfangen?

In diesem Teil stellt sich stärker als bei der Familie, deren sozialisatorische Wirkungen unbestritten sind, die Frage der Kausalität: Wenn ein statistischer Zusammenhang zwischen Konsum von Gewalt- und Horrorfilmen zum Gewaltverhalten der Kinder und Jugendlichen festgestellt wird, ist das ein Hinweis auf die Wirkung der Medien oder ist der Konsum der Ausdruck eines bestimmten Status, z. B. als „Täter“?

Im dritten Teil (5.3) werden mögliche Prädiktoren der Peer-Sozialisation geprüft: die Organisation in Gruppen, die Binnenstruktur dieser Gruppen (Intoleranz, Unterordnung) und ihre Außenorientierung (aggressive und delinquente Verhaltensformen von Cliquen). Bei unseren Hypothesen gehen wir davon aus, daß hier ein Zusammenspiel von schulischen und außerschulischen Wirkungszonen und Milieus besteht, die es schwer machen, eine Trennschärfe zwischen Prädiktoren von Gewalt und Aspekten der Gewaltausübung herzustellen.

Bei den Analysen und den Darstellungen der Ergebnisse wird folgendermaßen vorgegangen: Zunächst werden die einzelnen Variablen mit dem Ziel der Beschreibung der drei Sozialisationsbereiche (Familie, Medien, Peers) in ihren Verteilungen vorgestellt. Dabei wird geprüft, ob die Sozialisationsbedingungen im außerschulischen Bereich in Ost- und Westdeutschland übereinstimmen bzw. differieren und anschließend untersucht, ob und welche Auswirkungen diese Erfahrungen auf Gewalthandeln - und insbesondere auf die verschiedenen Aspekte des Syndroms gewaltförmiger und gewaltaffiner Verhaltensweisen - besitzen.

\subsection{Bedingungen familialer Sozialisation}

Die Bedeutung der Familie für die Entwicklung von Kindern und Jugendlichen wird von kaum einem Wissenschaftler ernsthaft in Zweifel gezogen. Daher erscheint es geboten, auch mögliche Auswirkungen familialer Sozialisation auf Gewalthandeln zu untersuchen. In der Familienforschung wird einerseits die zunehmende gesellschaftliche Differenzierung und ihre Auswirkung auf die Familien in Form einer Pluralisierung der Lebens- und Familienformen thematisiert (vgl. u.a. Vaskovics 1997, Nave-Herz 1997, Lukesch 1996) - eine Entwicklung, die mit einer Krise und Umstrukturierungen im „Inneren“ und „Äußeren“ der Familie verbunden ist -, auf der anderen Seite wird die Konstanz der Familie und die dauerhafte Wertschätzung von Partnerschaft, Ehe und insbesondere der Elternschaft betont (vgl. Vaskovicz 1996, Bertram 1997). Andere Autoren zeigen veränderte Rollenbilder von Erwachsenen und Heranwachsenden in der Familie, die bei aller verbleibenden und z.T. berechtigten feministischen Kritik eine Tendenz zu mehr Gleichberechtigung zwi- 
schen Männern und Frauen einschließlich einer modifizierten Männerrolle (vgl. Parke 1982, Fthenakis 1988) sowie im Beziehungsgefüge von Eltern und Heranwachsenden zeigen und mit dem Stichwort „Verhandlungshaushalt" charakterisiert werden können (Melzer 1992, Büchner/Fuchs/Krüger 1996). In der familienbezogenen Sozialisationsforschung besteht eine Kontinuität der Erziehungsstilforschung, in der gleichzeitig die Wirkungsweise von autoritärem, demokratischem und Laisser-faire-Stil herausgearbeitet und der Kontext des Familienklimas, in dem dieses erzieherische Handeln mit den entsprechenden Interventionen und Sanktionen stattfindet, betont wird (vgl. zusammenfassend Kreppner 1980, S. 399ff).

Auch der Zusammenhang von Familie und Gewalt ist vielfach untersucht und die Forschungslage eindeutig: $\mathrm{Da} ß$ die familiale Sozialisation ein Risikound damit gleichzeitig ein protektiver Faktor im Feld des Gewalthandelns von Kindern und Jugendlichen ist, daran kann nach Durchmusterung der relevanten Literatur keinerlei Zweifel bestehen. In bezug auf makrosoziales und politisches Verhalten waren in zwei klassischen Studien Zusammenhänge zur Familie hergestellt und z.T. auch nachgewiesen worden: Es sind dies die 1950 in den USA erschienene Studie von Adorno/Frenkel-Brunswik/Levinson/Sanford (1950) und eine qualitative Untersuchung von Mantell (1972). In der Tradition der Autoritarismusforschung, in der die erstgenannte Untersuchung steht, wird die Familie als eine latente politische Sozialisationsinstanz begriffen (vgl. Hopf/Hopf 1997, S. 23ff). Adorno wie auch Reich, Fromm oder Horkheimer erklären die faschistische Folgebereitschaft eines Großteils der Bevölkerung in Deutschland nach 1933 mit den in der Familienerziehung aufgebauten und verfestigten Charakterstrukturen, dem System der autoritären Persönlichkeit, die sich gegenüber dem Mächtigen als gehorsam und unterwürfig, gegenüber den Schwächeren als dominant und aggressiv zeige. Auch Mantell demonstriert in seiner Vergleichsstudie von Kriegsdienstverweigerern und Kriegsfreiwilligen, daß die Familienatmosphäre, die Art der Kommunikation der Eltern untereinander sowie die der Eltern mit den Kindern von nachhaltiger Bedeutung für politisches Verhalten ist und unter Umständen sogar dazu beiträgt, daß jemand für sein Vaterland freiwillig in den Krieg zieht oder den Dienst mit der Waffe generell verweigert. Bei den „Green Berets“ wurde in etwa zwei Drittel der Fälle eine Dominanz der Väter, zu einem Viertel eine Dominanz der Mütter und nur zu etwa $8 \%$ ein gleicher Einfluß beider Elternteile ermittelt. In allen Konstellationen von Dominanz (z.B. des Vaters gegenüber der Mutter, der Eltern gegenüber den Kindern) basierte diese auf "Strenge, Strafen und Furcht“. In bezug auf das Erziehungsverhalten der Eltern der Kriegsfreiwilligen wurde festgestellt: „So gut wie alle Eltern waren strenge Erzieher, drohten ihren Kindern mit körperlichen Strafen, teilten diese aus, verlangten unerschütterlichen Gehorsam und erreichten ihre Kontrolle über die Kinder hauptsächlich durch Einflößung von Furcht“" (Mantell 1972, S. 46).

Unter Bezug auf das Syndrom von Gewalt und gewaltaffinem Verhalten bei Kindern und Jugendlichen im schulischen und außerschulischen Raum 
sind die Forschungen zum delinquenten Verhalten von Heranwachsenden von Interesse. Pfeiffer/Wetzels (1997) haben in einer Analyse der „Polizeilichen Kriminalstatistik“ bei der Gruppe der 8- bis 14jährigen im Zeitraum von 1984 bis 1996 einen Anstieg der Tatverdächtigen im Bereich der Gewaltkriminalität um das 2,7fache festgestellt und kommen bei der Suche nach Ursachen an Hand einer repräsentativen Opferbefragung sowie einer umfassenden Sekundäranalyse anderer Studien zu dem Ergebnis, ,daß Kindheitserfahrungen mit Gewalt ein relevanter Risikofaktor für Gewalt und Kriminalität sind. (...) So hat sich beispielsweise die Verfügbarkeit einer vertrauensvollen, emotionale Wärme vermittelnden Bezugsperson als ein wichtiger protektiver Faktor erwiesen“ (ebd., S. 363). Die Autoren führen u.a. lerntheoretische Argumente für diesen Zusammenhang an, sehen in der funktionierenden Familie ein Unterstützungsnetzwerk für Kinder und Jugendliche und kommen zusammenfassend - ähnlich wie wir - zu einem synergistischen Modell von Gewalthandeln: „Demnach tragen innerfamiliäre Sozialisationserfahrungen, insbesondere die direkt gegen Kinder gerichtete Gewalt, aber auch die Beobachtung gewaltförmiger Konfliktaustragung seitens bedeutsamer primärer Bezugspersonen, über soziale Lernprozesse zur Entwicklung einer Risikogruppe bei, in der sich ein zunächst noch latentes Potential der Gewalt- und Delinquenzbereitschaft herausbildet. Im Falle des Eintritts zusätzlicher Risiken, wie sie z.B. mit der ökonomischen Belastung von Familien und der prekären Arbeitsmarktsituation für Jugendliche - insbesondere für diejenigen mit fehlendem oder niedrigem Schulabschluß - gegeben sind, besteht die Gefahr des Umschlagens eines solchen latenten Potentials in manifeste Delinquenz und Gewalt" (ebd., S. 363).

Zum Thema „Familie und Gewaltverhalten in der Schule“ liegen in der Tendenz ebenfalls diesen Zusammenhang bestätigende Befunde vor, die allerdings nicht in allen Fällen so eindeutig sind. In einer Befragung der Schwind-Gruppe zur Gewalt in der Schule aus der Perspektive unterschiedlicher Gruppen werden von jeweils etwa 90\% der Lehrer die Familienverhältnisse mit für das gewalttätige Verhalten verantwortlich gemacht. Beklagt werden „ungünstige familiale Verhältnisse“ $(92,9 \%)$, „,emotionale Kälte in der Eltern-Kind-Beziehung“ (91,0\%); außerdem wird in der „Berufstätigkeit beider Eltern“ $(89,1 \%)$ ein Risikofaktor gesehen (vgl. Schwind/ Roitsch/Gielen 1997, S. 95). Mit Krumm (1997) ist allerdings darauf hinzuweisen, daß diese Fremdattribuierungen von Lehrern auch die Funktion der Entlastung haben und von eigenen Defiziten im Verhalten gegenüber Schülern ablenken. In der Nürnberger Gewalt-Studie wurden verschiedene Parameter primärer Sozialisation geprüft (vgl. Funk/Passenberger 1997, S. 248f); der Einfluß der perzipierten Erziehungsstile wird von den Autoren aber eher als gering bewertet. Allerdings kommen auch sie zu dem Ergebnis, daß sich der Einfluß subjektiv als positiv erlebter Sozialbeziehungen in der Familie gewaltmindernd niederschlägt, und zwar bei Formen verbaler Aggression und vandalistischen Verhaltens (ebd., S. 258). Deutlicher dagegen werden von Olweus als relevante Prädiktoren des Familienkontextes betont: die emotionale Beziehung zur er- 
sten Bezugsperson während der frühen Kindheit, das billigende Verhalten der Erziehungsberechtigten bei Aggressionen der Kinder gegenüber Geschwistern, anderen Kindern oder Erwachsenen und die „Anwendung von machtbetonten Erziehungsmethoden, wie z.B. körperliche Züchtigung und heftigen Gemütsausbrüchen. Kinder, deren Eltern häufiger Gebrauch dieser Methoden machen, werden wahrscheinlich aggressiver als der Durchschnitt der Kinder. 'Gewalt erzeugt Gewalt'“ (Olweus 1997, S. 290).

Auch in unseren eigenen Studien haben sich Aspekte der familialen Sozialisation als erklärungsrelevant für schulisches Gewaltverhalten erwiesen. Bei ersten Untersuchungen zur Täter-Opfer-Typologie - mit einem anderen Datensatz, anderen Items und anderen statistischen Verfahren als in dieser Studie - werden in der Analyse als Konstituenzien sowohl für den Täter- als auch für den Opferstatus das Familienklima und bestehende Leistungskonflikte zwischen Kindern und Eltern deutlich. Familiale Sozialisationserfahrungen sind zwar nicht die wichtigste, stellen aber im Kontext eines umfassenderen Modells eine wichtige Erklärungsvariable für gewaltförmiges und gewaltaffines Verhalten dar - so die Ergebnisse von früheren und unsere Hypothese für die hier vorgestellte Untersuchung.

Dieser Gesamtkomplex familialer Sozialisationseinflüsse soll in bezug auf Gewalt unter folgenden Gesichtspunkten geprüft werden:

- Lassen sich Auswirkungen des angesprochenen gesellschaftlichen Strukturwandels der Familie auf Gewalthandeln feststellen? Sind Kinder aus Scheidungs-, Stief- und Ein-Eltern-Familien anfälliger für Gewalt? Welche Bedeutung hat die Geschwisterreihe, die Einschränkung der Sozialerfahrung als Einzelkind, das Aufwachsen von Jungen bzw. Mädchen nur mit Jungen und in anderen Geschwisterkonstellationen?

- Lassen sich Risikofaktoren für Gewalthandeln im Bereich der materiellen Situation der Familien (z.B. Höhe des Einkommens, Arbeitslosigkeit der Eltern) feststellen?

- Lassen sich Zusammenhänge von Familienklima und intergenerativem Übereinstimmungsgrad von Eltern und Heranwachsenden zum Gewalthandeln empirisch nachweisen?

- Lassen sich Auswirkungen des Erziehungsstils der Eltern und von Erfahrungen der Kinder mit aggressiven Verhaltensweisen der Eltern auf das Gewalthandeln der Heranwachsenden in der Schule nachweisen?

- Und wenn derartige Einflüsse der familialen Sozialisation bestehen: Bei welchen Aspekten gewaltförmigen und gewaltaffinen Verhaltens sind sie stärker, bei welchen weniger stark und warum ist dieses so?

Ziel dieser Analyse ist die Herausarbeitung von Risikofaktoren für Gewalthandeln durch die familiale Sozialisation, die auch für die in der Prävention und Intervention tätigen Lehrer von Interesse sein dürften. Gleichzeitig besteht ein theorieproduktives Interesse. Da wir ein Gesamtmodell entwickeln, in dem der Ost-West-Vergleich eine zentrale Rolle spielt, wird in dieser Perspektive zu 
prüfen sein, ob hinsichtlich der dargestellten Parameter Unterschiede zwischen unseren beiden Stichproben in Sachsen und Hessen bestehen. Eine unmittelbar nach der Wende durchgeführte Untersuchung hatte das Ergebnis gebracht, daß die familialen Lebenswelten in Ost- und Westdeutschland zwar unterschiedlich in die Biographien der Heranwachsenden - vor allem in bezug auf den Zeitpunkt der Eheschließung und der Geburt der Kinder - eingebettet sind, daß aber sowohl der elterliche Erziehungsstil als auch das Familienklima von Jugendlichen aus Ost- und Westdeutschland sehr ähnlich beurteilt wurden. Allerdings zeigte sich in Ostdeutschland eine geringere Übereinstimmung der Befragten mit dem Lebensstil ihrer Eltern bei einer aber insgesamt wie im Westen bestehenden hohen intergenerativen Übereinstimmung in Erziehungsfragen (vgl. Melzer 1992, Kap. 2). Von daher spricht alles für die Hypothese, daß die familialen und Sozialisationsbedingungen in Ost- und Westdeutschland sehr ähnlich sind und gleichermaßen als Prädiktoren gewaltförmigen und gewaltaffinen Handelns von Schülern und Jugendlichen wirken. In bezug auf die Strukturen werden z.T. ähnliche Bedingungen erwartet (z.B. bei den Familienformen), gleichzeitig ist aber von Unterschieden hinsichtlich der materiellen Möglichkeiten und insbesondere der Betroffenheit von Arbeitslosigkeit auszugehen. Was die Familienstrukturen und Lebensbedingungen anbelangt, stellt sich aber die Frage, ob diese Aspekte für unser Thema überhaupt erklärungsrelevant sind. Bisherige Versuche mit Mehrebenenanalysen (vgl. Funk/ Passenberger 1997) lassen diesbezüglich das Gegenteil vermuten.

In bezug auf die Familienformen sind die Ergebnisse in Hessen und Sachsen nahezu identisch. Die große Mehrheit der Kinder lebt trotz beachtlicher Scheidungsziffern mit beiden Elternteilen zusammen (je 79,8\%) und ca. 20\% in anderen Konstellationen (z.B. Stief-Familien, Ein-Elternteil-Familien). Anhand unserer Daten läßt sich aber zeigen, daß die Familienform keinen Einfluß auf das Gewalthandeln der Kinder in der Weise hat, daß die Unvollständigkeit der Familie als Risikofaktor bezeichnet werden könnte. Bei der „Schuldevianz“ und den „Beschimpfungen gegen Lehrer“ schneiden die Kinder, die mit beiden Elternteilen zusammen leben, sogar signifikant schlechter ab, so daß hier bei einem kleinen Teil der Eltern als Ursache Kumulationseffekte durch ein extrem negatives Erziehungsverhalten von Mutter und Vater vorliegen könnten. Meistens bestehen aber keine nachweisbaren Zusammenhänge zwischen den Familienformen auf der einen Seite und dem Täter- oder Opferstatus, der Devianz und Delinquenz sowie den Einstellungen zur Gewalt auf der anderen Seite. Entsprechendes gilt für die Geschwisterkonstellation: Es ist unerheblich für alle Facetten von Gewalthandeln, ob die befragten Schüler in ihren Ursprungsfamilien als Einzelkinder, mit mehreren Geschwistern oder nur unter Jungen aufwachsen.

Die materielle Situation der Familien ist in Ostdeutschland deutlich schlechter als in Westdeutschland - das zeigen die allgemeinen Wirtschaftsdaten ebenso wie Studien zur „,sozialen Ungleichheit bei Kindern“ (vgl. Mansel/Neubauer 1998). Unsere Vergleichsdaten spiegeln dies in Form von höhe- 
rer Arbeitslosigkeit der Schülereltern in Sachsen wider. In der ostdeutschen Stichprobe haben wir zusätzlich nach der finanziellen Situation der Familien gefragt. Etwa $11 \%$ der Probanden sagen, daß es ihren Familien finanziell „schlechter“ (2\% davon ,viel schlechter") geht als dem Durchschnitt, $12 \%$ geht es nach eigenen Angaben „,viel besser“ und ca. $20 \%$ „etwas besser“ als den durchschnittlichen Familien. Zwar lassen sich bei diesen und anderen materiellen und sozialen Indikatoren Unterschiede zwischen Ost- und Westdeutschland nachweisen, sie schlagen sich jedoch nicht in einer höheren Gewaltbereitschaft oder einer stärkeren Beteiligung am Gewalthandeln nieder, so daß unsere Hypothese, die wir in Übereinstimmung mit anderen Untersuchungsergebnissen formuliert hatten, als vorläufig bestätigt gelten kann: Im Bereich der Familienformen, der Geschwisterreihe, der materiellen und sozialen Lage der Familien ist kein deutliches Risikopotential für Gewalthandeln erkennbar. ${ }^{1}$

Das Gegenteil wird für die Sozialisationsbedingungen, das Familienklima und das Erziehungsverhalten in den Familien vermutet, wobei sich gleichzeitig die Frage stellt, ob diese Bedingungen in Ost- und Westdeutschland unterschiedlich sind. Zu diesem Themenbereich wurden insgesamt zwölf Einzelitems zur Beantwortung vorgelegt, die im folgenden an Hand eines Mittelwertvergleichs für Sachsen und Hessen dokumentiert und interpretiert werden sollen. Gleichzeitig wurde eine Faktorenanalyse durchgeführt und drei Faktoren herausgefunden, die als (1) positives Familienklima, (2) restriktives Verhalten der Eltern und als (3) aggressiver Erziehungsstil der Eltern bezeichnet werden. Die Ergebnisse hierzu sind unter Einbeziehung dieser Systematik im Ost-West-Vergleich in Tabelle 5-1 ausgewiesen.

Im Faktor 1 wird ein positives Familienklima beschrieben, man fühlt sich zu Hause wohl, erhält bei Problemen von den Eltern Unterstützung, stimmt mit dem Erziehungsstil der Eltern überein und würde die eigenen Kinder genauso erziehen, wie man von den Eltern erzogen worden ist und wird; bei Fehlern versuchen die Eltern eher durch Gespräche als durch Strafen auf die Kinder einzuwirken. In diesem Faktor ist gleichzeitig ein hoher intergenerativer Übereinstimmungsgrad in Verbindung mit einem starken Vertrauensverhältnis und positiven Gefühlen füreinander thematisiert.

Mit Ausnahme des Items zur Übereinstimmung mit dem elterlichen Erziehungsstil (V63), bei dem die Unterschiede nicht signifikant sind, wird von den hessischen Schülern ein positiveres Familienklima berichtet. Allerdings basieren diese Differenzen auf einem für beide Seiten hohem Niveau der Akzeptanz der Familie und der Eltern. Auch in Sachsen sind es über drei Vier-

1 Allerdings haben Kinder, die nicht mit beiden Elternteilen oder bei den Eltern aufwachsen, die arbeitslos sind, ein etwas niedrigeres Bildungsaspirationsniveau und besuchen überdurchschnittlich häufig eine niedrigere Schulform - haben somit etwas schlechtere Bildungschancen. Daher sind sie auch häufiger - über den Schülerkontext - mit Gewalt konfrontiert und zusätzlichen Risiken für eigenes Gewalthandeln ausgesetzt. Entscheidend ist also auch hier die Problemkumulation; vgl. zur Geschwisterreihe den abweichenden Befund in Kap. 7. 
tel der Kinder und Jugendlichen, die Unterstützung von ihren Eltern in allen Lebenslagen erhalten, und über $80 \%$, die sich zu Hause wohlfühlen. Gleichwohl ist der Faktor „Familienklima“ in Westdeutschland stärker akzentuiert.

\section{Tab. 5-1: Familiale Sozialisationsbedingungen im Ost-West-Vergleich (Mittelwertvergleich)}

Variable Ladung

Sachsen

Hessen

Mittelwert-

unterschied

Faktor 1: "Positives Familienklima“

Zu Hause fühle ich mich sehr wohl. (V62)

.82

4,23

4,38

$-, 1439 * * *$

Wenn ich mal Probleme habe, sind meine

.81

3,95

4,31

$-, 3620 * * *$

Eltern für mich da. (V58)

Meine Kinder würde ich so erziehen, wie

, 0359

(V63)

Wenn ich etwas falsch gemacht oder angestellt habe, reden meine Eltern eher

mit mir als sie mich strafen. (V52)

Faktor 2: "Restriktives Verhalten der Eltern"

Meine Eltern schreiben mir sehr stark vor,

was ich zu tun habe. (V60)

Die hohen Erwartungen meiner Eltern an

meine Schulleistungen setzen mich unter

Druck. (V59)

Meine Eltern erlauben mir viel weniger, als

andere in meinem Alter dürfen. (V57)

Meine Eltern sind gar nicht streng. (V54)

Faktor 3: "Aggressiver Erziehungsstil der Eltern"

Bei uns zu Hause schreien und schimpfen

Zu Hause hat es für mich schon so manches

\section{Einzelitems}

Wenn in meiner Familie etwas wichtiges 
Beim zweiten Faktor geht es um den Erziehungsstil der Eltern, den Grad der Restriktivität; die Eltern haben hohe Erwartungen in bezug auf die Schulleistung und wollen diese den Kindern gegenüber auch durchsetzen. Dazu wird der Erziehungsalltag stark reglementiert; die Eltern sind streng, und die Kinder haben das Gefühl, daß sie weniger Freiheiten besitzen als andere in ihrem Alter.

Mit Ausnahme der elterlichen Strenge (V54), die in Sachsen etwas größer ist, bestehen bei diesem Faktor keinerlei signifikante Ost-West-Unterschiede. D.h., daß es auf beiden Seiten in etwa gleich große Elterngruppen gibt, die ihre Kinder in der beschriebenen Weise - vor dem Hintergrund hoher Leistungserwartungen - steuern, einengen und reglementieren. Die Mittelwerte zeigen, daß dies aber nur für kleinere Gruppen gilt: so sind es bei den beiden Items mit den stärksten Faktorladungen in Sachsen etwa 8\%, die zu starke Vorschriften der Eltern (V60), und etwa 15\%, die zu hohe und Druck erzeugende Erwartungen der Eltern an die Schulleistungen (V59) beklagen. Etwa 20 bis $25 \%$ zeigen sich in beiden Fällen ambivalent, während die Mehrheit dieses zurückweist.

In einem dritten Faktor geht es um Aggressionen der Eltern beim Umgang mit den Kindern, um einen durch Schimpfen, Schreien und zum Teil auch Prügel bestimmten Erziehungsstil. Die Mittelwerte zeigen, daß nur eine kleine - beim Prügeln sehr kleine - Minderheit der Kinder von derartigem Erziehungsverhalten betroffen ist. Bei beiden Items sind die Werte für Hessen aber etwas bzw. deutlich höher, d.h., daß aggressives Erziehungsverhalten in Westdeutschland etwas verbreiteter ist als in Ostdeutschland. Wir führen diesen Befund auf die höhere Emotionalität der Eltern-Kind-Beziehung zurück, die im Positiven (siehe Faktor 1) wie hier im Negativen offenbar für westdeutsche Erziehungsmilieus und hier insbesondere für die Familiensituation typisch ist, während für die sächsischen Familien im Durchschnitt ein etwas distanzierteres Verhalten - ohne diese Extreme - vorzuherrschen scheint.

Bei den beiden Einzelitems (vgl. Tab. 5-1) ergibt die Dateninterpretation günstigere Bedingungen für die Mitbestimmung der Kinder in hessischen Familien, während die sächsischen Kinder und Jugendlichen signifikant häufiger meinen, eine größere Gleichgültigkeit der Eltern ihnen gegenüber registrieren zu können.

Es mag sein, daß diese Befunde in ihrer Differenziertheit bei der hermeneutischen Interpretation von Einzelaspekten hilfreich sein können. Insgesamt überwiegen jedoch die Gemeinsamkeiten in Ost und West. Die Bestätigung für diese Behauptung bringt eine Clusteranalyse, in der alle Facetten der familialen Sozialisation (vgl. Tab. 5-1) berücksichtigt wurden. Nach einer Zwei-Cluster-Lösung differieren die Gruppen mit den negativen bzw. positiven Bedingungen in Sachsen und Hessen nur um wenige Prozentpunkte; zudem sind die Unterschiede lediglich aufgrund der Stichprobengröße signifikant - dies zeigen auch die bivariaten Zusammenhänge (Cluster by Ost/West), die bei nur $\mathrm{r}=.04$ (Phi-Koeffizient) liegen. Bei einer Gesamtbilanz des Familienklimas und der 
familialen Sozialisationsbedingungen gilt also der Grundsatz einer prinzipiellen Übereinstimmung in Ost und West, der auch als Leitlinie für die weitere Elaborierung eines Mehr-Ebenen-Modells berücksichtigt werden muß.

Die noch offene Frage ist die, ob durch derartige Sozialisationsarrangements gewaltförmige und gewaltaffine Verhaltensweisen überhaupt begünstigt bzw. verhindert werden können. Um diese Hypothese zu testen, wurden die bivariaten Zusammenhänge von Familie und Gewalt überprüft (vgl. Tab. 5-2).

Tab. 5-2: Familiale Sozialisation und Gewalthandeln von Kindern (bivariate Zusammenhänge)

\begin{tabular}{|c|c|c|c|c|}
\hline $\begin{array}{l}\text { Familie } \\
\text { Gewalt }\end{array}$ & $\begin{array}{c}\text { Positives } \\
\text { Familienklima }\end{array}$ & $\begin{array}{l}\text { Restrikt. } \\
\text { Elternverhalten }\end{array}$ & $\begin{array}{l}\text { Aggressiver } \\
\text { Erziehungsstil }\end{array}$ & $\begin{array}{l}\text { Gleichgültigkeit der } \\
\text { Eltern }\end{array}$ \\
\hline $\begin{array}{l}\text { Weichere Aggressionen } \\
\text { (Täterstatus) }\end{array}$ & $-.23 * *$ & $.22 * *$ & $.19^{* *}$ & $.10^{* *}$ \\
\hline $\begin{array}{l}\text { Härtere Aggression } \\
\text { (Täterstatus) }\end{array}$ & $-.14 * *$ & $.18^{* *}$ & $.15^{* *}$ & $.11^{* *}$ \\
\hline Opfer sachbezogener Angriffe & $-.09 * *$ & $.13^{* *}$ & $.12^{* *}$ & $.06^{* *}$ \\
\hline $\begin{array}{l}\text { Opfer personenbezogener } \\
\text { Angriffe }\end{array}$ & $-.10^{* *}$ & $.21^{* *}$ & $.19^{* *}$ & .01 \\
\hline $\begin{array}{l}\text { Unbeteiligtsein } \\
\text { (Cluster) }\end{array}$ & $.21 * *$ & $-.23^{* *}$ & $-.20^{* *}$ & $-.05 * *$ \\
\hline Schuldeviantes Verhalten & $-.22 * *$ & $.19 * *$ & $.16^{* *}$ & $.09 * *$ \\
\hline Delinquentes Verhalten & $-.14 * *$ & $.16^{* *}$ & $.11^{* *}$ & $.13^{* *}$ \\
\hline Gewaltbefürwortung & $-.18 * *$ & $.25^{* *}$ & $.19^{* *}$ & $.15^{* *}$ \\
\hline Aggressionsbereitschaft & $-.20 * *$ & $.28^{* *}$ & $.24^{* *}$ & $-.06 * *$ \\
\hline $\begin{array}{l}\text { Diskursive Streitkultur } \\
\text { (V123) }\end{array}$ & $.15^{* *}$ & $-.17^{* *}$ & $-.13^{* *}$ & $.07^{* *}$ \\
\hline
\end{tabular}

** $\quad p<.01$

Alle nicht näher bezeichneten Variablen sind Faktorvariablen.

Bereits auf den ersten Blick bestätigen sich die in unseren Hypothesen formulierten Zusammenhänge durch eine Vielzahl von mehr oder weniger deutlichen Korrelationen auf relativ hohem Signifikanzniveau. Auffällig ist, daß familiale Sozialisationseffekte eher bei weicheren als bei härteren Gewaltformen (z.B. Täter-Selbstreport: weiche Aggressionen, Opfer sachbezogener Angriffe, 
schuldeviantes Verhalten) auftreten, sich deutlich auf die Aggressionsbereitschaft der Kinder auswirken und umgekehrt im Hinblick auf den Status als Unbeteiligter positiv wirken. Je gravierender die Delikte, Normverstöße und Gewalthandlungen sind, um so vergleichsweise geringer ist der familiale Anteil daran. Geht man von einem Kumulations- oder Synergiemodell aus, flieBen bei diesen extremen Formen verschiedene Faktoren zusammen - und die familialen Einflüsse sind nur ein Teil davon.

Differenziert man zwischen den Familienvariablen, lassen sich in erster Priorität Wirkungsfaktoren im Bereich des restriktiven elterlichen Verhaltens erkennen. Dieser Faktor weist in fast allen Fällen die stärkeren Zusammenhangsmaße mit Gewalt auf und ist im Vergleich mit den beiden anderen Faktoren besonders wirksam bei der Entstehung des Opferstatus (personenbezogene Angriffe) und der Aggressionsbereitschaft. Neben den bislang schon eingeführten und bekannten Gewaltvariablen wird auch die soziale Verhaltenskompetenz der Schülerinnen und Schüler bei sich anbahnenden Konflikten, die wir als „,diskursive Streitkultur“ bezeichnen (,Wenn ich mit anderen Streit bekomme, kann ich das meist durch Reden klären“), mit in den Blick genommen; unserer Daten zeigen, daß diese Fähigkeit in Ansätzen bereits in der Familie erworben wird. Auf der anderen Seite kann die Gleichgültigkeit elterlichen Verhaltens (,Meinen Eltern ist es egal, was ich mache“) bei einzelnen Gewaltaspekten ebenfalls als Prädiktor gelten, insbesondere für die Gewaltbefürwortung, das delinquente Verhalten und den Täterstatus. ${ }^{2}$

Zusammenfassend bleibt festzustellen: Es zeigt sich in den Analysen eine eindrucksvolle Bestätigung der Einflüsse familialer Sozialisation auf das Gewaltverhalten der Kinder in schulischen und außerschulischen Lebenszusammenhängen, wobei die verschiedenen Aspekte des Sozialisationsarrangements auf unterschiedliche Formen des Gewaltverhaltens spezifische Wirkungen zeigen.

Das Problem von Korrelation und Kausalität stellt sich bei der familialen Sozialisation weniger, da Eltern für sich bestimmte Lebensziele und in Auseinandersetzung mit ihrer Biographie auch bestimmte Erziehungsvorstellungen entwickelt haben und diese Einstellungen und Orientierungen nach der Geburt ihrer Kinder auf die konkrete Erziehungssituation anwenden und ggf. später modifizieren. Interaktion und Wechselbezüge werden mit zunehmendem Alter der Heranwachsenden bedeutsamer, so daß auch in diesem Falle nicht auszuschließen ist, daß das Verhalten der Kinder den Erziehungsstil der Eltern mit prägt und die Eltern z.B. in dem Maße ,die Zügel stärker anziehen“ und sich strenger zeigen, in dem das Verhalten der Kinder in der Schule problematischer wird und sich diese dort vielleicht zu devianten und delinquenten Verhaltensweisen hinreißen lassen.

$2 \mathrm{Da} \beta$ die Korrelationen in diesen Fällen nicht so deutlich ausfallen, liegt daran, daß es sich um Einzelitems und nicht um Faktorvariablen handelt. 
In der Tabelle 5-2 wurde die Aggressionsbereitschaft als Variable des Gewaltsyndroms eingeführt - ein Faktor, der aus fünf Einzelitems besteht, in denen in der Regel unabhängig von Situationen und Interaktionen die Reizbarkeit als Charakteristikum der Persönlicheitsstruktur thematisiert wird (z.B. „Ich gerate schnell in Wut.“, „Ich steigere mich schnell in einen Streit hinein.“). Eine hohe Aggressionsbereitschaft von Kindern wurde als mögliche Folge - vor allem - des restriktiven Verhaltens der Eltern angesehen und ist z.B. lerntheoretisch begründbar. Gleichzeitig zeigt das restriktive Erziehungsverhalten der Eltern Auswirkungen auf den Täterstatus und trägt dazu bei, daß in dieser Weise erzogene und reglementierte Kinder eher dazu neigen, Gewalt gegen andere auszuüben. Ebenso dürfte der Zusammenhang von Aggressionsbereitschaft zu diesen aggressiven Handlungen von Interesse sein; in Abb. 5-1 ist diese Gewaltkonfiguration dokumentiert.

\section{Abb. 5-1: Familiale Sozialisation, Aggressionsbereitschaft und Gewalthandeln (Bivariate Zusammenhänge)}

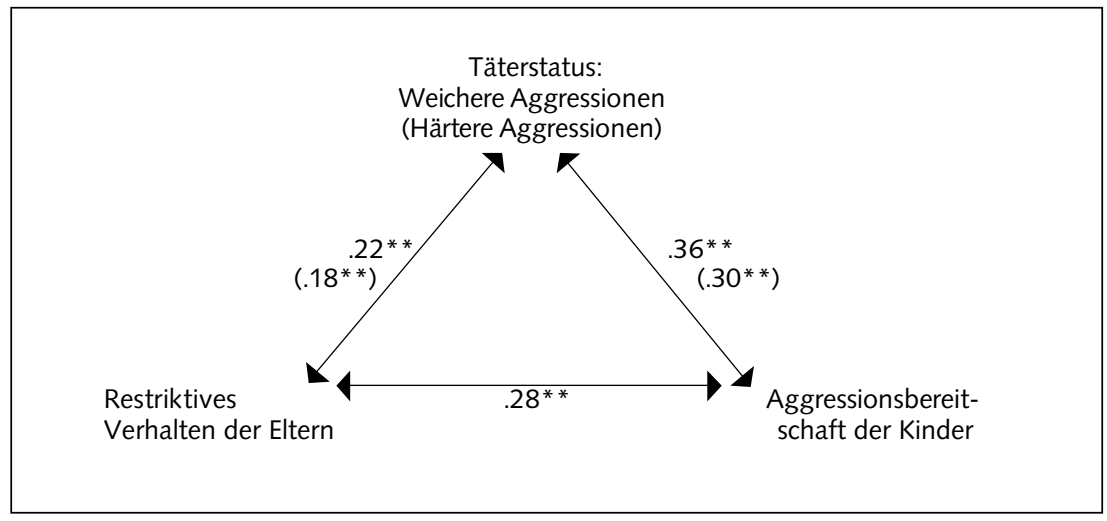

In der Graphik zeigt sich eine - unter steuerungstheoretischen Gesichtspunkten deutlich werdende Relevanz der Aggressionsbereitschaft als einer individuellen Prädisposition, die zwar z.T. auf das restriktive elterliche Erziehungsverhalten mit zurückgeführt werden kann, die aber erst in ihrer biographischen Synthese einen relevanten und gewichtigen Prädiktor für aggressive Gewalthandlungen darstellt. Die Zusammenhänge von Aggressionsbereitschaft - als einem Merkmal der Persönlichkeitsstruktur - und dem manifesten Aggressionshandeln sind deutlich höher als die durch das restriktive Erziehungsverhalten der Eltern verursachten Aggressionen. Im Hinblick auf unser theoretisches Gesamtmodell kommt daher der Aggressionsbereitschaft, die zu einem großen Teil durch die familialen Sozialisationsbedingungen mit geprägt ist, in ihrer Funktion 
als persönlichkeitsinterner Antriebs- und Steuerungskomponente, wie sie in psychoanalytischen Aggressionstheorien betont wird, ein zentraler Stellenwert zu (vgl. Kap.7).

Um die Analyse familialer Sozialisationsbedingungen und ihrer Auswirkungen auf schulisches Gewalthandeln abzurunden, wollen wir zum Abschluß die Bedeutung von Familienklima und Erziehungsstil im Hinblick auf die Konstituierung einer Täter-Opfer-Typologie herausarbeiten. Dazu wurden in einer gesamtdeutschen Stichprobe alle klimatischen und sozialisatorischen Bedingungen der Familie in eine Clusteranalyse eingebracht und die Ergebnisse einer Zwei-Cluster-Analyse mit den fünf im Kapitel 4 entwickelten Handlungstypen gekreuzt. Die Ergebnisse sind in Abb. 5-2 und Tab. 5-3 dargestellt.

Abb. 5-2: Familienklima und familiale Sozialisationsbedingungen (Ergebnisse der Clusteranalyse)*

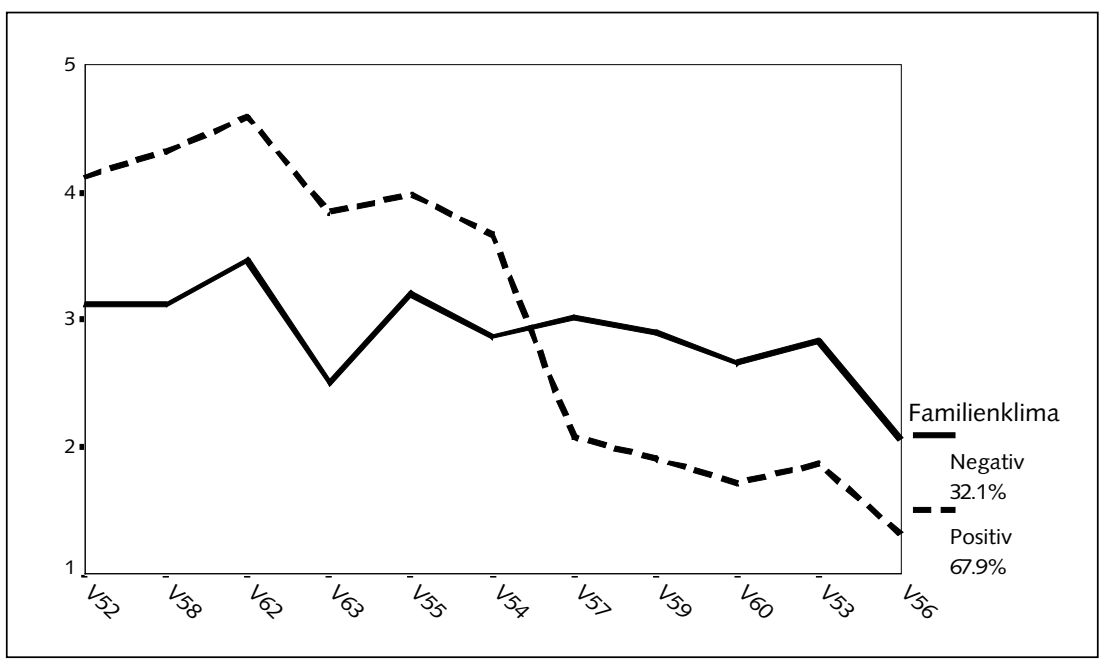

* Legende vgl. Tab. 5-1

Wie aus den Randsummen der Tab. 5-3 und der Clusterdarstellung in der Abb. 5-2 ersichtlich, zählt in Sachsen etwa ein Drittel der befragten Schülerinnen und Schüler zu dem Cluster mit negativen klimatischen und sozialisatorischen Bedingungen in den Familien, während drei Viertel von ihnen unter günstigeren Einflüssen aufwachsen. Während also im Durchschnitt etwa $32 \%$ in problematischen Familienkontexten aufwachsen, gilt dieser Sozialisationshintergrund für 53\% der ,harten“ Tätergruppe (,Täter-Opfer“) und fällt auch bei den übrigen Gruppen der Gewaltbeteiligten - also auch der Opfer - überdurch- 
schnittlich aus, während nur etwa ein Viertel der Unbeteiligten aus derartigen Verhältnissen stammt. Umgekehrt sind es drei Viertel dieser nicht in Gewalthandlungen involvierten Schüler, die einen positiven familialen Hintergrund haben. Damit ist der Einfluß der häuslichen Umgebung und der familialen Erziehungsverhältnisse auf das Gewaltverhalten der Kinder evident; statistisch drückt sich dies in einer deutlichen Korrelation der beiden Cluster-Variablen „Familienkontext“" und „Täter-Opfer-Status“ aus (Cramer’s V=.19**).

Tab. 5-3: Familienkontext und Täter-Opfer-Status (Kreuztabelle, Angaben in Prozent)

\begin{tabular}{lcccccc}
\hline Familienklima & Opfer & $\begin{array}{c}\text { Täter- } \\
\text { Opfer }\end{array}$ & Täter & $\begin{array}{c}\text { Episoden- } \\
\text { Täter }\end{array}$ & Unbeteiligte & Total \\
Negativ & 43,0 & 53,0 & 43,7 & 39,2 & 24,5 & 32,1 \\
Positiv & 57,0 & 47,0 & 56,3 & 60,8 & 75,5 & 67,9 \\
\hline
\end{tabular}

Sowohl dieser Wert mit seinen „Residuen“3 ${ }^{\circ}$ als auch die Verteilungen in der Kreuztabelle (vgl. Tab. 5-3) verweisen darauf, daß eine Reihe weiterer gewichtiger Prädiktoren für das Gewalthandeln der Kinder existieren müssen, die hinzukommen und möglicherweise einschlägiger sind, denn: über $60 \%$ der „Episodentäter“ und auch die Mehrheit in den Gruppen der „Täter“ und der „Opfer" besitzt diesen Status, obwohl die Rollenträger in günstigen familiären Verhältnissen aufgewachsen sind.

Abschließend zu dem Familienkapitel wäre noch die „Schicht“ als makrosozialer Aspekt der Herkunftsfamilie in ihrer Bedeutung für das Gewalthandeln zu untersuchen. Die Forschungslage hierzu ist widersprüchlich: häufig ist dieser Aspekt gar nicht untersucht worden, in einem anderen Teil der Studien werden Zusammenhänge von sozialer Herkunft und Gewalthandeln ermittelt (vgl. u.a. Brusten/Hurrelmann 1973, Mansel/Hurrelmann 1991, Würtz/Hamm/ Willems/Eckert 1996). Die differenzierten Theoriedebatten um die Relevanz von "Schicht“ und „Milieu“ in „pluralistischen Wohlfahrtsgesellschaften“ (Hradil 1994) bzw. in einer Epoche der „Transformation der industriegesellschaftlichen Sozialstruktur" (Vester 1997) sowie das Zusammenspiel dieser beiden Modelle (vgl. Berger/Vester 1998) lassen sich u.E. in einer empirischen Untersuchung wie dieser, die ganz anderen Zielsetzungen folgt, nicht einfach nebenbei prüfen. Dies müßte das Thema von Spezialstudien sein, die zu unserem Themenbereich bereits vorgelegt worden sind (vgl. u.a. Heitmeyer/ Ulbrich-Herrmann 1997) - allerdings mit einem hochgradig spezialisierten In-

3 Denn gleichzeitig kommt in der Höhe dieses Wertes seine eingeschränkte Erklärungskraft zum Ausdruck. 
strumentarium, das den Rahmen unserer Untersuchung gesprengt hätte. Dennoch wollen wir diese Problematik nicht ganz ausklammern, auch wenn wir sie aus methodischen Gründen nicht sehr tiefschürfend bearbeiten können.

Mit Hilfe der „Nicht-linearen Hauptkomponenten-Analyse“ (PRINCALS) wurde auf der Basis von vier Variablen (Schul- und Berufsabschluß von Mutter und Vater, Beruf von Mutter und Vater) ein Schicht-Konstrukt gebildet. Die Angaben dazu stammen allerdings von Schülern, die z.T. über diese Dinge nicht immer hinreichend informiert sind. Das Schichtmodell hat sich, so sehr wir es aus den genannten Gründen kritisch betrachtet haben, bei der Untersuchung einiger wichtiger bildungssoziologischer Zusammenhänge bewährt. So konnte u.a. ein starker Zusammenhang von sozialer Herkunft zur Bildungsaspiration $\left(\mathrm{r}=.41^{* *}\right)$ sowie zur besuchten Schulform $\left(\mathrm{r}=.38^{* *}\right)$ nachgewiesen werden, wie er auch in vielen anderen Studien bestätigt wird. Dieses - so bewährte - Schichtmodell wurde in eine bivariate Zusammenhangsanalyse mit dem Gewalthandeln eingebracht. Die Ergebnisse waren für die hier zugrundegelegten Individualdaten auf ganzer Linie negativ. Lediglich bei den Items, in denen Gewalt gerechtfertigt, abgelehnt oder als animierend bezeichnet wird, und bei der Kompetenz der Streitbewältigung (V123) gibt es nennenswerte Zusammenhänge $\left(r>.10^{* *}\right)$ in der Weise, daß Kinder aus unteren Sozialschichten etwas anfälliger für diese Deutungsmuster sind bzw. geringere Bewältigungskompetenzen besitzen. Bei allen Formen der Gewaltbeobachtung, des Täterbzw. Opferselbstreports, der Schuldevianz und Delinquenz lassen sich jedoch keine direkten Schichteinflüsse nachweisen; alle Werte sind nicht signifikant.

Allerdings zeigen sich bei den Variablen zum Familienkontext leichte Schichtspezifika, die differenziertere Konfigurationen vermuten lassen, insbesondere wenn Kumulationseffekte auf Klassenebene auftreten (vgl. Kap. 6). Daher sollen im folgenden alle zu untersuchenden Sozialisations- und Kontextvariablen im außerschulischen Bereich auf mögliche schichtspezifische Unterschiede untersucht werden. Zum Abschluß des Kapitels wird versucht, die Gesamtstruktur von Kontexteinflüssen und Sozialschichtzugehörigkeit zusammenfassend in den Blick zu nehmen.

\subsection{Medieneinflüsse}

Eine weitere Sozialisationsinstanz neben der Familie und in enger Verbindung mit ihr sind die Medien, insbesondere die elektronischen Medien. Wegen der immer stärker werdenden Mediatisierung des Alltags gewinnt diese Instanz immer mehr an Bedeutung. Meister und Sander (1998) kommen bei der Rezension von elf aktuellen Veröffentlichungen im Bereich „Kindheit und Jugend in der Mediengesellschaft“ zu folgendem Ergebnis: „Die Massenmedien, die neuen multimedialen und vernetzten Medien sowie die medial angereicher- 
ten Freizeit- und Konsumräume gelten heute neben Elternhaus, Schule und den Gleichaltrigengruppen als weitere wichtige Sozialisationsinstanzen, die wesentlich das Leben in den Phasen Kindheit und Jugend bestimmen“ (ebd., S. 6). Danach nimmt der Einfluß von Eltern und Lehrern in vielen Alltagssituationen ab, weil die Kinder ihre Deutungs- und Handlungsmuster vermehrt u.a. durch den Medienkonsum entwickeln. Schorb, Mohn und Theunert (1991) stellen fest, daß die neuen Informations- und Kommunikationstechniken zu einer Stärkung der Rolle der Medien im Sozialisationsprozeß führen. „Die Medien sind Arbeits-, Informations-, Kommunikations- und Unterhaltungsmittel in einem. (...) Sie sind Bestandteil aller Lebensbereiche, organisieren ihre Vernetzung, ihr Ineinandergreifen und z.T. ihr Ineinandergehen“" (ebd., S. 507f). In diesem Zusammenhang ist zu fragen, ob und inwieweit Gewaltdarstellungen in den Medien einen Einfluß auf die Einstellungen der Kinder und Jugendlichen zur Gewalt und ihre Konfliktlösungsstrategien haben. Wir werden in diesem Abschnitt die Zusammenhänge hauptsächlich auf einer bivariaten Analyseebene prüfen. Wir berücksichtigen dabei mehrere Dimensionen des Medienkonsums (Häufigkeit des Video- und Fernsehens, Art der Filme und Sendungen, Einmischung der Eltern bei der Bestimmung der zu konsumierenden Filme und Serien sowie die Medienausstattung des Haushalts) und mehrere Gewaltindikatoren (Täterverhalten, Opfer-Status, Unbeteiligtsein, Schuldevianz, Delinquenz, Aggressionsbereitschaft und Gewaltbefürwortung; vgl. Kap. 4).

\section{Häufigkeit des Video- und Fernsehkonsums bei verschiedenen Filmarten und Sendungen}

Die Häufigkeit des Video- und Fernsehens wurde (in Sachsen) für die Werktage und Wochenendtage getrennt erhoben. Die Befragten haben angegeben, wie lange sie durchschnittlich am Tag mit Fernsehen oder Video verbringen (V34a und V34b). Das ergab einen Mittelwert von drei Stunden an einem Werktag und knapp viereinhalb Stunden an einem Wochenendtag. Hinsichtlich eines eventuellen Zusammenhangs zwischen der Mediennutzung der Kinder und Jugendlichen und ihrer Gewalttätigkeit ist nicht nur die Dauer des Video- und Fernsehens, sondern auch die Art der konsumierten Sendungen von Bedeutung. Deshalb wurden die Probanden nach der Häufigkeit des Sehens von neun Film- und Sendungsarten während der letzten zwei Wochen gefragt. Tabelle 5-4 zeigt die Mittelwerte der entsprechenden Items. Die Sendungen, die normalerweise keine oder nur selten Gewaltszenen beinhalten, werden insgesamt öfter gesehen als Gewalt- und Sexfilme (vgl. die obere Hälfte der Tabelle mit der unteren). ${ }^{4}$

4 Die Liste der Filmarten und Sendungen ist nicht vollständig. Es fehlen einige wichtige Genres wie Sport und Werbung. 
Tab. 5-4: Durchschnittliche Video- und Fernsehdauer bei neun Sendungsarten

\begin{tabular}{|c|c|c|c|}
\hline $\begin{array}{l}\text { Bezogen auf die letzten } 2 \text { Wochen: } \\
\text { Wie oft hast Du folgende Filmarten und } \\
\text { Sendungen gesehen? }\end{array}$ & Sachsen & Hessen & Differenz \\
\hline V36. Spielfilme, Serien & 3,40 & 3,19 &, $21^{* * *}$ \\
\hline V40. Unterhaltung, Musik, Quiz & 2,48 & 2,33 &, $15^{* * *}$ \\
\hline V42. Kinder- und Jugendfilme, Comics & 2,27 & 2,37 &,$- 10^{* *}$ \\
\hline V38. Informationsfilme, Tierfile & 1,88 & 1,81 &, $07 * *$ \\
\hline V43. Science Fiction (Zukunftsfilme, Weltall) & 2,06 & 1,97 &, $10 * *$ \\
\hline V35. Krimis, Thriller, Western & 1,67 & 1,79 &,$- 12 * * *$ \\
\hline V37. Actionfilme (z.B. Rambo, Karate) & 1,80 & 1,92 &,$- 12 * * *$ \\
\hline V39. Horror- und Gewaltfilme (z.B. Zombies) & 1,63 & 1,75 &,$- 11 * * *$ \\
\hline V41. Porno- und Sexfilme & 1,41 & 1,43 &,- 02 \\
\hline
\end{tabular}

Skala: $1=0 \mathrm{mal}, 2=1-2 \mathrm{mal}, 3=3-5 \mathrm{mal}, 4=6-10 \mathrm{mal}, 5=\mathrm{mehr}$ als $10 \mathrm{mal}$

\section{Medienkonsum und Familienklima}

Da die Schüler vor allem in der elterlichen Wohnung Video und Fernsehen konsumieren und viele Eltern versuchen, den (vermeintlich oder tatsächlich) negativen Einflüssen bestimmter Filme entgegenzuwirken, gibt es häufig Konflikte zwischen den Eltern und Kindern bezüglich des Fernsehverhaltens der Kinder. Viele Eltern beispielsweise verbieten ihren Kindern, bestimmte Serien, Fernseh- oder Videofilme zu schauen. Das gilt für die Eltern von 33,6\% der sächsischen und $41,1 \%$ der hessischen Befragten (V44). ${ }^{5}$ Eine Überprüfung in der sächsischen Stichprobe hat gezeigt, daß es zwischen den oberen und unteren Bevölkerungsschichten keinen Unterschied bezüglich dieser Verbote gibt.

Die Befragten, bei denen bestimmte Verbote gelten, weisen eine kürzere Sehdauer auf (in Sachsen werktags 12 Minuten, am Wochenende etwa 35 Minuten weniger). Diese Abnahme der Sehdauer geht nicht nur zu Lasten der Gewalt- und Sexfilme, auch der Konsum von Serien, Unterhaltungs-, Musik-

5 V44: „Verbieten Dir Deine Eltern, bestimmte Fernseh-/Videofilme (nur in Sachsen: oder Serien) zu schauen?“ (1=ja; $2=$ nein). 
und Spielfilmen reduziert sich dadurch. Comics, Kinder- und Jugendfilme, Informations- und Tierfilme werden dagegen häufiger gesehen.

Mehr als ein Drittel der Kinder, die solchen Verboten ausgesetzt sind, ignoriert diese - eigenen Angaben zufolge - und schaut die umstrittenen Filme trotz Verbot an (V45). ${ }^{6}$ Die meisten Befragten, bei denen es keine Verbote gibt und die deshalb die Frage 45 eigentlich nicht zu beantworten hatten, haben dennoch geantwortet $(63,2 \%$ in Sachsen und $68,8 \%$ in Hessen). Unter diesen „,ungültigen“ Antworten gibt es in der sächsischen Stichprobe 47,8\% und in der hessischen 39,5\% Ja-Antworten. Das bedeutet, daß sich viele Schüler, bei denen es keine elterlichen Video- und Fernsehverbote gibt, über solche Verbote hinweggesetzt hätten. Es ist nicht auszuschließen, daß es in vielen Familien elterliche Verbote nicht (mehr) gibt, weil sie nicht befolgt werden bzw. wurden. In der Tat weist diese Gruppe in den beiden Stichproben die höchste Sehdauer auf. Sie konsumieren mehr als alle anderen Gewalt- und Sexfilme sowie Spielfilme, Serien, Unterhaltung, Musik und Quiz. Dafür sehen sie sich am wenigsten Informations- und Tierfilme sowie in Sachsen Comics, Kinderund Jugendfilme an.

Tab. 5-5: Einfluß der familialen Sozialisation auf das Sehverhalten der Kinder (bivariate Korrelationen; Stichprobe Sachsen)

\begin{tabular}{lccccc}
\hline Genre & $\begin{array}{c}\text { Positives } \\
\text { Familienklima }\end{array}$ & $\begin{array}{c}\text { Restrikt. } \\
\text { Elternver- } \\
\text { halten }\end{array}$ & $\begin{array}{c}\text { Aggr. } \\
\text { Erziehungsstil }\end{array}$ & $\begin{array}{c}\text { Gleich- } \\
\text { gültigkeit } \\
\text { der Eltern }\end{array}$ & $\begin{array}{c}\text { Schicht- } \\
\text { zugehörigkeit }\end{array}$ \\
Spielfilme, Serien & -.01 & $-.04^{*}$ & -.01 & .01 & -.03 \\
Unterhaltung, Musik, Quiz & -.03 & $-.06^{* *}$ & .00 & .04 & $-.05^{* *}$ \\
Kinder-/Jugendfilme, Comics & $.07^{* *}$ & .02 & $.06^{* *}$ & .00 & $-.12^{* *}$ \\
Informationsfilme, Tierfilme & $.09^{* *}$ & .01 & .03 & -.01 & -.02 \\
Science Fiction & -.02 & $.09^{* *}$ & $.05^{*}$ & $.04^{*}$ & -.01 \\
Krimis, Thriller, Western & .00 & .04 & $.06^{* *}$ & $.07^{* *}$ & -.01 \\
Actionfilme & -.03 & $.11^{* *}$ & $.08^{* *}$ & $.11^{* *}$ & $-.11^{* *}$ \\
Horror- und Gewaltfilme & $-.13^{* *}$ & $.14^{* *}$ & $.10^{* *}$ & $.14^{* *}$ & $-.09^{* *}$ \\
Porno- und Sexfilme & $-.10^{* *}$ & $.13^{* *}$ & $.09^{* *}$ & $.11^{* *}$ & $-.06^{* *}$ \\
Sehdauer Werktags & $-.09^{* *}$ & .02 & $.08^{* *}$ & $.09^{* *}$ & $-.20^{* *}$ \\
Sehdauer Wochenende & $-.10^{* *}$ & $.06^{* *}$ & $.10^{* *}$ & $.07^{* *}$ & $-.18^{* *}$ \\
\hline
\end{tabular}

6 V45: „Wenn ja, schaust Du trotz Verbot?“ (1=ja; 2=nein) 
Die Mediennutzung der Kinder hängt nicht nur mit den elterlichen Verboten zusammen, sondern auch mit dem Familienklima insgesamt. Wie die Tabelle 5-5 zeigt, führt ein positives Familienklima zur einer Abnahme der gesamten Sehdauer und des Konsums von Gewalt- und Sexfilmen. Die Sehdauer bei den Informations- und Tierfilmen sowie Kinder- und Jugendfilmen in Familien mit positivem Erziehungsklima ist dagegen etwas überdurchschnittlich. Restriktives Verhalten der Eltern geht auch mit vermehrtem Konsum von Action-, Gewalt- und Sexfilmen einher. Ähnlich verhält es sich auch, wenn die Eltern den Kindern gegenüber aggressiv und/oder gleichgültig sind. Die Zugehörigkeit zu den oberen Schichten senkt den Video- und Fernsehkonsum insgesamt. Das gilt vor allem für die Kinder- und Jugendfilme, Actionfilme sowie für Horror- und Gewaltfilme.

Die Fernsehdauer und die Art der konsumierten Filme hängt auch damit zusammen, welche elektronischen Medien zu Hause vorhanden sind bzw. zur alleinigen Verfügung der Kinder stehen. Wir haben nicht nach dem Vorhandensein eines Fernsehgerätes zu Hause gefragt, weil es sowohl im Westen als auch im Osten kaum Familien gibt, die kein Fernsehgerät haben. Ein eigenes Fernsehgerät haben knapp zwei Drittel der Schüler (64,3\% in Sachsen und 63,8\% in Hessen). 79,4\% der sächsischen und 86,4\% der hessischen Schüler haben ein Videogerät zu Hause; 20,4\% bzw. 28,6\% haben ein eigenes Videogerät. Auch die „,neuen“ Medien sind für die meisten Familien nicht mehr neu. 55,6\% der sächsischen und 65,3\% der hessischen Befragten geben an, zu Hause einen Homecomputer bzw. PC zu haben. $34,8 \%$ bzw. $44,2 \%$ besitzen selber ein solches Gerät. Etwa zwei Drittel der Schüler besitzen ein Telespiel bzw. einen Gameboy (64,9\% in Sachsen und 67,6\% in Hessen).

Um zu überprüfen, welchen Einfluß die Medienausstattung auf die Video- und Fernsehdauer und auf die Art der konsumierten Programme hat, vergleichen wir das Sehverhalten der Schüler, die ein bestimmtes Gerät zu Hause bzw. zur eigenen Verfügung haben, mit dem derjenigen, bei denen dies nicht der Fall ist. Dazu haben wir die Mittelwerte der Sehdauer aller Genres und die gesamte Sehdauer in jeder Gruppe berechnet und beide miteinander verglichen. Mittels statistischer t-Tests wurden die Unterschiede auf ihre Signifikanz geprüft. Die Ergebnisse haben gezeigt, daß die Ausstattung mit allen Medien (mit Ausnahme des Homcomputers/PCs zu Hause) die gesamte Sehdauer in den beiden Stichproben signifikant erhöht. Das gilt insgesamt eher für Gewalt- und Sexfilme. Das Vorhandensein eines Videogeräts zu Hause erhöht die Sehdauer insbesondere bei den Action-, Horror- und Gewaltfilmen sowie den Science Fiction und etwas moderater im Bereich Unterhaltung, Musik, Quiz, Spielfilme, Serien und Porno. Der Konsum von Kinder- und Jugendfilmen, Comics, Informations und Tierfilmen, Krimis, Thrillern und Western hängt dagegen mit dem Vorhandensein des Videogeräts zu Hause nicht zusammen. Offensichtlich wird bei diesen Genres ein solches Gerät nicht bevorzugt eingesetzt. Wenn ein Videogerät den Kindern zur alleinigen Verfügung steht, verringert sich sogar ihre Sehdauer bei den Kinder- und Jugendfilmen und 
Comics (nur in Sachsen) sowie Informations- und Tierfilmen. Dafür werden um so mehr Gewalt- und Sexfilme (in Hessen auch Unterhaltung, Musik und Quiz) geschaut. Die Verfügung über einen eigenen Fernseher verringert die Sehdauer bei den Informations- und Tierfilmen und erhöht sie für alle anderen Gattungen (mit Ausnahme der Comics, Kinder- und Jugendfilme in Sachsen) und insbesondere bei den Gewaltdarstellungen.

\section{Medienkonsum und Gewaltverhalten}

Im folgenden prüfen wir den Zusammenhang zwischen dem Mediennutzungsverhalten und dem Gewaltverhalten der Schüler. Die Analyse basiert auf sächsischen Daten. Als Gewaltindikatoren dienen dieselben Faktoren und Variablen wie in Kapitel 5.1. Tab. 5-6 zeigt die Korrelationskoeffizienten zwischen den Gewaltfaktoren und der Sehdauer an Werktagen bzw. am Wochenende. Die Sehdauer korreliert am stärksten mit der Gewaltbefürwortung: je häufiger die Schüler Video und/oder Fernsehen konsumieren, desto mehr sind sie der Meinung, daß die Gewalt etwas selbstverständliches, normales und notwendiges ist. Längere Sehdauer geht auch mit mehr Täterverhalten, Delinquenz, Schuldevianz und Aggressionsbereitschaft einher. Bei den OpferVariablen ist dagegen kein bzw. nur ein schwacher Zusammenhang mit der Sehdauer festzustellen. Die an der schulischen Gewalt Unbeteiligten zeichnen sich durch eine unterdurchschnittliche Sehdauer aus. Das gilt auch für die Schüler mit hoher „Streitkompetenz“, d.h. diejenigen, die meistens ihre Streitigkeiten durch Reden klären können.

Tab. 5-6: Zusammenhänge zwischen Gewaltvariablen und Konsum von Video/Fernsehen (bivariate Korrelationen; Stichprobe Sachsen)

\begin{tabular}{lcc}
\hline & Sehdauer werktags & Sehdauer wochenendtags \\
Täter: weichere Aggressionen & $.14^{* *}$ & $.18^{* *}$ \\
Täter: härtere Aggressionen & $.13^{* *}$ & $.14^{* *}$ \\
Opfer: sachbezogene Angriffe & $.05^{* *}$ & $.06^{* *}$ \\
Opfer: personenbezogene Angriffe & .03 & .01 \\
Unbeteiligtsein (Cluster) & $-.09^{* *}$ & $-.12^{* *}$ \\
Schuldeviantes Verhalten & $.11^{* *}$ & $.15^{* *}$ \\
Delinquentes Verhalten & $.08^{* *}$ & $.14^{* *}$ \\
Gewaltbefürwortung & $.20^{* *}$ & $.23^{* *}$ \\
Aggressionsbereitschaft & $.10^{* *}$ & $.11^{* *}$ \\
Diskursive Streitkultur (V123) & $-.13^{* *}$ & $-.11^{* *}$ \\
\hline
\end{tabular}


Tab. 5-7: Korrelative Zusammenhänge zwischen den Gewaltvariablen und dem Konsum von verschiedenen Filmarten und Sendungen (Sachsen)

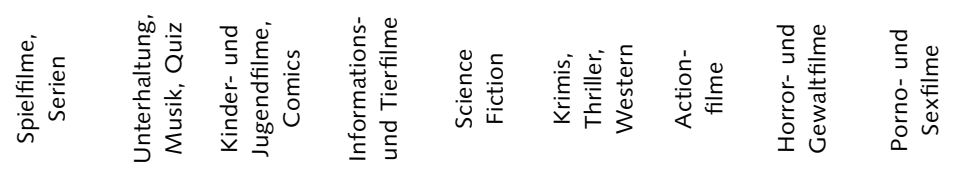

\begin{tabular}{|c|c|c|c|c|c|c|c|c|c|}
\hline $\begin{array}{l}\text { Täter: } \\
\text { weichere } \\
\text { Aggres- } \\
\text { sionen }\end{array}$ & .02 & $.04^{*}$ & $-.06 * *$ & $-.13^{* *}$ & $.16^{* *}$ & $.12^{* *}$ & $.28 * *$ & $.41^{* *}$ & $.36 * *$ \\
\hline $\begin{array}{l}\text { Täter: } \\
\text { härtere } \\
\text { Aggres- } \\
\text { sionen }\end{array}$ & $-.05^{* *}$ & -.03 & -.03 & $-.06 * *$ & $.15^{* *}$ & $.11 * *$ & $.28 * *$ & $.40^{* *}$ & $.37 * *$ \\
\hline $\begin{array}{l}\text { Opfer: } \\
\text { sachbe- } \\
\text { zogene } \\
\text { Angriffe }\end{array}$ & -.02 & .02 & $.04^{*}$ & $.04^{*}$ & $.08 * *$ & $.09 * *$ & $.15^{* *}$ & $.18^{* *}$ & $.17^{* *}$ \\
\hline $\begin{array}{l}\text { Opfer: } \\
\text { personen- } \\
\text { bezogene } \\
\text { Angriffe }\end{array}$ & -.01 & -.03 & $.11 * *$ & $.07 * *$ & $.08 * *$ & $.09 * *$ & $.10^{* *}$ & $.11 * *$ & $.13^{* *}$ \\
\hline $\begin{array}{l}\text { Unbe- } \\
\text { teiligtsein } \\
\text { (Cluster) }\end{array}$ & -.01 & -.01 & .01 & $.05^{* *}$ & $-.15^{* *}$ & $-.12^{* *}$ & $-.23^{* *}$ & $-.29 * *$ & $-.28^{* *}$ \\
\hline $\begin{array}{l}\text { Schuld- } \\
\text { eviantes } \\
\text { Verhalten }\end{array}$ & -.01 & $.04^{*}$ & $-.08^{* *}$ & $-.14^{* *}$ & $.14^{* *}$ & $.11^{* *}$ & $.24^{* *}$ & $.38^{* *}$ & $.37 * *$ \\
\hline $\begin{array}{l}\text { Delin- } \\
\text { quentes } \\
\text { Verhalten }\end{array}$ & $-.08 * *$ & -.02 & -.00 & $-.07 * *$ & $.14^{* *}$ & $.08^{* *}$ & $.22 * *$ & $.37^{* *}$ & $.34 * *$ \\
\hline $\begin{array}{l}\text { Gewalt- } \\
\text { befür- } \\
\text { wortung }\end{array}$ & -.02 & .01 & $-.07^{* *}$ & $-.13 * *$ & $.16^{* *}$ & $.11 * *$ & $.34^{* *}$ & $.47^{* *}$ & $.42 * *$ \\
\hline $\begin{array}{l}\text { Aggressi- } \\
\text { onsbereit- } \\
\text { schaft }\end{array}$ & $.04^{*}$ & .03 & .02 & $-.04^{*}$ & $.09 * *$ & $.06^{* *}$ & $.19 * *$ & $.24 * *$ & $.20^{* *}$ \\
\hline $\begin{array}{l}\text { Diskursive } \\
\text { Streitkultur } \\
\text { (V123) }\end{array}$ & -.01 & $.05^{* *}$ & $-.08^{* *}$ & .01 & $-.11^{* *}$ & $-.05 *$ & $-.19 * *$ & $-.15^{* *}$ & $-.15^{* *}$ \\
\hline
\end{tabular}


Nicht nur die Sehdauer insgesamt sondern auch die Art der konsumierten Filme hängt mit den Gewaltindikatoren zusammen. Tab. 5-7 zeigt analog zu Tab. 5-6 die Korrelationen zwischen den Gewaltvariablen einerseits und den einzelnen Filmarten andererseits. Der häufige Konsum von Gewalt- und Sexfilmen - und in geringerem Maße Science Fiction, Krimis, Thriller und Western - hängt mit einer positiveren Einstellung zur Gewalt, einem stärkeren Aggressionspotential, einem aggressiveren Verhalten sowie mehr Schuldevianz und Delinquenz zusammen. Auch die Opfer konsumieren diese Filmgattungen überdurchschnittlich häufig, wenn auch weniger als die Täter. Die Opfer zeichnen sich auch durch eine leicht überdurchschnittliche Sehdauer bei Kinder- und Jugendfilmen sowie Informations- und Tierfilmen aus. Das hängt aber in erster Linie damit zusammen, daß die Opfer eher jünger sind. ${ }^{7}$ Die Unbeteiligten weisen eine unterdurchschnittliche Sehdauer bei Gewalt- und Sexfilmen sowie Science Fiction, Krimis, Thriller und Western auf. Auch eine diskursive Streitkultur geht mit einem niedrigeren Konsum von Gewalt- und Sexfilmen einher.

Wir haben bereits festgestellt, daß die alleinige Verfügung über einen Fernseher, ein Videogerät oder einen Homcomputer/PC sowie das Vorhandensein eines Videogeräts oder eines Homcomputers/PCs zu Hause die Sehdauer insgesamt und insbesondere bei den Gewalt- und Sexfilmen erhöht. Wir überprüfen jetzt den Zusammenhang zwischen der Ausstattung mit den genannten Geräten und dem Gewaltverhalten der Schüler. Tabelle 5-8 zeigt die entsprechenden Ergebnisse. Die Eintragungen sind die Mittelwertunterschiede bei den Gewaltfaktoren und -variablen zwischen den Schülern, die das entsprechende Gerät besitzen bzw. zu Hause haben, und den Schülern, bei denen dies nicht der Fall ist.

Wie die Tabelle 5-8 zeigt, sind Gewaltbefürwortung, Täterverhalten, Schuldevianz und Delinquenz stärker, wenn die genannten Geräte zu Hause vorhanden sind und noch stärker, wenn sie im eigenen Besitz sind. Ein eigenes Videogerät hat den stärksten Zusammenhang mit den Gewaltindikatoren. Die Aggressionsbereitschaft ist ebenfalls höher, wenn diese Geräte (mit Ausnahme von Homecomputer/PC) vorhanden bzw. im persönlichen Besitz der Schüler sind. Der Opferstatus bei den personenbezogenen Angriffen ist mit der Medienausstattung kaum verbunden. Bei den sachbezogenen Angriffen sind zwar signifikante Zusammenhänge zu verzeichnen, weitere Analysen haben aber gezeigt, daß diese in erster Linie auf den Einfluß der Gruppe der Täter-Opfer zurückzuführen sind. Die Opfer unterscheiden sich kaum vom Gesamtdurchschnitt. Sie besitzen sogar signifikant seltener einen eigenen Fernseher als die anderen Befragten. Tabelle 5-8 zeigt außerdem, daß die Unbeteiligten bei allen genannten Geräten signifikant kleinere Werte aufweisen und daß eine diskursive Streitkultur beim eigenen Besitz der Geräte schwächer ausgeprägt ist.

7 Das wurde durch die partiellen Korrelationen zwischen den entsprechenden Variablen unter der Kontrolle für Alter bestätigt (vgl. auch Kap. 4). 
Tab. 5-8: Zusammenhang der Medienausstattung und Gewaltindikatoren in (Mittelwertunterschiede; t-Test; Stichprobe Sachsen)

\begin{tabular}{|c|c|c|c|c|c|c|}
\hline & \multicolumn{2}{|c|}{ zu Hause } & \multicolumn{4}{|c|}{ zur eigenen Verfügung } \\
\hline & Videogerät & PC & Fernseher & Videogerät & PC & $\begin{array}{l}\text { Telespiel/ } \\
\text { Gamboy }\end{array}$ \\
\hline $\begin{array}{l}\text { Täter: weichere } \\
\text { Aggressionen }\end{array}$ & $.13^{* *}$ & $.17 * * *$ & $.37 * * *$ & $.47^{* * *}$ & $.36 * * *$ & $.31 * * *$ \\
\hline Täter: härtere Aggressionen & $.18^{* * *}$ & $.16^{* * *}$ & $.33 * * *$ & $.43 * * *$ & $.37 * * *$ & $.32 * * *$ \\
\hline $\begin{array}{l}\text { Opfer: sachbezogene } \\
\text { Angriffe }\end{array}$ & .08 & .07 & $.13^{* * *}$ & $.24 * * *$ & $.14 * * *$ & $.17^{* * *}$ \\
\hline $\begin{array}{l}\text { Opfer: personenbezogene } \\
\text { Angriffe }\end{array}$ & .04 & .03 & -.00 & -.02 & .07 & $.12^{* *}$ \\
\hline Unbeteiligtsein (Cluster) & $-.06 * *$ & $-.08^{* * *}$ & $-.13 * * *$ & $-.13 * * *$ & $-.17 * * *$ & $-.13^{* * *}$ \\
\hline Schuldeviantes Verhalten & $.14^{* *}$ & $.13^{* *}$ & $.34 * * *$ & $.42 * * *$ & $.32 * * *$ & $.21 * * *$ \\
\hline Delinquentes Verhalten & $.13^{*}$ & $.20^{* * *}$ & $.30 * * *$ & $.43 * * *$ & $.35 * * *$ & $.25 * * *$ \\
\hline Gewaltbefürwortung & $.15^{* *}$ & $.14 * * *$ & $.41 * * *$ & $.45 * * *$ & $.36 * *$ & $.41 * * *$ \\
\hline Aggressionsbereitschaft & $.10^{*}$ & -.04 & $.24 * * *$ & $.16^{* *}$ & .07 & $.22 * * *$ \\
\hline $\begin{array}{l}\text { Diskursive Streitkultur } \\
\text { (V123) }\end{array}$ & -.09 & -.03 & $-.17^{* * *}$ & $-.16^{* *}$ & $-.12 * *$ & $-.24 * * *$ \\
\hline
\end{tabular}

Analog zu Kapitel 5-1 haben wir hier eine Clusteranalyse (k-Means-Verfahren) mit den Variablen zur Sehdauer der verschiedenen Filmarten durchgeführt, um in der Stichprobe die Schüler nach der Struktur der konsumierten Filme aufteilen zu können. Abbildung 5-3 zeigt die Mittelwerte dieser Variablen und der Variablen der Sehdauer (Werktags und an den Wochenenden) in den Clustern einer 2-Cluster-Lösung. 23,5\% der Befragten fallen in eine Gruppe, die eine durchschnittliche Sehdauer für die in der Regel gewaltfreien Filme und Sendungen (die ersten vier Kategorien: Spielfilme, Serien, Unterhaltung, Musik, Quiz, Kinder-, Jugend-, Informations- und Tierfilme) und eine überdurchschnittliche Sehdauer für Gewalt- und Sexfilme (die letzten fünf Kategorien) aufweisen. Die restlichen 76,5\% der Befragten sind in einer Gruppe, die sich bei den ersten vier Kategorien nur geringfügig (wenn auch statistisch hochsignifikant) von der ersten Gruppe unterscheidet, bei den Gewalt- und Sexfilmen aber eine deutlich geringere Sehdauer aufweist. Diese Abbildung zeigt deutlich, daß der Unterschied zwischen Vielsehern und Wenigsehern nicht nur ein quantitativer (bezüglich der Sehdauer), sondern auch ein qualitativer (bezüglich der Struktur der konsumierten Filme und Sendungen) Unter- 
schied ist. Vielseher haben vor allem einen höheren Konsum an Gewalt- und Sexfilmen. Bei den gewaltfreien Filmen aber unterscheiden sie sich kaum von den anderen.

Der Anteil der Schüler, die häufigen Fernseh- und Videokonsum angeben und dabei das Gewaltgenre bevorzugen, ist bei beiden Täter-Gruppen überproportional hoch, während die Episoden-Täter und Opfer weniger Gewaltfilme konsumieren. Dies gilt erst recht für die an Gewalt unbeteiligten Schüler: über $85 \%$ von ihnen zeigen kein problematisches Fernseh- und Videoverhalten und werden somit auch nicht durch Gewalt-, Horror- und Sexfilme negativ sozialisiert.

Abb. 5-3: Mittelwerte der Sehdauer bei verschiedenen Filmarten und Sendungen sowie an den Werktagen und Wochenendtagen in den Clustern einer 2-Cluster-Lösung (Stichprobe Sachsen)*

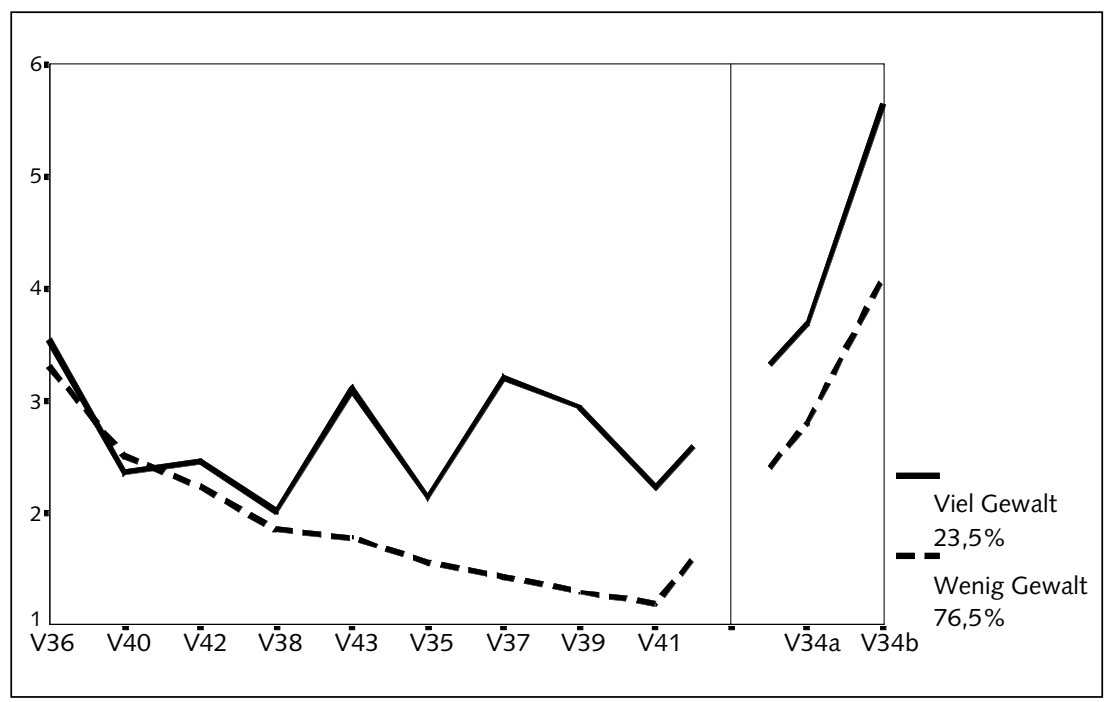

* Legende vgl. Tab. 5-4

Tab. 5-9: Zusammenhang zwischen der Präferenz für Gewaltfilme und dem Täter-Opferstatus (Kreuztabelle, Angaben in Prozent; Sachsen)

\begin{tabular}{lcccccc}
\hline Filmarten & Opfer & $\begin{array}{c}\text { Täter/ } \\
\text { Opfer }\end{array}$ & Täter & $\begin{array}{c}\text { Episoden- } \\
\text { Täter }\end{array}$ & Unbeteiligte & Total \\
Mit wenig Gewalt & 78,6 & 41,7 & 43,9 & 71,5 & 85,5 & 76,6 \\
Mit viel Gewalt & 21,4 & 58,3 & 56,1 & 28,5 & 14,5 & 23,4 \\
\hline
\end{tabular}


Die dargestellten Daten zeigen insgesamt einen deutlichen Zusammenhang zwischen Medienkonsum und Gewaltverhalten. Eine eindeutige Kausalrichtung läßt sich bivariat aber nicht bestimmen. Die beiden folgenden Interpretationen sind plausibel:

1. Imitation: Ein problematischer Medienkonsum trägt zu einer Erhöhung des Aggressionspotentials bei und dazu, daß die Hemmschwelle für die eigene Gewaltanwendung gesenkt wird. Je häufiger der Konsum solcher Filme, desto mehr prädestiniert sind die Kinder und Jugendlichen für die Gewaltbefürwortung und -anwendung.

2. Selektion: Kinder- und Jugendliche, die aus anderen Gründen aggressiver und gewalttätiger sind, haben eine stärkere Neigung zum Konsum von Gewaltfilmen. Je aggressiver, desto mehr Gewaltfilme.

Eine andere These jedoch kann als widerlegt gelten: Es trifft nicht zu, daß die Kinder durch den Konsum von Gewaltfilmen ihre Aggressionen abreagieren und dadurch Katharsiseffekte eintreten würden.

Um auszuschließen, daß die beobachteten Zusammenhänge nur dadurch zustande kommen, daß sowohl die Gewalttätigkeit als auch das Medienverhalten von anderen Variablen (etwa im Bereich Familie, Peers, Schule usw.) abhängen, sollen in einem multivariaten Verfahren alle relevanten Variablen für die Gewalttätigkeit gleichzeitig berücksichtigt werden. Ein solches Modell wird im Kapitel 7 vorgestellt.

\subsection{Einflüsse der Peer-Sozialisation}

Jugend als eine eigenständige Lebensphase mit den Peers als Gesellungsform und Sozialisationsinstanz kann als Produkt der Moderne begriffen werden - das belegen sowohl historische als auch kulturvergleichende Untersuchungen (vgl. u.a. Zinnecker 1987, Zinnecker/Molnar 1988, Zinnecker 1991, Melzer/ Lukowski/Schmidt 1991). Synonym zu dem Begriff der Peers wird in der Literatur auch die Bezeichnung der Gleichaltrigengruppe verwandt, obwohl nicht alle zu dieser Altersgruppe Zählenden gemeint sind, sondern nur diejenigen unter ihnen, die als Interaktionspartner akzeptiert sind und mit denen sich das Kind ,in Anerkennung der jeweiligen Interessen prinzipiell zu einigen bereit ist. Verlangt wird eine gewisse 'Soziabilität', also die Disposition, Handlungspläne miteinander abzustimmen, und zwar ohne das Streben einander zu dominieren, und dem Vorsatz, grundlosen Streit zu unterlassen“ (Krappmann 1991, S. 364).

Die Interaktion in Gleichaltrigengruppen entwickelt sich etwa vom 10. Lebensjahr an (vgl. Mansel/Hurrelmann 1991, S. 16) und ist im Freizeitsektor am stärksten entwickelt (Friebel 1985, S. 104). Bereits in der mittleren Kind- 
heitsphase differenzieren die Heranwachsenden zunehmend nach der Qualität der Beziehungen und heben Freundschaften, die auf besondere Intensität und ein wechselseitiges Vertrauens- und Unterstützungsverhältnis gründen, von bloßen Bekanntschaften ab (Krappmann 1991, S. 365). Mit Beginn der Jugendphase (etwa ab dem 15. Lebensjahr) gewinnen die „Cliquen“ an Bedeutung, eine informelle jugendliche Gesellungsform, die in erster Linie auf gemeinsame Freizeitaktivitäten unterschiedlichster Art gerichtet ist. Diese verschiedenen Formen von Peer-Sozialisation stellen für die Jugendlichen ein flexibles, gestaltbares Beziehungsgefüge dar, das ihnen gleichzeitig Rückhalt und ein Gefühl der Solidarität geben kann. Baacke weist unter Bezug auf Eisenstadt auf die besondere Funktion der Peer-Groups im Lebenslauf der Jugendlichen hin (vgl. auch Eisenstadt 1966, 1990, Oerter 1987, S. 318ff), auf ihre Systemähnlichkeiten mit der familialen Lebenswelt auf der einen und den Funktionsähnlichkeiten universalistischer Industriegesellschaften auf der anderen Seite.

„So sind die Beziehungen der Familie durch Nähe und Emotionalität bestimmt, und die Rollenzuschreibungen sind noch diffus und offen, wenig durch festgelegte Aufgaben geordnet und festgeschrieben: das verbindet die Jugendgruppe mit der Familie. Andererseits ist ein Status in ersterer nicht zugeschrieben, sondern muß erworben werden (Achtung und Anerkennung gleichaltriger Peers stellen sich nicht von selbst ein, sondern aufgrund von in Jugendgruppen anerkannten Leistungen): Diese Art des Statuserwerbs und der Statussicherheit ist wiederum etwas, was man in der Familie nicht, wohl aber später im 'Erwachsenenleben' beherrschen muß. Dazu gehört auch das Arrangieren eines eigenen Lebensstils. (...) Insofern dient die Jugendgruppe individueller Orientierung. In der Solidargemeinschaft von Gleichaltrigen, die sich gleichen Problemen gegenüber sehen, kann der Jugendliche biologisch bedingte Reifungsprozesse (Pubertät), interne Entwicklungsspannungen (kognitive und emotionale Entwicklung, Entstehung von Selbstreflexion) und sozial-kontextuelle Entwicklungen (Ausbildungserfahrungen, soziale Placierungschancen) derart zu integrieren versuchen, daß Identität entsteht, die gleichsam sozial produziert wird im Kontext der Interaktion von Jugendgruppen. Diese werden geradezu zur Prüfstelle gelungener Sozialisation“" (Baacke 1983, S. 235f). Zugleich stellt die Peer-Integration einen wichtigen Aspekt politischer Sozialisation dar, da sich in diesen Gruppen der Erwerb öffentlicher Kompetenzen und Rollen politischen Handelns zunächst im Kleinen vollzieht und sich im positiven Falle verantwortliches Handeln, das nicht wie in der Schule oder der Familie durch eine Kontrollinstanz von Erwachsenen erzwungen wird, entwickeln kann (vgl. Melzer 1992, S. 48ff).

Es hat wissenschaftliche Debatten darüber gegeben, ob die Peers sich „,in Konkurrenz“ zur Familie (Mitterauer 1986) etablieren und ob sie wirklich so positiv zu bewerten sind, wie eingangs dargestellt. Richtig ist, daß Gleichaltrigengruppen in dem Maße an Bedeutung gewinnen, wie sich die Loslösung der Heranwachsenden vom Elternhaus vollzieht. In einer früheren 
Untersuchung konnte aber nachgewiesen werden, daß eine Komplementarität von Elternhaus und Peers als Erfahrungsräume und als Teile des Unterstützungsnetzwerkes von Jugendlichen besteht (Melzer 1992, S. 55ff). Die emotionale Reproduktion in der Familie, die ausbildungs- und berufsbezogenen Stützleistungen, Bestärkungen und Ratschläge von Eltern sind nicht durch unverbindliche Hinweise oder einen Austausch mit Gleichaltrigen zu ersetzen. Die filiationistische Liebe scheint einstweilen noch verläßlicher, ermöglicht erst die sukzessive Ablösung vom Elternhaus und hilft, emotionale Rückschläge bei der Bewältigung von Entwicklungsaufgaben zu verarbeiten.

Die Anerkennung im Kontext der Peers ist wichtig für die jugendliche Identitätsentwicklung; was aber, wenn Gleichaltrige sie aus ökonomischen, sozialen oder ethnischen Gründen verweigern? Auch gibt es negative Bestimmungselemente der Peer-Konstituierung. Urie Bronfenbrenner weist darauf hin, daß im Zusammenhang mit Jugenddelinquenz eine höhere Anfälligkeit bei denjenigen Jugendlichen zu verzeichnen ist, deren Eltern häufiger abwesend sind und sich als weniger liebevoll bzw. weniger fest in Disziplinfragen ihren Kindern gegenüber verhalten (vgl. auch 5.1). So scheint die „Bindung an Gleichaltrige eher von mangelnder Aufmerksamkeit und Beachtung im Elternhaus als von irgendeiner Anziehungskraft der Kameradengruppe selbst abhängig zu sein“ (Bronfenbrenner 1976, S. 166) und möglicherweise eine problematische und für Jugendliche nicht oder nur schwer zu bewältigende Lebenssituation zu erzeugen. Auch in anderen Studien werden negative Aspekte der Peer-Sozialisation deutlich. Es wird darauf hingewiesen, daß in diesen Gruppen ein erhebliches internes, die Grenzen der Selbstregulierung der einzelnen Jugendlichen zum Teil übersteigendes Konfliktpotential besteht. Daneben wird die relative Abschottung gegenüber anderen gesellschaftlichen Bereichen und damit eine Beschneidung des Anregungspotentials und der Lernmöglichkeiten beklagt. Giesecke geht sogar soweit, von einer "GhettoSituation“ der Jugendlichengruppen zu sprechen, die er mit der der Rentner vergleicht. Er kritisiert die fehlenden Orientierungs- und Reibungspunkte an der Erwachsenenwelt (Giesecke 1985). Problematisch erscheint auch, daß Jugendliche in Peer-Gruppen oft zu stark einer gesellschaftlichen Entfremdung (z.B. unreflektiertes Konsum- oder Konkurrenzdenken, Orientierung an Medienleitbildern) ausgeliefert sind.

Diese Ambivalenz der Peers kommt auch im Phänomen des „Jugendzentrismus“, einem zentralen Konstrukt der Jugendwerkstudien seit 1991, zum Ausdruck (vgl. Jugendwerk 1982, S. 622ff, Jugendwerk 1992, S. 15ff). In der Skala verbinden sich positive Aspekte der Selbständigkeit, die auf gelungene Ablösungsprozesse von der Familie schließen lassen, mit einem starken Widerstandspotential und münden in kritisch zu bewertende Einstellungen, nach denen alle Polizisten als unfair und die Gesellschaft als feindselig erlebt wird. Hier wird der Zusammenhang zur Gewalt bereits deutlich. In einer eigenen Untersuchung konnte nachgewiesen werden, daß gesellschaftsbezogene Gewaltakzeptanz bei den Befragten mit einer jugendzentristischen Orientierung 
signifikant stärker ausgeprägt ist, während eine positive Einbindung in Cliquen und den privaten Freundeskreis ein Indikator für demokratisches politisches Handeln ist (Melzer 1992, S. 110ff).

In bezug auf Gewalthandeln von Schülern innerhalb und außerhalb der Schule ist die Einbindung in Peers in dreierlei Hinsicht von Bedeutung:

- Erstens haben die Täter-Opfer-Analysen ergeben, daß Isolation und fehlende soziale Einbindung in den Klassenverband und die Gleichaltrigengruppen charakteristische Merkmale für den Opferstatus sind.

- Zweitens können die Taten von Einzelnen oder Gruppen im schulischen und außerschulischen Raum verübt werden, wobei die Gewaltorientierung der Gleichaltrigen einen Verstärkungsfaktor darstellen kann.

- Drittens kann die Binnenstruktur einer Gruppe (z.B. eine von Intoleranz geprägte Interaktion) im Sinne der Autoritarismus-Hypothese einen Risikofaktor für Gewalthandeln darstellen.

Diese Annahmen werden im folgenden systematisch geprüft, wobei unsere Hypothesen dahin gehen, daß der Organisationsgrad in Gruppen als solcher kein Risiko für Gewalthandeln darstellt, wohl aber zur Erklärung des Opferstatus hilfreich ist, während als Prädiktoren schulischen Gewalthandelns die Gewaltorientierung von Freunden oder Peers, mit denen man auch im außerschulischen Bereich in Kontakt ist, ebenso wirken könnte wie das intolerante Binnenklima von Peer-Groups, sofern man ihnen angehört. Die Plausibilität und die Wahrscheinlichkeit des Zutreffens dieser Hypothesen wird gestützt durch die Nürnberger Studie: „Hinsichtlich aller (geprüften) Gewalthandlungen fällt die starke Determinationskraft der Gewalttätigkeit der Peergroups auf. Allerdings ist die Richtung der Beziehung, also die Kausalität, in diesem Fall nicht unbedingt eindeutig: Zwar kann eine gewalttätige Gleichaltrigengruppe die Gewaltaffinität der Jugendlichen erhöhen. Umgekehrt ist jedoch auch denkbar, daß sich gewalttätige Jugendliche eher gewalttätigen Peergroups anschließen. Trotzdem kann betont werden, daß insbesondere hinsichtlich des Prügelns die Prädiktoren aus dem Sozialkontext der Gleichaltrigengruppe eindeutig gewaltverstärkend zur Geltung kommen. Neben dem Prügeln werden auch vandalistische Handlungen stark durch die sozialen Beziehungen zur Gleichaltrigengruppe beeinflußt“" (Funk/ Passenberger 1997, S. 259).

\section{Gruppenzugehörigkeit und Gruppeneigenschaften}

Was die Zugehörigkeit zu Gleichaltrigengruppen anbelangt (V24), hat die Analyse der Daten ergeben, daß 47,3\% der Befragten in Sachsen zu einer festen Gruppe von Freundinnen oder Freunden gehören, die sich untereinander gut kennen und vieles gemeinsam machen (im folgenden „feste Gruppe“). Weitere 36,3\% sind mit verschiedenen Leuten oder Gruppen locker befreundet, mit denen sie nur selten etwas gemeinsam unternehmen (im folgenden „lok- 
kere Gruppe“). 12,5\% der Befragten sind in keiner Gruppe, haben aber eine/ n Freund/in, mit der/dem sie oft zusammen sind. Die restlichen 3,8\% sind meistens allein und haben keinen festen Freundeskreis. In Hessen ist der Anteil der Schüler in den festen Gruppen höher und in den anderen Kategorien niedriger. 14,5\% der Gruppen in Sachsen bestehen nur aus Jungen und 13,7\% nur aus Mädchen. Die restlichen 71,8\% sind gemischte Gruppen. In Hessen ist der Anteil der gemischten Gruppe kleiner (63,8\%). Die meisten Gleichaltrigengruppen bestehen gänzlich bzw. mehrheitlich aus den Schülern derselben Schule (vgl. Tab. 5-10). Falls alle bzw. keine Mitglieder der Freundesgruppe Mitschüler sind, ist die Freundschaft enger als in den Fällen, in denen ein Teil der Mitglieder aus derselben Schule kommt und ein anderer Teil nicht.

Tab. 5-10: Zusammenhang zwischen der Gruppenzusammensetzung (Mitschüler vs. Nichtmitschüler) und ihrer Festigkeit (Kreuztabelle; Angaben in Prozent; beide Stichproben)

\begin{tabular}{|c|c|c|c|c|c|c|}
\hline \multirow{2}{*}{$\begin{array}{l}\text { Sind die Mitglieder Deiner } \\
\text { Freundesgruppe aus Deiner } \\
\text { Schule? }\end{array}$} & \multicolumn{2}{|c|}{ Feste Gruppen } & \multicolumn{2}{|c|}{ Lose Gruppen } & \multicolumn{2}{|c|}{ Feste u. lose Gruppen } \\
\hline & Sachsen & Hessen & Sachsen & Hessen & Sachsen & Hessen \\
\hline Ja, alle & 19,2 & 26,1 & 12,2 & 21,5 & 16,2 & 24,5 \\
\hline Ja, die meisten & 46,0 & 52,5 & 52,2 & 54,5 & 48,7 & 53,2 \\
\hline Ja, nur einige & 28,7 & 14,7 & 31,1 & 18,2 & 29,8 & 15,9 \\
\hline Nein, niemand & 6,0 & 6,8 & 4,4 & 5,7 & 5,3 & 6,4 \\
\hline
\end{tabular}

Die aggressive Außenorientierung, das intolerante Binnenklima und das Wohlbefinden des Befragten in der Gruppe wurden anhand von sechs Items ermittelt (vgl. Tab. 5-11). Alle Mittelwerte der ersten fünf Items sind kleiner als der Skalenmittelwert (=3). Das zeigt, daß die meisten Gruppen von ihren Mitgliedern als eher nicht aggressiv und tolerant eingestuft werden. Die hohen Mittelwerte des Items „Wohlbefinden in der Gruppe“ zeigen auch, daß sich die überwiegende Mehrheit der Schüler in ihrer Gruppe richtig wohl fühlt. Das war zu erwarten, weil die Mitgliedschaft in den Gleichaltrigengruppen in der Regel freiwillig ist. Wer sich nicht wohl fühlt, bleibt auch nicht in der Gruppe.

Die Aggressivität der Gruppen nach außen ist in Sachsen und Hessen fast gleich. Nur die diskursive Streitkultur ist in Sachsen etwas schwächer ausgeprägt als in Hessen. Bei der internen Intoleranz sind aber hochsignifikante Unterschiede zu verzeichnen. Hier weisen die sächsischen Gruppen eine stärkere Intoleranz auf. Trotzdem fühlen sich die sächsischen Befragten in ihren Gruppen praktisch genauso wohl wie die hessischen. 
Tab. 5-11: Items der Aggressivität und internen Intoleranz der Gruppen und ihre Mittelwerte (beide Stichproben)

\begin{tabular}{|c|c|c|c|}
\hline $\begin{array}{l}\text { Im folgenden werden Aussagen zu Gruppen gemacht. Inwieweit } \\
\text { stimmen die Aussagen auch für Deine Freundes- und Bekannten- } \\
\text { gruppe? }\end{array}$ & Sachsen & Hessen & Differenz \\
\hline \multicolumn{4}{|l|}{ I. Aggressive Außenorientierung der Gruppe: } \\
\hline $\begin{array}{l}\text { V28. Wenn wir Probleme mit anderen Gruppen haben, lösen wir } \\
\text { die nicht mit Diskutieren. }\end{array}$ & 2,51 & 2,43 &, $08^{*}$ \\
\hline $\begin{array}{l}\text { V29. Im letzten Jahr hat es mit anderen Gruppen Kämpfe, Prüge- } \\
\text { leien oder ähnliche Auseinandersetzungen gegeben. }\end{array}$ & 1,75 & 1,80 &,- 05 \\
\hline $\begin{array}{l}\text { V30. Um die Ziele und Interessen unserer Gruppe durchzusetzen, } \\
\text { pfeifen wir auch schon mal aufs Gesetz. }\end{array}$ & 2,19 & 2,16 &, 03 \\
\hline \multicolumn{4}{|l|}{ II. Intolerantes Binnenklima: } \\
\hline $\begin{array}{l}\text { V27. Wer motzt oder kritisiert, wird in unserer Gruppe nicht } \\
\text { geduldet. }\end{array}$ & 2,62 & 2,31 &, $31 * * *$ \\
\hline $\begin{array}{l}\text { V31. Wer nicht vollständig mit unserer Gruppe übereinstimmt, ist } \\
\text { kein richtiges Gruppenmitglied. }\end{array}$ & 2,12 & 1,96 &, $16^{* * *}$ \\
\hline \multicolumn{4}{|l|}{ III. Wohlbefinden in der Gruppe: } \\
\hline V32. In meiner Freundesgruppe fühle ich mich richtig wohl. & 4,38 & 4,41 &,- 04 \\
\hline
\end{tabular}

Skala: $1=$ stimmt gar nicht $\ldots 5=$ stimmt ganz genau

Weitere Analysen haben ergeben, daß die festen Gruppen eine aggressivere Außenorientierung haben als die mit geringem Zusammenhalt. Hinsichtlich der internen Toleranz unterscheiden sich die beiden Gruppen aber nur geringfügig und die Befragten fühlen sich in den festen Gruppen wohler als in den losen. Die Langeweile in der Freizeit ist in den festen Gruppen geringer als bei den anderen. Sie ist aber in den aggressiven Gruppen größer. Es wurde weiterhin festgestellt, daß die Gruppen, deren Mitglieder aus derselben Schule sind, weniger aggressiv sind als die anderen und daß die Schüler aus Familien mit positivem Erziehungsklima eher zu den festen Gruppen gehören als die Schüler aus problematischen Familien. Sie schätzen ihre Kumpels auch weniger aggressiv und intolerant ein und fühlen sich in ihrer Gruppe wohler. Die Korrelationen zwischen den Gruppenvariablen und Medienvariablen zeigen ebenfalls, daß die Schüler, die in den aggressiven Gruppen sind, mehr als die anderen Video und Fernsehen (insbesondere Gewalt- und Sexfilme) konsumieren. 


\section{Gruppenzugehörigkeit und Gewaltverhalten}

Wir überprüfen nun anhand der sächsischen Daten, inwieweit die Gruppeneigenschaften und das Gewaltverhalten ihrer Mitglieder zusammenhängen. Dabei verwenden wir dieselben Gewaltindikatoren wie in den letzten beiden Abschnitten. Die Analysen haben ergeben, daß Täterverhalten, Schuldevianz und Delinquenz bei den Befragten, die in den Gruppen und insbesondere in den festen Gruppen integriert sind, häufiger zu verzeichnen sind. Diejenigen, die meistens allein sind, werden viel häufiger als die anderen Opfer personenbezogener Angriffe. Zwischen der Aggressionsbereitschaft und der Mitgliedschaft in den Gruppen wurde kein signifikanter Zusammenhang festgestellt. Die Befürwortung der Gewalt ist aber in den festen Gruppen etwas stärker.

Nicht aber die bloße Mitgliedschaft in einer Gruppe ist in diesem Zusammenhang ausschlaggebend. Es kommt vielmehr darauf an, wie sich die Gruppe nach außen und nach innen verhält. Tabelle 5-12 zeigt die bivariaten Korrelationen zwischen den individuellen Gewaltindikatoren und den Items der Gruppe (Aggressivität, Intoleranz und Wohlbefinden in der Gruppe). Es gibt starke Zusammenhänge zwischen der aggressiven Außenorientierung und der Aggressivität und Gewalttätigkeit der Mitglieder. Am stärksten sind die Delinquenz und Gewaltbefürwortung mit der Mitgliedschaft in einer aggressiven Gruppe verbunden. Es folgen das Täterverhalten in der Schule und die Aggressionsbereitschaft. Die Unbeteiligten und die Schüler mit ausgeprägter diskursiver Streitkultur sind am wenigsten in aggressiven Gruppen vertreten. Interessant ist, daß auch die Wahrscheinlichkeit, Opfer zu werden, in den aggressiven Gruppen größer ist. Die Frage bleibt aber offen, ob die Opfer in derartigen Gruppen einen Schutz suchen oder die Opfererfahrungen eine Reaktion auf die eigenen und Gruppenaggressionen sind.

Tab. 5-12: Korrelationen zwischen den individuellen Gewaltindikatoren und Gruppenvariablen (Stichprobe Sachsen)

\begin{tabular}{|c|c|c|c|c|c|c|c|}
\hline \multirow{2}{*}{$\begin{array}{l}\text { Individuelle } \\
\text { Gewaltindikatoren: }\end{array}$} & \multicolumn{3}{|c|}{$\begin{array}{c}\text { Aggressive Außenorientierung } \\
\text { der Gruppe }\end{array}$} & \multicolumn{2}{|c|}{$\begin{array}{c}\text { Intolerantes } \\
\text { Binnenklima der } \\
\text { Gruppe }\end{array}$} & \multirow{2}{*}{$\begin{array}{c}\text { Wohl- } \\
\text { befinden } \\
\text { in der } \\
\text { Gruppe } \\
\text { V32 }\end{array}$} & \multirow{2}{*}{$\begin{array}{l}\text { Gewalt- } \\
\text { tätigkeit } \\
\text { des } \\
\text { Kumpels }\end{array}$} \\
\hline & V28 & V29 & V30 & V27 & V31 & & \\
\hline $\begin{array}{l}\text { Täter: weichere } \\
\text { Aggressionen }\end{array}$ & $.22 * *$ & $.37^{* *}$ & $.39 * *$ & $.07 * *$ & $.19 * *$ & .02 & $.50 * *$ \\
\hline $\begin{array}{l}\text { Täter: härtere } \\
\text { Aggressionen }\end{array}$ & $.22 * *$ & $.37^{* *}$ & $.39 * *$ & $.09 * *$ & $.20^{* *}$ & -.03 & $.40^{* *}$ \\
\hline $\begin{array}{l}\text { Opfer: sachbezogene } \\
\text { Angriffe }\end{array}$ & $.07 * *$ & $.13^{* *}$ & $.18^{* *}$ & $.07 * *$ & $.11 * *$ & $-.04^{*}$ & $.18^{* *}$ \\
\hline
\end{tabular}




\begin{tabular}{|c|c|c|c|c|c|c|c|}
\hline \multirow{2}{*}{$\begin{array}{l}\text { Individuelle } \\
\text { Gewaltindikatoren: } \\
\text { Opfer: personenbez. } \\
\text { Angriffe }\end{array}$} & \multicolumn{3}{|c|}{$\begin{array}{c}\text { Aggressive Außenorientierung } \\
\text { der Gruppe }\end{array}$} & \multicolumn{2}{|c|}{$\begin{array}{c}\text { Intolerantes } \\
\text { Binnenklima der } \\
\text { Gruppe }\end{array}$} & \multirow{2}{*}{$\begin{array}{l}\text { Wohl- } \\
\text { befinden } \\
\text { in der } \\
\text { Gruppe } \\
-.06^{* *}\end{array}$} & \multirow{2}{*}{$\begin{array}{c}\text { Gewalt- } \\
\text { tätigkeit } \\
\text { des } \\
\text { Kumpels } \\
.11^{* *}\end{array}$} \\
\hline & $.08^{* *}$ & $.14 * *$ & $.08 * *$ & $.08^{* *}$ & $.10^{* *}$ & & \\
\hline Unbeteiligtsein (Cluster) & $-.18^{* *}$ & $-.26^{* *}$ & $-.30 * *$ & $-.10^{* *}$ & $-.16^{* *}$ & .02 & $-.38^{* *}$ \\
\hline Schuldeviantes Verhalten & $.17^{* *}$ & $.31 * *$ & $.37 * *$ & $.04^{*}$ & $.14^{* *}$ & .03 & $.45^{* *}$ \\
\hline Delinquentes Verhalten & $.30^{* *}$ & $.46^{* *}$ & $.48^{* *}$ & $.07^{* *}$ & $.18^{* *}$ & -.04 & $.43^{* *}$ \\
\hline Gewaltbefürwortung & $.33^{* *}$ & $.42 * *$ & $.43 * *$ & $.11^{* *}$ & $.26^{* *}$ & .01 & $.37 * *$ \\
\hline Aggressionsbereitschaft & $.16^{* *}$ & $.29 * *$ & $.23 * *$ & $.12^{* *}$ & $.19 * *$ & -.03 & $.22 * *$ \\
\hline $\begin{array}{l}\text { Diskursive Streitkultur } \\
\text { (V123) }\end{array}$ & $-.23 * *$ & $-.21 * *$ & $-.15^{* *}$ & $-.06 * *$ & $-.14^{* *}$ & $.11 * *$ & $-.09 * *$ \\
\hline
\end{tabular}

Wenn wir die ersten drei Spalten der Tabelle 5-12 miteinander vergleichen, können wir feststellen, daß am stärksten die Verstöße der Gruppe gegen die Gesetze (V30) mit den individuellen Gewaltindikatoren zusammenhängen. Es folgen die Kämpfe mit den anderen Gruppen (V29) und Diskussionsunfähigkeit der Gruppe nach außen (V28).

Die interne Intoleranz der Gruppe hängt mit den individuellen Gewaltindikatoren schwächer zusammen als die Aggressivität nach außen. Sie zeigt aber in groben Zügen dasselbe Muster: Gewaltbefürwortung, Aggressionsbereitschaft, Täterverhalten, Delinquenz, Schuldevianz und Opfererfahrungen sind in den intoleranteren Gruppen stärker zu beobachten. Unbeteiligte sind eher in toleranten Gruppen mit höherer diskursiver Streitkultur integriert.

Zwischen dem Wohlbefinden in der Gruppe und Gewaltfaktoren gibt es kaum einen Zusammenhang. Die Befragten mit stärkerer diskursiver Streitkultur fühlen sich in ihren Gruppen wohler als die anderen. Das ist auch zu erwarten, weil sie die Eskalation möglicher Konflikte in der Gruppe verhindern können. Die Opfer fühlen sich in ihren Gruppen etwas weniger wohl als die anderen. Sie bleiben trotzdem in ihren Gruppen, weil sie ansonsten vermutlich unangenehmere Erfahrungen befürchten müssen.

Wir haben die Schüler nicht nur nach der Aggression und Intoleranz der Freundes- und Bekanntengruppe gefragt, sondern auch nach der Gewalttätigkeit der Freunde, ohne Spezifizierung auf eine Gruppe. Die Befragten haben angegeben, ob sie bei ihren engsten Freunden oder Freundinnen folgende Aggressionsformen beobachtet haben: Vandalismus (V135a), Beschimpfung oder „Telefonterror" gegen Lehrer (V148a), Bedrohung, Schlagen oder sachbezogene Attacken gegen Lehrer (V152a), psychische Aggressionen gegen die 
Mitschüler (V159a), ausländerfeindliche Sprüche (V161a) sowie Erpressung (V165a). Wir haben für jeden Befragten die Anzahl der Ja-Antworten bei diesen sechs dichotomen Items aufsummiert und damit eine Variable für die Gewalttätigkeit der engsten Freunde gebildet. 43,1\% der Befragten haben ihre Freunde bei keiner der genannten Aggressionsformen beobachtet, 25,2\% nur bei einer Form, 14,0\% bei zwei Formen, 8,2\% bei drei Formen, 5,1\% bei vier Formen, 2,3\% bei fünf Formen und 2,2\% bei allen sechs Formen. Die letzte Spalte der Tabelle 5-12 beinhaltet die Korrelationen zwischen dieser Variable und den individuellen Gewaltindikatoren. Es gibt einen starken positiven $\mathrm{Zu}$ sammenhang zwischen der Gewalttätigkeit der Freunde einerseits und dem Täterverhalten des Befragten andererseits (härtere und weichere Aggressionen, Schuldevianz, Delinquenz, Gewaltbefürwortung und Aggressionsbereitschaft). Auch die Freunde der Opfer sind überdurchschnittlich aggressiv. Bei den Unbeteiligten ist es dementsprechend umgekehrt. Der (negative) Zusammenhang zwischen der diskursiven Streitkultur und der Gewalttätigkeit der Freunde ist aber nicht so stark. Gleichwohl zeigen die Daten, daß man weniger in der Lage ist, Streitigkeiten durch Reden zu klären, wenn man gewalttätige Freunde hat.

Analog zu den vorherigen Abschnitten können wir auch hier anhand einer Clusteranalyse (k-Means-Verfahren) die Befragten nach der Zugehörigkeit zu den Gruppen mit hoher bzw. niedriger Aggressivität und Intoleranz klassifizieren. Als Klassifikationsvariablen dienen alle sechs Gruppenvariablen (vgl. Tab. 5-11). Abbildung 5-4 zeigt die Mittelwerte dieser Variablen in einer 2-Cluster-Lösung. 34,4\% der Befragten fallen in ein Cluster mit höheren Mittelwerten bei den Items der Aggressivität und Intoleranz. Die restlichen 65,6\% befinden sich in einem anderen Cluster mit niedrigeren Werten bei diesen Items. Diese zwei Cluster unterscheiden sich hinsichtlich des Wohlbefindens in der Freundesgruppe (V32) nicht. Somit kann geschlußfolgert werden, daß sich die Jugendlichen in den aggressiveren und intoleranteren Gruppen im Schnitt genauso wohl fühlen wie in den anderen Gruppen.

Tab. 5-13: Zusammenhang zwischen der Aggressivität/Intoleranz der Gruppe und dem Täter-Opferstatus (Kreuztabelle, Angaben in Prozent)

\begin{tabular}{lcccccc}
\hline Gruppenprofile & Opfer & Täter-Opfer & Täter & $\begin{array}{c}\text { Episoden- } \\
\text { Täter }\end{array}$ & Unbeteiligte & Total \\
aggressiv/intolerant & 31,4 & 71,6 & 73,2 & 42,2 & 22,0 & 34,0 \\
friedlich/tolerant & 68,6 & 28,4 & 26,8 & 57,8 & 78,0 & 66,0 \\
\hline
\end{tabular}

Wenn wir nun diese Klassifizierung mit den fünf Handlungstypen aus dem Kapitel 4 in Zusammenhang bringen (vgl. Tab. 5-13), können wir einen starken Zusammenhang zwischen der Gruppenaggressivität und dem individuellen Status feststellen (Cramer's V=,35). Es zeigt sich, daß nicht nur die Täter, 
Abb. 5-4: Mittelwerte der Items der Gruppeneigenschaften in den Clustern einer 2-Cluster-Lösung (Sachsen)

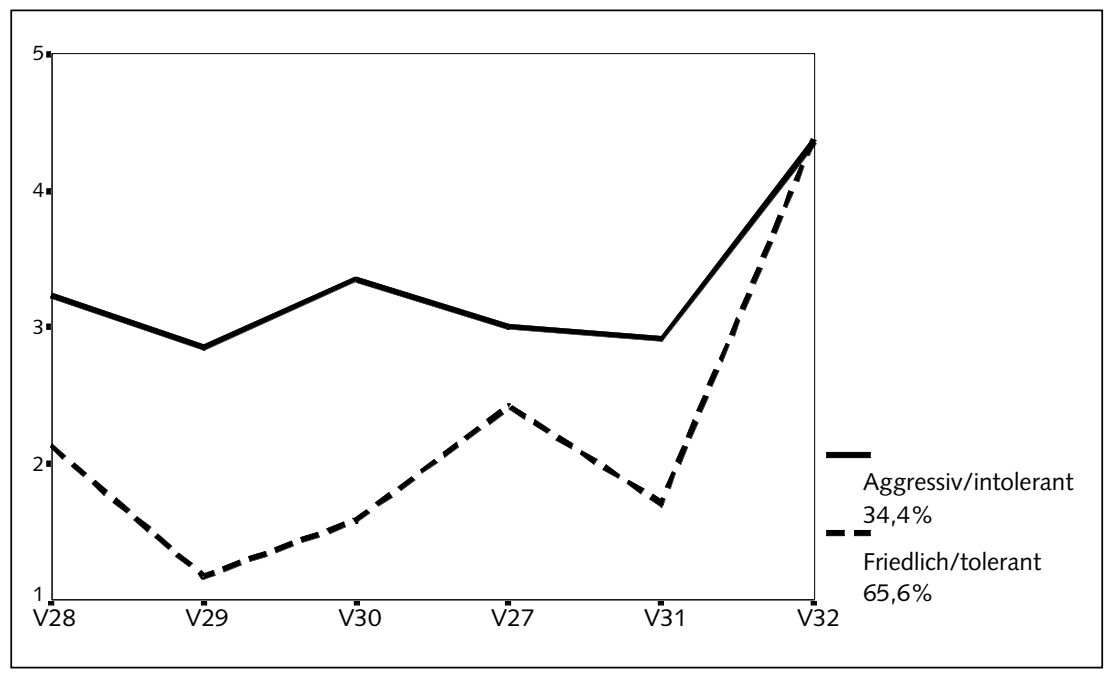

* Legende vgl. Tab. 5-11

sondern auch die Täter-Opfer überwiegend in den aggressiven und intoleranten Gruppen zu suchen sind: 73,2\% der Täter und 71,6\% der Täter/ Opfer sind in derartigen Gruppen, während dies nur für $22,0 \%$ der Unbeteiligten gilt. Die Episoden-Täter $(42,4 \%)$ nehmen eine mittlere Position zwischen den Tätern und Unbeteiligten ein. Die Opfer (31,4\%) sind zwar mehr als die Unbeteiligten aber immerhin leicht unterdurchschnittlich in den aggressiven und intoleranten Gruppen vertreten.

Zusammenfassend läßt sich sagen, daß die Ergebnisse auf einen hohen Stellenwert der Peer-Sozialisation in bezug auf das Gewaltverhalten hindeuten. Nicht die Gruppenzugehörigkeit an sich ist gewaltfördernd, sondern die $\mathrm{Zu}-$ gehörigkeit zu aggressiven und intoleranten Gruppen. Als Gegenstück zur Isolation senkt die Gruppenzugehörigkeit die Wahrscheinlichkeit der Viktimisierung bei den personenbezogenen Angriffen. Der starke Zusammenhang zwischen der individuellen und der Gruppenaggressivität ist nicht als eine eindeutige Kausalbeziehung zu betrachten. Es ist plausibel, daß das eigene Verhalten und die individuellen Einstellungen von denen der Freunde beeinflußt wird. Ebenso plausibel ist es aber, daß sich die gewalttätigen Jugendlichen eher den gleichgesinnten Gruppen anschließen. Somit ist die Aggressivität der Gruppe weniger als eine primäre Ursache und mehr als ein Verstärkungsfaktor zu betrachten. 


\subsection{Zusammenfassung}

Vergleicht man die Einflußstärke der drei untersuchten Sozialisationsbereiche, so läßt sich auf der Grundlage unserer bivariaten Modelle (Zwei-Cluster-Lösungen für: 1. familiale Sozialisation, 2. Medieneinflüsse, 3. Verstärkungen durch Peers) feststellen, daß die lebensweltlichen Einflüsse der Jugendkultur, des Umgangs mit anderen Jugendlichen (Cramer's $\mathrm{V}=.35^{* *}$ ) und die Medieneinflüsse (Cramer's V=.31**) in dieser Lebensphase (12- bis 17jährige) stärker auf Gewalthandeln durchschlagen, als die familialen Einflüsse (Cramer's $\left.\mathrm{V}=.19^{* *}\right)$. Insgesamt wird deutlich, daß die außerschulischen Sozialisationserfahrungen sehr prägend sind, nicht nur für die Persönlichkeitsentwicklung im allgemeinen, sondern auch im konkreten für den Umgang mit Gewalt in der Schule, den Täter- und Opferstatus, das Unbeteiligtsein oder auch die ablehnende oder befürwortende Einstellung zur Gewalt. Diese Zusammenhänge von Erziehungsmilieu und Gewalt haben die Analysen insgesamt belegt.

Am Ende des Familienabschnittes, als sich dieses Ergebnis an Hand der Bedeutung familialer Prädiktoren bereits andeutete, wurde die Frage nach der Bedeutung des Einflusses der Sozialschichtzugehörigkeit aufgeworfen und in den weiteren Untersuchungen verfolgt. Dabei zeigte sich, daß keine direkten sozialstrukturellen Einflüsse der Schülerinnen und Schüler auf das Gewalthandeln bestehen, also durch die soziale Herkunft als Individualdatum allein Gewaltemergenz nicht erklärt werden kann. Weder funktionierte ein globales Modell nach dem Muster: Unterschichtzugehörigkeit birgt ein stärkeres Gefährdungspotential für Gewalthandeln in sich, während Mittel- und Oberschichtzugehörigkeit einen protektiven Faktor darstellt, noch ein differenzierteres Modell, das auf der Annahme beruht, daß bestimmte Formen des Gewalthandelns (z.B. körperliche Aggressionen) von Kindern aus unteren Sozialschichten, andere Formen (z.B. psychische und verbale Aggressionen) von Heranwachsenden aus oberen Sozialschichten bevorzugt werden. Allerdings haben wir bei den Bildungsaspirationen und bestimmten Aspekten der Einflüsse des Erziehungsmilieus leichte schichtspezifische Unterschiede gefunden, so daß eine abschließende Überprüfung des Zusammenhangs von sozialer Herkunft und Erziehungsmilieu und des Einflusses dieser Faktoren auf Gewalthandeln naheliegt. Dabei war der Versuch leitend, der auch in der aktuellen Sozialstrukturdebatte eine Rolle spielt, die prägende Kraft des Milieus zu betonen, aber gleichzeitig eine Einordnung dieser Milieus in die Sozialstrukturen vorzunehmen und damit auf klassische Schichtmodelle nicht zu verzichten. So unterscheiden Vester u.a. (1992, S. 15) neun Typen von Milieus, die sie drei Soziallagen (obere Lagen, mittlere Lagen, Arbeiterlagen) zuordnen. Die oberen Lagen bestehen danach aus dem „konservativ-gehobenen Milieu“, dem „technokratisch-liberalen Milieu“ und dem ,alternativen Milieu“. Ebenso werden die anderen beiden Soziallager in jeweils drei Typen von Milieus ausdifferenziert. 
Wir nehmen das aus diesem Grundmodell resultierende Konzept der „Milieu-Biographie“ (vgl. Hradil 1994, S. 112) auf und untersuchen dementsprechend den Zusammenhang von Erziehungsmilieu und Schicht. Dabei geht es um die Überprüfung der Hypothese, ob die festgestellten sozialisatorischen Einflüsse durch den sozialen Status verstärkt bzw. gemindert werden, in der Weise, daß z.B. die ungünstigen Milieueinflüsse durch einen niedrigen sozialen Status zu einem erhöhten Risikofaktor zusammenfließen. Auf der Grundlage von Cluster-Analysen wird ein dreistufiges Schichtmodell (untere, mittlere, höhere Sozialschicht) konstruiert, während beim Erziehungsmilieu wie in den bisherigen Analyseschritten von einer Zwei-Cluster-Lösung (günstigere/restriktive Sozialisationsbedingungen) ausgegangen wird. In die Variable Erziehungsmilieu fließen alle Parameter ein, die sich in den Kap. 5.1 bis 5.3 als erklärungsrelevant für Gewalt erwiesen hatten. Die Clusteranalyse ergibt für etwa zwei Drittel der Schülerschaft günstigere und für gut ein Drittel ungünstigere Bedingungen des Aufwachsens und der Sozialisation. Aus der Kombination dieser Milieutypen mit der Schicht ergeben sich sechs Gruppen (G1 = untere Sozialschicht/restriktives Erziehungsmilieu bis G6 = obere Sozialschicht/günstigeres Erziehungsmilieu). Dieses Modell wurde am Beispiel des Täter-Status (weiche und härtere Aggressionen) durch einen Mittelwertvergleich überprüft (vgl. Tab. 5-14).

Tab. 5-14: Mittelwerte der Faktoren des Täterverhaltens in den sechs Kombinationsgruppen des Erziehungsmilieus und der Schicht

\begin{tabular}{lcccccc}
\hline & G6 & G4 & G5 & G1 & G2 & G3 \\
Täter: Weiche Aggressionen &,- 21 &,- 16 &,- 10 &, 28 &, 35 &, 40 \\
Täter: Harte Aggressionen &,- 18 &,- 16 &,- 13 &, 29 &, 30 &, 39 \\
\hline
\end{tabular}

Legende:

G1 = Untere Sozialschicht/ungünstiges Erziehungsmilieu

G2 = Mittlere Sozialschicht/ungünstiges Erziehungsmilieu

G3 = Obere Sozialschicht/ungünstiges Erziehungsmilieu

G4 = Untere Sozialschicht/günstigeres Erziehungsmilieu

$\mathrm{G} 5=$ Mittlere Sozialschicht/günstigeres Erziehungsmilieu

G6 = Obere Sozialschicht/günstigeres Erziehungsmilieu

Da die Gruppen nach aufsteigenden Mittelwerten sortiert sind und die einzelnen Werte positive bzw. negative Vorzeichen haben, läßt sich leicht erkennen, daß die Gruppen 1 bis 3 bei beiden Gewaltparametern und ähnlich hohen Werten einen positiven Zusammenhang zum Täterstatus aufweisen, d.h. Risikofaktoren für Gewalthandeln darstellen. Diese drei Gruppen sind aber durch das ungünstige Erziehungsmilieu geprägt und nicht durch die Sozialschicht. Auf keinen Fall bestätigen sich die erwarteten Effekte, da im Vergleich 
der drei Gruppen, die Kinder aus oberen Sozialschichten, die in ungünstigen Erziehungsmilieus aufwachsen (G3), am meisten gefährdet sind, allerdings ist dieser Unterschied nicht signifikant. Demgegenüber haben die Gruppen 4 bis 6 bei ihren Mittelwerten ein negatives Vorzeichen, d.h., sie verfügen aufgrund ihres positiven Erziehungsmilieus über einen positiven Faktor in bezug auf das Gewalthandeln - unabhängig davon, welcher Sozialschicht sie zugehören. Die ersten drei Gruppen unterscheiden sich bei allen möglichen neun Vergleichen untersuchter Gewaltparameter signifikant von den drei anderen Gruppen. $\mathrm{Da}$ innerhalb dieser beiden Dreiergruppen keine signifikanten Unterschiede bestehen, bedeutet dies, daß die Schichtzugehörigkeit als individuelles Merkmal über das Erziehungsmilieu hinaus keinen Einfluß auf Gewalttätigkeit hat. Zwar ist der Anteil der Schüler aus negativen Erziehungsmilieus in den oberen Schichten etwas geringer als in den unteren, aber dieser Zusammenhang ist statistisch sehr schwach (Cramer's $\left.V=.05^{*}\right)^{8}$.

Wie deutlich im Gegensatz dazu das Erziehungsmilieu in seiner Gesamtheit (Familie, Medien, Peers) auf die Gewalt in der Schule durchschlägt, zeigt der Rückbezug zur Täter-Opfer-Typologie. Die Mehrheit der Täter und der Täter-Opfer ist in ungünstigen Erziehungsmilieus aufgewachsen, während etwa drei Viertel aller an Gewalt Unbeteiligten in einem positiven Erziehungsmilieu aufgewachsen sind. Da aber das Aufwachsen in einem guten Familienklima, ein kindorientiertes und -gemäßes Erziehungsverhalten der Eltern oder ein bewußter Medienumgang und soziale Umgangsformen im Kreise von Jugendlichen kein Garant dafür sind, daß es nicht trotzdem zu einem Gewaltinvolvement - sei es als Täter, sei es als Opfer - kommt, müssen weitere mögliche Prädiktoren des Gewalthandelns und ihr Zusammenwirken geprüft werden. Im nächsten Kapitel sind dies Aspekte des Schulalltags und der Schulkultur. Dort werden die Analysen mit Daten fortgesetzt, die auf Klassenebene aggregiert sind, um auf diese Weise die Einzelaspekte möglicher Prädiktoren, wie sie in Gruppensituationen zusammentreffen, in ihrer Gesamtheit und ihren Synergismen erfassen zu können. In diesem Zusammenhang soll auch noch einmal die Schichtzugehörigkeit als Aggregatvariable überprüft werden.

8 Diese z.T. überraschenden Befunde erklären sich u.E. daraus, daß mit der Wende im Osten ein Wandel in der Schichtzugehörigkeit eingetreten ist und die Formierung einer relativ stabilen Sozialstruktur noch längst nicht abgeschlossen ist. 


\section{Schulkultur und ihre Auswirkung auf Gewalt (Wolfgang Melzer, Manuela Mühl, Christoph Ackermann)}

In den vorausgegangenen Kapiteln wurde Gewalt in phänomenologischer Perspektive vor allem auf der Individualebene (Kap. 3 bis 5) bzw. unter der spezifischen Fragestellung des Ost-West-Vergleichs (Kap. 2) auf einer makrosozialen Systemebene betrachtet. Schulbezogene Gewaltforschung hat aber ebenso die Institutionen-, also die Mesoebene mit in den Blick zu nehmen. Eine Durchmusterung der vorliegenden schulbezogenen Gewaltstudien der neunziger Jahre (vgl. ausführlich Schubarth 1998b) zeigt, daß eine erste, stark deskriptiv angelegte Phase der schulischen Gewaltforschung, in der man sich vor allem der Untersuchung von allgemeiner Gewaltbelastung und Ausmaß einzelner Gewaltformen an Schulen widmete, einen vorläufigen Abschluß gefunden hat. In einem zweiten Schritt gilt es nun, eine auf die Schule bezogene Ursachenanalyse hinsichtlich des Gewalthandelns von Schülern voranzutreiben, um auf der Grundlage entsprechender Ergebnisse zu wirkungsvollen Präventionsansätzen für die Schule zu gelangen. Auch hierzu liegen - neben älteren Studien (vgl. z.B. Fend 1977) - neuere Erkenntnisse aus empirischen Untersuchungen vor - allerdings in weitaus geringerem Umfang als es die Vielzahl der Studien, die sich dezidiert der Gewalt an Schulen widmen, erwarten lassen würde.

Eine erste Annäherung an mögliche gewaltfördernde Problemkonstellationen in Schulen ergibt sich aus der subjektiven Beurteilung der Gewaltursachen durch die Betroffenen, die in einigen der neueren Untersuchungen erhoben wurden (vgl. z.B. Schubarth/Melzer 1994, Fuchs/Lamnek/Luedtke 1996, Schwind/Roitsch/Gielen 1997). Daß dabei methodische Zweifel hinsichtlich einer fundierten innerschulischen Ursachenklärung anzumelden sind, wurde von Krumm im Rahmen einer umfassenden Methodenkritik der schulischen Gewaltforschung unter dem Stichwort „Fragwürdige Auskunftspersonen“ bereits ausführlich dargestellt (Krumm 1997, S. 73). Bemerkenswert sind jedoch folgende Ergebnisse unserer Schulleiterbefragung (vgl. Melzer/Schubarth 1994, S. 43ff): Schulpädagogische und schulkulturelle Ursachen spielen nach dem Dafürhalten der Schulleiter und Lehrer eine deutlich untergeordnete Rolle; dagegen werden Belastungen des Schullebens - etwa durch Erziehungsversagen oder sozioökonomische Probleme in der Familie, durch schulorganisatorische Probleme (Klassenstärke usw.), einen allgemeinen Normen- und Werteverfall, u.a. - als dominante Prädiktoren angegeben. Die Schulleiter und Lehrer sehen die Schule und sich selbst eher in der Rolle der Leidtragenden, die mit diesen defizitiven Lernbedingungen konfrontiert werden und sie pädagogisch kompensieren sollen. Wurde - wie beispielsweise in der Berliner (Dettenborn/Lautsch 1993), der Nürnberger (Funk 1995) oder der Bochumer 
Studie (Schwind/Roitsch/Gielen 1997) auch die Meinung der Schüler erfragt, ergaben sich erhebliche Differenzen zu den Lehrerurteilen - gerade in bezug auf die Schule, die im Schülerurteil stärker als ein Gewaltauslöser bewertet wurde (z.B. schulbezogene Frustration, Leistungsdruck u.a.).

Einen weiteren Hinweis auf schuleigene gewaltfördernde Problemlagen liefert der in vielen Studien durchgeführte Schulformvergleich, der durchgängig für die Gymnasien die niedrigste und für die Förderschulen die höchste Gewaltbelastung ermittelt hat (vgl. u.a. Meier/Melzer/Schubarth/ Tillmann 1995, Kolbe 1996, Fuchs/Lamnek/Luedtke 1996, Schubarth 1997). Die Erklärungskraft der Schulform ist aber mit einiger Berechtigung in jüngster Zeit in Zweifel gezogen worden, da in der Schulform letztlich die Individualmerkmale der Schüler (vgl. Funk/Passenberger 1997, S. 260) bzw. milieuspezifische Merkmale der Schülerpopulationen (vgl. Heitmeyer/Ulbrich-Herrmann 1997) mit ihrem jeweiligen Einfluß auf Gewalthandeln gebündelt werden und sich ein direkter Einfluß des Schultyps kaum nachweisen läßt. Damit soll die Betrachtung schulformspezifischer Gewaltbelastung nicht als ein marginales Problem abgewertet werden - im Gegenteil: den stärker gewaltbelasteten Schulformen muß nach wie vor ein besonderes Augenmerk der Gewaltforschung, insbesondere bei der Konzeptualisierung kontextbezogener Präventionsansätze, gelten. Gleichwohl zeigt sich aber - ähnlich wie in der Schulqualitätsdebatte -, daß der Blick auf das Gesamtsystem von Schule und die Schullandschaft allein zu kurz greift und um eine Auseinandersetzung mit dem innerschulischen Bedingungsgefüge für Gewalt ergänzt werden muß. Dies bestätigt auch die geringe Erklärungskraft anderer organisationsbezogener Merkmale, wie beispielsweise die Schul- und die Klassengröße, deren unmittelbarer Einfluß in mehrebenenanalytischen Modellen eher bescheiden ausfällt (vgl. Funk/Passenberger 1997, S. 259). ${ }^{1}$

Neben diesen deskriptiven Befunden liegen seit jüngster Zeit einige weiterführende Untersuchungsergebnisse zu innerschulischen Problemkonstellationen mit potentiell gewaltförderndem Charakter vor. Die Untersuchung der innerschulischen Wirklichkeit hinsichtlich ihres gewaltfördernden (bzw. -mindernden) Potentials ist dabei auf verschiedenen Ebenen betrieben worden, von denen die drei wichtigsten vorgestellt werden sollen: ${ }^{2}$

1 Allerdings haben Fuchs/Lamnek/Luedtke (1996, S. 304ff) nach Schulformen getrennte Regressionsanalysen unter Einbeziehung einer Vielzahl schulstruktureller Merkmale (Schülerzahl, Lehrerzahl, Schüler-Lehrer-Relation, Klassengröße, Jungen-Mädchen-Relation, Anteil ausländischer Schüler, Stadt-Land-Relation u.a.) mit hoher Erklärungskraft für die physische Gewalt als unabhängiger Variable durchgeführt. Daß sich für die Schulformen jeweils unterschiedliche Faktoren für die Erklärung als bedeutsam erwiesen, zeigt, daß die Evidenz organisationsstruktureller Aspekte der Schulorganisation auch weiterhin nicht unterschätzt werden sollte.

2 Nicht einbezogen werden hier Überlegungen zu Folgen der sog. „strukturellen Gewalt“, der Schüler in der Institution Schule ausgesetzt sind (hierarchischer Charakter von Schule, Erleben von Zwang, Fremdbestimmung und Unterdrückungsmechanismen in der Schule 
- In interaktionstheoretischer Perspektive wird auf die Bedeutung der Qualität sozialer Beziehungen zwischen den Schülern sowie zwischen Lehrern und Schülern verwiesen. Dabei scheint neben problematischen Interaktionszusammenhängen innerhalb der Schulklasse (Klassenkohäsion, Konkurrenz und Integrationsfähigkeit von Schulklassen) insbesondere ein die Schüler stigmatisierendes bzw. etikettierendes Lehrerverhalten im Sinne des „Labeling-approach-Ansatzes“ deviante Verhaltensformen zu fördern und unter Umständen dauerhaft zu manifestieren (vgl. Holtappels 1993, Meier 1997). Problembelastete Lehrer-Schüler-Beziehungen werden auch von Funk (1995, S. 201ff) als Indikator für Gewalthandeln und Gewalteinstellungen hervorgehoben, während er den Einfluß problematischer Schüler-Schüler-Beziehungen auf Gewalt nicht bestätigen konnte. Allerdings weist der von ihm nachgewiesene Zusammenhang zwischen negativen Schüler-Schüler-Beziehungen und dem Opferstatus auf die besondere Bedeutung von Desintegrationserfahrungen innerhalb der Schule hin.

- Die Bedeutung der Lehrer-Schüler-Beziehung wird auch hinsichtlich der Professionalität des Lehrerhandelns hinterfragt. Hier treten zunehmend Aspekte des Unterrichtens und der Unterrichtsgestaltung neben sozial-erzieherische Dimensionen, die über den Unterricht hinausweisen. So konnte von Meier unter Verwendung des Begriffs der „Lernkultur“ die Relevanz eines lebensweltbezogenen und schülerorientierten Unterrichts und eines an den Schülerbedürfnissen ausgerichteten Förderengagements der Lehrer für das deviante Verhalten der Schüler nachgewiesen werden (Meier 1997, S. 235).

- In der subjektiven Perspektive der Schüler werden schulische Belastungen und Problemlagen als devianzfördernde Dimensionen hervorgehoben. Dabei wird insbesondere auf die Bedeutung der an die Schüler gestellten Leistungsanforderungen im Verhältnis zu ihren subjektiven Bewältigungsmöglichkeiten (Leistungsdruck) hingewiesen (Mansel/Hurrelmann 1991, Meier 1997, S. 235). Auch hier zeigen sich Differenzen zwischen einzelnen Studien: Nach den Befunden Funks haben schulische Problembelastungen keinen Einfluß auf gewaltförmige Handlungen und Einstellungen (1997, S. 207).

Schließlich haben die komparatistisch angelegten Fallstudien, die unsere Forschungsgruppe durchgeführt hat (vgl. Schubarth/Stenke 1996, Schubarth/ Darge/Mühl/Bergelt/Börner/Hannich 1997), wichtige Hinweise auf schulspezifische Bedingungsfaktoren für Aggression und Gewalt geliefert. Im Ver-

usw.). Diese sind in jüngster Zeit eher ins Abseits empirischer Gewaltforschung geraten. $\mathrm{Da}$ die von Schülern erlebte strukturelle Schulgewalt unter Umständen in gewalthaltigen Handlungsweisen verarbeitet wird bzw. - vorgelebt als erfolgreich praktiziertes Interaktionsmuster - zur Nachahmung einlädt, wird von Holtappels (1993, S. 127) betont (vgl. auch Kap. 1). 
gleich von zwei Schulen gleicher Schulform mit ähnlichem sozialen Umfeld, die hinsichtlich der Gewaltentwicklung bzw. des Gewaltausmaßes Differenzen aufweisen, zeigten sich auch Unterschiede in der pädagogischen Ausgestaltung des Schullebens und der Schulkultur, die mit der Gewaltbelastung korrespondierten. Im einzelnen waren das der Führungsstil der Schulleitung, die Professionalität des Lehrerhandelns im unterrichtlich-didaktischen wie auch im außerunterrichtlich-erzieherischen Bereich und die Gestaltung der Sozialbeziehungen, insbesondere des Lehrer-Schüler-Verhältnisses. Diese Bereiche wurden als bedeutsam für die Gewaltbelastung an den Schulen und ebenso als Ausgangspunkte für die Präventionsarbeit im Sinne einer Schulentwicklungsarbeit bewertet (vgl. dazu Kap. 8).

Als Resümee der vorliegenden Ergebnisse schulbezogener Gewaltforschung kann festgestellt werden, daß für deviantes Verhalten unter Schülern die Schule als Sozialisationsfeld - entgegen der zu einiger Popularität gelangten Vermutung, daß es sich bei der Schule nur um den Austragungsraum andernorts verursachter Gewalterscheinungen handeln würde - eine deutliche Mitverantwortung trägt. Einzelne „Risikobereiche“ (Lehrer-Schüler-Beziehung, professionelles Lehrerhandeln, schulische Belastung der Schüler) sind anhand der referierten Ergebnisse zwar erkennbar, sie bedürfen aber einer weitergehenden systematischen Analyse. Auch hinsichtlich der Vernetzung schulischer Sozialisationseffekte in bezug auf Gewalthandeln mit den Effekten anderer Sozialisationsinstanzen (Familie, Peers, Medien) in Mehrebenenanalysen besteht noch Klärungsbedarf. ${ }^{3}$ Mit einer isolierten Betrachtung schulischer Auslösungsfaktoren für Gewalthandeln würde die schulbezogene Gewaltforschung Gefahr laufen, zu einer einseitigen „Belastungsforschung für die Institution Schule“ zu werden. Daher erfordert die Aufklärung von Gewaltursachen bei der Betrachtung der Institutionsebene eine doppelte Perspektive: eine differenzierte Untersuchung innerschulischer Problemlagen und deren Einbindung in ein komplexes makrosoziales Erklärungstableau (vgl. Kap. 1.4).

In diesem Kapitel ist die Untersuchung der schulischen Bedingungsfaktoren für Gewalt Gegenstand einer detaillierten Analyse. Die Befunde sind als Zwischenergebnis zu werten und werden dann in ein Gesamtmodell zur Erklärung gewalthaltigen und gewaltaffinen Handelns einfließen (vgl. Kap. 7). Unsere Argumentation baut sich wie folgt auf: In einem ersten Schritt werden wir uns vor dem Hintergrund der gegenwärtig geführten Schulkultur-Debatte mit der Tragfähigkeit des „Schulkultur-Theorems“ zur Erklärung des Zusammenhangs von schulbezogenen Merkmalen und gewaltförmigem Verhalten von Schülern auseinandersetzen. Damit soll gleichzeitig versucht werden, die

3 Erste diesbezügliche Ergebnisse, die auf eine eher untergeordnete, allenfalls gleichrangige Bedeutung gewaltfördernder Dimensionen innerhalb der Schule verweisen, finden sich bei Funk/ Passenberger (1997, S. 256ff) und Kühnel/Matuschek (1997, S. 279). 
weitgehend separat geführte Gewaltdiskussion an schulpädagogische Fragestellungen - insbesondere an Fragen der Schulqualitäts- und Schulentwicklungsforschung - anschlußfähig zu machen. Wir werden in diesem Zusammenhang ein Schulkultur-Modell vorstellen, das im wesentlichen auf einer von unserer Forschungsgruppe in einem anderen Forschungszusammenhang empirisch gewonnenen Konzept basiert (vgl. Melzer/Stenke 1996, S. 326), das jedoch aufgrund unterschiedlicher Instrumentarien und Forschungsintentionen einige Modifikationen erfährt (6.1). Dieses Modell mit seinen - wie wir meinen - auch für Gewalt erklärungsrelevanten Bereichen der sozialräumlichen Schulumwelt und der Lehrerprofessionalität sowie der mit der Schulkultur korrespondierenden Merkmale der Schülerbefindlichkeit wird anhand der Schülerdaten überprüft und die Ergebnisse in einer deskriptiven Darstellung aus der Individualperspektive vorgestellt (6.2). Dazu werden StellvertreterItems für die Faktoren aus den drei genannten Bereichen interpretiert, die den jeweiligen Faktor inhaltlich und statistisch gut repräsentieren. Diesen Abschnitt abschließend sollen im Ost-West-Vergleich mögliche Unterschiede und Gemeinsamkeiten der Schulkultur in den sächsischen und hessischen Schulen herausgearbeitet werden.

Im Zentrum dieses Kapitels (6.3) steht die Überprüfung unseres Modells mit Hilfe einer zunächst bivariaten Zusammenhangsanalyse zwischen schulkulturellen Merkmalen und gewaltförmigen bzw. gewaltaffinen Handlungen und Wahrnehmungen. Dazu werden die Daten auf Klassenebene ${ }^{4}$ aggregiert, um die schulkulturellen Aspekte über die Ebene individueller Bewertung von Einzelpersonen hinaus zu kontrollieren und einer intersubjektiven Bewertung zu unterziehen und damit zu ,verobjektivieren“. Neben einer grundsätzlichen Prüfung des Einflusses schulkultureller Aspekte auf die Gewaltemergenz richtet sich unser Interesse auf eine möglichst differenzierte Exploration des $\mathrm{Zu}$ sammenhangs einzelner Schulkulturbereiche mit bestimmten Ausprägungen gewalthaltigen und gewaltaffinen Wahrnehmens und Handelns (z.B. Schuldevianz, Selbstreport härterer und weicherer Gewaltformen und außerschulische Delinquenz). Abschließend werden wir das Zusammenwirken von Aspekten der Schulkultur und des Syndroms gewalthaltiger und gewaltaffiner Verhaltensweisen - unter Einbeziehung protektiver bzw. verstärkender Regulationsmechanismen - überprüfen.

4 Die Aggregierung der Daten bezieht sich auf 178 verschiedene Schulklassen, in denen die Befragung durchgeführt wurde. 


\subsection{Schulkultur und Gewalt - ein Modell}

Bei der Analyse schulinterner Bedingungsfaktoren für gewalthaltige und gewaltaffine Einstellungen und Verhaltensweisen sind wir einerseits davon ausgegangen, daß spezifische Problemkonstellationen an Einzelschulen mit ausschlaggebend für das Gewaltausmaß ihrer Schülerschaft sein können. So ist es beispielsweise vorstellbar, daß an Schulen an denen ein negatives Lehrer-Schüler-Verhältnis besteht, das durch mangelndes Vertrauen, fehlende gegenseitige Anerkennung oder mangelnde Rücksicht auf die Interessen und Bedürfnisse des jeweils anderen gekennzeichnet ist, sich dieser Problembereich begünstigend für deviantes Verhalten von Schülern auswirkt. Wir haben uns dabei auf zwei zentrale Bereich des Schulalltags konzentriert: Mit dem Aspekt der Lehrerprofessionalität werden didaktische und pädagogische Aspekte der Lehrerrolle auf ihre Relevanz für das Gewalthandeln unter Schülern überprüft. Unter dem Leitbegriff der sozialräumlichen Schulumwelt wurden schulklimatische, schul-ökologische und partizipatorische Dimensionen des Schulalltags zusammengefaßt. Im übrigen gehen wir aber davon aus, daß die isolierte Betrachtung eines dieser Teilbereiche oder gar der darunter subsumierten konkreten Merkmalsausprägungen kaum ausreichenden Aufschluß über innerschulische Gewaltursachen geben kann. Vielmehr scheint uns die Gesamtheit der an einer Schule sich vollziehenden pädagogischen Handlungskonzepte, Lern- und Erziehungsprozesse und der Interaktionszusammenhänge zwischen allen Schulbeteiligten, d.h. die institutionelle, pädagogische und organisatorische Ausgestaltung der Schule als Ganzer für das Ausmaß abweichenden und gewalthaltigen Verhaltens evident. Das Konzept, in dem diese Einzelaspekte zusammenfließen, ist das der Schulkultur, das sich diesbezüglich durch seine „Integrationsleistung“ (Wiater 1997, S. 39) auszeichnet: „Schulkultur" bezieht die Perspektiven aller an Schule Beteiligten in bezug auf den Schulalltag ein, es integriert einzelne Aufgabenfelder und Funktionsbereiche von Schule sowie ihre pädagogische Ausgestaltung und die innerschulischen Voraussetzungen für diesen Ausgestaltungsprozeß einschließlich ihrer äußeren Umfeldbedingungen.

Ein zentraler integrativer Aspekt besteht darüber hinaus in der Verknüpfung zweier neuerer schulpädagogischer Topoi: dem der Schulqualität und dem der Schulentwicklung. Schulkultur soll einerseits aufmerksam machen auf die Verfaßtheit der an einer Schule vorzufindenden Aspekte der Lernkultur, Erziehungskultur und Organisationskultur (vgl. Holtappels 1995, S. 12). ${ }^{5}$ Daneben beinhaltet Schulkultur aber auch einen Entwicklungsaspekt, der die übergrei-

5 In unserer Studie wurden bei der Schülerbefragung lediglich die beiden ersten Bereiche der Lern- und Erziehungskultur, nicht aber der der Organisationskultur thematisiert, weil sich dieser in der Regel der Beurteilung der Schüler entzieht. 
fenden pädagogischen Handlungskonzepte und Problemlösungsversuche - verstanden als ein permanenter Prozeß - umfaßt. Mit einem derartigen Rahmenkonzept werden die im folgenden vorzustellenden Zusammenhangsanalysen zwischen Elementen der Schulkultur und dem gewalthaltigen Schülerhandeln als Ausdruck einer problematischen Schulqualität interpretiert. Wenn sich die vermuteten Zusammenhänge bestätigen, wäre dies gleichzeitig ein Hinweis auf geeignete Ausgangspunkte für eine Gewaltprävention im Sinne von Schulentwicklung (vgl. Kap. 8).

Bei einer Schulkultur-Analyse scheint uns ein in der Schulkultur-Debatte konträr diskutierter Aspekt besonders wichtig zu sein: Schulkultur wird einerseits betrachtet als ein Modell, das den Konsens aller an Schule Beteiligten betont (vgl. z.B. Aurin 1993, S. 17ff, Seibert 1997, S. 441), wobei die Perspektive der Schüler - zumal in der empirischen Umsetzung des Konsenspostulats - bisher eher zu wenig beachtet wird. Dem steht ein sich auf „Konflikt, genauer, (...) die Gestaltung von Modalitäten der Konfliktaustragung“ (Terhart 1994, S. 695) beziehender Schulkultur-Begriff gegenüber. Für eine Einbettung des Schulkultur-Konzepts in die Gewaltproblematik ist es naheliegend, den Aspekt der Konflikt-Lösung als Zielkategorie zu übernehmen, zumal nach unserem Verständnis bei einer schulumfassenden Perspektive ein pluralistisches und gewaltreguliertes Schulleben eine sowohl realistische als auch hinsichtlich eines lebensweltbezogenen Ansatzes anstrebenswerte Perspektive darstellt. Von daher stellen der Umgang mit Gewalt und die äußeren Regulierungsmechanismen als interaktionale Komponenten eine vermittelnde Dimension zwischen der Schulkultur und dem Syndrom gewaltaffiner und gewalthaltiger Einstellungen und Verhaltensweisen dar. In Abbildung 6-1 haben wir den Versuch unternommen, die angesprochenen Theorieelemente in einem Schema zusammenzufassen (vgl. Abb. 6-1).

Mit diesem Modell soll eine an der Schulkulturdebatte orientierte und kontextbezogene Analyse für das Bedingungsgefüge von Gewalt und ihren schulinternen Bedingungsfaktoren ermöglicht werden. Grundlegend ist dabei die Annahme, daß Schulkultur und Gewalt nicht miteinander kompatibel sind und sich gegenseitig ausschließen. Schulen, die massiv durch Gewalt belastet sind, verfügen kaum über eine positive Schulkultur, und umgekehrt ist davon auszugehen, daß Schulen mit positiver Schulkultur in der Lage sind, Gewalt wirksam zu begegnen und zu verhindern (eine erste empirische Annäherung an diese Hypothese liefern Stenke/Melzer 1996 und Melzer/Rostampour 1996). Damit begreifen wir Gewalt an Schulen als einen Indikator für eine negative Schulkultur. Um diesen Wirkungszusammenhang zu verdeutlichen, haben wir die Schülerbefindlichkeiten als einen vermittelnden Bereich eingeführt. Wir betrachten Schülerbefindlichkeiten als subjektive Reaktionen der Schülerschaft auf das schulkulturelle Entwicklungsniveau und die entsprechenden Handlungskonzepte, die an der jeweiligen Schule vorherrschen. Wir sehen sie aber auch vor dem Hintergrund der oben referierten Ergebnisse aus der schulbezogenen Ursachenforschung für Gewalt als mögliche Auslösefaktoren 
für deviante Verhaltensweisen. In unserem Modell sind die Schülerbefindlichkeiten daher als ein integraler Bestandteil der Schulkultur dargestellt worden, der aber wenigstens teilweise als eine selbständige Erklärungsdimension in Betracht zu ziehen ist.

Abb. 6-1: Modell - Schulkultur und Gewalt

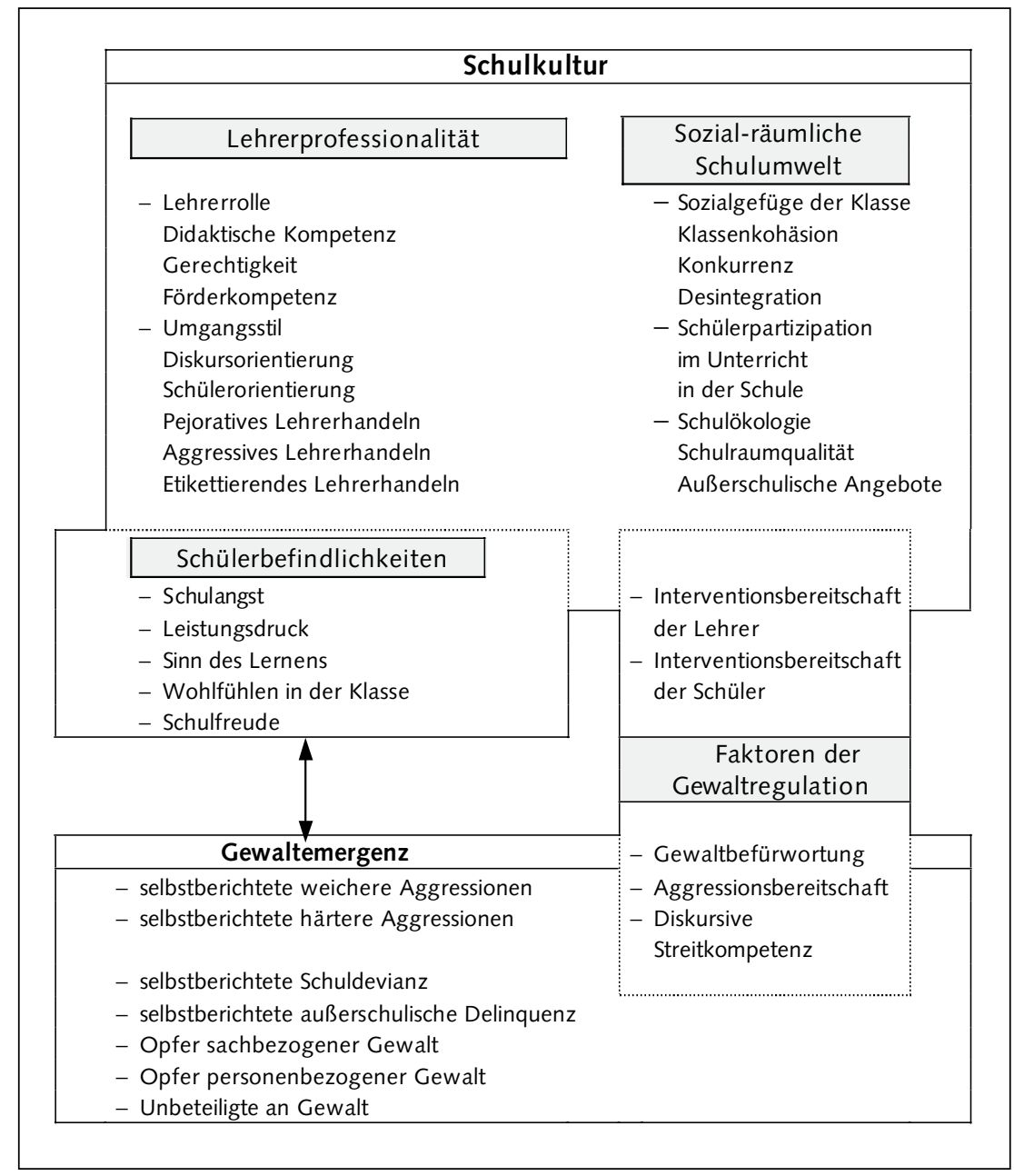


Als Indikatoren für das Syndrom gewaltförmiger und gewaltaffiner Verhaltensweisen (vgl. Kap. 1.4, 2, 4) unterscheiden wir zwischen härteren und weicheren Gewaltformen, zwischen ausgeübter und erlittener Gewalt sowie zwischen Gewalt innerhalb der Schule und Formen außerschulischer Delinquenz. Wir gehen dabei davon aus, daß der Wirkungszusammenhang zwischen schulkulturellen Merkmalen und Gewaltmerkmalen - ähnlich wie bei den familialen Sozialisationseinflüssen - im Bereich der innerschulischen, weicheren Gewaltformen besonders hoch, im Bereich der außerschulischen, härteren Gewaltformen dagegen niedriger ausfällt. Weiterhin vermuten wir Regulationseffekte durch eine äußere Steuerung (z.B. durch Lehrer- und Schülerintervention) sowie eine persönlichkeitsinterne Steuerung, bei der z.B. die Aggressionsbereitschaft verstärkend und die negativen Einstellungen zur Gewalt und eine diskursive Streitkompetenz protektiv intervenieren.

Unser Schulkultur-Begriff schließt also interaktionale und auch Besonderheiten bzw. Unterschiede der einzelnen Klassen einer Schule ein. Wenn wir die folgenden Analysen auf der Basis von auf Klassenebene aggregierten Daten durchführen, so hat das neben methodisch-statistischen (größere Anzahl der Untersuchungseinheiten im Vergleich zur Anzahl der Schulen in der Stichprobe) vor allem theoretische Gründe. Es sind dieselben, die Parsons zu einer Untersuchung der „Schulklasse als soziales System“ veranlaßt haben: die Klasse sei der Ort, „wo das eigentliche 'Geschäft' der formalen Erziehung tatsächlich besorgt wird“" (Parsons 1968, S. 161).

\subsection{Lehrerprofessionalität, Sozial-räumliche Schulumwelt und Schülerbefindlichkeiten als Aspekte der Schulkultur}

Für die inhaltliche Konkretisierung des Bereichs Schulkultur wurden Faktorenanalysen durchgeführt, in deren Ergebnis sich für die Lehrerprofessionalität, die sozialräumliche Schulumwelt und die subjektiven Schülerbefindlichkeiten mehrere Subkategorien abzeichneten. Anhand von Stellvertreter-Variablen, die den jeweiligen subkategorialen Faktor inhaltlich und statistisch gut repräsentieren, zeigen die Tabellen 6-1 und 6-2 die Beurteilungen der jeweiligen schulkulturellen Aspekte durch die sächsischen Schüler, während die Tabelle 6-3 die aus diesen Bedingungen resultierenden Schülerbefindlichkeiten ausweist. ${ }^{6}$ Die sich daran anschließende Deskription der einzelnen schulkulturellen Aspekte

6 Für das Interventionsverhalten der Lehrer und Schüler verweisen wir auf die ausführliche Deskription der entsprechenden Variablen in Kapitel 2. Die persönlichkeitsintegralen Steuerungselemente sind bereits in den Kapiteln 4 und 5 mit in die Analyse eingeflossen. 
berücksichtigt noch weitere in den jeweiligen Faktor eingeflossene Items, die zwar nicht tabellarisch ausgewiesen sind, aber in Form von Trendaussagen in die Interpretation eingehen.

\section{Schulkultur im Urteil der Schüler}

\section{Lehrerprofessionalität:}

Um die Qualität des Lehrerhandelns zu erfassen, wurden Faktoren gebildet, die inhaltlich die Ausgestaltung des unterrichtlichen und außerunterrichtlichen Lehrerverhaltens gegenüber den Schülern beschreiben: die didaktische Kompetenz, die Gerechtigkeit und die Förderkompetenz als allgemeine Aspekte der Lehrerrolle sowie die sozialen Kompetenzen der Lehrer im Umgang mit den Schülern sind die beiden großen inhaltlichen Dimensionen der Lehrerprofessionalität. Mit diesen insgesamt 8 Faktorvariablen wird das didaktische und pädagogisch-erzieherische Konzept des Lehrerhandelns bzw. seine Realisierung - aus der Perspektive der Schüler - in den Blickpunkt genommen. ${ }^{7}$ Die aufgeführten Bereiche betreffen jeweils professionsspezifische Aspekte des Lehrerberufs, die über das allgemeine Verhältnis zwischen Lehrern und Schülern hinausweisen (vgl. Tab. 6-1).

In den Faktor Didaktische Kompetenz sind insgesamt fünf Items eingeflossen, die auf die Anschaulichkeit des Unterrichts, ein zu schnelles Lerntempo, die Erklärungskompetenz der Lehrer und ihre Fähigkeit, einen abwechslungsreichen, nicht langweiligen Unterricht zu gestalten, abzielen. Das Urteil der Schüler über die didaktischen Fähigkeiten der Lehrer fällt ambivalent aus: Neben der großteils (45\%) positiv wahrgenommenen Erklärungskompetenz der Lehrer, wird ihrem Unterricht vor allem Langeweile (54\%) bescheinigt. Ein als zu hoch wahrgenommenes Lerntempo (34\%) steht einem als anschaulich (31\%) und abwechslungsreich (34\%) erlebten Unterricht gegenüber.

Die Gerechtigkeit des Lehrerhandelns, die mit zwei Items erfaßt wurde, wird von den Schülern bezüglich der gerechten Leistungsbeurteilung nicht sehr positiv beurteilt. Lediglich $37 \%$ von ihnen meinen, daß die Lehrer ihre Schulleistungen angemessen beurteilen würden; zudem zeigt sich die Mehrheit

7 Dies hat ein methodisches Dilemma zur Folge: Die Professionalität des Lehrerhandelns ist streng genommen nur auf der Individualebene (der Lehrer) erfaßbar, diese aber läßt sich aus forschungspragmatischen und Datenschutzgründen nicht erheben - zumindest nicht in der Perspektive der Schüler. Die Schüler wurden also nicht nach dem Verhaltensrepertoire eines konkreten Lehrers gefragt, sondern nach Lehrern im allgemeinen. Daß die Ausweichkategorie „teils teils“ bei der Bewertung des Lehrerverhaltens bevorzugt gewählt wurde, könnte auf die Schwierigkeiten der Schüler hinweisen, aus den Verhaltensweisen, Interaktionsformen u.ä. von - in der Regel - über zehn Lehrern, mit denen sie allein im Fachunterricht konfrontiert sind, ein übergreifendes Verhaltensmuster zu abstrahieren. 
der Schüler wiederum unentschlossen (47\%). Noch weniger Schüler geben ein positives Votum bei einer allgemeinen Beurteilung des Lehrerverhaltens bezüglich der Gerechtigkeit ab (27\%). Insgesamt verweist die eher negative Beurteilung des Lehrerhandelns somit auf eine Störung des Lehrer-SchülerVerhältnisses in einem zentralen gesellschaftlichen Funktionsbereich von Schule.

Tab. 6-1: Faktoren zur Beurteilung von Aspekten der Lehrerprofessionalität durch die Schüler

\begin{tabular}{|c|c|c|c|c|}
\hline \multirow[b]{2}{*}{ Faktor } & \multirow[b]{2}{*}{ Stellvertreter-Item* } & \multicolumn{3}{|c|}{ Häufigkeit (in \%)** } \\
\hline & & $\begin{array}{l}\text { Zustim- } \\
\text { mung }\end{array}$ & teils teils & Ablehnung \\
\hline \multicolumn{5}{|l|}{ Lehrerrolle } \\
\hline $\begin{array}{l}\text { Didaktische Kompetenz } \\
\text { (V69, V70, V72-V74) }\end{array}$ & Lehrer erklären gut (V69) & 45,2 & 39,1 & 15,7 \\
\hline Gerechtigkeit (V171, V245) & $\begin{array}{l}\text { Lehrer beurteilen Schulleistungen } \\
\text { gerecht (V171) }\end{array}$ & 37,4 & 47,4 & 15,1 \\
\hline $\begin{array}{l}\text { Förderkompetenz (V78, V80, } \\
\text { V81, V174-V177, V179) }\end{array}$ & $\begin{array}{l}\text { Lehrer fördern lernschwächere } \\
\text { Schüler (V176) }\end{array}$ & 46,4 & 37,8 & 15,9 \\
\hline \multicolumn{5}{|l|}{ Umgangsstil } \\
\hline $\begin{array}{l}\text { Diskursorientierung } \\
\text { (V236, V237, V184) }\end{array}$ & $\begin{array}{l}\text { Lehrer diskutieren mit Schülern } \\
\text { (V236) }\end{array}$ & 48,9 & 38,2 & 12,9 \\
\hline $\begin{array}{l}\text { Schülerorientierung } \\
\text { (V239-V244) }\end{array}$ & $\begin{array}{l}\text { Lehrer gehen auf Probleme der } \\
\text { Schüler ein (V243) }\end{array}$ & 22,9 & 49,0 & 28,1 \\
\hline $\begin{array}{l}\text { Pejoratives Lehrerhandeln } \\
\text { (V180, V185, V234) }\end{array}$ & $\begin{array}{l}\text { Lehrer blamieren Schüler vor der } \\
\text { Klasse (V185) }\end{array}$ & 31,8 & 34,0 & 34,2 \\
\hline $\begin{array}{l}\text { Aggressives Lehrerhandeln } \\
\text { (V181, V182) }\end{array}$ & $\begin{array}{l}\text { Lehrern werden handgreiflich } \\
\text { gegen Schüler (V182) }\end{array}$ & 9,3 & 14,1 & 76,6 \\
\hline $\begin{array}{l}\text { Etikettierendes Lehrerhandeln } \\
\text { (V100, V112, V113, V129) }\end{array}$ & $\begin{array}{l}\text { Lehrer behalten mich } \\
\text { dauernd im Auge (V100) }\end{array}$ & 16,6 & 27,8 & 55,6 \\
\hline
\end{tabular}

* Die genauen Item-Formulierungen finden sich im Anhang. Aus Platzgründen wurde hier eine verkürzte Formulierung gewählt.

* Skalen: 5 = stimmt ganz genau ... 1 = stimmt gar nicht. Die Kategorien "stimmt ganz genau“ und "stimmt überwiegend" wurden als Zustimmung, die Kategorien "stimmt überwiegend nicht" und "stimmt gar nicht" als Ablehnung zusammengefaßt.

In der Kategorie Förderkompetenz wurden insgesamt acht Items zu einem Faktor zusammengefaßt, die einem wichtigen Bereich pädagogisch-didaktischen Lehrerhandelns im Unterricht, nämlich der angemessenen Förderung 
von Schülern unterschiedlicher Leistungsniveaus, zuzuordnen sind. Mit der Förderung, Binnendifferenzierung und individuellen Unterstützung wird ein außerordentlich sensibel zu handhabendes Aufgabenfeld von Lehrern untersucht, das im Spannungsfeld zwischen Selektionsfunktion von Schule und Herstellung von Chancengleichheit steht, und dadurch für die Zukunftsperspektiven von Schülern eine außerordentlich hohe Bedeutung erhält. Im Mittelpunkt des Faktors steht die besondere Förderung lernschwächerer Schüler bzw. die angemessene Berücksichtigung aller Schüler. Ein Item widmet sich dem allgemeinen Bildungsengagement der Lehrer. Von den Schülern wird der Bereich der binnendifferenzierten Förderung verhältnismäßig positiv bewertet. Etwa die Hälfte der Schüler bestätigt beispielsweise eine besondere Förderung bei Lernproblemen und ein prinzipielles Interesse am Lernerfolg einzelner Schüler. Dieser Gruppe steht allerdings eine beinahe ebenso große Zahl von Schülern gegenüber, die sich unentschlossen bei der Bewertung des Lehrerengagements zeigen - die Kategorie ,teils/teils“ ist gerade im Bereich der binnendifferenzierten Förderung sehr hoch besetzt.

Mit fünf Subkategorien, die sich in statistischen Faktoren niederschlagen, wurde der Umgangsstil der Lehrer gegenüber den Schülern erfaßt. Mit dem diskursorientierten Lehrerhandeln soll die Offenheit der Lehrer gegenüber den Vorschlägen und Meinungen der Schüler einerseits beurteilt und andererseits ihre Fähigkeit, eigene Fehler einzugestehen, geprüft werden. Die Bereitschaft der Lehrer, sich auf Diskussionen und Vorschläge der Schüler einzulassen, ist - nach den Angaben der Schüler - relativ weit verbreitet. $40 \%$ bis 50\% der Schüler bestätigen das Lehrerinteresse an den Meinungen bzw. Diskussionsbeiträgen der Schüler. Dagegen beurteilen Schüler die Fähigkeit der Lehrer zur Selbstkritik deutlich negativ - lediglich $15 \%$ der Schüler bestätigen selbstkritisches Verhalten von Lehrern.

Lehrer begegnen Schülern in gestaltbaren Rollenkonzeptionen. Dieser formelle und informelle Bereich der Beziehung der Lehrer zu ihren Schülern ist in unserer Untersuchung durch einen Faktor repräsentiert, der sich unter dem Gesichtspunkt der Schülerorientierung zusammenfassen läßt und Aspekte des Vertrauens von Schülern gegenüber Lehrern, der Anerkennung der Schüler mit ihren besonderen Problemen durch Lehrer und des privaten Interesses der Lehrer für die Schüler umfaßt oder allgemein den Charakter dieser Beziehung beschreibt. Auch wenn das Verhältnis der Lehrer zu ihren Schülern im allgemeinen von immerhin $40 \%$ der Befragten eher positiv eingeschätzt wird und sich ein großer Teil der Schüler (45\%) von den Lehrern ernst genommen fühlt, registrieren sie das Bemühen der Lehrer um einen persönlichen Kontakt und ein Eingehen auf ihre Probleme eher selten. Eine Folge davon könnte sein, daß die wenigsten von ihnen (je 15\%) im Lehrer eine Vertrauensperson sehen, mit der man private Probleme bereden möchte. Dies zeigt eine Begrenzung der schülerorientierten Haltung der Lehrer auf den Unterricht, die im außerunterrichtlichen Bereich - wenigstens in der Wahrnehmung der Schüler - keine Entsprechung findet. 
Der Faktor pejoratives Lehrerhandeln umfaßt das „Vorführen“ einzelner Schüler vor der gesamten Klasse, das Schlechtmachen von Schülern bei Fehlern und die „Abstrafung“ bei Disziplinproblemen mittels Noten. Das Auftreten solcher Vorgehensweisen an Schulen wird zwar nur von einer Minderheit der Schüler (überwiegend oder ganz) bestätigt, jedoch handelt es sich hierbei immerhin noch um zwischen 17\% und 32\% der Schülerschaft. Besonders häufig tritt dabei das Blamieren von Schülern auf. Somit zeigt sich ein relativ hohes Potential an bedenklichen Umgangsformen der Lehrer mit Schülern, das auf der Ausnutzung der intellektuellen Überlegenheit und der Statusvorteile der Lehrer beruht. Das pejorative Lehrerhandeln kann somit auch als ein subtil-aggressive Verhaltensweise von Lehrern gewertet werden. Eine so hohe Verbreitung haben Handgreiflichkeiten von Lehrern nicht. Gemeinsam mit dem Anschreien wurden sie zum Faktor aggressives Lehrerhandeln zusammengefaßt. Das Anschreien durch Lehrer wird allerdings von einem Drittel der Schüler bestätigt, was auf eine weit verbreitete empfindliche Störung der Lehrer-Schüler-Kommunikation verweist (vgl. zu den Lehreraggressionen auch Kap. 2). In den Bereich des Umgangsstils fallen weiterhin Erscheinungsformen etikettierenden Lehrerhandelns, die in einem vier Items umfassenden Faktor enthalten sind. Damit werden solche Verhaltensweisen von Lehrern bezeichnet, die durch eine vorurteilsgeleitete Stigmatisierung von Schülern bestimmt sind. Die Schüler nehmen etikettierendes Lehrerverhalten aber relativ selten wahr. Zwar meinen ca. 17\% der Schüler, von den Lehrern ständig beobachtet zu werden, jedoch haben nur $4 \%$ das Gefühl, von den Lehrern aufgegeben worden zu sein, $7 \%$ sehen sich als „Störenfriede“ stigmatisiert, $12 \%$ fühlen sich durch Lehrer ständig beobachtet. Im Vergleich mit den meisten Formen offener, subtiler und manifester Aggressionen (Ausnahme: Handgreiflichkeiten) scheinen etikettierende Verhaltensweisen von Lehrern seltener aufzutreten bzw. von Schülern seltener wahrgenommen zu werden.

Insgesamt zeigt sich ein eher kritisches Bild der Qualität des didaktischmethodischen sowie erzieherisch-pädagogischen professionellen Lehrerhandelns an sächsischen Schulen. Folgt man dem Urteil der Schüler, so werden konkrete Fördermaßnahmen einzelner Schüler durch die Lehrer noch relativ positiv bewertet. Auch das selten auftretende Etikettierungsverhalten sowie das Interesse an Schülermeinungen lassen sich als positiv ausgeprägte Komponenten des Lehrerhandelns hervorheben. Einen eher problematischen Bereich stellen dagegen das nicht zu unterschätzende Potential abwertender Verhaltensformen der Lehrer gegenüber Schülern und das von Schülern relativ häufig registrierte Anschreien dar. Darüber hinaus tragen die Zweifel an der Gerechtigkeit des Lehrerhandelns zu einer Belastung des Lehrer-SchülerVerhältnisses bei. Das Nebeneinander von Schülerzugewandtheit im Unterricht und autoritätsorientierter Kontroll- und Disziplinierungsmechanismen verweist somit auf eine eher brüchige als konsistente Ausgestaltung des Lehrund Erziehungsarrangements durch die sächsischen Lehrer, das sich mit dem Nachwirken spezifischer Elemente der Erziehungskonzeption an DDR-Schulen 
einerseits und der unter Umständen noch nicht vollzogenen Entwicklung neuer Lehr- und Erziehungskonzeptionen in einer gewandelten und sich wandelnden Schule und Schulumwelt andererseits erklären läßt (vgl. auch Kap. 2).

\section{Sozialräumliche Schulumwelt:}

Drei Hauptaspekte kennzeichnen die sozialräumliche Schulumwelt der Kinder und Jugendlichen: das Sozialgefüge der Klasse, das in drei Faktoren (Klassenkohäsion, Konkurrenz und Desintegration) erfaßt ist, die Partizipationsmöglichkeiten von Schülern auf Schul- wie Unterrichtsebene und schließlich in schulökologischer Perspektive die Qualität von Schulgebäuden und Schulräumen sowie die außerschulischen Angebote, die für die Schüler bereitgestellt werden (vgl. Tab. 6-2).

Das Sozialgefüge der Klasse wird durch drei Subkategorien erfaßt: $\mathrm{Zu}$ sammenhalt und Konkurrenz innerhalb der Klasse sowie die dort gemachten Desintegrationserfahrungen. Neben drei Items, die ein positives, auf Gemeinsamkeiten und gute Konfliktlösungsmuster der Klasse abhebendes Klassenklima implizieren, wurde im Rahmen des Faktors Klassenkohäsion mit einem Item die Integration von einzelnen Schülern in die Klasse erfragt. Über den allgemeinen Zusammenhalt in der Klasse urteilt eine Mehrheit (65\%) der Schüler positiv. Die Fähigkeit der Klasse zur Konfliktlösung wird mit 45\% Zustimmung - nach Angaben der Schüler - bereits niedriger veranschlagt. Die Integrationskraft der Klasse schließlich wird von noch weniger Schülern positiv eingeschätzt: $37 \%$ der Schüler gaben an, daß in ihren Klassen alle leicht Anschluß finden. Bei dieser insgesamt positiven Beurteilung läßt sich auf eine hohe Bedeutung der Klasse als Bezugsgruppe für die Schüler schließen; kein anderer Bereich der Schulkultur hat so hohe Zustimmungsquoten erhalten.

Weniger eindeutig ist die Beurteilung von Konkurrenzerscheinungen innerhalb der Klasse. Ein allgemeines Konkurrenzgefühl wird zwar noch von einer Mehrheit verneint (38\% Ablehnung gegenüber 21\% Zustimmung), aber immerhin je 30\% der Schüler haben in der Klasse Neid auf bessere Leistungen oder Rücksichtslosigkeit erlebt (gegenüber je 27\%, die dies nicht erlebt haben). Trotz des insgesamt positiv bewerteten Klassenzusammenhalts scheinen also Konkurrenz- und Neidgefühle keine Ausnahme zu bilden. Mit dem Aspekt der Desintegration wurde eine dritte, stärker an der Perspektive des Einzelnen orientierte Dimension der Schülerinteraktionen auf Klassenebene in die Analyse einbezogen. Dabei wurde in zwei Items nach dem Außenseiterstatus und der Nicht-Beachtung einzelner Schüler gefragt. Die Möglichkeit, daß man in der eigenen Klasse zum Außenseiter abgestempelt wird, sieht die Mehrheit der Schüler (53\%) allerdings nicht gegeben. Gleichwohl scheint die abgeschwächte Variante des Außenseitertums, die Nicht-Beachtung einzelner Schüler, eine größere Rolle zu spielen: immerhin 32\% der Schüler gestehen diesen Sachverhalt ein. Die Existenz konkurrierender, verfeindeter Gruppen innerhalb der Schule, die in diesem Faktor mit berücksichtigt wurde, wird aber von $46 \%$ der Schüler verneint. 
Tab. 6-2: Faktoren zur Beurteilung von Aspekten der sozialräumlichen Schulumwelt durch die Schüler

\begin{tabular}{|c|c|c|c|c|}
\hline \multirow[b]{2}{*}{ Faktor } & \multirow[b]{2}{*}{ Stellvertreter-Item* } & \multicolumn{3}{|c|}{ Häufigkeit (in \%)** } \\
\hline & & $\begin{array}{l}\text { Zustim- } \\
\text { mung }\end{array}$ & teils teils & Ablehnung \\
\hline \multicolumn{5}{|l|}{ Sozialgefüge der Klasse } \\
\hline $\begin{array}{l}\text { Klassenkohäsion } \\
\text { (V247, V249, V251, V252) }\end{array}$ & $\begin{array}{l}\text { Schüler der Klasse halten prima } \\
\text { zusammen (V249) }\end{array}$ & 67,4 & 24,6 & 8,0 \\
\hline $\begin{array}{l}\text { Konkurrenz } \\
\text { (V257-V259) }\end{array}$ & $\begin{array}{l}\text { in der Klasse will jeder besser als } \\
\text { andere sein (V259) }\end{array}$ & 21,3 & 40,7 & 38,0 \\
\hline $\begin{array}{l}\text { Desintegration } \\
\text { (V246, V248, V250) }\end{array}$ & $\begin{array}{l}\text { in der Klasse werden Schüler } \\
\text { wenig beachtet (V246) }\end{array}$ & 24,9 & 43,4 & 31,7 \\
\hline \multicolumn{5}{|l|}{ Schülerpartizipation } \\
\hline $\begin{array}{l}\text { auf Unterrichtsebene } \\
\text { (V65, V75) }\end{array}$ & $\begin{array}{l}\text { etwas eigenes in den Unterricht } \\
\text { einbringen (V65) }\end{array}$ & 18,6 & 45,2 & 36,1 \\
\hline $\begin{array}{l}\text { auf Schulebene } \\
\text { (V235, V238) }\end{array}$ & $\begin{array}{l}\text { Beschwerde über Verbote nützt } \\
\text { nichts (V238) }\end{array}$ & 52,9 & 33,6 & 13,5 \\
\hline \multicolumn{5}{|l|}{ Schulökologie } \\
\hline $\begin{array}{l}\text { Schulraumqualität } \\
\text { (V311-V315) }\end{array}$ & $\begin{array}{l}\text { man hält sich gern im Schul- } \\
\text { gebäude auf (V314) }\end{array}$ & 12,8 & 32,9 & 54,4 \\
\hline $\begin{array}{l}\text { Außerschulische Angebote } \\
\text { (V82-V91)*** }\end{array}$ & $\begin{array}{l}\text { Zufriedenheit mit dem } \\
\text { Schülercafé }\end{array}$ & 23,3 & - & 17,1 \\
\hline
\end{tabular}

* Die genauen Item-Formulierungen finden sich im Anhang. Aus Platzgründen wurde hier eine verkürzte Formulierung gewählt.

* Skalen: 5 = stimmt ganz genau ... 1 = stimmt gar nicht. Die Kategorien "stimmt ganz genau“ und "stimmt überwiegend" wurden als Zustimmung, die Kategorien "stimmt überwiegend nicht" und "stimmt gar nicht" als Ablehnung zusammengefaßt.

** Es wurden zehn außerschulische Angebote vorgestellt, bei denen erfragt wurde, ob es ein solches Angebot an der Schule gibt und darum gebeten, dieses auf einer vierstufigen Skala zu beurteilen ( 1 = bin damit sehr unzufrieden $\ldots 4=$ bin damit sehr zufrieden $)$.

Somit ergibt die Beurteilung der Schulklasse als wichtigster Bezugsgruppe innerhalb der Schule ein heterogenes Bild: Ein allgemein positiv eingeschätzter Zusammenhalt innerhalb der Klasse steht den nicht zu vernachlässigenden Konkurrenzerscheinungen und Desintegrationsphänomenen gegenüber. Auch innerhalb der Schulklasse scheinen sich also die Insignien der alten und der neuen Gesellschaft (Gruppenorientierung vs. Individualismus) zu überlagern und zu einer schwer durchschaubaren, in sich brüchigen Sichtweise auf die eigene Bezugsgruppe zu vermischen. 
Als ein weiterer Bereich der sozialräumlichen Schulumwelt sind die innerschulischen Interaktionsstrukturen in Form von Partizipationsmöglichkeiten der Schülerschaft auf der Unterrichtsebene und auf der Schulebene thematisiert (vgl. Melzer 1998). Folgt man den Aussagen der Schüler, so sind in beiden Bereichen deutliche Defizite zu verzeichnen. Ein Drittel der Schüler sieht keine Möglichkeit, eigene Vorschläge in den Unterricht einzubringen (gegenüber ca. 20\%, die diese Möglichkeit sehen) und sogar die Hälfte aller Schüler bestätigt, daß die Beschwerde über Schulregeln und -verbote nichts nütze.

Die Schulraumqualität, als eine der beiden schulökologischen Dimensionen, wird von sächsischen Schülern stark kritisiert. Zwischen 50\% und 70\% der Schüler äußern ihre Unzufriedenheit hinsichtlich der Gestaltung des Schulgeländes, des Schulgebäudes und der Klassenräume. Den Schulen fehlen - so die Meinung von $68 \%$ der Schüler - gemütliche Ecken oder Rückzugsnischen. Der bauliche und ästhetisch-gestalterische Bereich sächsischer Schulen stellt somit ein außerordentlich problematisches Erbe der DDR dar.

Neben den räumlichen Bedingungen wurde die Zufriedenheit mit außerunterrichtlichen Angeboten der Schule in den schulökologischen Bereich einbezogen. Hierzu wurde den Schülern eine Liste mit zehn Angeboten bzw. Veranstaltungen (Klassenfahrten, Klassenfeste, Förderstunden, Arbeitsgemeinschaften usw.) vorgelegt, bei der sie Auskunft geben sollten, ob diese an der Schule existieren und wie zufrieden sie mit dem jeweiligen Angebot sind. Solche Veranstaltungen und Angebote sind an den sächsischen Schulen stärker verbreitet als es die öffentliche Kritik an der ,reinen Unterrichtsschule“ erwarten läßt. Selbst von Schülercafés berichten immerhin $40 \%$ der Schüler. Wenn entsprechende Angebote an den Schulen gemacht werden, äußern sich die Schüler mehrheitlich auch zufrieden mit deren Qualität.

\section{Schülerbefindlichkeiten:}

Mit dem Bereich der subjektiven Schülerbefindlichkeiten werden positive bzw. problematische Reaktionsweisen und Einstellungen der Schüler auf die Anforderungen des Schulalltags (Schulfreude, Schulangst), des Unterrichts (Leistungsdruck, Sinn des Lernens) und der Sozialbeziehungen (Wohlfühlen in der Klasse) erfaßt (vgl. Tab. 6-3).

Angst oder Nervosität bei Leistungsüberprüfungen im Rahmen des Unterrichts sind die Situationen, anhand derer wir den Faktor Schulangst konstitutiert haben. Zusätzlich dazu bezieht sich eine Frage auf die allgemeine Angst, im Unterricht etwas zu sagen, weil es falsch sein könnte. Mit Ausnahme schriftlicher Klassenarbeiten ist die Angst vor (mündlichen) Prüfungen im Unterricht bei einer Mehrheit der Schüler verbreitet. Dagegen äußern 55\% der Schüler, daß sie sich auch auf die Gefahr, etwas Falsches zu sagen, am Unterricht beteiligen, was auf eine insgesamt offene Atmosphäre des Unterrichts schließen läßt, die aber scheinbar nicht die gefürchteten mündlichen Einzelprüfungen einbezieht. 


\section{Tab. 6-3: Faktoren zur Schülerbefindlichkeit}

\begin{tabular}{|c|c|c|c|c|}
\hline \multirow[b]{2}{*}{ Faktor } & \multirow[b]{2}{*}{ Stellvertreter-Item* } & \multicolumn{3}{|c|}{ Häufigkeit (in \%)** } \\
\hline & & $\begin{array}{l}\text { Zustim- } \\
\text { mung }\end{array}$ & teils teils & Ablehnung \\
\hline $\begin{array}{l}\text { Schulangst } \\
\text { (V316-V320) }\end{array}$ & $\begin{array}{l}\text { Herzklopfen, wenn ein Lehrer } \\
\text { abfragt (V317) }\end{array}$ & 40,4 & 31,6 & 27,9 \\
\hline $\begin{array}{l}\text { Leistungsdruck } \\
\text { (V169, V170) }\end{array}$ & $\begin{array}{l}\text { Belastung durch zu viel Lernstoff } \\
\text { u. Hausaufgaben (V170) }\end{array}$ & 50,9 & 28,2 & 21,0 \\
\hline $\begin{array}{l}\text { Sinn des Lernens } \\
\text { (V64, V67, V68) }\end{array}$ & $\begin{array}{l}\text { der Sinn des Unterrichtsstoffs ist } \\
\text { unklar (V67) }\end{array}$ & 31,0 & 36,5 & 33,5 \\
\hline $\begin{array}{l}\text { Wohlfühlen in der Klasse } \\
\text { (V253-V256) }\end{array}$ & $\begin{array}{l}\text { Wohlfühlen unter den Schülern } \\
\text { der Klasse (V253) }\end{array}$ & 52,1 & 35,9 & 12,0 \\
\hline $\begin{array}{l}\text { Schulfreude (V94, V98, V109, } \\
\text { V120, V125, V127) }\end{array}$ & gern zur Schule gehen (V98) & 16,3 & 35,4 & 48,3 \\
\hline
\end{tabular}

* Die genauen Item-Formulierungen finden sich im Anhang. Aus Platzgründen wurde hier eine verkürzte Formulierung gewählt.

* Skalen: 5 = stimmt ganz genau ... 1 = stimmt gar nicht. Die Kategorien "stimmt ganz genau“ und "stimmt überwiegend" wurden als Zustimmung, die Kategorien "stimmt überwiegend nicht" und "stimmt gar nicht" als Ablehnung zusammengefaßt.

Mit der Schulangst steht der subjektiv empfundene Leistungsdruck, dem sich Schüler ausgesetzt sehen, eng in Verbindung. Dieser Faktor bezieht sich auf die bereits vorgestellte Angst vor Überprüfungen im Unterricht, die hier als eine dauerhafte Bedrohungssituation verstanden wird. Außerdem werden von den befragten Schülern die Belastungen durch eine Überfrachtung mit Lernstoff und Hausaufgaben als gravierend angesehen. Diese beiden Aspekte werden von einer Mehrheit von $46 \%$ bis $51 \%$ der Schüler als Belastungssituationen empfunden.

Der Faktor Sinn des Lernens betrifft den von den Schülern subjektiv empfundenen Lebensweltbezug oder Sinn des Unterrichts bzw. die Frage nach der Verwendbarkeit des Lernstoffs. Mit 40\% der Befragten erschließt sich einem relativ großen Teil der Schüler zwar der Bezug des Unterrichtsstoffs zur außerschulischen Wirklichkeit, einen Sinn mögen 32\% dennoch nicht darin sehen (gegenüber $31 \%$, denen sich ein Sinn erschließt). Negativ fällt auch das Urteil über die Praxisrelevanz des Lernstoffs aus - nur 23\% sehen die Möglichkeit, das Erlernte außerhalb der Schule anzuwenden (gegenüber 34\%, die diese Möglichkeit nicht sehen).

Die Individuum-Klasse-Beziehung, die wir mit dem Faktor Wohlfühlen in der Klasse erfaßt haben, gehört zum Kern der Schülerbefindlichkeiten, da die Klasse für das Erleben des Schulalltags zentraler Bezugspunkt im Unterricht 
und darüber hinaus ist. Neben dem allgemeinen Wohlbefinden in der Klasse sollten sich die Schüler über das Interesse an den Klassenkameraden, über freundschaftliche Beziehungen und ihre eigene Position in der Klasse äußern. Die Mehrheit der Schüler (52\% bis 69\%) berichtet von einer guten Integration innerhalb der Klasse, wobei Freundschaftsbeziehungen eine herausragende Bedeutung haben.

Der Bereich der Schulfreude umfaßt positive Einstellungen zur Schule und einen positiv erlebten Schulalltag bzw. entsprechende negative Einstellungen, wie Mißmut u.ä.. Wie - mit Blick darauf, daß Schulunlust eine häufige Erscheinungsform im Schulalltag ist - zu erwarten war, äußert nur ein sehr kleiner Teil der sächsischen Schüler (16\%), gern zur Schule zu gehen. Neben dieser umfassenden Einschätzung der Institution Schule gewinnen die Schüler dem Schulalltag durchaus positive Seiten ab: Immerhin 37\% aller Befragten sehen viele Dinge im Schulalltag, die Spaß machen; und $43 \%$ äußern, in der Schule meist gut gelaunt zu sein.

Im Rahmen dieser Schülerbefindlichkeiten, die auf gelungene Verarbeitungsmechanismen und Belastungen des erlebten Schulalltags aufmerksam machen, könnte die eigene Klasse eine Nischenfunktion einnehmen. Sowohl hinsichtlich der Faktoren aus dem Bereich der Schulkultur als auch der damit korrespondierenden Schülerbefindlichkeiten zeigt sich die einzelne Schulklasse als im Durchschnitt eher positiv bewertete soziale Bezugsgruppe innerhalb der mehrheitlich problematisch beurteilten Institution Schule. Die insgesamt kritische Bewertung des professionellen Lehrerhandelns und der meisten Aspekte der sozialräumlichen Schulumwelt spiegelt sich entsprechend in den Reaktionen und Bewertungen zum erlebten Schul- und Unterrichtsalltag: Es dominieren - mit Ausnahme der Einschätzung der Situation in der eigenen Klasse - problematische und negative Einstellungen, wie z.B. die hohe Schulangst und die niedrige Schulfreude.

Aus Untersuchungen zur Schulfreude ist bekannt, daß diese mit zunehmender Schulzeit sinkt und in den Schulformen am relativ höchsten ist, in denen sich den Schülern die besten Zukunftschancen bieten. Auf der anderen Seite wissen wir aus der Schulqualitätsdebatte und auch aus eigenen Untersuchungen (vgl. Melzer 1997a), daß viele Qualitätsparameter schulformunabhängig sind. Wir wollen daher im folgenden kurz überprüfen, ob sich in unserer Stichprobe bei den unterschiedlichen Aspekten der Schulkultur Unterschiede zwischen den Schulformen zeigen (vgl. Tab. 6-4).

Die Parameter, bei denen signifikante Schulformunterschiede bestehen, sind in der Tab. 6-4 dunkel markiert. Dadurch wird ein sehr differenziertes Gesamtbild sichtbar. Bei den Aspekten der Lehrerrolle besteht nur bezüglich der Förderkompetenz ein signifikanter Unterschied. D.h., daß die didaktische Kompetenz und die Gerechtigkeit der Notengebung, aber auch die Diskursorientierung und mögliche pejorative Verhaltensweisen gegenüber Schülern - als Merkmale des Umgangsstils der Lehrer - unabhängig von der Schulform auftreten. Bei der Schülerpartizipation verhält es sich ebenso. In 


\section{Tab. 6-4: Schulkultur im Schulformvergleich} (Mittelwerte der Stellvertreter-Items)

\begin{tabular}{|c|c|c|c|}
\hline Faktor & Stellvertreter-Item & MS & GY \\
\hline Didaktische Kompetenz & $\begin{array}{l}\text { Lehrer erklären gut } \\
\text { (V69) }\end{array}$ & 3,39 & 3,37 \\
\hline Gerechtigkeit & $\begin{array}{l}\text { Lehrer beurteilen Schulleistungen gerecht } \\
\text { (V171) }\end{array}$ & 3,29 & 3,28 \\
\hline Förderkompetenz & $\begin{array}{l}\text { Lehrer fördern lernschwächere Schüler } \\
\text { (V176) }\end{array}$ & 3,53 & $3,27^{+}$ \\
\hline Diskursorientierung & $\begin{array}{l}\text { Lehrer diskutieren mit Schülern } \\
\text { (V236) }\end{array}$ & 3,45 & 3,51 \\
\hline Schülerorientierung & $\begin{array}{l}\text { Lehrer gehen auf Probleme der Schüler ein } \\
\text { (V243) }\end{array}$ & 2,97 & 2,87 \\
\hline Pejoratives Lehrerhandeln & $\begin{array}{l}\text { Lehrer blamieren Schüler vor der Klasse } \\
\text { (V185) }\end{array}$ & 3,02 & 2,99 \\
\hline Aggressives Lehrerhandeln & $\begin{array}{l}\text { Lehrern werden handgreiflich gegen Schüler } \\
\text { (V182) }\end{array}$ & 2,14 & 1,44 \\
\hline $\begin{array}{l}\text { Etikettierendes } \\
\text { Lehrerhandeln }\end{array}$ & $\begin{array}{l}\text { Lehrer behalten mich dauernd im Auge } \\
\text { (V100) }\end{array}$ & 2,51 & 2,32 \\
\hline Klassenkohäsion & $\begin{array}{l}\text { Schüler der Klasse halten prima zusammen } \\
\text { (V249) }\end{array}$ & 3,84 & 3,97 \\
\hline Konkurrenz in der Klasse & $\begin{array}{l}\text { in der Klasse will jeder besser als andere sein } \\
\text { (V259) }\end{array}$ & 2,81 & 2,79 \\
\hline Desintegration & $\begin{array}{l}\text { in der Klasse werden Schüler wenig beachtet } \\
\text { (V246) }\end{array}$ & 3,00 & 2,85 \\
\hline $\begin{array}{l}\text { Partizipation auf } \\
\text { Unterrichtsebene }\end{array}$ & $\begin{array}{l}\text { etwas eigenes in den Unterricht einbringen } \\
\text { (V65) }\end{array}$ & 2,75 & 2,79 \\
\hline $\begin{array}{l}\text { Partizipation } \\
\text { auf Schulebene }\end{array}$ & $\begin{array}{l}\text { Beschwerde über Verbote nützt nichts } \\
\text { (V238) }\end{array}$ & 3,55 & 3,59 \\
\hline Schulraumqualität & $\begin{array}{l}\text { man hält sich gern im Schulgebäude auf } \\
\text { (V314) }\end{array}$ & 2,26 & 2,54 \\
\hline Schulangst & $\begin{array}{l}\text { Herzklopfen, wenn ein Lehrer abfragt } \\
\text { (V317) }\end{array}$ & 3,11 & 3,31 \\
\hline Leistungsdruck & $\begin{array}{l}\text { Belastung durch zu viel Lernstoff } \mathrm{u} \text {. } \\
\text { Hausaufgaben (V170) }\end{array}$ & 3,40 & 3,56 \\
\hline Sinn des Lernens & $\begin{array}{l}\text { Sinn des Unterrichtsstoffs ist unklar } \\
\text { (V67) }\end{array}$ & 3,10 & 3,00 \\
\hline Wohlfühlen in der Klasse & $\begin{array}{l}\text { Wohlfühlen unter den Schülern der Klasse } \\
\text { (V253) }\end{array}$ & 3,47 & 3,69 \\
\hline Schulfreude & $\begin{array}{l}\text { gern zur Schule gehen } \\
\text { (V98) }\end{array}$ & 2,36 & 2,54 \\
\hline
\end{tabular}

$+\quad$ Differenzen mit einem Signifikanzniveau von $p<0.01$ sind fett gedruckt. 
bezug auf die Schüler-Schüler-Beziehungen fällt der Vergleich unterschiedlich aus: Bei einigen Parametern gibt es Vorteile für das Gymnasium (besserer Klassenzusammenhalt, weniger Außenseiter). Hinsichtlich der Konkurrenzsituation bestehen keine signifikanten Schulformunterschiede. Dagegen scheint an Mittelschulen, deren hoher Grad an Desintegration auffällig ist, das Bewußtsein der Lehrer für diese Situation höher zu sein; denn Bemühungen um eine Individualisierung von Unterricht und Schule im Sinne einer umfassenden Schülerorientierung sind hier ebenso signifikant stärker verbreitet wie Bemühungen um Binnendifferenzierung und Förderung lernschwacher Schüler. Auffällig sind die - ohne Ausnahmen - vorliegenden Signifikanzen bei den Schülerbefindlichkeiten. Gymnasiasten empfinden mehr Leistungsdruck und Schulangst, sehen aber gleichwohl den Sinn des Lernens eher ein, fühlen sich in der Schule wohler und empfinden eher Schulfreude. Sehr signifikante Unterschiede zwischen den Schulformen zeigen sich auch bei jenen Aspekten des Lehrerverhaltens, die als subtile oder manifeste Gewaltausübung gegen Schüler bzw. als Reaktion auf entsprechendes Schülerverhalten interpretiert werden können. Sowohl das aggressive Lehrerverhalten als auch ein vorurteilsgeleitetes, etikettierendes Lehrerhandeln gegenüber potentiellen Tätern sind in der Mittelschule wesentlich stärker ausgeprägt. Insgesamt sind die herausgefundenen Schulformunterschiede, vor allem in den Bereichen, die schulische Gewalt direkt betreffen, bedenkenswert und müssen im Hinblick auf unsere Modellbildung weiter verfolgt werden. Unter diesem Blickwinkel werden auch mögliche Ost-West-Unterschiede untersucht, die in Abbildung 6-2 dargestellt sind.

Bereits auf den ersten Blick zeigen sich erhebliche Unterschiede der Schulkultur, die bei aller Zurückhaltung in der Bewertung der einzelnen Kriterien, für das sächsische Bildungssystem insgesamt sehr ungünstig ausfallen. Defizite im Bereich der Lehrerprofessionalität sind auffällig, sie gibt es vor allem in bezug auf die didaktische, die Beurteilungs- und die Förderkompetenz. Ostdeutsche Lehrer stellen sich offenbar weniger auf die differenzierten $\mathrm{Ge}$ gebenheiten und individuellen Bedingungen des Unterrichts ein. Auch die Beteiligung von Schülern im Unterricht und auf Schulebene prägt den Schulalltag in Sachsen weniger als in Hessen. Bei den Schülerbefindlichkeiten sind Leistungsdruck und Schulangst eher Charakteristika des ostdeutschen Schulsystems, während Schulfreude und Wohlbefinden in westdeutschen Schulen ausgeprägter sind und auch der Sinn des Lernens eher eingesehen wird. Bei den gewaltbezogenen Kriterien der Schulkultur lassen sich dagegen keine OstWest-Unterschiede feststellen.

Was folgt aus diesen im großen und ganzen doch erheblichen Unterschieden der Schulkultur in Ost- und Westdeutschland, wenn gleichzeitig bei den Erscheinungsformen von Gewalt nur noch geringfügige Differenzen bestehen (vgl. Kap. 2)? Gehören die Parameter der Schulkultur vielleicht doch nicht zu den bedeutsamen Prädiktoren, wie wir es auf Grund der Forschungslage in unseren Hypothesen vermutet hatten? Oder gibt es Spezifika und gravierende 
Unterschiede in anderen Bereichen, die durch die Differenzen der Schulkultur ausgeglichen werden? Um diese Fragen zu beantworten, haben wir eine Reihe weiterer Datenanalysen durchgeführt.

Abb. 6-2: Schulkultur im Ost-West-Vergleich (Mittelwerte der Faktoren)

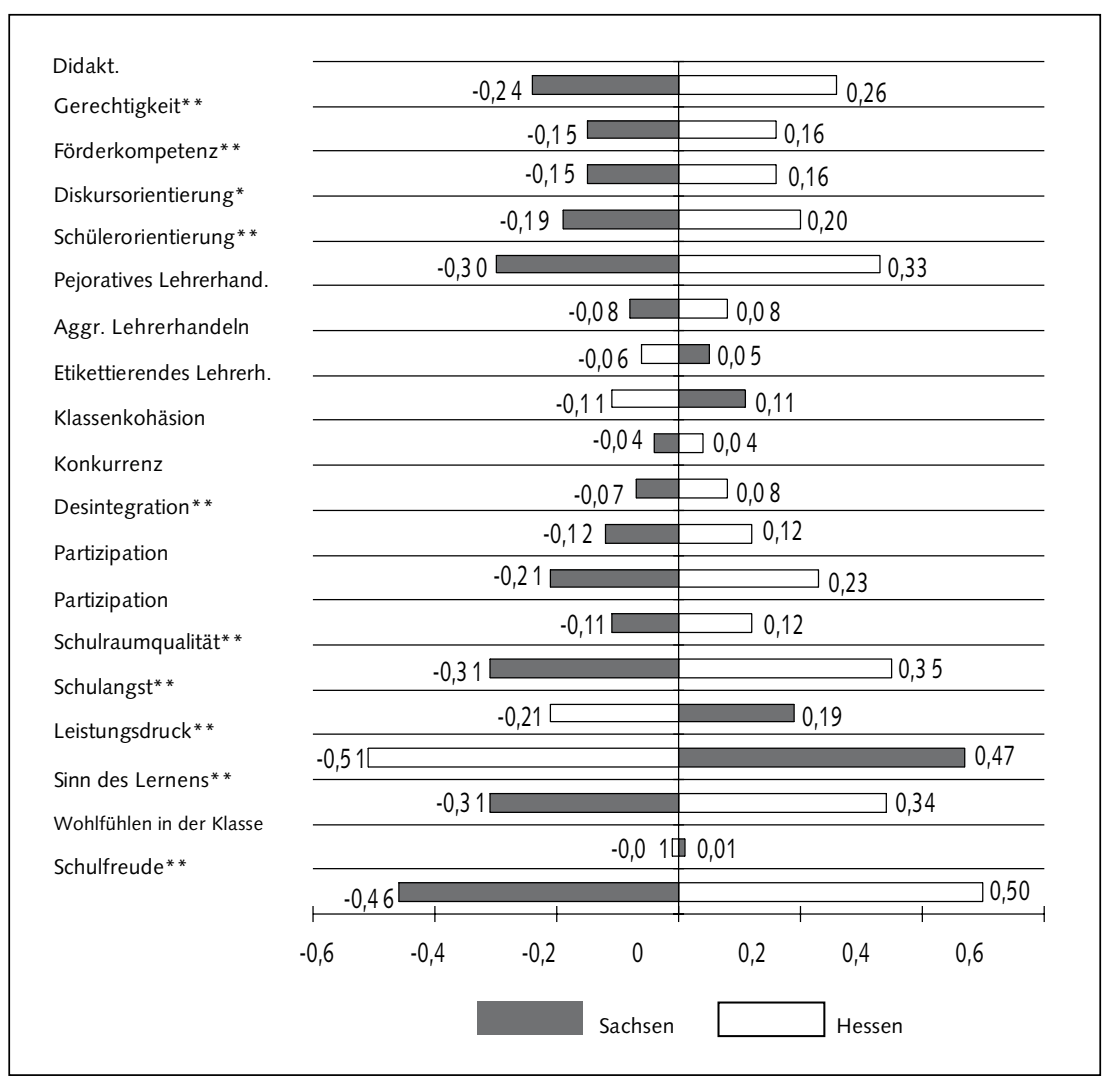

\subsection{Schul- und Erziehungskultur als Prädiktoren für das Gewalthandeln von Schülern}

Wie in der theoretischen Einführung zu diesem Kapitel dargelegt, kommt der Schulkultur eine hohe Bedeutung in bezug auf die Qualität und Entwicklung von Schule zu, so daß wir auch für die Gewaltthematik - trotz der zuletzt aufgeworfenen Fragen - von einer Relevanz dieses Einflußfaktors ausgehen (vgl. 
unser Theoriemodell, Abb. 6-1). Um dies zu überprüfen, wurde untersucht, ob und welche Zusammenhänge sich zwischen Parametern der Schulkultur, wie sie im vorangegangenen Teil entwickelt und beschrieben worden sind, und Aspekten des Gewaltsyndroms feststellen lassen. Bei den Indizes für Gewalt halten wir uns ebenfalls an die bereits eingeführte Systematik, die Aspekte des Selbstreports als Täter oder Opfer, das Unbeteiligtsein umfaßt (vgl. Kap. 5), verzichten aber aus Gründen der Übersichtlichkeit auf die Darstellung der Ergebnisse für die Einstellungen zur Gewalt bzw. zu den Kompetenzen des Umgangs mit Gewalt, für die sich dieselben Antwortstrukturen ergeben. Die Ergebnisse der bivariaten Analyse sind in der folgenden Tabelle 6-5 zusammengefaßt.

Die hier dargestellten Korrelationen basieren auf aggregierten Daten, das heißt beispielsweise, daß der Faktor „harte Aggressionen“ nicht wie bei Individualdaten den Täterstatus ausdrückt, sondern ein bestimmtes Niveau der Belastung durch harte Aggressionen in bezug auf die jeweilige Klasse, der die Schüler angehören. Ebenso gibt der Faktor „Unbeteiligte“ die diesbezüglichen Bedingungen in den Klassen an und nicht das individuelle Unbeteiligtsein als Täter oder Opfer.

Die durchgehend hoch ausfallenden Korrelationsmaße und das Signifikanzniveau der festgestellten Zusammenhänge dokumentieren, daß ein evidenter Zusammenhang von Schulkultur und Gewalt besteht. In den Klassen mit hohem Anteil des Unbeteiligtseins an Gewalt ist auch die Lehrerprofessionalität hoch, das Klassengefüge intakt, es bestehen überdurchschnittliche Beteiligungsmöglichkeiten für die Schüler, und die Schülerbefindlichkeit ist sehr positiv. Außerdem wird das mittel- und unmittelbar auf Gewalt bezogene Verhalten der Lehrer als wichtiger Einflußfaktor deutlich: Sowohl abwertendes als auch manifest-aggressives und etikettierendes Lehrerhandeln wirken verstärkend auf Gewalt. Zwar ergibt sich dieses Verhalten aus Interaktionen mit den Schülern und ist, da es sich um Korrelationen handelt, prinzipiell in beide Richtungen - als Aktion oder Reaktion - zu interpretieren. Da aber in Täterwie in Opferperspektive gleichermaßen ein solches Lehrerhandeln registriert wird, kann man nicht sagen, daß dieses nur eine Reaktion auf Schüleraggressionen ist, sondern daß Lehrer zum Teil auch mit ihrem eigenen Verhalten zur Gewaltemergenz beitragen.

Neben dem auf Gewalt bezogenen Lehrerhandeln erweisen sich zwei weitere Bereiche als bedeutsame Indikatoren für alle hier untersuchten Merkmale der Gewaltemergenz: das Sozialgefüge der Klasse und zwei Aspekte der Schülerbefindlichkeiten, das Wohlfühlen in der Klasse und die Schulfreude. Ansonsten sind die beiden folgenden Muster auffällig:

- Die meisten Parameter der Schulkultur wirken - sei es verstärkend, sei es präventiv - stärker auf die weicheren Gewaltformen als auf die härteren. Dieses Phänomen hatten wir bereits bei den Einflüssen der familialen Sozialisation festgestellt. 
Tab. 6-5: Zusammenhang zwischen Elementen der Schulkultur und Gewalt (Korrelationen)

\begin{tabular}{|c|c|c|c|c|c|c|c|}
\hline & 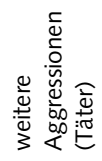 & 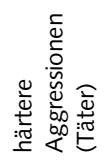 & 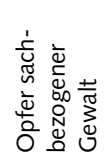 & 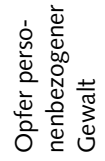 & 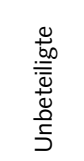 & 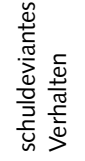 & 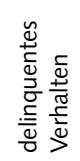 \\
\hline Didaktische Kompetenz & $-.57^{* *}$ & $-.41 * *$ & $-.19 *$ & .04 & $.50^{* *}$ & $-.64 * *$ & $-.31 * *$ \\
\hline Pejoratives Lehrerhandeln & $.56^{* *}$ & $.48^{* *}$ & $.27^{* *}$ & $.18^{*}$ & $-.50 * *$ & $.50 * *$ & $.25 * *$ \\
\hline Aggressives Lehrerhandeln & $.68^{* *}$ & $.64^{* *}$ & $.39 * *$ & .15 & $-.58 * *$ & $.64 * *$ & $.52 * *$ \\
\hline Etikettierendes Lehrerh. & $.55^{* *}$ & $.60^{* *}$ & $.44^{* *}$ & $.34 * *$ & $-.54 * *$ & $.54 * *$ & $.56 * *$ \\
\hline Förderkompetenz & $-.42 * *$ & $-.27 * *$ & -.06 & .13 & $.32 * *$ & $-.50 * *$ & -.15 \\
\hline Gerechtigkeit & $-.45^{* *}$ & $-.37 * *$ & -.14 & .03 & $.37^{* *}$ & $-.48^{* *}$ & $-.33 * *$ \\
\hline Diskursorientierung & $-.45^{* *}$ & $-.37 * *$ & -.14 & .02 & $.38^{* *}$ & $-.50^{* *}$ & $-.24^{*}$ \\
\hline Schülerorientierung & $-.43 * *$ & $-.31 * *$ & -.08 & .04 & $.37^{* *}$ & $-.43 * *$ & -.18 \\
\hline Klassenkohäsion & $-.31 * *$ & $-.30^{* *}$ & -.15 & -.14 & $.29 * *$ & $-.28^{* *}$ & $-.29 * *$ \\
\hline Konkurrenz in der Klasse & $.26^{* *}$ & $.28^{* *}$ & $.21 * *$ & $.28 * *$ & $-.24 * *$ & .13 & $.23^{*}$ \\
\hline Desintegration & $.46^{* *}$ & $.45^{* *}$ & $.31 * *$ & $.32 * *$ & $-.41 * *$ & $.35^{* *}$ & $.37 * *$ \\
\hline Partizip. Unterrichtsebene & $-.42 * *$ & $-.22 * *$ & .01 & .08 & $.30 * *$ & $-.44^{* *}$ & -.04 \\
\hline Partizipation Schulebene & $-.40^{* *}$ & $-.18^{*}$ & -.03 & .11 & $.27^{* *}$ & $-.36 * *$ & -.10 \\
\hline Schulraumqualität & $-.29 * *$ & $-.27 * *$ & -.09 & -.09 & $.23 *$ & $-.19 *$ & $-.29 * *$ \\
\hline außerschulische Angebote & $-.23 * *$ & $-.18^{*}$ & -.05 & -.01 & $.19^{*}$ & $-.20 * *$ & -.12 \\
\hline Schulfreude & $-.55^{* *}$ & $-.49 * *$ & $-.29 * *$ & $-.17^{*}$ & $.48^{* *}$ & $-.54 * *$ & $-.43^{* *}$ \\
\hline Schulangst & $-.31^{* *}$ & $-.26^{* *}$ & -.16 & .08 & $.28^{* *}$ & $-.31^{* *}$ & $-.36^{* *}$ \\
\hline Leistungsdruck & $.26^{* *}$ & .11 & -.03 & -.15 & $-.19 *$ & $.29 * *$ & -.14 \\
\hline Sinn des Lernens & $-.53 * *$ & $-.38 * *$ & -.15 & .09 & $.41^{* *}$ & $-.61 * *$ & $-.33 * *$ \\
\hline Wohlfühlen in Klasse & $-.33^{* *}$ & $-.43 * *$ & $-.28 * *$ & $-.39 * *$ & $.30 * *$ & $-.32 * *$ & $-.42^{* *}$ \\
\hline Lehrerintervention & $-.27^{* *}$ & $-.32 * *$ & $-.18^{*}$ & .00 & $.15^{*}$ & $-.42 * *$ & $-.22^{*}$ \\
\hline
\end{tabular}

${ }^{*} \mathrm{p}<.05 ;{ }^{* *} \mathrm{p}<.01$ 
- Mit Ausnahme der drei Bereiche, die auf alle Facetten von Gewalt wirken (gewaltbezogenes Lehrerhandeln, Klassensituation, schulklimatische Aspekte der Schülerbefindlichkeiten) ist die Effektstärke der Schulkultur deutlich höher in bezug auf den Täterstatus als in bezug auf den Opferstatus.

Um diese Unterschiede zu verdeutlichen wurden die Kategorien weichere und härtere Aggressionen zum Indikator „Täterbelastung einer Klasse“ und die beiden Kategorien Opfer sachbezogener und personenbezogener Gewalt zum Indikator „Opferbelastung in einer Klasse“ zusammengefaßt. Die folgende Tabelle 6-6 zeigt die Aspekte der Schulkultur, die am deutlichsten in bezug auf die Täter- bzw. Opferbelastung einer Klasse diskriminieren.

Tab. 6-6: Einfluß ausgewählter Aspekte der Schulkultur auf die Täter- und Opferbelastung einer Klasse

\begin{tabular}{lcc}
\hline \multicolumn{1}{c}{ Täter-Opfer-Belastung } & Opfer \\
Schulkultur & Täter & -.05 \\
Didaktische Kompetenz & $-.48^{* *}$ & -.04 \\
Gerechtigkeit & $-.41^{* *}$ & .02 \\
Förderkompetenz & $-.37^{* *}$ & -.02 \\
Diskursorientierung & $-.46^{* *}$ & -.01 \\
Schülerorientierung & $-.37^{* *}$ & .06 \\
Partizipation im Unterricht & $-.30^{* *}$ & .06 \\
Partizipation in der Schule & $-.27^{* *}$ & -.04 \\
Außerunterrichtliche Angebote & $-.27^{* *}$ & -.07 \\
Lehrerintervention & $-.29^{* *}$ & \\
\hline
\end{tabular}

** $\quad p<.01$

Die Daten belegen, daß die allgemeinen Aspekte der Schulkultur, wie die Lehrerprofessionalität, das Verhältnis der Lehrer zu den Schülern, die Partizipationsmöglichkeiten und das außerunterrichtliche Angebot einen großen Einfluß auf die Täterbelastung in den Klassen haben. Im Hinblick auf Präventionsstrategien kann man daraus ableiten, daß eine Verbesserung der Lernkultur und der Schulkultur insgesamt einen geeigneten Ansatzpunkt für Präventions- und Interventionsstrategien darstellen. Die zuletzt vorgestellten Daten zeigen aber ebenso deutlich, daß Strategien der Entwicklung von Schulund Lernkultur - so geeignet sie sind, Gewaltemergenz insgesamt zu begegnen - wenig in bezug auf den Opferstatus bewirken. Offenbar ist dieser Status 
biographisch nachhaltiger durch eine Kontinuität der Sozialisations- und Opfererfahrungen geprägt, die mit entsprechenden Persönlichkeitseigenschaften konvenieren, als dies für die Täterrolle zutrifft. Hinzu kommt, daß die Täter als Störenfriede von den Lehrern schnell erkannt sind und man ihrem Treiben Einhalt gebieten kann, während die Opfer schulischer Gewalt von den Lehrern häufig gar nicht erkannt werden, weil sie im Sozialverhalten wenig auffällig sind, vielleicht sogar zu den besseren Schülern gehören und sich mit ihrem geringen Selbstwertgefühl eher im Hintergrund halten (vgl. Kap. 5 und 8). So gesehen ist es auch nicht verwunderlich, daß die Lehrerintervention bei Gewalt zwar die Täter-, nicht aber die Opferbelastung einer Klasse eindämmt. Die Konsequenz für Prävention und Intervention lautet, daß eine Doppelstrategie erfolgen muß, die einerseits auf eine Optimierung von Schulqualität im Bereich von Unterricht und Schule insgesamt zielt und geeignete Kommunikationsformen mit auffälligen Schülern einschließt, andererseits opferbezogene Maßnahmen einbeziehen muß, die eher auf der Individual- und der Klassenebene anzusiedeln sind. Dafür spricht auch die relativ starke Auswirkung der Variablen zur Beschreibung der Qualität des Klassengefüges, die auch für den Opferstatus gelten (vgl. Tab 6-5). Wir versuchen daher, mit einem auf diese doppelte Anforderungsstruktur abgestimmten Präventionskonzept in Modellschulen zu arbeiten (vgl. Kap. 8).

Um dem Einwand zu entgehen, daß Korrelationen nicht als Kausalitäten zu interpretieren sind, haben wir mehrere multiple Regressionsanalysen durchgeführt und festgestellt, daß bei der Entstehung schulischer Gewalt das aggressive und etikettierende Lehrerverhalten eine große Rolle spielt, aber auch Aspekte der Klassensituation, wie Desintegration, und Aspekte der Schülerbefindlichkeit (Wohlfühlen in der Klasse, Sinn des Lernens, Schulfreude). Immer spielt auch ein hoher Jungenanteil in den Klassen gewaltunterstützend mit hinein, während der Leistungsdruck nur bei der Wahrnehmung härterer Aggressionen eine leichte Effektstärke zeigt.

\section{Offene Fragen zu Ost-West-Unterschieden in bezug auf Schulkultur und Gewalt}

Kehren wir noch einmal zu unseren Ausgangsfragen zurück, die wir angesichts der großen schulkulturellen Unterschiede in Ost- und Westdeutschland gestellt haben. Wenn die Schulkultureffekte so stark sind, wie wir zuletzt belegen konnten, besteht ein Erklärungsbedarf in bezug auf das fast gleiche Gewaltniveau in Sachsen und Hessen. Unsere Interpretation dieser scheinbar widersprüchlichen Datenlage lautet wie folgt:

- Die Ausgangsbedingungen bei den außerschulischen Gewaltprädiktoren und auch einigen Persönlichkeitsmerkmalen sind nicht völlig gleich. So bestehen beim Familienklima, dem intoleranten Binnenklima in den Peers 
und bezüglich der Aggressionsbereitschaft gewisse Unterschiede, die wir als Risikofaktoren für die sächsischen Schüler interpretieren.

- Wir haben in all unseren Untersuchungen festgestellt, daß in den Schulen Ostdeutschlands - trotz der Veränderung der gesellschaftlichen Rollen der Kinder in der Familie und der Jugendkultur sowie der Gleichheit der Heranwachsenden auf dem Markt - auf Grund der besonderen Kontinuität der Lehrerschaft und der Systemkontinuität als Staatsschule tradierte Vorstellungen von pädagogischem Handeln und aus der Sicht der Lehrer bewährte Strukturen und Rollen fortgeschrieben wurden. Diese Beharrlichkeit des Systems Schule wird von den Schülern nur zum Teil in Frage gestellt. D.h., daß die Schulkultur, wenn man sie als Gesamtkomplex der äußeren Gewaltregulation begreift, in Ostdeutschland noch funktioniert, während die Akzeptanz von äußeren Regulationsmechanismen bei Schülern in Westdeutschland geringer ist.

- Ein weiterer Grund für die Gemeinsamkeiten der Gewaltbelastung in Ost und West besteht darin, daß sich nur bestimmte und eher die allgemeinen Aspekte der Schulkultur unterscheiden, dagegen bei den eher gewaltbezogenen Aspekten keine oder nur geringe Unterschiede bestehen (vgl. Abb. 6-2); dies ist sowohl beim pejorativen, aggressiven und etikettierenden Lehrerhandeln der Fall wie auch bei mehreren Aspekten, die die Klassensituation (Klassenkohäsion und Konkurrenz) betreffen, also bei den beiden Erklärungsdimensionen, die sich multivariat als besonders einschlägig erwiesen hatten.

\section{Faktoren der Gewaltregulation}

Ein letzter Aspekt der Modellprüfung bleibt noch offen: die Frage nach den intervenierenden Variablen der äußeren Regulation, die z.B. in den Studien von Olweus immer wieder herausgestrichen wird (vgl. u.a. Olweus 1995) und die der Selbstregulation, die z.B. in den Arbeiten zur moralischen Sozialisation unter dem Stichwort der „Transformation des moralischen Denkens“ (vgl. zusammenfassend Oser/Althof 1994) ausführlich diskutiert worden ist. Nun haben wir nicht all die Variablen in unserer Untersuchung erfragt, die zur Prüfung derart anspruchsvoller und komplexer Theorien notwendig wären, allerdings wollen wir einzelne Aspekte der äußeren Gewaltregulation in Form der Interventionsbereitschaft der Lehrer und der Schüler bei auftretenden Konflikten und beobachteten Gewalthandlungen sowie der internen Steuerung (Gewaltbefürwortung, Aggressionsbereitschaft, diskursive Streitkompetenz) in ihren Auswirkungen auf Gewalthandeln überprüfen.

$\mathrm{Zu}$ den äußeren Regulationsmechanismen wurde festgestellt, daß Lehrerund Schülerintervention nicht konvenieren und keine sinnvolle Grundlage für eine Clustereinteilung bilden. Dies ist u.E. darauf zurückzuführen, daß Schülerinterventionen nicht immer in friedvoller Absicht oder im Sinne positiver 
Interventionen erfolgen, sondern z.T. auch ein „Mitmischen“ bedeuten können bzw. im Verlauf der Interaktion in aggressive Verhaltensweisen umschlagen. Daraus folgt, daß die äußere Regulation besser durch die Lehrerintervention gemessen werden kann. Diese wirkte auf Täter stärker als auf Opfer und wird aus Schülersicht bei den manifesten Gewaltsituationen in Ostdeutschland als wirksamer eingeschätzt (vgl. Tab. 6-5). Betrachtet man zwei Gruppen von Schulklassen mit hohem bzw. niedrigem Grad der Außenregulierung durch Lehrerintervention und führt für diese beiden Gruppen getrennte Korrelationsanalysen für den Zusammenhang von Schulkultur und Gewalt durch, so ergibt sich, daß in den Klassen mit hoher Lehrerintervention auch die schulkulturellen Einflüsse auf Gewalt höher sind. Beispielsweise beträgt in den Klassen mit hoher Intervention der Zusammenhang von Lehrerprofessionalität (vgl. Abb. 6-1) und Täter-Gewalt $\mathrm{r}=-.64^{* *}$ und in den Klassen mit geringerer Intervention durch die Lehrer nur $\mathrm{r}=-.54 * *$. Dies bedeutet, daß Lehrerinterventionen bei gewaltförmigen Verhaltensweisen unter Schülern angemessen, notwendig und wirkungsvoll sind, da sie die Effekte einer positiven Schulkultur verstärken bzw. im Sinne unserer Grundannahmen z.T. selbst als ein Aspekt der Schulkultur zu begreifen sind.

Die Funktion interner Steuerungsmechanismen, d.h. eine hohe Kompetenz beim Umgang mit Gewalt, eine geringe Aggressionsbereitschaft und eine ablehnende Haltung gegenüber gewaltförmigen und gewaltaffinen Verhaltensweisen wird im Rahmen der Mehrebenenanalyse (vgl. Kap. 7) untersucht.

\subsection{Zusammenfassung}

Materialreich und mit verschiedenen Analysestrategien konnte in diesem Kapitel die Relevanz der Schulkultur für die Entstehung und das Ausmaß von Gewalt unter Schülern nachgewiesen werden. Insbesondere die Lehrerprofessionalität, der Umgang von Lehrern mit Gewalt und das intakte Klima bzw. die Interaktionen innerhalb einer Klasse sind wichtige Prädiktoren bzw. Protektoren. Allerdings ist ebenso deutlich geworden, daß Lehrer nicht nur mittelbar über die Schulkultur für die sozialen Belange von Schule und Unterricht mit verantwortlich sind, sondern auch unmittelbar durch ihr eigenes latent oder manifest gewalthaltiges Verhalten Schülergewalt verursachen oder sich durch das Gewalthandeln von Schülern herausfordern lassen, z.B. indem sie diese abwerten, etikettieren - und ihnen somit keine Chance zur Besserung geben -, anschreien oder gar schlagen. Lehrer, die sich so verhalten, sind zwar eher Ausnahmen; daß sie sich innerhalb ihrer Schule so verhalten können, ist aber gleichzeitig Ausdruck der Kultur der betreffenden Schule.

Eine weitere, insbesondere für die Prävention wichtige Erkenntnis war die Tatsache, daß allgemeine Aspekte der Schulkultur sich stark auf den Täter- 
status, aber so gut wie gar nicht auf den Opferstatus auswirken. Daher haben wir für eine doppelte Präventionsstrategie plädiert, die schulumfassende sowie klassenspezifische und individuumbezogene Maßnahmen umfaßt und die Fürsorge für die Opfer in besonderer Weise berücksichtigt.

Um die Zusammenfassung inhaltlich fortzuführen, wird - unserem Theoriemodell folgend - zum Abschluß der Versuch einer Abschätzung vorgenommen, wie stark die in diesem Kapitel analysierten Aspekte der Schulkultur (Kap. 6) im Vergleich zu denen der Erziehungskultur (vgl. Kap. 5) mit dem Gewalthandeln zusammenhängen. Dazu wurden die in Kapitel 5 analysierten Individualdaten, ebenso wie bei der Schulkultur geschehen, auf Klassenebene aggregiert; entsprechend wurde mit den Variablen des Schichtmodells verfahren, dessen Erklärungskraft auf Klassenebene noch einmal überprüft werden sollte. Die Ergebnisse des bivariaten Vergleichs dieser Parameter in ihrer möglichen Wirkung auf das Gewalthandeln zeigt die Tab. 6-7.

Tab. 6-7: Außerschulische und schulische Prädiktoren des Gewalthandelns im Vergleich (auf Klassenebene aggregierte Daten; bivariate Zusammenhänge)

\begin{tabular}{lccc}
\hline Sozialisationseinflüsse & Täter-Niveau & $\begin{array}{c}\text { Niveau der } \\
\text { Schuldevianz }\end{array}$ & Delinquenzniveau \\
Niveau des Erziehungsmilieus & $.71^{* *}$ & $.56^{* *}$ & $.64^{* *}$ \\
Familie & $.42^{* *}$ & $.38^{* *}$ & $.33^{* *}$ \\
Medien & $.66^{* *}$ & $.61^{* *}$ & $.53^{* *}$ \\
Peers & $.50^{* *}$ & $.29^{* *}$ & $.66^{* *}$ \\
Sozialstrukturelles Niveau & $-.38^{* *}$ & $-.25^{* *}$ & $-.32^{* *}$ \\
Niveau der Schulkultur & $-.54^{* *}$ & $-.51^{* *}$ & $-.41^{* *}$ \\
Lehrerprofessionalität & $-.61^{* *}$ & $-.66^{* *}$ & $-.39^{* *}$ \\
Sozial-räumliche Schulumwelt & $-.45^{* *}$ & $-.37^{* *}$ & $-.34^{* *}$ \\
Schülerbefindlichkeiten & $-.40^{* *}$ & $-.40^{* *}$ & $-.33^{* *}$ \\
\hline
\end{tabular}

** $\quad p<.01$

Zunächst ist augenfällig, daß die Prädiktoren in den Feldern der Sozialisation deutlich stärker mit dem Niveau der Gewalt, der Schuldevianz und der (außerschulischen) Delinquenz zusammenhängen als die schulspezifischen und - nochmals abgestuft - die sozialstrukturellen Einflüsse. Innerhalb des Erziehungsmilieus kommt dem biographisch aktuellen Hintergrund der PeerSozialisation und vor allem des Medienkonsums eine etwas höhere Bedeutung 
zu als den familialen Einflüssen, obwohl zweifellos Synergien zwischen diesen Bereichen bestehen.

Interessant ist weiterhin das Ergebnis, daß - im Unterschied zu den Befunden auf der Basis von Individualdaten - auch die soziale Herkunft der Schüler einen relevanten Erklärungsfaktor für Gewalt darstellt. Das bedeutet, daß in Klassen, in denen beispielsweise auf Grund des Einzugsgebietes der Schule oder innerschulischer Segregierungen Kinder aus bestimmten Sozialschichten dominant vertreten sind, auch die Gewaltbelastung entsprechend höher bzw. niedriger ausfällt. Dies ist ein wichtiger Teil der Erklärung des Phänomens, daß in Gymnasien (mit ihrem überproportional hohen Anteil von Kindern aus mittleren und höheren Sozialschichten) Gewalt seltener und in Förderschulen für Erziehungshilfe sowie den Hauptschulklassen der Mittelschulen (mit ihrem überproportional hohen Anteil von Kindern aus unteren Sozialschichten) Gewalt häufiger vorkommt. Die bloße Schichtzugehörigkeit als Individualmerkmal läßt keine Rückschlüsse auf Gewaltverhalten zu; Effekte treten erst in der Verbindung von Sozialstruktur mit schulischen Selektions- und Differenzierungsprozessen sowie vorgängigen Sozialisationserfahrungen auf.

Demgegenüber wirkt die Schulkultur stärker, sie zeigt aber im Vergleich mit dem Erziehungsmilieu geringere Effektstärken in bezug auf Gewalt in der Schule. Innerhalb der Schulkultur ist die Lehrerprofessionalität der mit Abstand wichtigste Faktor, der offenbar auch am besten geeignet ist, der Schuldevianz zu begegnen.

Im Vergleich der Gewaltformen ist zu registrieren, daß bei der Delinquenz, die im außerschulischen Raum stattfindet, in erster Linie strukturelle, verstärkende und regulierende Einflüsse von Gleichaltrigen und Freunden wirksam werden. Medienverhalten und -konsum ist bei der Entstehung aller Formen des Gewaltsyndroms wichtig. Die Schuldevianz als Sektor alltäglicher Schülerstrategien und -verweigerungen wird im Vergleich mit den beiden anderen, gravierenderen Formen von Gewalt etwas weniger durch Sozialisation beeinflußt - weil jeder Schüler „mal so etwas macht“ wie den Unterricht stören oder die Schule schwänzen.

Bezieht man die Individualvariablen, die in Kapitel 4 überprüft worden sind, mit in unsere abschließenden Überlegungen ein, so ist im Hinblick auf die beabsichtigte multivariate Modellprüfung (vgl. Kap. 7) zu erwarten, daß diese Merkmale gemeinsam mit den individuellen Sozialisationserfahrungen, die die Entwicklung dieser Persönlichkeitsstruktur beeinflußt haben, deutlich stärker durchschlagen als die Effekte der Schulkultur.

Gleichwohl ist die Schule für die untersuchte Thematik wichtig, weil sie ein Ort ist, an dem die Schülerinnen und Schüler einen großen Teil des Tages verbringen, in Kooperation mit andern lernen, sich mit Gleichaltrigen und Erwachsenen verständigen, Konflikte lösen müssen, kommunikative Erfahrungen sammeln und entsprechende Kompetenzen entwickeln können. Dies funktioniert aber nur im Rahmen einer auf Transparenz der Wissens- und Anforderungsstruktur, der Komplementarität der Rollen von Lehrenden und 
Lernenden sowie der Offenheit der Kommunikationsstrukturen basierenden und in diesem Sinne entwickelten Schulkultur. 


\section{Schulische Gewalt im Lebenszusammenhang von Schülern - Gesamtmodell (Mehrebenenanalyse) \\ (Parviz Rostampour, Wolfgang Melzer, Wilfried Schubarth)}

Im Einführungskapitel ist unser Theoriemodell dargestellt, das auf die Erklärung von Gewaltemergenz ausgerichtet ist. Prädiktoren für schulisches Gewalthandeln wurden im Individualbereich, im Erziehungsmilieu (Familie, Medien, Peers) und in der Schule selbst (Schulkultur) vermutet sowie in den folgenden Kapiteln untersucht. Die bivariaten Analyseschritte sind schwerpunktmäßig in den Kapiteln 5 und 6 unternommen worden und dort auch dargestellt. Am Ende der Kapitel 2 und 6 wurde der Ost-West-Vergleich zusammenfassend behandelt; er wird daher in der abschließenden multivariaten Modellprüfung keine Rolle mehr spielen.

Ziel der multivariaten Modellprüfung ist eine theoriegeleitete empirische Abschätzung und Abgleichung möglicher Effekte für Gewalthandeln auf verschiedenen Strukturebenen. Das Beispiel der Schichtvariable hat die Bedeutung der Unterscheidung dieser Ebenen gezeigt: Auf Individualebene tendierte der Erklärungswert für Gewalthandeln durch die Zugehörigkeit der einzelnen Schüler zu einer bestimmten Sozialschicht in Richtung Null, wenn aber auf Klassenebene durch schulische Differenzierungs- und Selektionsprozesse Schüler bestimmter Schichten kumuliert auftreten, sind deutliche Zusammenhänge zum Gewaltverhalten in diesem Gruppenkontext bzw. zur Gewaltbelastung von Lerngruppen nachweisbar. Bei unserem Modell haben wir zunächst zwischen drei Ebenen unterschieden: der Schulebene, der Klassenebene und der Individualebene. Sozialisationseinflüsse (z.B. durch das Familienklima, den elterlichen Erziehungsstil, den Medienkonsum oder die Peer-Sozialisation) sind bei dem verwandten multivariaten Verfahren nur auf Individualebene einzubringen, so daß mit einem deutlich größeren Gewicht dieser erweiterten Individualebene im Vergleich zur Schul- und Klassenebene gerechnet werden kann.

In allen vorliegenden Gewaltstudien werden zumindest bei den deskriptiven Analysen Unterschiede zwischen den Schulformen, aber auch zwischen den Einzelschulen innerhalb des jeweiligen Schultyps festgestellt. Ebenso bestehen bei Gewalt und anderen Schulqualitätsparametern innerhalb dieser Einzelschulen erhebliche Differenzen von Klasse zu Klasse. In unserem Modell soll die Trennschärfe und Reichweite dieser beiden schulischen Ebenen (Einzelschule, Klasse) im Vergleich zur Individual- und Sozialisationsebene geprüft werden. Daher müssen zunächst einige Informationen zur Bedeutung 
der verschiedenen Ebenen im Hinblick auf eine Mehrebenenanalyse vorausgeschickt werden.

Insgesamt waren 30 für den Freistaat Sachsen repräsentativ ausgewählte Schulen, 178 Klassen und 3.147 Schüler in die Untersuchung einbezogen. Um die Relevanz der Schulform für die Gewaltemergenz zu verdeutlichen und gleichzeitig die Bedeutung der Unterschiede der Einzelschulen innerhalb der Schulformen zu zeigen, haben wir eine Rangreihe der Gewaltbelastung der Schulen auf der Basis von Mittelwerten vorgenommen. Die Gewaltbelastung wurde durch die Häufigkeit des Täterverhaltens auf Schulebene, wie es von den Schülern im Selbstreport angegeben worden ist, gemessen. Tab. 7-1 zeigt die Mittelwertrangreihe der 30 untersuchten Schulen (Förderschulen, Mittelschulen und Gymnasien) in unserer Stichprobe.

Die Rangreihe zeigt neben einer schwer einzuschätzenden Unterschiedlichkeit bei den Förderschulen, daß die Gewaltbelastung der einzelnen Mittelschulen in der Regel deutlich höher ist als die der Gymnasien. Nur drei der Mittelschulen (Rang 20, 24, 26) können in die Phalanx der besten Schulen vordringen, die - außer zwei Schulen für Lernbehinderte - sonst hauptsächlich aus Gymnasien besteht. Das schlechteste Gymnasium taucht erst auf Platz 19 der Rangreihe auf. Bis dahin werden in der Liste der Gewaltbelastung nur Mittelschulen und Förderschulen geführt. Regionale Muster lassen sich wie in den bisherigen Untersuchungen nicht erkennen.

Die Mittelschule mit der größten Gewaltbelastung (Rang 2) liegt im Ballungsraum Leipzig in einer Kleinstadt mit etwa 25.000 Einwohnern und wird von über 500 Schülern besucht; sie ist eine der größten Mittelschulen in ganz Sachsen und hat einen leicht überdurchschnittlichen Jungenanteil. Beim Gebäude handelt es sich um eine sogenannte "Neubauschule" im Plattenbaustil, die am Stadtrand in einem Plattenbauviertel gelegen ist. Die Schüler kommen aus der Stadt und den umliegenden Dörfern, so daß ein sehr weiter Einzugsbereich besteht. Im Vergleich mit den übrigen Sekundarschulen am Ort hat diese Schule einen schwierigen Stand. Sie konkurriert mit zwei Gymnasien (je etwa 600 Schüler) und zwei weiteren Mittelschulen (220 und 270 Schüler), die bessere Lernbedingungen haben, so daß ein deutlicher Creaming-Effekt zugunsten dieser Schulen feststellbar ist. Der Schulleiter ist sich seines schwierigen Schülerklientels bewußt. Schüler dieser Schule waren vor einiger Zeit an Ausschreitungen gegen ein Asylbewerberheim, das mittlerweile geschlossen ist, beteiligt und zeigen auch im Schulalltag häufig ein problematisches Verhalten. Da nach Aussage des Schulleiters der Teamgeist im Kollegium fehlt und auch die Eltern sich nicht sonderlich engagieren, sieht die Schule die einzige Möglichkeit zum Umgang mit Gewalt in der Schule in einer strikten pädagogischen Intervention und einer konsequenten Bestrafung der Täter. Aktivitäten zur Entwicklung der Schulkultur oder spezifische Interventionsprogramme gegen Schülergewalt gibt es an dieser Schule keine. 


\section{Tab. 7-1: Rangreihe der Gewaltbelastung* im Vergleich der Einzelschulen (nach Schulformen und Regionen; Faktormittelwerte)}

\begin{tabular}{|c|c|c|c|}
\hline Rang & Schulform & Ort/Region & Mittelwert \\
\hline 1 & Förderschule $(E H)^{* *}$ & Mittelgroße Stadt im Ballungsraum Leipzig & 2,71 \\
\hline 2 & Mittelschule & Mittelgroße Stadt im Ballungsraum Leipzig & ,73 \\
\hline 3 & Mittelschule & Ländliche Gemeinde (Westerzgebirge) & ,68 \\
\hline 4 & Mittelschule & Ländliche Gemeinde (Westerzgebirge) & ,66 \\
\hline 5 & Mittelschule & Ländliche Gemeinde (Westerzgebirge) & ,65 \\
\hline 6 & Mittelschule & Kleinstadt im ländlichen Bereich (Westerzgebirge) &, 60 \\
\hline 7 & Förderschule (LB)** & Kleinstadt im ländlichen Bereich (Westerzgebirge) &, 58 \\
\hline 8 & Förderschule (LB)** & Mittelgroße Stadt im Ballungsraum Leipzig &, 44 \\
\hline 9 & Mittelschule & Großstadt (Dresden) & ,39 \\
\hline 10 & Mittelschule & Mittelgroße Stadt im Ballungsraum Leipzig & ,35 \\
\hline 11 & Mittelschule & Mittelgroße Stadt im Ballungsraum Leipzig &, 21 \\
\hline 12 & Mittelschule & Großstadt (Dresden) &, 21 \\
\hline 13 & Förderschule $(E H)^{* *}$ & Großstadt (Dresden) & 13 \\
\hline 14 & Mittelschule & Kleinstadt im ländlichen Bereich (Westerzgebirge) & 10 \\
\hline 15 & Mittelschule & Ländliche Gemeinde (Westerzgebirge) & ,08 \\
\hline 16 & Mittelschule & Kleinstadt im ländlichen Bereich (Westerzgebirge) &, 05 \\
\hline 17 & Förderschule (LB)** & Großstadt (Dresden) &,- 02 \\
\hline 18 & Mittelschule & Ländliche Gemeinde (Westerzgebirge) &,- 07 \\
\hline 19 & Gymnasium & Kleinstadt im ländlichen Bereich (Westerzgebirge) &,- 14 \\
\hline 20 & Mittelschule & Ländliche Gemeinde (Westerzgebirge) &,- 17 \\
\hline 21 & Gymnasium & Mittelgroße Stadt im Ballungsraum Leipzig &,- 17 \\
\hline 22 & Förderschule (LB)** & Kleinstadt im ländlichen Bereich (Westerzgebirge) &,- 25 \\
\hline 23 & Förderschule (LB) ${ }^{* *}$ & Kleinstadt im ländlichen Bereich (Westerzgebirge) &,- 28 \\
\hline 24 & Mittelschule & Großstadt (Dresden) &,- 38 \\
\hline 25 & Gymnasium & Mittelgroße Stadt im Ballungsraum Leipzig &,- 51 \\
\hline 26 & Mittelschule & Kleinstadt im ländlichen Bereich (Westerzgebirge) &,- 57 \\
\hline 27 & Gymnasium & Kleinstadt im ländlichen Bereich (Westerzgebirge) &,- 58 \\
\hline 28 & Gymnasium & Kleinstadt im ländlichen Bereich (Westerzgebirge) &,- 60 \\
\hline 29 & Gymnasium & Großstadt (Dresden) &,- 65 \\
\hline 30 & Gymnasium & Großstadt (Dresden) & $-1,13$ \\
\hline
\end{tabular}

* Gewaltbelastung beinhaltet die Schülerangaben über selbst ausgeführte härtere und weichere Aggressionen.

** Förderschule $(\mathrm{EH})=$ Förderschulen für Erziehungshilfe; Förderschule $(\mathrm{LB})=$ Förderschulen für Lernbehinderte 
$\mathrm{Zu}$ den besseren Mittelschulen (Rang 24) gehört eine Dresdner Mittelschule, die in einem Nobelviertel direkt an der Elbe und am Fuße des Elbhanges liegt. Sie ist in einem schönen alten Gebäude untergebracht und wird von etwa 320 Schülern besucht. Eine Besonderheit der Schule ist das sehr seltene musische Profil, von dem vor allem die Mädchen angesprochen werden. Daher ist es nicht verwunderlich, daß die Schülerschaft zu etwa zwei Dritteln weiblich ist. Eine weitere Besonderheit sind die leistungsheterogenen Klassen (Differenzierung ab Klasse 7 nur in den Hauptfächern); von diesem Differenzierungsmodell macht nur eine Minderheit der Schulen Gebrauch. In drei Viertel der Fälle führen die sächsischen Schulen nach der Orientierungsstufe getrennte Bildungsgänge für Hauptschüler und für Realschüler ein. Diese Schule hat also sehr gute Ausgangsbedingungen, bemüht sich nach Auskunft des Schulleiters und unserem Gesamteindruck nach sehr stark um die individuellen Belange der Schüler und besitzt ein reges Schulleben, in das auch die Eltern einbezogen werden.

In einer westerzgebirgischen Kleinstadt mit etwa 20.000 Einwohnern finden wir die interessante Konstellation der besten Mittelschule (Rang 26) und des schlechtesten Gymnasiums (Rang 19). Hier ist die Gewaltbelastung im Gymnasium - im Gegensatz zum Untersuchungstrend - höher als die der Mittelschule. Das Gymnasium ist mit knapp 1000 Schülern eines der größten im Lande und besteht an zwei Standorten. Die Sekundarstufe I, in der wir unsere Untersuchungen durchgeführt haben, ist in einem "Neubau", der in einem Plattenbauviertel am Stadtrand gelegen ist, die älteren Schüler der Sekundarstufe II in einem stilvollen Altbau mehr zur Stadtmitte hin untergebracht. Die Schule hat sich aus einer ehemaligen Polytechnischen Oberschule (vgl. Fußnote 5, S. 247) entwickelt, hat also als Gymnasium keinerlei Tradition. Die Standorte sind etwa einen Kilometer voneinander entfernt, so daß die Lehrer z.T. pendeln müssen und darüber auch klagen. Trotz der beiden Gebäude sind die räumlichen Möglichkeiten für die Schüler der Klassen 5 bis 10 so begrenzt, daß die Klassen keine eigenen festen Räume haben. Die Schulleiterin bewertet diese Gesamtsituation kritisch und berichtet über eine geringe Schülermotivation und eine mangelnde Unterstützung durch die Eltern. Zwar sind im sozial-räumlichen Bereich im Rahmen der Sanierung des Neubaus Fortschritte erzielt worden (Einrichtung von Sitzecken und Rückzugsmöglichkeiten), im pädagogischen Bereich finden sich aber kaum Ansätze zur Entwicklung der Schulkultur.

Demgegenüber kommt dem Beobachter die Situation an der untersuchten Mittelschule in derselben Kleinstadt (Rang 26) wie eine "Pädagogische Provinz" vor. Die Schule wird von ca. 360 Schülern besucht und ist in einem stilvollen Altbau im alten Stadtzentrum untergebracht. Es bestehen vier Profile (Regelfall zwei Profile) zur Neigungswahl der Schüler ab Klasse 7, darunter auch das musische Profil. Dadurch ist der Mädchenanteil leicht überdurchschnittlich. Die pädagogische Arbeit begrenzt sich nicht nur auf den Unterricht, sondern bezieht das Schulleben und das schulische Umfeld mit ein. Es gibt 
viele Freizeitangebote in der Schule, die auch in der Stadt nicht so selten sind wie andernorts, Projektunterricht und Projektwochen sowie Schulsozialarbeit. Der Schulleiter ist äußerst engagiert, wünscht sich mehr jüngere Lehrer in seinem Kollegium und ist für alle Maßnahmen der Schulentwicklung offen einschließlich der Kooperation mit der Wissenschaft. Das Lehrerverhalten scheint sehr schülerzentriert und partizipativ zu sein; so wurde der Beratungslehrer von den Schülern gewählt. Auf der anderen Seite schätzen die Schüler die Lehrerintervention bei problematischem Schülerverhalten als sehr bestimmt und den Erziehungsstil der Lehrer als eher streng ein. Insgesamt machen das Kollegium und die Schulleitung einen sehr engagierten Eindruck.

Auch innerhalb dieser einzelnen Schulen haben wir große Unterschiede von Klasse zu Klasse festgestellt. Dieses wird im folgenden am Beispiel einer großstädtischen Mittelschule verdeutlicht. Die Schule befindet sich innerhalb unserer Rangreihung der Gewaltbelastung auf Platz 9, gehört also zu den eher gewaltbelasteten Schulen.

Abb. 7-1: Klassenunterschiede innerhalb einer großstädtischen Mittelschule (Faktorenmittelwerte)

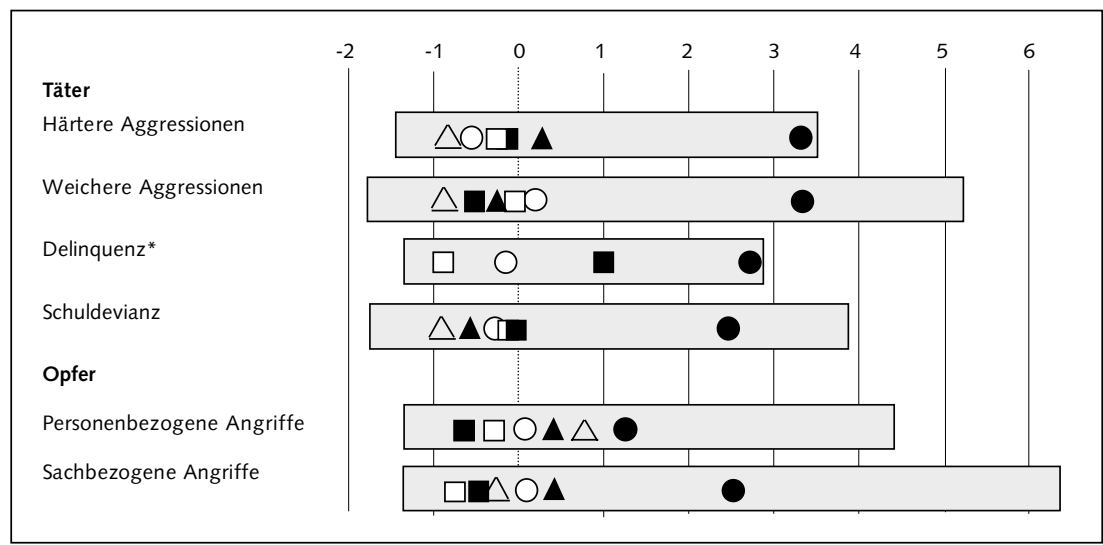

* In den 6. Klasse wurden keine Delinquenz-Items erhoben.
$\Delta$ 6. Klasse
- 8. Hauptschulklasse
9. Hauptschulklasse
$\triangle 6$. Klasse
Realschulklasse
$\square$ 10. Realschulklasse

Die Symbole zeigen die Situation der jeweiligen Klasse. Die jeweilige Positionierung des Symbols zeigt die Bedeutung dieses Kriteriums im Vergleich zu den anderen Klassen dieser Schule. Die Spanne des grauen Balkens verdeutlicht, wie groß die Differenz zwischen den extremsten Klassen der Gesamtstichprobe ist (Minimum-, Maximumwert).

Daß die schulischen Selektionsprozesse nicht ohne Folgen für das Gewalthandeln bleiben, läßt sich anhand dieser Graphik besonders deutlich bei den Täter-Items zeigen. Hier ist bei den Hauptschulklassen eine wesentlich stärkere 
Belastung zu registrieren als bei den Realschulklassen. Die 8. Hauptschulklasse stellt bei allen dargestellten Gewaltindikatoren die extremste Gruppe innerhalb dieser Mittelschule dar. Am auffälligsten aber ist die Unterschiedlichkeit der Klassen überhaupt, die auch an den Differenzen der beiden 6. Klassen, in denen noch nicht nach Bildungsgängen differenziert ist, deutlich wird (vgl. Kap. 8.3). ${ }^{1}$

Ob und wie stark sich die Differenzen der Einzelschulen, die Unterschiedlichkeit der einzelnen Klassen oder die ebenfalls beträchtlichen Unterschiede zwischen den über 3000 untersuchten Individuen als erklärungsrelevant für Gewaltemergenz erweisen, soll die Mehrebenenanalyse zeigen.

\section{Zum Verfahren der Mehrebenenanalyse}

In den Kapiteln 1 bis 5 haben wir die Analysen auf der Individualebene durchgeführt, weil die Daten in den untersuchten Bereichen hauptsächlich die individuellen Merkmale der Befragten bzw. Angaben über ihre Familien und Peers betrafen. Im Kapitel 6, in dem die schulischen Bedingungen der Gewaltemergenz im Vordergrund standen, haben wir aber die Daten nicht mehr auf Individualebene, sondern auf Aggregatebene (Klassenebene) analysiert. Dafür wurde bei jeder untersuchten Variable der entsprechende Mittelwert für die Klasse gebildet und als Klassenmerkmal betrachtet. Die Fallzahl auf Aggregatebene war somit identisch mit der Anzahl der Klassen und nicht mehr mit der Anzahl der befragten Schüler. Der eigentliche Grund für die Verwendung auf Klassenebene aggregierter Daten war, daß die Angaben der Befragten über ihre Schule, Klasse, Lehrer und Mitschüler stark mit ihren individuellen Einstellungen und Erfahrungen zusammenhängen und insoweit keine objektiven Messungen für diese Schulvariablen darstellen. So kommt es z.B. dazu, daß in derselben Klasse ein Teil der Schüler ihre Lehrer als aggressiv einstuft und ein anderer Teil nicht. Genauso ist es bei der Beurteilung des Klassenklimas usw. Um diese subjektive Komponente soweit wie möglich aus den Daten herauszupartialisieren und objektivere Messungen der Schulvariablen zu erzielen, kann man (wie in Kap. 6) für jede Variable den Durchschnitt der Antworten der Schüler in der Klasse als eine intersubjektive (und somit objektivere) Kontextvariable bilden und die Analyse auf einer höheren Ebene (in unserem Fall Klasse) durchführen. Die Analyse auf Aggregatebene hat aber neben dem genannten Vorteil auch bestimmte Nachteile. Denn bei diesem Verfahren müssen - gezwungenermaßen - auch die "eigentlich" individuellen Merkmale der Befragten aggregiert werden. Damit verlieren diese ihren Charakter als Individualvariablen und werden als Durchschnittswerte der Klasse

1 Eine Differenzierung nach Hauptschul- und Realschulbildungsgang erfolgt in der sächsischen Mittelschule erst ab dem 7. Jahrgang. 
verwendet, so daß man den Zusammenhang zwischen dem Kontext und dem Individuum nicht angemessen untersuchen kann. Das Problem stellt sich insbesondere dann, wenn man in einem multivariaten Modell gleichzeitig mit Individual- und Kontextvariablen eine individuelle abhängige Variable erklären will. Mit der Aggregierung einer solchen abhängigen Variable verschwinden die individuellen Unterschiede innerhalb der Gruppe und das Problem wird dadurch nicht mehr differenziert in seinen personalen Ausprägungen, sondern pauschalisierend als Gruppenphänomen untersucht. Um dies zu verhindern, dürfen die abhängige Variable (Täterverhalten, Opferstatus) und die individuellen erklärenden Variablen (z.B. Selbstwertgefühl) nicht aggregiert werden. Daher ist ein Verfahren erforderlich, bei dem man die Analyse gleichzeitig mit aggregierten und nicht-aggregierten Daten durchführen kann. Die aggregierten Daten (Gruppenvariablen) dürfen nicht - so z.B. in einem einfachen Regressionsmodell (OLS) - wie die Individualvariablen auf Individualebene eingeführt werden, weil dadurch bei diesen Variablen die Fallzahl (in unserem Beispiel die Anzahl der Klassen bzw. Schulen) fälschlicherweise erhöht würde. Diese künstliche Erhöhung der Fallzahl führt dazu, daß die entsprechenden Schätzungen des Modells eine Genauigkeit suggerieren, die keineswegs gerechtfertigt ist (Unterschätzung der Standard-Errors). Dadurch können zufällige Ergebnisse als statistisch signifikante Ergebnisse erzielt werden und falsche Schlußfolgerungen gezogen werden. Um einerseits die Aggregierung der Individualdaten und andererseits die "Individualisierung" der Kontextvariablen zu vermeiden, ist die Mehrebenenanalyse anstelle von OLS zu verwenden. In einer Mehrebenenanalyse liegt die abhängige Variable auf der niedrigsten Ebene (Individualebene); die erklärenden Variablen können aber sowohl auf dieser ersten Ebene (Individualvariablen) als auch auf einer höheren Ebene (Gruppen- bzw. Kontextvariablen) angesiedelt sein. ${ }^{2}$

Die abhängigen Variablen in diesem Kapitel sind die zwei Faktorvariablen "Täter" und "Opfer"3 auf der Individualebene, die wir in zwei getrennten Modellen analysieren. Die Mehrebenenanalyse ist nicht auf zwei Ebenen beschränkt, sondern kann Daten analysieren, die in mehreren hierarchisch strukturierten Ebenen organisiert sind (z.B. Schüler, Klassen, Schulen usw.). Als erklärende Variablen auf Individualebene werden sowohl Individualmerkmale als auch Variablen aus dem Bereich außerschulischer Sozialisation (Elternhaus, Medien und Peers) eingeführt. Bei den Individualmerkmalen verzichten wir auf diejenigen erklärenden Variablen, die das Gewaltsyndrom mit repräsentieren (wie Gewaltbefürwortung, Aggressionsbereitschaft), weil erstens diese Variablen selbst von den anderen erklärenden Variablen abhängig sind

2 Für eine Einführung in die Mehrebenenanalyse wird auf Goldstein (1995) verwiesen.

3 Die Faktorvariablen "Täter" und "Opfer" beinhalten alle Täter- bzw. Opfer-Items aus dem Bereich Selbstreport (vgl. Tab. 4-1 und 4-2). Die Analysen in diesem Kapitel werden ausschließlich mit den sächsischen Daten durchgeführt. 
und zweitens mit den abhängigen Variablen stark korrelieren, so daß ihre Berücksichtigung als erklärende Variablen dazu führt, daß die Effekte weiterer relevanter erklärender Variablen verdeckt werden.

\section{Ergebnisse der Mehrebenenanalyse}

Zunächst haben die vorbereitenden Untersuchungen ergeben, daß sich auf der institutionellen Ebene im Vergleich der Bedingungen und Unterschiede auf Schul- und Klassenebene nur ein geringer Teil der Gesamtvarianz der abhängigen Variable auf die Schulebene verteilt und diese bereits durch das einfachere Zwei-Ebenen-Modell (Klassen- und Individualebene) mit erklärt wird. Wir können daher die Schulklasse als das für die Erklärung der Gewaltthematik relevantere Sozialsystem im Sektor Schule betrachten, das gleichzeitig zu einem beträchtlichen Anteil die Spezifika der entsprechenden Einzelschule mit repräsentiert (vgl. Kap. 6). Daher bezeichnen wir diese institutionelle Ebene auch als "Schul- und Klassenebene", obwohl in unserem Modell - rein rechnerisch betrachtet - auf Klassenebene verfahren wird.

Ein zweiter Befund bezieht sich auf die Abschätzung der Relevanz dieser Schul- und Klassenebene im Vergleich zur Individualebene, die - wie bereits dargelegt - die biographisch-sozialisatorischen Erfahrungen im außerschulischen Raum mit einschließt. Dazu hat eine einfache Varianzanalyse ergeben, daß 16,3\% der Gesamtvarianz der Variable "Täter" in der Stichprobe auf die Varianz zwischen den 153 Klassen $^{4}$ und 83,7\% auf die Varianz zwischen den Individuen innerhalb der Klassen zurückzuführen ist. Für die Variable "Opfer" sind es $11,3 \%$ bzw. 88,7\%. Eine Mehrebenenanalyse ohne erklärende Variablen - ein sogenanntes "intercept-only model" (vgl. Hox 1995, S. 15) oder "random effects analysis of variance" (vgl. Snijders/Bosker 1998, S. 16) hat für die Grundgesamtheit folgende Varianzzerlegungen geschätzt: für die Variable "Täter" $10,7 \%$ auf Klassenebene und 89,3\% auf Individualebene und für die Variable "Opfer" 5,2\% auf Klassenebene und 94,8\% auf Individualebene.

Als eine Zwischenstufe zum Endmodell werden zuerst die Ergebnisse eines Modells vorgestellt, in dem ausschließlich Kontextvariablen auf Klassenebene als erklärende Variablen eingeführt werden, während die abhängigen Variablen (Täter bzw. Opfer) - wie bereits erwähnt - Individualvariablen sind. Die Tabellen 7-2 und 7-3 zeigen die entsprechenden Ergebnisse für das Täterverhalten ("Täter") und den Opferstatus ("Opfer"). Von den Klassen- und Schulvariablen, die in bivariaten Analysen signifikante Zusammenhänge mit der jeweiligen abhängigen Variable aufgewiesen hatten, bleiben sechs bzw. fünf erklärende Variablen in einer multivariaten Analyse signifikant. Das Vorzeichen der (un-

4 Die Förderschulen konnten nicht in die Analyse einbezogen werden, weil dort mehrere erklärende Variablen nicht erhoben worden sind. 
standardisierten) Koeffizienten zeigt, ob die betreffende erklärende Variable einen positiven oder einen negativen Zusammenhang mit der abhängigen Variable hat. Diese Koeffizienten sind aber wegen der unterschiedlichen Skalen der betreffenden Variablen miteinander nicht vergleichbar. Im Hinblick auf eine Abschätzung der relativen Einflüsse der einzelnen erklärenden Variablen können die $\mathrm{Chi}^{2}$-Werte miteinander verglichen werden. ${ }^{5} \mathrm{Im}$ Modell Täter weist der Anteil der Jungen in der Klasse den stärksten Zusammenhang mit dem Täterverhalten der Schüler auf. Das bedeutet, daß die Schüler in den Klassen mit einem überdurchschnittlichen Jungenanteil gewalttätiger sind.

Weiterhin ist festzustellen, daß ein aggressives Verhalten der Lehrer (Lehrer schreien, werden handgreiflich) und ein mangelnder Lebensweltbezug des Unterrichts $^{6}$ - der Sinn des Lernens ist für Schüler nicht klar, da die Lerngegenstände wirklichkeitsfremd sind und man für die Lernergebnisse im späteren Leben kaum eine Verwendung findet - einen positiven Zusammenhang mit dem Täterverhalten der Schüler haben. Darüber hinaus ist das Täterverhalten in den Klassen niedriger, in denen die Lehrer bereit sind, eigene Fehler zuzugeben und in den Klassen, in denen die Lehrer bei einem Fehlverhalten der Schüler eher bereit sind, mit ihnen zu reden als sie zu strafen. Wie bereits in den Korrelationsanalysen (vgl. Kap. 6) setzt sich also auch multivariat die Lehrerprofessionalität und -kompetenz als eine entscheidende Variable durch. Daneben spielt auch die Atmosphäre unter den Mitschülern in der Klasse (Feindschaften, Mißachtungen und Ausgrenzungen in der Klasse) eine Rolle und trägt zu einer Verstärkung des Täterverhaltens bei (vgl. ebenfalls Kap. 6).

Tab. 7-2: Ergebnisse des Mehrebenenmodells mit erklärenden Variablen ausschließlich auf Klassenebene (abhängige Variable: Täter)

\begin{tabular}{lccc}
\hline Variable & Koeffizient & $\begin{array}{c}\text { Standard- } \\
\text { abweichung }\end{array}$ & Chi $^{2}$-Wert \\
Anteil der Jungen in der Klasse & .71 & .12 & $33.11^{* * * * * *}$ \\
Aggressives Lehrerhandeln & .19 & .06 & $11.06^{* * *}$ \\
Fehlender Lebensweltbezug des Unterrichts & .19 & .06 & $10.66^{* *}$ \\
Desintegration in der Klasse & .17 & .05 & $10.49^{* *}$ \\
Lehrer reden statt strafen (V184) & -.18 & .08 & $4.47^{*}$ \\
Lehrer geben ihre Fehler nicht zu (V183) & .13 & .06 & $4.00^{*}$ \\
\hline
\end{tabular}

${ }^{*} \mathrm{p}<.05 ;{ }^{* *} \mathrm{p}<.01 ; \quad{ }^{* * *} \mathrm{p}<.001 ; \quad{ }^{* * *} . . \mathrm{p}<.0001$ usw.

5 Die Chi²-Werte in der letzten Spalte der Tabellen 7-2 bis 7-5 sind die Differenzen der Minimierungsfunktion (-2*Log Likelihood) zwischen einem Modell mit allen aufgeführten erklärenden Variablen und einem Modell, in dem die entsprechende erklärende Variable ausgeschlossen wird.

6 Der Faktor ist gebildet aus den drei Variablen zum "Sinn des Lernens" (V68, V67, V64) und der Variable V122: "Das meiste, was man in der Schule lernt, kann man später doch nicht gebrauchen." 
Tab. 7-3: Ergebnisse des Mehrebenenmodells mit erklärenden Variablen ausschließlich auf Klassenebene (abhängige Variable: Opfer)

\begin{tabular}{lccl}
\hline Variable & Koeffizient & $\begin{array}{c}\text { Standard- } \\
\text { abweichung }\end{array}$ & Chi²-Wert \\
Jahrgang & -.10 & .01 & $44.21^{* * * * * *}$ \\
Etikettierungsverhalten der Lehrer & .27 & .07 & $12.02^{* * *}$ \\
Niveau der ausländerfeindlichen Sprüche in der & .09 & .03 & $10.07^{* *}$ \\
Klasse (V160) & & & $4.62^{*}$ \\
Wohlbefinden in der Klasse & -.14 & .06 & $4.43^{*}$ \\
Schülereinmischung & .14 & .07 & \\
\hline
\end{tabular}

Siginifikanzniveau vgl. Tab. 7-2

* Uns ist bewußt, daß die Einordnung dieser Variable als erklärende Variable aufgrund ihrer Nähe zur Gewalt nicht unumstritten ist.

Im Modell "Opfer" (Tab. 7-3) schlägt die Jahrgangsstufe am stärksten durch. Die jüngeren und körperlich noch weniger entwickelten Schüler werden signifikant häufiger zum Opfer. Zu diesem Befund mag auch die zuvor festgestellte stärkere Offenheit der Rollen bei jüngeren Gewaltakteuren beigetragen haben. Mehr Opfer gibt es auch in den Klassen mit starkem Etikettierungsverhalten der Lehrer und in den Klassen mit häufigen ausländerfeindlichen Sprüchen. Weniger Opfer gibt es dagegen, wenn die Schüler sich in der Klasse wohl fühlen, Anerkennung finden und Freundschaft erleben. Bemerkenswert ist, $\mathrm{da} ß$ es in den Klassen, in denen Schüler überdurchschnittlich häufig in Gewaltsituationen eingreifen (im Sinne von Mitmischen), mehr Opfer gibt. Die Einmischung der Schüler muß also nicht in allen Fällen zur Schlichtung, sondern kann auch zur Eskalation führen.

Das Modell “Täter" erklärt 95,6\% und das Modell "Opfer" 83,1\% der Varianz auf der Klassenebene. Die Varianzen auf der Individualebene bleiben aber unerklärt, da die beiden vorläufigen Modelle keine erklärenden Individualvariablen enthalten.

In einem nächsten Schritt wurden die erklärenden Individualvariablen mit in die Analysen einbezogen. Tabelle 7-4 zeigt die Ergebnisse für das Modell mit der abhängigen Variable "Täter". Im Bereich der Individualmerkmale erweisen sich das Interesse für den Unterricht und das Geschlecht als die stärksten Erklärungsgrößen. Hier können wir nochmals feststellen, daß das Täterverhalten am meisten für die Jungen zutrifft, und zwar auch dann, wenn alle anderen erklärenden Variablen des Modells konstant gehalten werden. Ein geringes Interesse für den Unterricht geht ebenfalls mit mehr aggressiven Handlungen einher. Der positive Koeffizient der "Leistungsattribuierung" zeigt, daß die aggressiveren Schüler selbst genau wissen, daß sie in der Schule weniger zustande bringen als die anderen, und daß sie die Aufgaben nicht gut bewältigen. Das ist aber auf keinen Fall als ein geringeres allgemeines Selbst- 
wertgefühl bei den aggressiveren Schülern zu interpretieren. Das Gegenteil ist der Fall; das zeigen die positiven Koeffizienten von "Selbstwertgefühl" und "Selbstvertrauen". Aggressive Schüler beziehen ihr Selbstwertgefühl somit nicht über schulische Leistungen sondern aus anderen Bereichen, z.B. der Freizeit.

Im Bereich des Familienkontextes zeigen die Ergebnisse, daß ein positives Familienklima zur Verminderung des Täterverhaltens der Kinder führt. Dieser Faktor hatte sich bereits bei den Korrelationsanalysen neben zwei anderen Faktoren, die das Erziehungsverhalten der Eltern betreffen, als erklärungsrelevant erwiesen (vgl. Kap. 5.1). Im Gegensatz zu den bivariaten Berechnungen setzen sich im multivariaten Modell auch Aspekte der Familienkonstellation durch: Kinder aus Familien mit mehreren Kindern verhalten sich danach vergleichsweise aggressiver als andere. Vermutlich verbergen sich hinter dieser Familienkonstellation eine Reihe anderer Faktoren und Bedingungen, z.B. Aspekte des Milieus, des Erziehungsstils und sozioökonomischer Belastungen.

Der Zusammenhang zwischen dem Medienkonsum und aggressivem Verhalten ist - wie schon in Kapitel 5.2 und am Ende von Kapitel 6 in der vergleichenden Analyse der Parameter der Erziehungskultur und der Schulkultur festgestellt - insgesamt stärker als der zwischen den Familienvariablen und dem aggressiven Verhalten. Der Konsum von Gewaltfilmen hat einen stark positiven, der Konsum von Informations- und Kinderfilmen einen negativen Zusammenhang mit aggressivem Verhalten. Allerdings müssen mit Blick auf das Sozialverhalten der Heranwachsenden die Peers als eine ebenfalls einflußreiche Sozialisationsinstanz begriffen werden. In der bivariaten Analyse war das Medienverhalten etwas stärker als der Einfluß der Gleichaltrigengruppen ausgefallen, in der multivariaten Analyse ist es umgekehrt: Die Gewalttätigkeit der engsten Freunde und die Aggressivität der Freundesgruppe erweisen sich in unserem Modell als die stärksten Erklärungsvariablen für das aggressive Verhalten der Schüler. Auch die interne Intoleranz der Freundesgruppe zeigt einen positiven Zusammenhang mit dem Täterverhalten. In der Biographie der Schüler fließen all diese heuristisch getrennten Prädiktoren als Risikofaktoren des Verhaltens jedoch zusammen, dabei ist allerdings zu beachten, daß der Einfluß der Medien und der Peers stark durch die familiale Sozialisation geprägt ist. Somit läßt sich zwar der Einfluß der Familie auf Gewalthandeln als Kausalzusammenhang interpretieren, bei den außerfamilialen Bedingungen liegt jedoch u.E. eher eine wechselseitige Einflußnahme vor.

Die genannten Individualvariablen erklären nicht nur die Varianz auf der Individualebene, sondern auch zum Teil die Varianz auf der Klassenebene, weil sie in verschiedenen Klassen unterschiedliche Niveaus haben. Somit bleibt wenig Spielraum für die eigentlichen Klassenvariablen, zumal der Varianzanteil auf der Klassenebene ohnehin geringer ist als auf der Individualebene. Deshalb kann sich im Modell "Täter" nur eine Klassenvariable durchsetzen. Trotzdem wird 95,6\% der Varianz von "Täter" auf Klassenebene durch das Modell erklärt, während auf der Individualebene 40,3\% der Varianz erklärt 
Tab. 7-4: Ergebnisse des Mehrebenenmodells (abhängige Variable: Täter)

Variable

Koeffizient $\begin{gathered}\text { Standard- } \\ \text { abweichung }\end{gathered} \quad$ Chi²-Wert $^{2}$

1. Individualebene

1.1. Individualmerkmale

Geschlecht (Junge)

Interesse für Unterrichtsstoff (V66)

negative Leistungsattribuierung

Selbstwertgefühl

Selbstvertrauen (V117)

1.2. Familienkontext

Positives Familienklima

Anzahl der Geschwister

\section{3: Medienkonsum}

Konsum von Gewaltfilmen

Konsum von Informations- und Kinderfilmen

1.4. Peer-Einflüsse

Gewalttätigkeit der engsten Freunde

Aggressivität der Freundesgruppe

Intoleranz der Freundesgruppe

2. Schul- und Klassenebene

Pejoratives und aggressives Lehrerhandeln

Siginifikanzniveau vgl. Tab. 7-2

wird. Als Kontextvariable können mehrere aggregierte Klassen- oder Lehrervariablen alternativ berücksichtigt werden. Wir haben uns aus inhaltlichen und rechnerischen Gründen für einen gemeinsamen Faktor aus pejorativem und aggressivem Verhalten des Lehrers entschieden. Der positive Koeffizient dieses Faktors zeigt, daß das Lehrerverhalten auch unabhängig von individuellen Voraussetzungen und außerschulischen Sozialisationsbedingungen das aggressive Verhalten der Schüler beeinflußt - dies liegt auf der Linie bisheriger Befunde und Interpretationen. 
Das Mehrebenenmodell für "Opfer" (vgl. Tab. 7-5) zeigt, daß auch bei den Opfern der Anteil der Jungen größer ist als der der Mädchen, auch wenn die anderen erklärenden Variablen des Modells konstant gehalten werden. Viel wichtiger als das Geschlecht und als alle anderen Variablen für die Erklärung der abhängigen Variable "Opfer" ist aber das Außenseitergefühl, wobei die Frage offen bleibt, ob der Opferstatus ein solches Gefühl erzeugt oder die Täter ihre Angriffe eher gegen die Außenseiter richten, die in der Regel auch wehrloser sind. Wir sind der Meinung, daß es hier eine Wechselwirkung gibt, weil beide Kausalrichtungen theoretisch haltbar sind. Neben dem Außenseitergefühl ist ein positiver Zusammenhang auch zwischen der "Opfer"-Variable und dem Gefühl der Hilflosigkeit bei ungerechter Behandlung durch den Lehrer zu beobachten. D.h., Opfer sind mit ihrem primären Merkmal als Außenseiter und durch Hilflosigkeit zu kennzeichnen und können sich genauso wenig gegen Übergriffe von seiten der Schüler wie gegen Lehrerwillkür durchsetzen.

Tab. 7-5: Ergebnisse des Mehrebenenmodells (abhängige Variable: Opfer)

Variable

Koeffizient $\begin{gathered}\text { Standard- } \\ \text { abweichung }\end{gathered}$ Chi²-Wert $^{2}$

1. Individualebene

1.1. Individualmerkmale

Geschlecht (Junge)

Außenseitergefühl

Hilflosigkeit gegenüber den Lehrern (V93)

1.2: Familienkontext

Aggressiver Erziehungsstil der Eltern

1.3: Medienkonsum

Konsum von Gewaltfilmen

1.4. Peer-Einflüsse

Gewalttätigkeit der engsten Freunde

2. Schul- und Klassenebene

Jahrgang

Etikettierungsverhalten der Lehrer

Siginifikanzniveau vgl. Tab. 7-2
.04

.16

.26

.05

.08

.09

.02

$14.67 * * *$

01

.01

.06

$14.26^{* * *}$

$162.25 * * * * * * *$

$7.49 * *$

$16.23^{* * * *}$

$10.70 * *$

$-.08$

.12 
Unter den familialen Merkmalen hat sich in diesem multivariaten Modell der aggressive Erziehungsstil der Eltern als entscheidend herausgestellt. Je mehr die Kinder zu Hause Opfer von Aggressionen sind, desto mehr sind sie es auch in der Schule. Die Aggressionen der Eltern führen offensichtlich zur Wehrlosigkeit ihrer Kinder. In verschiedenen anderen Zusammenhängen waren wir diesem Phänomen schon begegnet und hatten es als eine Kontinuität der Opfererfahrung interpretiert.

In den Bereichen des Medienkonsums und der Peer-Einflüsse stellt sich heraus, daß auch die Opfer überdurchschnittlich häufig Gewaltfilme konsumieren und gewalttätige Freunde haben, auch wenn diese Erklärungsfaktoren im Modell "Täter" bei weitem stärker sind als im Modell "Opfer". Bei der Erklärung dieses Ergebnisses ist wiederum auf den nicht seltenen Übergang und die partielle Austauschbarkeit von Täter- und Opferverhalten zu verweisen, ein Befund der insbesondere durch die Gruppe der Täter-Opfer verursacht wird (vgl. Kap. 4).

Unter den Schul- und Klassenvariablen stellt sich die Jahrgangsstufe als der stärkste Zusammenhangsfaktor heraus. Danach gibt es in den höheren Klassenstufen weniger Opfer als in den niedrigeren (s.o.). Darüber hinaus hat sich das Etikettierungsverhalten der Lehrer als relevant erwiesen. Der Opferstatus zeigt einen positiven Zusammenhang mit dem Verhalten der Lehrer, die bestimmte Schüler in ihrer Klasse ständig in Verdacht haben und ihnen das Gefühl vermitteln, sie bereits aufgegeben zu haben. Hätte es sich bei diesen Einstellungen und Einschätzungen um Täter gehandelt, hätte man derartiges Lehrerverhalten als Reaktion auf das Gewalthandeln der Schüler interpretieren können. Daß auch Opfer - und gerade sie - dies feststellen, dokumentiert, daß ein problematisches Lehrerverhalten in relevanter Größenordnung auch unabhängig von der Interaktion mit den Schülern existiert. Das zeigen auch die bereits referierten Ergebnisse, die auf der Seite der Opfer ein Gefühl der Hilflosigkeit gegenüber den Lehrern und auf Seiten der Täter ein stark pejoratives und z.T. auch aggressives Lehrerhandeln dokumentiert haben.

Das Modell "Opfer" erklärt 79,6\% der Varianz der abhängigen Variable auf Klassenebene und 14,2\% der Varianz auf Individualebene. Das ist viel weniger als beim Modell "Täter". Das bedeutet, daß es insgesamt leichter ist, das Täterverhalten zu erklären als den Prozeß des Opferwerdens. Das macht auch die Schwierigkeit der Lehrer deutlich, mit der Gruppe der Opfer angemessen umzugehen und die einzelnen Opfer in den Schulklassen überhaupt erst einmal zu identifizieren.

Mit den auf Klassenebene aggregierten Daten wurde am Ende von Kapitel 6 die Funktion der Außensteuerung im Prozeß der Gewaltemergenz am Beispiel der Lehrerintervention untersucht und Verstärkungseffekte gefunden, die uns zu der Interpretation veranlaßten, daß das Interventionsverhalten Effekte der Schulkultur moderiert, vielleicht sogar selbst als Aspekt der Schulkultur begriffen werden kann. Die internen Steuerungsprozesse konnten auf der Klassenaggregatebene nicht untersucht werden, da es sich um individuelle 
Regulationsmechanismen handelt. Die Mehrebenenanalyse erlaubt allerdings eine vergleichende Betrachtung solcher intrapersonaler Kompetenzen im Kontext der Einflüsse von Erziehungsmilieu und Schulkultur.

Um dies zu ermöglichen, wurde zunächst auf der Basis von Individualdaten eine Clusteranalyse (k-Means-Verfahren) anhand der Variablen der intrapersonalen Steuerung von aggressiven Handlungsweisen vorgenommen und die Stichprobe in zwei Gruppen von Schülern mit folgenden individuellen Merkmalen unterschieden: (1) Schüler mit hoher Aggressionsbereitschaft, starker Gewaltbefürwortung und geringer diskursiver Streitkultur, also niedriger Selbstregulationskompetenz und (2) Schüler mit den entgegengesetzten Merkmalen und Eigenschaften, also hoher Selbstregulationskompetenz. Für diese beiden Gruppen wurden zwei getrennte multivariate Täteranalysen (nach dem Muster von Tab. 7-4) durchgeführt und die entsprechenden Koeffizienten miteinander verglichen. Das Ergebnis ist folgendes: Bei allen Paarvergleichen wurden in der Teilstichprobe der Schüler mit geringer Steuerungskompetenz stärkere Zusammenhänge festgestellt als in der anderen Stichprobe. Alle überprüften erklärenden Variablen (Individualmerkmale, Familienkontext, Medienkonsum, Peer-Einflüsse und Lehrerverhalten) zeigen eine Interaktion mit den internen verhaltensregulierenden Merkmalen in bezug auf Gewalt in der Weise, daß bei den Schülern mit hoher Steuerungskompetenz (Gruppe 2) die Einflußfaktoren eine geringere Rolle spielen als bei der Vergleichsgruppe. Bei dieser handelt es sich um die unter pädagogischen Gesichtspunkten problematischeren Schüler mit niedriger Steuerungskompetenz (Gruppe 1), bei denen die Einflüsse des Erziehungsmilieus und der Schulkultur stärker zum Tragen kommen. Für die Prävention signalisiert dieser Befund einen pädagogischen Optimismus: denn die Ergebnisse zeigen, daß sich bei einem schwierigen Schülerklientel - z.B. durch gemeinsame Anstrengungen von Schule und Elternhaus - wenigstens teilweise eine Kompensation personaler Defizite erreichen läßt.

In diesem Zusammenhang stellt sich die Frage nach dem möglichen Beitrag der Institution Schule und der professionellen Pädagogik zur Gewaltprävention und -intervention. Die bisherigen Analysen haben gezeigt, daß 16,3\% der Varianz der Täter-Variable und 11,3\% der Varianz der Opfer-Variable in der Stichprobe auf die Unterschiede der Schul- und Klassenebene zurückzuführen sind. D.h., daß der größere Anteil bei der Beeinflussung möglichen Gewaltverhaltens auf außerschulische Sozialisationsbedingungen und individuelle Eigenschaften zurückgeführt werden kann. Bei anderen Indikatoren des gewaltförmigen und gewaltaffinen Verhaltens stellt sich diese Verteilung der Varianz etwas anders dar: während die Einflußmöglichkeit der Schule bei außerschulischem Gewalthandeln noch begrenzter ist $(14,1 \%)$, lassen sich Phänomene der Schuldevianz $(18,0 \%)$ und der Aggression in konkreten schulischen Situationen $(21,8 \%)$ im schulischen Raum vergleichsweise besser bearbeiten. Auf der Linie bisheriger Befunde zeigt sich eine Abstufung von härteren Gewaltformen, auf die der schulische Einfluß geringer ist, und 
weicheren Gewaltformen, für die eine schulische Prävention und Intervention aussichtsreicher erscheint. Dies ist ein erneuter Hinweis darauf, daß schulische Präventions- und Interventionskonzepte bei den weicheren Gewaltformen und den gewaltaffinen Verhaltensweisen ansetzen müssen, um dem Beginn und der Verfestigung von "Gewaltkarrieren" entgegenzuwirken. 


\section{Gewaltprävention im Kontext von Schulentwicklungsprozessen - eine Pilotstudie (Kerstin Darge)}

Bereits im Ansatz unseres Forschungsprojektes, das im Sonderforschungsbereich 227 ,Prävention und Intervention im Kindes- und Jugendalter“ angesiedelt ist, wird die Frage nach Maßnahmen der Gewaltprävention und -intervention thematisiert. Wissenschaftliche Analysen und Präventionskonzepte sollten eng verbunden sein, wobei ebenfalls auf vorliegende Programme und Erfahrungen zurückgegriffen werden konnte. Daraus entwickelte sich im Verlaufe unserer Tätigkeit ein spezifisches Programm zur Gewaltreduktion, das derzeit exemplarisch im Rahmen einer Pilotstudie an einer Schule durchgeführt wird. Aus den daraus resultierenden Erfahrungen sollen verallgemeinerbare, über die Einzelschule hinausführende Empfehlungen für die Präventions- und Interventionspraxis gewonnen werden.

Die Konzeptionierung unserer Präventions- und Interventionsarbeit basiert auf von uns selbst erhobenen Analysedaten, die nach Schul-, Klassen- und Individualebene spezifiziert wurden. Neben quantitativen Untersuchungsbefunden, wie sie in dieser Studie in den Kapiteln 2 bis 7 dargestellt worden sind, wurden im Rahmen zweier Fallstudien weitere qualitative Untersuchungsverfahren (in Form von Schülerbeurteilungen durch die Lehrer, Interviews und eines soziometrischen Tests) umgesetzt und für die Präventionsarbeit nutzbar gemacht. Unser Modell fußt weiterhin auf solchen Präventions- und Interventionskonzepten, die sich bereits in der Praxis etabliert haben sowie auf konzeptionellen Überlegungen, die im Rahmen der Schulqualitäts- bzw. weiterführend der Schulentwicklungsdebatte entwickelt wurden (Steffens/Bargel 1993, Rolff/ Bauer/Klemm/Pfeiffer 1996). Die Auswahl der Programme erfolgt dabei unter Berücksichtigung ihrer Zielebenen (Schul-, Klassen- und Individualebene) und nach spezifischen Bedürfnissen in den jeweiligen Bereichen. Für diese Vorgehensweise sprechen die unterschiedlichen Erklärungsfaktoren für einen Täter- bzw. Opferstatus (vgl. Kap. 4 bis 6 sowie Melzer/Rostampour 1996, Melzer 1997, Rostampour/Melzer 1997). Demnach üben Täter massive Kritik an vielen Aspekten der Schulkultur, während Opfer deutlich unter sozialer Isolation, negativem Selbstkonzept und Ausgrenzung in den Klassen leiden. Diesen Ergebnissen entsprechend müßten Präventions- und Interventionsprogramme gleichermaßen die Opfer- als auch die Täterperspektive berücksichtigen (vgl. Kap. 6.3). Allerdings beziehen sich die meisten Programme eher auf die Täter-Problematik, ohne auf die Arbeit mit den Opfern einzugehen. Unser Ansatz greift dieses Defizit auf und verfolgt daher eine Doppelstrategie: Zum einen wird mit der Integration einzelner Elemente - besonders aus schulumfassenden Programmen - in die gewaltpräventive und -intervenierende Arbeit 
die Problematik der Täter berücksichtigt; zum anderen versuchen wir mit der Implementation weiterer Elemente in die gewaltpräventive Arbeit - besonders aus Ansätzen auf Klassen- bzw. Individualebene - auf die Opferperspektive zu zielen.

Das Ziel unseres Präventions- und Interventionsmodells besteht darin, die personalen und sozialen Ressourcen einer Schule zu aktivieren, um dadurch zu einer Gewaltreduktion und zu einer Verbesserung des reflektierten und konstruktiven Umgangs der Lehrer und Schüler mit Aggressionen beizutragen. Beides versteht sich dabei als Beitrag zur Schulentwicklung auf dem Weg zu einer ,guten Schule“. Insgesamt verfolgen unsere Arbeiten einerseits theorieproduktive Zwecke und andererseits handlungsorientierte pädagogische Zielsetzungen für die Arbeit mit einzelnen Schulen.

Im ersten Teil dieses Beitrags werden die Orientierungspunkte für unser Konzept der Gewaltprävention und -intervention dargestellt. Neben dem international anerkannten Olweus-Programm werden Strategien zur Schulentwicklung und zur schulinternen Lehrerfortbildung (SchiLF) beschrieben sowie ein auf psychologischer Theorie aufbauendes Gewaltpräventionsmodell vorgestellt. Mittlerweile liegt ein reichhaltiger Fundus an Präventions- und Interventionsstrategien vor. Eine Auswahl der vorgestellten Ansätze erfolgt unter dem Gesichtspunkt der Kompatibilität, d.h., im Hinblick darauf, ob die in den Datenanalysen deutlich gewordenen möglichen Ansatzpunkte durch diese Vorgehensweise abgedeckt sind. Der zweite Teil stellt die Ergebnisse der Fallanalysen auf Schul-, Klassen- und Individualebene dar, die im Rahmen einer Pilotstudie gewonnen wurden. Im Gegensatz zum SchiLF-Konzept, das auf Alltagstheorien von Lehrern basiert, operieren wir in unserer Zusammenarbeit mit Schulen - ähnlich wie Olweus - auf der Grundlage von wissenschaftlichen Datenanalysen. Weiterhin wird der Prozeß der aktiven Schulgestaltung und entwicklung am Beispiel der Modellschule widergespiegelt. Der abschließende dritte Teil stellt eine erste Bilanzierung des bisherigen Schulentwicklungsprozesses dar. Darüber hinaus werden die positiven Auswirkungen einer gezielten Hauptschulpädagogik dargestellt. Dieses geschieht auf der Grundlage repräsentativer Einzelitems: So vergleichen wir die Daten einer Haupt- und einer Realschulklasse unserer Modellschule mit den entsprechenden Daten der Haupt- und Realschulklasse einer weiteren Schule. Eine Evaluation unseres Programms ist im Schuljahr 1998/99 nach dem klassischen Modell eines Vorher-Nachher-Vergleichs unter Einbeziehung von Kontrollgruppen an mindestens zwei weiteren gewaltbelasteten Schulen vorgesehen, und schließt eine Überprüfung der sozialökologischen Bedingungen der Schule und der Effekte der pädagogischen Arbeit - u.a. über die Wirkungen der bisher durchgeführten Maßnahmen - ein. Gesicherte Evaluationsergebnisse zur Wirksamkeit des Programms lassen sich daher zum jetzigen Zeitpunkt noch nicht vorstellen. Im Anschluß an diese Darstellungen wird ein Ausblick über die gewaltpräventive und intervenierende Arbeit an Schulen insgesamt gegeben. 


\subsection{Prävention und Intervention - Definitionen und Programme}

Wie im internationalen Raum liegen in Deutschland Erfahrungen mit gewaltmindernden Präventions- und Interventionsprogrammen vor, die - ihren zugrundeliegenden Konzepten entsprechend - nach zwei Kriterien gruppiert werden können: 1. dem Stadium, in dem die Maßnahme ansetzt (präventiv oder korrektiv) und 2. der Dimension, auf welche sie zielt: auf personale Ressourcen (der Adressat ist die Einzelperson, z.B. bei Verhaltenstherapien) oder auf soziale Ressourcen (durch Modifikationen verschiedener Umweltfaktoren werden indirekt Verhaltensänderungen einzelner Personen bewirkt). In der wissenschaftlichen Literatur wird auch nach Primär-, Sekundär- und Tertiärpräventionen unterschieden, wobei präventive Maßnahmen den Primärpräventionen entsprechen und Sekundär- bzw. Tertiärpräventionen den korrektiven Maßnahmen zuzuordnen sind. Kontext- bzw. lebensweltbezogene Ansätze, die sich auf eine Verbesserung des gesamten sozialen Gefüges der Schule beziehen und primärpräventiv erfolgen (d.h. problematische Interaktionen im Vorfeld verhindern), sind z.B. die Verbesserung des Schulklimas und der Partizipationsmöglichkeiten der Schüler. Sekundärpräventive Maßnahmen sollen ein Ausufern bereits bestehender Probleme durch direkte Interventionen verhindern (z.B. Schulsozialarbeit). Primärpräventive Maßnahmen auf personaler Ebene sind z.B. schulische Leistungsförderung oder Kompetenztrainings; dagegen beziehen sich sekundärpräventive Maßnahmen z.B. auf Verhaltensmodifikationen. Tertiärpräventive Maßnahmen (z.B. Resozialisierung, Psychotherapie) betreffen therapeutische und rehabilitative Vorgehensweisen und setzen entsprechende Qualifikationen voraus. Besonders mit Blick auf die Opfer verwendet Korte den Begriff der Postvention. In diesem Zusammenhang verweist er auf die Notwendigkeit der nachsorgenden Betreuung schulischer Gewaltopfer, die ,bei der nachträglichen Aufarbeitung der Fälle stets zu kurz kommen“ (Korte 1995, S. 30). Anstelle der Einteilung in Primär-, Sekundär- und Tertiärprävention ist der Gebrauch der Termini Prävention, Intervention und Rehabilitation eindeutiger. Insgesamt sind die begrifflichen Abgrenzungen problematisch, da jede Prävention auch eine Intervention ist. Ebenfalls sind die Übergänge der Präventionsarten fließend, und eine gezielte Intervention problembelasteter Schüler hat meistens auch sekundärpräventive Effekte (Dann 1997, S. 354f, Hurrelmann 1990, S. 370f, Schwarzer 1997, S. 780ff, Stark 1992, Wambach 1990).

Wichtiger als eine enge begriffliche Abgrenzung in präventive und intervenierende Maßnahmen ist die Trennung der Ebenen, auf denen sie ansetzen bzw. auf die sie zielen. Unseren Analysen zufolge benötigen manche Schulen, auch wenn sie einer überproportional belasteten Schulform ${ }^{1}$ angehören,

1 Statistischen Analysen zufolge sind Gymnasien im Vergleich zu Mittel- und Förderschulen weniger gewaltbelastet. 
kein Programm, andere hingegen dringend. Ebenso sind oftmals nur einzelne Klassen problembelastet. Und auch auf dieser Ebene ist zu prüfen, ob die beabsichtigten Maßnahmen die gesamte Klasse oder einzelne Schüler betreffen sollen. D.h., die unterschiedlichen Problembelastungen auf Schul-, Klassen- und Individualebene machen spezifische Handlungskonzepte erforderlich. Von diesen Voraussetzungen her ergibt sich ein die spezifischen Probleme betreffendes Präventions- und Interventionskonzept, das in den Rahmen eines Schulentwicklungsprozesses gestellt sein sollte. Unser Verständnis und Konzept der Gewaltprävention und -intervention basiert auf empirischen Ergebnissen von Analysen auf unterschiedlichen Ebenen. Auf dieser Grundlage werden etablierte Interventions- und Präventionskonzepte ausgewählt und für unsere Konzeption nutzbar gemacht. Letztlich sollen den unterschiedlichen Problemen spezifische Maßnahmen entgegenstehen, die in einem Schulentwicklungsprozeß über einen längeren Zeitraum integriert werden - so die Idealvorstellung unseres Ansatzes.

Folgende bewährte Ansätze dienen unserer Präventions- und Interventionsarbeit als theoretische und praktische Orientierungspunkte:

- Das Konstanzer Trainingsprogramms (KTM) nach Tennstädt/Krause/ Humpert/Dann $(1991,1994)$ ist auf der Lehrerebene angesiedelt. Es beinhaltet neben theoretisch-empirischen Grundlagen konkrete Hilfen für Probleme im Schulalltag. Im Mittelpunkt stehen Alltagstheorien über Aggressionen im Unterricht, welche Lehrer im Laufe ihrer Berufszeit ausgebildet haben. Ziele des Programms sind die Erhöhung pädagogischer Handlungskompetenzen durch den Aufbau eines reflektierten und angemessenen Handlungsrepertoires sowie die Verminderung störender Interaktionen im Unterricht zugunsten kooperativer und konstruktiver Umgangsformen. Das Konzept fußt auf einem handlungstheoretischen Modell von Lehrerreaktionen auf Störungen und Aggressionen. Jedes Handeln ist in vier Stufen eines Reaktionsprozesses unterteilt (Situationsauffassung, Handlungsauffassung, Handlungsausführung, Handlungsergebnisauffassung). Diesen Stufen sind insgesamt zehn Trainingselemente zugeordnet. Einen inhaltlichen Schwerpunkt bildet jenes Element, das - nach Einführung in lerntheoretische Grundlagen und die Bedeutung von konsequentem Verhalten - 27 mögliche Lehrerreaktionen bei störendem und aggressivem Verhalten beschreibt. Diesen 27 Maßnahmen lassen sich fünf pädagogischpsychologische Handlungsstrategien zuordnen. Darüber hinaus nimmt der Kommunikationsstil des Lehrers eine zentrale Rolle ein; daher werden Kenntnisse der Kommunikationspsychologie als Anstöße zur Überprüfung und zur Verbesserung des Kommunikationsstils des Lehrers vermittelt. Das KTM ist als „Hilfe zur Selbsthilfe“-Programm so konzipiert, daß es in Form von Lehrer-Partnerarbeit jederzeit angewendet werden kann. Die Trainierenden wählen dabei die Trainingselemente entsprechend ihrer Probleme. Nach der gemeinsamen Bearbeitung des schriftlichen Trai- 
ningsmaterials und der Diagnose und Planung des Unterrichtsverlaufs werden neue Handlungsmöglichkeiten im Unterricht geübt und letztlich auf ihre Wirkung hin überprüft. Das KTM ist problemlos im Rahmen einer Gesamtkonzeption der Schulentwicklung in ein von der Schule selbst entwickeltes pädagogisches Konzept einzubetten. Damit ist es im Zusammenhang mit der schulinternen Lehrerfortbildung (SchiLF) zu sehen. Das Programm hat in der Lehrerfort- und Weiterbildung einen hohen Verbreitungsgrad. Bisherige Evaluationsstudien konnten positive Trainingseffekte bei Schülern und Lehrern nachweisen. So fühlen sich Lehrer im Umgang mit Aggressionen kompetenter, haben mehr Selbstvertrauen und einen engeren Kontakt zum Kollegium. Die Schüler stören weniger, sind weniger aggressiv und arbeiten engagierter mit. Nachuntersuchungen, die Aufschluß über langfristige Wirkungen geben, stehen aus.

- Auf Erfahrungen von Petermann/Petermann (1996, 1997) aufbauend entwickelten Petermann/Jugert/Tänzer/Verbeek (1997) das Sozialtraining in der Schule, das auf Schülerebene angesiedelt ist. Ziel ist die Förderung sozial-kompetenten Verhaltens bei Schülern. Anlaß für die Entwicklung war der Gedanke einer frühen, gezielten Förderung sozialer Fähigkeiten von Kindern, um Verhaltensstörungen präventiv entgegenzuwirken. Das Programm bezieht sich auf Schüler der Grundschule und Orientierungsstufe, da sie sich in einer Entwicklungsphase befinden, in der sie ein hohes Maß an sozialer Orientierung benötigen und in der viele Schüler Verhaltensauffälligkeiten zeigen, die sich verfestigen können. Sozial-kompetentes Verhalten setzt kognitive Fähigkeiten (Wahrnehmung, Interpretation, Suche nach Bewertung von Handlungsalternativen) sowie soziale Fertigkeiten (Erkennen/Äußern von Gefühlen, angemessene Selbstbehauptung, Kooperation und Einfühlungsvermögen) voraus. Da Verhaltensauffälligkeiten Einschränkungen der Handlungskompetenz bewirken und dem Kind die Möglichkeit nehmen, ein differenziertes und situationsangemessenes Verhaltensrepertoire zu entwickeln, gilt es, diese Fertigkeiten und Fähigkeiten zu trainieren. Der Ansatz ist in die sozial-kognitive Informationsverarbeitungstheorie nach Dodge (1986) sowie die Theorie der Selbstwirksamkeit nach Bandura (1979) eingebettet. Dodge beschreibt die Verarbeitung sozialer Informationen von der Wahrnehmung über die Speicherung und Interpretation, Suche und Bewertung der Handlungsmöglichkeiten bis zur Reaktion. Nach Bandura sind die Ergebnisse der jeweiligen Handlung Grundlage erneuter Situationsbewertungen. Kinder mit Verhaltensstörungen nehmen auf allen Stufen soziale Informationen fehlerhaft wahr und verarbeiten sie falsch. Das Sozialtraining greift dies auf, indem kognitive Prozesse in spielerische Übungen des sozialen Lernens eingebettet werden. Im Anschluß einer jeweiligen Trainingssitzung werden die Übungen mit den Schülern reflektiert (Petermann/Jugert/Verbeek/Tänzer 1997).

- Lerchenmüller (1986, 1987) konzipierte ein Unterrichtsprogramm für soziales Lernen in der Schule im Rahmen eines eigenen Curriculums. 
Mit dem Ziel, delinquenz-prophylaktisch zu wirken, werden Themen wie Vorurteile und Diskriminierung von Minderheiten, Konflikte mit Autoritätspersonen und Gleichaltrigen, Hintergründe und Folgen einer Straftat etc. bearbeitet. Als Methoden werden Rollenspiele, Klassengespräche sowie Gruppen- und Partnerarbeit angewendet. Die Durchführung des Programms trägt zur Förderung von Handlungskompetenz der Schüler und zur Verbesserung des Lernklimas innerhalb der Klasse bei. Die Programmevaluation konnte positive soziale Lerneffekte nachweisen, die aber nach einem halben Jahr weitgehend nivelliert waren. Da zur Umsetzung des Programms ein sozial-integrativer Unterrichtsstil der Lehrer erforderlich war, den sie jedoch nur z.T. verwirklichen konnten, fordert Lerchenmüller Trainings der pädagogischen Handlungskompetenzen von Lehrern.

- Wegweisend sind die Forschungen über Gewalt in der Schule und über Interventions- und Präventionsmaßnahmen des Norwegers Olweus (1995). Er entwickelte ein in Norwegen und Schweden seit den 80er Jahren eingesetztes und evaluiertes Interventionsprogramm mit gleichzeitig präventiver Wirkung. Es umfaßt mögliche Interventionen auf drei Ebenen: 1. der Schulebene (Fragebogenerhebung, pädagogischer Tag, Schulkonferenz, verbesserte Pausenaufsicht, Schulhofgestaltung, Kontakttelefon, Kooperation zwischen Lehrern und Eltern, Lehrergruppen zur Entwicklung des sozialen Milieus an der Schule, Elternarbeitsgruppen), 2. der Klassenebene (z.B. Klassenregeln gegen Gewalt, Klassengespräche, Rollenspiele, Literatur, kooperatives Lernen, gemeinsame Klassenaktivitäten, Zusammenarbeit zwischen Klassenelternbeirat und Lehrkräften) und 3. der persönlichen Ebene (Gespräche mit Tätern und Opfern, Lehrern, Eltern und Schülern, Hilfe von neutralen Schülern, Hilfe und Unterstützung von Eltern, Diskussionsgruppen für Eltern von Gewalttätern und -opfern, Klassen- und Schulwechsel). Übergeordnetes Ziel ist die Steigerung der sozialen Kompetenz der Schüler. Ausgangspunkt der Umsetzung des Programms in die Praxis ist a) eine Fragebogenerhebung, b) ein pädagogischer Tag, an dem die Ergebnisse rückgekoppelt werden und c) eine Schulkonferenz, bei der Maßnahmen vereinbart werden. Das Programm wurde inzwischen auch in Schulen Schleswig-Holsteins angewendet und evaluiert (Hanewinkel/Knaak 1997). Die Untersuchungsergebnisse beider direkt miteinander vergleichbarer Studien weisen eine Reduzierung der Gewalt und eine Verbesserung des Klassen- und Schulklimas nach.

- Da die gewaltpräventive und intervenierende Arbeit in der einzelnen Schule ansetzen muß und es kein Patentrezept für die Problemlösung gibt, sollte jede Schule ein an Risikofaktoren ansetzendes pädagogisches Profil entwickeln. Bei dieser spezifischen schulinternen Gestaltung ist das Konzept der Schulinternen Lehrerfortbildung (vgl. auch Greber/ Maybaum/Priebe/ Wenzel 1993) hilfreich. Dabei werden ausgewählte Maßnahmen von möglichst vielen an der Schule beteiligten Personen umgesetzt. ,SchiLF ist 
ein systematischer Arbeitsprozeß eines Kollegiums mit Schülerinnen und Schülern wie Eltern zur Entwicklung der eigenen Schule im Hinblick auf drängende Aufgaben und Probleme“ (Priebe 1995, S. 11). Die Prozesse orientieren sich an Konzepten und Methoden der schulischen Organisationsentwicklung und an schulpädagogischer Theorie und Praxis. Innerhalb der Schulinternen Lehrerfortbildung setzen sich Lehrer, Schüler und Eltern mit einem Thema auseinander. Ziel ist der Austausch über Lehr- und Lernerfahrungen, die Erweiterung von Kenntnissen, der Abbau von Unsicherheiten sowie letztlich die Festlegung gemeinsamer Handlungsschritte für die Gestaltung einer demokratisch partizipativen Schulentwicklung. Externe Berater bzw. Moderatoren (z.B. ausgebildete Lehrer der jeweiligen Lehrerfortbildungsinstitute) begleiten den Arbeitsprozeß, indem sie Theorien, Untersuchungen und Konzepte einbringen, bei inhaltlichen, motivationalen und gruppendynamischen Schwierigkeiten helfen und darauf achten, daß verbindliche Handlungsvorschläge erarbeitet werden und deren Umsetzung realisiert wird. Je mehr Personen an der Schulentwicklung aktiv werden, desto aussichtsreicher sind die Erfolgschancen. Wird eine Maßnahme im Anschluß positiv bewertet, ist das Vorhaben abgeschlossen. Meist resultieren aus der Bearbeitung eines Themas Folgethemen, die in weiteren Schulinternen Lehrerfortbildungen bearbeitet werden, so daß letztlich ein SchiLF-Projekt entsteht. Positiv sind die methodische Vielfalt und Flexibilität sowie die Eigenverantwortlichkeit der Lehrer bei der im Rahmen von SchiLF durchgeführten Schulentwicklung. Bestehende Konzepte werden nicht einfach übernommen, vielmehr wählen die Lehrer Handlungsstrategien nach ihren Wünschen und entsprechend ihres Schulprofils aus, so daß sich spezifische Arbeitspläne ergeben. Letztlich entsteht ein „Konzept mit pädagogischem Eigensinn, das dem spezifischen Bildungs- und Erziehungsauftrag der Schule entspricht" (Schubarth/Akkermann 1997, S. 88). Unabhängig von den Formen Schulinterner Lehrerfortbildung (Intervallseminar, Fachkonferenzen, Trainingsgruppen etc.) haben SchiLF-Prozesse ein typisches Verlaufsmuster ${ }^{2}$ (vgl. Miller 1995, S. 80f, Miller 1996, S. 71ff): 1. Phase: Ausgangspunkt (z.B. der Wunsch nach Schulinterner Lehrerfortbildung); 2. Phase: Vorbereitung (z.B. Konkretisierung des Themas, Festlegung des weiteren Vorgehens [Schulkonferenz, Wahl von Verantwortlichen, Analyse des Ist-Zustandes der Schule etc.]); 3. Phase: Durchführung der SchiLF-Maßnahme (z.B. Vorstellen erster Ergebnisse, Vereinbarung von Handlungsschritten); 4. Phase: Auswertung der Maßnahme.

2 Miller stellt Strukturen und Verlaufspläne verschiedener SchiLF-Maßnahmen vor, die Grundmuster darstellen und Schwerpunkte angeben, aber ,je nach Bedarf, Situationen, Rahmenbedingungen, Zielen u.ä. verkürzt, erweitert, umgestellt werden“ können (Miller 1995, S. 83). 
Über diese Konzepte hinaus existieren weitere Programme (z.B. Hensel 1993, 1994) sowie unzählige Berichte über und Anregungen für Handlungsmöglichkeiten. ${ }^{3}$ Die Vorschläge sind vielfältig und basieren auf theoretischen Überlegungen, empirischen Ergebnissen und Alltagserfahrungen. Eine umfassende Sekundäranalyse von Handlungskonzepten gegen Schulgewalt haben Schwind u.a. (1995) vorgenommen. Sie differenzieren und klassifizieren diese hinsichtlich verschiedener Zielebenen: Programme für Schüler, für Klassen und Schulprogramme. Auf die Vielzahl bestehender Modellversuche zur Gewaltprävention reagierten 22 Projekte und Experten aus verschiedenen Wissenschaftsbereichen mit einer Vernetzungs-Fachtagung. Ziel der Tagung und des daraus resultierenden Sammelbands ist die Kooperation verschiedener Modellversuche zum Zwecke des Erfahrungsaustausches, die Entwicklung von Perspektiven bereits bestehender und geplanter Modellversuche sowie die breite Veröffentlichung ihrer Ergebnisse (Balser/Schrewe/ Schaaf 1997).

Ohne die Notwendigkeit präventiver Bemühungen in Zweifel zu ziehen, wird die derzeitige Präventions- und Interventionspraxis z.T. kritisiert: „Sowohl die Ratgeberliteratur für Lehrkräfte als auch die wissenschaftliche Fachliteratur ist in dieser Hinsicht ausgesprochen unübersichtlich; die meisten Vorschläge erscheinen durchaus plausibel, wenn auch eher beliebig ausgewählt oder unzureichend begründet" (Dann 1997, S. 354). Manche Autoren gehen in ihrer Einschätzung bestehender Konzepte so weit zu behaupten: „Praktikable Vorschläge zur Verringerung des Problems an Schulen sind immer noch Mangelware“ (Berger/Finze 1997). Letztlich läßt sich eine Bewertung bisheriger Erfahrungen mit Gewaltpräventions- und -interventionsmaßnahmen in drei Punkten zusammenfassen:

1. Präventions- und Interventionsmaßnahmen basieren oft auf allgemeinen Prämissen der jeweiligen wissenschaftlichen Disziplin (Psychologie, Pädagogik) oder auf Alltagserfahrungen, nicht aber auf spezifischen und wissenschaftlichen Analysen der Bedingungskonstellationen von Gewalt einer einzelnen Schule oder Klasse mit ihren einzelnen Schülern. Eine sorgfältige Recherche und Anamnese vor Ort über die Ursachen von Gewalt sollte allerdings dem jeweiligen pädagogischen Handeln vorausgehen, da wirksame Maßnahmen zur Prävention bzw. zum Abbau von Gewalt in enger Beziehung zu aggressionsauslösenden und -fördernden Ursachen stehen müssen.

2. Die Zielebenen und Anwendungssituationen sind sehr heterogen. Viele Vorschläge beziehen sich auf einmalige, punktuelle Maßnahmen, bleiben

3 Z.B. AJS 1994, Braun/Hünicke 1995, Die Grundschulzeitschrift 100/1996, Gades 1995, Gruschka/Hesse/Michely/Schomacher 1995a, 1995b, Hensel 1995, Hurrelmann/Rixius/ Schirp 1996, Kultusministerium des Landes Sachsen-Anhalt 1996, Korte 1995, Möhle 1997, Reitmajer 1996, Schultze 1995. 
dadurch episodenhaft und sind von wenig dauerhafter Wirkung. Schulische Prävention und Intervention ist allerdings nur dann optimal wirksam, wenn Einzelmaßnahmen unterschiedlicher Ebenen in den Rahmen eines Gesamtkonzepts der spezifischen Schulentwicklung eingebettet sind, wie es bei der schulinternen Lehrerfortbildung und in den Olweus-Projekten geschieht. Nur langfristige und auf mehreren Ebenen ansetzende Maßnahmen bewirken, daß neue Verhaltensweisen - bei Lehrern wie Schülern verinnerlicht und Bestandteil der eigenen sozialen Kompetenz werden.

3. Nicht zuletzt stellt sich die Frage nach den Resultaten solcher Bemühungen. Auch wenn verschiedene Maßnahmen nicht direkt auf die Gewaltproblematik zielen, werden ihre positiven Auswirkungen hervorgehoben. $\mathrm{Ob}$ es sich um kurzfristige Verbesserungen oder um Langzeiterfolge handelt, bleibt z.T. unerwähnt. Außerdem unterliegen viele Effektivitätskontrollen allein den subjektiven Empfindungen der Teilnehmer. Ob eine Maßnahme als gelungen gilt oder nicht, wird dann primär mit dem persönlichen Gewinn, nicht aber mit dem Erreichen der Ziele in Verbindung gebracht. Erforderlich wäre eine systematische Programmevaluation, die aus Kosten- und Zeitgründen oft nicht durchgeführt wird. Nur so kann die Wirksamkeit einzelner Maßnahmen festgestellt und die Ergebnisse im Sinne einer systematischen Gewaltprävention auf andere Projekte übertragen werden (Dann 1997, Hesse 1993, S. 225ff, Lerchenmüller-Hilse 1996).

\subsection{Ergebnisse der Pilotstudie an einer Mittelschule}

Um gewaltfördernde und -hemmende Bedingungen transparent zu machen, wurden mit verschiedenen Instrumentarien (quantitative und qualitative Erhebungen, Schülerbeurteilungen durch Lehrer, soziometrische Tests) Analysen auf Schul-, Klassen- und Individualebene durchgeführt. Ein besonderer Akzent lag dabei auf der Entwicklung entsprechender Präventions- und Interventionskonzepte als Bestandteil von Schulentwicklung. Die in diesem Teil dargestellten Ergebnisse beziehen sich auf die Modellschule, die im folgenden kurz vorgestellt werden soll.

Die Schule wurde 1966 erbaut und liegt in einem Arbeiterviertel einer sächsischen Großstadt, dessen Bild von Häusern aus der Gründerzeit und nach dem Krieg entstandenen Wohnblocks geprägt ist. Die schlechten Wohnverhältnisse (viele nicht sanierte Häuser), eine gegenüber dem Stadtdurchschnitt höhere Arbeitslosenquote sowie eine hohe Anzahl von Sozialhilfeempfängern kennzeichnen die Lebenssituation der hier lebenden Menschen. Der Anteil der Familien mit drei oder mehr Kindern und der Haushalte Alleinerziehender liegt im Vergleich zu dem der Gesamtstadtbevölkerung über den Durch- 
schnittswerten. ${ }^{4}$ Im Unterschied zum Altersdurchschnitt der Gesamtstadtbevölkerung (etwa 40 Jahre) handelt es sich um einen Stadtteil mit vorwiegend junger Bevölkerung (Durchschnittsalter: 36 Jahre). Offene Kinder- und Jugendeinrichtungen sind im Viertel zwar vorhanden, dennoch sind solche Plätze, an denen die Schüler unbeaufsichtigt ihre Freizeit verbringen können, begrenzt. So gibt es im Stadtteil nur einen Spielplatz. Das äußere Erscheinungsbild der Schule ist - bedingt durch die graue Fassade - eher trist. Allerdings wurde durch die Teilnahme an einem Projekt ,Schule ohne Gewalt“ der Schulhof mit Sitzbänken und verschiedenen Sportgeräten ausgestattet.

Im Schuljahr 1995/96 wurden die 130 Mädchen und 177 Jungen von nur 15 Lehrern (davon acht Gastlehrer) unterrichtet. Folglich stellte der Personalmangel und die damit verbundene Abdeckung des Unterrichts ein Problem dar. Ebenso fehlte ein Beratungslehrer; Arbeitsgemeinschaften konnten nicht angeboten werden. Diese Situation erschwerte die erzieherische Arbeit, so daß der Wunsch der Lehrer nach zusätzlichem Personal deutlich wurde. Im Schuljahr 1996/97 entspannte sich die Situation, da seither 19 Lehrer, drei Gastlehrer und eine Referendarin an der Schule unterrichten. Die Klassenstufen werden meist zweizügig geführt. Die beiden 8. Klassen des Schuljahrs 1995/96 gliederten sich - auch im Schuljahr 1996/97 - in eine Haupt- und eine Realschulklasse. Die Schule bietet das technische, das sozial-hauswirtschaftliche und das sportliche Profil an. Eine besondere Fördermaßnahme ist der Deutschkurs für die insgesamt neun ausländischen Schüler. Ebenso versucht man, Patenschaften zwischen deutschen und ausländischen Kindern herzustellen. Im Schuljahr 1995/96 wechselten zwölf und ein Schuljahr später drei Schüler vom Gymnasium zur Mittelschule. Daß umgekehrt Mittelschüler auf das Gymnasium wechseln, kommt selten vor. Zur Öffnung der Schule trägt eine Gruppe von Frauen bei, die Hauswirtschaftsarbeitsgemeinschaften organisieren. Durch die Existenz des schuleigenen Fördervereins können „kleinere Projekte“ finanziert werden.

Vor der Wende war die Schule eine POS. ${ }^{5}$ Nach Inkrafttreten des neuen Schulgesetzes blieben die meisten Lehrer an der Schule. Der neue Schulleiter übt sein Amt seit 1992 aus - während sein Vorgänger seither „einfaches“ Mitglied des Kollegiums ist. Vorher waren der Schulleiter und seine Stellvertreterin nicht Lehrer an dieser Schule. Im selben Schulgebäude ist neben der Mittelschule eine Grundschule ansässig, allerdings ist das Raumangebot für die Gesamtschülerzahl zu gering. Dadurch hat nicht jede Klasse einen eigenen Klassenraum. Ein Aufenthaltsraum, ein Schülercafé bzw. Sitzecken existieren nicht. Die Schüler können in der Schulmensa essen. Klassen- und

4 Amt für Informationsverarbeitung, Statistik und Wahlen. Stand 30.06.1996.

5 POS ist die Abkürzung für die in der DDR obligate zehnklassige Polytechnische Oberschule, in der Schüler von der 1. bis zur 10. Klasse unterrichtet wurden (vgl. auch Döbert 1995b, S. 24ff). 
Fachräume sowie die Flure und Toiletten wurden seit Bestehen der Schule nicht modernisiert. Der Informatikunterricht findet in einer Nachbarschule statt, da ein Computerkabinett aus Kosten- und Platzmangel bisher nicht eingerichtet werden konnte.

Schon dieses kurze Schulportrait ${ }^{6}$ macht die Schulsituation und die Bedingungen des schulischen Umfeldes deutlich, die nun im einzelnen auf der Basis der quantitativen Erhebungen dargestellt werden sollen. ${ }^{7}$ Bei dieser empirischen Beurteilung der Schul- und Klassensituation fließen die Ergebnisse der Analysen auf Individualebene und der qualitativen Erhebung mit ein. Die quantitative Analyse erfolgt - neben prozentualen Auswertungen - hauptsächlich in Analogie zu dem von Melzer/Stenke (1996) entwickelten Schulqualitätsindex bzw. den entsprechenden Schulentwicklungsstrategien, in denen diese Idee zum Einsatz kommt. Der seiner Zeit entwickelte Index umfaßte (auf Grundlage einer Schülerbefragung) Faktorvariablen zu den Bereichen: verschiedene Aspekte der Schulkultur, außerschulische Sozialisationskontexte Familie und Peers, Persönlichkeitsmerkmale sowie Gewaltwahrnehmung, Verhaltensformen und Einstellungen von Schülern zur Gewalt. Es entstehen „pädagogische Benchmarks“ von Einzelkriterien, die im Gesamtbild ein typisches Profil der Schule bzw. Klassen ergeben und anhand derer Ansätze für eine Präventions- und Interventionsarbeit gewonnen werden können: Über den Mittelwert des jeweiligen Qualitätkriteriums für die Einzelschule ist ein Vergleich mit den Mittelwerten aller Schulen bzw. dem der eigenen Schulform möglich. Die Minimum- und Maximumwerte, die die jeweilige Streuung begrenzen, zeigen, in welchen Bereichen - im Vergleich mit anderen Schulen - eine Entwicklungsarbeit nötig ist. Benchmarkingstrategien stammen aus dem wirtschaftswissenschaftlichen Bereich: Benchmarks als Grundlage der Ziele des Entwicklungsprozesses einer Institution sind vorteilhaft, weil sie (1.) „ein Unternehmen mit den Besten seiner Branche“ vergleichen und damit (2.) „,im ganzen Unternehmen ein Bewußtsein für die tatsächliche Position des Unternehmens im Vergleich mit der Konkurrenz" schaffen (Brenner/Keller 1995, S. 26f, vgl. auch Metzen 1994). Auf die Schule übertragen ermöglicht ein entsprechendes Instrumentarium den Lehrern, ihnen als wichtig erscheinende Kriterien zu betrachten und läßt ihnen Spielraum für Interpretationen. In ähnlicher Weise sind wir auch in unserer Pilotstudie vorgegangen und haben der Modellschule Gewalt- und Qualitätsprofile vorgelegt, die eine Grundlage der weiteren pädagogischen Arbeit setzten (vgl. Abb. 8-1 bis 8-3).

Die zusätzlichen Ergebnisse der Analysen auf Individualebene wurden mittels zweier Instrumente erhoben: Zum einen nahmen die Schüler an einem

6 Die Informationen erschließen sich aus Interviews, die mit dem Schulleiter im Oktober 1995 sowie September und November 1996 geführt worden sind sowie aus einer Stadtteilanalyse.

7 Die Daten wurden auf Schul- bzw. Klassenebene aggregiert, zu Faktoren zusammengefaßt, durch Mittelwerte ausgedrückt und z.T. graphisch dargestellt. 
soziometrischen Test teil. Sie wurden gebeten, Klassenkameraden auf ihr soziales Verhalten hin zu beurteilen. Zum anderen wurden die Lehrer um eine Schülerbeurteilung gebeten. ${ }^{8}$ Die qualitativen Erhebungen fanden im Rahmen der Fallstudien zur gleichen Thematik an der Modellschule sowie an einer weiteren Mittelschule statt. Sie wurden ca. ein Jahr nach der standardisierten Befragung im Rahmen eines zweisemestrigen Lehrforschungsprojekts realisiert. An beiden Schulen wurden insgesamt sechs leitfadengestützte Gruppen-, 13 Einzelinterviews (vgl. Lamnek 1989) und zweitägige Hospitationen (vgl. Ingenkamp 1973) durchgeführt, die sich jeweils auf Schüler einer achten Haupt- und einer Realschulklasse bezogen. An der Modellschule fanden drei Gruppengespräche und sechs Einzelinterviews statt. Von den Realschülern nahmen vier und von den Hauptschülern zwei Jungen an den Einzelinterviews teil. Die Schüler wurden entsprechend der Lehrerurteile ausgewählt und sind ihren Einschätzungen nach den „Tätern“ zuzuordnen. Ebenso unterlag die Auswahl der Schüler, die an den Gruppengesprächen teilnahmen, den Urteilen der Lehrer. Es wurden solche Schüler gewählt, die nach Meinung der Lehrer als „Unbeteiligte“ gelten. Mit den Realschülern wurden zwei Gruppengespräche geführt: An dem einen nahmen sieben Jungen, an dem anderen acht Mädchen teil. Da die Hauptschulklasse im Vergleich zur Realschulklasse weniger Schüler hatte, wurde nur ein Gruppengespräch geführt, an dem zwei Mädchen und vier Jungen teilnahmen. Thematische Kategorien der Interviews waren die Bereiche: Schul(er)leben, Schüler-Schüler-Verhältnis, Lehrer-Schüler-Verhältnis und Umgang mit Gewalt. ${ }^{9}$

\section{Ergebnisse der quantitativen und qualitativen Analysen unter Berücksichtigung verschiedener Analyseebenen}

Die folgenden Ergebnisse gliedern sich in die Themenbereiche Gewalt, Schulkultur, Klassenklima und außerschulische Sozialisationskontexte, wobei ein Rückbezug zu früheren Analysen (Rostampour/Melzer 1997) und den in den vorangegangenen Kapiteln dargestellten Ergebnissen hergestellt wird. Es werden also neben der Erscheinungsebene von Gewalt auch ihre schulischen und außerschulischen Prädiktoren im Rahmen einer Bedingungsanalyse für die Modellschule thematisiert.

8 Der soziometrische Test für die Schüler wurde in Kooperation mit Prof. Dollase (Universität Bielefeld), das Instrumentarium der Schülerbeurteilung durch Lehrer wurde in Kooperation mit Prof. Lösel (Universität Erlangen-Nürnberg) entwickelt (siehe Anhang).

9 Ausführliche Ergebnisse der Fallanalysen liegen in einem Forschungsbericht der Forschungsgruppe Schulevaluation vor (Schubarth/Darge/Mühl/Bergelt/Börner/Hannich u.a. 1997). 


\section{Gewalt auf Schul- und Klassenebene:}

In Kapitel 1 wurde bereits gezeigt, daß Schüler - anders als Lehrer - einen engen Gewaltbegriff haben..$^{10}$ Dies wird durch die unterschiedlichen Gewaltdefinitionen der Schüler und Lehrer dieser Mittelschule belegt: Die Schüler beurteilen Körperverletzungen, Erpressungen sowie grobe Sachbeschädigungen als Gewalt; dagegen schätzen sie mehrheitlich leichte Formen von Vandalismus oder verbale Aggressionen nicht als Gewalt ein. ${ }^{11}$ In den Interviews zeigt sich der geschlechtsspezifische Blick auf Gewalt: Mädchen unterscheiden - eher als die Jungen - Spaßkämpfe von ernsthaften Prügeleien.

Vandalismus und psychische Aggressionen gegen Mitschüler nehmen die Schüler der Modellschule mehr als andere (Mittel-)Schüler wahr (vgl. Abb. 8-1): $62 \%$ von ihnen registrieren fast täglich Beschimpfungen sowie Spotten und Auslachen von Mitschülern (gegenüber 49\% der Gesamtschülerschaft). Ernsthafte Prügeleien sind die Ausnahme: Nur 6\% der Schüler sind im letzten Jahr mindestens mehrmals im Monat Zeuge von Prügeleien zwischen zwei Gruppen geworden. Das Beobachten von Erpressung, sexueller Belästigung und ernsthafter körperlicher Auseinandersetzung gehört für die Schüler nicht zum Schulalltag. Dazu die Meinung eines Schülers der Hauptschulklasse: „Na kleinere Prügeleien, gut, die gibt es immer. Aber das ist selten, daß hier einer blutet oder so." Die Realschüler nehmen alle Aggressionsformen weniger wahr als die Hauptschüler. Derartige Vorkommnisse werden aber in den Interviews von beiden Gruppen nicht thematisiert. ${ }^{12}$ Trotz des hohen Gewaltniveaus richten sich die Schüleraggressionen kaum gegen Lehrer. Hier hebt sich die Schülerschaft positiv von der Gesamtschülerschaft ab. Hinsichtlich der Interven-

10 Lehrer haben ,einen beträchtlich weiteren Begriff von Gewalt als Schüler. Schüler orientieren sich mit ihrem Gewaltverständnis mehr am Alltagsbegriff von Gewalt also an 'schwerer Gewalt'. Mit anderen Worten, Lehrer bezeichnen Schülerverhaltensweisen als 'Gewalt', die für Schüler keine 'Gewalt' sind“" (Krumm/Lamberger-Baumann/Haider 1997, S. 261, vgl. auch Krumm 1993, S. 161 und 1997, S. 70). Die Gewaltbegriffsanalyse von Schwind u.a. (1995), in die Lehrer, Schüler, Hausmeister und Sekretärinnen einbezogen waren, zeigt - im Vergleich zu Ergebnissen von Krumm - bzgl. der Schülermeinungen ähnliche, aber hinsichtlich der Lehrermeinungen unterschiedliche Ergebnisse: „Der Gewaltbegriff der befragten Schüler erweist sich als relativ eng gefaßt und stark an der physischen Gewalt orientiert. Ähnlich wie von Schulleitern und Lehrern werden auch von den Schülern verbale Aggressionen, Spaßkloppe und unabsichtliche Auseinandersetzungen überwiegend nicht als 'Gewalt' eingeschätzt“" (Schwind u.a. 1995, S. 163). Insgesamt verdeutlichen die Ergebnisse die Notwendigkeit, sich verstärkt mit dem Gewaltbegriff zu beschäftigen. Unsere Gewaltbegriffsanalyse stimmt darin mit den Ergebnissen von Schwind überein, daß mit zunehmender Härte der Aggressionsform die Anerkennung der Verhaltensweisen als „Gewalt“ bei Schülern wie Lehrern steigt.

11 Die Ergebnisse resultieren aus der Gewaltbegriffsanalyse (vgl. Kap. 1), an der auch Schüler und Lehrer dieser Mittelschule beteiligt waren.

$12 \mathrm{Ob}$ die interviewten Schüler sozial erwünscht geantwortet haben, bleibt offen. 
tionsbereitschaft unterscheidet sich die Modellschule nicht vom Durchschnitt der Mittelschulen: 66\% der Schüler geben an, ihre Lehrer würden sehr oft bei Prügeleien einschreiten. Die Schüler der Hauptschulklasse beobachten Lehrerinterventionen - im Vergleich zu den Schülern der Realschulklasse - seltener. Lehrer, die bei ernsten Kämpfen nicht eingreifen, verlieren die Achtung der Schüler: „Wir hatten einen Lehrer, der ist echt ein Schrank. Da haben die sich ständig gekloppt und der stand vorne und hat gegrient. Da ist eine Lehrerin reingekommen und hat die zwei auseinander gerissen. Und der stand da: Ha, ha, ha! Bei dem konntest du nicht viel erwarten! " Die statistischen Analysen auf Schulebene zu den Reaktionen der Schüler auf Gewalt zeigen ihre eingreifende Haltung und durch Ablehnung gekennzeichnete Einstellung. In der Bereitschaft, Gewalt nachzuahmen sowie im Empfinden, Gewalt sei normal, unterscheiden sie sich nicht von anderen Mittelschülern. Allerdings haben sie im Vergleich zu ihnen weniger Angst vor Gewalt. Nach Aussagen der Interviewten existiert vereinzelt Angst vor Auseinandersetzungen mit Waffen, obwohl diese bisher selten in die Schule mitgebracht wurden und noch nie zum Einsatz gekommen sind. Verglichen mit den Realschülern sind die Einstellungen der Hauptschüler tendenziell stärker gewaltbefürwortend. Sie empfinden Gewalt eher als normal, haben weniger Angst vor Gewalt, ärgern sich weniger über Gewaltvorkommen und mischen sich dementsprechend bei Konflikten seltener ein. Mädchen fühlen sich oft aufgrund ihres mangelnden Durchsetzungsvermögens den Jungen gegenüber benachteiligt: ,Wenn ich ein Junge wäre, ich hätte dem schon lange die Meinung gesagt, als Mädchen traut man sich das nicht so." ${ }^{13}$

Gewalthaltige Auseinandersetzungen entstehen oftmals durch Gruppendynamik. Durch gegenseitiges Provozieren schaukeln sich die Gewaltakteure so lange hoch, bis spaßhaftes „Kräftemessen“ zu ernsten Kämpfen führt und ein anfangs harmloser Konflikt zu eskalieren droht. „In der Klasse gibt es eigentlich keine Schlägereien, vielleicht so aus Spaß. Aber irgendwann hört der Spaß auf." Diese Dynamik mag mit dem Aggressionspotential der Schülerschaft zusammenhängen: So stimmen mehr Schüler - im Vergleich zur Gesamtschülerschaft - der Aussage zu, schnell in Wut zu geraten (28\% vs. $23 \%$ ). Auch vertreten die Schüler vergleichsweise häufig nationalistische Einstellungen (vgl. Abb. 8-3). Daß Meinungen und Persönlichkeitsmerkmale der Schüler auch geschlechtsspezifisch bedingt sind, zeigen die diesbezüglichen Einstellungen der Schüler. Bei den Jungen sind diese besonders stark ausgeprägt, wobei die Jungen der Realschulklasse weniger als die männlichen Hauptschüler der Aussage zustimmen, sie würden sich gern schlagen, wenn Mädchen dabei zuschauen. Mädchen mögen zwar weniger (oder anders?) als Jungen an Gewalthandlungen beteiligt sein, tragen aber dennoch einen Anteil

13 Nach Mohr/Becker (1997) zeigen Mädchen in Gewaltsituationen häufiger aggressionsvermeidende Strategien und zeigen Verhaltensweisen, die auf Hilflosigkeit hinweisen (S. 357). 
zum Gewaltverhalten bei: Sie schauen gern zu, wenn sich Jungen prügeln und fühlen sich bei Schlägereien ihretwegen geschmeichelt (vgl. Abb. 8-3). ${ }^{14}$

Besonders bedenkenswert ist die Problembelastung durch die Anzahl der Schüler, die dem Selbstreport zufolge als Täter und/oder Opfer an Gewalt beteiligt sind (vgl. Abb. 8-1). Obwohl 46\% der Schüler zu den Unbeteiligten zählen, geben jeweils mehr Schüler - im Gegensatz zu den anderen Schülern - an, als Täter-Opfer (5\% vs. 3\%), als Täter (11\% vs. $8 \%$ ) und als Episoden-Täter (32\% vs. $26 \%$ ) an den oben genannten Handlungen beteiligt zu sein. ${ }^{15}$ Hier kristallisiert sich die Problemlage der Hauptschulklasse heraus. In der Häufigkeit als Täter härterer Aggressionen und delinquenten Verhaltens (fremdes Eigentum zerstören oder entwenden etc.) weist diese Klasse den höchsten Wert aller befragten Klassen auf. Auch wenn sich die Schülerschaft in ihrer Anzahl an Opfern nicht von den anderen befragten Schülern abhebt (7\%), ist diesen Schülern, weil sie oftmals übersehen werden, ein besonderes Interesse entgegenzubringen. Um diesen Problembereich näher zu analysieren, wurden Lehrer um eine Beurteilung der Schüler gebeten: Auf die Frage, welche Schüler der Hauptschulklasse ihnen als Täter, Opfer und Täter-Opfer auffallen, wird die Mehrzahl der Schüler allerdings als Unbeteiligte eingeschätzt. Die Täter und diejenigen, die sowohl als Täter als auch als Opfer in Erscheinung treten, können genau bezeichnet werden, nicht aber die Opfer. Diese Ergebnisse stimmen tendenziell mit den quantitativen Daten der Schülerbefragung überein, und zeigen gleichzeitig die Diskrepanz zwischen den quantitativen Ergebnissen und den Lehrerbeurteilungen hinsichtlich der Wahrnehmung der Opfer. Laut quantitativer Analyse sind überdurchschnittlich viele Schüler der Hauptschulklasse Gewaltopfer. Allerdings kann nur ein Lehrer einen einzigen Schüler als Opfer angeben. Dies verdeutlicht, daß Lehrer sich auf „Aktivposten“ und Problemkinder in der Klasse konzentrieren, wobei die Wahrnehmung der Opfer zu kurz kommt. ${ }^{16}$ In der Charakteristik der Täter stimmen die Aussagen der Lehrer und Schüler überein: Die Täter gelten als unbeherrscht und dominant. Die Schüler mit den meisten Täterzuschreibungen erfahren von ihren Mitschülern überwiegend Ablehnung und nehmen daher den soziometrischen Status ${ }^{17}$ eines Außenseiters bzw. Abgelehnten ein - auch wenn ihnen einzelne Schüler Sympathie entgegenbringen. Hier unterscheiden

$14 \mathrm{Zu}$ geschlechtsspezifischen Facetten von (schulischer) Gewalt vgl. Kap. 3, sowie Popp 1997; Euler 1997.

15 Es handelt sich hier um Ergebnisse aus Clusteranalysen, vgl. auch Kap. 4.

16 Auch Krumm (1993) verweist auf die Vernachlässigung der Opfer: „Opfer stören Lehrer viel weniger als hyperaktive, freche, unverschämte, bösartige Schüler. Auch die deutschsprachigen Untersuchungen widmen sich einseitig der Frage, warum Kinder aggressiv sind. Welche Bedingungen Kinder zu 'Opfern' machen und was auf Seiten der Opfer pädagogisch dagegen getan werden kann, ist selten ein Thema“ (Krumm 1993, S. 174).

17 Der soziometrische Status wurde in Anlehnung an die Normentabellen nach Petillon 1980 ermittelt. 
Abb. 8-1: Merkmale der Gewalt (auf Schulebene aggregierte Daten)

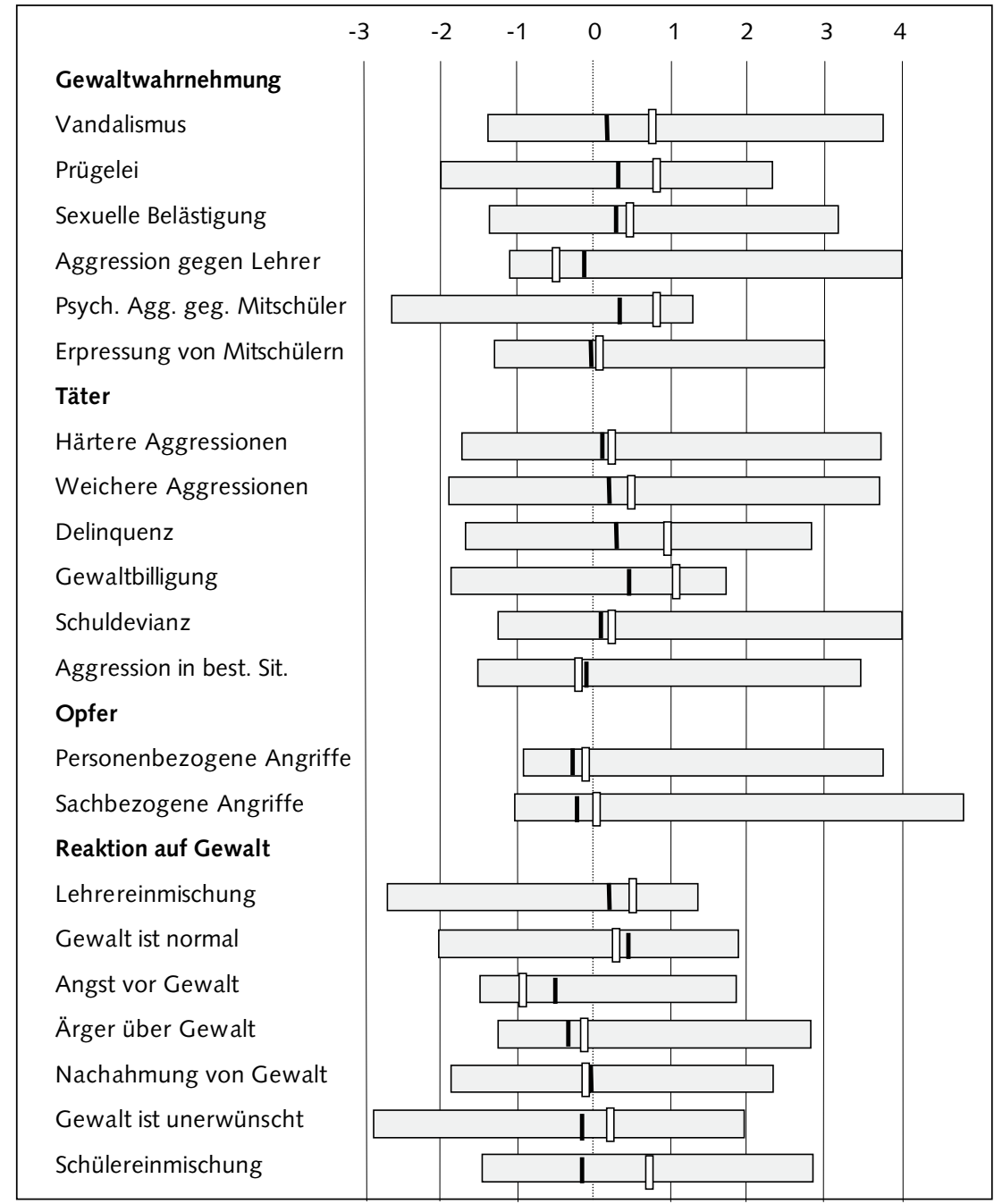

Die weißen Striche zeigen die jeweilige Situation der Modellschule. Ein Vergleichskriterium auf Schulebene ist der schwarze Strich. Er repräsentiert den Durchschnittswert aller Mittelschulen. Die jeweilige Positionierung zeigt die Bedeutung dieses Kriteriums im Vergleich mit den anderen Schulen. Die Spanne des grauen Balkens verdeutlicht, wie groß die Differenz zwischen den extremsten Schulen (auf beiden Seiten) ist. Die Abweichung des Wertes der Einzelschule vom Mittelwert aller Schulen (Nullinie) ermöglicht eine Einschätzung des Qualitätskriteriums als unter- oder überdurchschnittlich. 
sich die Lehrerurteile von denen der Schüler, da die Lehrer der Meinung sind, diese Schüler seien gänzlich in den Klassenverband integriert. Lehrern scheint es demnach schwer zu fallen, die Beziehungen der Schüler untereinander richtig zu deuten.

Im Schulalltag erleben die Schüler - besonders die Hauptschüler - ebenfalls Aggressionen, die von Lehrern ausgehen. ${ }^{18}$ Solches Verhalten nehmen die Schüler - laut Gewaltbegriffsanalyse - mehrheitlich nicht als Gewalt wahr. $\mathrm{Da}$ Schüler institutionelle Gewalt erfahren, zeigen die qualitativen und quantitativen Ergebnisse über solche Lehrerreaktionen wie Strafen mit schlechten Noten oder mit Klassenbucheinträgen etc. Die häufigsten Lehreraggressionen sind verbaler Art (Schreien, verletzende Anrede etc.) „Dummer Haufen! “, „Du Oberpflaume! “. Solche Äußerungen lehnen die Schüler mehrheitlich ab. „Frau X hat mal gesagt Affenpinscher, seitdem hat der den Namen Affe. Das ist ihr so rausgerutscht. Seit dem geht's immer: Affe. In der Stunde, wenn der etwas sagt, geht's: Huh, huh, huh! Da fragen wir, ob der 'ne Banane will. Na, bloß ich meine, irgendwo ist es Frau Xs Schuld, wenn sie mit Affenpinscher ankommt. Da wird gelacht. Der leidet darunter. Ich finde das nicht o.k., aber lachen muß man trotzdem. Na, so lange es einen nicht selber betrifft, sage ich mal, rührt es einen nicht.“ Daß 22\% der Schüler der Aussage „Ich kann mich so gut benehmen wie ich will, man glaubt immer nur Schlechtes von mir." zustimmen, mag mit solchen Etikettierungsprozessen zusammenhängen. Prinzipiell verweisen die Schüler in den Interviews darauf, daß sie derartige Lehreraggressionen in Abhängigkeit von der Persönlichkeit und dem Image des einzelnen Lehrers bewerten. Bestimmten Lehrern wird ein solches Verhalten nicht verübelt, anderen sehr. ${ }^{19}$

\section{Schulkultur:}

Die Schüler erleben Schule als „,normal“, ohne im Lernen und im Schulbesuch einen Sinn zu sehen - mit Ausnahme der Notwendigkeit des Schulabschlusses im Hinblick auf einen Beruf (vgl. Abb. 8-2 und 8-3). Dennoch gehen sie im Durchschnitt gern zur Schule, sicherlich auch, weil dies ein sozialer Treffpunkt ist. Allerdings fallen die Urteile der Hauptschüler deutlich negativer aus. Es verwundert, daß gerade diejenigen Mädchen gern zur Schule gehen, die den soziometrischen Status einer „Unbeachteten“ bzw. einer „Ausgestoßenen“ haben. Sie mögen Mitschülerinnen, ohne daß ihre Sympathie erwidert wird.

18 „Verletzende Handlungen von Lehrern gegen Schüler kommen den Aussagen zufolge im Durchschnitt häufiger vor als verletzende Handlungen von Schülern gegen Schüler und Lehrer“ (Krumm 1997, S. 266). Krumm verdeutlicht ferner wie die Opfer unter diesen Lehrerreaktionen leiden (ebd., 267; vgl. auch Kap. 2).

19 Von Spaun (1997) kommt zu dem Ergebnis, daß einem Hauptfachlehrer, der einen Leistungsdruck ausüben kann, nicht mehr Respekt seitens der Schülern entgegengebracht wird als einem Nebenfachlehrer. Sie schließt daraus, daß die Persönlichkeit des Lehrers vorrangiger als das Unterrichtsfach das Verhalten der Schüler beeinflußt (von Spaun 1997, S. 213). 
Entweder haben sie ihre randständige Rolle verinnerlicht und akzeptieren sie oder sie gehen wenigstens in die Schule, um dadurch ihre Isolation zu überwinden. Als Ursachen für ihre Schulunlust nennen die interviewten Schüler u.a. Mangel an außerunterrichtlichen Freizeitangeboten. „Ich würde erst einmal verändern, daß man so in der Schule immer sein kann. Vielleicht Tischtennis oder richtig etwas machen kann. Damit das so richtig Spaß machen würde, hier in der Schule zu sein." Gerade im Hinblick auf die Räumlichkeiten der Schule und die außerunterrichtlichen Angebote hat die Modellschule nur Durchschnittliches zu bieten. So kritisieren z.B. 35\% der Schüler, daß viele Dinge im Schulgebäude schmutzig und kaputt seien. „Ich will nicht in einem verdreckten Zimmer sitzen und auch noch rumbüffeln müssen." Ein weiterer Defizitbereich ist die Schülerpartizipation bei schulischen Veränderungen. Demgegenüber sind die Schüler durchschnittlich - ebenso wie die Schüler beider Klassen - der Meinung, auf die Unterrichtsgestaltung Einfluß nehmen zu können. Mitbestimmung heißt für die Schüler - so das Fazit der Interviews - Lehrer zu bitten, den Unterricht interessanter zu gestalten, an der Organisation von Klassenfahrten beteiligt zu sein und am Elternabend teilnehmen zu können.

Die didaktische Kompetenz der Lehrer wird von den Schülern weder negativ noch positiv bewertet. Sie sind der Meinung, daß die Lerninhalte nur einen durchschnittlichen Bezug zu ihrem Leben haben. Die Realschüler finden den Unterricht anschaulicher und sehen eher einen Sinn im Unterrichtsstoff bzw. im Lernen. An ihre Lehrer haben die Schüler die Erwartung eines abwechslungsreichen Unterrichts, der persönliche Bezüge zuläßt.

Insgesamt bewerten die Schüler die Beziehung zu ihren Lehrern positiv, wobei die Schüler je nach Persönlichkeit des Lehrers differenzieren. Im allgemeinem können sich die Schüler in Alltagssituationen mit Lehrern gut verständigen und sind der Meinung, daß ihre Lehrer auch ein persönliches Interesse an ihnen haben. Die Schüler der Realschulklasse haben eine positivere Beziehung zu ihren Lehrern als die Hauptschüler.

Das Bemühen der Lehrer um Förderung und Binnendifferenzierung (ihre Förderkompetenz) heben die Schüler - besonders die Realschüler - positiv hervor. Sie sind der Meinung, die Lehrer würden bei Aufgaben darauf achten, niemanden zu überfordern und würden lernschwächere Schüler fördern. Von allen befragten Schülern äußern sich die Schüler dieser Mittelschule besonders positiv über das partizipative Lehrerverhalten: ihre Lehrer diskutieren, wenn den Schülern etwas nicht gefällt, und fragen sie bei Entscheidungen oft nach ihrer Meinung. Dies korrespondiert mit überdurchschnittlichen Werten für Schülerpartizipation im Unterricht. Allerdings verdeutlichen die negativen Aussagen über die Schülerpartizipation auf institutionalisierter Ebene, daß die Partizipation nicht gleichbedeutend ist mit „Mit-Entscheiden“. Weiterhin fühlen sich die Schüler von ihren Lehrern gerecht behandelt, sind der Meinung, jeder habe die gleichen Chancen und es gäbe genügend Möglichkeiten, gute Zensuren zu bekommen. Die Realschüler vertreten diese Meinungen eher als 
die Schüler der Hauptschulklasse. Trotzdem empfinden die Realschüler überdurchschnittlich viel Leistungsdruck. Diese Bemühungen der Lehrer tragen dazu bei, daß den Schülern das Lernen insgesamt eher leicht fällt und sie wenig Schulangst empfinden.

\section{Klassenklima:}

Auffällig ist das schlechte Klassenklima (vgl. Abb. 8-2). Manche Schüler bekommen schwer Anschluß und einige Schüler verstehen sich nicht gut mitein-

Abb. 8-2: Merkmale der Schulkultur (auf Schulebene aggregierte Daten)

Schulkultur
Soz.-räuml. Schulumwelt
Klassenkohäsion
Konkurrenz
Desintegration
Partizipation im Unterricht
Partizipation in der Schule
Schulraumqualität
Lehrerprofessionalität
Didaktische Kompetenz
Chancengleichheit
Binnendifferenzierte
Förderndes
Diskursorientierung
Schülerorientierung
Pejoratives Lehrerhandeln
Aggressives Lehrerhandeln
Schülerbefindlichkeiten
Selbstwahrn. der Etikettierung
Wohlfühlen in der Klasse
Außenseitergefühl
Sinn des Lernens
Leistungsdruck
Schulhektik

* $\quad$ Legende vgl. Abb. 8-1 
ander. Ebenso konkurrieren die Schüler stark, so daß viele von ihnen meinen, in der Klasse wäre jeder auf seine Vorteile bedacht. Dementsprechend fühlen sich $14 \%$ der Schüler (gegenüber 12\% der Gesamtschülerschaft) innerhalb des Klassenverbandes nicht wohl und werden von ihren Klassenkameraden nicht anerkannt. Dieses schlechte Klassenklima bedeutet für viele Schüler eine Desintegration. So meinen 27\% der Schüler (gegenüber 21\% der Gesamtschülerschaft) leicht zum Außenseiter zu werden, wenn sie nicht tun, was die Klasse für richtig hält, und ein Drittel der Schüler stimmt der Aussage zu, daß es in ihrer Schule verschiedene Gruppen gibt, zwischen denen eine Feindschaft besteht. Die soziometrischen Ergebnisse spiegeln die Situation der Hauptschulklasse wider: Zwischen einzelnen Schülern besteht zwar eine Freundschaft, dennoch existieren Außenseiter. Viele Schüler bezeichnen solche Klassenkameraden als Freunde, obwohl das Soziogramm - ebenso wie die quantitativen Daten - dies nicht zeigt. Mitschüler werden möglicherweise als Freunde bezeichnet, weil man diese gern als Freunde hätte, evtl. um die eigene Position aufzuwerten. Außenseiter sind - nach Aussagen der interviewten Hauptschüler - einerseits diejenigen, die durch aggressives Verhalten auffallen und andererseits die Opfer von Gewalthandlungen. Der Opferstatus anderer wird durch alle möglichen Attribuierungen zu rechtfertigen versucht, darunter auch äußere Merkmale und Verhaltensweisen, die anderen nicht angelastet werden. „Auf die Sachen kommt es trotzdem drauf an, wenn einer keine Markenklamotten hat, wird mit dem nicht diskutiert. Der ist Außenstehender."; „Na, die sprüht sich immer ein, aber sie tut sich nicht den Schweiß abwischen. Und die spielt sich auf und erreicht bei uns damit genau das Gegenteil. Die hat einen Spitznamen: Tauchsieder, weil sie sich in alles reinhängt."

\section{Außerschulischer Sozialisationskontext:}

Die Jugendlichen kommen aus Familien, in denen häufiger ein negatives Familienklima besteht und ein rigider, auf Strenge aufbauender Erziehungsstil vorherrscht (14\% Schüler vs. 9\% der Gesamtschülerschaft). Sie fühlen sich vergleichsweise weniger wohl zu Hause, werden angeschrien, manchmal auch geschlagen. Ebenso wird ihnen stärker vorgeschrieben, was sie zu tun haben (vgl. Abb. 8-3). Daher ist Schule für einige Schüler attraktiver als der Aufenthalt zu Hause. „Ich würde sagen, ich gehe ganz gern zur Schule weilman sitzt nicht nur dumm zu Hause rum und faulenzt. Das ist einfach mal eine Abwechslung in der Schule." Ebenfalls gravierend sind die Probleme im Freizeitbereich. Viele Schüler gehören Cliquen an, in denen gewaltsame Auseinandersetzungen an der Tagesordnung sind (19\% Schüler vs. 7\% der Gesamtschülerschaft). So prügelten sich Mitglieder der Freundesgruppe mit anderen Gruppen. Allerdings thematisierten die Schüler diese Vorkommnisse in den Interviews nicht (zur Relevanz der Peergroup für Gewaltbilligung, Gewaltbereitschaft und Gewalttäigkeit vgl. Funk u.a. 1995, S. 172f.). 
Abb. 8-3: Außerschulische und individuelle Merkmale (auf Schulebene aggregierte Daten)

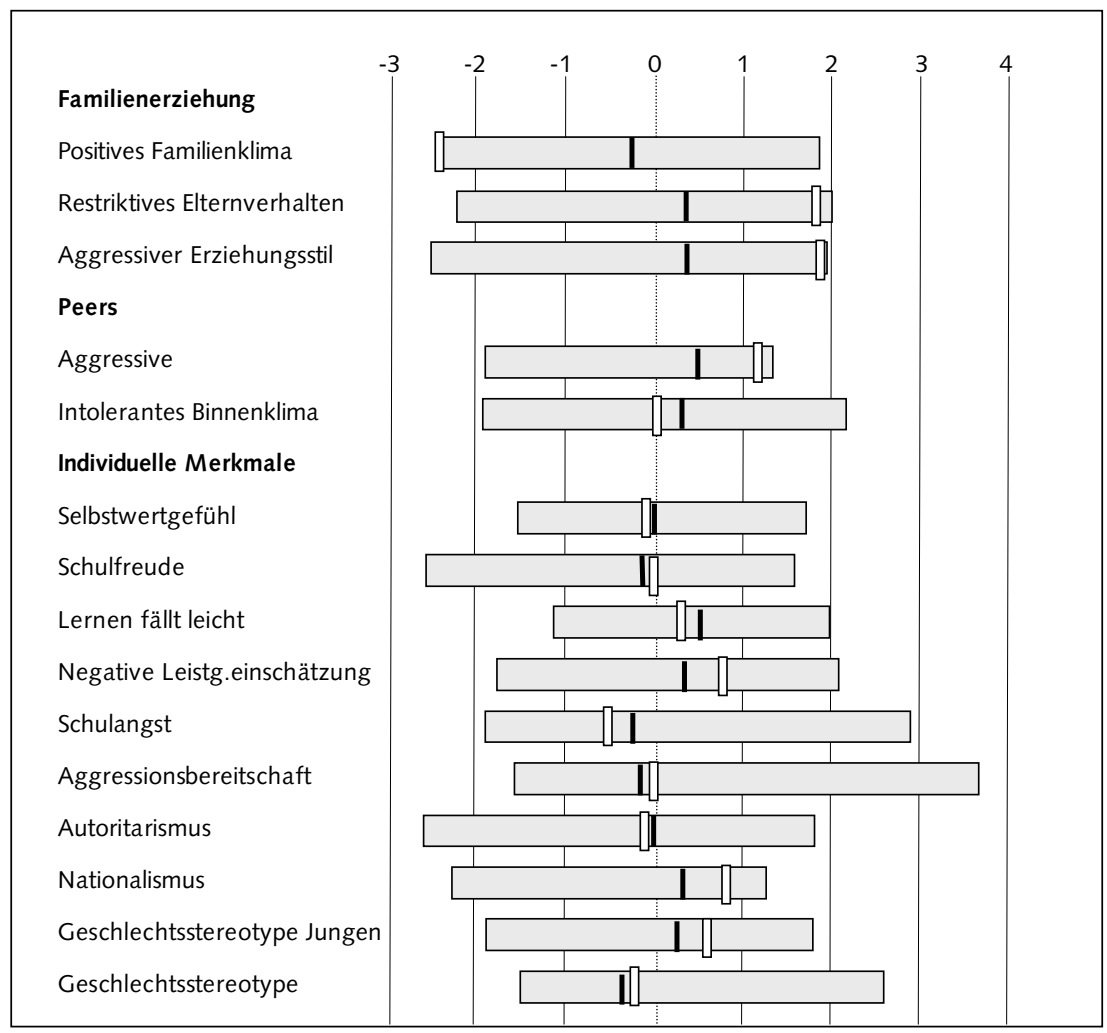

* $\quad$ Legende vgl. Abb. 8-1

\section{Konsequenzen der Analyse für die Prävention und Intervention}

Im folgenden werden aus der vorgenommenen Bedingungsanalyse Schlußfolgerungen für mögliche, auf die Situation der Modellschule abgestimmte Präventions- und Interventionsstrategien gezogen. Dabei wird auf die im ersten Teil vorgestellten Programme mit zurückgegriffen. Diese Darstellungen sind als Empfehlungen zu verstehen, da für die Lösung eines Problems meist mehrere Handlungsmöglichkeiten in Betracht kommen. Welches Programm bzw. welche Maßnahme zur Anwendung kommt, ist letztlich davon abhängig, auf welche Probleme ein Lehrer sein Augenmerk richtet und über welche 
Kompetenzen er verfügt. Je differenzierter die Ergebnisse der Analysen aber bei der Auswahl eines Programms reflektiert werden und je höher die Bereitschaft eines Lehrers ist, Zeit in die gewaltpräventive Arbeit zu investieren, desto höher sind die Erfolgschancen für eine Gewaltreduktion.

\section{Schulische Maßnahmen:}

Die Schüler üben z.T. massive Kritik am Zustand des Schulgebäudes und der Unterrichtsräume. Eine gemeinsame Gestaltung des Schulgebäudes und -geländes durch Lehrer, Schüler und evtl. Eltern könnte demnach zu einer Verbesserung der Lebens- und Lernqualität in der Schule beitragen. Dies würde insofern Erfolg für die Gewaltprävention versprechen, als die Schüler dazu veranlaßt wären, mehr Verantwortung für ihren Lebensbereich zu übernehmen. Ebenso könnte durch die gemeinsame Tätigkeit ein stärkeres Gemeinschaftsgefühl entwickelt werden. Beide Faktoren hätten letztlich Auswirkung auf die Identifikation der Schüler mit der Schule. Damit zeigt sich die Weiterentwicklung der Schulqualität in der Gestaltung von Schule über den Kernbereich des Unterrichts hinaus. Besonders die Probleme im Freizeitbereich legen nahe, die Schule zu einem kulturellen Zentrum im Stadtteil, der den Jugendlichen sonst wenig bietet, auszubauen. ${ }^{20}$ Derartige Maßnahmen könnten wiederum einen positiven Einfluß auf die Schulfreude der Schüler haben und auch motivationssteigernd für den Unterricht wirken.

\section{Maßnahmen für Lehrer:}

Die problematischen Familienbedingungen, die z.T. zweifelhaften Freundesbeziehungen der Schüler sowie ihre Einstellungen zu Gewalt machen eine Kooperation zwischen Schule, Elternhaus und anderen Institutionen ratsam. In diesem Rahmen könnten Erfahrungen ausgetauscht werden, ebenso wie überforderte Eltern oder Lehrer auf diese Weise Unterstützung erhalten würden. Die Schülerorientierung der Lehrer bietet sich als Grundstein für Gespräche mit Gewalttätern, besonders aber mit Gewaltopfern an (Olweus). Diese Aussprachen können - je nach Situation - auch unter Einbeziehung der Eltern stattfinden. Daß viele Schüler Kritik an der Schülerpartizipation üben und Lehreraggressionen wahrnehmen, macht - ebenso wie die Tatsache, daß sich Lehrer häufig auf die „Aktivposten“ konzentrieren und die Opfer sowie die Außenseiter nicht erkennen - deutlich, daß Lehrer ihren Umgang mit und ihr Wissen über abweichendes Verhalten reflektieren sollten. Hierfür bietet sich als eine geeignete Methode die Arbeit mit dem KTM an.

20 Die konzeptionelle und strukturelle Entwicklung einer Schule hin zu einem sozialen und kulturellen Zentrum einer Gemeinde ist Ziel der Community Education. Die Multifunktionalität der Einrichtung verbessert die Infrastruktur der Gemeinde und ermöglicht die Kooperation und Koordination von Bildungsangeboten von alt und jung (lifelong learning) (vgl. Buhren 1997). 


\section{Maßnahmen für Schüler bzw. eine Klasse:}

Viele Probleme verweisen auf Lösungsstrategien, die die Klassenebene betreffen. Insbesondere die hohe Täter- und Opferzahl, das Aggressionspotential der Schüler sowie die Desintegration von Schülern in den Klassenverband machen die Verbesserung des Klassenklimas nötig. Da besonders die Gewaltopfer den Schutz ihrer Mitschüler benötigen, wäre es ratsam, pädagogisches Handeln an der Interventionsbereitschaft der Schüler anzusetzen. Die Lehrer sollten intervenierendes Verhalten ebenso wie gewaltablehnende Einstellungen der Schüler durch positive Verstärkung stützen, da sich so weitere Schüler am sozial erwünschten Verhalten als Norm orientieren und abweichendes Verhalten stärker ablehnen würden. ${ }^{21} \mathrm{Im}$ allgemeinen empfehlen sich Trainings, in denen alternative Verhaltensformen vermittelt werden, wie sie z.B. von der Petermann-Arbeitsgruppe vorgelegt wurden. So erlangt das aggressive Kind Fähigkeiten und Fertigkeiten, sozial kompetent zu handeln, ebenso wie das unsichere Kind mehr Selbstvertrauen bekommt, wodurch es vor Angriffen geschützt wird. Besonders für Mädchen sind solche Sozialtrainings gewinnbringend, da sie sich oftmals hilflos gegenüber gewalttätigem Verhalten fühlen. Darüber hinaus würden klare Regeln, auf deren Mißachtung Konsequenzen folgen, den Opfern ein größeres Sicherheitsgefühl und den Tätern mehr Orientierung für ihr Verhalten geben (Olweus). Der Einsatz von Streitschlichtermodellen kann nach Vorfällen zur Wiedergutmachung beitragen: Durch Verträge kommt es hier zu einem fairen Täter-Opfer-Ausgleich (Braun/Hünicke 1995). Auch das Einrichten eines Kontakttelefons bietet für Gewaltopfer erste Hilfe (Olweus).

\section{Maßnahmen auf Unterrichtsebene:}

Die Situation, daß die Schüler in hohem Maße mit Gewalt konfrontiert sind und sie die Lerninhalte z.T. als lebensfern empfinden, spricht für eine Integration des Gewaltthemas in den Unterricht. Somit hätte der Unterrichtsstoff unmittelbaren Bezug zum Alltag vieler Schüler. Das Unterrichtsprogramm nach Lerchenmüller bietet in diesem Zusammenhang vielfältige Möglichkeiten. Gleichzeitig werden den Schülern Konsequenzen von abweichendem Verhalten verdeutlicht, was wiederum zu einer höheren Sensibilität gegenüber Gewalt führt und somit gewaltpräventiv wirkt.

Aus der Tatsache, daß die Hauptschulklasse einen sozialen Brennpunkt innerhalb der Schule darstellt und in fast allen Bereichen schlechter abschneidet,

21 Auf die Bedeutung der Peers verweist z.B. Hesse (1993). Obwohl sie die Thematik der Suchtprävention in der Schule anspricht, lassen sich ihre Ergebnisse auch auf die schulische Gewaltprävention beziehen: So verweisen Studien darauf, „daß der Einsatz von Gleichaltrigen als Programmvermittler größeren Einfluß auf die Entwicklung von drogenbezogenem Verhalten und Einstellungen hat; insgesamt aber zu selten angewandt wurde (lediglich 5\% der Programme setzen sie ein)“ (Hesse 1993, S. 231). 
resultiert der Bedarf einer speziellen Hauptschulpädagogik, die damit gleichzeitig gewaltpräventiv wirken kann (vgl. 8.3).

\section{Ablauf des Präventions- und Schulentwicklungsprozesses}

Im folgenden wird der Schulentwicklungsprozeß der Modellschule dokumentiert, dessen schwerpunktmäßige Zielsetzung u.a. die Gewaltreduktion ist. Ausgangspunkt der Kooperation dieser Mittelschule und der Forschungsgruppe war die 1995 durchgeführte Befragung. Eine Zusammenarbeit entwickelte sich 1996, als erneut Kontakt zur Schule aufgenommen wurde, da Fallanalysen die quantitativen Ergebnisse ergänzen sollten. Zeitgleich plante die Schulleitung eine Lehrerfortbildung. Dies wurde durch innerschulische Probleme (z.B. aggressives Schülerverhalten, wenig Kooperation innerhalb des Lehrerkollegi$\mathrm{ums}^{22}$ ) motiviert. Da sich das Gefühl eines fruchtbaren Erfahrungsaustausches zwischen Praxis und Wissenschaft verdichtete, beschloß man die Präsentation der Forschungsergebnisse auf einer Schulinternen Lehrerfortbildung (SchiLF). Die Analyse des Ist-Zustands der Schule war also ein wesentlicher Impuls für den Schulentwicklungsprozeß. Der Schulleitung fiel die Rolle des Initiators zu, allerdings galt es, für eine erfolgreiche SchiLF-Maßnahme die Unterstützung vieler Kollegen zu aktivieren. Unter den möglichen Formen Schulinterner Lehrerfortbildung wurde der Einstieg in den Schulentwicklungsprozeß mit einem „Pädagogischen Tag“ gewählt. Die Vorbereitung übernahmen die Schulleitung, vier Lehrer, eine externe Beraterin der Sächsischen Akademie für Lehrerfortbildung (SALF) und zwei Projektvertreter. Ihre Aufgabe bestand in der Konkretisierung der Themen. Ebenso wurde ein externer Berater ${ }^{23}$ eingeladen, welcher der Schule als „Prozeßbegleiter“ beratend zur Seite steht. Bei der Durchführung des pädagogischen Tages stellten zwei Mitarbeiter der Forschungsgruppe die Untersuchungsergebnisse sowie die daraus abgeleiteten Vorschläge für Präventions- und Interventionsmaßnahmen vor. Auf dieser Grundlage wurden mögliche Strategien zur Schulentwicklung überdacht und reflektiert. Nach der Fortbildung erfolgte die Auswertung unter dem Aspekt der Umsetzung und Wirksamkeit der Ergebnisse im Schulalltag. Ziel der Veranstaltung war es, die Ergebnisse so darzustellen, daß ein für die Lehrer befriedigender Lernprozeß stattfinden konnte und sie nach der Veranstaltung motiviert waren, Schulleben aktiv zu gestalten. Die Evaluation des Fortbildungstages wurde mittels eines Auswertungsbogens (Miller 1996, S. 82) durchgeführt. Es wurden die Effektivität der Prozesse, das Befinden der Lehrer und ihre

22 Zu Kooperationsbeziehungen zwischen Lehrern siehe Rolff (1995, S. 33f).

23 Es handelt sich um einen von der SALF ausgebildeten Lehrer. 
Bewertung der inhaltlichen Arbeit erhoben. ${ }^{24}$ Die Auswertung der Ergebnisse wurde der Schulleitung mitgeteilt. Insgesamt legte die Lehrerfortbildung - wie intendiert - einen Grundstein für ,die Bewältigung schulischer/erzieherischer Schwierigkeiten und Probleme“ (Miller 1995, S. 36). Viele Lehrer wollen sich weiterhin mit bestehenden Problemen beschäftigen. Damit wurde ein zweites Ziel von SchiLF, ,die Erhaltung, Aktualisierung und Verbesserung bereits erworbener Qualifikationen“ (ebd.) sowie die Vermittlung neuer Informationen, erreicht. Ebenso kam es zur Reflexion von „Arbeits-, Lehr- und Lernprozessen“, was eine „Verbesserung (bzw.) (...) Veränderung der Unterrichtswirklichkeit" bewirken kann (ebd.).

Die positive Bilanz der Fortbildung resultiert aus folgenden inhaltlichen, sozialen sowie organisatorischen Faktoren: Die Lehrer akzeptierten die Fortbildung, weil sie in den Prozeß der Problemlösung einbezogen waren. Sie wählten das Thema, beteiligten sich an den Vorbereitungen und der Durchführung. Eine praxisnahe Darstellung, die als problem- und handlungsorientiert sowie situations- und personenbezogen empfunden wurde, war ein weiterer wichtiger Aspekt. Durch das Stattfinden der Veranstaltung außerhalb der Schule war den Lehrern ein kurzzeitiger Abstand zum Schulalltag bei gleichzeitiger Chance zur gemeinsamen Kommunikation möglich. Die Lehrer zeigten sich nach Abschluß der Maßnahme für Innovationen aufgeschlossen und motiviert, „Schule selbst zu gestalten“. Dieses Resultat war nicht von vornherein zu erwarten, denn ,SchiLF bedeutet auch, zur ohnehin schon beträchtlichen Unterrichtsverpflichtung zusätzliche Zeit aufzuwenden“ (ebd., S. 43). Die Entschlüsse der Lehrer zeigen ihr Denken im Sinne Millers: „Nicht nur Freizeit ist Lebenszeit, sondern auch Arbeitszeit und somit ein hoher Wert in sich" (ebd.). Im Anschluß an den pädagogischen Tag haben sich die Lehrer - entsprechend ihrer Interessen - in eine Liste mit Vorschlägen zu Maßnahmen der Schulentwicklung eingetragen, ebenso brachten sie eigene Vorschläge ein. Daneben wurden der Schulleitung die während der Fortbildung vorgeschlagenen Programme als Literaturempfehlung übergeben. Es bestand das Angebot, diese darüber hinaus auf weiteren pädagogischen Konferenzen den Lehrern vorzustellen und zu erläutern.

Insgesamt kristallisierten sich drei Arbeitsbereiche heraus:

1. Verbesserung des Schulklimas,

2. Veränderung der Freizeitgestaltung sowie

3. Intensivierung der Elternarbeit.

Folgende Methoden der Gewaltprävention und Schulentwicklung wurden in Absprache mit der Schulleitung, vier Lehrern und zwei externen Moderatoren ausgewählt:

24 Auf einer siebenstufigen Skala (+3 bis -3 ) äußerten die Lehrer ihre Meinungen. Die Gründe konnten zusätzlich erläutert werden. 
a) Die Organisationsentwicklung 25 und

b) das Sozialtraining in der Schule (Petermann u.a.).

Dem Schulkollegium wurde fünf Monate später während einer pädagogischen Konferenz das Sozialtraining in der Schule vorgestellt, das seit April 1998 in einer Klasse in Zusammenarbeit einer Lehrerin und einer Projektmitarbeiterin durchgeführt wird. Weiterhin fanden zwei pädagogische Tage - unter Einbeziehung interessierter Schüler und Eltern - zur Organisationsentwicklung der Schule statt. Unter Begleitung zweier externer Berater wurde eine „Zukunftswerkstatt“ durchgeführt mit dem Thema „Miteinander-Füreinander“. Im Anschluß daran wurden konkrete Maßnahmen zur Veränderung der Schulwirklichkeit mit verbindlichen Verantwortlichkeiten und Terminen festgelegt. Bis zum Sommer 1998 sind folgende Veränderungen geplant:

- Gestalten verschiedener Klassen- und Fachräume sowie der Flure,

- Einrichten einer Bibliothek,

- Gestalten des Schulhofes,

- Einrichten eines Schülerradios,

- Schaffen von Rückzugsmöglichkeiten.

Alle Maßnahmen sind als Kooperationsprojekte von Schülern, Lehrern und Eltern geplant. Weitere Kooperationspartner (Schulpsychologen, Vertreter der Jugendarbeit, Firmen etc.) sollen - im Sinne der Community Education - in die Arbeit involviert werden. Es wird eine Zusammenarbeit angestrebt, bei der jede beteiligte Person einen individuellen Beitrag zur Lösung eines Problems leistet. Es geht dabei um die Integration verschiedener Aktivitäten, deren gemeinsames Ziel die Entwicklung einer ,gute Schule“ ist. Die externen Moderatoren stehen während der Zeit allen am Schulprozeß beteiligten Personen beratend zur Seite. ${ }^{26}$ Regelmäßige Treffen, bei denen jedes Team über Erfahrungen und Ergebnisse berichtet, finden statt. Mit der Konkretisierung der Ziele in bestimmten Handlungsfeldern und der Übernahme von Verantwortlichkeiten haben sich die Lehrer, Schüler und Eltern auf einen Prozeß der Schulentwicklung eingelassen, ,der nach vorne hin offen ist“ (Miller 1995, S. 27).

25 „Unter Organisationsentwicklung verstehen wir im Anschluß an Matthew und Miles u.a. die systematisch geplante Anstrengung einer Organisation zum Zwecke der Selbstanalyse und Erneuerung. Ziel ist sowohl die Verbesserung der Arbeitszufriedenheit, der Arbeitsfreude und der Arbeitseffizienz der einzelnen Organisationsangehörigen als auch die Verbesserung der Funktionsfähigkeit und der Resultate der Organisation“ (Osswald 1993, S. 330; vgl. auch Greber/ Maybaum/Priebe/Wenzel 1993; Fatzer 1996).

26 Besonders beim Auftreten von schwierigen Situationen ist die beratende Tätigkeit der Moderatoren wichtig. Beispielsweise können sie den Beteiligten die Angst nehmen, Erwartungen (ihre eigenen oder die anderer Personen) nicht erfüllen zu können (zur Bedeutung pädagogischer Supervision vgl. auch Petermann 1995). 


\subsection{Perspektiven der Gewaltprävention und -intervention sowie der Präventionsforschung}

Bei der schulischen Gewaltfrage steht nicht nur die Institution Schule auf dem Prüfstand, oft liegen die Ursachen auch in außerschulischen Bereichen. Grund dafür, warum Veränderungen von Schulwirklichkeit zunehmend an Bedeutung gewinnen, ist zum einen, daß viele Kinder heute mehr Zeit in der Schule verbringen als in ihrer Familie - womit die Schule über ein enormes, sinnvoll zu nutzendes Zeit- und Einflußbudget verfügt; zum anderen, weil Schule selbst Bedingungsfaktor von Gewalt sein kann (vgl. Kap. 6). Welche Maßnahmen der Gewaltprävention und -intervention angewandt werden, ist dabei ebenso von den spezifischen Problemen einer einzelnen Schule wie von den Kompetenzen der in ihr unterrichtenden Lehrer abhängig. Die Auswahl und Umsetzung von Aktivitäten ist somit von spezifischen personalen und situativen Faktoren abhängig und kann nicht im Sinne einer allgemeinen Rezeptologie erfolgen. Auch der hier beschriebene Schulentwicklungsprozeß ist kein „Königsweg“. Dennoch verdeutlicht er Rahmenbedingungen, deren Berücksichtigung zur Gewaltreduktion beitragen: So haben die schulspezifischen wissenschaftlichen Analysen - im Gegensatz zu Konzepten, die auf Alltagserfahrungen basieren - Gewaltursachen transparent gemacht, woraus sich Präventions- und Interventionsempfehlungen ableiten ließen: Schulische Maßnahmen in Anlehnung an Olweus: Gestaltung des Schulgebäudes und des -geländes, Schaffen eines schulischen Freizeitangebotes, Intensivierung der Elternarbeit; Maßnahmen für Schüler bzw. Klassen nach Petermann u.a. und Olweus: Verbesserung des Klassenklimas durch Verhaltenstrainings, Erstellen von Regeln, TäterOpfer-Ausgleich; Maßnahmen auf Unterrichtsebene nach Lerchenmüller: Behandlung des Themas „Gewalt“ im Unterricht; Maßnahmen für Lehrer nach Tennstädt u.a.: Das KTM als Grundlage zur Reflexion der Alltagstheorien von Lehrern. Ebenso konnte - im Sinne einer Primärprävention - durch Untersuchungsergebnisse in Bereichen, in denen keine Probleme existierten, verdeutlicht werden, ob und warum die Schule bei der Gestaltung des Schullebens auf dem richtigen Weg ist (Schülerorientierung der Lehrer, binnendifferenzierte Förderung, diskursorientiertes Lehrerhandeln, Chancengleichheit, Gewaltablehnung der Schüler, Aggressionen richten sich nicht gegen Lehrer etc.).

Im Anschluß an die erste Lehrerfortbildung kristallisierten sich Arbeitsbereiche heraus, die sich auf die Verbesserung des Schulklimas und der Freizeitgestaltung sowie auf die Intensivierung der Elternarbeit beziehen. Die Realisierung eines Bündels von Maßnahmen schien allen Beteiligten angemessen und machbar. Allerdings konnte davon nur ein Teil in die Praxis umgesetzt werden. Das Kollegium konkretisierte die Arbeitsschwerpunkte auf das Sozialtraining und auf schulische Maßnahmen, wie das Gestalten von Klassenräumen und des Schulhofs, das Schaffen von Rückzugsmöglichkeiten sowie die Einrichtung einer Bibliothek und eines Schülerradios. Es handelt sich also um 
Aktivitäten, welche die Lehrer mit ihrer ohnehin schon bestehenden Belastung im Schulalltag vereinbaren können. Dabei werden sie durch externe Berater unterstützt. Konzepte wie das KTM oder das Unterrichtsprogramm nach Lerchenmüller fließen zunächst nicht mit in die Arbeit ein, da sie spezifische Kompetenzen und eine längere Einarbeitungszeit voraussetzen. Dies verweist auf die Notwendigkeit der Mitarbeit weiterer Experten, die den Schulentwicklungsprozeß beraten und unterstuitzen.

Obwohl zum jetzigen Zeitpunkt keine Aussagen über die Wirkungen der schulischen Bemühungen gemacht werden können, sind positive Effekte zu erwarten. Dafür sprechen die im Kap. 6 beschriebenen Zusammenhänge zwischen Schulkultur und Gewalt. Perspektivisch sind von der Präventionsarbeit der Modellschule folgende Ergebnisse zu erwarten: Die gemeinsamen Aktivitäten tragen zur Verbesserung der Kommunikationsstrukturen und Beziehungen der am Schulentwicklungsprozeß beteiligten Personen bei, so daß sie sich verstärkt mit der Schule als Lebens- und Erfahrungsraum identifizieren können. Durch den attraktiv gestalteten Schulhof gewinnen die Schüler einen zusätzlichen Ort, an dem sie sich vermehrt in ihrer Freizeit aufhalten und entspannen können. Die Einbeziehung der Eltern in den Prozeß der Schulentwicklung ist gleichzeitig ein Ansatz der Elternarbeit. Sie begünstigt einen gegenseitigen Erfahrungsaustausch und beeinflußt möglicherweise das Erziehungsverhalten der Eltern. Dadurch, daß diese auf Schulebene ansetzenden Maßnahmen mit dem Sozialtraining in der Klasse kombiniert werden sollen, wird man der Problematik der Täter ebenso wie der der Opfer, die oftmals zu kurz kommen oder gar unberücksichtigt bleiben, gerecht. Darüber hinaus erwarten wir, daß der Schulentwicklungsprozeß - vorausgesetzt die Maßnahmen werden von allen Beteiligten positiv empfunden - dynamisch ist, d.h., sich weitere Maßnahmen an ein gelungenes Projekt anschließen werden.

Einen bereits positiven Einfluß von pädagogischen Maßnahmen auf die Schulkultur sowie im Hinblick auf die Reduzierung der Gewaltbelastung zeigen unsere Analysen einzelner Klassen (vgl. Tab. 8-1 und 8-2). Die Tabellen weisen die wichtigsten Parameter der Gewaltemergenz und der Schulkultur unserer Modellschule im Vergleich mit einer ähnlich gewaltbelasteten Mittelschule (Mittelschule B) in einem ebenfalls städtischen Umfeld aus. Die Daten beziehen sich in beiden Fällen auf jeweils eine 8. Haupt- und Realschulklasse. Die Abstufungen zwischen diesen beiden Klassen mit den deutlich ungünstigeren Bedingungen, wie wir sie in unserer Modellschule vorfinden, stellen den Regelfall dar. So schneiden die achten Hauptschulklassen in Bezug auf die Gewaltbelastung und die Schulkultur tendenziell schlechter ab als die Realschulklassen bzw. Klassen der Gymnasien (vgl. Tab. 8-3). Bei dieser zum Vergleich herangezogenen Schule zeigt sich eine umgekehrte Tendenz. Hier sind es die Schüler der Realschulklasse, die sich im Gegensatz zu denen der Hauptschulklasse negativer äußern: Die Gewaltwahrnehmung der Schüler der Hauptschulklasse ist zwar immer noch überdurchschnittlich, aber wesentlich 
Tab. 8-1: Merkmale der Gewalt an zwei Einzelschulen (Mittelwertvergleich)

\begin{tabular}{|c|c|c|c|c|c|}
\hline & \multicolumn{3}{|c|}{ Modellschule } & \multicolumn{2}{|c|}{ Mittelschule B } \\
\hline & Item* & RS & HS & RS & $\mathrm{HS}$ \\
\hline \multicolumn{6}{|l|}{ Wahrnehmung von Gewalt } \\
\hline $\begin{array}{l}\text { Aggressionen gegen Lehrer } \\
(\mathrm{V} 147, \mathrm{~V} 149, \mathrm{~V} 151)\end{array}$ & V150 & 1,1 & 1,4 & 1,2 & 1,1 \\
\hline $\begin{array}{l}\text { Psychische Aggressionen gegen Mitschüler } \\
\text { (V156-V158a) }\end{array}$ & V157 & 4,9 & 5,3 & 4,4 & 4,3 \\
\hline \multicolumn{6}{|l|}{ Täter } \\
\hline $\begin{array}{l}\text { Weichere Aggressionen } \\
\text { (V260-V262, V267, V273, V274, V278) }\end{array}$ & V274 & 1,8 & 3,8 & 2,2 & 2,1 \\
\hline $\begin{array}{l}\text { Schuldevianz } \\
\text { (V269-V271, V276, V277) }\end{array}$ & V277 & 1,9 & 4,5 & 2,6 & 2,4 \\
\hline \multicolumn{6}{|l|}{ Opfer } \\
\hline $\begin{array}{l}\text { Personenbezogene Angriffe } \\
\text { (V280-V284) }\end{array}$ & V283 & 1,8 & 2,6 & 2,0 & 1,5 \\
\hline $\begin{array}{l}\text { Sachbezogene Angriffe } \\
\text { (V285-V287) }\end{array}$ & V286 & 1,4 & 2,2 & 1,7 & 1,3 \\
\hline
\end{tabular}

* Es handelt sich hierbei um Mittelwertvergleiche repräsentativer Stellvertreter-Items. Skala: $1=$ nie $\ldots 6$ = fast täglich

geringer als bei den Schülern der Hauptschulklasse in unserer Modellschule; die Häufigkeit der eigenen Beteiligung als Täter und/oder Opfer ist in der Realschulklasse höher als in der Hauptschulklasse; die Realschüler ärgern sich weniger als die Hauptschüler über Gewalt und mischen sich bei Gewalt weniger ein; die Urteile über die Lernkultur und das Klassenklima der Realschüler sind schlechter als die der Hauptschüler. Diese positiven Effekte werden von uns und der Schulleitung der betreffenden Schule, mit der wir die Befunde diskutiert haben, mit auf das seit dem Schuljahr 1994/95 durchgeführte Projekt „Besondere Förderung und Integration der Hauptschüler“ zurückgeführt, in dem folgende Maßnahmen umgesetzt wurden und werden: Geringe Klassenstärke; Einsatz weniger, engagierter Lehrer; Transparenz in der Unterrichtsorganisation und der Benotung; gute Schüler-Lehrer-Beziehung; individuelle Förderung; Erziehung zum selbständigen Arbeiten z.B. durch Benutzen der schuleigenen Bibliothek, zur Verantwortung und Zuverlässigkeit; verschiedene Sportangebote zum Entspannen und Austoben; intensive Kontakte zu Eltern mit regelmäßigen Treffen und Unterstützungsangebote für die Eltern; Freizeitangebote durch Arbeitsgemeinschaften und durch die Öffnung des Schulclubs; Kooperation mit dem Arbeitsamt, mit Schulsozialarbeitern und mit den Sportvereinen des Stadtteils; Weiterbildungen der Lehrer; enge Zusammenarbeit der Lehrer. Perspektivisch sind folgende Aktivitäten geplant: Organisation der Profilunterrichtsstunden in den Tagesablauf als Randstunden, 
Tab. 8-2: Merkmale der Schulkultur an zwei Einzelschulen (Mittelwertvergleich)

\begin{tabular}{|c|c|c|c|c|c|}
\hline \multirow{3}{*}{ Schulkultur } & \multirow[b]{2}{*}{ Item* } & \multicolumn{2}{|c|}{ Modellschule } & \multicolumn{2}{|c|}{ Mittelschule B } \\
\hline & & RS & HS & RS & HS \\
\hline & & & & & \\
\hline $\begin{array}{l}\text { Schulraumqualität } \\
\text { (V311-V315) }\end{array}$ & V314 & 2,7 & 2,0 & 2,8 & 3,1 \\
\hline $\begin{array}{l}\text { Schülerorentierter Unterricht } \\
\text { (V69, V70, V72-V74) }\end{array}$ & V69 & 3,6 & 3,2 & 3,3 & 4,0 \\
\hline $\begin{array}{l}\text { Binnendifferenzierte Förderung } \\
\text { (V78, V80, V81, V174) }\end{array}$ & V80 & 3,9 & 3,7 & 3,5 & 4,3 \\
\hline $\begin{array}{l}\text { Chancengleichheit } \\
\text { (V171, V172) }\end{array}$ & V172 & 4,1 & 3,9 & 3,8 & 4,2 \\
\hline $\begin{array}{l}\text { Pejoratives Leherhandeln } \\
\text { (V180, V185, V234) }\end{array}$ & V180 & 2,6 & 3,1 & 2,8 & 2,0 \\
\hline $\begin{array}{l}\text { Förderndes Lehrerengagement } \\
\text { (V175-V177, V179) }\end{array}$ & V176 & 4,2 & 3,7 & 3,1 & 3,4 \\
\hline $\begin{array}{l}\text { Diskursorientiertes Lehrerhandeln } \\
\text { (V236, V237) }\end{array}$ & V236 & 4,3 & 3,7 & 3,4 & 3,8 \\
\hline $\begin{array}{l}\text { Schülerorientierung der Lehrer } \\
\text { (V239-V244) }\end{array}$ & V243 & 3,3 & 2,6 & 2,7 & 3,5 \\
\hline $\begin{array}{l}\text { Klassenkohäsion } \\
\text { (V247, V249, V251, V252) }\end{array}$ & V252 & 3,6 & 3,3 & 3,3 & 3,7 \\
\hline $\begin{array}{l}\text { Außenseitergefühl } \\
\text { (V106, V119, V126) }\end{array}$ & V106 & 2,1 & 2,3 & 1,7 & 1,4 \\
\hline $\begin{array}{l}\text { Schulfreude } \\
\text { (V94, V98, V109, V120, V125, V127) }\end{array}$ & V98 & 2,8 & 2,0 & 2,4 & 2,5 \\
\hline
\end{tabular}

* Es handelt sich hierbei um Mittelwertvergleiche repräsentativer Stellvertreter-Items. Skala: 1 = stimmt gar nicht $\ldots 6=$ stimmt ganz genau

von Freizeitaktivitäten, Schulfesten und Projektwochen sowie Hospitationen an anderen Schulen. Diese Initiativen zeigen, daß schon kleinere Projekte, die sich auf die Schulentwicklung im allgemeinen beziehen und einige pädagogische Akzente setzten, geeignet sind, Gewalthandeln und Gewaltrisiken in der Schule zu reduzieren und damit präventiv zu wirken.

$\mathrm{Ob}$ die umfangreichen Bemühungen des Kollegiums und der Schulleitung in Verbindung mit Aktivitäten unserer Forschungsgruppe, den Moderatoren und weiteren Experten im Rahmen einer umfassenden Präventions- und Interventionsstrategie sich an der Modellschule bestätigen und welche Effekte sie erbringen, wird eine im ersten Halbjahr 1998 durchgeführte Replikationsstudie zeigen. Diese ist als Trenduntersuchung, d.h. als Vortest-Nachtest-Untersuchung geplant. Im einzelnen werden Präventions- und Interventionsmaßnahmen oder andere pädagogische Innovationen, die im Zeitraum zwischen den beiden 
Tab. 8-3: Gewaltstatus und Schulkultur nach Bildungsgang (Mittelwertvergleiche von Faktoren zweiter Ordnung)

\begin{tabular}{|c|c|c|c|c|c|c|}
\hline & \multirow[b]{2}{*}{$\begin{array}{c}\text { 8. Klassen } \\
\text { HS }\end{array}$} & \multirow[b]{2}{*}{$\begin{array}{l}\text { 8. Klassen } \\
\text { RS }\end{array}$} & \multirow[b]{2}{*}{$\begin{array}{l}\text { 8. Klassen } \\
\text { GY }\end{array}$} & \multicolumn{3}{|c|}{ signifkante Differenzen bestehen zwischen } \\
\hline & & & & $\begin{array}{c}\text { GY \& } \\
\text { RS }\end{array}$ & $\begin{array}{c}\text { GY \& } \\
\text { HS }\end{array}$ & $\begin{array}{l}\text { RS \& } \\
\text { HS }\end{array}$ \\
\hline Täterstatus $^{+}$ & 0,55 & 0,24 & $-0,07$ & * & * & \\
\hline Opferstatus $^{++}$ & 0,31 & 0,09 & $-0,05$ & * & & \\
\hline $\begin{array}{l}\text { Beobachtete } \\
\text { Gewalt }{ }^{+++}\end{array}$ & 0,49 & 0,33 & $-0,16$ & * & * & \\
\hline $\begin{array}{l}\text { Schulkulturelle } \\
\text { Merkmale }\end{array}$ & $-0,30$ & 0,01 & 0,01 & * & * & \\
\hline
\end{tabular}

Die Faktoren zweiter Ordnung beinhalten folgende Faktoren:

+ Täterstatus: Harte Aggressionen, Weiche Aggressionen, Schuldevianz, Aggressionen in konkreten Situationen

++ Opferstatus: Opfer personenbezogener Angriffe, Opfer sachbezogener Angriffe

+++ Beobachtete Gewalterscheinungen: Vandalismus, Prügelei, Prügelei in konkreten Situationen, Sexuelle Belästigung, Aggressionen gegen Lehrer, Psychische Aggressionen, Erpressung

++++ Schulkulturelle Merkmale: Didaktische Kompetenz, Binnendifferenzierte Förderung, Schülerorientierung der Lehrer, Chancengerechtigkeit, Pejoratives Lehrerhandeln, Aggressives Lehrerhandeln, Lehrerengagement, Schülerpartizipation in der Schule, Schülerpartizipation im Unterricht, Lebensweltbezug der Lerninhalte

Untersuchungen an der Modellschule sowie an einer weiteren gewaltbelasteten Schule durchgeführt wurden, als intervenierende Variablen kontrolliert. Bezugsebene ist die Schul- und Klassenebene. Als Kontrollgruppe fungieren Schulen der Stichprobe, an denen keine gezielten Interventionen bzw. intentionale Entwicklungsprozesse stattfanden. Anhand des zeitversetzten Vergleichs altersäquivalenter Klassen kann kontrolliert werden, ob die Gewaltentwicklung einer einzelnen Schule dem allgemeinem Entwicklungstrend entspricht oder ob Veränderungen durch geplante Interventionen bedingt sind. Ebenso wird die Wirksamkeit der Präventions- und Interventionsarbeit selbst erfaßt. Es werden subjektive Einschätzungen (durch Schüler- und Lehrergespräche, Hospitationen und durch die Mitarbeit der Forschungsgruppe am Schulentwicklungsprozeß) bezüglich der Wirkung des Programms im Praxisfeld erhoben. Ziel dabei ist die Verbesserung der Konzeption und der Durchführung des Programms. Dieser aus der Handlungsforschung resultierende Ansatz geht davon aus, daß eine gute Programmimplementation nur erreicht wird, wenn eine Zusammenarbeit zwischen Schule und externen Beratern in der Form besteht, daß Informationssammlung, Erarbeiten von Handlungsstrategien und Interventionen abwechselnd in den Prozeß der Schulentwicklung integriert sind (vgl. Hesse 1993, S. 103ff). Insgesamt wird keine Repräsentativität sondern ein Fallstudiencharakter der Evaluation angestrebt, d.h., die Auswahl 
der Interventionsklassen erfolgt nicht unter repräsentativen Gesichtspunkten, sondern ist davon abhängig, ob Interventions- und Präventionsmaßnahmen durchgeführt wurden oder nicht.

Zusammenfassend kristallisieren sich für eine Gewaltprävention und intervention, die den allgemeinen Prämissen der Schulentwicklung und der Zielgruppenorientierung (Täter, Opfer, Unbeteiligte) verpflichtet ist, folgende curriculare, individuelle, soziale, kulturelle, sozial-ökologische, ökonomische und politische Faktoren heraus: ${ }^{27}$ Gewaltpräventive Maßnahmen sind um so erfolgreicher, je früher sie einsetzen, je kind- bzw. jugendspezifischer sie ausgerichtet sind, je länger sie durchgeführt werden, je mehr Personen beteiligt sind und je umfassender die Zielebenen sind (Schul-, Klassen- und Individualebene). Die Auswahl der Maßnahmen soll dabei auf empirischen Datenanalysen fußen und in einem konkreten Bezug zu den Alltagssituationen der Schüler stehen. Schule muß daher alltägliche Fertigkeiten und Fähigkeiten vermitteln, wie sie umgekehrt einen Lebens- und Erfahrungsraum darstellen soll, in dem Schüler ihre außerunterrichtlichen Erfahrungen einbringen können. Daher muß sie sich auch ihrem äußeren Umfeld ein Stück weit öffnen, andere Lernorte mit einbeziehen und als Institution Funktionen im Stadtteil bzw. der Gemeinde mit übernehmen (Community Education). Eine „gute Schule“ wird in beträchtlichem Maße durch ihre Lernkultur, die fachliche und didaktische Kompetenz der Lehrer, ihre Integrations- und Kommunikationsfähigkeiten definiert. Außerdem bestimmen soziale Faktoren die Schulqualität wie Partizipationsmöglichkeiten der Schüler am Schulleben, die Schülerorientierung der Lehrer sowie räumliche Gegebenheiten. Diese Aspekte schulischen Lernens haben insofern Einfluß auf das Verhalten der Schüler, als sie im positiven Fall zu einer Identifikation der Schüler und Lehrer mit der Schule beitragen können und diese als Lebens- und Erfahrungsraum annehmen.

Unabdingbar für eine langfristige Wirkung von Präventions- und Interventionsbemühungen sind Fortbildungen und Supervisionen für Lehrer. Zur Entlastung der Lehrer und zur Lösung vieler Probleme würde auch eine Lehrerausbildung beitragen, die neben der fachwissenschaftlichen und didaktischen Ausbildung mindestens genau so viel Wert auf die pädagogische und psychologische Ausbildung legt.

Die Integration des Gewaltthemas in das schulische Curriculum (z.B. nach Lerchenmüller) vermittelt Schülern Kenntnisse, die Voraussetzungen für Verhaltensänderungen sind. In bezug auf die Wissensvermittlung verzeichnen die meisten Programme gute Effekte, hingegen sind Einstellungsänderungen schwieriger zu bewirken. Für langfristige Verhaltensmodifikationen sind allerdings Einstellungsänderungen sowie die Förderung von Fertigkeiten und Fähigkeiten unabdingbar. Präventions- und Interventionsprogramme sollten

27 Sie stimmen in vielen Punkten mit den Ergebnissen von Evaluationsstudien zur Erfassung gesundheitsrelevanter Strukturen in Schulen überein (vgl. Hesse 1993). 
daher - neben der Vermittlung von kognitiven Elementen - handlungssteuernde und handlungsauslösende Motive ansprechen (z.B. bei Trainingsprogrammen nach Petermann u.a.).

Wünschenswert ist weiterhin eine verstärkte Zusammenarbeit von Kindergarten und Grundschule sowie von Grund- und weiterführenden Schulen. Die Entwicklung gemeinsamer pädagogischer Konzepte ist notwendig und unter Einbeziehung der Eltern auch aussichtsreich. Der gewaltpräventiven Arbeit der Grundschule und des Kindergartens kommt daher eine große Bedeutung zu, da sie als familiennahe Institutionen eng mit Eltern zusammenarbeiten können und darüber hinaus eine frühe Gewaltprävention/-intervention die besten Voraussetzungen dafür bietet, Mehrfachstörungen entgegenzuwirken (vgl. Krumm 1997, S. 74, S. 78f).

Trotz zahlreicher Erfahrungen und Bemühungen im Bereich der Gewaltreduktion existieren noch viele Entwicklungsaufgaben und Defizite. Die Verbreitung und Veröffentlichung etablierter Programme sowie der Erfahrungsaustausch über die Entwicklung und Durchführung verschiedener Modellprojekte sind verbesserungsbedürftig. An die Wissenschaftler stellt sich die Forderung nach Evaluationsstudien (Mutzeck 1993, Posse 1993, Schratz 1995), in denen die erwarteten Effekte und die Grenzen der Programme überprüft werden. Mit der Einrichtung regionaler Koordinierungsstellen könnte eine Kooperation verschiedener Institutionen begünstigt werden. Über diese regionalen Bemühungen hinaus ist der Aufbau eines bundesweiten und internationalen Erfahrungsaustausches voranzutreiben. Die Bemühungen um eine solche Vernetzung aller Gewaltpräventionskonzepte, wie sie im Projekt Lahn-Dill-Kreis (Wetzlar) realisiert werden, sind unter dieser Perspektive beispielhaft (Balser/ Schrewe/Schaaf 1997). Ebenso liefern internationale Gewaltpräventions- und Interventionsprogramme wichtige Erkenntnisse, die es auszutauschen lohnt. Länderübergreifende Fachtagungen über Gewalt an Schulen - wie sie beispielsweise im September 1996 vom Sonderforschungsbereich 227 „Intervention und Prävention im Kindes- und Jugendalter" der DFG an der Universität Bielefeld (vgl. Holtappels/Heitmeyer/Melzer/Tillmann 1997) sowie im Februar 1997 zum Thema „safe(r) at School“ im Rahmen einer EU-Konferenz (Mooij 1997) stattfanden - geben Einblicke in spezifische Problemsituationen und die Möglichkeiten der Prävention und Intervention.

Solange aber auf der Ebene der Schuladministration keine strukturellen Veränderungen erfolgen, wird die Gestaltung von Schulwirklichkeit weiterhin zu einem beträchtlichem Anteil vom Engagement einer einzelnen Schule mitsamt ihren Lehrern, Schülern und deren Eltern abhängen. 



\section{Literatur}

Ackermann, Ch.: Interventions- und Präventionspraxis an Schulen - Ergebnisse einer vergleichenden Schulleiterbefragung. In: Schubarth/Kolbe/ Willems 1996, S. $205-215$

Adomat, D.: Differenzierung und Durchlässigkeit an der sächsischen Mittelschule. Empirische Ergebnisse und pädagogische Folgerungen. Dresden 1997 (unveröffentl. Examensarbeit)

Adorno, Th. W./Frenkel-Brunswik, E./Levinson, D. J./Sanford, R. N.: The authoritarian personality. New York 1950

Arbeitsgemeinschaft Kinder- und Jugendschutz (AJS). Landesstelle Nordrhein-Westfalen e.V.: Materialien zum Thema Gewalt und Gewaltprävention. Köln 1994

Arbeitsgruppe Bielefelder Jugendforschung: Das Individualisierungs-Theorem - Bedeutung für die Vergesellschaftung von Jugendlichen. In: Heitmeyer/Olk 1990, S. 11-34

Aurin, K. (Hrsg.): Auffassung von Schule und pädagogischer Konsens. Fallstudien bei Lehrerkollegien, Eltern- und Schülerschaft von fünf Gymnasien. Stuttgart 1993

Baacke, D.: Unsere Ambivalenz-Kultur. In: Böllert, K./Otto, H.-U. (Hrsg.): Soziale Arbeit auf der Suche nach Zukunft. Bielefeld 1989, S. 63-72

Baacke, D.: Die 13- bis 18jährigen. Weinheim 1983

Balser, H./Schrewe, H./Schaaf, N. (Hrsg.): Schulprogramm Gewaltprävention. Ergebnisse aktueller Modellversuche. Neuwied 1997

Bandura, A.: Sozial-kognitive Lerntheorie. Stuttgart 1979

Beck, U.: Risikogesellschaft. Auf dem Weg in eine andere Moderne. Frankfurt/M. 1986

Bell, P.: Zur Auflösung der Widersprüche von Modernität und Modernismus. Das Beispiel Amerikas. In: Meier, H.: Diagnose der Moderne. München 1990, S. 21-67

Bergelt, S.: Geschlechtsspezifische Ausdrucksformen von Gewalt innerhalb der Schule. Dresden 1997 (unveröffentlichte Vordiplomarbeit)

Berger, J./Finze, F.-R.: Entwicklung und Erprobung eines Trainings zum Aggressionsabbau und zur Verhaltensmodifikation bei Kindern der dritten Klasse (Forschungsbericht des Instituts für Allgemeine Psychologie und Methoden der Psychologie an der TU Dresden, Bd. 39). Dresden 1997

Berger, P. A./Vester, M. (Hrsg.): Alte Ungleichheit Neue Spaltungen. Opladen 1998

Bertram, H.: Die Familie: Solidarität oder Individualität? In: Vaskovics 1997, S. 370_ 381

Blackham, G. J.: Der auffällige Schüler. Weinheim 1971

Böhnisch, L.: Ist Gewalt männlich? In: Thiersch, H./Wertheimer, J./Grunwald, K.: überall in den Köpfen und Fäusten.“ Darmstadt 1994, S. 103-113

Böhnisch, L.: Schule als anomische Struktur. In: Schubarth/Melzer 1995, S. 141-151

Braun, G./Hünicke, W.: Schülerinnen und Schüler als „Streitschlichter“. In: Praxis Schule 5-10 H. 5/1995, S. 24-27

Brenner, W./Keller, G. (Hrsg.): Business reengineering mit Standardsoftware. Frankfurt 1995

Bronfenbrenner, U.: Ökologische Sozialisationsforschung. Stuttgart 1976

Bronfenbrenner, U.: Die Ökologie menschlicher Entwicklung. Frankfurt/M. 1989 
Brusten, M./Hurrelmann, K.: Abweichendes Verhalten in der Schule. München 1973

Büchner, P./Fuchs, B./Krüger, H.-H. (Hrsg.): Vom Teddybär zum ersten Kuß. Wege aus der Kindheit in Ost- und Westdeutschland. Opladen 1996

Buhlmann, T.: Vereinigungsbilanzen. Die deutsche Einheit im Spiegel der Sozialwissenschaften. In: Aus Politik und Zeitgeschichte B 40-41/1997, S. 29-37

Buhren, C.: Community Education. Münster 1997

Bundesministerium für Frauen, Familie, Jugend und Senioren (Hrsg.): Neunter Jugendbericht. Bonn 1994

Busch, L./Todt, E.: Aggressionen in Schulen. Möglichkeiten ihrer Bewältigung. In: Holtappels/Heitmeyer/Melzer/Tillmann 1997, S. 331-350

Dann, H.-D.: Aggressionsprävention im sozialen Kontext der Schule. In: Holtappels/ Heitmeyer/Melzer/Tillmann 1997, S. 351-366

Dettenborn, H./Lautsch, E.: Aggression in der Schule aus der Schülerperspektive. In: Zeitschrift für Pädagogik H. 5/1993, S. 745-774

Die Grundschulzeitschrift H. 100/1996 (Themenheft: „Kinderrechte“)

Döbert, H.: Befindlichkeit der Lehrerschaft im Osten: teilsteils. In: Deutsche Lehrerzeitung Nr. 6/1995a

Döbert, H.: Das Bildungswesen der DDR in Stichworten. Inhaltliche und akademische Sachverhalte und ihre Rechtsgrundlagen. Berlin 1995b

Döbert, R./Nunner-Winkler, G.: Adoleszenzkrise und Identitätsbildung. Frankfurt/M. 1975

Dodge, A.: A social information processing model of social competence in children. In: Perlmutter, M. (Ed.): Eighteenth Annual Minnesota symposium on child psychology. Hillsdale 1986

Dollard, J. u.a.: Frustration und Aggression. Weinheim, Basel $1973^{5}$

Dudek, P./Tenorth, H.-E.: Transformation der deutschen Bildungslandschaft. Rückblick in prospektiver Absicht. In: Dies. (Hrsg.): Transformation der deutschen Bildungslandschaft. Lernprozeß mit ungewissem Ausgang (Zeitschrift für Pädagogik, 30. Beiheft). Weinheim, Basel 1993, S. 301-327

Dümcke, W./Vilmar, F. (Hrsg.): Kolonialisierung der DDR. Kritische Analysen und Alternativen des Einigungsprozesses. Münster 1995

Eisenstadt, S. N.: Von Generation zu Generation. Altersgruppen und Sozialstruktur. München 1966

Eisenstadt, S. N.: Der Wandel der Lebensphase Jugend in modernen Gesellschaften. In: Melzer/Ferchhoff/Neubauer 1990, S. 22-32

Eisenstadt, S. N.: Revolution und die Transformation von Gesellschaften. Eine vergleichende Untersuchung verschiedener Kulturen. Opladen 1982

Elias, N.: Über den Prozeß der Zivilisation. 2 Bde. Frankfurt 1976

Erikson, E.: Identität und Lebenszyklus. Frankfurt/M. 1973

Etzioni, A.: Die Verantwortungsgesellschaft. Individualismus und Moral in der heutigen Demokratie. Frankfurt/M., New York 1997

Euler, H. A.: Geschlechtsspezifische Unterschiede und die nicht erzählte Geschichte in der Gewaltforschung. In: Holtappels/Heitmeyer/Melzer/ Tillmann 1997, S. 191-206

Fatzer, G. (Hrsg.): Organisationsentwicklung und Supervision: Erfolgsfaktoren bei Veränderungsprozessen. Zürich 1996

Fend, H.: Schulklima: Soziale Einflußprozesse in der Schule. Weinheim, Basel 1977

Flösser, G./Otto, H.-U./Tillmann, K.-J. (Hrsg.): Schule und Jugendhilfe. Neuorientierungen im deutsch-deutschen Übergang. Opladen 1996 
Frehsee, D.: Sozialer Wandel und Jugendkriminalität. In: DVJJ-Journal. Zeitschrift für Jugendkriminalrecht und Jugendhilfe H. 3-4/1995, S. 269-278

Friebel, H.: Berufliche Qualifikation und Persönlichkeitsentwicklung. Alterserfahrungen Jugendlicher und sozialwissenschaftliche Deutung. Opladen 1985

Friedrich, W./Förster, P.: Jugend im Osten. Politische Mentalität im Wandel. Leipzig 1996

Fthenakis, W. E.: Väter. 2 Bde. München 1988

Fuchs, M./Lamnek, S./Luedtke, J.: Schule und Gewalt. Realität und Wahrnehmung eines sozialen Problems. Opladen 1996

Funk, W. (Hrsg.): Nürnberger Schüler Studie 1994: Gewalt an Schulen. Regensburg 1995

Funk, W./Passenberger, J.: Determinanten der Gewalt an Schulen. Mehrebenenanalytische Ergebnisse aus der Nürnberger Schüler-Studie 1994. In: Holtappels/Heitmeyer/Melzer/Tillmann 1997, S. 243-260

Gades, G.: Schulen brauchen Partner. Eine Initiative zur Umweltbildung und Prävention von Gewalt in der Schule: „StUPS“. In: Praxis Schule 5-10, H. 5/1995, S. 17-19

Galtung, J.: Strukturelle Gewalt. Beiträge zur Friedens- und Konfliktforschung. Reinbek 1975

Geulen, D./Hurrelmann, K.: Zur Programmatik einer umfassenden Sozialisationstheorie. In: Hurrelmann, K./Ulich, D. (Hrsg.): Handbuch der Sozialisationsforschung. Weinheim, Basel 1980, S. 51-67

Giesecke, H.: Das Ende der Erziehung. Stuttgart 1985

Giesen, B./Leggewie, C. (Hrsg.): Experiment Vereinigung. Ein sozialer Großversuch. Berlin 1991

Gille, M: Wertorientierungen und Geschlechtsrollenorientierungen im Wandel. In: Hoffmann-Lange 1995, S. 109-158

Goldstein, H.: Multilevel Statistical Models. 2nd ed. London 1995

Gottschalch, W.: Männlichkeit und Gewalt. Weinheim, München 1997

Grauer, G./Zinnecker, J.: Schülergewalt. Über unterschlagene und dramatisierte Seiten des Schülerlebens. In: Reinert/Zinnecker 1978, S. 282-348

Greber, U./Maybaum, J./Priebe, B./Wenzel, W. (Hrsg.): Auf dem Weg zur „Guten Schule": Schulinterne Lehrerfortbildung. Weinheim, Basel 1993

Greiffenhagen, M.: Die Bundesrepublik Deutschland 1945 - 1990. Reformen und Defizite der politischen Kultur. In: Aus Politik und Zeitgeschichte B 1-2 1991, S. $16-26$

Gruschka, A./Hesse, C./Michely, H./Schomacher, H.: Aus der Praxis lernen. Methodenhandbuch für Lehrer und Pädagogen. Berlin 1995a

Gruschka, A./Hesse, C./Michely, H./Schomacher, H.: Aus der Praxis lernen. Arbeitsbuch für die Ausbildung in Erziehungsberufen. Berlin 1995b

Habermas, J.: Legitimationsprobleme im Spätkapitalismus. Frankfurt 1973

Habermas, J.: Der philosophische Diskurs der Moderne. Frankfurt/M. 1985a

Habermas, J.: Die neue Unübersichtlichkeit. Frankfurt/M. 1985b

Habermas, J.: Die nachholende Revolution. Kleine politische Schriften VII. Frankfurt/M. 1990

Hanewinkel, R./Knaack, R.: Prävention von Aggression und Gewalt an Schulen. Ergebnisse einer Interventionsstudie. In: Holtappels/Heitmeyer/ Melzer/Tillmann 1997, S. 299-313 
Heitmeyer, W./Olk, Th. (Hrsg.): Individualisierung von Jugend. Gesellschaftliche Prozesse, subjektive Verarbeitungsformen, jugendpolitische Konsequenzen. Weinheim, München 1990

Heitmeyer, W./Ulbrich-Herrmann, M.: Verschärfung sozialer Ungleichheit, soziale Milieus und Gewalt. In: Holtappels/Heitmeyer/Melzer/Tillmann 1997, S. 45-62

Heitmeyer, W. u.a.: Gewalt. Schattenseiten der Individualisierung bei Jugendlichen aus unterschiedlichen sozialen Milieus. Weinheim, München 1995

Hellwig, G./Nickel, H. M.: Frauen in Deutschland 1945-1992. Bonn 1993

Hensel, R. u.a.: Schule und Gewalt. In: Senatsverwaltung für Inneres (Hrsg.): Endbericht der unabhängigen Kommission zur Verhinderung und Bekämpfung von Gewalt in Berlin. Berlin 1994

Hensel, R.: Auf dem Weg zu weniger Gewalt - auch durch das Leben in der Schule. In: Spreiter, M. (Hrsg.): Waffenstillstand im Klassenzimmer. Vorschläge, Hilfestellungen, Prävention. Weinheim 1993

Hensel, R.: Lebenswelt Schule. In: Praxis Schule 5-10, H. 5/1995, S. 20-23

Hesse, S.: Suchtprävention in der Schule. Evaluation der Tabak- und Alkoholprävention. Opladen 1993

Hoffmann-Lange, U. (Hrsg.): Jugend und Demokratie in Deutschland. Opladen 1995

Höhn, E.: Der schlechte Schüler. München 1967

Holtappels, H. G.: Aggression und Gewalt als Schulproblem - Schulorganisation und abweichendes Verhalten. In: Schubarth/Melzer 1993, S. 116-146

Holtappels, H. G.: Schulkultur und Innovation - Ansätze, Trends und Perspektiven der Schulentwicklung. In: Ders. (Hrsg.): Entwicklung von Schulkultur. Ansätze und Wege schulischer Erneuerung. Neuwied 1995, S. 6-36

Holtappels, H. G./Heitmeyer, W./Melzer, W./Tillmann, K.-J. (Hrsg.): Forschung über Gewalt an Schulen. Erscheinungsformen und Ursachen, Konzepte und Prävention. Weinheim, München 1997

Holtappels, H. G./Meier, U.: Gewalt an Schulen. Erscheinungsformen von Schülergewalt und Einflüsse des Schulklimas. In: Die Deutsche Schule H. 1/1997, S. 50-62

Holtappels, H. G./Meier, U./Tillmann, K.-J.: Auf dem Schulhof mangelt es an Zivilcourage. In: Frankfurter Rundschau vom 8. August 1996

Holzkamp, Ch./Rommelspacher, B.: Frauen und Rechtsextremismus. Wie sind Mädchen und Frauen verstrickt? In: Sozial Extra H. 6/1991, S. 17-19

Hopf, Ch./Hopf, W.: Familie, Persönlichkeit, Politik. Weinheim, München 1997

Hox, J.J.: Applied Multilevel Analysis. 2nd ed. Amsterdam 1995

Hoyer, H.-D.: Modernisierung von Bildung, Schule und Pädagogik? In: Deutsche Lehrerzeitung Nr. 51-52/1996

Hradil, St.: Sozialisation und Reproduktion in pluralistischen Wohlfahrtsgesellschaften. In: Sünker, H./Timmermann, D./Kolbe, F.-U. (Hrsg.): Bildung, Gesellschaft, soziale Ungleichheit. Frankfurt/M. 1994, S. 89-119

Hurrelmann, K.: Gewalt in der Schule. In: Schwind, H.-D. u.a. (Hrsg.): Ursachen, Prävention und Kontrolle von Gewalt. Band III. Berlin 1990, S. 363-380

Hurrelmann, K./Pollmer, K.: Gewalttätige Verhaltensweisen von Jugendlichen in Sachsen - ein speziell ostdeutsches Problem. In: Kind - Jugend - Gesellschaft H. 1/1994, S. 3-12

Hurrelmann, K./Rixius, N./Schirp, H. u.a.: Gegen Gewalt in der Schule. Ein Handbuch für Elternhaus und Schule. Weinheim, Basel 1996 
Hurrelmann, K./Ulich, D. (Hrsg.): Neues Handbuch der Sozialisationsforschung. Weinheim, Basel $1991^{4}$

Ingenkamp, K. (Hrsg.): Beobachtung und Analyse von Unterricht. Teilausgabe des Handbuchs der Unterrichtsforschung. Weinheim, Basel 1973

Jugendwerk der Deutschen Shell (Hrsg.): Jugend '81. Lebensentwürfe, Alltagskulturen, Zukunftsbilder. Opladen 1982

Jugendwerk der Deutschen Shell (Hrsg.): Jugend '92. Lebenslagen, Orientierungen und Entwicklungsperspektiven im vereinigten Deutschland. Opladen 1992

Jugendwerk der Deutschen Shell (Hrsg.): Jugend '97. Zukunftsperspektiven, gesellschaftliches Engagement, politische Orientierungen. Opladen 1997

Kavemann, B.: Gewalt gegen Mädchen findet auch in der Schule statt. In: Senatsverwaltung für Arbeit und Frauen (Hrsg.): Gewalt gegen Mädchen in Schulen. Berlin 1992, S. 11-35

Kersten, K.: Sozialwissenschaftliche und politische Anmerkungen zum Thema „Jungen und Gewalt“، In: Behn, S./Heitmann, H./Voß, S. (Hrsg.): Jungen, Mädchen und Gewalt - ein Thema für geschlechtsspezifische Jugendarbeit?! (IFFJ-Schriften Nr. 8). Berlin o. J., S. 20-38

Knopf, H. (Hrsg.): Aggressives Verhalten und Gewalt in der Schule. München 1996

Kolbe, F.-U.: Schulformspezifische Belastung durch abweichendes Verhalten in bundeslandeigener Problemkonstellation. In: Schubarth/Kolbe/ Willems 1996, S. 4870

Korte, J.: Nicht reden, sondern handeln. In: Praxis Schule 5-10, H. 5/1995, S. 28-33

Krappmann, L.: Sozialisation in der Gruppe der Gleichaltrigen. In: Hurrelmann/Ulich 1991, S. 355-375

Kreppner, K.: Sozialisation in der Familie. In: Hurrelmann, K./Ulich, D.: Handbuch der Sozialisationsforschung. Weinheim, Basel 1980, S. 395-422

Krumm, V.: Aggression in der Schule. Lehrer können mehr tun, als sie glauben. In: Schmälzle, U. (Hrsg.): Mit Gewalt leben. Arbeit am Aggressionsverhalten in Familie, Kindergarten und Schule. Frankfurt/M. 1993, S. 153-202

Krumm, V.: Methodenkritische Analyse schulischer Gewaltforschung. In: Holtappels/ Heitmeyer/Melzer/Tillmann 1997, S. 63-79

Krumm, V./Lamberger-Baumann, B./Haider, G.: Gewalt in der Schule - auch von Lehrern. In: Empirische Pädagogik H. 2/1997, S. 257-274

Kultusministerium des Landes Sachsen-Anhalt (Hrsg.): Schulstress - Aggression Entspannung. Grundsätze und Anregungen für die Schulpraxis. Magdeburg 1996

Kury, H./Obergfell-Fuchs, J.: Kriminalität Jugendlicher in Ost und West - Auswirkungen gesellschaftlicher Umwälzungen auf psychisches Erleben und Einstellungen. In: Lamnek, S. (Hrsg.): Jugend und Gewalt. Devianz und Kriminalität in Ost und West. Opladen 1995, S. 291-314

Lamnek, S.: Qualitative Sozialforschung. Bd. 2. München 1989

Lerchenmüller, H.: Evaluation eines Lernprogramms in der Schule mit delinquenzpräventiver Zielsetzung. Köln 1986

Lerchenmüller, H.: Soziales Lernen in der Schule: zur Prävention sozial-auffälligen Verhaltens. Ein Unterrichtsprogramm für die Sekundarstufe I. Bochum 1987

Lerchenmüller-Hilse, H.: Möglichkeiten der Delinquenzprävention im Schulbereich - Überlegungen und Erfahrungen aus der Praxis. In: Tenczek, T./Pfeiffer, H. (Hrsg.): Kommunale Kriminalprävention. Paradigmenwechsel und Wiederentdekkung alter Weisheiten. Bonn 1996, S. 278-294 
Lösel, F./Averbeck, M./Bliesener, T.: Erlebens- und Verhaltensprobleme von Tätern und Opfern. In: Holtappels/Heitmeyer/Melzer/Tillmann 1997, S. 137-153

Luhmann, N.: Soziale Systeme. Grundriß einer allgemeinen Theorie. Frankfurt/M. 1984

Lukesch, H.: Leitbilder der Familienerziehung. In: Vaskovics/Lipinski 1996, S. 153184

Mansel, J.: Quantitative Entwicklung von Gewalthandlungen Jugendlicher und ihre offizielle Registrierung. Ansätze schulischer Prävention zwischen Anspruch und Wirklichkeit. In: Zeitschrift für Sozialisationsforschung und Erziehungssoziologie H. 2/1995, S. 101-121

Mansel, J./Hurrelmann, K.: Alltagsstreß bei Jugendlichen. Eine Untersuchung über Lebenschancen, Lebensrisiken und psychosoziale Befindlichkeiten im Statusübergang. Weinheim, München 1991

Mansel, J./Hurrelmann, K.: Außen- und innengerichtete Formen der Problemverarbeitung Jugendlicher. In: Soziale Welt H. 2/1994, S. 147-179

Mansel, J./Hurrelmann, K.: Aggressives und delinquentes Verhalten Jugendlicher im Zeitvergleich. Bielefeld 1997 (Manuskript)

Mansel, J./Neubauer, G. (Hrsg.): Armut und soziale Ungleichheit bei Kindern. Opladen 1998

Mantell, D. M.: Familie und Aggression. Frankfurt/M. 1972

Mead, M.: Der Konflikt der Generationen. Jugend ohne Vorbild. Freiburg 1973

Meier, U.: Gewalt im sozialökologischen Kontext der Schule. In: Holtappels/Heitmeyer/Melzer/Tillmann 1997, S. 225-242

Meier, U./Melzer, W./Schubarth, W./Tillmann, K.-J.: Schule, Jugend und Gewalt. Ergebnisse einer Schulleiterbefragung in Ost- und Westdeutschland. In: Zeitschrift für Sozialisationsforschung und Erziehungssoziologie H. 2/1995, S. 168-182

Meister, D./Sander, U.: Kindheit und Jugend in der Mediengesellschaft. In: Sozialwissenschaftliche Literaturrundschau H. 36/1998, S. 5-16

Melzer, W.: Familie und Schule als Lebenswelt. Zur Innovation von Schule durch Elternpartizipation. München 1987

Melzer, W.: Jugend und Politik in Deutschland. Gesellschaftliche Einstellungen, Zukunftsorientierungen und Rechtsextremismus-Potential Jugendlicher in Ost- und Westdeutschland. Opladen 1992

Melzer, W.: Ziel ist ein überzeugendes pädagogisches Profil. Entwicklung von Schulqualität. Ein Modellversuch in Sachsen. In: Deutsche Lehrerzeitung H. 37/38, 1997a, S. 22

Melzer, W.: Zweigliedrig oder wie? Erfahrungen in Ostdeutschland - Konsequenzen für die gesamte Bundesrepublik? In: Praxis Schule 5-10, H. 1/1997b, S. 57-59

Melzer, W.: Das Selbstbild von Opfern und Tätern. Ergebnisse einer Gewaltstudie in Sachsen. In: Praxis Schule 5-10, H. 1/1997c, S. 37-39

Melzer, W.: Schülerpartizipation: Ansprüche, Realität und Möglichkeiten einer Beteiligung von Schülern im Schulalltag. In: Güthoff, F./Sünker, H. (Hrsg.): Partizipation von Kindern - Demokratisierung von Gesellschaft. Münster 1998 (in Vorbereitung)

Melzer, W./Adomat, D.: Der Hauptschulbildungsgang in den neuen Bundesländern - Entwicklungen und Erfahrungen. In: Bronder, D. J./Ipfling, H. J./Rekus, J./ Zenke, K. (Hrsg.): Handbuch Hauptschulbildungsgang. Bad Heilbrunn 1998 (im Erscheinen) 
Melzer, W./Ferchhoff, W./Neubauer, G. (Hrsg.): Jugend in Israel und in der Bundesrepublik. Sozialisationsbedingungen im Kulturvergleich. Weinheim, München 1990

Melzer, W./Heitmeyer, W./Liegle, L./Zinnecker, J. (Hrsg.): Osteuropäische Jugend im Wandel. Ergebnisse vergleichender Jugendforschung in der Sowjetunion, Polen, Ungarn und der ehemaligen DDR. Weinheim, München 1991

Melzer, W./Hurrelmann, K.: Individualisierungspotentiale und Widersprüche in der schulischen Sozialisation von Jugendlichen. In: Heitmeyer/ Olk 1990, S. 35-59

Melzer, W./Lukowski, W./Schmidt, L.: Deutsch-polnischer Jugendreport. Lebenswelten im Kulturvergleich. Weinheim, München 1991

Melzer, W./Oelze, H./Priebe, B./Tillmann, K.-J./Werning, R. (Hrsg.): GewaltLösungen. Jahresheft Schüler. Seelze 1995

Melzer, W./Rostampour, P.: Schulische Gewaltformen und Täter-Opfer-Problematik. In: Schubarth/Kolbe/Willems 1996, S. 131-148

Melzer, W./Sandfuchs, U. (Hrsg.): Schulreform in der Mitte der 90er Jahre. Opladen 1996

Melzer, W./Schubarth, W./Tillmann, K.-J.: Gewalt in der Schule: zum Forschungsstand eines (wieder) aktuellen Themas. In: Schubarth/Melzer 1995, S. 15-38

Melzer, W./Stenke, D.: Schulentwicklung und Schulforschung in den ostdeutschen Bundesländern. In: Rolff u.a. 1996, S. 307-337

Melzer, W./Tillmann, K.-J.: Viele Sachbeschädigungen, kaum Erpressungen. Wie Schulleitungen in Sachsen und Hessen die Lage sehen. In: Melzer/Oelze/Priebe/ Tillmann/Werning 1995, S. 120-123

Metzen, H.: Schlankheitskur für den Staat: Lean Management in der öffentlichen Verwaltung. Frankfurt 1994

Meyer, B.: Offene Fragen zum Thema: Frauen und Rechtsextremismus. In: Feministische Studien H. 2/1993, S. 117-127

Miller, R.: Schulinterne Lehrerfortbildung. Der SCHiLF-Wegweiser. Weinheim, Basel 1995

Miller, R. (Hrsg.): Schule selbst gestalten. Bd. 1: Beziehung und Interaktion. Kopiervorlagen mit Informationen, Kommentaren und Aufgaben/ Anleitungen. Weinheim, Basel 1996

Mitterauer, M.: Sozialgeschichte der Jugend. Frankfurt/M. 1986

Möhle, N.: Erziehung zur Verantwortung. Verträge als Mittel pädagogischer Arbeit. In: Praxis Schule 5-10, H. 1/1997, S. 42-45

Mohr, A./Becker, P.: Strategien von Schülerinnen und Schülern im Umgang mit Gewalt in der Schule. In: Empirische Pädagogik H. 2/1997, S. 351-366

Mooij, T.: Schule und Sicherheit. Zusammenfassender Bericht zur Vorbereitung auf die EU-Konferenz vom 24. bis 26. Februar 1997 in Utrecht, Niederlande. Institut für angewandte Sozialwissenschaften/ Universität Nijmwegen 1997

Mutzeck, W.: Transferorientierte Evaluation. In: Greber/Maybaum/Priebe/ Wenzel 1993, S. 481-513

Nachtigall, Ch.: Selbstorganisation und Gewalt. Münster, New York 1998

Nave-Herz, R.: Pluralisierung familialer Lebensformen - ein Konstrukt der Wissenschaft? In: Vaskovics 1997, S. 36-49

Nolte, C.: Die Würde der Kinder. In: Sächsische Zeitung vom 25. September 1997

Oerter, R.: Jugendalter. In: Oerter, R./Montada, L.: Entwicklungspsychologie. München und Weinheim $1987^{2}$, S. 265-338 
Olweus, D.: Gewalt in der Schule. Was Lehrer und Eltern wissen sollten und tun können. Bern 1995

Olweus, D.: Täter-Opfer-Probleme in der Schule: Erkenntnisstand und Interventionsprogramm. In: Holtappels/Heitmeyer/Melzer/Tillmann 1997, S. 281-297

Oser, F./Althof, W.: Moralische Selbstbestimmung. Stuttgart $1994^{2}$

Osswald, E.: Organisationsentwicklung in Schulen - Ein Beitrag aus Basel. In: Greber/Maybaum/Priebe/Wenzel 1993, S. 310-333

Otto, H.-U./Merten, R. (Hrsg.): Rechtsradikale Gewalt im vereinigten Deutschland. Jugend im gesellschaftlichen Umbruch. Bonn 1993

Pädagogik und Ethik, hrsg. v. Beutler, K./Horster, D. Stuttgart 1996

Parke, R. D.: Erziehung durch den Vater. Stuttgart 1982

Parsons, T./Bales, R. F.: Family, socialization and interaction process. Glencoe (Ill.). 1955

Parsons, T.: Sozialstruktur und Persönlichkeit. Frankfurt/M. 1968

Paschen, H.: Schulautonomie und Erziehungswissenschaft. In: Melzer/Sandfuchs 1996, S. 197-207

Petermann, F. (Hrsg.): Pädagogische Supervision. Salzburg 1995

Petermann, F./Jugert, G./Tänzer, U./Verbeek, D.: Sozialtraining in der Schule. Materialien für die psychosoziale Praxis. Weinheim 1997

Petermann, F./Jugert, G./Verbeek, D./Tänzer, U.: Verhaltenstraining mit Kindern. In: Holtappels/Heitmeyer/Melzer/Tillmann 1997, S. 315-329

Petermann, F./Petermann, U.: Training mit sozial unsicheren Kindern. Einzeltraining, Kindergruppen, Elternberatung. Weinheim $1996^{6}$

Petermann, F./Petermann, U.: Training mit aggressiven Kindern. Einzeltraining, Kindergruppe, Elternberatung. Weinheim $1997^{8}$

Petillon, H.: Soziometrischer Test. (Reihe „Deutsche Schultests“). Weinheim 1980

Pfeiffer, Ch./Wetzels, P.: Kinder als Täter und Opfer. Eine Analyse auf der Basis der PKS und einer repräsentativen Opferbefragung. In: DVJJ-Journal H. 4/1997, S. 346-366

Popp, U.: Gewalt an Schulen - ein „Jungenphänomen“? In: Die Deutsche Schule H. 1/1997, S. 77-87

Posse, N.: Konzepte zur formativen Evaluation schulinterner Lehrerfortbildung. In: Greber/Maybaum/Priebe/Wenzel 1993, S. 457-480

Priebe, B.: Eine Herausforderung für alle. Gewaltprävention durch schulinterne Lehrerfortbildung. In: Praxis Schule 5-10, H. 5/1995, S. 10-16

Prüß, F./Bettmer, F.: Schule und Jugendhilfe? Neue Kooperationschancen im Osten? In: Flösser/Otto/Tillmann 1996, S. 238-252

Rammstedt, O.: Subjektivität und Sozialwissenschaften. In: Schülein, J. u.a.: Politische Psychologie. Frankfurt/M. 1981

Reinert, B./Zinnecker, J. (Hrsg.): Schüler im Schulbetrieb. Reinbek 1978

Reitmajer, V.: Pädagogischer Leitfaden zum Abbau ausgrenzender und abwertender (verbaler) Praxen bei Jugendlichen im Kontext von rechtsextremistischer Orientierung, Ausländer- und Minderheitenfeindlichkeit. München 1996

Reuband, K.-H.: Gesellschaftlicher Wandel, Kriminalität und Kriminalitätsfurcht. In: Neue Praxis H. 6/1996, S. 494-504

Richter, E./Sünker, H.: Jugendliche zwischen gesellschaftlichen Gewaltpotentialen und Verständigungsverhältnissen. In: Neue Praxis H. 3/1997, S. 221-236

Richter, H.-E. (Hrsg.): Russen und Deutsche. Hamburg 1990 
Rolff, H.-G.: Schule als soziale Organisation. Von der aktuellen Gestalt der Schule zum Modell der professionellen Vertrauensorganisation. In: Buchen, H./Horster, L./Rolff, H.-G.: Schulleitung und Schulentwicklung. Berlin 1995, S. 26-36

Rolff, H.-G.: Autonomie von Schule - Dezentrale Schulentwicklung und zentrale Steuerung. In: Melzer/Sandfuchs 1996, S. 209-227

Rolff, H.-G./Bauer, K.-O./Klemm, K./Pfeiffer, H. (Hrsg.): Jahrbuch der Schulentwicklung Bd. 9. Daten, Beispiele und Perspektiven. Weinheim, München 1996

Rostampour, P./Melzer, W.: Täter-Opfer-Typologien im schulischen Gewaltkontext. Forschungsergebnisse unter Verwendung von Cluster-Analyse und multinomialer logistischer Regression. In: Holtappels/Heitmeyer/Melzer/Tillmann 1997, S. $169-189$

Rostampour, P./Schubarth, W.: Gewaltphänomene und Gewaltakteure. Befunde aus einer Schülerbefragung in Sachsen. In: Empirische Pädagogik H. 2/1997, S. 135150

Scherer, D.: Gewalt in der Schule. Eine Studie in der Interregion Saarland - Lothringen - Luxemburg. Beiträge der Arbeitskammer des Saarlandes H. 1/1996

Schluchter, W.: Aspekte bürokratischer Herrschaft. Frankfurt/M. 1985

Schmidtchen, G.: Wie weit ist der Weg nach Deutschland? Sozialpsychologie der Jugend in der postsozialistischen Welt. Opladen 1997

Schorb, B./Mohn, E./Theunert, H.: Sozialisation durch (Massen-)Medien. In: Hurrelmann/Ulich 1991, S. 493-508

Schratz, M.: Durch Evaluation den Stand schulinterner Entwicklungsprozesse widerspiegeln. In: Buchen, H./Horster, L./Rolff, H.-G.: Schulleitung und Schulentwicklung. Berlin 1995, S. 116-120

Schröder, H./Melzer, W./Salustowicz, P.: Jugend in der Republik Polen, in Ost- und Westdeutschland. Strukturwandel, Kulturunterschiede und interkulturelle Kommunikation. DFG-Abschlußbericht. 1995

Schubarth, W.: Gewalt an Schulen als Medienereignis. In: Schubarth/Melzer 1995, S. $104-111$

Schubarth, W.: Je liberaler, desto mehr Gewalt an Schulen? In: Schubarth/ Kolbe/Willems 1996, S. 29-47

Schubarth, W.: Gewaltphänomene aus Sicht von Schülern und Lehrern. In: Die Deutsche Schule H. 1/1997, S. 63-76

Schubarth, W.: Von der Unterrichtsschule zum „Haus des Lernens“. Schule zwischen Offenheit und Halt. In: Rudolph, M./Wolf, B./Böhnisch, L. (Hrsg.): Lebensort Jugendarbeit. Eine Standortbestimmung der Jugendarbeit angesichts der Janusköpfigkeit postmoderner Sozialisation. Weinheim, München 1998a, S. 235-248

Schubarth, W.: Analyse und Prävention von Gewalt. Der Beitrag interdisziplinärer Forschung zur Gewaltprävention in Schule und Jugendhilfe (Habilitationsschrift). Dresden 1998b

Schubarth, W./Ackermann, Ch.: 45 Fragen und Projekte zur Gewaltprävention. Eine Handreichung für Lehrer, Sozialpädagogen und Eltern. Dresden 1997

Schubarth, W./Darge, K./Mühl, M./Ackermann, Ch.: Im Gewaltausmaß vereint? Erste Ergebnisse einer vergleichenden Schülerbefragung in Sachsen und Hessen. In: Holtappels/Heitmeyer/Melzer/Tillmann 1997, S.101-118

Schubarth, W./Darge, K./Mühl, M./Bergelt, S./Börner, F./Hannich, K. u. a.: Schulische Entstehungsbedingungen für Gewalt und pädagogische Handlungsmöglichkeiten. Eine vergleichende Fallstudie. Dresden 1997 (Forschungsbericht) 
Schubarth, W./Kolbe, F.-U./Willems, H. (Hrsg.): Gewalt an Schulen. Ausmaß, Bedingungen und Prävention. Opladen 1996

Schubarth, W./Melzer, W. (Hrsg.): Schule, Gewalt und Rechtsextremismus. Opladen 1993/2. Aufl. 1995

Schubarth, W./Melzer, W. unter Mitarbeit von Ackermann, Ch. und Stenke, D.: Gewalt an Schulen. Ergebnisse einer Schulleiterbefragung zum abweichenden Verhalten in Sachsen. (Forschungsbericht). Dresden 1994

Schubarth, W./Stenke, D.: Gewaltintervention und -prävention als Merkmale von Schulqualität: Zwei Schulbeispiele. In: Schubarth/Kolbe/Willems 1996, S. 173204

Schubarth, W./Stenke, D./Melzer, W.: Schule und Schüler-Sein unter neuen gesellschaftlichen Bedingungen. In: Flösser/Otto/Tillmann 1996, S. 101-117

Schultze, A.: Über Psychodrama zum Aggressionsabbau. In: Praxis Schule 5-10, H. 5/1995, S. 33-39

Schütze, F.: Emergenz. In: Fuchs, W. u. a. (Hrsg.): Lexikon der Soziologie. Opladen $1978^{2}$, S. 185

Schwarzer, C.: Beratung in der Schule. In: Weinert, F. E. (Hrsg.): Enzyklopädie der Psychologie. Psychologie des Unterrichts und der Schule. Göttingen 1997, S. 771804

Schwind, H.-D./Baumann, J. u. a. (Hrsg.): Ursachen, Prävention und Kontrolle von Gewalt. Analysen und Vorschläge der unabhängigen Gewaltkommission zur Verhinderung und Bekämpfung von Gewalt. Bd. IV. Berlin 1990

Schwind, H.-D. u.a. (Hrsg.): Gewalt in der Schule. Mainzer Schriften zur Situation von Kriminalitätsopfern. Mainz 1995

Schwind, H.-D./Roitsch, K./Ahlborn, W./Gielen, B.: Gewalt in der Schule - am Beispiel von Bochum. Schriftreihe des Weissen Rings, Bd. 10. Mainz 1995

Schwind, H.-D./Roitsch, K./Gielen, B.: Gewalt in der Schule aus der Perspektive unterschiedlicher Gruppen. In: Holtappels/Heitmeyer/Melzer/ Tillmann 1996, S. $81-100$

Seibert, N.: Warum und wozu brauchen wir die „Schulkultur“? In: Pädagogische Welt H. $10 / 1995$, S. 438-441

Seibert, N. (Hrsg.): Anspruch Schulkultur. Interdisziplinäre Darstellung eines neuzeitlichen schulpädagogischen Begriffs. Bad Heilbrunn 1997

Snijders, T./Bosker, R.: The Introduction to Multilevel Analysis. 1998 (in Vorbereitung)

v. Spaun, N.: Spurensuche der 'kleinen Gewalt' in der Schule (anhand der Auswertung alter Klassenbücher 1963; 1982 bis 1994/95). In: Empirische Pädagogik H. 2/1997, S. 209-216

Stark, W.: Prävention. In: Asanger, R./Wenninger, G.: Handwörterbuch der Psychologie. Weinheim $1992^{4}$, S. 563-567

Steffens, U./Bargel, T.: Erkundungen zur Qualität von Schule. Neuwied 1993

Stenke, D./Melzer, W.: Hat das Zwei-Säulen-Modell eine bildungspolitische Zukunft. In: Melzer/Sandfuchs 1996, S. 67-85

Stenke, D.: Das Grapschen lassen die sich doch gefallen. Annäherung und Abgrenzung in der Pubertät. In: Biermann, C./Etschenbach, K./Koch, F./ Nowak, C./Tillmann, K.-J. (Hrsg.): Schüler 1996 - Liebe und Sexualität. Seelze 1996, S. 52-55.

Stenke, D.: Mädchen, Gewalt und Rechtsextremismus. Kritische Anmerkungen zur Thematisierung des Geschlechterverhältnisses in der Auseinandersetzung mit 
Jugendgewalt. In: Mädchen mit oder gegen Gewalt. AgAG-Informationsdienst H. 3/1994, S. 19-29

Tennstädt, K.-Ch./Krause, F./Humpert, W./Dann, H.-D.: Das Konstanzer Trainingsmodell (KTM). Neue Wege im Schulalltag: Ein Selbsthilfeprogramm für zeitgemäßes Unterrichten und Erziehen. Einführung. Bern 19912

Tennstädt, K.-Ch./Krause, F./Humpert, W./Dann, H.-D.: Das Konstanzer Trainingsmodell (KTM). Neue Wege im Schulalltag: Ein Selbsthilfeprogramm für zeitgemäßes Unterrichten und Erziehen. Bd. 1: Trainingshandbuch. Bern $1994^{2}$

Terhart, E.: SchulKultur. Hintergründe, Formen und Implikationen eines schulpädagogischen Trends. In: Zeitschrift für Pädagogik. H. 5/1994, S. 685-699

Tillmann, K.-J.: Gewalt an Schulen. Öffentliche Diskussion und erziehungswissenschaftliche Forschung. In: Die Deutsche Schule H. 1/1997, S. 36-49

Tillmann, K.-J.: Gewalt an Schulen: Öffentliche Diskussion und erziehungswissenschaftliche Forschung. In: Holtappels/Heitmeyer/Melzer/Tillmann 1997, S. $11-25$

Tillmann, K.-J. u. a.: Gewalt in der Schule. Die Bielefelder Studie. Weinheim, München 1998 (in Vorbereitung)

Utzmann-Krombholz, H.: Rechtsextremismus und Gewalt: Affinitäten und Resistenzen von Mädchen und jungen Frauen. Düsseldorf 1994

Vaskovicz, L.: Veränderte Familien- und Lebensformen: Entscheidungsfeld und Optionen. In: Vaskovics/Lipinski 1996, S. 35-68

Vaskovics, L. (Hrsg.): Familienleitbilder und Familienrealitäten. Opladen 1997

Vaskovics, L./Lipinski, H. (Hrsg.): Familiale Lebenswelten und Bildungsarbeit Bd. 1. Opladen 1996

Vaskovics, L./Lipinski, H. (Hrsg.): Familiale Lebenswelten und Bildungsarbeit Bd. 2. Opladen 1997

Vester, M.: Klassengesellschaft ohne Klassen: Auflösung oder Transformation der Industriegesellschaft. In: Widersprüche H. 4/1997, S. 25-50

Vester, M. u.a.: Neue soziale Milieus und pluralistische Klassengesellschaft. Forschungsbericht Hannover 1992

Vobruba, G.: Jenseits der sozialen Fragen. Frankfurt/M. 1991

Wambach, M.: Prävention. In: Grubitzsch, S./Rexilius, G. (Hrsg.): Psychologische Grundbegriffe. Mensch und Gesellschaft in der Psychologie. Ein Handbuch. Reinbek 1990, S. 778-783

Weber, M.: Gesammelte Aufsätze zur Soziologie und Sozialpolitik. Tübingen 1924

Weishaupt, H./Zedler, P.: Aspekte der aktuellen Schulentwicklung in den neuen Ländern. In: Rolff, H.-G. u.a. (Hrsg.): Jahrbuch der Schulentwicklung. Bd. 8. Weinheim, München 1994, S. 395-429

Wiater, W.: Schulkultur - ein Integrationsbegriff für die Schulpädagogik? In: Seibert 1997, S. 21-43

Willems, H.: Fremdenfeindliche Gewalt. Opladen 1993

Würtz, St./Hamm, S./Willems, H./Eckert, R.: Gewalt und Fremdenfeindlichkeit in der Erfahrung von Schülern und Lehrern. In: Schubarth/Kolbe/ Willems 1996, S. $85-130$

Zapf, W.: Der Untergang der DDR und die soziologische Theorie der Modernisierung. In: Giesen/Leggewie 1991, S. 38-51

Zinnecker, J.: Jugendkultur 1940 - 1985. Opladen 1987 
Zinnecker, J.: Jugend als Bildungsmoratorium. Zur Theorie des Wandels der Jugendphase in west- und osteuropäischen Gesellschaften. In: Melzer/ Heitmeyer/Liegle/ Zinnecker 1991, S. 9-24

Zinnecker, J: Jugendforschung in Deutschland. Bilanz und Perspektiven. In: Edelstein, W./ Sturzbecher, D. (Hrsg.): Jugend in der Krise. Ohnmacht der Institutionen. Potsdam 1996, S. 189-207

Zinnecker, J./Molnar, P.: Lebensphase Jugend im historisch-interkulturellen Vergleich: Ungarn 1985, Westdeutschland 1954 - Westdeutschland 1984. In: Ferchhoff, W./ Olk, T. (Hrsg.): Jugend im internationalen Vergleich. Weinheim, München 1988, S. $181-206$

Züghart, E.: Disziplin-Konflikte in der Schule. Köln 1963 


\section{Anhang}

1. Schülerfragebogen

2. Fragebogen zur Schülerbeurteilung durch Lehrer

3. Soziometrischer Test

4. Fragebogen zum Gewaltbegriff 

Technische Universität Dresden

Fakultät Erziehungswissenschaften

Forschungsgruppe Schulevaluation

Leitung: Prof. Dr. Wolfgang Melzer

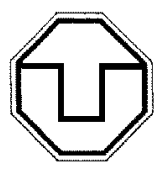

\section{Fragebogen \\ für Schülerinnen und Schüler \\ des 8., 9. und 10. Jahrgangs}

Liebe Schülerin, lieber Schüler,

wir sind eine Gruppe von Forscherinnen und Forschern, die sich mit der Schule beschäftigen. Wir möchten mit diesem Fragebogen einiges über Deine Schule und die Schülerinnen und Schüler erfahren.

Auf den folgenden Seiten befinden sich einige Aussagen und Fragen, die alle mit Deiner Schule zu tun haben. In den Fragen geht es um Deine persönliche Sicht und Meinung. Es gibt keine falschen oder richtigen Antworten. Wichtig ist allein Deine Meinung.

Die Beantwortung der Fragen ist freiwillig (und aus der Nichtbeantwortung können Dir keine Nachteile entstehen) und erfolgt selbstverständlich ohne Angabe Deines Namens. Wir bitten Dich um Deine Mithilfe, denn nur wenn sich viele Schülerinnen und Schüler beteiligen, können wir zu aussagekräftigen Ergebnissen gelangen.

Weder Deine Lehrerinnen und Lehrer noch Deine Eltern erhalten Einblick in Deinen Fragebogen. Die mit der Auswertung befaßten Forscherinnen und Forscher sind zu absoluter Vertraulichkeit verpflichtet und geben keine Angaben über Deine Person weiter. Die Auswertung der Fragebögen dient ausschließlich wissenschaftlichen Zwecken. Die Fragebögen werden nach der Auswertung vernichtet.

Herzlichen Dank für Deine Mitarbeit!

7. Dein Geschlecht:

männlich: $\square$ (1)

weiblich: $\square(2)$

8. Wie alt bist Du?

Jahre

10a. Bitte beantworte diese Frage nur dann, wenn Du ein/e Deutsche/r bist:

Wo bist Du geboren? 
$\square(1)$ in Ostdeutschland; $\square(2)$ in Westdeutschland; $\square(3)$ anderswo und zwar ...(10b.)

11a. Man sagt, Jugendliche stehen zwischen Kindsein und Erwachsenensein.

Fühlst Du Dich eher als Kind oder als Erwachsene/r?
$\square(1)$
(2)
(3)
(4)
(5)
(6) $\square(7)$
(8)
$\square(9) \square(10)$
Kind
Erwachsene/r

12. Ich lebe ...

$\square$ (1) mit beiden Eltern zusammen;

- (3) bei meiner Mutter und ihrem Partner;

$\square$ (5) bei meinem Vater und seiner Partnerin;

$\square(7)$ in einer Pflegefamilie;

$\square(2)$ bei meiner Mutter;

$\square(4)$ bei meinem Vater;

$\square(6)$ bei Verwandten;

$\square(8)$ woanders, und zwar

\begin{tabular}{|c|c|c|c|}
\hline $13 a$. & Hast Du Schwestern? & 13b. & Hast Du Brüder? \\
\hline$\square(1)$ & nein & $\square(1)$ & nein \\
\hline$\square(2)$ & ja, eine & $\square(2)$ & ja, einen \\
\hline$\square(3)$ & ja, zwei & $\square(3)$ & ja, zwei \\
\hline ل & ja, drei oder mehr & 口(4) & ja, drei oder mehr \\
\hline
\end{tabular}

14a. Wie sind Deine schulischen Pläne?

Ich will ...

$\square$ (1) ohne Abschluß die Schule verlassen; $\square(2)$ den Hauptschulabschluß, KI. 9;

口 (3) den qualifizierten Hauptschulabschluß (KI. 9 und Prüfung);

- (4) den Realschulabschluß, Kl. 10; $\quad \square(5)$ mittleren Abschluß an einer beruflichen Schule;

$\square(6)$ die Fachhochschulreife; $\square(7)$ das Abitur; $\quad \square(8)$ weiß nicht

15. Wie sicher oder unsicher bist Du Dir, daß Du den Schulabschluß bekommst, den Du haben willst?

(5) ganz sicher

$\square$ (4) ziemlich sicher

$\square$ (3) teils/teils

$\square$ (2) ziemlich unsicher

$\square$ (1) ganz unsicher

Sind in Deiner bisherigen Schulzeit schon folgende Situationen für Dich aufgetreten?

$$
\text { Skala: } 1=j a ; 2=\text { nein }
$$

16. Ich wäre beinahe sitzengeblieben.

17. Ich mußte einmal eine Klasse wiederholen.

18. Ich mußte zweimal eine Klasse wiederholen.

19. Ich habe wegen schlechter Schulnoten schon einmal die Schule gewechselt.

20.a Ich konnte wegen guter Schulnoten eine höhere Schulform besuchen. 
Welche Zensur hattest Du im letzten Zeugnis in folgenden Fächern?
21. Deutsch
(1) (2)
(3) (4)
(5) (6)
weiß nicht mehr
घ $\square$
]
$\square(8)$
22. Mathematik
(1) (2) (3) (4) (5) (6)
weiß nicht mehr
$\square(8)$
23. Englisch
(1) (2) (3) (4) (5) (6)
weiß nicht mehr
$\square(8)$

24. Manche haben mehrere Freunde/Freundinnen, andere haben nur eine/n Freund/in, andere sind viel allein. Was trifft bei Dir am ehesten zu?

$\square$ (1) Ich gehöre zu einer festen Gruppe von Freunden oder Freundinnen. Wir kennen uns untereinander gut und machen vieles gemeinsam. $\Rightarrow$ weiter bei Frage 25

$\square$ (2) Ich bin mit verschiedenen Leuten oder Gruppen locker befreundet. Alle zusammen machen wir aber nur selten etwas gemeinsam. $\Rightarrow$ weiter bei Frage 25

$\square$ (3) Ich habe eine Freundin/einen Freund. Wir sind oft zusammen. $\Rightarrow$ weiter bei Frage 33

$\square$ (4) Ich bin meistens allein. Einen festen Freundeskreis habe ich eigentlich nicht. $\Rightarrow$ weiter bei Frage 33

Falls Du einer Gruppe angehörst oder mit verschiedenen Leuten oder Gruppen befreundet bist, beantworte bitte die folgenden Fragen 25-32 auf dieser Seite.

Alle anderen machen bitte bei Frage 33 auf der nächsten Seite weiter.

25. Sind die Mitglieder Deiner Freundesgruppe aus Deiner Schule?

$\square(1) \quad j a$, alle; $\quad \square(2) \quad j a$, die meisten;

$\square(3)$ ja, nur einige; $\quad \square(4) \quad$ nein, niemand

26. Wie sieht Deine Freundesgruppe aus?

o(1) In unserer Gruppe sind nur Mädchen;

o(3) In unserer Gruppe sind Mädchen und Jungen

$o(2)$ In unserer Gruppe sind nur Jungen;

Im folgenden werden Aussagen zu Gruppen gemacht. Inwieweit stimmen die Aussagen auch für Deine Freundes-/Bekanntengruppe? Bitte kreuze jeweils nur eine der fünf Antwortvorgaben an!

Skala: 1 = stimmt gar nicht; 2 = stimmt überwiegend nicht; 3 = teils teils; 4 = stimmt überwiegend; 5 = stimmt ganz genau

27. Wer motzt oder kritisiert, wird in unserer Gruppe nicht geduldet.

28. Wenn wir Probleme mit anderen Gruppen haben, lösen wir die nicht mit Diskutieren.

29. Im letzten Jahr hat es mit anderen Gruppen Kämpfe, Prügeleien oder ähnliche Auseinandersetzungen gegeben.

30. Um die Ziele und Interessen unserer Gruppe durchzusetzen, pfeifen wir auch schon mal aufs Gesetz.

31. Wer nicht vollständig mit unserer Gruppe übereinstimmt, ist kein richtiges Gruppenmitglied.

32. In meiner Freundesgruppe fühle ich mich richtig wohl. 
33. Weißt Du manchmal nicht, was Du in Deiner Freizeit tun sollst?
$\square$ (1) häufig;
$\square$ (2) manchmal;
$\square$ (3) so gut wie nie

Wieviel Zeit verbringst Du durchschnittlich am Tag mit Fernsehen oder Video?

34a. werktags (Mo. - Fr.) .................... Stunden

34b. am Wochenende (Sa./So.) .............. Stunden

Bezogen auf die letzten 2 Wochen: Wie oft hast Du folgende Filmarten und Sendungen gesehen?

Skala: Omal; 1-2mal; 3-5mal; 6-10mal; mehr als 10mal

35. Krimis, Thriller, Western

36. Spielfilme, Serien

37. Actionfilme (z.B. Rambo, Karate)

38. Informationsfilme, Tierfilme

39. Horror- und Gewaltfilme (z.B. Zombies)

40. Unterhaltung, Musik, Quiz

41. Porno- und Sexfilme

42. Kinder-und Jugendfilme, Comics

43. Science Fiction (Zukunftsfilme, Weltall)

44. Verbieten Dir Deine Eltern, bestimmte Fernseh-/Videofilme oder Serien zu schauen?

\begin{tabular}{|c|c|c|c|c|}
\hline & $\square(1)$ & ja; & $\square(2)$ & nein \\
\hline Wenn ja, schaust Du trotz Verbot? & 口(1) & ja; & $\square(2)$ & nein \\
\hline
\end{tabular}

Welche der folgenden Fernsehprogramme könnt Ihr bei Euch zu Hause empfangen?

$\begin{array}{llll}\text { 45a. } & \text { ARD } & \square(1) \mathrm{ja} & \square(2) \text { nein } \\ \text { 45b. } & \text { ZDF } & \square(1) \mathrm{ja} & \square(2) \text { nein } \\ 45 \mathrm{c} . & \text { MDR } & \square(1) \mathrm{ja} & \square(2) \text { nein } \\ 45 \mathrm{~d} . & \text { SAT1 } & \square(1) \mathrm{ja} & \square(2) \text { nein } \\ 45 \mathrm{e} . & \text { RTL-plus } & \square(1) \mathrm{ja} & \square(2) \text { nein } \\ 45 f . & \text { Pro 7 } & \square(1) \mathrm{ja} & \square(2) \text { nein }\end{array}$

Welche der folgenden elektronischen Geräte gibt es bei Euch zu Hause?

46. Videogerät: 47. Homecomputer/PC: $\square(1)$ ja $\square(2)$ nein : $\square(1)$ ja $\square(2)$ nein
Welche der folgenden elektronischen Geräte hast Du selbst?

48. Fernsehgerät:

$\square(1)$ ja $\square(2)$ nein

49. Videogerät:

$\square(1)$ ja $\square(2)$ nein

50. Home Computer/PC: $\square(1)$ ja $\square(2)$ nein

51. Telespiel, Gameboy: $\square(1)$ ja $\square(2)$ nein 
Treffen die folgenden Aussagen auch für Dich zu?

Bitte kreuze jeweils eine der fünf Antwortvorgaben jeweils für Deine Mutter und für Deinen Vater an. Wenn Du nicht bei Deiner Mutter oder Deinem Vater wohnst, brauchst Du die entsprechenden Fragen nicht zu beantworten.

Skala: 1 = stimmt gar nicht ... 5 = stimmt ganz genau

52a. Wenn ich was falsch gemacht oder angestellt habe, redet meine Mutter eher mit mir, als daß sie mich straft.

52b. Wenn ich was falsch gemacht oder angestellt habe, redet mein Vater eher mit mir, als daß er mich straft.

53a. Bei uns zuhause schreit und schimpft meine Mutter oft.

53b. Bei uns zuhause schreit und schimpft mein Vater oft.

54a. Meine Mutter ist gar nicht streng.

54b. Mein Vater ist gar nicht streng.

55. Wenn in meiner Familie etwas wichtiges entschieden werden soll, dann darf ich auch mitbestimmen.

56a. Zuhause hat es für mich schon so manches Mal Prügel gegeben (seitens meiner Mutter).

56b. Zuhause hat es für mich schon so manches Mal Prügel gegeben (seitens meines Vaters).

57a. Meine Mutter erlaubt mir viel weniger als andere in meinem Alter dürfen.

57b. Mein Vater erlaubt mir viel weniger als andere in meinem Alter dürfen.

58a. Wenn ich mal Probleme habe, ist meine Mutter für mich da.

58b. Wenn ich mal Probleme habe, ist mein Vater für mich da.

59a. Die hohen Erwartungen meiner Mutter an meine Schulleistungen setzen mich unter Druck.

59b. Die hohen Erwartungen meines Vaters an meine Schulleistungen setzen mich unter Druck.

60a. Meine Mutter schreibt mir sehr stark vor, was ich zu tun habe.

60b. Mein Vater schreibt mir sehr stark vor, was ich zu tun habe.

61a. Meiner Mutter ist es egal, was ich mache.

61b. Meinem Vater ist es egal, was ich mache.

62a. Zuhause bei meiner Mutter fühle ich mich sehr wohl.

62b. Zuhause bei meinem Vater fühle ich mich sehr wohl.

63a. Meine Kinder würde ich so erziehen, wie ich von meiner Mutter erzogen wurde.

63b. Meine Kinder würde ich so erziehen, wie ich von meinem Vater erzogen wurde.

Im folgenden werden Aussagen über die Schule gemacht. Überlege bei jeder Aussage, inwieweit sie auch für Deine Schule zutrifft. Bitte kreuze bei jeder Aussage jeweils nur eine der 5 Antwortmöglichkeiten an! Wähle die aus, die am ehesten zutrifft.

Skala: 1 = stimmt gar nicht ... 5 = stimmt ganz genau

64. Mit dem, was wir im Unterricht lernen, kann ich außerhalb der Schule zur Zeit wenig anfangen.

65. Im Unterricht kann ich vieles einbringen, was ich außerhalb der Schule mache.

66. Der Unterrichtsstoff interessiert mich meistens sehr.

67. Bei einem großen Teil des Unterrichtsstoffs weiß ich nicht, welchen Sinn das haben soll.

68. Das Lernen in der Schule hat oft nichts mit der Wirklichkeit zu tun.

69. Die meisten unserer Lehrer/innen können gut erklären.

70. Der Unterricht wird von den meisten Lehrern/innen abwechslungsreich gestaltet.

71. Die Lehrer/innen lassen uns im Unterricht oft selbständig arbeiten. 
Im folgenden werden Aussagen über die Schule gemacht. Überlege bei jeder Aussage, inwieweit sie auch für Deine Schule zutrifft. Bitte kreuze bei jeder Aussage jeweils nur eine der 5 Antwortmöglichkeiten an! Wähle die aus, die am ehesten zutrifft.

$$
\text { Skala: } 1 \text { = stimmt gar nicht ... } 5 \text { = stimmt ganz genau }
$$

72. Die meisten Lehrer/innen machen den Unterricht nicht anschaulich, so daß man keinen guten Durchblick bekommt.

73. Im Unterricht gehen die Lehrer/innen oft viel zu schnell vor, man kommt gar nicht mit.

74. In den meisten Unterrichtsstunden kommt bei den Schülern/innen Langeweile auf.

75. Auf Vorschläge der Schüler/innen zur Durchführung des Unterrichts gehen unsere Lehrer/ innen meistens nicht ein.

76. Filme oder Computer kommen in unserem Unterricht häufig zum Einsatz.

77. Im Unterricht haben wir neben unseren Schulbüchern eine Menge an hilfreichem Lern- und Übungsmaterial.

78. In den normalen Unterrichtsstunden haben die Lehrer/innen kaum Zeit, sich mit den Fragen und Problemen von einzelnen Schülern zu befassen.

79. Die Lehrer/innen geben manchmal einzelnen Schülern unterschiedlich schwierige Aufgaben zu bearbeiten. Das hängt davon ab, wie gut jeder den Stoff beherrscht.

80. Wenn einzelne Schüler/innen beim Lernen Probleme haben, geben ihnen die Lehrer/innen meistens Tips und Ratschläge, wie sie am besten weiterkommen.

81. Die meisten Lehrer/innen achten bei den Aufgaben darauf, daß niemand in der Klasse überfordert wird.

Im folgenden sind verschiedene Angebote und Veranstaltungen aufgeführt. Bitte gib jedesmal an, ob es diese an Deiner Schule gibt und wenn ja, wie zufrieden Du damit bist!

Skala: $1=$ bin sehr unzufrieden ... $4=$ bin sehr zufrieden; $5=$ gibt's an unserer Schule nicht

82. Klassenfahrten/Schullandheimaufenthalte

83. Schulfeste/Klassenfeste

84. Schulaufführungen (z.B. Theater, Tanz, Musik, Schulchor)

85. Arbeitsgemeinschaften, Wahlkurse, Hobbykurse

86. Projektwochen

87. Hausaufgabenhilfe/Förderstunden („Arbeitsstunden“)

88. Freizeitmöglichkeiten in Schulräumen (z.B. Spiele, Film, Disco)

89. Räume zum Treffen und Reden (z.B. Teestube, Schülercafè)

90. Aufenthaltsräume zum Zurückziehen (z.B. Sitzecken)

91. Verkauf von Frühstücksverpflegung 
Im folgenden werden einige persönliche Aussagen gemacht. Überlege bei jeder Aussage, inwieweit sie auch für Dich zutrifft. Bitte kreuze bei jeder Aussage jeweils nur eine der 5 Antwortmöglichkeiten an! Wähle die aus, die am ehesten zutrifft.

$$
\text { Skala: } 1 \text { = stimmt gar nicht ... } 5 \text { = stimmt ganz genau }
$$

92. Im großen und ganzen bin ich mit mir zufrieden.

92a. Ich bin mit meinem Aussehen zufrieden.

93. Wenn ich von einer Lehrperson ungerecht behandelt werde, fühle ich mich zu hilflos, um mich dagegen zu wehren.

94. Schon der Gedanke an die Schule macht mich oft mißmutig.

95. Ich wünsche mir oft, eine andere/ein anderer zu sein.

96. Ich kann mich so gut benehmen wie ich will, man glaubt immer nur Schlechtes von mir.

97. Auch wenn ich nichts ausgefressen habe, hat man mich immer gleich in Verdacht.

98. Ich gehe sehr gern zur Schule.

99. Ich fühle mich oft unglücklich.

100. Ich habe das Gefühl, die Lehrer/innen behalten ich dauernd im Auge.

101. Ich glaube fast, ich bin immer dabei, wenn Strafen verteilt werden.

102. In der Schule habe ich oft das Gefühl, daß ich weniger zustande bringe als andere.

103. Ich glaube, ich habe eine Reihe guter Eigenschaften.

104. Schon bevor ich mit einer Aufgabe anfange, weiß ich, daß ich sie nicht gut mache.

105. Ich fühle mich leicht angegriffen.

106. Ich habe das Gefühl, Lehrer/innen und andere Schüler behandeln mich wie einen Außenseiter.

107. Ich habe Grund, auf mich stolz zu sein.

108. Ich steigere mich leicht in einen Streit hinein.

109. In der Schule bin ich meist gut gelaunt.

110. Manchmal komme ich mir wirklich nutzlos vor.

111. Ich muß mich nur anstrengen, dann schaffe ich schon die Aufgaben, die mir in der Schule gestellt werden.

112. Ich habe den Eindruck, hinter meinem Rücken reden Lehrer/innen und andere Schüler schlecht über mich.

113. Ich glaube, die Lehrer/innen haben mich schon aufgegeben.

114. Ich gerate schnell in Wut.

115. Ich habe manchmal Angst vor Gewalt in der Schule durch Mitschüler/innen (z.B. Prügeleien).

116. Aus Angst vor Gewalt nehme ich eine Waffe (z.B. Messer) mit in die Schule.

117. Ich kann mir selbst helfen, wenn eine Aufgabe schwierig ist.

118. Ich wollte, ich könnte von mir eine bessere Meinung haben.

119. Ich glaube, niemand versteht mich so recht.

120. Es gibt in der Schule eigentlich viele Dinge, die mir Spaß machen.

121. Es bringt mich zum Kochen, wenn andere sich über mich lustig machen.

122. Das meiste, was man in der Schule lernt, kann man später doch nicht gebrauchen.

123. Wenn ich Streit mit anderen Schüler/innen bekomme, kann ich dies meist durch Reden klären.

124. Im großen und ganzen halte ich mich für erfolgreich.

125. Ich bin froh, wenn ich nicht mehr zur Schule gehen muß.

126. Ich habe das Gefühl, viele in der Schule meiden den Kontakt mit mir. 
Im folgenden werden einige persönliche Aussagen gemacht. Überlege bei jeder Aussage, inwieweit sie auch für Dich zutrifft. Bitte kreuze bei jeder Aussage jeweils nur eine der 5 Antwortmöglichkeiten an! Wähle die aus, die am ehesten zutrifft.

$$
\text { Skala: } 1 \text { = stimmt gar nicht ... } 5 \text { = stimmt ganz genau }
$$

127. Ich könnte meine Zeit besser außerhalb der Schule nutzen.

128. Bei Streit mit anderen raste ich richtig aus und habe mich nicht mehr unter Kontrolle.

129. Ich glaube, die meisten Lehrer/innen und Schüler/innen betrachten mich als Störenfried.

Im folgenden zählen wir Handlungen auf, die bei Schülerinnen und Schülern manchmal vorkommen. Wie oft hast Du selber in den letzten 12 Monaten beobachtet, daß andere Schüler/innen an Deiner Schule folgendes getan haben? Bitte kreuze jeweils eine der Antwortvorgaben an:

Skala: $1=$ nie; 2 = seltener; $3=$ alle paar Monate; $4=$ mehrmals im Monat;

$5=$ mehrmals wöchentlich; 6 = fast täglich

130. Einrichtungsgegenstände wurden absichtlich beschädigt.

131. Sachen, die anderen Schülern gehören, wurden absichtlich beschädigt.

132. Wände wurden absichtlich beschmiert oder besprüht.

133. Unterrichtsmaterial wurde absichtlich beschädigt.

134. Toiletteneinrichtungen wurden absichtlich beschädigt.

135. So etwas habe ich fast nur bei Jungen $\quad \square ; \quad$ bei Jungen und Mädchen beobachtet: fast nur bei Mädchen 135a. So etwas habe ich auch bei meinen engsten Freunden oder $\quad \square_{(1)}$ ja $\quad \square_{(2)}$ nein
Freundinnen beobachtet:

Wie oft hast Du selber in den letzten 12 Monaten an Deiner Schule oder auf dem Schulweg folgendes beobachtet ? Bitte kreuze jeweils eine der Antwortvorgaben an:

$$
\text { Skala: } 1=\text { nie } \ldots .6 \text { = fast täglich }
$$

136. Spaßkampf zwischen zwei Jungen.

137. Ernsthafte Prügelei zwischen zwei Jungen.

138. Spaßkampf zwischen zwei Mädchen.

139. Ernsthafte Prügelei zwischen zwei Mädchen.

140. Prügelei zwischen einem Mädchen und einem Jungen.

141. Zwei Gruppen von Schülern (Jungen oder Mädchen) prügeln sich.

142. Eine größere Zahl von Schüler/innen feuert zwei an, die sich prügeln.

143. Jemand wird von einem/einer anderen mit einer Waffe (Schlagring, Messer usw.) angegriffen.

144. Ein oder mehrere Jungen bedrängen ein Mädchen und fassen es gegen ihren Willen an (z.B. an den Busen).

145. Ein Junge wird von einem oder mehreren Mädchen bedrängt und gegen seinen Willen angefaßt (z.B. zwischen den Beinen). 
Kommt es an Deiner Schule vor, daß Schüler/innen sich in folgender Weise gegenüber Lehrer/innen verhalten?

$$
\text { Skala: } 1=\text { nie } \ldots \text {. } 6 \text { fast täglich }
$$

146. Schüler/innen beschimpfen oder beleidigen Lehrpersonen in deren Gegenwart.

147. "Telefonterror" gegen Lehrpersonen.

148. So etwas habe ich fast nur bei Jungen $\square$; bei Jungen und Mädchen $\square$; beobachtet: fast nur bei Mädchen

148a. So etwas habe ich auch bei meinen engsten Freunden oder $\square_{(1)}$ ja $\quad \square_{(2)}$ nein Freundinnen beobachtet:

149. Schüler/innen bedrohen Lehrer/innen (z.B. mit Schlägen).

150. Schüler/innen schlagen absichtlich eine Lehrperson.

151. Schüler/innen entwenden oder beschädigen absichtlich Sachen, die einer Lehrperson gehören.

152. So etwas habe ich fast nur bei Jungen $\quad \square$; bei Jungen und Mädchen $\square$; beobachtet: fast nur bei Mädchen

152a. So etwas habe ich auch bei meinen engsten Freunden oder $\square_{(1)}$ ja $\square_{(2)}$ nein Freundinnen beobachtet.

Nur für Jungen zu beantworten

Skala: 1 = stimmt gar nicht ... 5 = stimmt ganz genau

153. Ich schlage mich besonders gerne, wenn Mädchen dabei zuschauen.

154. Meistens gerate ich mit einem anderen Jungen wegen Mädchen in Streit.

155. Die Mädchen nehmen die schwachen und hilflosen Jungen gerne in Schutz.

\section{Wieder für alle!}

Wie oft hast Du selbst folgende Verhaltensweisen zwischen Schülerinnen und Schülern in den letzten 12 Monaten an Deiner Schule beobachtet?

$$
\text { Skala: } 1=\text { nie } \ldots 6 \text { = fast täglich }
$$

156. Spotten/Auslachen

157. Beschimpfen/Gemeine Ausdrücke

158. Gemeine Gesten/Zeichen

158a. Daß jemand von anderen ausgeschlossen (geschnitten) wird.

159. So etwas habe ich fast nur bei Jungen $\square$; bei Jungen und Mädchen $\square$; beobachtet: fast nur bei Mädchen

159a. So etwas habe ich auch bei meinen engsten Freunden oder $\square_{\text {(1) }}$ ja $\square_{\text {(2) }}$ nein Freundinnen beobachtet:

160. Ausländerfeindliche Sprüche

161. So etwas habe ich fast nur bei Jungen $\quad \square$; bei Jungen und Mädchen $\quad \square$; beobachtet: fast nur bei Mädchen

161a. So etwas habe ich auch bei meinen engsten Freunden oder $\square_{(1)}$ ja $\quad \square_{(2)}$ nein Freundinnen beobachtet: 
Hast Du davon gehört - oder hast Du selbst beobachtet - , daß in den letzten 12 Monaten folgende Ereignisse an Deiner Schule vorgekommen sind?

$$
\text { Skala: } 1=\text { nie } \ldots \text {. } 6 \text { = fast täglich }
$$

162. Jemand fordert Geld und droht mit Strafe (z.B. Prügel), wenn nicht gezahlt wird.

163. Jemand fordert, Sachen abzugeben ( $z$ B. eine schöne Jacke) und droht mit Strafe (z.B. Prügel), wenn nicht abgegeben wird.

164. Jemand droht mit Strafe (z.B. Prügel), falls dem Lehrer etwas mitgeteilt wird.

165. Wenn so etwas an Eurer Schule vorgekommen ist, von wem gingen die Erpressungen aus?

fast nur von Jungen von Jungen und Mädchen fast nur von Mädchen

165a. So etwas habe ich auch bei meinen engsten Freunden oder Freundinnen beobachtet:

Im folgenden werden Aussagen über die Schule gemacht. Überlege bei jeder Aussage, inwieweit sie auch für Deine Schule zutrifft. Bitte kreuze bei jeder Aussage jeweils nur eine der 5 Antwortmöglichkeiten an! Wähle die aus, die am ehesten zutrifft.

$$
\text { Skala: } 1 \text { = stimmt gar nicht ... } 5 \text { = stimmt ganz genau }
$$

166. Ich finde, man muß sich kaum anstrengen, um in unserer Schule mitzukommen.

167. In unserer Schule kann man sich kaum leisten, krank zu werden, da man sonst den Anschluß verpaßt.

168. Mir macht es nicht viel aus, daß man laufend Klassenarbeiten schreiben muß.

169. Mir macht es zu schaffen, daß man dauernd damit rechnen muß, im Unterricht abgeprüft zu werden.

170. Oft schafft man es gar nicht, für alle Fächer zu lernen und auch die Hausaufgaben zu machen.

171. Unsere Lehrer/innen beurteilen uns und unsere Schulleistungen äußerst gerecht.

172. Bei etwas Anstrengung hat in unserer Schule jeder eine gute Chance durchzukommen.

173. Ich kann lernen, soviel ich will: Das, was die Schule verlangt, kann ich gar nicht schaffen.

174. Auf Schüler/innen, die beim Lernen nicht mitkommen, wird in unserer Schule wenig Rücksicht genommen.

175. Den meisten Lehrern/innen ist es gleichgültig, ob wir etwas lernen.

176. Die meisten Lehrer/innen strengen sich an, um lernschwächere Schüler/innen zu fördern.

177. Bei uns setzen sich Lehrer/innen für Schüler/innen ein, wenn sie mal in Schwierigkeiten geraten.

178. Die meisten Lehrer/innen kümmern sich nicht um persönliche Probleme der Schüler/innen.

179. Unsere Lehrer/innen sind daran interessiert, daß alle Schüler/innen beim Lernen Fortschritte machen.

180. Wenn bei uns jemand etwas falsch gemacht oder etwas angestellt hat, dann stellen ihn die Lehrer/innen als schlecht hin.

181. Bei uns kommt es nur selten vor, daß uns Lehrer/innen anschreien.

182. Bei uns gibt es Lehrer/innen, die gegen Schüler/innen auch schon mal handgreiflich werden (z.B. schlagen).

183. Bei uns würde nie ein Lehrer/eine Lehrerin zugeben, daß er/sie sich einmal geirrt hat.

184. Die meisten Lehrer/innen reden eher mit uns, als daß sie uns strafen.

185. Es gibt Lehrer/innen, die einen vor der ganzen Klasse blamieren. 
In folgenden Ausagen werden Meinungen vertreten. Wie stehst Du dazu?

Skala: 1 = stimmt gar nicht ... 5 = stimmt ganz genau

186. Gewalt gehört selbstverständlich zur menschlichen Natur.

186a. Gewalt ist geil.

186b. Um seine Interessen druchzusetzen, muß man manchmal Gewalt anwenden.

187. Der Stärkere muß sich durchsetzen, sonst gibt es keinen Fortschritt.

188. Gewalt ist etwas völlig normales, weil sie überall vorkommt.

189. Es ist völlig normal, wenn Männer sich im körperlichen Kampf mit anderen selbst beweisen wollen.

190. Auge um Auge, Zahn um Zahn, so ist nun einmal das Leben.

Nur für Mädchen zu beantworten

Skala: 1 = stimmt gar nicht ... 5 = stimmt ganz genau

191. Wenn sich Jungen prügeln, schaue ich gerne zu.

192. Wenn sich zwei Jungen meinetwegen schlagen, fühle ich mich geschmeichelt.

193. Ich habe oft Mitleid mit Jungen, die sich nicht so richtig wehren können.

\section{Wieder für alle! \\ Auf den folgenden Seiten werden vier Situationen vorgestellt, zu denen Du Stellung beziehen sollst.}

1. Situation:

Ein Junge/ein Mädchen tritt jemanden in böser Absicht.

3. Situation:

Zwei prügeln sich so stark, daß es Verletzungen (z.B. blutige Nasen, blaue Augen) gibt.
2. Situation:

Ein Junge/ein Mädchen fordert Geld von jemandem und droht ihm/ihr Strafe an, wenn er/ sie nicht bezahlt.

4. Situation:

Ein Junge bedrängt ein Mädchen und faßt es gegen ihren Willen an den Busen oder zwischen die Beine.

Skala: 1 = nie; 2 = selten; 3 = manchmal; 4 = oft; 5 = sehr oft

194./204./214./224. So etwas habe ich in meiner Klasse in den letzten 12 Monaten beobachtet.

195./205./215./225. So etwas habe ich außerhalb meiner Klasse in unserer Schule (vor und nach dem Unterricht, in der Pause) in den letzten 12 Monaten beobachtet.

196./206./216./226. Wenn unsere Lehrer/innen solches Verhalten beobachten, dann greifen sie ein und versuchen dieses Verhalten zu beenden.

197./207./217./227. So etwas habe ich in den letzten 12 Monaten selbst getan.

Skala: 1 = stimmt gar nicht $\ldots .5$ = stimmt ganz genau

Wenn ich so etwas beobachte, dann ...

198./208./218./228. sehe ich das als ganz normal an; ich denke mir nichts dabei.

199./209./219./229. empfinde ich Angst.

200./210./220./230. ärgere ich mich.

201./211./221./231. reizt es mich (ich würde am liebsten das Gleiche machen).

202./212./222./232. wünsche ich mir, daß sich Schüler nicht so verhalten.

203./213./223./233. mische ich mich ein und versuche, das Verhalten zu beenden. 
Im folgenden werden Aussagen über die Schule gemacht. Überlege bei jeder Aussage, inwieweit sie auch für Deine Schule zutrifft. Bitte kreuze bei jeder Aussage jeweils nur eine der 5 Antwortmöglichkeiten an! Wähle die aus, die am ehesten zutrifft.

$$
\text { Skala: } 1 \text { = stimmt gar nicht ... 5 = stimmt ganz genau }
$$

234. Wer sich bei unseren Lehrer/innen nicht an die Regeln hält, riskiert zur Strafe auch noch schlechte Noten.

235. In unserer Schule haben die Schüler/innen kaum Einfluß auf die entscheidenden Dinge.

236. Unsere Lehrer/innen sind bereit, mit uns zu diskutieren, wenn uns etwas nicht gefällt.

237. Die Lehrer/innen fragen uns häufig nach unserer Meinung, wenn etwas entschieden oder geplant werden soll.

238. Es nützt meistens nichts, sich in der Schule über sinnlose Regeln und Verbote zu beschweren.

239. Die meisten Lehrer/innen bemühen sich, uns auch persönlich kennenzulernen.

240. Ich würde mich trauen, mit einigen von unseren Lehrer/innen auch persönliche Probleme zu besprechen.

241. Man wird an dieser Schule von den meisten Lehrer/innen ernst genommen.

242. Mit einigen Lehrer/innen reden wir häufig auch außerhalb des Unterrichts über Privates.

243. Die meisten Lehrer/innen versuchen, auf die Eigenarten und Probleme einzelner Schüler/innen einzugehen.

244. Wir kommen mit unseren Lehrer/innen gut aus.

245. Die Lehrer/innen hier behandeln nicht alle Schüler/innen gerecht.

246. In unserer Klasse gibt es eine ganze Reihe von Schüler/innen, die bei den anderen wenig Beachtung finden.

247. In unserer Klasse ist es für alle Schüler/innen einfach, Anschluß und Kontakt zu bekommen.

248. Bei uns wird man leicht zum Außenseiter, wenn man nicht tut, was die Klasse für richtig hält.

249. Wenn es einmal drauf ankommt, halten die Schüler/innen unserer Klasse prima zusammen.

250. In unserer Schule gibt es verschiedene Gruppen von Schüler/innen, zwischen denen eine richtige Feindschaft besteht.

251. Die meisten Schüler/innen verstehen sich richtig gut miteinander.

252. Auch wenn wir in der Klasse mal richtig Streit haben, werden die Konflikte rasch und gut gelöst.

253. Unter den Schüler/innen meiner Klasse fühle ich mich richtig wohl.

254. Die Schüler/innen meiner Klasse sind mir völlig gleichgültig.

255. In meiner Klasse habe ich überhaupt keine guten Freund/innen.

256. Ich werde von den Schüler/innen meiner Klasse anerkannt.

257. In unserer Klasse sieht jeder nur auf seinen eigenen Vorteil, wenn es um die Noten geht.

258. Viele Schüler/innen sind hier manchmal neidisch, wenn ein anderer bessere Leistungen hat als sie.

259. In unserer Klasse versucht unter den Schüler/innen jeder besser zu sein als der andere. 
Wie oft hast Du selbst an Deiner Schule oder auf dem Schulweg in den letzten 12 Monaten folgendes gemacht?

$$
\text { Skala: } 1 \text { = stimmt gar nicht ... } 5 \text { = stimmt ganz genau }
$$

260. Andere im Unterricht geärgert, beworfen oder beschossen.

261. Einen Lehrer oder eine Lehrerin geärgert oder provoziert.

262. Andere gehänselt oder mich über sie lustig gemacht.

263. Im Schulgebäude etwas absichtlich beschädigt.

264. Mich mit einem/einer anderen geprügelt.

265. Mit anderen einen Jungen/ein Mädchen verprügelt.

266. Sachen von anderen absichtlich kaputtgemacht.

267. Andere unter Druck gesetzt.

268. Anderen auf dem Schulweg aufgelauert, sie belästigt, bedroht oder verprügelt.

269. Den Unterricht erheblich gestört.

270. Bei Klassenarbeiten erheblich gemogelt.

271. Die Schule ohne Entschuldigung geschwänzt.

272. Waffen (Schreckschußpistole, Reizgas) mit in die Schule gebracht.

273. Mit anderen bewußt Streit angefangen, sie angeschrien, beschimpft.

274. Andere mit Sachen (zum Beispiel Lineal, Mäppchen) beworfen.

275. Sachen absichtlich zerstört, die der Schule gehören (z.B. Stühle, Bücher).

276. Anderen Sachen versteckt, so daß sie sie nicht wiederfinden konnten.

277. Den Unterricht absichtlich gestört.

278. Andere mit gemeinen Ausdrücken beschimpft.

279. Anderen etwas gewaltsam weggenommen.

279a. Alkohol getrunken.

279b. Zigaretten geraucht.

279c. Drogen genommen (z.B. Haschisch).

Wie oft bist Du selbst an Deiner Schule oder auf dem Schulweg in den letzten 12 Monaten...

$$
\text { Skala: } 1 \text { = nie } \ldots .6 \text { = fast täglich }
$$

280. von anderen geschlagen worden?

281. von anderen gehänselt oder geärgert worden?

282. auf dem Schulweg belästigt, bedroht worden?

283. von anderen angeschrien, beschimpft, beleidigt worden?

284. von anderen unter Druck gesetzt, erpreßt worden?

Wie oft wurde(n) Dir an Deiner Schule oder auf dem Schulweg in den letzten 12 Monaten...

285. von anderen Sachen absichtlich kaputtgemacht?

286. von anderen gewaltsam etwas weggenommen?

287. von anderen Sachen versteckt, so daß Du sie nicht wiederfinden konntest?

288a. Welchen Schulabschluß hat Deine Mutter 289a. Welchen Schulabschluß hat Dein Vater erreicht? erreicht?

$\square$ (1) Sie hat keinen Abschluß.

- (2) Abschluß 8. Klasse POS

口 (3) Abschluß 10. Klasse POS

$\square$ (4) Hochschulreife, Abitur $\square$ (1) Er hat keinen Abschluß.

(2) Abschluß 8. Klasse POS

$\square$ (3) Abschluß 10. Klasse POS

$\checkmark$ (4) Hochschulreife, Abitur 


\begin{tabular}{|c|c|c|c|}
\hline 290a. & Was Ist Deine Mutter von Beruf? & $291 a$. & Was Ist Dein Vater von Beruf? \\
\hline$\square(1)$ & ganztags berufstätig als ... & $\square(1)$ & ganztags berufstätig als ... \\
\hline$\square(2)$ & halbtags berufstätig als ... & $\square(2)$ & halbtags berufstätig als ... \\
\hline$\square(3)$ & $\begin{array}{l}\text { stundenweise/gelegentlich berufstätig } \\
\text { als ... }\end{array}$ & ](3) & $\begin{array}{l}\text { stundenweise/gelegentlich berufstätig } \\
\text { als ... }\end{array}$ \\
\hline$\square(4)$ & zur Zeit arbeitslos; zuletzt berufstätig & 口) (4) & zur Zeit arbeitslos; zuletzt berufstätig als .. \\
\hline & als ... & $\square(5)$ & nicht berufstätig (Hausmann, Rentner) \\
\hline $\begin{array}{l}(5) \\
(6)\end{array}$ & $\begin{array}{l}\text { nicht berufstätig (Hausfrau, Rentnerin) } \\
\text { in Ausbildung }\end{array}$ & $\square(6)$ & in Ausbildung \\
\hline
\end{tabular}

293a. Wieviel Taschengeld hast Du zur Deiner freien Verfügung?

..... DM im Monat

Skala: 1 = viel schlechter $\ldots 5=$ viel besser

293b. Wie ist die finanzielle Situation Deiner Familie im Vergleich mit den anderen?

293c. Viele Leute verwenden die Begriffe LINKS und RECHTS, wenn es darum geht, unterschiedliche politische Einstellungen zu kennzeichnen. Wenn Du an Deine eigenen politischen Ansichten denkst, wo würdest Du Dich einstufen?

$$
\begin{aligned}
& \begin{array}{lllllllllllll}
\text { LINKS } & \square & \square & \square & \square & \square & \square & \square & \square & \square & \square & \square & \text { RECHTS } \\
& 5 & 4 & 3 & 2 & 1 & 0 & 1 & 2 & 3 & 4 & 5 &
\end{array} \\
& \square \text { Kann ich nicht sagen, habe noch nie darüber nachgedacht. }
\end{aligned}
$$

Hast Du selbst in den letzten 12 Monaten folgendes getan?

$$
\text { Skala: } 1=\text { keinmal; } 2 \text { = einmal; } 3=2-4 \text { mal; } 4 \text { = über } 4 \text { mal }
$$

294. Dich mit anderen gerauft, geschlagen.

295. Schlägereien mitgemacht und dabei jemanden zusammengeschlagen oder arg zugerichtet.

296. Einer Bande (Gruppe, Club, Clique) angehört, in der manche unerlaubten "Dinge gedreht" werden.

297. Anderen Gegenstände oder Geld im Wert von mehr als 10 DM entwendet.

298. Irgendwo eingebrochen (z.B. in ein Gebäude, ein Auto usw.), um etwas zu stehlen.

299. Einen Automaten aufgebrochen oder "gefilzt ${ }^{\mu}$.

300. Fremdes (auch öffentliches) Eigentum mit Absicht zerstört oder erheblich beschädigt. 
In folgenden Aussagen werden Meinungen vertreten. Wie stehst Du dazu?

Skala: 1 = stimmt gar nicht ... 5 = stimmt ganz genau

301. Das Wichtigste in der heutigen Zeit ist die Aufrechterhaltung von Ruhe und Ordnung.

302. Unser Volk sollte wieder einen Führer haben, der mit starker Hand regiert.

303. Die Deutschen waren schon immer die Größten in der Geschichte.

304. Es ist höchste Zeit, daß die Jugend wieder Disziplin lernt.

305. In jeder Gesellschaft gibt es Konflikte, die nur mit Gewalt gelöst werden können.

306. Wer sich in Deutschland nicht anpassen kann, soll das Land am besten verlassen.

307. Ich bin durchaus bereit, Fremde schlechter zu behandeln, damit sie wissen, wo hier ihre Grenzen sind.

307a. Nur für die Deutschen: Ich bin stolz, ein/e Deutsche/r zu sein.

Im folgenden werden Aussagen über die Schule gemacht. Überlege bei jeder Aussage, inwieweit sie auch für Deine Schule zutrifft. Bitte kreuze bei jeder Aussage jeweils nur eine der 5 Antwortmöglichkeiten an! Wähle die aus, die am ehesten zutrifft.

$$
\text { Skala: } 1 \text { = stimmt gar nicht ... } 5 \text { = stimmt ganz genau }
$$

308. Die meisten Schultage erlebe ich von der Zeit her als sehr gehetzt.

309. Wir haben in den einzelnen Unterrichtsstunden meistens genügend Zeit und Ruhe für das Lernen.

310. An den meisten Schultagen gibt es zuwenig Zeit für persönliche Gespräche unter Mitschülern.

311. Unser Schulgelände ist langweilig. Man kann wenig Interessantes unternehmen.

312. Unser Klassenraum ist irgendwie gemütlich und einladend gestaltet.

313. An unserer Schule geht manches drunter und drüber: Vieles ist seit langem schmutzig oder kaputt.

314. Unser Schulgebäude ist innen so schön gestaltet, daß sich die meisten Schüler/innen gerne darin aufhalten.

315. In unserer Schule gibt es gemütliche Ecken, wo man seine Ruhe hat und sich zurückziehen kann.

Im folgenden werden einige persönliche Aussagen gemacht. Überlege bei jeder Aussage, inwieweit sie auch für Dich zutrifft. Bitte kreuze bei jeder Aussage jeweils nur eine der 5 Antwortmöglichkeiten an! Wähle die aus, die am ehesten zutrifft.

$$
\text { Skala: } 1 \text { = stimmt gar nicht ... } 5 \text { = stimmt ganz genau }
$$

316. Ich werde nervös, wenn ich an die Tafel gerufen werde, unabhängig davon, ob ich vorbereitet bin oder nicht.

317. Ich bekomme Herzklopfen, wenn ein Lehrer/eine Lehrerin mich abfragen will.

318. Aus Angst, etwas Falsches zu sagen, beteilige ich mich selten am Unterricht.

319. Es kommt häufig vor, daß ich am Anfang einer Prüfung keinen klaren Gedanken fassen kann.

320. Ich habe Angst vor Klassenarbeiten.

321. Am Morgen vor einer Prüfung kann ich nichts essen.

Vielen Dank für Deine Mitarbeit ! 
Technische Universität Dresden

Fakultät Erziehungswissenschaften

Forschungsgruppe Schulevaluation

Leitung: Prof. Dr. Wolfgang Melzer

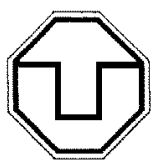

\section{Klassenbeurteilung}

Im folgenden bitten wir Sie um eine Einschätzung Ihrer Schüler hinsichtlich einiger Merkmale sozialen Verhaltens. Tragen Sie dazu die Codenummern der Schüler Ihrer Klasse in die vorgegebenen Schemata ein (jeweils ein Schüler pro Kästchen). Gehen Sie so vor, daß Sie die Schüler entsprechend ihrer Merkmalsausprägung eintragen: Schüler, auf die die Beschreibung in sehr hohem Maße zutrifft, oben; Schüler, für die das Gegenteil gilt, unten; Schüler mit durchschnittlicher Merkmalsausprägung in der Mitte.

Fangen Sie am besten mit den Extremen an und berücksichtigen Sie alle Schüler. Eventuell überzählige Kästchen lassen Sie frei.

Verwenden Sie bitte für eventuelle Korrekturen einen Bleistift.

Diese Befragung bezieht sich auf die Klasse

Wieviele Stunden unterrichten Sie pro Woche in dieser Klasse?

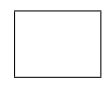

Wieviele Schuljahre - das laufende mitgerechnet unterrichten Sie bereits in dieser Klasse?
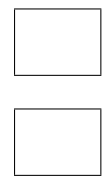
1. Wie verhalten sich die Schüler dieser Klasse: wie egoistisch bzw. gemeinschaftsorientiert (solidarisch) sind sie?

egoistisch

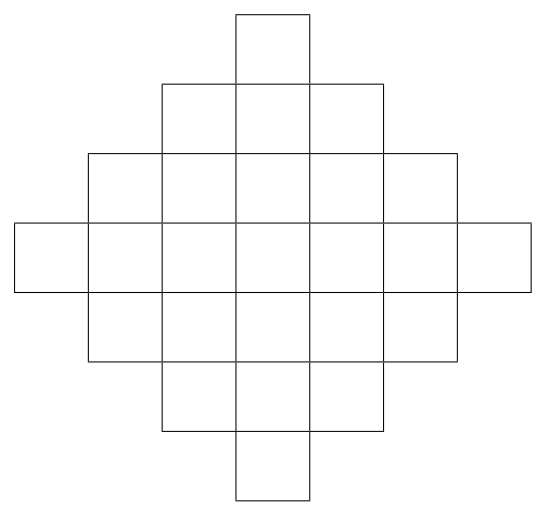

gemeinschaftsorientiert (solidarisch)

2. Wie ist das Temperament der einzelnen Schüler: sind sie impulsiv und unbeherrscht oder sehr ruhig und ausgeglichen?

hoch impulsiv und unbeherrscht

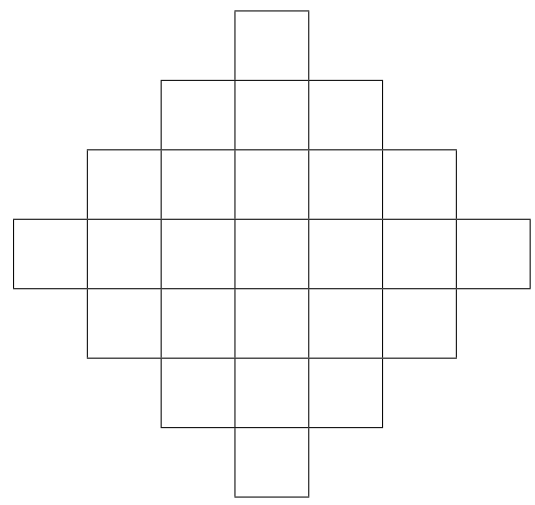

sehr ruhig und ausgeglichen

3. Welches Auftreten und welches Durchsetzungsvermögen haben die Schüler in der Klasse: sind sie eher dominant oder orientieren sie sich eher an anderen? 
dominant

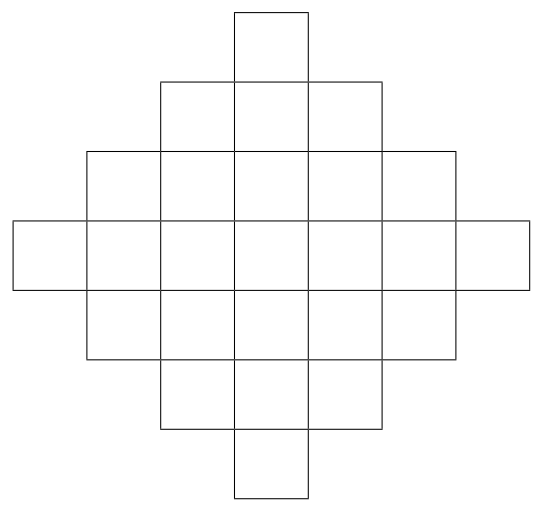

sich unterordnend

4. Welche Stellung nehmen die einzelnen Schüler in der Klasse ein: sind sie eher integriert oder eher Außenseiter?

integriert

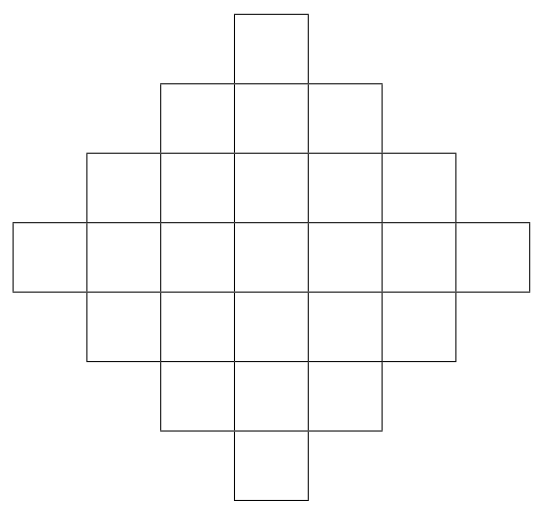

Außenseiter

5. Wie beurteilen Sie die Beteiligung Ihrer Schüler an schulischer Gewalt (Prügelei, Zerstörung von Sachen, Beschimpfung und Beleidigung anderer Schüler usw.)? 
- Wer tritt als Täter in Erscheinung?

- Wer ist „Opfer“ solcher Handlungen?

- Wer ist sowohl Opfer als auch Täter?

- Wer ist unbeteiligt?

$$
\text { nur Täter nur Opfer Täter/Opfer unbeteiligt }
$$
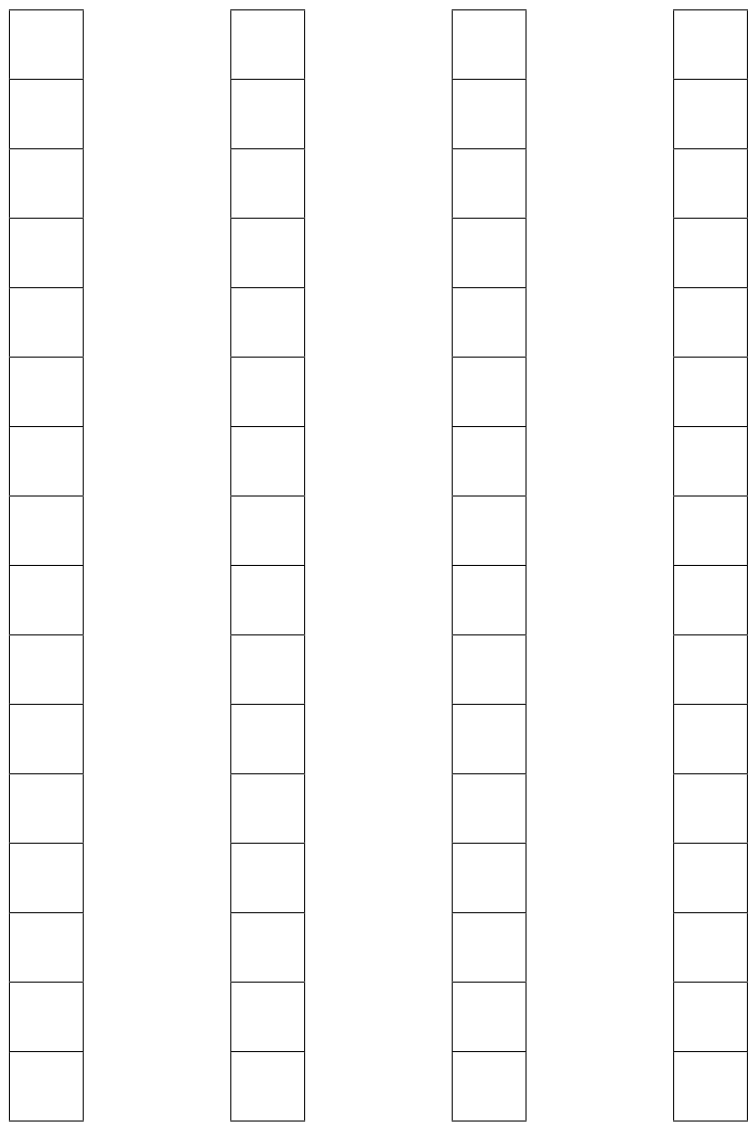

Vielen Dank für Ihre Mitarbeit! 
Technische Universität Dresden

Fakultät Erziehungswissenschaften

Forschungsgruppe Schulevaluation

Leitung: Prof. Dr. Wolfgang Melzer

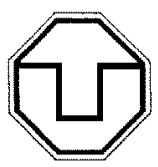

\section{Liebe Schülerinnen und Schüler,}

wir möchten Euch heute einige Fragen zu Euch selbst und zu Eurer Schulklasse stellen. Dabei geht es darum, wie es Euch in der Klasse gefällt, mit wem Ihr gerne zusammen seid, wen Ihr nicht so gut findet und einiges mehr. Ihr habt dafür die ganze Schulstunde Zeit.

Lies Dir bitte jede Frage genau durch und schreibe das hin, was Dir als erstes dazu einfällt. Du brauchst nicht lange zu überlegen. Es gibt keine „richtigen“ und ,falschen“ Antworten. Wir wollen einfach Deine Meinung wissen.

Bitte nenne keine Namen, weder Deinen eigenen noch andere, damit die Befragung anonym bleibt. Es werden nur die Code-Nummern eingetragen, die auf der Klassenliste stehen, die Du vorne siehst.

Es geht los!

Meine Code-Nummer ist (Bitte eintragen!)

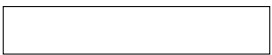

Ich bin $\square$ Jahre

1. Ich fühle mich in meiner Klasse (Zutreffendes bitte ankreuzen):
$(++)$
$(+)$
( 0 )
$(-)$
$(--)$
sehr wohl
teils/teils
sehr unwohl

2. Ich gehe gern zur Schule (Zutreffendes bitte ankreuzen):
$(++)$
$(+)$
( 0 )
$(-)$
$(--)$
sehr gern
teils/teils
sehr ungern

3. Mit wem hast Du in Deiner Klasse am meisten zu tun?

a.) Bitte nenne die Mitschüler und Mitschülerinnen, mit denen Du am häufigsten sprichst und zusammen bist, trage ihre Code-Nummer in die Kästchen ein! 
Wenn es weniger als fünf sind, läßt Du die restlichen Kästchen einfach frei.

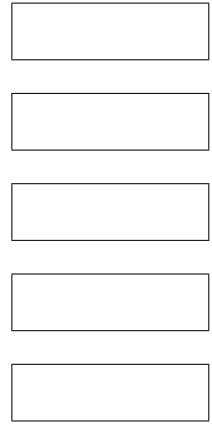

b) Schreibe hinter jede Zahl, was Du mit dieser Person zu tun hast, was Ihr zusammen macht. Denke dabei an die Schule, den Schulweg und auch an Deine Freizeit!

c) Wieviel Freunde und Bekannte hast Du in Deiner Klasse, in der Schule, außerhalb der Schule? Trage die Anzahl der Freunde in die Kästchen ein. Wenn Du dort keine Freunde hast, kreuze das an!

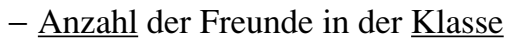

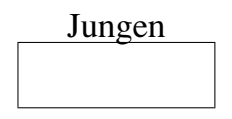

\section{Mädchen}

- Anzahl der Freunde in anderen Klassen meiner Schule

- $\underline{\text { Anzahl der Freunde außerhalb }}$ meiner Schule
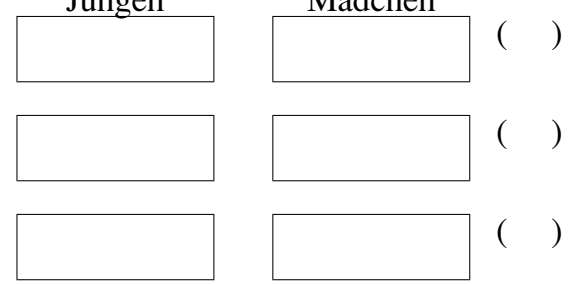

4. Wen magst Du in Deiner Klasse am liebsten?

a) Trage nicht die Namen, sondern wieder die Code-Nummern für diese Personen in die Kästchen ein! Du kannst bis zu 5 Personen nennen.
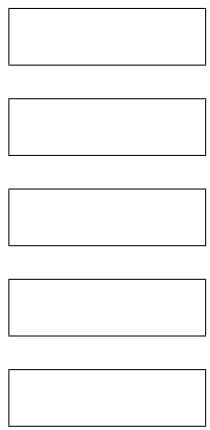
b) Schreibe jetzt hinter die Kästchen, was Du an der jeweiligen Person gut findest!

5. Schreibe jetzt die Code-Nummern der Mitschüler/innen auf, die Du gar nicht magst; und dahinter wieder, warum! Wenn es weniger sind als fünf, läßt Du die restlichen Kästchen einfach frei.

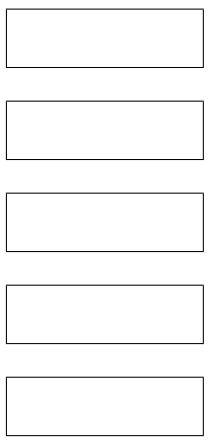

6. Bitte schreibe jetzt auf, wer von Deinen Mitschülern Dich oder andere Klassenkameraden häufiger beleidigt hat. Trage die Code-Nummern für die betreffenden Personen in die Kästchen ein!
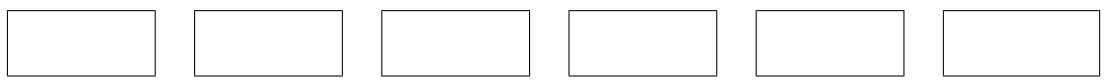

7. Bitte schreibe auf, wer von Deinen Mitschülern Dich oder andere Klassenkameraden absichtlich getreten, geschlagen oder sonst irgendwie körperlich angegriffen hat.
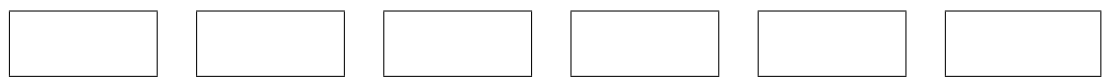

8. Bitte schreibe nun auf, wer von Deinen Mitschülern Dich häufiger beleidigt hat (mit Gesten oder Worten).
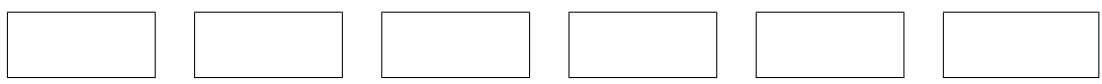

9. Bitte schreibe ebenfalls auf, wer von Deinen Mitschülern Dich absichtlich körperlich angegriffen hat (getreten, geschlagen etc.).
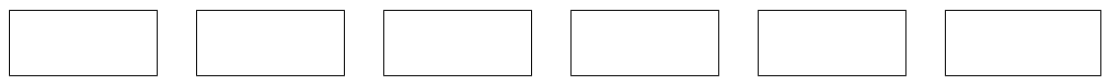
Falls Du zu den einzelnen Fragen und dem Thema insgesamt noch etwas aufschreiben möchtest, kannst Du das nachfolgend tun.

Vielen Dank für Deine Mitarbeit! 
Technische Universität Dresden

Fakultät Erziehungswissenschaften

Forschungsgruppe Schulevaluation

Leitung: Prof. Dr. Wolfgang Melzer

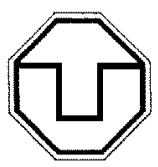

\section{Einschätzung von Situationen im Schulalltag}

Die folgenden Situationen haben Schülerinnen und Schüler geschildert; die Namen wurden jedoch geändert!

Bitte kreuzen Sie an, ob es sich Ihrer Meinung nach bei den Beispielen um „Gewalt“ handelt oder nicht?

Wenn Christian nach der Pause in das Klassenzimmer zurückkommt, liegt der Inhalt seiner Tasche oft verstreut am Boden

Johannes wird von den meisten Schülern gehänselt, weil er keine Markenklamotten trägt.

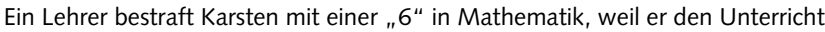
gestört hat.

Seit einigen Wochen besucht ein vietnamesischer Junge die Klasse. Jeden Morgen, wenn er die Klasse betritt, rufen die anderen: „Fidschi“

Thomas und Philipp halten Claudia in einer Ecke fest und versuchen, sie zu küssen, obwohl sie sich wehrt und nicht will.

Die Mitglieder zweier Cliquen von Jungen prügeln sich fast täglich während der großen Pause auf dem Schulhof.

Paul ritzt mit dem Zirkel für seine Freundin eine Nachricht in die Schulbank.

Stefan hält den 2 Jahre jüngeren David die Faust unter die Nase und sagt zu ihm: Morgen will ich Geld sehen, sonst passiert etwas! ”

Eine Lehrerin sagt zu Michael, nachdem er mehrmals falsch geantwortet hat: "Bei Dir ist doch Hopfen und Malz verloren!“

Eine Lehrerin wird während des Unterrichts von den Schülern so geärgert, daß sie weinend das Klassenzimmer verläßt.

Einige Jugendliche schlitzen im Bus die Sitze mit Rasierklingen auf.

\begin{tabular}{|l|l|}
\hline Gewalt & $\begin{array}{c}\text { keine } \\
\text { Gewalt }\end{array}$ \\
\hline & \\
\hline & \\
\hline & \\
\hline & \\
\hline & \\
\hline & \\
\hline & \\
\hline & \\
\hline & \\
\hline & \\
\hline & \\
\hline & \\
\hline & \\
\hline & \\
\hline & \\
\hline & \\
\hline & \\
\hline & \\
\hline & \\
\hline
\end{tabular}


Mitschüler der kleinen Angela, die seit kurzem eine Brille trägt, sagen nur noch "Brillenschlange" zu ihr.

Steffen und Robert sperren Bernd in der Toilette ein und verbarrikadieren die Tür so, daß er nicht pünktlich zur Stunde kommt.

Eine Lehrerin meint zu Marko, der die Klassenarbeit verhauen hat: „Du bis die größte Oberpflaume, die mir jemals untergekommen ist. "

Eine Clique zersticht die Autoreifen eines Lehrers, weil sie ihn nicht leiden können.

Martin u. Sven quatschen während des Unterrichts. Nachdem der Lehrer sie einmal ermahnt hat, geht er zu Sven, reißt ihn vom Stuhl hoch u. schüttelt ihn kräftig durch.

Falk tritt mit voller Wucht gegen die Tür des Klassenzimmers, so daß sie zersplittert.

Dirk und Kai bedrängen Katja auf dem Schulweg und versuchen, ihr den Pullover hochzuziehen.

Eine Lehrerin ist bekannt für ihre Gutmütigkeit. Die Schülerinnen und Schüler nutzen das aus und verlassen einfach den Raum.

Marie und Beate schubsen ihre Mitschülerin Susanne die Schultreppe hinunter, weil diese ihnen nicht die Hausaufgaben überlassen will.

Im Streit schmeißt Lutz die Federmappe seines Freundes Frank auf den Boden und trampelt darauf herum.

Auf dem Weg nach Hause verstellen Matthias und Christoph ihrem Mitschüler Peter den Weg, holen ein Messer raus und sagen: „Los, rück die Jacke raus! ”

Eine Lehrerin stellt Schüler vor die Tür, wenn diese quatschen.
Ich bin
Schülerin
Lehrerin
Schulform:
Mittelschule
Schüler
Lehrer
Gymnasium

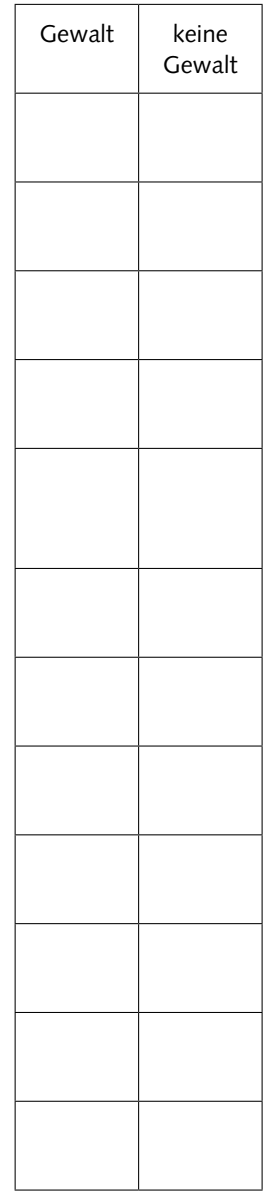

Vielen Dank für Ihre Mitarbeit! 